\title{
Zwischen Sorgerecht und Unterhaltspflicht.
}

\section{Determinanten väterlichen Sorgehandelns in Nachtrennungs- familien an den Beispielen Deutschland, Großbritannien und Norwegen.}

Dissertation zur Erlangung des sozialwissenschaftlichen Doktorgrades der Sozialwissenschaftlichen Fakultät der Georg-August-Universität Göttingen

Vorgelegt von

Carina Marten aus Oldenburg (Oldenb.)

Göttingen, März 2008 
1. Gutachter:

2. Gutachterin:

Tag der mündlichen Prüfung:
Prof. Dr. Steffen-Matthias Kühnel

Prof. Dr. Ilona Ostner

17.04.2008 


\section{Zusammenfassung}

Männer als Väter befinden sich in einer inkonsistenten Situation. Zum einen haben soziale Veränderungen zu einer Neu-Definition der männlichen Rolle innerhalb der Familie geführt. Väter werden nicht länger ausschließlich als Ernährer konzeptionalisiert, darüber hinaus wird von ihnen ein „neues“ intensives Engagement in der Betreuung und Erziehung ihrer Kinder erwartet und gewünscht. Gleichzeitig gewinnt aufgrund zunehmender Trennungen bzw. Scheidungen von Eltern eine Familienform an Bedeutung, die durch die Abwesenheit des biologischen Vaters gekennzeichnet ist. Beide Entwicklungen - die wachsenden sozio-emotionalen Anforderungen an Väter sowie ihre zunehmende Abwesenheit innerhalb des familialen Haushalts aufgrund der elterlichen Trennung - stehen sich konträr gegenüber. Untersuchungen zu Nachtrennungsvätern zeigen unterschiedliche individuelle Strategien mit dieser Widersprüchlichkeit umzugehen. Nachtrennungsväter, ihre Wahrnehmung und Ausgestaltung der Sorge um ihre getrennt lebenden Kinder in Form von Care und Cash sind Gegenstand der vorliegenden Arbeit. Es stellt sich die Frage, nach der qualitativen und quantitativen Ausgestaltung des sozio-emotionalen Kontakts sowie der finanziellen Unterstützung. Welche Determinanten bestimmen das väterliche Engagement nach der elterlichen Trennung? Zur Beantwortung der forschungsleitenden Frage werden Trennungsväter in verschiedenen Kontexten in den Blick genommen. Die Untersuchung basiert auf der Sekundäranalyse dreier quantitativer Datensätze, die unabhängig voneinander in Deutschland, Großbritannien und Norwegen entstanden sind. Nach dem Prinzip "parallel demonstration of theory" werden zunächst basierend auf einem Rational Choice-Modell Hypothesen über mögliche Einflussfaktoren väterlichen Sorgehandelns entwickelt, die in einem zweiten Schritt anhand der nationalen Studien überprüft werden. Die Ergebnisse deuten auf eine recht unterschiedliche Ausgestaltung väterlichen Sorgehandelns - Care wie Cash - in deutschen, britischen und norwegischen Nachtrennungsfamilien hin. Das jeweilige Niveau wird durch z.T. andere Determinanten bestimmt. Insgesamt erweist sich für das sozio-emotionale Sorgen in Form von Care das Verhältnis der Eltern vor, während und insbesondere nach der Trennung als relevant. Darüber hinaus wird Care wesentlich durch die Entfernung zwischen den elterlichen Wohnorten sowie der jeweiligen Beziehungssituation der getrennt lebenden Elternteile bestimmt. Die Ergebnisse für Cash zeigen eine stärkere länderspezifische Heterogenität. Die Zahlungspraxis deutscher Väter wird wesentlich durch ihre ökonomische Situation sowie den Aushandlungsprozess des Unterhalts beeinflusst. In Großbritannien und Norwegen stellt sich die Erklärung der Cash-Variablen deutlich komplexer dar. Hier erweisen sich darüber hinaus Faktoren, wie z.B. das freundschaftliche Verhältnis der Eltern nach der Trennung im britischen Fall oder die Form des Umgangs im norwegischen Fall, als erklärungsrelevant. 


\section{Abstract}

Nowadays, some fathers find themselves in an inconsistent situation. On the one hand, demographic changes have caused a re-definition of men's role within families who are no longer exclusively considered as providers. From a 'new' kind of 'active' father a family orientated, child centred attitude, a higher allocation of time towards and a more intense contact with their children is expected as well as wanted. Simultaneously, the emerging social change - inter alia expressed in increasing divorce rates - leads to an augmenting number of families characterised by absent fathers due to parental break-up. Thus, both developments - increasing expectations towards and from fathers as well as decreasing time spend with their children within one household - create an inconsistent situation for absent fathers. Studies on non-resident fathering show different individual strategies to cope with this contradiction. Despite a growing debate about post-separation families, so far, little is known about the actual non-resident fathering and its variation. The following research questions are yet to be answered: Which form and extent has fathering in terms of care and cash within post-separated families from fathers' own perspective? How - based on which economical, socio-demographic and institutional determinants - can the variations in non-resident fathering be explained? Answering the research question the focus lies on non-resident fathers in different contexts. The secondary analysis of three independently developed studies from Germany, Great Britain and Norway follows the principle of "parallel demonstration of theory". That is, from a Rational Choice-approach different hypotheses about determinants of non-resident fathering are generally derived. These are tested on the empirical material of each nation. The research findings show a rather different form and extend of fathering for German, British and Norwegian separated fathers. This holds true for the care as well as the cash dimension. Each model of explanation shows varying determinants. Overall, the social-emotional fathering depends highly on the relationship between the parents before, during and after separation. Further, the fathers' caring is determined by the distance between the parents' home and the parents' new partnership situation. The cash aspects cannot as easily be explained by the applied theoretical model. Findings have to be characterized as rather nationally heterogeneous. German fathers' paying moral is based on their economical situation as well as by negotiation processes of maintenance. In Great Britain and Norway the explanation of maintenance practices is more complex. Other determinants proof relevance like the amicable relationship between the separated parents in the British case or the extent of contact agreed on between Norwegian parents. 


\section{Inhaltsverzeichnis}

Zusammenfassung ....................................................................................... II

Abstract ........................................................................................................... III

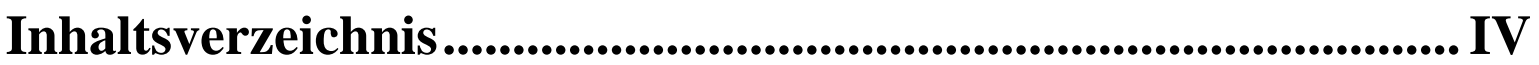

Tabellenverzeichnis ...................................................................... VIII

Abbildungsverzeichnis ......................................................................

\section{TEIL I: PROBLEMBEZOGENER HINTERGRUND}

1 Fragestellung und methodischer Aufbau der Arbeit...............1

2 Das Konzept väterlichen Sorgehandelns in Nachtrennungsfamilien .....................................................................7

2.1 Väterliches Sorgehandeln in Form von Care und Cash ..................................... 7

2.2 Nachtrennungsfamilien: das Phänomen abwesender Väter ............................... 13

3 Die sozialwissenschaftliche Väterforschung - Eine kurze

Bestandsaufnahme ...................................................................16

3.1 (Nachtrennungs-)Väter in der wissenschaftlichen Literatur............................... 16

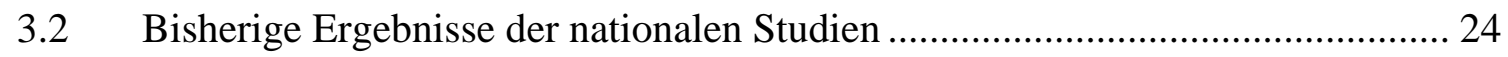

3.2.1 Zusammenfassung der Ergebnisse der Forsa-Studie................................. 25

3.2.2 Zusammenfassung der Ergebnisse der Bradshaw-Studie............................ 27

3.2.3 Zusammenfassung der Ergebnisse der NOVA-Studie ............................... 28

3.3 Einordnung der Fragestellung in die aktuelle Diskussion................................. 31 


\section{TEIL II: THEORETISCHER RAHMEN}

\section{Die Handlungstheorie ......................................................35}

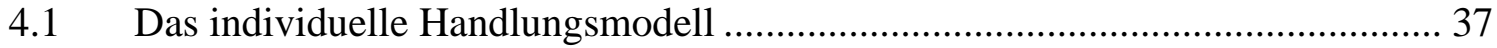

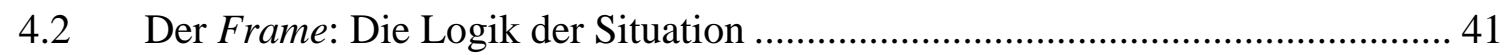

4.2.1 Innere Bedingungen: Das Überzeugungssystem...................................... 46

4.2.2 Äußere Bedingungen: Der soziale Kontext............................................. 48

4.2.2.1 Die physische Umwelt: Ressourcen und Restriktionen ........................ 49

4.2.2.2 Die mikro-soziale Umwelt: Beziehungen und Einflussnahmen ............ 52

4.3 Die Handlungsauswahl: Die Logik der Selektion .............................................. 54

4.3.1 Rational Choice und väterliches Sorgehandeln.......................................... 54

4.3.1.1 Sorgehandeln als Entscheidung zwischen Alternativen......................... 55

4.3.1.2 Handlungskonsequenzen als Kosten und Nutzen .............................. 57

4.3.1.3 Die Präferenzordnung als Bewertungen und Erwartungen.................... 61

4.3.1.4 Die Handlungswahl als Nutzenmaximierung...................................... 63

4.3.2 Familiales Handeln als rationale Abwägung? - Eine kritische Betrachtung 64

4.3.2.1 Familiales Handeln als soziales Handeln ............................................ 65

4.3.2.2 Familiales Handeln als norm-orientiertes Handeln.............................. 67

4.3.2.3 Familiales Handeln als emotionales Handeln .................................... 74

4.3.2.4 Familiales Handeln als rationales Handeln ...................................... 77

4.3.2.5 Zwischenfazit I: Das Rationalitätsverständnis ...................................... 79

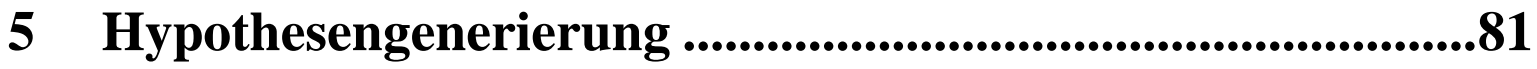

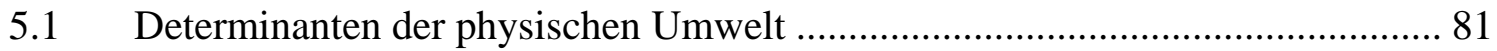

5.2 Determinanten der mikro-sozialen Umwelt ..................................................... 85

5.3 Determinanten innerhalb des Überzeugungssystems ....................................... 92 


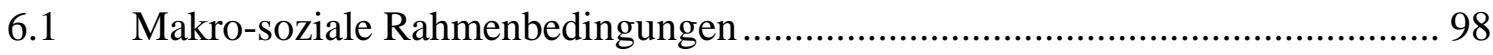

6.1.1 Der makro-soziale Kontext von Nachtrennungsvätern ................................ 99

6.1.2 Der Einfluss des makro-sozialen Kontextes............................................. 105

6.2 Der deutsche, britische und norwegische makro-soziale Kontext.................... 109

6.2.1 Institutioneller Kontext deutscher, britischer und norwegischer (Nachtrennungs-)Väter ......................................................................... 109

6.2.2 Gesetzliche Nachtrennungsregelungen ................................................. 119

6.2.2.1 Unterhaltsregelungen ............................................................... 119

6.2.2.2 Sorge- und Umgangsrechte ....................................................... 127

6.2.3 Väterliche Rechte und Pflichten - ein zusammenfassender Vergleich..... 132

\section{TEIL III: EMPIRISCHE ANALYSE}

\section{$7 \quad$ Datengrundlage der Sekundäranalyse....................................137}

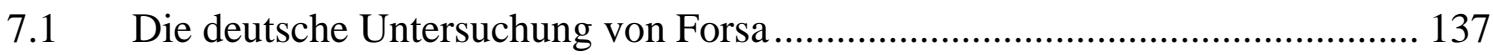

7.2 Die britische Untersuchung von Bradshaw und Mitarbeitern ........................... 138

7.3 Die norwegische Untersuchung von NOVA .................................................. 141

\section{Operationalisierung und Ausgestaltung väterlichen} Sorgehandelns

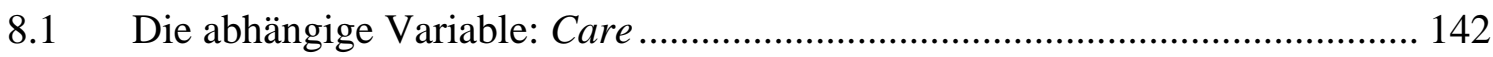

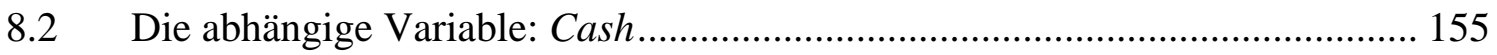

8.3 Statistisches Vorgehen: Modelle und Maßzahlen ............................................. 162

\section{$9 \quad$ Ergebnisse: Darstellung und Interpretation .........................169}

9.1 Operationalisierung der Prädiktoren und bivariate Zusammenhänge ................ 169

9.1.1 Der Einfluss der Prädiktoren der physischen Umwelt ............................... 169

9.1.2 Der Einfluss der Prädiktoren der mikro-sozialen Umwelt ........................ 182

9.1.3 Der Einfluss der Prädiktoren des individuellen Überzeugungssystems .... 206

9.1.4 Ergebnisse der bivariaten Zusammenhangsanalyse ............................... 212 
9.1.4.1 Bivariate Zusammenhänge: Care

9.1.4.2 Bivariate Zusammenhänge: Cash ................................................. 219

9.1.4.3 Zwischenfazit II: Die bivariaten Ergebnisse ...................................... 223

9.2 Ergebnisse der multiplen Zusammenhangsanalyse ......................................... 225

9.2.1 Determinanten im multiplen Regressionsmodell für Care ........................ 226

9.2.2 Determinanten im multiplen Regressionsmodell für Cash ....................... 229

9.3 Interpretation der bivariaten und multiplen Ergebnisse ................................. 233

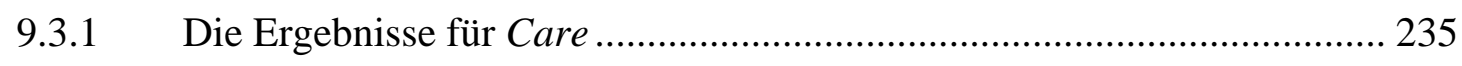

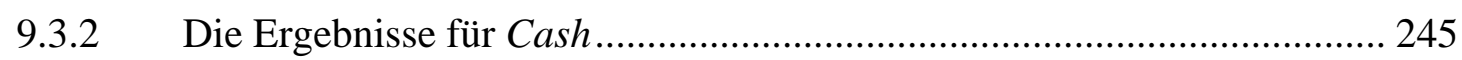

9.4 Väterliches Sorgehandeln: Das Verhältnis von Care und Cash......................... 254

\section{TEIL IV: ABSCHLIESSENDE BETRACHTUNG}

10 Was bestimmt väterliches Sorgehandeln? - Die Ergebnisse im Überblick.

\section{Einordnung der Ergebnisse: Eine kritische Würdigung...264}

11.1 Theoretische Implikationen der Ergebnisse ................................................. 264

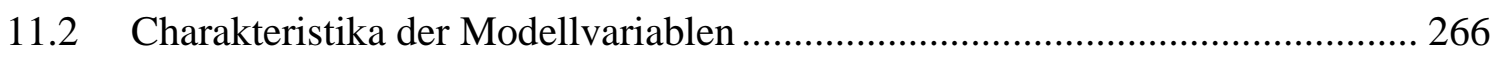

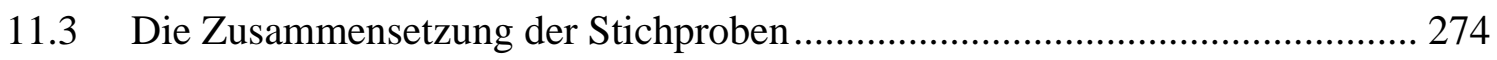

12 Ausblick: Weiterführende Forschungsansätze .................279

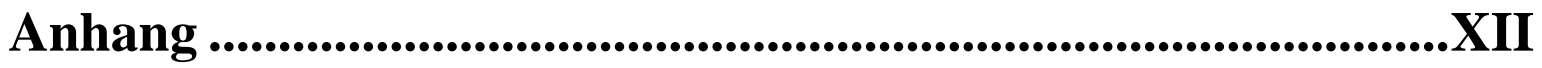

Literaturverzeichnis ................................................................. XXI

\section{Lebenslauf}




\section{Tabellenverzeichnis}

Tab. 5.1: Hypothesen zur Entfernung zwischen den Wohnorten................. 81

Tab. 5.2: Hypothesen zum Einkommen des Vaters........................ 81

Tab. 5.3: Hypothesen zum Ausbildungsniveau des Vaters...................... 83

Tab. 5.4: Hypothesen zur Arbeitszeit des Vaters............................ 84

Tab. 5.5: Hypothesen zur Zahl der Kinder im väterlichen Haushalt ............... 85

Tab. 5.6: Hypothesen zur Zahl der Kinder außerhalb des väterlichen Haushalts.... 86

Tab. $\quad$ 5.7: Hypothesen zur Partnersituation des Vaters.......................................... 87

Tab. 5.8: Hypothesen zur Partnersituation der Mutter......................................... 88

Tab. 5.9: Hypothesen zur Familienform der Eltern vor der Trennung.................... 89

Tab. 5.10: Hypothesen zur Dauer der Beziehung vor der Trennung......................... 90

Tab. 5.11: Hypothesen zur Unterhalts- und Sorge- bzw. Umgangsfestlegung.......... 91

Tab. 5.12: Hypothesen zur festgelegten Form des Sorge- bzw. Umgangsrechts....... 91

Tab. 5.13: Hypothesen zum freundschaftlichen Verhältnis der Eltern...................... 92

Tab. 5.14: Hypothesen zur ökonomischen Situation der Mutter................................ 93

Tab. 5.15: Hypothesen zur Dauer seit der Trennung.............................................. 93

Tab. 5.16: Hypothesen zur Anzahl der Beziehungen mit externen Kindern............. 94

Tab. 5.17: Übersicht der theoretisch generierten Determinanten väterlichen Sorgehandelns und ihrer Wirkungsrichtung getrennt nach Care und Cash

Tab. 6.1: Väterliche Rechte und Pflichten nach der elterlichen Trennung in unterschiedlichen nationalstaatlichen Kontexten.

Tab. 8.1: Care-Dimensionen erfasst in den einzelnen Studien................................ 143

Tab. 8.2: Komponenten der nationalen Care-Indizes.............................................. 150

Tab. 8.3: Häufigkeitsverteilungen der nationalen Care-Indizes............................... 154

Tab. 8.4: Cash-Dimensionen erfasst in den einzelnen Studien............................... 156

Tab. 8.5: Kategorien und Häufigkeiten der Cash-Variablen.................................... 160

Tab. 9.1: Operationalisierungen der Entfernung zwischen den Wohnorten............ 170

Tab. 9.2: Kreuztabellen zur Entfernung zwischen den Wohnorten.......................... 172

Tab. 9.3: Operationalisierungen des Einkommens des Vaters............................... 173

Tab. 9.4: Kreuztabellen zum Einkommen des Vaters............................................. 175

Tab. 9.5: Operationalisierungen des Ausbildungsniveaus des Vaters...................... 178

Tab. 9.6: Kreuztabellen zum Ausbildungsniveau des Vaters................................... 179

Tab. 9.7: Operationalisierungen der Arbeitszeit des Vaters.................................... 180

Tab. 9.8: Kreuztabellen zur Arbeitszeit des Vaters............................................... 181

Tab. 9.9: Operationalisierungen der Zahl der Kinder im väterlichen Haushalt....... 182 
Tab. 9.10: Kreuztabellen zur Zahl der Kinder im väterlichen Haushalt. 183

Tab. 9.11: Operationalisierungen der Zahl der Kinder außerhalb des väterlichen Haushalts

Tab. 9.12: Kreuztabellen zur Zahl der Kinder außerhalb des väterlichen Haushalts. 186

Tab. 9.13: Operationalisierungen der Partnersituation des Vaters. 187

Tab. 9.14: Kreuztabellen zur Partnersituation des Vaters. 189

Tab. 9.15: Operationalisierungen zur Partnersituation der Mutter..... 190

Tab. 9.16: Kreuztabellen zur Partnersituation der Mutter.. 191

Tab. 9.17: Operationalisierungen der Familienform der Eltern vor der Trennung.... 192

Tab. 9.18: Kreuztabellen zur Familienform der Eltern vor der Trennung................. 193

Tab. 9.19: Operationalisierungen der Dauer der Beziehung vor der Trennung......... 195

Tab. 9.20: Kreuztabellen zur Dauer der Beziehung vor der Trennung...................... 195

Tab. 9.21: Operationalisierungen der Unterhaltsfestlegung..................................... 196

Tab. 9.22: Kreuztabellen zur Unterhaltsfestlegung............................................... 197

Tab. 9.23: Operationalisierungen der Sorgerechts- bzw. Umgangsfestlegung.......... 200

Tab. 9.24: Kreuztabellen zur Sorgerechts- bzw. Umgangsfestlegung....................... 201

Tab. 9.25: Operationalisierungen der festgelegten Form des Sorgerechts................. 202

Tab. 9.26: Kreuztabellen zur festgelegten Form des Sorgerechts............................. 203

Tab. 9.27: Operationalisierungen des freundschaftlichen Verhältnisses der Eltern... 204

Tab. 9.28: Kreuztabellen zum freundschaftlichen Verhältnis der Eltern .................. 205

Tab. 9.29: Operationalisierungen der ökonomischen Situation der Mutter............... 206

Tab. 9.30: Kreuztabellen zur ökonomischen Situation der Mutter........................... 207

Tab. 9.31: Operationalisierungen der Dauer seit der Trennung................................ 208

Tab. 9.32: Kreuztabellen zur Dauer seit der Trennung............................................ 209

Tab. 9.33: Operationalisierung der Anzahl der Beziehungen mit externen Kindern. 210

Tab. 9.34: Kreuztabellen zur Anzahl der Beziehungen mit externen Kindern.......... 212

Tab. 9.35: Übersicht der bivariaten Regressionskoeffizienten der einzelnen Prädiktoren für Care bzw. Cash nach Datensätzen.....

Tab. 9.36: Übersicht der Ergebnisse der bivariaten Thesentestung getrennt nach Care und Cash.

Tab. 9.37: Übersicht der bivariaten und multiplen Ergebnisse für Care getrennt nach Datensätzen.

Tab. 9.38: Übersicht der bivariaten und multiplen Ergebnisse für Cash getrennt nach Datensätzen. 
Tab. 9.39: Zusammenfassende Gegenüberstellung der durch die bivariaten bzw. multiplen Ergebnisse bestätigten Hypothesen nach Datensatz und Care bzw. Cash getrennt.

Tab. 9.40: Relevante Determinanten für Care und Cash im Vergleich nach Datensätzen.

Tab. 9.41: Regressionskoeffizienten für jeweils Care und Cash als abhängige Variable.

Tab. 9.42: Häufigkeitsverteilung der Typologie väterlichen Sorgehandelns nach Datensätzen getrennt 259

Tab. 12.1: Überblick der theoretisch denkbaren und tatsächlich erfassten Variablen nach Datensätzen.

\section{Abbildungsverzeichnis}

Abb. 4.1: Individuelles Handlungsmodell - die Logik der Situation und Selektion 39

Abb. 4.2: Handlungsalternativen der Nachtrennungsväter.................................. 55

Abb. 6.1: Mehrebenen-Modell der sozialen Situation......................................... 97

Abb. 8.1: Graphische Häufigkeitsverteilung der abhängigen Variable Care nach Datensatz......

Abb. $\quad$ 8.2: Graphische Häufigkeitsverteilung der abhängigen Variable Cash nach Datensatz..

Abb. 9.1: Graphische Häufigkeitsverteilung der Typologie väterlichen Sorgehandelns nach Datensätzen getrennt..... 


\section{TEIL I: PROBLEMBEZOGENER HINTERGRUND}

\section{Fragestellung und methodischer Aufbau der Arbeit}

Männer als Väter befinden sich in einer inkonsistenten Situation. Zum einen haben demographische, soziale und ökonomische Veränderungen zu einer Neu-Definition der männlichen Rolle innerhalb der Familie geführt. Insgesamt hat sich die Organisation männlichen Lebens in den letzten Jahrzehnten wesentlich verändert: Die Möglichkeiten der Ausgestaltung des "Fathering" haben zugenommen, das Ausfüllen der Rolle ist zunehmend frei gewählt. Väter werden nicht länger ausschließlich als Ernährer konzeptionalisiert, darüber hinaus wird von ihnen ein „neues“ intensives Engagement in der Betreuung und Erziehung ihrer Kinder ${ }^{1}$ erwartet und gewünscht. Sie verbringen mehr Zeit mit ihren Kindern und bauen enge Bindungen zu ihnen auf (u.a.: Cabrera et al. 2000: 132; Knijn/Selten 2002: 171; Marsiglio et al. 2000: 1174; Tölke 2005: 3f.; Walter 2002b: 27).

Gleichzeitig führen ähnliche soziale, demographische und ökonomische Veränderungen zu einer Ausdifferenzierung familialer Lebensformen (Clarke/Roberts 2002: 165; Tölke 2005: 3; Jaursch 2003: 18; Oláh et al. 2002: 25). Diese weisen eine zunehmende Fragmentierung und Flexibilisierung traditioneller familialer Normen auf (Leira 1999: ix; Lewis 2003: 66; Kudera 2002: 145). Durch die elterliche Trennung und Scheidung wird ferner ein wachsender Anteil an Familien von der väterlichen Abwesenheit gekennzeichnet (Knijn et al. 2007: 190; Bray/Berger 1993: 156). Aufgrund von lange unhinterfragten geschlechtsspezifischen Rollenzuschreibungen verbleiben die Kinder häufig im Haushalt der Mutter, während Männer ihre Vaterschaft über Haushaltsgrenzen hinweg ausgestalten (müssen). Beide Entwicklungen, die wachsenden Anforderungen, die von Vätern erwarten sich über finanzielle Pflichten hinaus um ihre Kinder zu kümmern, und die zunehmende Abwesenheit der Väter innerhalb des familialen Haushalts aufgrund der elterlichen Trennung stehen sich konträr gegenüber (Skevik 2006b: 182).

Untersuchungen zu Nachtrennungsvätern zeigen eine hohe Variation an väterlichen Strategien mit dieser Widersprüchlichkeit umzugehen. Ein Teil der Väter kommt seinen elterlichen Pflichten, finanziell und sozio-emotional, über die elterliche Trennung hinaus nach. Andere Väter verlieren den Kontakt zu ihren getrennt lebenden Kindern kurz nach ihrem

\footnotetext{
1 Alle Ausführungen beziehen sich in gleichem Maße auf einzelne wie mehrere Kinder. Zur besseren Lesbarkeit wird auf die Darstellung beider Formen verzichtet. Es wird entweder der Singular oder der Plural verwendet.
} 
Auszug. Einige erfüllen ihre Unterhaltsverpflichtungen nicht oder nur begrenzt (Hobson/Morgan 2002: 4; Forsa 2002; Bradshaw et al. 1999, Skevik/Hyggen 2002). Offensichtlich bestehen Unterschiede in der Ausgestaltung des väterlichen Sorgehandelns in Nachtrennungsfamilien.

Trotz eines wachsenden Anstiegs allein erziehender Mütter und damit verbunden eines steigenden öffentlichen wie wissenschaftlichen Interesses an getrennt lebenden Familien, trotz einer vielfältigen (populär-)wissenschaftlichen Literatur zu moderner Vaterschaft liegen wenig Informationen über die Ausgestaltung und Rahmenbedingungen des Vaterseins in Nachtrennungsfamilien vor. Die vorliegende Arbeit beschäftigt sich daher mit der Frage, welche Bedeutung dem Mann als Vater nach der elterlichen Trennung zukommt. Gesetzlich wird zumindest die Gewährleistung einer finanziellen Versorgung vom Vater erwartet. Doch die Konzentration auf ökonomische Aspekte des Vaterseins verstellt den Blick u.a. auf sozio-emotionale Dimensionen. Sowohl die finanzielle Versorgung als auch die Beteiligung an Betreuungs- und Erziehungsaufgaben kann dabei sehr unterschiedlich ausgestaltet sein. Es bleibt offen, welche Determinanten das Handeln getrennt lebender, unterhaltspflichtiger Väter bestimmen.

Zur Beantwortung der forschungsleitenden Frage gliedert sich die Untersuchung in vier Teile. Der erste beschäftigt sich mit dem thematischen Rahmen der Arbeit. An die Einleitung schließt das Kapitel 2 mit der Konzeptionalisierung des väterlichen Sorgehandelns in Nachtrennungsfamilien an. Dabei wird zunächst der Begriff des väterlichen Sorgehandelns bestimmt (Kap. 2.1). Es wird eine Differenzierung zwischen sozio-emotionalen (Care) und finanziellen Aspekten (Cash) der väterlichen Fürsorge entwickelt und begründet. Die Klärung, wer als Nachtrennungsvater im Sinne der Forschungsfrage gilt und welche Besonderheiten mit dieser Form der Vaterschaft verbunden sind, dient in Kapitel 2.2 ferner der Spezifikation des Analysegegenstands. Dem folgt in Kapitel 3 eine kurze Bestandsaufnahme des aktuellen Forschungsstandes mit Blick auf Väter generell und Nachtrennungsväter im Speziellen (Kap. 3.1). Dies impliziert u.a. die Darstellung der Ergebnisse aus den Primäranalysen (Kap. 3.2), jener Datensätze, die der Sekundäranalyse (Kap. 8 bis 11) zugrunde gelegt werden. Den ersten Teil der Arbeit schließt eine Einordnung der Fragestellung in den derzeitigen Forschungsstand ab (Kap. 3.3).

Nach der Klärung wesentlicher Begrifflichkeiten sowie der Einordnung der Arbeit in den aktuellen Forschungsstand umfasst der zweite Teil der Arbeit die theoretische Fundierung der vorliegenden Untersuchung. Zur Beantwortung der Fragestellung wird dem Prinzip "parallel demonstration of theory" nach Skocpol und Somers (1980) gefolgt. Danach werden zunächst theoretische Annahmen entwickelt, die in einem zweiten Schritt anhand unterschiedlicher „Fälle“ überprüft werden (ebd.: 177). Dieses Vorgehen stellt für die Autorinnen eine von drei Methoden der vergleichenden Geschichtsforschung dar, mit 
deren Hilfe historische Entwicklungen in nationalstaatlichen Kontexten erklärt werden sollen. In dieser Logik können die „Fälle“ z.B. unterschiedliche Wohlfahrtsstaaten darstellen. In Anlehnung an Skocpol/Somers wird zur Bestimmung der Determinanten väterlichen Sorgehandelns in Nachtrennungsfamilien zunächst eine Handlungstheorie entwickelt, die sich jedoch nicht primär auf makro-soziale Strukturen, sondern auf individuelle Akteure bezieht. Damit sind die wesentlichen Analyseeinheiten („Fälle“) hier im Sinne des methodologischen Individualismus die individuell handelnden Väter. Die Gültigkeit der aufgestellten Theorie wird dann in einem zweiten Schritt in unterschiedlichen makro-sozialen Kontexten geprüft. Dies erfolgt mittels einer parallelen Sekundäranalyse dreier unabhängiger Datensätzen, die in verschiedenen nationalstaatlichen Kontexten entstanden sind.

Kapitel 4 stellt die individuelle Handlungstheorie dar, aus der theoretische Annahmen über die Bestimmungsfaktoren väterlichen Sorgehandelns in Nachtrennungsfamilien abgeleitet werden. Gemäß der Fragestellung steht dabei die Perspektive der Väter im Vordergrund. Ausgangspunkt der theoretischen Überlegungen stellt das zweistufige Handlungsmodell nach Kühnel und Bamberg dar (Kap. 4.1), das um Annahmen der integrativen Sozialtheorie von Hartmut Esser ergänzt wird. Die erste Modellstufe besteht aus der Rahmung der Situation, d.h. der Definition des Akteurs, in welcher Situation er sich befindet (Kap. 4.2), während die zweite Stufe die Handlungsauswahl betrifft (Kap. 4.3). Auf beiden Handlungsstufen wird von einer rationalen Wahl des Akteurs ausgegangen. Diese Annahme wird in Kapitel 4.3.2 kritisch mit Blick auf seine Anwendung innerhalb der Familiensoziologie generell und auf das väterliche Sorgehandeln speziell beleuchtet. Aus der Handlungstheorie werden in Kapitel 5 Hypothesen jeweils für die beiden Komponenten väterlichen Sorgehandelns, Care und Cash, abgeleitet.

Theorien besitzen Raum und zeitlich ungebunden Gültigkeit. Zur Testung der theoretischen Annahmen und damit der Güte des Handlungsmodell wird das Handeln der Nachtrennungsväter in unterschiedlichen „Räumen“, sprich makro-sozialen Kontexten, und anhand von Daten, die sich in ihren Erhebungszeitpunkten unterscheiden, untersucht. Die Fallauswahl erfolgt nach Skocpol/Somers kontrastiv, um möglichst viele denkbare „Falltypen“ abzudecken (Skocpol/Somers 1980: 176). Erweist sich die Theorie oder das theoretische Argument in allen Kontexten als valide, sichert dies die Allgemeingültigkeit der Theorie insgesamt ab. Als makro-soziale Fälle dienen dabei nationale Untersuchungen aus Deutschland, Großbritannien und Norwegen. Alle drei Länder sind durch ähnliche Entwicklungen, wie z.B. der Zunahme des Anteils allein erziehender Mütter oder veränderten geschlechtspezifischen Rollenerwartungen, gekennzeichnet. Der jeweilige Wandel unterscheidet sich jedoch nach Ausmaß, Zeitraum und Ausgestaltung. Des Weiteren können unterschiedliche institutionelle Rahmenbedingungen des Sorgehandelns von 
Nachtrennungsvätern ausgemacht werden. Neben der Unterschiedlichkeit der Kontexte besteht ein - eher forschungspragmatisches - Argument für die Länderauswahl in der Verfügbarkeit von quantitativen Datensätzen. Kapitel 6 widmet sich zunächst der Bedeutung unterschiedlicher makro-sozialer Kontexte für das individuelle Sorgehandeln theoretisch, indem die Dimensionen genannt werden, die die väterliche Fürsorge in Nachtrennungsfamilien rahmen (Kap. 6.1). In einem zweiten Schritt (Kap. 6.2) werden die theoretischen Überlegungen mit den jeweiligen nationalen Gegebenheiten empirisch gefüllt.

Zur Länderauswahl ist einschränkend zu sagen, dass sich die Ausführungen zum deutschen Fall ausschließlich auf Westdeutschland beziehen. ${ }^{2}$ Aufgrund der historisch sehr unterschiedlichen Entwicklung sind die Ausführungen zu Westdeutschland nur bedingt auf Ostdeutschland zu übertragen (Ostner et al. 2003: 3; Ostner 2002: 152). Auch knapp 20 Jahre nach der Wiedervereinigung bleiben immer noch einige Unterschiede zwischen den deutschen Teilen bestehen. So finden sich nach der Wende weiterhin Differenzen in der Haushaltszusammensetzung (z.B. höhere Ehe- und Scheidungsraten im Osten, weniger Alleinerziehende im Westen und eine stärkere Dual Carer/Dual Earner-Norm im Osten), in der Infrastruktur (Ostdeutschland weist eine höhere Bereitstellung an institutioneller Kinderbetreuung auf) und in der höheren Erwerbsbeteiligung von Müttern im Osten (Clasen 2005: 159). Des Weiteren wurden einige gesetzliche Nachtrennungsregelungen erst mit der Kindschaftsrechtsreform 1998 angeglichen. Neben rechtlichen Unterschieden bestehen weiterhin verschiedene Lebensverhältnisse und Lebenshaltungskosten in Ost und West, die z.B. in der Höhe des festgelegten Unterhalts berücksichtigt werden (Forsa 2002: 15ff.). Es kann argumentiert werden, Ostdeutschland als vierten makro-sozialen Kontext zu verstehen und auch hier die Gültigkeit der aufgestellten Thesen empirisch zu testen. Dagegen wird angeführt, dass der Erhebungszeitpunkt und der Zeitraum des Bestehens der DDR zu weit auseinander liegen. Die befragten ostdeutschen Nachtrennungsväter sind nicht mehr in den makro-sozialen Kontext der ehemaligen DDR eingebettet, dennoch wirken die historischen Rahmenbedingungen in die Gegenwart hinein. Seit der Wiedervereinigung haben weitreichende Anpassungsprozesse stattgefunden, die eine klare Abgrenzung der beiden Teile erschwert. Insgesamt bestehen Unterschiede fort, doch auch Angleichungen sind zu beobachten. Die Beschreibung der Länder bezieht sich

\footnotetext{
${ }^{2}$ Dies gilt auch für die Berechnungen im empirischen Teil. Es werden nur die Ergebnisse für die westdeutschen unterhaltspflichtigen Männer der Forsa-Stichprobe dargestellt. Erste Berechnungen mit dem gesamten Forsa-Datensatz zeigen einen signifikanten Effekt der Variable, die zwischen Ost- und Westdeutschland differenziert, sowohl auf die Care- wie auch auf die Cash-Variable. Damit steigt die Wahrscheinlichkeit von ausbleibenden Unterhaltszahlungen sowie eines Kontaktrückgangs, wenn der Vater aus Ostdeutschland stammt.
} 
im Wesentlichen auf generelle Trends der Institutionalisierung väterlicher Rechte und Pflichten, aufgrund des starken Transformationsprozesses der ostdeutschen Bundesländer in den letzten 20 Jahren beschränken sich die Ausführungen auf Westdeutschland, wo die meisten Regelungen und Rahmenbedingungen aufgrund des ausbleibenden Regimewechsels gleich geblieben sind. Die Berücksichtigung der ostdeutschen Spezifika würde die Darstellung verkomplizieren und nur bedingt zusätzlichen Erkenntnisgewinn leisten.

Die Länderdarstellung der institutionellen Kontexte zielt nicht darauf ab, wie in der vergleichenden Wohlfahrtsstaatsforschung üblich, einen systematischen Ländervergleich mit dichten Beschreibungen der jeweiligen Kontexte zu leisten. Auch wird keine strenge Typologie von Vaterschaftsregimen entwickelt. Es geht primär um die Verdeutlichung der Unterschiedlichkeit makro-sozialer Rahmenbedingungen, in denen die handelnden Nachtrennungsväter eingebettet sind. Dies gilt in gleichem Maße für die rechtliche Institutionalisierung väterlichen Sorgehandelns. Eine rechtsvergleichende Untersuchung würde schon deshalb den Rahmen der vorliegenden Arbeit sprengen, weil verschiedene Väterkohorten in den Samples vertreten sind. Das bedeutet, aufgrund von Reformen und Übergangsregelungen gelten nicht für alle Väter eines Landes die gleichen gesetzlichen Regelungen nach der elterlichen Trennung. Darüber hinaus ist eine ländervergleichende Perspektive neben Sprachbarrieren mit weiteren methodischen Schwierigkeiten verbunden (dazu u.a. Corden 2001: 289; Kilkey 2000: 33). Selbst wenn ein gemeinsames Verständnis von (juristischen) Konzepten und Begrifflichkeiten ausgemacht werden kann und dies die Vergleichbarkeit administrativer Arrangements sowie Outcomes für die betroffenen Eltern und Kinder erleichtert, bildet es nur einen Teil des rechtsstaatlichen Kontexts ab. Darüber hinaus müssten z.B. (rechts-) historische und kulturelle Entwicklungen berücksichtigt werden. Das würde den Rahmen der Arbeit sprengen und keinen zusätzlichen Erkenntnisgewinn zur Beantwortung der Forschungsfrage liefern. Ziel der Länderbeschreibungen ist es daher, generelle Tendenzen in der Institutionalisierung väterlicher Rechte und Pflichten in den nationalen Gesetzen aufzuzeigen. Zum Nachweis der Allgemeingültigkeit der Theorie reicht es aus einzelne Dimensionen - wie die nationale Familienpolitik oder Scheidungsgesetzgebung - oberflächlich zu skizzieren und daraus unterschiedliche Institutionalisierungsformen und -grade väterlicher Rechte und Pflichten generell abzuleiten. Damit wird das Argument gestützt, dass obwohl die Väter in sehr unterschiedlichen makro-sozialen Kontexten agieren, die mikro-soziologische Handlungstheorie empirisch Stand hält. Dazu ist es insgesamt weniger wichtig, wie verschieden die nationalen Kontexte sind, sondern vielmehr entscheidend, dass sie sich unterscheiden.

Des Weiteren ist darauf hinzuweisen, dass sich die Ausführungen mit Blick auf den britischen Kontext auf den Zeithorizont bis 1995 beziehen, während die deutschen und norwegischen Rahmenbedingungen bis 2002 beschrieben werden. Dies ist den Erhe- 
bungszeitpunkten der einzelnen Studien geschuldet, die in die Sekundäranalyse eingehen. Für die Untersuchung des väterlichen Sorgehandelns ist es entscheidend, in welchen Rahmenbedingungen sich die Väter im Zeitpunkt der Befragung befunden haben. Seitdem haben sich in allen drei Ländern interessante institutionelle Entwicklungen für (Nachtrennungs-)Väter vollzogen, diese sind für die Erklärung des angegebenen Handelns zum Zeitpunkt der Befragungen jedoch nicht relevant. Deswegen wird an dieser Stelle explizit darauf hingewiesen, dass sich die Länderbeschreibungen nicht am aktuellen Status Quo orientieren, sondern vielmehr primär die Situation im und die Entwicklung bis zum Erhebungszeitraum schildern.

Die forschungsleitende Fragestellung wird anhand einer parallel an drei unabhängig voneinander entstandenen Datensätzen durchgeführten quantitativen Sekundäranalyse empirisch untersucht. Im dritten Teil der vorliegenden Arbeit wird auf den deutschen, britischen und norwegischen Datensatz eingegangen, auf denen die statistische Analyse basiert. Dabei wird zunächst das methodische Handwerkszeug dargestellt. Dazu zählen die Beschreibung des jeweiligen Untersuchungsdesigns der drei Datensätze (Kap. 7), die Operationalisierung der erklärenden und zu erklärenden Variablen (Kap. 8 und 9) sowie das statistische Vorgehen der Analyse (Kap. 8.3). In den folgenden Kapiteln werden die statistischen Ergebnisse zunächst auf bivariater (Kap. 9.1) und dann auf multipler Ebene (Kap. 9.2) beschrieben und interpretiert. Dabei erfolgt die Darstellung der Ergebnisse entsprechend der Fragestellung nicht in einer ländervergleichenden Perspektive - die aufgrund der Datenlage insgesamt nur sehr begrenzt möglich ist - sondern vielmehr thesenweise. Das bedeutet, es werden an maximal drei Datensätzen - je nach Datenlage - die einzelnen theoretischen Annahmen nacheinander geprüft. Abschließend wird auf das Verhältnis der beiden theoretisch entwickelten Komponenten väterlichen Sorgehandelns, Care und Cash, eingegangen (Kap. 9.4).

Der letzte und vierte Teil, das Fazit der Arbeit, besteht aus drei Kapiteln. Zunächst werden die Ergebnisse im Überblick zusammengestellt. Kapitel 12 widmet sich der kritischen Betrachtung der Ergebnisse. Zum einen wird die Bedeutung der Ergebnisse für die aufgestellte Theorie thematisiert. Des Weiteren wird der Frage nachgegangen, welche methodischen und auch inhaltlichen Mängel aufgetreten sein könnten. Im letzten Kapitel werden die kritischen Aspekte als Anhaltspunkte für weiterführende Forschungsfragen genutzt. 


\section{Das Konzept väterlichen Sorgehandelns in Nachtrennungsfamilien}

In der Literatur finden sich zahlreiche Begriffe zur Umschreibung des Handelns als Vater. So wird in der deutschen Diskussion z.B. von väterlichem Engagement, von väterlicher Partizipation, von väterlicher Beteiligung an Erziehungsaufgaben, von väterlichem Erziehungsverhalten oder väterlicher Fürsorge gesprochen (Knijn 1995: 176; Fthenakis 1999, 2002). Zu den in der englischen Literatur verwendeten Begriffe zählen z.B. paternal engagement, paternal involvement, paternal generativity, paternal behavior, fatherhood activity, men's fathering attitudes and behavior oder fatherwork (Cabrera et al. 2000; Palkovitz 1997; Snarey 1993; Matzner 2004: 19, Dollahite et al. 1997: 17ff.). Entsprechend der Fragestellung konzentriert sich die Analyse auf die Handlungsebene. ${ }^{3}$ Ein in diesem Zusammenhang verwendeter Begriff aus dem Englischen ist das "Fathering“. Dieser ist insofern umfassend, als dass er sich nicht auf den Status des Mannes - wie der Begriff der Vaterschaft - bezieht, sondern das Handeln des Vaters in Verbform ausdrückt. Damit bezieht er sich auf die Rolle des Vaters als Akteur, oder in anderen Worten auf das Ausüben der Vaterschaft bzw. des Vaterseins. Leider gibt es in der deutschen Sprache keine adäquate Entsprechung (- Vatern?). Es wird der etwas umständliche Begriff des väterlichen Sorgehandelns verwendet, der synonym mit dem englischen Begriff Fathering gebraucht wird. Dabei wird zwischen einer Care- und einer Cash-Dimension des Sorgehandelns unterschieden.

\subsection{Väterliches Sorgehandeln in Form von Care und Cash}

Ausgangspunkt der Überlegungen ist ein von Amato (1998: 243ff.) eingeführter theoretischer Ansatz, der den Blick auf die Ressourcen lenkt, die Eltern ihren Kindern bereitstellen. Kinder bedienen sich im Verlauf ihrer Entwicklung sämtlicher Ressourcen ihrer

\footnotetext{
${ }^{3}$ Es kann dabei zwischen dem äußerlich sichtbaren Ausführen einer Handlung und einem inneren Tun, das insbesondere auf kognitive und emotionale Prozesse zurückgreift, unterschieden werden (Esser 1999: 179; Palkovitz 1997: 208ff.; Nauck 1989: 50, Marsiglio et al. 2000: 1182). Letztere gehen nur bedingt in die Analyse ein. Ferner wird nicht die Interaktion mit dem Kind im Vordergrund des Interesses steht, d.h. die Aktion-Reaktion-Sequenz zweier Akteure, sondern vielmehr das Handeln aus der einseitigen Perspektive des Vaters. Zwar impliziert dies ein soziales Handeln, insofern sich das Tun des Vaters auf sein Kind bezieht, doch nicht die Interaktion zwischen Vater und Kind wird analysiert, sondern vielmehr die Motivation und Initiative des Vaters. Der Fokus liegt auf dem getrennten Vater und seiner Handlungsentscheidung. Die Interaktion mit dem Kind - ebenso wie mit der Kindesmutter werden nur berücksichtigt, wenn sie in die „Berechnungen“ des Vaters über die Konsequenzen seines Tuns eingehen (siehe dazu ausführlich Kap. 4 Die Handlungstheorie).
} 
physischen und sozialen Umwelt, innerhalb derer grundsätzlich beiden Elternteilen eine bedeutende Rolle zukommt. Es können dabei drei Arten der Ressourcenquellen differenziert werden: das Finanz-, Human- und Sozialkapital. Zum Finanzkapital zählen v.a. das Einkommen und materielle Güter. Die kindliche Entwicklung wird z.B. durch die Gewährleistung einer guten Ernährung, einer adäquaten, sicheren Unterkunft oder dem Zugang zu guten Bildungsstätten unterstützt. Ressourcen basierend auf dem elterlichen Humankapital sind u.a. verbale und numerische Fähigkeiten, Fertigkeiten, Wissen oder Charaktereigenschaften. Ein Indikator, der auf das Niveau des Humankapitals hinweist, ist die elterliche Bildung in Jahren. Eltern mit hohem Humankapital können u.a. kognitive Fähigkeiten und sozioökonomische Erfolge durch eine stimulierende Umwelt fördern. Aus dem elterlichen Sozialkapital werden Ressourcen vermittelt, die zum Führen zwischenmenschlicher Beziehungen befähigen. Innerhalb des familialen Systems sind dabei v.a. die Beziehungsebene zwischen den Eltern sowie zwischen einem Elternteil und dem Kind von Bedeutung. Die dyadische Interaktion der Eltern, z.B. in Form von emotionaler Unterstützung, respektvollem Umgang, offener Kommunikation oder adäquater Konfliktlösestrategien, dient dem Kind als Vorbild. Innerhalb der Ebene der Eltern-Kind-Beziehung ist nicht die Quantität der gemeinsam verbrachten Zeit entscheidend, sondern vielmehr die Qualität. Maßgebliche Komponenten sind hier die elterliche Unterstützung einerseits, dazu zählen z.B. emotionale Zuwendung, Ermutigung und alltägliche Unterstützung, und andererseits die Kontrolle, darunter sind z.B. die Formulierung von Regeln oder Disziplinierungen zu fassen (Amato 1998: 243ff.; siehe ebenso: Marsiglio et al. 2000: 1176; Fthenakis 2002: 107; Matzner 2004: 31).

Die kindliche Entwicklung basiert demnach auf der Verfügbarkeit, Intensität und Qualität des elterlichen Human-, Finanz- und Sozialkapitals. Dabei können prinzipiell alle drei Formen sowohl von der Mutter als auch vom Vater bereitgestellt werden. Kulturelle, historische und biologische Entwicklungen haben jedoch u.a. in einigen westlichen Gesellschaften eine starke männliche Ernährernorm forciert, die eine geschlechtsspezifische Arbeitsteilung impliziert (Amato 1998: 245). Väter sind danach v.a. über die Versorgerrolle in die Familienstruktur eingebunden (worden). ${ }^{4}$ Damit kam ihnen lange Zeit in erster Linie die Funktion der Bereitstellung des Finanzkapitals zu. Gleichzeitig hat dies die Entbindung von binnenfamilialen Aufgaben impliziert, wie z.B. der Haushaltsführung oder der Betreuung und Versorgung der Kinder. Diese Tätigkeiten wurden stattdessen grundsätzlich der Rolle der Mutter zugeschrieben (Rollett/Werneck 2002: 325; Cohen 1993: 2).

\footnotetext{
${ }^{4}$ Zur Bedeutung des wohlfahrtsstaatlichen Ernährermodells im makro-sozialen Kontext siehe ausführlich Kap. 6.2.1 Institutioneller Kontext deutscher, britischer und norwegischer (Nachtrennungs-)Väter.
} 
Sie umfassten folglich v.a. die Bereitstellung von Ressourcen aus dem Human- und Sozialkapital. Doch die lange Zeit fraglos akzeptierte Selbstverständlichkeit eines dominanten bürgerlichen Leitbildes schwindet (Kudera 2002: 148). Mit der Entwicklung zunehmend gleichberechtigter Rollenerwartungen an die Geschlechter innerhalb der letzten Jahrzehnte geht allmählich die Veränderung der Ausgestaltung der Vater-Kind-Beziehung einher (Herlth 2002: 586f.). Von den „neuen“ Vätern wird eine familienfreundlichere, kindzentrierte Haltung erwartet, die sich in der Bereitschaft zur Annahme von egalitären Rollenmustern innerhalb und außerhalb der Familie manifestiert. Dies bedeutet konkret, dass Väter stärkeres Interesse am Alltag der Kinder zeigen, mehr Zeit mit ihnen verbringen, sowie vermehrt Formen der Fürsorge und liebevollen Zuwendung übernehmen (sollen) (Herlth 2002: 587; Rollett/Werneck 2002: 325, Knijn 1995).

Abgeleitet aus der traditionellen Aufgabenteilung in "Breadwinning " und "Caregiving" (Amato 1998: 242; Cabrera et al. 2000: 133), werden im Folgenden vereinfacht zwei Dimensionen des väterlichen Sorgehandelns unterschieden: "Care" und "Cash" (Ostner et al. 1995: 419; Millar 1999: 33). Beide Komponenten stellen jeweils eine Seite derselben Medaille dar. Die Cash-Komponente umfasst dabei insgesamt die finanzielle Versorgung von und Investitionen in Kinder (u.a. Becker 1985: 102) - die Bereitstellung von Finanzkapital. Innerhalb von Nachtrennungsfamilien wird dies primär durch den Transfer formalisierter Unterhaltszahlungen geleistet. Darüber hinaus können informelle Zahlungen vom Vater erfolgen, wie das Finanzieren der Klassenfahrt oder eines neuen Kinderfahrrads während eines Besuches. Zur Care-Komponente zählt dagegen insbesondere die sozioemotionale Unterstützung - die Verfügbarkeit von Human- und Sozialkapital. Diese kann sehr unterschiedliche Formen annehmen von Kommunikation (wie Zuhören, Ausdruck von Sorge, Emotionen oder Interesse) und Lehraspekten (wie das Disziplinieren, Fördern und Unterrichten), über die Versorgung und Pflege (z.B. Essen, Baden oder Wickeln) bis hin zur Ausübung gemeinsamer Aktivitäten in Alltag und Freizeit (u.a. Einkaufen, Sport, Hobbys, Spielen usw.) (Palkovitz 1997: 209f.).

Die analytische Differenzierung väterlichen Sorgehandelns in "Care" und "Cash" erscheint aus mehreren Gründen sinnvoll: Zunächst ist darauf zu verweisen, dass die in der Literatur bisher im Vordergrund stehende Rolle des Vaters als „Ernährer“ nur einen Aspekt guter Vaterschaft darstellt. Darüber hinaus sind noch andere emotionale und soziale Aspekte väterlicher Beteiligung in Studien väterlicher Praxis einzubeziehen (Fthenakis 1999: 39). Mit der Veränderung der Rollenerwartungen an Väter wird auch in der Literatur zunehmend die Angemessenheit vorliegender Vaterschaftskonzepte neu diskutiert (z.B. Booth/Crouter 1998; Dollahite et al. 1997; Fthenakis 2002: 100). Die Einführung eines erweiterten Vaterschaftskonzeptes führt zur Ablehnung einer ausschließlichen Definition der Väter als „Ernährer“, denn nur unter Berücksichtigung aller 
Bereiche, in denen sich Väter engagieren, kann die Bedeutung väterlicher Beteiligung für Väter und ihre Familien verstanden werden. Bisher wurde diese Differenzierung in empirischen Studien vernachlässigt. Sie konzentrierten sich i.d.R. vornehmlich auf „traditionell männliche“ oder „traditionell weibliche“ Aspekte. Nur wenige Untersuchungen integrieren beide Komponenten (Fthenakis 2002: 100ff.). ${ }^{5}$

Eine besondere Bedeutung kommt der Differenzierung ferner mit Blick auf Nachtrennungsfamilien zu. Hier wird per Gesetz eine klare Trennung zwischen der Care- und Cash-Komponente väterlichen Sorgehandelns hergestellt, indem zwischen Unterhaltspflichten und Sorge- bzw. Umgangsrechten differenziert wird. Bei Scheidungen ist die unterstellte strikte Trennung zwischen verschiedenen elterlichen Pflichten an der geschlechtsspezifischen Arbeitsteilung orientiert geblieben. Lange wurde z.B. in Deutschland im Falle einer Scheidung einem Elternteil - meist der Mutter - das alleinige Sorgerecht zugesprochen, während dem getrennt lebenden Vater das Leisten von Unterhaltszahlungen auferlegt wurde. Damit wurde er in seinen Elternrechten und in seiner Verantwortung eingeschränkt (Napp-Peters 1995: 110). Väter wurden so im Scheidungsverfahren vom emotionalen und sozialen Sorgehandeln für ihre Kinder in einem Ausmaß ,freigesetzt', wie dies ihrer Alltagsroutine möglicherweise gar nicht (mehr) entsprach. Zum Teil besteht diese geschlechtsspezifische Differenzierung heute fort.

Es ist darüber hinaus darauf zu verweisen, dass verschiedene Wohlfahrtsstaaten väterliches Sorgehandeln in Form von Care-Rechten und Cash-Pflichten in unterschiedlicher Weise fordern und fördern. ${ }^{6}$ Es ist zu fragen, ob sich Väter weniger kümmern, wenn der Staat ihre Abwesenheit kompensiert und sie damit aus ihrer Verantwortung entlässt. Dies entspricht der aktuellen sozialpolitischen Debatte in Europa. Die aktuellen staatlichen Interessen gehen über die bisher diskutierten familialen Verpflichtungen hinaus. Sie weiten sich ferner auf familiale Beziehungen und Praktiken aus, besonders mit dem Blick auf die Ausübung elterlicher Rollen (Daly 2004: 138). So formulieren Hobson/Morgan (2002: 2):

"In policy discourse the question who pays for the kids is now paired with that who cares for the kids." (Hervorhebungen C.M.).

\footnotetext{
${ }^{5}$ Siehe dazu ausführlich Kap. 3 Die sozialwissenschaftliche Väterforschung - Eine kurze Bestandsaufnahme.

${ }^{6}$ Zur Institutionalisierung väterlicher Rechte und Pflichten in unterschiedlichen nationalen Kontexten vergleiche ausführlich Kap. 6.2 Der deutsche, britische und norwegische makro-soziale Kontext.
} 
Wie bereits angeführt, wurde von Vätern lange primär die Bereitstellung materieller Mittel erwartet. In einigen europäischen Ländern wird Vaterschaft zunehmend auch als CareAufgabe definiert (Daly 2004: 138).

Die Differenzierung in Care und Cash trägt ferner den Unterschieden der beiden Dimensionen Rechnung. So kann die sozio-emotionale Auseinandersetzung mit dem eigenen Kind auf der Handlungsebene diverse Formen annehmen. Neben der Kommunikation sind hier u.a. auch Erziehung, Planung, Kommunikation oder Handlungen zum Schutze des Kindes zu nennen (Palkovitz 1997: 209). Die Gestaltungsmöglichkeiten der Form der Unterhaltszahlung sind demgegenüber begrenzt. Zwar gibt es auch verschiedene Formen Unterhaltsraten zu zahlen, für das Kind bzw. die betreuende Mutter ist es jedoch lediglich von Bedeutung, dass finanzielle Mittel transferiert werden; die Form der Zahlung ist dabei unerheblich. Viele Formen väterlichen Engagements, wie z.B. das Beaufsichtigen, Betreuen, Pflegen, Beschäftigen oder Spielen machen ferner die unmittelbare Zuwendung erforderlich (Herlth 2002: 586). Während Care auf direktem Kontakt mit und zu dem Kind basiert, können Unterhaltszahlungen ohne direkte Face-to-Face-Interaktion aus der Ferne erfolgen. Ein weiterer entscheidender Unterschied zwischen väterlichem Care und Cash besteht in der Motivation des Vaters, sozio-emotionale bzw. finanzielle Unterstützung (nicht) zu leisten. Das Niveau des väterlichen Sorgehandelns basiert auf einer gewissen Freiwilligkeit des Vaters. Bei der Zahlung von Unterhalt handelt es sich im Idealfall dagegen um eine regelmäßige automatische Abbuchung vom Konto des Vaters, so dass dieser wenig Eigeninitiative aufbringen muss. Ferner kann die Kindsmutter die Unterhaltsansprüche des gemeinsamen Kindes juristisch einklagen. Entzieht sich der Vater dem kindlichen Wunsch nach gemeinsam verbrachter Zeit, sind rechtliche Schritte zur Forcierung des Kontakts mit Blick auf das Kindeswohl nur begrenzt möglich.

Ein weiterer Unterschied zwischen den Dimensionen besteht in der zugrunde liegenden Motivation, eines von beidem nicht zu gewährleisten. Kümmert ein Vater sich nicht um sein Kind, kann er dies z.B. mit der emotionalen Belastung für sich und das Kind begründen. Die durch die Trennung und den Verlust der Familie erlittenen Schmerzen und Leiden werden mit Besuchen der Kinder aktualisiert (Braver et al. 1993: 93). Der Vater kann es für die Erziehung als schädlich empfinden, wenn das Kind aus seiner alltäglichen Routine herausgerissen wird. ${ }^{7}$ Ein anderer Grund für das Nicht-Kümmern wäre das subjektive Gefühl, als Vater nicht zu genügen (Amendt 2004: 220f.). Auch ein generelles

${ }^{7}$ Amendt spricht in diesem Zusammenhang von einem heroischen Verzicht dem Kind zuliebe (Amendt 2004: 216ff.). 
Desinteresse am Kind kann ein Grund für den Vater sein, sich nicht um das Kind zu kümmern. ${ }^{8}$ Deswegen wäre hier die Vater-Kind-Beziehung vor der Trennung der Eltern genauer zu betrachten. Dies sind nur einige generelle Überlegungen zur väterlichen Motivation, den Kontakt zu seinem Kind abzubrechen. Die Unterlassung von Unterhaltszahlungen dagegen ist durch zwei zentrale Begründungsmuster geprägt (Forsa 2002: 129ff.): Zum einen können Unterhaltsprobleme aufgrund von Zahlungsunfähigkeit auftreten, d.h. es fehlen die finanziellen Mittel. Eine derartige Begründung ist für das Ausbleiben des väterlichen Kümmerns in Form von Care eher ungewöhnlich. Zum anderen kann die Verweigerung ein Grund für ausbleibende Zahlungen darstellen, wenn der Vater keine Einsicht in seine finanzielle Verantwortung zeigt oder die Höhe der Unterhaltszahlungen für ungerechtfertigt hält. Die Wahrscheinlichkeit der Zahlung von Unterhaltsleistungen sinkt ferner, wenn der Vater annimmt, dass das Geld zugunsten der ExPartnerin und nicht zum Wohle des Kindes verwendet wird. ${ }^{9}$ Es wird deutlich, dass väterliches Kümmern neben dem direkten Kontakt v.a. zeitliche, emotionale und soziale Ressourcen erfordert. Ein Mangel an diesen kann ein Grund sein, sich nicht ausreichend zu kümmern, bedingt jedoch nicht gleichzeitig das Ausbleiben von Unterhaltszahlungen. Für die Überweisung von Unterhalt sind primär finanzielle Ressourcen von Bedeutung. Fehlt es dagegen an finanziellen Mitteln, muss das nicht zwangsläufig bedeuten, dass das väterliche Kümmern zurückgeht. Die Ausführungen zeigen, dass die Care- und CashKomponente väterlichen Sorgehandelns durch sehr unterschiedliche Faktoren bestimmt werden. ${ }^{10}$ Eine Differenzierung in die beiden Dimensionen wird diesen Unterschieden gerecht. Zum besseren Verständnis werden im Folgenden beide Komponenten primär separat voneinander dargestellt. Dies erleichtert sowohl die Argumentation als auch die Präsentation der Ergebnisse.

Einige der wesentlichen Differenzen zwischen der finanziellen und sozio-emotionalen Unterstützung der Nachtrennungsväter hängen eng mit der besonderen Vater-KindSituation nach der elterlichen Trennung zusammen, auf die im folgenden Kapitel im Einzelnen eingegangen wird.

\footnotetext{
${ }^{8}$ Tatsächliches Desinteresse, Verantwortungslosigkeit und Egoismus der Väter sind jedoch eher selten angegebene Gründe für den Kontaktabbruch (Matzner 1998: 101).

${ }^{9}$ In der englischen Literatur spricht man vom sog. "Child Support Abuse“ (Braver et al. 1993: 100f.).

${ }^{10}$ Zur genauen theoretischen Fundierung der jeweiligen Determinanten von Care bzw. Cash siehe ausführlich Kap. 5 Hypothesengenerierung.
} 


\subsection{Nachtrennungsfamilien: das Phänomen abwesender Väter}

Es gibt verschiedene Ursachen für die Abwesenheit des Vaters innerhalb der Familie. ${ }^{11}$ Die Mehrzahl der aktuellen Fälle getrennt lebender Väter basiert - unabhängig von der gemeinsamen Haushaltsführung und dem Vorliegen eines Trauscheins - auf dem Scheitern einer Partnerschaft, aus der mindestens ein Kind hervorgegangen ist. Dieses verbleibt mit der (räumlichen) Trennung der Eltern i.d.R. im Haushalt der Mutter. Darüber hinaus sind Fälle zu berücksichtigen, bei denen nie eine partnerschaftliche Beziehung zwischen den Eltern bestand (Bradshaw et al. 1999: 23). Die vorliegende Untersuchung nimmt solche Väter in den Blick, die zwei entscheidende Merkmale simultan aufweisen: Zum einen muss die elterliche Paarbeziehung gescheitert oder nie existent gewesen sein. Zum anderen muss damit einhergehen, dass der Vater in einem anderen Haushalt lebt als seine unterhaltsberechtigten Kinder. ${ }^{12}$ Im Folgenden werden unter dem Begriff „getrennt lebender Vater“13 die Väter zusammengefasst, die Unterhaltsverpflichtungen gegenüber mindestens einem minderjährigen Kind haben, das im Haushalt der Mutter lebt.

Mit der räumlichen Abwesenheit des Vaters und der Auflösung der elterlichen Paarbeziehung gehen wesentliche Veränderungen für das gesamte Familien- und Verwandtschaftssystem einher, die sich auf das väterliche Sorgehandeln auswirken (Bray/Berger 1993: 161; Matzner 1998: 89). Kinder bedienen sich, wie dargestellt, in ihrer Entwicklung sämtlicher Ressourcen ihrer physischen und sozialen Umwelt. Der uneingeschränkte Zugang zu den elterlichen Ressourcen wird mit der räumlichen Trennung erschwert. So sind

\footnotetext{
${ }^{11}$ Insgesamt gibt es - insbesondere mit der in der Literatur postulierten Pluralisierung der Familienformen weder in der Familienforschung noch im alltäglichen Sprachgebrauch ein einheitliches Verständnis, was als Familie zu bezeichnen ist (u.a. Nave-Herz 1989: 2). Fraglich ist z.B., ob bereits kinderlose Ehepaare als Familien zu bezeichnen sind. Offen bleibt ferner, wie es sich mit der Anwesenheit eines neuen Partners im Haushalt der allein erziehenden Mutter verhält, und ob der getrennt lebende Elternteil gegebenenfalls zu dieser Familie zählt. Des Weiteren bleiben die Grenzen unscharf, wer zum weiteren Familienkreis gehört. Diese kurzen Anmerkungen skizzieren die unterschiedlichen Formen familialen Zusammenlebens und deuten die Schwierigkeit einer konsistenten Definition der Familie an. Diese Herausforderungen wirken sich auch auf die statistische Erfassung von Familien aus (Hantrais 1999: 103). Für die vorliegende Arbeit erscheint eine abschließende Definition der Familie generell nicht erforderlich. Gemäß der Fragestellung wird im Folgenden expliziert, wer als Nachtrennungsvater bezeichnet wird und damit in der vorliegenden Sekundäranalyse Berücksichtigung findet.

${ }^{12}$ Wichtig ist, dass beide Merkmale gleichzeitig auftreten. Hier nicht berücksichtigt werden (i.d.R. erwachsene) Kinder, die den elterlichen Haushalt bereits verlassen haben, oder Eltern, die sich zwar in einer Beziehung befinden, aber zwei Haushalte unterhalten, wobei die gemeinsamen Kinder bei der Mutter leben.

13 Andere Bezeichnungen wären z.B. „abwesende“ oder „unterhaltspflichtige“ Väter. Der Begriff des „abwesenden Vaters“ ist dabei kritisch zu betrachten, da er suggeriert, dass der andere Elternteil sich vollständig aus der Erziehung des Kindes zurückgezogen hat, was, wie die empirischen Ergebnisse zeigen (werden), in vielen Nachtrennungsfamilien nicht der Fall ist (u.a. Corden 2001: 297). Ein besserer Ausdruck besteht im Englischen mit "non-resident father". Im Folgenden werden die Begriffe synonym verwendet.
} 
Begegnungen zwischen Vater und Kind grundsätzlich nur noch durch eine Verabredung möglich. Besuche beim oder vom Kind erfolgen nach mehr oder weniger strengen Zeitplänen, erfordern eine gewisse organisatorische Vorbereitung v.a. bei einer großen räumlichen Distanz zwischen den Wohnorten und finden gegebenenfalls im öffentlichen Raum statt (Matzner 1998: 106).

Neben den Schwierigkeiten der konkreten Besuche führt die elterliche Trennung dazu, dass die Eltern nicht mehr als Einheit in Erscheinung treten. Die Umgangsregelung birgt die Gefahr in sich, dass Alltag und Freizeit nicht in gleicher Weise von Mutter und Vater repräsentiert werden (Amendt 2004: 164ff.). Der Kontakt zwischen Kind und abwesendem Vater vollzieht sich - zumeist begrenzt auf das Wochenende - v.a. auf der Freizeitund Erholungsebene. Die alltägliche Erziehungsfunktion des Vaters geht mit der räumlichen Trennung zurück. Das bedeutet z.B., dass der abwesende Vater weniger Regeln für das alltägliche Zusammenleben aufstellt und kontrolliert oder dass er seltener bei den Hausaufgaben hilft. Alltägliche Selbstverständlich- und Gemeinsamkeiten gehen für den Vater verloren (Amendt 2004:167; Amato 1998: 257).

Der starke Regelungsbedarf von Fragen, die wichtige Belange des Kindes betreffen, erfordert Kommunikation und Interaktion der beiden getrennten Elternteile (Amendt 2003: 62). Gegenseitig zugefügte Demütigungen und Verletzungen innerhalb der Paarbeziehung erschweren die Kommunikation auf der elterlichen Ebene (Amendt 2004: 180; Matzner 1998: 101). Das Kind stellt die letzte bestehende Verbindung zwischen den ehemaligen Partnern dar. Die Begegnung mit dem Kind kann bei dem Vater Gefühle der Wut, des Schmerzes, der Trauer und des Verlustes, die die räumliche Trennung von der Familie mit sich bringt, wecken (Matzner 1998: 102). Innerhalb dieser stark konfliktären Situation zwischen den Elternteilen ist es schwer eine jahrelang gewachsene Vater-Kind-Beziehung in die neue Konstellation hinüberzuretten. Je nachdem wie stark die Elternbeziehung von den Konflikten auf der Partnerschaftsebene belastet ist, kann dies das regelmäßige und intensive Kümmern des Vaters negativ beeinträchtigen (Braver et al. 1993: 93). Der Vater ist darüber hinaus darauf angewiesen, dass die Mutter sein Kümmern zulässt, d.h. seine Bemühungen eines intensiven Kontakts nicht untergräbt oder boykottiert. ${ }^{14}$ Die Störung und Behinderung der väterlichen Besuche beeinträchtigt die Beziehung zwischen dem

\footnotetext{
${ }^{14}$ Ungefähr der Hälfte der Eltern gelingt es nicht, im Interesse des Kindes eine, die kindliche Entwicklung fördernde, praktische Gestaltung des Umgangs des nicht sorgeberechtigten Elternteils zu gewährleisten. Eine Befragung des Deutschen Jugendinstituts ergab, dass 56\% der befragten alleinerziehenden Mütter versuchen, den nicht sorgeberechtigten Vater „soweit wie möglich aus der Erziehung der Kinder herauszuhalten“ (Matzner 1998:105f.).
} 
getrennt lebenden Elternteil und seinem Kind (Braver et al. 1993: 96). Amendt (2004: 206) geht davon aus, dass der väterliche Kontakt zum Kind mithin abhängig ist vom Unterstützungsverhalten der Mutter und dem Durchhaltevermögen des Vaters.

Während der Besuchszeit trägt der Vater aufgrund der i.d.R. abwesenden Mutter die alleinige Verantwortung für das Wohl des Kindes, die ihm je nach Rollenverteilung innerhalb der ehemaligen Partnerschaft mehr oder weniger vertraut ist. Für einige Väter gestaltet sich die mit dem Kind gemeinsam verbrachte Zeit schwierig, weil sie eine ungewohnte Zweisamkeit darstellt (Amendt 2004: 164). Dies widerspricht vordergründig der zuvor getroffenen Annahme, dem Vater ginge Alltäglichkeit mit dem Kind verloren. Dem ist zum einen zu entgegnen, dass die väterlichen Besuche in Trennungsfamilien häufig auf das Wochenende oder in die Ferien fallen. Zwar wird auch hier ein Stück Alltag gelebt, aber dieser ist durch einen hohen Anteil an Freizeit geprägt. Des Weiteren sind hier keinerlei Aussagen über die Dauer der Besuchszeit getroffen. Das bedeutet, auch stundenweiser Kontakt kann für den Vater eine ungewohnte Zweisamkeit darstellen und wenig Alltäglichkeit bereithalten.

Insgesamt zeigt sich, dass das väterliche Sorgehandeln in Nachtrennungsfamilien in Abgrenzung zum Sorgehandeln in „Normalfamilien“ durch einige Besonderheiten charakterisiert ist. 


\section{Die sozialwissenschaftliche Väterforschung - Eine kurze Bestandsaufnahme}

Innerhalb der sozialwissenschaftlichen und öffentlichen Diskussion kommt dem Mann als Vater verstärkt Aufmerksamkeit zu. Dabei werden sowohl die abwesenden wie anwesenden Väter in den Blick genommen. Zur Einordnung der forschungsleitenden Fragestellung der vorliegenden Untersuchung erfolgt im nachstehenden Kapitel eine kurze Bestandsaufnahme der aktuellen Väterforschung (in Nachtrennungsfamilien).

\section{1 (Nachtrennungs-)Väter in der wissenschaftlichen Literatur}

Die traditionelle Perspektive und damit verbundene Prämissen der geschlechtsspezifischen Rollenverteilung spiegeln sich auch im aktuellen Forschungsstand zu Vätern wieder. Lange wurde dem Vater und Mann, der auch über die öffentlichen Dimensionen hinaus Aufgaben in der Familie erfüllt, wenig Aufmerksamkeit geschenkt. Dementsprechend konzentrier(t)en sich Studien und theoretische Auseinandersetzungen mit der Familie v.a. auf die Perspektive der Frauen und/oder Kinder (Cohen 1993: 1f., Rollett/Werneck 2002: 324; Walter 2002b: 47f.; Forste 2002: 594; Tölke/Hank 2005: 7f.; Amato 1998: 251ff.; Smart/Neale 1999: 2; Marsiglio et al. 2000: 1183; Kolbe 2001: 190; Knijn 1995: 179). Geraten Väter in den Blick, dienen sie als Erklärungsgrößen für mütterliches Handeln (Tölke/Hank 2005: 9). Als Gründe für die Vernachlässigung der Väter in der Familienforschung sind häufig die - unhinterfragte - Dominanz des (männlichen) Ernährermodells und die mit ihr verbundene geschlechtsspezifische Teilung von Erwerbs- und Familienarbeit genannt worden. Diese führe zur Abwesenheit des Vaters im kindlichen Alltag. Damit müsse sein Einfluss z.B. auf die Betreuung und Erziehung des Kindes nicht geklärt werden. Das Argument ist eng verbunden mit der lange in Frage gestellten eigenen Beziehung zwischen Vater und Kind (Knijn et al. 2007: 192f.; Tölke/Hank 2005: 7).

Heute besteht wissenschaftlich kein Zweifel mehr an der eigenständigen - von der Mutter unabhängigen - Qualität der Vater-Kind-Beziehung. Die zahlreichen Studien zur kindlichen Entwicklung und zu den Dimensionen des väterlichen Engagements (auch in Nachtrennungsfamilien) zeigen, dass ein engagiertes väterliches Sorgehandeln die kindliche Entwicklung fördert (Marsiglio et al. 2000: 1182f.; Hartl 2002a: 150; Smart/Neale 1999: 36). Dennoch entwickelt sich nur langsam ein selbstständiges wissenschaftliches Interesse am Vater. So wird beispielsweise das männliche Reproduktionsverhalten nicht in gleichem Maße in der amtlichen Statistik erfasst, wie dies für Frauen der Fall ist. Das Wissen über die Gründe, warum Männer zu Vätern werden und wie sie diese Vaterschaft im Handeln ausgestalten, ist lange gering und wenig systematisiert worden (Knijn et al. 
2007: 191; Ostner 2002: 150; Hobson 2002, Bradshaw et al. 1999). Zunächst fanden sich in der Literatur v.a. zahlreiche populär-wissenschaftliche Ratgeber für (werdende) Väter. Sie wurden verstärkt als Figuren moderner Belletristik, in Film und Fernsehen sowie in der Werbung genutzt. Jüngst ist aufgrund ökonomischer, sozialer und demographischer Veränderungen darüber hinaus ein wissenschaftliches Interesse am Mann und Vater entstanden. Dabei stand insbesondere seine An- bzw. Abwesenheit innerhalb der Familie im Vordergrund.

Die Aussagen und Ergebnisse der jüngeren Väterforschung in den Sozialwissenschaften werden durch eine starke Ambivalenz gekennzeichnet (Walter 2002b: 24; Matzner 1998: 12). Einige Wissenschaftler ${ }^{15}$ konstatieren heute eine „Krise der Vaterschaft“ aufgrund ihrer Abwesenheit, während andere Autoren eine Generation „neuer Väter“ beobachten, die eine neuartige Anwesenheit aufweisen. Anfänglich wurden v.a. „defizitäre“ Väter in den Blick genommen (Ostner 2002; Marsiglio et al. 2000: 1174). Darüber hinaus fanden sich in der Literatur häufig Thesen über eine „vaterlose Gesellschaft“ oder die „Krise des Paternalen“ (Knijn 1995: 171; O’Brien 2006: 3; Matzner 1998: 12; Drinck 2005: 8; Kudera 2002: 146). Die Vaterlosigkeit wurde häufig mit der Funktionslosigkeit des Vaters begründet (Walter 2002b: 24). Andere Autoren gehen heute weniger dramatisch nicht vom gänzlichen Untergang aber dennoch vom „Rückgang der Väterlichkeit“ aus (Jensen 1999) oder sprechen positiv gewendet von einer „transition of fatherhood“ bzw. einer „Detraditionalisierung des Vaters“ (O’Brien 2006: 2f.).

Unabhängig davon, wie die Entwicklung benannt wird, kann insgesamt ein Wandel der väterlichen Rechte und Pflichten innerhalb der öffentlichen und privaten Rollenerwartungen - in Europa - ausgemacht werden. Die Aufgabe des Vaters wurde lange - ausschließlich - als Ernährer definiert. Diese Rollenzuschreibung wird aufgrund verschiedener sozialer Entwicklungen, wie z.B. einer zunehmenden Bedeutung der Gleichberechtigung zwischen den Geschlechtern, einer alternden Gesellschaft, veränderten Familienstrukturen und -formen oder sich wandelnden Arbeitsmarktanforderungen und bedingungen, zunehmend in Frage gestellt (Drinck 2005: 9; Scheiwe 1999: 283; Knijn 1995: 182). Frauen und Mütter sind verstärkt selbst in den Arbeitsmarkt integriert. Ihre ökonomische Abhängigkeit vom männlichen Versorger hat an Bedeutung verloren (Ellingsæter 2003: 420, Knijn 1995: 186; O’Brien 2006: 2; Kudera 2002: 147). Dies führt

${ }^{15} \mathrm{Zu}$ Gunsten einer besseren Lesbarkeit wird im Folgenden auf die gesonderte Darstellung der weiblichen Form verzichtet. 
zu einer Marginalisierung des Ehemanns und Vaters als Ernährer sowie insbesondere zu der Forderung nach einer egalitären Aufgabenteilung.

Gleichzeitig wird es für Männer zunehmend schwieriger das Familieneinkommen allein zu sichern. Die traditionelle Arbeitsteilung basierte auf der Annahme eines stabilen Arbeitsmarktes, der die Erwirtschaftung eines sicheren und v.a. ausreichenden Einkommens für eine ganze Familie ermöglicht. Die hohe Arbeitslosigkeit, sinkende Löhne und steigende Flexibilisierungs- und Mobilitätsanforderungen an globalisierten Arbeitsmärkten erschweren es Vätern wie Männern, ihrer finanziellen Verantwortung als Alleinversorger nachzukommen (Ostner 2002: 166; Lewis 2003: 62; Hobson et al. 2006: 267f., Tölke 2005 101f.; Kudera 2002: 147). Auch diese Entwicklung erfordert eine NeuDefinition der Vaterrolle in der Familie.

Darüber hinaus erfordert die männliche Ernährerrolle ein weibliches Pendant. Dies setzt stabile Partnerschaften und Ehen voraus, eine weitere Prämisse neben der Arbeitsmarktstabilität, die zunehmend nicht gegeben ist (Lewis 2003: 62; Jensen 2006: 41; Bradshaw et al. 1999; Skevik 2006a: 114; Kolbe 2001: 190). Mit der steigenden Zahl allein erziehender Mütter sind unweigerlich auch Väter verbunden, die nicht mit ihren Kindern in einem Haushalt leben. Dies erschwert ferner die Neu-Definition der Vaterrolle. Die Zahl der Männer, die ihre Vaterschaft aufschieben, steigt ebenso wie die Zahl der getrennt lebenden Väter, so dass Väter insgesamt weniger Zeit mit ihren Kindern in einer Haushaltsgemeinschaft verbringen (Jensen 1999: 26). Dieses Phänomen wird verstärkt durch die bereits skizzierten Flexibilisierungs- und Mobilitätsanforderungen am Arbeitsmarkt, die den familialen Verpflichtungen in Form einer aktiven Betreuung durch den Vater widersprüchlich gegenüber stehen (Hobson et al. 2006: 267f.; O’Brien 2006: 2). Besonders der Alleinverdiener ist von den Arbeitsmarktbedingungen abhängig. Lange und flexible Arbeitszeiten führen ebenso wie lange Arbeitswege zu einer verstärkten Abwesenheit der Väter im familialen Alltag (Knijn 1995: 185). Die Vereinbarkeit von Familie und Beruf lange eine Herausforderung, die nur erwerbstätige Mütter betraf - wird auch für Väter immer mehr zu einem Problem, insbesondere wenn sie sich über die Ernährerrolle hinaus definieren (Ostner 2002: 166; Ellingsæter 1999: 40; Lammi-Taskula 2006: 79). Die beschriebene Entwicklung stellt einen weiteren Aspekt der Neu-Definition der Vaterrolle dar, der innerhalb der Literatur diskutiert wird.

Der Verlust bzw. das Abschwächen eines Ernährermodells erfordert die Entwicklung eines neuen Rollenmusters, das - so die wissenschaftliche Annahme - jedoch noch aussteht (Tölke 2005: 100f.; Knijn 1995: 171; Leitner et al. 2003). Es wird u.a. argumentiert, dass nicht mehr von einem Vaterbild in der Gesellschaft ausgegangen werden kann. Vielmehr muss - u.a. aufgrund der steigenden Pluralisierung der Familien- und Lebensformen - eine Vielzahl verschiedener Rollenmuster angenommen werden. Es gibt nicht die 
Vaternorm (Drinck 2005: 8; Marsiglio et al. 2000: 1175). Vorhandene Krisenherde in der Partnerschaft und Unsicherheiten im eigenen Rollenverhalten als Vater sowie die Widrigkeiten des Arbeitsmarktes erschweren die Suche nach der väterlichen Funktion innerhalb der Familie zusätzlich (Tölke 2005: 100f.).

Neben der veränderten Definition der Vaterschaft hat sich auch der Anteil der Männer, die überhaupt zu Vätern werden, gewandelt. Lange wurde der Beitrag der Männer zum Rückgang der europäischen Fertilitätsraten weitestgehend vernachlässigt, obwohl Männer häufiger kinderlos bleiben (wollen) als Frauen. Männer schieben die Elternschaft länger auf und darüber hinaus wollen sie auch weniger Kinder als Frauen (Knijn et al. 2007, Schmitt 2005, Jensen 2006: 40, Goldscheider/Kaufman 1996, Dorbritz 2004). Dies stellt eine weitere Form des möglichen Bedeutungsverlustes der Vaterschaft dar.

In engem Zusammenhang mit den Ursachen der Kinderlosigkeit steht die Frage nach der Bedeutung der Männer für den Übergang in die Elternschaft, der jüngst in der Familiensoziologie und -politik verstärkt Aufmerksamkeit geschenkt wird. Auch hier haben bisher wissenschaftliche Untersuchungen primär die Heirats- und Fertilitätsentscheidungen von Müttern in den Blick genommen (Tölke/Hank 2005: 7). Neuere Studien beschäftigen sich mit der männlichen oder zumindest der Paarperspektive bei der Familiengründung (u.a. das Sonderheft von Tölke/Hank 2005; die Studien von Fthenakis et al. 2002; Rollett/Werneck 2002 oder international: Forste 2002, Goldscheider/Kaufman 1996).

Doch der Rückgang des männlichen Ernährers muss nicht zwingend zu einem Verlust der Väterlichkeit führen. Im Gegenteil, neben der Diskussion um die „Krise der Vaterschaft“ findet sich innerhalb der Literatur die Perspektive auf die sog. „neuen Väter“. Einige Wissenschaftler gehen davon aus, dass die Abschaffung des Patriarchats zu einer „,neuen“ Form von Väterlichkeit geführt hat (Walter 2002b: 24; Matzner 1998: 12; Jaursch 2003: 17f., Tölke 2005: 3f.). Verbunden mit zunehmend egalitär ausgestalteten Rollenerwartungen an Männer und Frauen bzw. Väter und Mütter führt die Re-Definition der Vaterrolle zu höheren sozialen Erwartungen an Väter wie auch zu einem stärkeren Wunsch der Väter selbst, sich aktiv in die Erziehung und Betreuung ihrer Kinder einzubringen (Hobson 2002; Marsiglio et al. 2000: 1183; Kolbe 2001: 190; Knijn 1995: 179; O’Brien 2006: 12; Jensen 2006: 37; Cabrera et al. 2000: 132; Knijn/Selten 2002: 171; Marsiglio et al. 2000: 1174; Hobson et al. 2006: 268; Matzner 1998). Von diesen "caring fathers“ wird eine starke Familienorientierung, eine höhere Kindzentrierung sowie das Verbringen von mehr Zeit mit und für das Kind erwartet und gewollt (O’Brien 2006: 12; Jensen 2006: 37; Cabrera et al. 2000: 132; Knijn/Selten 2002: 171; Marsiglio et al. 2000: 1174; Hobson et al. 2006: 268; Herlth 2002: 586f.; Forste 2002: 582; Rollett/Werneck 2002: 325; Kudera 2002: 148). 
Die Entwicklung wird häufig als Trend von der Betonung finanzieller Verpflichtungen hin zu Betreuungsrechten innerhalb der Vaterrolle charakterisiert. Dabei muss jedoch darauf hingewiesen werden, dass es sich weniger um das vollständige Ablösen eines Modells durch ein anderes handelt, sondern vielmehr um einen langfristigen Veränderungsprozess auf verschiedenen Ebenen. Mit der „neuen“ Vaterrolle haben sich in der Literatur zahlreiche Wissenschaftler beschäftigt (Tölke/Hank 2005: 9, z.B. Kalicki et al. 2002; Herlth 2002). Ebenso zahlreich sind dabei die Vatertypologien, die anhand von empirischen Untersuchungen ausgemacht werden. So identifizieren Rollet und Werneck (2002: 334) drei Väterformen: die „neuen Väter“, die „eigenständigen Väter“ und die „familienorientierten Väter“. Kudera (2002: 167ff.) entwickelt aus qualitativen Interviews mit Vätern vier wesentliche Muster der elterlichen Arbeitsteilung: ein „ländlich-traditionales“, ein „karrierezentriertes Arrangement“, ein „modernes Gegenmodell“ dazu sowie eine Familienform zwischen ,Modernität' und ,Traditionalität'. Eine weitere Typologie findet sich mit den Vaterschaftskonzepten des „traditionellen“ und des „modernen“ Ernährers, den „ganzheitlichen“ sowie den „familienzentrierten“ Vätern bei Matzner (2004: 350f.). Dies sind nur einige Beispiele der Rollenmodelle wie sie in der deutschen Literatur zu finden sind. In den meisten Fällen basieren sie auf Auswertungen qualitativer Interviews mit Vätern.

Offen bleibt, inwieweit sich die skizzierten Veränderungen in den (männlichen) Einstellungen zu Vaterschaftskonzepten im väterlichen Verhalten niederschlagen. In Deutschland sind zwar die Normen bzgl. der innerfamilialen Arbeitsteilung gelockert, haben sich aber nur vereinzelt in veränderten Verhaltensmuster ausgedrückt (Döge 2006: 149f.). Ähnliche Entwicklungen finden sich auch in den skandinavischen Ländern, die häufig als Vorreiter der Institutionalisierung einer egalitären Arbeitsteilung von Männern und Frauen innerhalb der Diskussion angeführt werden. Doch trotz veränderter Opportunitätsstrukturen für Männer, z.B. am Arbeitsmarkt, zeigen sich im täglichen Handeln der (nordischen) Väter nur moderate Veränderungen (Kitterød/Kjeldstad 2003: 49; Skrede 2001: 8; LammiTaskula 2006: 84f.; Ostner 2002: 164f.). Die Umsetzung veränderter Erwartungen an Väter auf der Handlungsebene muss wissenschaftlich stärker in den Blick genommen werden. Zwar finden sich zahlreiche Zeitverwendungsstudien insbesondere mit Blick auf die geschlechtsspezifische Arbeitsteilung in der Literatur (für Deutschland u.a. Döge 2006), doch die Differenzierung in Haus- und Familienarbeit bleibt meist offen. Des Weiteren werden unterschiedliche Dimensionen der Familienarbeit nicht ausreichend differenziert. Auch hier besteht Forschungsbedarf.

Die Möglichkeitsstrukturen für Männer, z.B. familiale und berufliche Pflichten in Einklang zu bringen, verweisen auf eine weitere Perspektive, die sich in den letzten Jahren in der Väterforschung entwickelt hat: die Untersuchung des Vaterkonzepts innerhalb 
politischer, öffentlicher und wissenschaftlicher Diskussionen. Das Interesse an Vätern findet sich auch auf der politischen Agenda der letzten Jahre wieder (Lewis 2002: 125). Die dargestellten Veränderungen und ihre Bedeutung für die Vaterrolle, ihre potentielle Funktionslosigkeit werden öffentlich und zwischen verschiedenen (politischen) Lagern kontrovers diskutiert (Marsiglio et al. 2000; Hobson 2002; Tölke/Hank 2005b; Ostner/Schmitt 2008). Väter werden verstärkt über den Ernährer hinaus konzeptionalisiert und als "carer" anerkannt (Hobson et al. 2006: 269; Skevik 2006a: 114). Dies lenkt die Perspektive auf die Institutionalisierung von Vaterschaft in unterschiedlichen Sozialmodellen. Hobson (2002) vereint in ihrem Sammelband unterschiedliche nationale Perspektiven auf die jeweilige rechtliche und sozialpolitische Begründung und Regulierung des Vaterseins. Der Band macht auf eine wichtige Forschungslücke aufmerksam: der Zusammenhang zwischen gesellschaftlicher Ausgestaltung des Vaterseins - der Institutionalisierung und Sanktionierung von väterlichen Rechten und Pflichten bzw. Pflichtverletzungen - und dem väterlichen Sorgehandeln. Im europäischen Vergleich dient immer wieder das skandinavische Modell des Dual Earner/Dual Carer, das ein aktives Vaterschaftsmodell impliziert, als wissenschaftliche Referenzkategorie (Hobson et al. 2006: 269; Jensen 2006: 44; Ostner/Schmitt 2008; Bradshaw/Hatland 2006).

Neben der Thematisierung anwesender Väter und ihrer (neuen) Funktion innerhalb der Familie findet sich ferner - entsprechend der beschriebenen Ambivalenz - die Diskussion um die Ausgestaltung der Abwesenheit des Vaters im aktuellen Forschungsstand. Mit dem Ansteigen der Scheidungszahlen und dem Rückgang der Geburten innerhalb der Institution Ehe und damit der gleichzeitigen Pluralisierung der Familienformen wird zunehmend die Frage diskutiert, wie Väter in ,anderen“ Familienarrangements ihre väterlichen Rechte und Pflichten ausfüllen. Mit dem gesellschaftlichen Wandel sind neue Formen väterlicher Abwesenheit entstanden. Es haben sich Ursachen, Formen und damit verbunden Folgen väterlicher Abwesenheit verändert. Längst wird das Fehlen des Vaters im familialen Kontext verstärkt durch die elterliche Trennung verursacht und nicht mehr durch den Tod des Vaters u.a. durch Kriegsbeteiligung (Jaursch 2003: 18; Goldscheider/Kaufman 1996: 91; Forste 2002: 581; Walter 2002a). Doch auch in der Scheidungsforschung dominierte lange Zeit die Perspektive auf die Konsequenzen der elterlichen Trennung für Kinder und allein erziehende (Ehe-)Frauen (Amato 1998: 251ff.; Martin 1995: 49; Marsiglio et al. 2000: 1174; als Beispiele u.a. Babka von Gostomski 1999; Hildenbrand 2002, BuMinJFG 1977, für die USA: Bray/Berger 1993).

Die wenigen Studien, die sich mit abwesenden Vätern beschäftigten, konzentrierten sich entweder auf bestimmte soziale Gruppen wie z.B. geschiedene Väter, bestimmte Altersklassen der Kinder oder begrenzte Erfassungs(zeit)räume (Clarke/Roberts 2002: 172; Depner/Bray 1993b: 182; Ostner 2002: 156; Forsa 2002: 26). In Deutschland untersuchte 
z.B. Moch (2002: 643ff.) die Gestaltung der Beziehung zwischen erwachsenen Töchtern und ihren geschiedenen Vätern einige Jahre nach der elterlichen Trennung. Die britische Studie "Family fragments?" von Smart und Neale (1999) basierte auf qualitativen Interviews mit insgesamt 60 Elternteilen aus verschiedenen Nachtrennungsfamilien im Raum West Yorkshire. Aus der Paarperspektive wurde der Frage nachgegangen, wie sich der Erziehungsstil der Eltern nach ihrer Trennung verändert. Durch die Erhebung (1994-96) von drei Zeitpunkten bis maximal 18 Monate nach der elterlichen Trennung konnte eine Verlaufsperspektive berücksichtigt werden (Smart/Neale 1999: 40ff.). Anhand der Differenzierung zwischen "Care" und "Authority" haben die Autorinnen drei Typen der Elternschaft nach Trennung und Scheidung unterschieden: 'Co-Parenting', 'Custodial Parenting' und 'Solo Parenting'(ebd.: 57ff.). Insgesamt standen also weniger Väter und ihr Sorgehandeln isoliert im Vordergrund des Interesses, sondern vielmehr der Aushandlungsprozess zwischen den Eltern. Die deutsche Studie von Napp-Peters (1995) untersuchte ebenso Paare, jedoch lediglich nach der Scheidung nicht generell nach der elterlichen Trennung. Mittels qualitativer Interviews mit Vätern und Müttern wurde die langfristige wirtschaftliche ebenso wie sozio-emotionale Entwicklung der Nachtrennungsbeziehungen rekonstruiert. Dabei gaben über die Hälfte der 109 Interviewten an, dass kein Kontakt zwischen den betroffenen Kindern und dem nicht sorgeberechtigten Elternteil besteht. Ein Großteil begründete dies mit der Unterbindung des Kontakts durch den anwesenden Elternteil (Matzner 1998: 113ff.).

Ein von Bien und Mitarbeitern (2002) herausgegebener Sammelband hat das wissenschaftliche Interesse auf die besondere Lebensform der Stieffamilien in Deutschland gelenkt, nicht zuletzt weil sie quantitativ an Bedeutung gewonnen hat. Die meist qualitativ angelegten Studien haben primär die Perspektive der Kinder berücksichtigt (Bien et al. 2002: 10; Teubner 2002: 69; Beckh/Walper 2002: 210ff.). Dies gilt auch für eine der wenigen quantitativen Untersuchungen in dem Band. Hartl (2002b) wertete die Daten aus dem Familiensurvey von 409 Stiefkindern und 586 Kindern Alleinerziehender unter 18 Jahren quantitativ aus. Im Mittelpunkt der Analyse stand der Kontakt zwischen dem getrennt lebenden Vater und seinem Kind. Die mit dem Kind zusammenlebenden Erwachsenen wurden nach der Beziehung des Kindes zum getrennt lebenden Elternteil und zu den Gründen für die Verringerung bzw. den Abbruch des Kontakts befragt. Die Ergebnisse machen deutlich, dass sich die Beziehung zwischen dem Stiefkind und seinem externen Elternteil sehr unterschiedlich gestalten kann. Ein beachtlicher Teil hat gar keinen Kontakt. Dabei ist die Chance, dass eine Verbindung zum „externen“ leiblichen Vater bestehen bleibt, bei Stiefkindern bedeutend geringer als bei Kindern aus Ein-ElternFamilien. Die multivariate Analyse ergab, dass die Trennungsdauer, die Verehelichung der Stieffamilie, eine Partnerschaft des externen Elternteils und das Leben in Ostdeutschland negativ auf die Kontaktwahrscheinlichkeit wirken. Ein hoher Schulabschluss der 
Mutter und die Regelung eines gemeinsamen Sorgerechts vergrößern dagegen die Wahrscheinlichkeit, dass eine Verbindung erhalten bleibt (Hartl 2002b: 185ff.). Insgesamt durchzieht ein Ergebnis die für das Thema „abwesende Vaterschaft“ relevanten Beiträge im Band: die Gründung einer Stieffamilie erhöht die Wahrscheinlichkeit des Kontaktabbruchs zwischen dem „externen“ Elternteil und dem Kind, das in der neuen Familienkonstellation lebt (Teubner 2002: 70; Hartl 2002b: 178).

Eine der wenigen quantitativen Studien zum Thema „Scheidungsväter“, ${ }^{16}$ wurde 2000/01 von Amendt und seinen Mitarbeitern für Deutschland erhoben. Innerhalb der Studie wurden insgesamt 3600 Männer zu ihren Erfahrungen als Nachtrennungsväter per Internet befragt (Amendt 2004: 10). Anhand von Häufigkeitstabellen stellt Amendt die Alters-, Bildungs- und Einkommensstruktur der Väter, den Familienstand, die Kontaktsituation zu ihren Kindern ${ }^{17}$ und zur ehemaligen Partnerin sowie Unterhaltsverpflichtungen und eventuelle gerichtliche Auseinandersetzungen dar. Darüber hinaus wird die berufliche und gesundheitliche Situation der Väter, sowie die Inanspruchnahme professioneller Hilfe in dieser krisenhaften Lebensphase erfasst. Sämtliche Variablen sind auf bivariate Zusammenhänge geprüft worden. Die Ergebnisse zeigen, dass insgesamt 23,7\% der Befragten nach der Trennung den Kontakt zu ihren Kindern abbrechen (ebd.: 209). Dabei ergibt sich, dass der Erhalt von Kommunikationsfähigkeit und eines gemeinsamen Verantwortungsbewusstsein als Elternpaar nach der Trennung begünstigende Voraussetzungen für den Fortbestand des Vater-Kind-Kontaktes sind (Amendt 2003: 49). Väter ohne Kontakt sind durchschnittlich älter, verfügen über eine unterdurchschnittliche Bildung, sind erwerbslos und erzielen nur ein unterdurchschnittliches Einkommen. Der Kontakt zum Kind verringert sich, wenn der abwesende Vater in einer neuen Partnerschaft lebt oder die Mutter das alleinige Sorgerecht hat. Mit der Anzahl der Kinder, für die Unterhalt gezahlt werden muss, nimmt das Risiko des Kontaktabbruchs zu. Der Kontakt bricht des Weiteren häufiger ab, wenn eine längere Beziehung zwischen den Eltern bestand, die zeitliche Distanz zur Trennung größer ist und das Alter der Kinder über 13 Jahre liegt (Amendt 2004: 209f.). Knapp 80\% zahlen laut der Bremer-Studie regelmäßig Unterhalt. 11,5\% geben an, gar keine Zahlungen zu leisten (Amendt 2003: 14). Dabei gilt: je höher die Schulbildung, umso regelmäßiger wird Unterhalt gezahlt. Ein positiver Zusammenhang besteht ferner zum Einkommen des Vaters (ebd.: 94f.). Die Ergebnisse der Studie weisen insgesamt auf einen Zusammenhang zwischen Unterhalt und Umgangsrecht hin. Viele Männer, die

\footnotetext{
${ }^{16}$ Auf diese wird im Folgenden mit der Kurzform „Bremer-Studie“ verwiesen.

${ }^{17}$ Dazu zählen das vereinbarte Sorge- und Umgangsrecht, die Häufigkeit des Kontaktes, sowie das väterliche Erleben des Zusammenseins mit den Kindern.
} 
regelmäßig Unterhalt zahlen, haben häufiger Kontakt zu ihren Kindern. Teilweise, unregelmäßige oder gar keine Zahlungen gehen häufiger einher mit keinem Kontakt (Amendt 2003: 100). Kritisch anzumerken ist, dass innerhalb der Bremer-Studie die Bildungs- und Einkommensstruktur der Befragten nicht dem gesellschaftlichen Durchschnitt entspricht. Die Befragung wurde über das Internet erhoben. Der typische Internetnutzer ist der junge, männliche Akademiker mit vergleichsweise hohem Einkommen. Generalisierende Aussagen sind daher stark begrenzt. Amendt selbst betrachtete die Studie als Basis für explorative Analysen sowie als Ausgangspunkt weiterer Untersuchungen (ebd.: 9).

Die väterliche Perspektive wurde bisher auch in der familiensoziologischen Wohlfahrtsstaatsforschung vernachlässigt. Eine der wenigen Ausnahmen stellt der bereits erwähnte Sammelband von Hobson (2002) dar. Hier finden sich in den Länderberichten auch Ausführungen darüber, welche Rechte und Pflichten Vätern je nach Familienform zugesprochen werden. Dabei kann vermutet werden, dass die Symmetrie bzw. Asymmetrie von Rechten und Pflichten der getrennten oder geschiedenen Väter deren Sorgehandeln durchaus mitbestimmen. Es fehlt jedoch an empirischen Studien, die den Zusammenhang zwischen institutionalisierter und wahrgenommener Reziprozität von Pflicht und Recht einerseits, dem väterlichen Sorgen andererseits analysieren (Marsiglio et al. 2000: 1175f.; Lange 2003: 320).

Neben den dargestellten inhaltlichen Desideraten eröffnet der Stand der Forschung zu (Nachtrennungs-)Vätern ferner einige theoretische Anknüpfungspunkte für weitere Untersuchungen. Wie andere Teildisziplinen leidet auch die - internationale - Familiensoziologie an einem Mangel an soziologisch erklärenden Theorien (Hill/Kopp 1999: 39; Smart/Neale 1999: 2, Matzner 2004: 18, Braver et al. 1993: 87; Walter 2002b: 50). Dies gilt ebenso für die aufgeführten Untersuchungen. Die wenigsten haben explizit theoretische Annahmen vorgegeben, die empirisch getestet wurden. Dabei besteht die Gefahr, dass Wissenschaftler das eigene Alltagswissen über familiale Praktiken und Elternschaften als „Plausibilitätsressource“ (Kolbe 2002: 17) nutzen. Eine theoretische Fundierung erscheint daher dringend notwendig.

\subsection{Bisherige Ergebnisse der nationalen Studien}

Als zweiten Teil des Forschungsstandes werden im folgenden Kapitel die wesentlichen Ergebnisse der Untersuchungen dargestellt, die in der vorliegenden Sekundäranalyse die Datengrundlage bilden. Dabei wird zunächst ein zusammenfassender Überblick über die zentralen Ergebnisse gegeben. In einem abschließenden Kapitel wird die forschungsleitende Fragestellung von den bereits bestehenden Erkenntnissen abgegrenzt. 


\subsubsection{Zusammenfassung der Ergebnisse der Forsa-Studie}

Innerhalb der Forsa-Studie „Unterhaltszahlungen für Kinder in Deutschland“, erhoben 2001/02, steht die Unterhaltssituation minderjähriger Kinder, deren Eltern nicht (mehr) zusammenleben, im Vordergrund des Interesses (Forsa 2002: 159). ${ }^{18}$ Dabei handelt es sich um eine repräsentative Umfrage, die sowohl unterhaltsberechtigte wie unterhaltspflichtige Elternteile berücksichtigt. ${ }^{19}$ Im Vorgrund stehen insbesondere die Festlegung, Regelung und Praxis von Unterhaltszahlungen (ebd.: 33). Ziel ist es gewesen, das Ausmaß und die Gründe für das Ausbleiben von Unterhaltsleistungen zu erfassen (ebd.: 154). Dabei werden die Ergebnisse getrennt für Unterhaltsberechtigte und -pflichtige dargestellt, die jedoch nicht nach Geschlecht differenziert werden. Für die vorliegende Forschungsfrage interessieren primär die Angaben der Väter, die innerhalb der Stichprobe der Unterhaltspflichtigen 96\% ausmachen (ebd.: 163).

Mit Blick auf die Unterhaltsfestlegung macht die Studie deutlich, dass die Regelung der Höhe zwischen den Eltern relativ unproblematisch erfolgt. Die Ergebnisse zeigen ferner, dass häufig die den Unterhaltsberechtigten zustehenden Unterhaltsleistungen in der Höhe nicht vollends ausgeschöpft werden. Es bleibt offen, ob es sich dabei um einen Unterhaltsverzicht der Unterhaltsberechtigten oder eine Leistungsunfähigkeit der Zahlungspflichtigen handelt (ebd.: 96). Neben dem Prozess der Festlegung wird in der Forsa-Studie ausführlich die Unterhaltspraxis getrennt lebender Elternteile untersucht. Dabei ergibt sich, dass verglichen mit den festgelegten Beträgen rund ein Drittel der Unterhaltsberechtigten die finanziellen Transfers nicht in festgelegter Form erhält. Dagegen geben rund ein Fünftel der Unterhaltspflichtigen an, dass sie Zahlungsschwierigkeiten haben bzw. gehabt haben (ebd.: 103ff.). Probleme, der Unterhaltspflicht nachzukommen, treten je nach Familienkonstellation (häufiger bei Unverheirateten), Dauer seit der Trennung (häufiger mit steigendem zeitlichen Abstand), räumlicher Entfernung zwischen den Elternteilen (seltener bei geringer Distanz), Kontakthäufigkeit (seltener bei regelmäßigem Umgang), Art der Unterhaltsfestlegung (häufiger bei Regelung per Gericht oder Jugendamt) und Erwerbstätigkeit (häufiger bei Erwerbslosigkeit des Unterhaltspflichtigen) auf. Es bestehen ferner Ost-West-Unterschiede. Im Osten bleibt der Unterhalt häufiger aus als im Westen (ebd.: 107). Die Begründungen für ausbleibende Zahlungen durch die Befragten sind je nach Perspektive unterschiedlich gestaltet: Unterhaltsberechtigte nennen im

\footnotetext{
${ }^{18}$ Die Studie wurde vom Bundesministerium für Familie, Senioren, Frauen und Jugend in Auftrag gegeben (Forsa 2002: 159).

${ }^{19}$ Zur genaueren Beschreibung des Untersuchungsdesigns siehe Kap. 7.1 Die deutsche Untersuchung von Forsa.
} 
gleichem Maße die Zahlungsunfähigkeit und -verweigerung des Pflichtigen, während Unterhaltspflichtige häufiger auf finanzielle Probleme verweisen (ebd.: 155).

Für die Erklärung der unterschiedlich ausgestalteten Unterhaltspraxis wird innerhalb der Forsa-Studie ferner eine multiple Analyse durchgeführt, der im Rahmen der Publikation der Ergebnisse jedoch nur ein sehr geringer Raum zukommt. ${ }^{20}$ Es wird lediglich darauf verwiesen, dass die allgemeinen Ergebnisse der deskriptiven Analyse insgesamt bestätigt werden konnten. Unterhaltsprobleme kommen dabei häufiger vor, wenn geringer Kontakt zwischen getrennt lebendem Elternteil und Kind besteht, wenn die Trennung bereits länger zurückliegt, wenn der Unterhalt durch behördliche Instanzen (Gericht oder Jugendamt) und nicht von den Eltern selbst festgelegt wurde und wenn der Unterhaltspflichtige erwerbslos ist (ebd.: 132f.).

Neben dem Finanziellen werden einige Aspekte der sozio-emotionalen Komponente des väterlichen Sorgehandelns nach der Trennung erhoben. Diese dienen jedoch - gemäß der forschungsleitenden Fragestellung der Untersuchung - primär der Erklärung auftretender Unterhaltsprobleme bzw. -zahlungen. Rund 44\% der unterhaltspflichtigen Elternteile teilen sich das Sorgerecht; in 52\% der Fälle hat der Unterhaltsberechtigte das alleinige Sorgerecht; bei 3\% ist das Sorgerecht noch nicht geregelt (ebd.: 165). Die Entscheidung über die Form der Festlegung wird dabei bestimmt durch die frühere Familienkonstellation der Eltern. Das gemeinsame Sorgerecht ist eng an die Ehe gebunden. Bei dem Auftreten gibt es ferner deutliche Unterschiede zwischen Ost- und Westdeutschland: $61 \%$ der ostdeutschen Unterhaltspflichtigen teilen sich die Sorge nicht, während dies im Westen nur 49\% sind. Mit Blick auf den Umgang geben 12\% der Unterhaltspflichtigen an, dass sie keinen Kontakt zum Kind haben (ebd.: 166). Auch hier bestehen Unterschiede zwischen den beiden deutschen Teilen: im Osten treten häufiger Kontaktabbrüche auf als im Westen (ebd.: 61). Des Weiteren besteht ein Zusammenhang zwischen der Familienkonstellation und der Dauer seit der elterlichen Trennung und der Kontakthäufigkeit. Kontaktabbrüche finden sich häufiger bei nicht verheirateten Elternteilen sowie bei den Eltern, die schon länger getrennt sind. Besteht ein gemeinsames Sorgerecht, so bleibt der Abbruch des Kontakts zwischen Kind und unterhaltspflichtigem Elternteil eher die Ausnahme. Der Rückgang des Umgangs wird ferner mit der räumlichen Entfernung zwischen den Wohnorten der Eltern begründet (ebd.: 64ff.). Eine weitere Form des Kontakts, der in der Studie berücksichtigt wird, sind gemeinsam verbrachte Ferien (ebd. 2002: 66f.). Dies

${ }^{20}$ Offen bleibt, wie der Aspekt der Unterhaltsprobleme konkret operationalisiert wurde, ebenso welche Erklärungsgrößen wie in die Berechnungen integriert wurden. Auch das statistische Vorgehen der Analyse wird nicht näher spezifiziert. 
kommt häufiger im Westen vor als im Osten. Seltener besteht Ferienkontakt, wenn die Eltern nie zusammengelebt haben. Öfter werden gemeinsame Urlaube verbracht, wenn die Eltern geschieden sind und/oder ein gemeinsames Sorgerecht besteht. Ferien werden jedoch nicht als Ersatz für einen regelmäßigen Kontakt gesehen. Es kann kein Zusammenhang zwischen der Kontakthäufigkeit und gemeinsamen Urlauben nachgewiesen werden. Neben der Häufigkeit des Umgangs werden die unterhaltspflichtigen Elternteile ferner nach der Ausgestaltung ihrer Besuche befragt (ebd.: 67). Zu den am häufigsten angegebenen Aktivitäten zählen Unterhaltungen, Ausflüge oder Spaziergänge, Spielen, Fernsehen und für die Schule lernen bzw. Hausaufgaben erledigen. Besteht eine geringe Kontakthäufigkeit, werden alltägliche Beschäftigungen seltener genannt.

Insgesamt zeigen die Ergebnisse Unterschiede in den Angaben zwischen Unterhaltspflichtigen und Unterhaltsberechtigten. Während unterhaltsberechtigte Elternteile häufiger Zahlungsschwierigkeiten und seltener Besuche des abwesenden Elternteils angeben, ist dies bei letzteren umgekehrt. Zahlungspflichtige schätzen die Probleme geringer und die Kontakthäufigkeit höher ein. Des Weiteren können deutliche Ost-West-Unterschiede nachgewiesen werden.

\subsubsection{Zusammenfassung der Ergebnisse der Bradshaw-Studie}

Bradshaw und Mitarbeiter ${ }^{21}$ veröffentlichen unter dem Titel “Absent Fathers?” die Ergebnisse ihrer 1995/96 erhobenen Studie zu Nachtrennungsvätern in Großbritannien. Die Untersuchung steht vor dem Hintergrund eines stark ansteigenden Anteils abwesender Väter, einer fast vollständigen Unwissenheit über ihre Lebens- und Rahmenbedingungen sowie der Tatsache, dass sie auf der politischen Agenda aufgrund einer steigenden Sozialhilfeabhängigkeit allein erziehender Mütter zunehmend thematisiert werden (Bradshaw et al. 1999: 2).

Ähnlich wie in der deutschen Studie zeigen die Angaben der britischen Nachtrennungsväter zu ihrer Kontakthäufigkeit ein höheres Niveau als dies in Studien mit Angaben von allein erziehenden Müttern nachgewiesen worden ist. Ein regelmäßiger Kontakt ist dabei wahrscheinlicher, wenn eine freundschaftliche Beziehung zwischen Kindsmutter und Nachtrennungsvater besteht, der Vater erwerbstätig ist, jüngere Kinder betroffen sind, keine „neuen“ Kinder im väterlichen Haushalt leben und die räumliche Entfernung zwischen den elterlichen Wohnorten gering ist. Das Verhältnis zur Kindsmutter ist unbelasteter, wenn der Vater keine neue Beziehung hat. Auch das Leisten von Unterhalts-

${ }^{21}$ Zur Vereinfachung wird im Folgenden auf diese Untersuchung als „Bradshaw-Studie“ verwiesen. 
zahlungen wirkt sich, laut den Ergebnissen, positiv auf die väterliche Kontaktpraxis aus, wobei die Wirkungsrichtung des Zusammenhangs offen bleiben muss. Die Gestaltung des Kontakts umfasst am häufigsten Fernsehen, Spiele, Einkäufe oder Aktivitäten draußen (ebd.: 95ff).

Die Ergebnisse der britischen Studie zeigen ferner, dass rund 57\% der befragten Väter derzeit Unterhalt zahlen. Nachtrennungsväter, die angeben ihren finanziellen Verpflichtungen nachzukommen, sind häufiger erwerbstätig; sind bereits älter als sie das erste Mal Vater werden; haben seltener Ex-Partnerinnen, die Sozialhilfe beanspruchen; haben Kontakt zur Kindsmutter; leisten über den festgelegten Unterhalt hinaus finanzielle Unterstützung und verfügen über eine formale Festlegung des Unterhalts. Als Hauptursache für ausbleibende Unterhaltsleistungen wird insbesondere die väterliche Arbeitslosigkeit und/oder Zahlungsunfähigkeit angeführt. Väter, die ihre Zahlungen eingestellt haben, begründen dies häufig mit dem Verlust des Arbeitsplatzes. Des Weiteren wurde das jeweilige Potential, Unterhalt leisten zu können, der Väter untersucht, die ihren finanziellen Verpflichtungen im Zeitpunkt der Befragung nicht nachkommen. Nur rund 9\% wiesen eine gewisse Zahlungsfähigkeit auf, doch nur ein Drittel dieser Väter hatte regelmäßigen Kontakt zu seinen Kindern. Die Ergebnisse verweisen einmal mehr auf den Zusammenhang zwischen finanziellen und sozio-emotionalen Aspekten väterlichen Sorgehandelns. Des Weiteren machen sie darauf aufmerksam, dass die unterhaltspflichtigen Nachtrennungsväter nur bedingt über weitere finanzielle Ressourcen verfügen, aus denen Unterhalt gezahlt werden könnte (ebd.: 144).

\subsubsection{Zusammenfassung der Ergebnisse der NOVA-Studie}

Die norwegische Untersuchung „Samværfedrenes situasjon: økonomiske og normative problemstillinger“, 22 2001/2002 durchgeführt vom Forschungsinstitut NOVA ${ }^{23}$, deckt im Wesentlichen drei Aspekte der Vaterschaft nach der elterlichen Trennung ab: den Kontakt zwischen abwesendem Elternteil und Kind, die Einstellung zu und die Praxis von Unterhaltszahlungen sowie die Lebensumstände der Befragten. Dabei wird explizit die Perspektive der Nachtrennungsväter eingenommen (Skevik/Hyggen 2002: 125).

Auch im norwegischen Fall gibt die Mehrheit der Väter an, dass sie einen regelmäßigen Umgang mit ihren Kindern pflegen. Nur 10\% haben ihr Kind im letzten halben Jahr nicht

\footnotetext{
${ }^{22}$ Frei zu übersetzen als „Situation von Teilzeitvätern - finanzielle und normative Problemstellungen“.

${ }^{23}$ NOVA steht kurz für „Norsk Institutt for Forskning om oppvekst, velferd og aldring“. Die Studie wurde finanziert vom norwegischen Ministerium für Kinder und Familien (Skevik/Hyggen 2002: 125).
} 
gesehen, 2\% hatten noch nie Kontakt. Dabei wird der Mangel an direktem Umgang nicht durch postalischen, elektronischen oder telefonischen Kontakt kompensiert. Der fehlende Kontakt innerhalb des letzten Monats wird primär mit drei Argumenten begründet: die Kindesmutter will keinen Umgang, die Entfernung zwischen den elterlichen Wohnorten ist zu groß und die Reisekosten können nicht aufgebracht werden (Skevik/Hyggen 2002: 126). Dennoch spielt bei der Mehrheit der Fälle die Distanz keine Rolle, weil die Eltern innerhalb einer Nachbarschaft leben. Die norwegischen Ergebnisse zeigen, dass die Anzahl der gemeinsam verbrachten Tage von der räumlichen Entfernung zwischen den Wohnorten (stärkerer Kontakt bei geringer Distanz), dem Alter des Kindes (mehr Kontakt zu jüngeren Kindern), der Familienkonstellation (Ehe oder Zusammenleben vor der Trennung fördert Kontakt danach), der Dauer seit der Trennung (mit zeitlichen Abstand sinkt der Kontakt), der Festlegung des Umgangs (einseitige Entscheidungen gehen zu Lasten des Kontakts), sowie der jeweiligen Partnersituation der Eltern (eine neue Partnerschaft wirkt negativ auf die Kontakthäufigkeit) abhängen (Skevik/Hyggen 2002: 126). Innerhalb einer linearen Regression ist der Einfluss des Alters des Kindes sowie des Vaters, die Partnersituation beider Elternteile, die Einigkeit über die elterliche Trennung, der Familienstand vor der Trennung, die Dauer der Partnerschaft sowie die Entfernung zwischen den elterlichen Wohnorten simultan überprüft worden. Das Gesamtmodell trägt zu 28\% der Varianzaufklärung mit Blick auf die Anzahl der gemeinsam verbrachten Tage zwischen getrennt lebendem Vater und Kind bei. Als signifikant erweisen sich dabei die Partnersituation der Mutter, das Kindesalter, das Zusammenwohnen der Eltern vor der Trennung sowie die Entfernung zwischen den Wohnorten (ebd.: 133). Bei der Ausgestaltung des Kontakts scheinen norwegische Trennungsväter die Besuche ihrer getrennt lebenden Kinder in ihren eigenen Alltag zu integrieren. Sie schauen mit ihren Kindern fern, gehen einkaufen, kochen und erledigen Hausarbeit, unternehmen Spaziergänge und spielen. Des Weiteren wird Zeit mit der neuen Familie des Vaters sowie den Großeltern verbracht (ebd.: 127).

In einem Aufsatz stellt Skevik (2006a) über die erste Analyse hinaus - die in einem in Norwegisch verfassten Ergebnisbericht dargestellt wurde - die Determinanten der beiden Kontaktextreme dar: die Einflussgrößen des Kontaktabbruchs werden denen eines regelmäßigen, sehr intensiven Kontakts gegenübergestellt. Die Ergebnisse der multiplen Regressionen weisen andere Determinanten für den Kontaktabbruch auf, als sie für einen intensiven Umgang ausgemacht werden. Regelmäßiger Kontakt wird bestimmt durch eine formale Festlegung des Umgangs, die elterliche Kohabitation vor der Trennung, ein geringes Kindesalter, relativ nahe Wohndistanz und die Abwesenheit eines Partners an der Seite der Mutter. Ein Abbruch des Kontakts wird begünstigt durch eine große räumliche Entfernung zwischen den elterlichen Wohnorten (sofern diese über 6 Stunden Reisezeit erfordert), einen neuen Partner der Mutter, die fehlende Kohabitation der Eltern vor der 
Trennung sowie durch den Sozialhilfebezug des Vaters. Die Beziehungssituation des Vaters ist dagegen nicht von Bedeutung für den Kontakt (Skevik 2006a: 125ff.).

Innerhalb des Untersuchungsberichts werden neben der Kontakthäufigkeit und der Ausgestaltung der Besuche ferner finanzielle Aspekte des Nachtrennungshandelns dargestellt. Norwegische Väter tendieren dazu, ihre Zahlungsverpflichtungen für zu hoch einzuschätzen. Darüber hinaus geben die Befragten häufig finanzielle Probleme an. Rund 25\% haben häufig und 25\% haben manchmal Schwierigkeiten, ihre laufenden Kosten aufzubringen. Der Anteil der Väter mit finanziellen Problemen ist höher als in anderen Bevölkerungsgruppen - mit Ausnahme allein erziehender Mütter (Skevik/Hyggen 2002: 127). Eine multiple Analyse hat ergeben, dass das Risiko finanzieller Probleme unter Nachtrennungsvätern bestimmt wird durch ein junges Alter des Vaters, den Mangel an Vollzeitbeschäftigung, einem niedrigen Einkommen und einer kurzen zeitlichen Distanz zur Trennung (ebd.: 128). Die weitere Analyse der Daten - über den Ergebnisbericht hinaus hat gezeigt, dass die väterliche Armut ein starker Indikator für den Kontaktverlust zwischen getrennt lebendem Vater und seinem Kind darstellt (Skevik 2006a: 114, 121).

Skevik (2006a) liefert in ihrem Aufsatz zur Bestimmung der Determinanten der beiden Extreme des väterlichen Kontakts als eine der wenigen Arbeiten in der (Nachtrennungs-) Väter-Forschung explizit theoretisch fundierte Annahmen, die am empirischen Material geprüft werden. Dabei entwickelt die Autorin drei Hypothesen basierend auf drei unterschiedlichen Theorien. Zunächst wird in Anlehnung an Becker (1981) von einem spezifischen Kapital ausgegangen, das Männer und Frauen innerhalb der Institution Ehe akkumulieren. Dabei gilt: je höher dieses Kapital ist, desto höher fallen auch die Investitionen in die Ehe aus und desto stärker ist die Entschlossenheit die Nähe zu den Kindern zu erhalten, die daraus entstanden sind. Dahinter steht die Annahme, dass Väter, die die Verpflichtung einer Ehe eingegangen sind, eher die Verbindlichkeit gegenüber ihren Kindern - entstanden aus dieser Ehe - wahrnehmen als uneheliche Väter. Die "marital involvement“-Hypothese, wie sie von Skevik aufgestellt wird, nimmt daher an, dass geschiedene Väter sich stärker nach der Trennung engagieren als vormals nicht verheiratete.

Als zweites vertritt die Autorin die "social parenting perspective“. Diese nimmt die Bedeutung der aktuellen Partnersituation beider Elternteile in den Blick. Die These lautet, dass der Kontakt zwischen Vater und Kind abnimmt, wenn dieser eine neue Beziehung eingegangen ist. Dieser Effekt wird vermutlich verstärkt, wenn die neue Partnerin weitere Kinder in die Partnerschaft mitbringt. Laut Hypothese wird damit die soziale Elternschaft innerhalb einer Haushaltsgemeinschaft über die biologische Elternschaft gestellt, die über Haushaltsgrenzen hinweg gestaltet werden muss. Die neue Beziehung ist für den Vater mit zeitlichen Restriktionen verbunden, die die Kontaktroutinen mit getrennt lebenden Kindern stören. Nach Skevik führen theoretische Überlegungen zur Bedeutung der mütter- 
lichen Partnersituation zu widersprüchlichen Annahmen. Zum einen kann die Mutter ein Interesse daran haben, dass die Kinder aus früheren Beziehungen mehr Zeit mit ihrem biologischen Vater verbringen, um damit zeitliche Freiräume für ihre neue Partnerschaft zu schaffen. Andererseits kann es ihr Ziel sein, den leiblichen Vater und seine Funktion innerhalb der getrennten Familie durch die Person des neuen - anwesenden - Partners zu ersetzen. Damit kann sie unangenehmen Kontakt zum Ex-Partner vermeiden. Die Autorin vermeidet es an dieser Stelle eine klare theoretische Position zu beziehen. Sie geht jedoch von der Bedeutung der mütterlichen Partnersituation - in welche Richtung auch immer aus (Skevik 2006a: 117ff.).

Im dritten Ansatz folgt Skevik (ebd.: 121) der "socioeconomic-advantaged parenting perspective“. Dabei geht sie davon aus, dass das Einkommen und das Bildungsniveau starke Einflussfaktoren für das Ausmaß väterlichen Kontakts sind. Insbesondere hohe Reisekosten müssen finanziert werden. Kindliche Besuche beim Vater sind darüber hinaus mit Kosten verbunden. Hinzu kommen immer noch stark ausgeprägte Erwartungen vieler Männer, dass “fathering“ mit "providing“ eng verbunden ist. Männer mit sozio-ökonomischen Schwierigkeiten, so die theoretische Annahme, brechen eher den Kontakt ab.

\subsection{Einordnung der Fragestellung in die aktuelle Diskussion}

Die Ausführungen zur Literatur der Vaterforschung weisen auf die Ambivalenz der Diskussion und der empirischen Ergebnisse hin. Einerseits wird zunehmend eine väterliche Abwesenheit oder gar eine Vaterlosigkeit postuliert, zum anderen findet sich eine verstärkte wissenschaftliche Auseinandersetzung mit „neuen“ Väterformen. Viele Studien beziehen sich primär auf die erlebte und weniger die gelebte Vaterschaft, wie die unterschiedlichen Vaterschaftskonzepte deutlich machen. Die alltägliche Ausgestaltung bleibt häufig dahinter verborgen. Die dargestellte Ambivalenz betrifft insbesondere die Situation getrennt lebender Väter. Auch sie sehen sich zunehmend mit gesteigerten Erwartungen an eine aktive Vaterschaft konfrontiert und stellen auch selbst gesteigerte Anforderungen an ihr Sorgehandeln; gleichzeitig verbringen sie jedoch aufgrund der räumlichen Trennung weniger Zeit mit ihren Kindern und verlieren ein Stück Alltäglichkeit. Insgesamt liegen nur wenige und wenig systematische Erkenntnisse darüber vor, wie Männer zu (aktiv handelnden) Vätern werden oder was sie vom Vatersein und aktiver Vaterschaft abhält (Hobson 2002; Knijn et al. 2007: 191; Ostner 2002: 150; Walter 2002b: 50). Trotz des Anstiegs des Anteils wissenschaftlicher Beiträge sowohl über allein erziehende Elternteile als auch über Vaterschaft, bestehen immer noch wenig gesicherte Erkenntnisse über getrennt lebende Väter. Das Wissen über ihr väterliches Sorgehandeln sowie dessen Rahmenbedingungen bleibt fragmentiert und wenig systematisiert (Forsa 2002: 26; Depner/Bray 1993b: 182; Walter 2002b: 50). Ferner konzentrieren sich die wenigen Studien, die sich mit den abwesenden Vätern auseinandersetzen, entweder auf bestimmte so- 
ziale Gruppen wie z.B. Ehepaare, bestimmte Altersklassen der Kinder, oder die Erfassungs(zeit)räume sind begrenzt. Neben methodischen Einschränkungen nehmen einige Studien eine inhaltlich restringierte Perspektive ein. So ist kaum eine Studie bekannt, die in ausreichendem Umfang Determinanten des väterlichen Sorgeverhaltens untersucht hätte (Jaursch 2003: 215; Depner/Bray 1993b: 182). Auf der anderen Seiten - der Erklärungsgröße - integrieren Untersuchungen häufig nur Teilaspekte väterlichen Sorgehandelns; ökonomische und sozio-emotionale Aspekte müssen jedoch verstärkt gemeinsam berücksichtigt werden (Forste 2002: 596). Methodisch basieren die dargestellten Untersuchungen entweder auf qualitativen Interviews (Matzner 2004; Moch 2002, Napp-Peters 1995) oder quantitativen Daten, die Mängel aufweisen (Amendt 2004).

Die Datensätze, die der vorliegenden Untersuchung als Datengrundlage dienen, stellen dabei die wenigen quantitativ erhobenen Ausnahmen dar. Sie verfügen über Datenmaterial, das sowohl die Betrachtung des väterlichen Care als auch des Cash ermöglicht. Dabei weicht die Perspektive der folgenden Untersuchung in einigen Teilen von denen der Primäranalysen ab. Insgesamt konzentriert sich die Forsa-Studie gemäß ihrer Fragestellung auf die ökonomische Dimension väterlichen Sorgehandelns. Die Analyse bleibt dabei v.a. der bivariaten Ebene, dargestellt in einfachen Kreuztabellen, verhaftet. Neben den finanziellen Regelungen werden zwar auch Aspekte des Sorgerechts und der Umgangsregelung berücksichtigt, doch diese werden primär als Erklärungsfaktoren (nicht) ausbleibender Unterhaltszahlungen verwendet. Die Studie bleibt eine theoretische Fundierung ihrer Analyse schuldig. Des Weiteren steht nicht explizit die Perspektive der getrennt lebenden Väter im Vordergrund, sondern vielmehr die Gegenüberstellung der Angaben von unterhaltsberechtigten und -pflichtigen Elternteilen nach der Trennung unabhängig von ihrem Geschlecht.

Innerhalb der britischen Analyse liegt der Fokus bei der sozio-emotionalen Komponente väterlichen Sorgehandelns auf der Erklärung eines regelmäßigen Kontakts. Kontaktabbrüche werden darüber hinaus nicht gesondert untersucht. Mit Blick auf die finanziellen Aspekte werden die Ergebnisse der drei Gruppen von Zahlungspraktiken gegenüber gestellt. Es wird differenziert zwischen derzeit zahlenden, früher zahlenden und nie zahlenden Vätern. Dabei wird auf multipler Ebene v.a. die Gruppe der aktuell Unterhaltsleistenden analysiert. Die Untersuchung der Väter, die ihrer finanziellen Verpflichtung nicht (mehr) nachkommen, bleibt rein deskriptiv. Anders als in der Forsa-Studie wird in der britischen Ergebnisdarstellung der multiplen Analyse stärkere Bedeutung beigemessen.

Entgegen der britischen und deutschen Studie werden im norwegischen Fall nicht die finanziellen Aspekte bei der Analyse in den Vordergrund gestellt. Unterhaltsprobleme werden gar nicht betrachtet; die Analyse wird auf finanzielle Schwierigkeiten der Trennungsväter generell beschränkt. Diesen kommt jedoch in der Darstellung der Ergeb- 
nisse nur eine untergeordnete Bedeutung zu. Die Kontakthäufigkeit in Form gemeinsam verbrachter Tage wird ausführlicher analysiert.

Die folgende Untersuchung wird explizit und systematisch beide Dimensionen väterlichen Sorgehandelns in Form von Care und Cash in den Blick nehmen. Das Care-Konzept wird dabei über die bisher rein quantitative Operationalisierung des Kontakts hinaus qualitative Aspekte, wie z.B. die Ausgestaltung des Kontakts, integrieren. Methodisch werden sowohl bivariate als auch multiple Analysen vorgenommen. Die bisherigen Ergebnisse in allen drei Datensätzen wurden primär auf bivariater Ebene dargestellt. Die z.T. durchgeführten multiplen Berechnungen werden nur unzureichend erläutert. Anders als in den bisherigen Analysen üblich dient die empirische Untersuchung hier explizit der Testung theoretisch fundierter Hypothesen als Teil einer Gesamttheorie.

Insgesamt schließt die vorliegende Arbeit damit an folgende Desiderate an:

- das Fehlen der väterlichen Perspektive

- die systematische Untersuchung väterlichen Sorgehandelns,

- die Differenzierung in Form von Care und Cash,

- die explizite Überprüfung von theoretisch fundierten Hypothesen in unterschiedlichen sozialen Kontexten,

- $\quad$ sowie die Analyse quantitativer Individualdaten auf bivariater und multipler Ebene.

Zur Stärkung der väterlichen Perspektive, die in der bisherigen Literatur häufig vernachlässigt worden ist, geht die Untersuchung explizit von den Angaben der Nachtrennungsväter aus. Das bedeutet, es werden Informationen der Akteure zu ihrem eigenen Handeln analysiert. Einige Studien gehen aufgrund der traditionellen Arbeitsteilung davon aus, dass Mütter die besseren Informantinnen mit Blick auf die familiale Alltagspraxis seien (Snarey 1993: 43). Die Konzentration auf spezifische Familienmitglieder widerspricht jedoch der traditionellen familiensoziologischen Herangehensweise (Kjeldstad 2000: 352). Gegen die Fokussierung allein auf väterliche Angaben kann ferner kritisch angeführt werden, dass die Perspektive der allein erziehenden Mütter zusätzlich notwendig sei, um ein gesichertes Gesamtbild des väterlichen Sorgehandelns zu erhalten (Hill 2005: 176). So zeigen die empirischen Ergebnisse starke Unterschiede im Niveau väterlichen Sorgehandelns sowohl beim Care als auch beim Cash, je nachdem welche elterliche Perspektive eingenommen wird (Braver et al. 1993; Bradshaw et al 1999; Skevik 2006a: 122; Marsiglio et al. 2000: 1180; Forsa 2002). Andere fordern die Untersuchung der Bedürfnisse, Wünsche und Forderungen aller beteiligten Familienmitglieder (Eltern wie Kinder), wenn aus den Forschungsergebnissen Handlungskonsequenzen abgeleitet werden sollen. 
Eine Optimierung der Rahmenbedingungen aus Sicht der Kinder ist noch lange keine für die Eltern und umgekehrt (Marsiglio et al. 2000: 1179).

Den Einwänden wird an dieser Stelle entgegengehalten, dass das väterliche Engagement in seiner Einzigartigkeit und nicht in Relation zum mütterlichen Sorgehandeln betrachtet werden muss. Die Angaben der Männer werden hier als valide männliche Erfahrungen akzeptiert - nicht mit den Geschichten der ehemaligen Partnerinnen und Mütter verglichen oder evaluiert (Smart/Neale 1999: 42; Fthenakis 1999: 40). Für den Vater und sein Handeln ist das relevant, was er selbst wahrnimmt. Das bedeutet, im Moment der Handlung sind für den Vater die subjektiven, jeweils real vorliegenden, wenn auch möglicherweise falsch wahrgenommenen Situationsbedingungen relevant (Esser 1999: 63). Zur Erklärung des väterlichen Sorgehandelns ist damit die Perspektive der Kindsmutter nur insofern von Bedeutung, wie sie selbst das Handeln mitbestimmt, indem sie beispielsweise den Kontakt zwischen Vater und Kind ermöglicht bzw. verhindert. Doch auch wenn Mutter und Kind den Kontakt zulassen müssen, sind es primär die Väter, die darüber entscheiden, wie sie sich verhalten (wollen). Des Weiteren ist anzumerken, dass sich die Analyse dem väterlichen Sorgehandeln über die reine Kontakthäufigkeit hinaus widmet. Zur Ausgestaltung des Kontakts zwischen getrennt lebenden Vater und Kind kann die Kindsmutter aufgrund ihrer fehlenden Anwesenheit selten ausreichend Auskunft geben. 


\section{TEIL II: THEORETISCHER RAHMEN}

\section{Die Handlungstheorie}

Ziel der Arbeit ist es nicht, das konkrete Verhalten eines bestimmten Vaters zu erklären. Vielmehr geht es um die Analyse generalisierten Handelns von Nachtrennungsvätern. Das bedeutet, es werden unterschiedliche Verhaltensmuster auf der Makro-Ebene ausgemacht und untersucht. Diese generellen Muster basieren auf individuellen Handlungsentscheidungen und -umsetzungen. Das entspricht den Annahmen des methodologischen Individualismus, nach dem die individuellen Akteure die ursprüngliche Quelle und die ausführenden Träger jeglichen Handelns darstellen (Lindenberg 1981: 20; Esser 1999: 27; Schimank 2004: 293; Greshoff/Schimank 2003: 3; Himmelweit 2002: 232). Selbst die Relation zwischen zwei Variablen, die auf kollektiver Ebene empirisch nachweisbar sind, kann nach diesem Grundtheorem nicht auf der Makro-Ebene bestimmt werden; vielmehr ist eine Mikrofundierung makro-soziologischer Phänomene notwendig (u.a. Esser 1999: 11; Miebach 2006: 397, 409; Kunz 2004: 25; Schmid 2004: 88). Die folgenden theoretischen Überlegungen stellen daher die generellen Beweggründe der Väter dar, nach der elterlichen Trennung den Kontakt zu ihrem Kind (nicht) aufrechtzuerhalten bzw. ihren Unterhaltsverpflichtungen (nicht) nachzukommen. Die individuellen Väter werden als Handlungseinheiten betrachtet.

Mit Hilfe eines allgemeinen Handlungsmodells werden Determinanten väterlichen Sorgehandelns in Nachtrennungsfamilien abgeleitet. Jedoch sind nicht alle Väter in ihrem Handeln gleich - es gibt nicht das väterliche Sorgehandeln - dennoch kann ihr Handeln nach gleichen Kriterien nachvollzogen werden. Die Analyse sozialen Handelns stellt daher einen Versuch dar, die vorliegenden subjektiven Ziele und Mittel der Akteure objektiv richtig zu (re-)konstruieren (Esser 1999: 203). Dabei wird die Mikrofundierung primär für die Generierung theoretisch plausibler Annahmen über relevante Einflussgrößen genutzt. Das bedeutet, die im Folgenden ausführlich dargestellte Theorie dient ausschließlich dem Aufstellen empirisch überprüfbarer Hypothesen. Damit ist es zweitrangig, inwieweit die entwickelte Theorie die Realität tatsächlich abbildet; vielmehr ist entscheidend, dass sie theoretisch nachvollziehbare Anhaltspunkte für die Suche nach den Bestimmungsfaktoren väterlichen Sorgehandelns in Nachtrennungsfamilien liefert (Kunz 2004: 151; Esser 1999: 249; Hill/Kopp 2004: 131). Sie dient als analytisches Instrument zur Reduktion der sozialen Komplexität.

Wie andere Teildisziplinen leidet auch die internationale Familiensoziologie an einem Mangel an sozialwissenschaftlich erklärenden Theorien (Smart/Neale 1999: 2; Matzner 
2004: 18; Braver et al. 1993: 87). Die vorliegende Analyse basiert daher auf der allgemeinen Handlungstheorie der rationalen Wahl - die nicht speziell für familiales Handeln entwickelt worden ist. ${ }^{24}$ Der Erklärungsansatz findet seine Ursprünge in der (neoklassischen) ökonomischen Theorie. Insbesondere bei der Erklärung ökonomischen Handelns, wie z.B. Kaufentscheidungen, wird das Menschenbild des homo oeconomicus unterstellt. Nach einer Phase der Resistenz gegen die Annahmen des Modells fanden sie letztlich doch Eingang in soziologische Theorien und erfreuen sich besonders in der jüngsten Entwicklung sozialwissenschaftlicher Debatten zunehmender Beliebtheit. Dabei ist die ursprüngliche „harte“ Version der Rational Choice Theorie (kurz: RC) durch zahlreiche Erweiterungen und Modifizierungen soziologisch „fit“ gemacht worden. Derzeit besteht innerhalb der Sozialwissenschaften eine große Vielfalt theoretischer Ansätze, die sich dem Rational Choice Paradigma verpflichtet sehen. ${ }^{25}$

Im Folgenden wird rationales Handeln verstanden als:

- $\quad$ eine Entscheidung zwischen Handlungsalternativen

- $\quad$ bestehend aus dem Abwägen von Konsequenzen, die mit Kosten und Nutzen verbunden sind, und

- $\quad$ die basierend auf (subjektiven) Bewertungen (Präferenzen und Interessen) und Erwartungen evaluiert werden.

${ }^{24}$ Eine ausführliche Diskussion der Anwendbarkeit rationaler Handlungsannahmen auf familiales Handeln findet sich in Kap. 4.3.2 Familiales Handeln als rationale Abwägung? - Eine kritische Betrachtung.

${ }^{25}$ Die im Folgenden dargestellten Prämissen repräsentieren das Grundmodell, über das in der Literatur relative Einigkeit herrscht (u.a. Opp 1999). Darüber hinaus gibt es zahlreiche Varianten die in Zahl und Form der Zusatzannahmen variieren. Unterschiede finden sich in Hinblick auf die entscheidungstheoretischen Annahmen. Es werden verschiedene Entscheidungsregeln modelliert, nach denen Akteure zwischen Handlungsalternativen auswählen. Damit verbunden sind je unterschiedliche Vorstellungen darüber, was für einen Akteur „bestmöglich“ heißt (Schnabel 2006: 177; Kunz 2004: 14). Des Weiteren ist der Umgang mit Entscheidungen unter Unsicherheit in der Literatur umstritten (u.a. Brüderl 2004: 166). Besonders im Vergleich zu den ökonomischen Ursprüngen der RC-Theorie lassen sich innerhalb sozialwissenschaftlicher Varianten entscheidende Unterschiede in den Annahmen finden. So werden beispielsweise Restriktionen und Ressourcen innerhalb der ökonomischen Version vornehmlich auf ökonomisch-materielle Bedingungen begrenzt anstelle der Berücksichtigung umfassender Norm-Wert-Systeme, wie sie sich in den Sozialwissenschaften finden (Opp 1999: 173ff.; Burkart 1994: 39). Auf die wesentlichen Unterschiede zwischen den ökonomischen und den hier vereinfacht als sozialwissenschaftliche Variante zusammengefassten „,weicheren“ Versionen der RC-Theorie wird an den gegebenen Stellen hingewiesen. Die Unterschiede der einzelnen sozialwissenschaftlichen Ansätze liegen in Details, denen hier keine entscheidende Bedeutung beigemessen wird. Die im Wesentlichen auf die Kernannahmen der Rational Choice-Theorie beschränkten theoretischen Prämissen werden hier als ausreichend erachtet, väterliches Sorgehandeln zu erklären. Auf zusätzliche Annahmen wird zugunsten der vorhandenen Sparsamkeit des Modells verzichtet. Aufgrund der eingeschränkten Datenlage führt eine theoretische Verkomplizierung des Modells zu wenig zusätzlichem Analysegewinn. 
- Die Auswahl einer Handlung erfolgt nach dem Prinzip der Nutzenmaximierung (u.a. Esser 1999: 199, 248; Schmid 2004: 149; Hill/Kopp 2004: 126f.).

Dabei wird ein weites Verständnis von Rationalität angenommen, das neben ökonomischen Präferenzen, Restriktionen und Ressourcen auch soziale Komponenten zulässt (Opp 1999: 174; Lindenberg 1981: 25). Das genaue Konzept der hier zugrunde gelegten Rationalität wird in den folgenden Abschnitten anhand des von Kühnel und Bamberg (1998) entwickelten zweistufigen Modells rationaler Handlungen konkretisiert und auf das väterliche Sorgehandeln übertragen. ${ }^{26}$

\subsection{Das individuelle Handlungsmodell}

Die Autoren Kühnel und Bamberg (1998) gehen in ihrem Handlungsmodell, dargestellt in Abbildung. 4.1, von zwei Entscheidungsschritten aus. Dies unterscheidet sie wesentlich vom klassischen Rational Choice-Denken insbesondere der Ökonomen, die nur eine Entscheidung für oder gegen eine Handlung annehmen. Dieser konkreten Auswahl zwischen Handlungsalternativen schalten Kühnel und Bamberg einen Schritt vor: die Festlegung des Entscheidungsrahmens bestehend aus der Festlegung der relevanten Handlungsalternativen und den Entscheidungskriterien. Dieser Rahmungsprozess wird im Modell als Frame bezeichnet. Bestimmt wird dieser erste Schritt durch das Überzeugungssystem des Akteurs basierend auf individuellen Werten und Einstellungen, der sozialen Umwelt, die die Beziehungen und sozialen Einflussnahmen umfasst sowie der physischen Umwelt, die sich aus Ressourcen und Restriktionen zusammensetzt. ${ }^{27}$ Beide Umwelten verweisen auf den Kontext- und Situationsbezug individuellen Handelns. So bestimmen relativ konstante Rahmenbedingungen - hier in soziale und physische Umwelt differenziert - unter variablen Wahrnehmungs- und Beeinflussungsbedingungen die unterschiedlichen Reaktionsweisen der Individuen (Schmid 2004: 148; Esser 1999: 27).

Die Verbindung zwischen individuellem Akteur und sozialem Kontext - im weitesten Sinne auf der makro-soziologischen Ebene - wird auch in Form der berühmten „Badewanne“ von McClelland (1961: 47), dem Standardmodell soziologischer Erklärungen,

\footnotetext{
${ }^{26}$ Die folgenden Ausführungen lassen sich theoretisch wie inhaltlich in weiten Teilen auf väterliches Sorgehandeln generell - auch in sog. Kernfamilien oder als soziale Vaterschaft - übertragen. Einige Aspekte gelten jedoch explizit für Väter und ihr Handeln in Nachtrennungsfamilien. Nachstehende Darstellung basiert auf der Perspektive abwesender Väter - unabhängig von ihrer Gültigkeit für andere Vaterschaftsformen. Es wird nicht explizit zwischen den einzelnen Formen väterlichen Sorgehandelns differenziert, d.h. es wird nicht weiter auf die (Nicht-)Gültigkeit der theoretischen Überlegungen für andere Vaterformen verwiesen.

${ }^{27}$ Die einzelnen Elemente des Modells werden in den folgenden Abschnitten im Detail dargestellt.
} 
dargestellt (u.a. Brüderl 2004: 175; Kunz 2004: 25; Miebach 2006: 396; Esser 1999: 17). Das Modell umfasst drei Schritte: die Logik der Situation als Verknüpfung der Makro- mit der Mikroebene, die Logik der Selektion auf der Akteursebene sowie die Logik der Aggregation als analytischer „Rückschritt“ von der Mikro- auf die Makroebene. ${ }^{28}$ Zwei der drei Schritte der soziologischen Erklärung finden sich im zweistufigen Modell von Kühnel und Bamberg wieder. Der von den Autoren als "Framing“ bezeichnete erste Schritt entspricht der Logik der Situation (innerhalb der Abbildung durch den größeren, dunkelgrauen Kasten links dargestellt, der die entsprechenden Elemente verbindet). Die Auswahl zwischen Handlungsmöglichkeiten nach einer Gesetzmäßigkeit im zweiten Modellschritt von Kühnel/Bamberg entspricht der Logik der Selektion (kleinerer, dunkelgrauer Kasten mittig im Modell).

Nach dem Modell der soziologischen Erklärung ist der Ausgangspunkt die Makroebene, eine soziale Situation mit ihren objektiven Bedingungen und Strukturen (Greshoff/Schimank 2003: 4). Innerhalb der Logik der Situation wird der Bezug zwischen situativen sozialen Bedingungen und den handelnden Individuen hergestellt (Kunz 2004: 26). Es wird rekonstruiert, welche Aspekte der Handlungssituation für die Akteure relevant sind, welche Interessen und Erwartungen sie verfolgen, sowie welche Handlungsoptionen sich ihnen bieten (Kunz 2004: 27; Esser 1999: 25).

Sowohl bei Kühnel und Bamberg als auch im Modell der soziologischen Erklärung wird zwischen einer individuellen Akteursebene und einer „sozialen Situation“ differenziert. Letztere kann dabei je nach Abstraktionsgrad mit unterschiedlichen Inhalten gefüllt werden. Das bedeutet, der Kontext, in dem der individuelle Vater handelt, umfasst sowohl wohlfahrtsstaatliche Institutionen, z.B. in Form von Gesetzen oder Normen, die im Folgenden als makro-soziale Ebene verstanden werden, als auch das soziale und physische

\footnotetext{
${ }^{28}$ Vom individuellen Handeln auf der Mikroebene wird die Perspektive im dritten Schritt der soziologischen Erklärung wieder auf die Makroebene gelenkt. Das Handeln zahlreicher individueller Akteure schließt aneinander an und fügt sich auf diese Weise zu einem aggregierten Ergebnis zusammen (Greshoff/Schimank 2003: 10). Mit Hilfe von Transformationsregeln werden die individuelle Handlungen mit dem kollektiven Explanandum verbunden, indem sie angeben, wann und wie bestimmte individuelle Handlungen zu bestimmten - häufig individuell nicht intendierten - kollektiven Effekten führen (Kunz 2004: 17, 28; Miebach 2006: 400; Esser 1999: 16). Die Art der Regeln kann sich mehr oder weniger komplex gestalten. Die einfachste Form stellt die simple Kumulation von Einzelhandlungen dar, die sich in formalen Operationen eines statistischen Mittel- oder Prozentwertes ausdrücken (Miebach 2006: 410; Brüderl 2004: 176). Ein Beispiel stellt hier die Geburtenrate als einfache Aufsummierung zahlreicher individueller Entscheidungen der Familienplanung dar. Die bisherigen Ausführungen haben deutlich gemacht, dass der zentrale Punkt der theoretischen Überlegungen zur Erklärung väterlichen Sorgehandelns gemäß dem methodologischen Individualismus die individuellen Handlungen der einzelnen Väter darstellt. Der Fokus der Untersuchung liegt damit weniger auf dem dritten Schritt der soziologischen Erklärung, der Logik der Aggregation, als vielmehr auf den ersten beiden Schritten, der Logik der Situation und der Selektion.
} 
Nahumfeld wie es bei Kühnel/Bamberg gemeint zu sein scheint. Beide Dimensionen wirken sich als Kontextbedingungen auf das Handeln aus, jedoch in unterschiedlichen Wirkungsgraden. Innerhalb der hier verwendeten Theorie wird in erster Linie vom Nahumfeld des Akteurs - eingebettet in einen makro-sozialen Kontext - ausgegangen. ${ }^{29}$

\section{Abb. 4.1: Individuelles Handlungsmodell - die Logik der Situation und Selektion.}

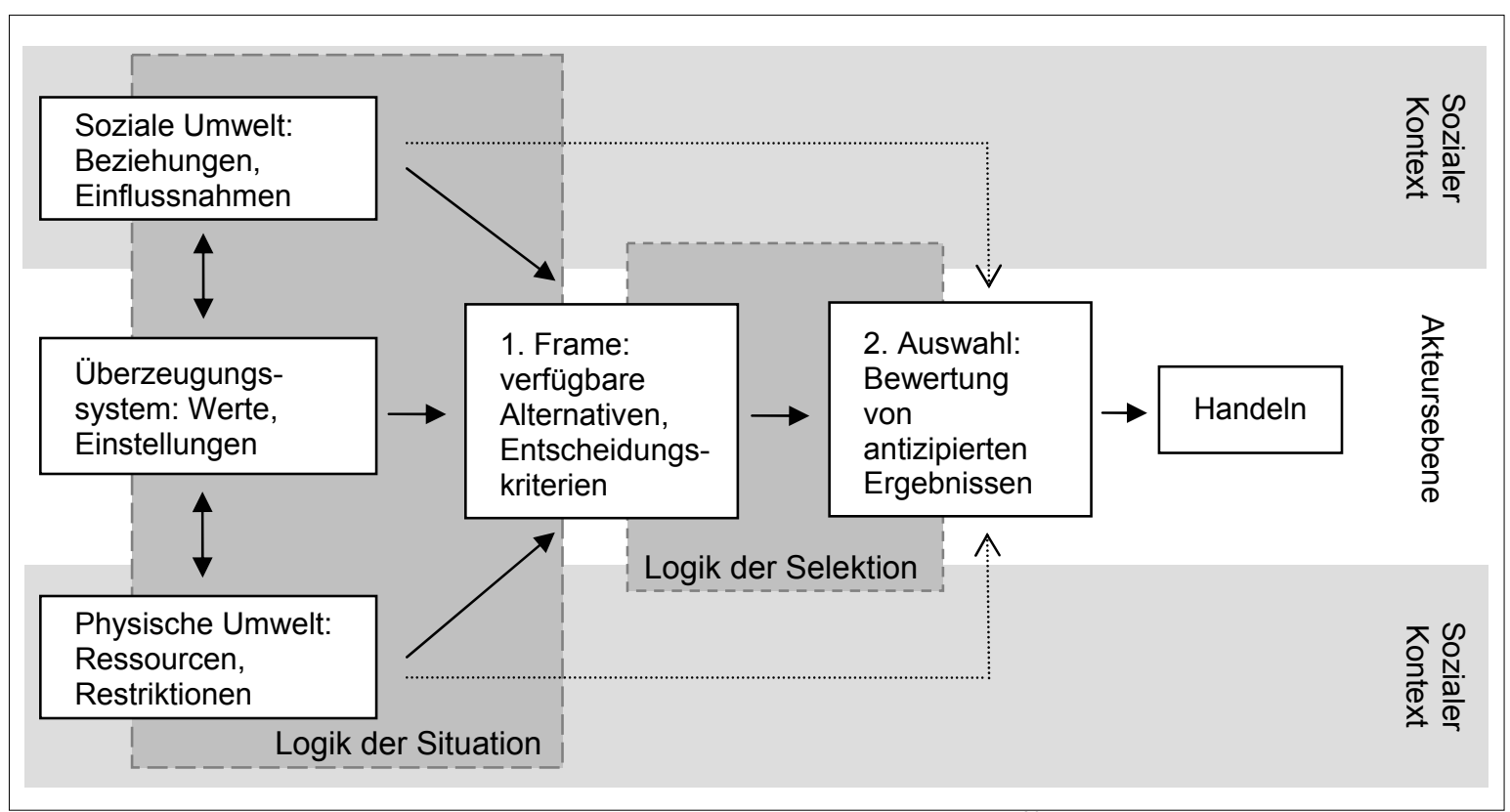

Quelle: in Anlehnung an Kühnel/Bamberg 1998: 258, eigene Erweiterung. ${ }^{30}$

Nach der Rahmung der Situation erfolgt in einem zweiten Schritt die Auswahl einer Handlungsalternative, die auf der Bewertung von antizipierten Handlungsergebnissen beruht. Dies entspricht dem klassischen Verständnis der Theorie der rationalen Wahl bestehend aus einer Entscheidung. Kühnel/Bamberg (1998: 256) gehen von einem rationalen Entscheidungsmodell auf beiden Stufen ihres Modells aus. Situationen sind komplexe Phänomene und bieten den Akteuren vielfältige Möglichkeiten zu handeln

29 Auf das Verhältnis der sozialen und physischen Umwelt zum makro-sozialen Kontext wird in einem gesonderten Kap. 6.1 Makro-soziale Rahmenbedingungen ausführlich eingegangen. Die Reihenfolge der Argumentation wird zum einen damit begründet, dass die Makro-Ebene im Modell von Kühnel/Bamberg nicht explizit differenziert wird und daher zunächst das Handlungsmodell in seiner ursprünglichen Version dargestellt wird. Zum anderen wird dem unmittelbaren sozialen Umfeld ein direkterer Einfluss auf das individuelle Handeln unterstellt als den makro-sozialen Rahmenbedingungen. Des Weiteren erscheint es inhaltlich sinnvoll, den theoretisch begründeten makro-sozialen Kontext mit seinen empirischen Ausgestaltungen, d.h. in Form der konkreten Beschreibung der hier untersuchten nationalen Kontexte, in einem Kapitel zu integrieren.

${ }^{30}$ Die Erweiterung umfasst die beiden dunklen Kästen der Logik der Situation und Selektion, die explizite Unterscheidung in die Ebenen sozialer Kontext und Akteur sowie die Pfeile, die den Einfluss der sozialen und physischen Umwelt auf die Handlungsumsetzung (Pfeil zwischen Auswahl und Handlung) darstellen. 
(Greshoff/Schimank 2003: 15). Handeln bedeutet die Auswahl zwischen verschiedenen Handlungsoptionen, deren simultane Umsetzung sich ausschließt (Esser 1999: 38ff.; Burkart 1994: 72). Die Ausführung einer Handlung impliziert so immer auch eine - dem Handeln vorweg genommene - (rationale) Entscheidung. ${ }^{31}$ Esser (1999: 21) fordert, dass die Erklärung des Handelns zunächst mit Annahmen der denkbar einfachsten Handlungstheorie - trotz aller Komplexität der „Wirklichkeit“ - beginnt. So kann z.B. davon ausgegangen werden, dass soziale Akteure innerhalb der jeweiligen Handlungssituation theoretisch unendlich viele verschiedene Handlungsmotive verfolgen können, letztlich sich jedoch auf das Mögliche und situationsgerecht Erscheinende konzentrieren. Die Herausforderung der Analyse besteht demnach nicht in der ausufernden Beschreibung einer bunten und komplexen Realität, sondern vielmehr in der abstrahierenden Darstellung einfacher und typischer Strukturen der realen Situation, die zu empirisch prüfbaren Hypothesen führt.

Die Auswahl einer Handlungsalternative wird sodann in Handeln umgesetzt. An dieser Stelle wird das ursprüngliche Modell um zwei Pfeile erweitert, die die Störung der Handlungsumsetzung repräsentieren. Die Wahl einer Handlung muss nicht zwingend zu ihrer Ausführung führen. Sie kann sowohl durch die soziale als auch durch die physische Umwelt be- oder gar verhindert werden.

Die beiden Entscheidungsschritte, die Logik der Situation und die Logik der Selektion, jeweils dargestellt in einem grauen Kasten, bilden den Kern des theoretischen Modells nach Kühnel und Bamberg. Entsprechend dem Entscheidungs- und Handlungsablaufes der Akteure werden im Folgenden die beiden Schritte chronologisch dargestellt und auf das konkrete Beispiel des väterlichen Sorgehandelns übertragen. Zwar gehen Kühnel/Bamberg bereits im ersten Schritt ihres Handlungsmodells von rationalen Abwägungen der Akteure aus, die insbesondere die Elaboriertheit des Nachdenkens und Wahrnehmens der Situation betreffen; ${ }^{32}$ die konkrete Auswahl zwischen Handlungsalternativen - auf die sich die klassischen Rational Choicer meist beziehen - findet jedoch primär im zweiten Schritt des Modells statt. Dieser Annahme folgt die nachstehende Darstellung. Zunächst wird auf die Situationskomponenten eingegangen, die den Akteur in seiner Rahmung der Situation beeinflussen (1. Schritt). In einem zweiten Abschnitt werden dann die bereits angedeuteten Annahmen des hier verwendeten Rational Choice-Ansatzes konkretisiert, die sich ins-

\footnotetext{
${ }^{31}$ Zur kritischen Reflexion der theoretischen Annahme einer Handlungsentscheidung siehe auch Kap. 4.3.2 Familiales Handeln als rationale Abwägung? - Eine kritische Betrachtung.

${ }^{32}$ Siehe dazu ausführlicher Kap. 4.2 Der Frame: Die Logik der Situation.
} 
besondere auf den 2. Schritt im Modell beziehen. Das zweistufige Modell von Kühnel und Bamberg dient dabei den Ausführungen als Ausgangspunkt und wird ergänzt durch generelle Überlegungen, die sich in der Rational Choice-Literatur finden, sowie speziellen Ausführungen zur integrativen Sozialtheorie von Hartmut Esser, die einige sinnvolle Anknüpfungspunkte liefern.

\subsection{Der Frame: Die Logik der Situation}

Der erste Schritt innerhalb des Handlungsmodells besteht aus der Eingrenzung des Entscheidungsproblems durch die Festlegung der in Frage kommenden Handlungsalternativen sowie der anzuwendenden Entscheidungskriterien. Dies erfolgt innerhalb der Definition der Situation als Framing (Kühnel/Bamberg 1998: 257; Esser 2001: 259). Dabei wird die vorliegende objektive Situation vom Akteur wahrgenommen. Er stellt sich - mehr oder weniger bewusst - die Frage, in welcher Art von Situation er sich gerade befindet (Esser 2001: 153). Das bedeutet, im Moment der Handlung sind vor allem die subjektiven, jeweils real vorliegenden, wenn auch möglicherweise falsch angenommenen Interpretationen der Situationsbedingungen handlungsrelevant (Esser 1999: 63). ${ }^{33}$ Damit ist nicht entscheidend, was aus der Perspektive wissenschaftlicher Prämissen das „objektiv richtige“ Handeln wäre, sondern vielmehr müssen die Erklärungen auf dem subjektiven Wissen, den Intentionen und Interpretationen der Akteure basieren (ebd.: 207). Danach kann die von außen betrachtet gleiche Situation von verschiedenen Akteuren ganz unterschiedlich definiert werden (ebd.: 64f.).

Trotz der zentralen Bedeutung, die dem individuellen Handeln und damit den einzelnen Akteuren innerhalb des Modells zukommt, wird darauf verwiesen, dass die individuellen Charakteristika - die subjektive Wahrnehmung und die inneren Bedingungen - hier nicht als psychologische Prozesse analysiert werden. Die subjektiv wahrgenommene Struktur der Situation bestimmt zwar das Interesse der Akteure objektiv und determiniert damit auch die daran anschließende Selektion zwischen den Handlungsalternativen. Der Akteur wird theoretisch jedoch als typischer sowie anonymer Repräsentant seiner sozialen Lage interpretiert und weniger als einzigartiges Individuum. Nicht die zahlreichen psychischen Dispositionen der jeweiligen Akteure, sondern vielmehr die sie umgebenden Strukturen

33 Dieses Argument unterstützt entscheidend das methodische Vorgehen dieser Arbeit, lediglich die väterliche Perspektive zur Beantwortung der forschungsleitenden Fragestellung zu berücksichtigen. Ausschließlich das, was die Väter wahrnehmen und wie sie ihre Situation dadurch definieren, bestimmt ihr eigenes Handeln. Es ist weniger relevant, ob die Ex-Partnerin tatsächlich, d.h. für einen außenstehenden Beobachter wahrnehmbar, den Kontakt zwischen Vater und Kind nach der elterlichen Trennung behindert, sondern vielmehr ist von Bedeutung, dass der Vater dies so wahrnimmt. 
gehen primär in die Erklärung väterlichen Sorgehandelns ein (Esser 1999: 23; Schmid 2004: 149; Miebach 2006: 431). Kurz: es geht um typische Handlungen typischer Akteure in typischen sozialen Kontexten.

Innerhalb der Rahmung der Situation aktiviert der Handelnde ein mentales inhaltliches Modell der Situation. Dieses Modell stellt den Frame der Situation dar, unter dem Akteure die Situation definiert sehen und der die Relevanzstruktur der Situation beschreibt (Esser 2006: 148; Hill/Kopp 2004: 144). Diese erste Festlegung des Handlungsspielraums dient der Orientierung in einer für den menschlichen Verstand zu komplexen Welt (Esser 1999: 190). Sie stellt eine Fokussierung auf einen in der Situation für den Handelnden wesentlichen Aspekt der Situation, dem Oberziel, dar. Dies trägt in zweifacher Hinsicht der begrenzten Kapazität des Menschen zur Informationsverarbeitung Rechnung: Zum einen stellen Frames drastisch vereinfachte und auf bestimmte Aspekte fokussierte Repräsentationen der vorliegenden Situation dar. Zum anderen werden alle anderen Aspekte, die nicht dem festgelegten Frame entsprechen systematisch ausgeblendet (Esser 2001: 262; Miebach 2006: 132, 420).

Mit der Festlegung der vorliegenden Situation geht die Definition der Handlungsalternativen, zwischen denen gewählt werden kann, einher. Es muss eine Auswahl der „richtigen“ Mittel zur Erreichung des wahrgenommenen Oberziels der Situation erfolgen. Das Individuum sucht innerhalb seines Reaktionsfundus nach einem gespeicherten Handlungsmodell, einem sog. Skript, d.h. der Akteur prüft, ob er schon einmal in einer ähnlichen Situation eine Handlung - erfolgreich - angewendet hat und ihre Anwendung auch in diesem Fall Erfolg versprechend erscheint (Esser 2001: 155; 261). ${ }^{34}$

„Es [das Skript, C.M.] ist das Programm des Handelns innerhalb eines bestimmten Frames. Das Programm enthält die auf die Situation bezogenen typischen Erwartungen und Alltagstheorien über die typische Wirksamkeit typischer Mittel“ (Esser 2001: 263).

Übertragen auf den Untersuchungsgegenstand bedeutet das, dass getrennt lebende Väter zunächst ihre Situation als Nachtrennungsväter definieren. Als Handlungsalternativen stehen ihnen unterschiedlichen Cash- und Care-Formen väterlichen Sorgens zur Verfügung, die in verschiedenen Kombinationsmöglichkeiten für sie in ihrer Situation subjektiv denkbar sind. ${ }^{35}$

${ }^{34}$ Ein häufig zitierter Kritikpunkt an der RC-Theorie besteht in der Tatsache, dass offen bleibt, wie diese Skripte entstehen.

${ }^{35} \mathrm{Zu}$ den genauen Handlungsformen siehe ausführlich Kap. 4.3.1.1 Sorgehandeln als Entscheidung zwischen Alternativen. 
Gewöhnlich - v.a. in Alltagsroutinen - wird mit der Rahmung gleichzeitig die Handlungsfolge festgeschrieben (Esser 2001: 156). Manchmal bleibt den Akteuren jedoch innerhalb des Frames ein mehr oder weniger weitreichender Handlungsspielraum oder es stehen keine Routinen für die vorgefundene Situation zur Verfügung, so dass bewusst über mögliche Handlungsalternativen nachgedacht werden muss. Die Bestimmung der Optionen innerhalb der subjektiven Definition der Situation kann mittels unterschiedlicher Verarbeitungsmodi getroffen werden. Der Modus legt die Art der Informationsverarbeitung fest, die ein Akteur innerhalb der spezifischen Situation angesichts der vorhandenen Daten und der vorliegenden Begrenzungen und Opportunitäten vornehmen kann (Esser 1999: 228).

Für die Selektion der jeweiligen Modelle für Situation und für das Handeln werden zwei Verarbeitungsmodi als Extrempunkte differenziert:

- $\quad$ Einerseits besteht die Möglichkeit der Anwendung einer spontan-automatischen Heuristik (kurz: as-Modus), die in der Aktivierung eines gedanklichen Modells ohne besondere Berücksichtigung der Konsequenzen erfolgt. Hier wird v.a. auf bewährte Alternativen zurückgegriffen, indem die Akteure bisher erfolgreiche Handlungsabläufe routiniert und unhinterfragt in als ähnlich erkannten Situationen anwenden (Esser 2001: 261ff; Hill/Kopp 2004: 134).

- $\quad$ Die reflexiv-kalkulierende Strategie (kurz: rc-Modus) andererseits besteht aus einer möglichst systematischen Berücksichtigung aller als relevant erachteten Konsequenzen einer Entscheidung (Esser 2001: 266). ${ }^{36}$

Die beiden „Heuristiken der Informationsverarbeitung“ unterscheiden sich in ihrem Grad der Elaboriertheit sowie der rationalen Durchdringung der Handlungssituation und damit in dem mit ihnen verbundenen kognitiven Aufwand (Esser 2006: 147). Bereits hier ist von einer Entscheidung nach rationalen Kriterien auszugehen. Die Anwendung des as-Modus ist v.a. innerhalb alltäglicher Routinen zu erwarten. In Alltagssituationen gekennzeichnet durch häufiges Wiederkehren sind Situations- und Handlungsmodelle eng miteinander verbunden, so dass eine bewusste Entscheidung für eine Handlung häufig ausbleibt. Der Akteur entscheidet demnach, ob er von seinen Gewohnheiten im Alltag abweichen muss oder möchte (Esser 2001: 152). Erst wenn er dies bejaht, kommt es zur Festlegung verschiedener Handlungsalternativen, Entscheidungskriterien und im zweiten Schritt des Modells zu einem Abwägungsprozess zwischen den verschiedenen Handlungsoptionen.

\footnotetext{
${ }^{36}$ Handlungsrelevant bedeutet in diesem Fall die Auftretenswahrscheinlichkeit der Konsequenz ist nicht zu gering und die Folge wird nicht neutral bewertet (siehe dazu im Detail Kap. 4.3 Die Handlungsauswahl: Die Logik der Selektion).
} 
Die aufwendigere Informationsverarbeitung wird ausgelöst durch ein Mismatch, d.h. gewissen Störungen innerhalb einer - alltäglichen - Situation, die automatisch und unkontrollierbar Irritationen und eine unspezifische Aufmerksamkeit verursachen (Esser 2001: 271; Burkart 1994: 99; Miebach 2006: 421; Greshoff/Schimank 2003: 18). Bisher Unhinterfragtes gerät in das Visier des Interesses:

„Zweifel und Fragen und alle geschilderten Vorgänge der Wiedergewinnung der Gewißheit sind aber für die Strukturen der Lebenswelt des Alltags nicht typisch. Sie sind für folgenschwere Entscheidungen, für nicht definierte Situationen, für Übergangszeiten, für Menschen in biographischen Übergängen und Krisen kennzeichnend“ (Esser 2001: 162, Hervorhebungen C.M.).

Zwar besteht väterliches Sorgehandeln generell in weiten Teilen aus Alltagsroutinen, deren Konsequenzen im täglichen Miteinander nicht immer wieder aufs Neue abgewogen werden, ${ }^{37}$ doch es kann zunächst argumentiert werden, dass auch Alltagsroutinen in ihrer ersten Umsetzung „entschieden“ worden sind, um durch wiederholte Anwendungen und Bewährungen zu Routinen institutionalisiert zu werden. Für Nachtrennungsväter kann aufgrund ihrer Lebenssituation ein weiteres Argument für die Annahme einer Informationsverarbeitung im rc-Modus angeführt werden. Mit der Trennung werden alltägliche Abläufe des familialen Zusammenlebens erschüttert und damit in Frage gestellt. Dem Vater stehen bisherige Interaktionsmuster mit dem Kind, u.a. aufgrund der räumlichen Trennung, nicht länger zur Verfügung. Die Ausführungen zu den Besonderheiten väterlichen Sorgehandelns über Haushaltsgrenzen hinweg machen deutlich, dass dem Vater ein Stück Alltag mit seinen Kindern verloren geht. ${ }^{38}$

Für die Anwendung eines elaborierten Informationsverarbeitungsmodus spricht ferner die Krisenhaftigkeit der Situation. Väter in Nachtrennungsfamilien empfinden besonders häufig die Phase kurz nach der elterlichen Trennung und damit kurz nach der räumlichen Trennung vom Kind als „Lebenskrise“ (Amendt 2004: 215ff.). Der Vater befindet sich in einer biographischen Übergangsphase, in der alte Handlungsgewohnheiten ihre Gültigkeit verlieren (können). Neben der Krisenhaftigkeit wird die Situation für den abwesenden Vater ferner durch Neuartigkeit gekennzeichnet. Es müssen neue Skripte für das väterliche Sorgehandeln entwickelt werden. Alte Handlungsskripte können nur noch bedingt angewandt werden, z.B. wenn die Kinder über einen längeren Zeitraum bei ihm sind und ein Alltag möglich ist. Ferner kann, wie dargestellt, eine neue Zweisamkeit entstehen. Das Handeln als Nachtrennungsvater ist somit (zunächst) neu und kann damit als reflexivkalkulierendes „Entscheiden“ konzeptionalisiert werden.

\footnotetext{
${ }^{37}$ Anders als z.B. die Entscheidung zur Elternschaft, die mit weitreichenden „lebenslangen“ Konsequenzen verbunden ist und wohl überlegt sein muss (Leip 2004: 61; Burkart 1994: 74).

${ }^{38}$ Siehe dazu ausführlich Kap. 2.2. Nachtrennungsfamilien: das Phänomen abwesender Väter.
} 
Eine hohe Motivation zur aufwendigen Abwägung von Alternativen ist ferner in so genannten High Cost-Situationen gegeben. Die mit diesen Situationen verbundene außergewöhnliche Wichtigkeit und gravierenden Folgen - gegebenenfalls aufgrund hoher (Opportunitäts-) Kosten im Fall einer Fehlentscheidung - bewegen den Akteur zum Innehalten (Kunz 2004: 158; Esser 2000b: 84). Auch väterliches Sorgehandeln nach der elterlichen Trennung oder Scheidung ist für den Vater von besonderer Bedeutung. So ist z.B. der Verlust einer guten Vater-Kind-Beziehung für einen engagierten Vater mit hohen emotionalen „Kosten“ verbunden. ${ }^{39}$

Abgesehen von dem erhöhten Nutzen einer bewussten Entscheidung muss der Akteur ferner die Möglichkeit zum Abwägen haben. Die Gelegenheitsstruktur ist maßgeblich von zeitlichen Ressourcen abhängig (Kunz 2004: 158; Esser 1999: 353). Gelegenheit über Handlungsalternativen und Entscheidungskriterien nachzudenken bietet sich für Nachtrennungsväter insofern, als dass sie nicht unter Zeitdruck stehen. Sie müssen nicht sofort, in einer spezifischen Situation, entscheiden, wie sie ihr Verhältnis zu ihren Kindern gestalten wollen. Durch die jeweiligen Einzelhandlungen, die in ihrer Gesamtheit das Sorgehandeln ausmachen, wird ein Prozess in Gang gesetzt und gehalten, der die Vater-KindBeziehung bzw. ihren Ausdruck im Handeln charakterisiert. ${ }^{40}$

Insgesamt kann demnach davon ausgegangen werden, dass Nachtrennungsväter sowohl Gründe wie auch Gelegenheiten haben, eine durchdachte Entscheidung mit Blick auf ihr väterliches Sorgehandeln zu treffen. Die folgenden theoretischen Überlegungen und v.a. die Operationalisierung basieren auf der Annahme, dass Nachtrennungsväter am Anfang oder an entscheidenden Schnittstellen im Zeitverlauf nach der Trennung die Situation im rc-Modus definieren.

Die subjektive Definition der Situation wird bestimmt durch die vorliegenden inneren und äußeren Bedingungen, wie Esser sie differenziert:

„Es geht bei der subjektiven Definition der Situation um ein inneres Tun eines einzelnen Akteurs im Moment einer gegebenen Situation, der auf der Grundlage der objektiven äußeren und inneren Bedingungen eine Selektion über eine bestimmte subjektive Sicht der Situation vornimmt“ (Esser 1999: 66).

${ }^{39}$ Zur ausführlichen kritischen Würdigung des „Kosten-“ bzw. „Nutzen“-Konzepts innerhalb familialer Beziehungen siehe Kap. 4.3.2 Familiales Handeln als rationale Abwägung? - Eine kritische Betrachtung.

${ }^{40}$ An dieser Stelle ist darauf zu verweisen, dass der Prozess im Zeitpunkt der Befragung des Vaters sicherlich nicht abgeschlossen ist, sondern sich auch darüber hinaus entwickelt. Zur Prozesshaftigkeit väterlichen Sorgehandelns siehe ausführlich Kap. 11.2 Charakteristika der Modellvariablen. 
Die äußeren Bedingungen umfassen dabei Opportunitäten, institutionelle Regeln und den externen Bezugsrahmen des Handelns, der klassischerweise auch als Struktur verstanden wird (Esser 1999: 425), während sich die inneren Bedingungen auf die individuellen Einstellungen der Akteure beziehen. Die Unterscheidung zwischen inneren und äußeren Bedingungen findet sich auch im Handlungsmodell von Kühnel/Bamberg, auch wenn die Ebenen im Original nicht explizit so benannt werden. Die Differenzierung zwischen dem „sozialen Kontext“ und der „Akteursebene“ wurden im vorliegenden Modell ergänzt. Dabei gehören die soziale und die physische Umwelt zu den externen Bedingungen, während das Überzeugungssystem akteursimmanent ist. Im Folgenden wird auf die inneren und äußeren Bedingungen mit Verweis auf das Anwendungsbeispiel des väterlichen Sorgehandelns näher eingegangen.

\subsubsection{Innere Bedingungen: Das Überzeugungssystem}

Nach Kühnel und Bamberg (1998) beinhaltet das individuelle Überzeugungssystem miteinander verknüpfte Wertvorstellungen und Einstellungen, die in allen Entscheidungssituationen determinieren, welche Alternativen relevant erscheinen und nach welchen Kriterien eine Auswahl getroffen wird. Wissenschaftlich interessant werden diese inneren Bedingungen erst, wenn verschiedene Akteure in vergleichbaren Entscheidungssituationen aufgrund variierender Überzeugungssysteme unterschiedliche Alternativen wahrnehmen und nach anderen Kriterien entscheiden. Prozesse, die im Inneren des Akteurs ablaufen, sind empirisch jedoch schwer zu fassen. Im Folgenden muss die Erklärung väterlichen Sorgehandelns darauf beschränkt werden, den inneren Entscheidungsprozess theoretisch zu (re-) konstruieren (Kühnel/Bamberg 1998: 257f.). ${ }^{41}$

Im konkreten Fall kann davon ausgegangen werden, dass subjektive Vaterschaftskonzepte als innere Bedingungen Einfluss auf die Definition der Situation nehmen. ${ }^{42}$ Dabei kann der Mann sich als Vater unterschiedlich begreifen. Männer mit einem eher traditionell ausgeprägten Rollenverständnis sehen v.a. Cash-Aspekte des väterlichen Sorgehandelns als Handlungsalternativen, während die sog. „neuen“ Väter (zusätzlich) ein hohes Maß an sozio-emotionalen Sorgehandeln zeigen (wollen). Der traditionelle Vater leistet damit kein oder wenig Care, nicht weil er es nicht kann, sondern weil er es innerhalb seines Entscheidungsspielraums nicht als eine Alternative sieht. Das Vaterschaftskonzept entsteht dabei nicht erst mit der elterlichen Trennung, sondern besteht schon vorher. Es kann ange-

\footnotetext{
${ }^{41}$ Hier gelten ähnliche Überlegungen wie zu den Präferenzen, die in Kap. 4.3.1.3 Die Präferenzordnung als Bewertungen und Erwartungen ausführlich thematisiert werden.

42 Zu unterschiedlichen Vaterschaftskonzepten siehe u.a. Matzner 1998 und 2004.
} 
nommen werden, dass die Ausgestaltung der Vaterrolle vor der Trennung auch von Bedeutung für das Handeln danach ist. Ein Vater, der eine intensive emotionale Beziehung zu seinem Kind aufgebaut hat, wird diese auch nach der Trennung suchen bzw. aufrecht erhalten. Wenn sich der Vater in erster Linie jedoch als Ernährer der Familie gesehen hat, wird er sein väterliches Engagement auch nach der Trennung auf finanzielle Aspekte konzentrieren. In diesem Zusammenhang ist auch die Arbeitsteilung innerhalb der Partnerschaft der Eltern von Bedeutung.

Mit dem Rollenverständnis eng verbunden ist die Einsicht des Vaters in seine Zahlungsverpflichtung. Ein Vater, der sich primär als Familienernährer sieht, wird ein anderes Verständnis für Unterhaltsforderungen aufbringen, als beispielsweise ein Vater, der sich darüber hinaus sozio-emotional kümmert. Letzterer wird eine alleinige Zahlungsverpflichtung möglicherweise als unfair erachten, wenn er die gleichen Zeitanteile mit dem Kind verbringt wie die Mutter, dieser aber formal keine finanziellen Pflichten zugesprochen werden. Das kann ausbleibende Unterhaltsleistungen zur Folge haben. Des Weiteren kann die Einsicht des Vaters in seine Zahlungspflichten eingeschränkt werden durch die aktuelle finanzielle Situation der Mutter. So kann das Einstellen von Unterhaltszahlungen mit der guten ökonomischen Lage der Mutter begründet werden. Ein zusätzliches Argument könnte hier die Anwesenheit eines neuen Partners im mütterlichen Haushalt sein. Der neue Mann im Haushalt des Kindes kann vom Vater als sein Substitut betrachtet werden, der das eigene väterliche Engagement weder finanziell noch sozio-emotional notwendig macht.

Eine weitere denkbare Determinante, die den inneren Bedingungen zuzurechnen ist und die die Anerkennung väterlicher Verpflichtungen bestimmt, ist die Verbindlichkeit der elterlichen Beziehung. Getrennt lebende Kinder, die aus einer langjährigen, formalisierten Beziehung hervorgegangen sind, können für den Vater einen anderen Stellenwert einnehmen, als Kinder, die ungeplant oder gar ungewünscht gezeugt wurden. Der Entstehungszusammenhang der jeweiligen Vaterschaft und die subjektive Einschätzung der damit verbundenen Handlungsverpflichtungen und -erwartungen können das väterliche Sorgehandeln, sowohl in Form von Cash wie auch von Care beeinflussen.

Zur Erklärung des väterlichen Sorgehandelns müssen ferner kognitive Überzeugungen hinsichtlich verschiedener Erziehungswerte, -einstellungen und -erwartungen berücksichtigt werden (Jaursch 2003: 4f). Diese dürften sich v.a. auf die Care-Komponente des väterlichen Engagements auswirken.

Eng verbunden mit dem subjektiven Vaterschaftskonzept sind andere individuelle Faktoren, die einen Teil des Überzeugungssystems ausmachen. Hierzu zählen z.B. väterliche Persönlichkeitsmerkmale oder die jeweilige Lebensphase des Vaters, in der unterschied- 
liche Prioritäten und 'Commitments' vorherrschen können (Palkovitz 1997: 213; Marsiglio 1991: 975). Väterliches Engagement steht anderen Lebensbereichen wie z.B. der Erwerbstätigkeit des Vaters, seiner beruflichen (Karriere-) Orientierung oder auch Freizeitpräferenzen konkurrierend gegenüber. ${ }^{43}$ Diese Konkurrenz kann je nach spezifischem Lebenskontext unterschiedlich ausgestaltet sein. Des Weiteren weist der Vater je nach Alter einen anderen Entwicklungsstand auf. Die Berücksichtigung des väterlichen Alters deutet auf einen wichtigen theoretischen Aspekt hin: die Verlaufsperspektive. Auch der Zeitpunkt der Vaterschaft im Lebenslauf kann unterschiedliche Implikationen für das väterliche Sorgehandeln haben. So könnte ein „später“ Vater andere Schwerpunkte im Ausfüllen seiner väterlichen Rolle setzen als z.B. ein Mann, der sehr jung - und vielleicht auch ungeplant - zum Vater geworden ist.

\subsection{2 Äußere Bedingungen: Der soziale Kontext}

Neben den inneren Bedingungen strukturieren die äußeren Rahmenbedingungen - der Kontext - die Wahl zwischen Handlungsalternativen vor. Die objektiven Gegebenheiten der Situation bilden materielle, institutionelle und kulturelle Grenzen, die durch die subjektive Wahrnehmung und Einstellung nur bedingt außer Kraft gesetzt werden können (Esser 1999: 216). Die Form der Bedürfnisbefriedigung, d.h. die Auswahl legitimer Mittel sowie auch die Festlegung der Bedürfnisse selbst, unterliegen dem Einfluss der objektiven Situation (ebd.: 110). Akteure sind in den allermeisten Fällen nicht perfekt, aber in den für sie relevanten Ausschnitten erstaunlich gut und objektiv richtig informiert, worum es in einer Situation primär geht (ebd.: 216f.). Und sie wissen darüber hinaus, welche Optionen und Mittel ihnen zur Erreichung bestimmter Ziele innerhalb dieser Situation zur Verfügung stehen. Nicht alle Optionen, die innerhalb einer Situation objektiv denkbar wären, sind für den individuellen Akteur vorstellbar, weil sie einen ersten Filter schon nicht passieren - die Logik der Situation (Esser 2000b: 1f.). Darüber hinaus findet die Definition der Situation nicht innerhalb eines sozialen Vakuums statt, sondern stellt einen sozialen Prozess dar, indem einzelne Akteure sich gegenseitig beeinflussen. Esser (1999: 167) geht von einer „gesellschaftlichen Konstruktion der Wirklichkeit“ aus. Frames sind kulturell verankerte Muster und damit „kollektive Repräsentationen“ typischer Situationen (Esser 2002: 473; Esser 2006: 148). Die folgenden beiden Kapitel beschäftigen sich mit der Bedeutung der äußeren Bedingungen für die individuelle Definition der Situation. Es wird nach Kühnel/Bamberg zwischen sozialer und physischer Umwelt differenziert.

${ }^{43}$ Dies gilt nicht zuletzt aufgrund der knappen Ressource Zeit. Siehe dazu ausführlich den Kap. 4.2.2.1 Die physische Umwelt: Ressourcen und Restriktionen. 


\subsubsection{Die physische Umwelt: Ressourcen und Restriktionen}

Die Gesamtheit der Handlungsoptionen, die sich aus den objektiv vorliegenden materiellen Knappheiten ergeben, wird als Opportunitäten bezeichnet (Esser 1999: 52; Greshoff/Schimank 2003: 16). Als Möglichkeitsraum bilden sie den weitesten Rahmen dessen ab, was an Alternativen überhaupt möglich ist (Esser 1999: 44). Die „Denkbarkeit“, d.h. die Verfügbarkeit einzelner Handlungsoptionen, wird dabei wesentlich bestimmt durch objektiv vorhandene bzw. subjektiv wahrgenommene Ressourcen und Restriktionen. Letztere wirken vergleichsweise sicher als Handlungsbarrieren, während verfügbare Ressourcen nicht zwingend zu einem bestimmten Verhalten führen. Inwieweit sich Ziele realisieren lassen, hängt somit wesentlich von den Handlungsbeschränkungen oder - in Umkehrung des Zusammenhangs - von den Handlungsermöglichungen innerhalb der Situation ab (Kunz 2004: 36f.). Das bedeutet, der Akteur kann nur jene Mittel, sprich Ressourcen, zur Zielerreichung in Erwägung ziehen, die ihm zur Verfügung stehen (Esser 2000b: 209f.). Alles, was nicht zum Ressourcenfundus eines Akteurs zählt, kann als Restriktion seines Handlungsspielraums interpretiert werden. Die relativen Knappheiten bestimmter Ressourcen steuern das Handeln, indem sie zu einem bedachten Mitteleinsatz zwingen, um so - den optimalen - Nutzen zu produzieren. Mit der Modifikation der Restriktionen ändert sich die Rahmung des Handelns (Esser 1999: 106f.).

Neben der fehlenden Kontrolle über bestimmte Ressourcen können jedoch die objektivmateriellen Gegebenheiten das Individuum auch an der Anwendung einer verfügbaren Ressource hindern. Welche Mittel für die Erreichung bestimmter Ziele zum Einsatz kommen, hängt nicht allein von den verfügbaren Mitteln ab, sondern auch davon, ob eine Ressource sich für die Anwendung eignet und als legitim gilt (Esser 1999: 106). ${ }^{44}$

Insgesamt können alle immateriellen und materiellen Dinge, Ereignisse, Zustände, Eigenschaften und Leistungen als Ressourcen bzw. Restriktionen individuellen Handelns wirken (Esser 1999: 38). Es können verschiedene Arten an Ressourcen bzw. Restriktionen differenziert werden (Leip 2004: 28; Greshoff/Schimank 2003: 16), wie z.B. materiellökonomisches Kapital, Humankapital, kulturelles, institutionelles und soziales Kapital. Letztere drei zählen zur sozialen Umwelt. Die physische Umwelt umfasst das materiellökonomische Kapital. ${ }^{45}$ Darunter sind insbesondere alle zu wirtschaftlichen Zwecken anwendbaren physischen und finanziellen Ressourcen zu verstehen, über die der Akteur ein

\footnotetext{
44 Die Legitimität der Mittel wird nicht zuletzt durch institutionelle Vorgaben bestimmt. Darauf wird ausführlich in Kap.6 Nationale Kontexte der Nachtrennungsväter eingegangen.

${ }^{45}$ Das jedoch nicht als von der sozialen Umwelt unabhängig verstanden werden kann.
} 
privates Eigentumsrecht verfügt (Esser 2000b: 213; Leip 2004: 66). Eine wesentliche Determinante väterlichen Sorgehandelns ist sein Einkommen. Dieses ist grundlegend von Bedeutung für das Leisten von Unterhaltszahlungen. Doch auch ein Einfluss auf CareAspekte des väterlichen Sorgehandelns ist zu vermuten. Finanzielle Ressourcen sind zur Aufrechterhaltung eines regelmäßigen Kontakts zwischen Vater und Kind über Haushaltsgrenzen hinweg grundlegend erforderlich. Dabei beeinflusst u.a. die räumliche Entfernung zwischen den elterlichen Wohnorten die mit einem Besuch verbundenen Kosten.

In diesem Zusammenhang können regionale Unterschiede angenommen werden. Elternschaft auf dem Land kann einen höheren logistischen und organisatorischen Aufwand erfordern, weil die Wege länger sind und eine andere Infrastruktur besteht als z.B. in der Stadt (Leip 2004: 173). Des Weiteren können generell regionale Unterschiede - neben den angesprochenen Stadt-Land-Differenzen - ausgemacht werden. Insbesondere im deutschen Fall ist hier auf Ost-West-Unterschiede zu verweisen. Auch knapp 20 Jahre nach der Wiedervereinigung herrschen immer noch soziodemographische und ökonomische Unterschiede, die sich auch auf das väterliche Sorgehandeln auswirken. Ostdeutsche Eltern sind häufiger von geringerem und/oder unsicherem Einkommen, von höherer Arbeitslosigkeit, von Wohnraumproblemen sowie auch veränderten Rahmenbedingungen für Partnerschaften betroffen (ebd.: 184).

Eng verbunden mit dem ökonomischen Kapital ist das Humankapital eines Akteurs, d.h. seine produktiven Eigenschaften und Fähigkeiten wie beispielsweise seine Intelligenz, Gesundheit, seine Fähigkeit zur Mobilität, sein Informationsstand, sein Wissen, seine emotionale Belastbarkeit oder seine Kreativität (Esser 2000b: 214f.; Leip 2004: 66; Kunz 2004: 37; Becker 1992: 22). ${ }^{46}$ Die Verbindung des Bildungskapitals zum ökonomischen Kapital liegt in zweierlei Hinsicht vor: Zum einen kann ökonomisches Kapital, v.a. Finanzkapital, in die eigene Bildung des Akteurs investiert werden. Zum anderen besteht ein enger Zusammenhang zwischen Bildungsniveau und erwirtschaftetem Finanzkapital mittels Erwerbstätigkeit. Die Ressource Bildung wirkt sich dabei sowohl auf Unterhaltszahlungen als auch auf Care-Aspekte aus.

${ }^{46}$ An dieser Stelle ist darauf zu verweisen, dass das Bildungsniveau des Vaters auch als Teil der inneren Bedingungen konzeptionalisiert werden kann, wenn davon ausgegangen wird, dass das Überzeugungssystem auf individuellem Wissen basiert. Dennoch ist das zu Grunde gelegte Verständnis von Bildung hier ein weitreichenderes als das generelle Wissen über Erziehungsstile oder moralische Erwartungen, die an den Akteur als Vater gestellt werden könnten. Beide Konzepte, Wissen und Bildung, sind eng miteinander verbunden. Hier wird das väterliche Bildungsniveau jedoch primär als Ressource der physischen Umwelt verstanden. 
Einen weiteren wichtigen Teil der physischen Umwelt, der zur Bestimmung väterlichen Sorgehandelns beiträgt, stellt die Ressource Zeit dar (Esser 2000b: 63; Becker 1992: 22). Ihre Bedeutung basiert nicht zuletzt auf ihrer Endlichkeit. Im Gegensatz zu anderen physischen wie materiellen Ressourcen kann sie nicht angehäuft werden. Ein Tag hat 24 Stunden, daran sind alle Akteure und gesellschaftliche Strukturen gebunden (Esser 1999: 107). Während das Geldeinkommen die Möglichkeiten des Konsums von Marktgütern begrenzt, reguliert die i.d.R. knappe reale Zeit, die nicht mit Erwerbsarbeit verbracht wird, andere mehr oder weniger zeitaufwendige Aktivitäten (Esser 2000b: 63f.). Einige Nutzen produzierende Güter, die ein hohes Interesse der Akteure auf sich ziehen, können nur unter der Verwendung eines hohen Zeitaufwandes hergestellt werden. In der vorliegenden Fragestellung ist eines dieser zeitaufwendigen Güter z.B. die vertrauensvolle Beziehung zwischen Vater und Kind. Esser (2000a: 62) formuliert es folgendermaßen:

„Nicht zuletzt gehören Kinder als für viele Eltern außerordentlich ,primäres’ Zwischengut zu den ,Erzeugnissen’, die nur dann Wertschätzung und Wohlbefinden erzeugen, wenn man sich sehr viel um sie kümmert. Ein Gameboy oder teure Klamotten helfen hier in keiner Weise“.

Zeit - wie andere Ressourcen auch -, die für eine Aktivität oder ein Gut aufgewandt wird, steht gleichzeitig für andere Aktivitäten bzw. Güter nicht mehr zur Verfügung (Esser 2000a: 62).

Werden diese bisher sehr allgemein gehaltenen Überlegungen konkret auf das väterliche Sorgehandeln übertragen, so sind zwei Aspekte mit Blick auf die knappe Ressource Zeit von besonderer Bedeutung: Zum einen gilt auch hier, die Zeit, die ein Vater in der Erwerbstätigkeit verbringt, steht ihm nicht mehr für seine Kinder zur Verfügung. Die Arbeitszeit des Vaters begrenzt damit sein väterliches Engagement. ${ }^{47}$ Neben dem absoluten Zeitanteil, den der Vater am Arbeitsplatz verbringt, ist ferner die Art der Arbeitszeit von Bedeutung. Vätern mit unregelmäßigen Arbeitszeiten, die Schicht arbeiten oder Wochenenddienste übernehmen, wird ein regelmäßiger direkter Kontakt zu ihren getrennt lebenden Kindern erschwert. Zum anderen gelten zeitliche Restriktionen ferner mit Blick auf eine neue Familie des Vaters. Zeit, die dieser mit seinen „externen“ Kindern verbringt, steht ihm nicht mehr für seine im Haushalt anwesenden „neuen“ Kinder aus einer bestehenden Partnerschaft und auch nicht mehr für weitere „externe“ Kinder aus anderen zerbrochenen Beziehungen zur Verfügung. Kinder aus verschiedenen Partnerschaften

${ }^{47}$ Gleichzeitig ermöglicht es aber auch das väterliche Sorgehandeln, indem Ressourcen erwirtschaftet werden, die dem Kind zugute kommen (können). So sollte sich daher die am Arbeitsplatz verbrachte Zeit positiv auf Cash, jedoch negativ auf Care auswirken. Siehe dazu im Einzelnen Kap.5 Hypothesengenerierung. 
konkurrieren somit nicht nur um die knappe Zeit des abwesenden Vaters, sondern insgesamt um seine begrenzten Ressourcen.

Die Verfügbarkeit bestimmter Ressourcen ist zunächst eine Frage individueller Eigenschaften. So bestimmen z.B. physische oder psycho-soziale Eigenschaften, Talente und Fähigkeiten wie Kreativität, welche Handlungsoptionen, dem Akteur zur Verfügung stehen. Doch Ressourcen wie Restriktionen sind nicht unabhängig von der sozialen Umgebung (Esser 1999: 140; Glover 2002: 263).

\subsubsection{Die mikro-soziale Umwelt: Beziehungen und Einflussnahmen}

Innerhalb der sozialen Umwelt kann ebenso von begünstigenden und einschränkenden Determinanten des Handelns ausgegangen werden. Die Opportunitäten der mikro-sozialen Umwelt umfassen nach Kühnel und Bamberg die sozialen Beziehungen zu und Einflussnahmen von anderen Akteuren. Zunächst besteht eine wesentliche Funktion der sozialen Umwelt in der Gewinnung sozialer Anerkennung. Damit sind die Beziehungen an sich schon von direktem Interesse für die Individuen (Esser 2000b: 236). Dies ist eng verbunden mit der Tatsache, dass innerhalb einer sozialen Situation durch die beteiligten Akteure unterschiedliche Erwartungen und Bewertungen an das Handeln herangetragen werden. Durch die Erfüllung dieser Erwartung kann u.a. soziale Anerkennung gewonnen werden. Des Weiteren bestimmen die Erwartungen der signifikanten Anderen die Handlungskonsequenzen entscheidend mit (Esser 2000a: 4ff.). Soziales Handeln bezieht sich immer auf andere Akteure und ihr Handeln (Brüderl 2004: 176; Himmelweit 2002: 245). Das Individuum benötigt Informationen darüber, wer als anderer Akteur innerhalb der Situation relevant ist, wie diese signifikanten Anderen agieren werden und welche Erwartungen an das eigene Handeln gestellt werden. Dies kann z.B. als Handlungsorientierung bzw. als Legitimierung eigener Handlungen dienen. Väter, die Scheidungserfahrungen in ihrem eigenen Elternhaus gesammelt haben, werden sich eventuell am Nachtrennungshandeln ihrer Väter orientieren. Als Handlungsorientierung können auch andere Scheidungsväter im persönlichen Umfeld dienen. Andere Akteure können ferner - direkt wie vermittelt - Einfluss auf die Struktur der Präferenzen nehmen (Schmid 2004: 152f.). Des Weiteren werden sie in die Kosten-Nutzen-Abwägung des Akteurs integriert. Neben den Erwartungen und Bewertungen dürften auch die vom Akteur wahrgenommenen Interessen der anderen Beteiligten Berücksichtigung finden. Dies gilt verstärkt für familiale Beziehung, insbesondere für die Eltern-Kind-Beziehung (Brüderl 2004: 176). ${ }^{48}$

\footnotetext{
${ }^{48}$ Siehe dazu Kap. 4.3.2 Familiales Handeln als rationale Abwägung? - Eine kritische Betrachtung.
} 
Mit Blick auf die Fragestellung bestehen die wesentlichen sozialen (alltäglichen) Beziehungen des Nachtrennungsvaters zu seinen Kindern, zur Ex-Partnerin und Kindesmutter, gegebenenfalls zur neuen Partnerin und zu Kindern, die aus der neuen Partnerschaft hervorgegangen sind. Wichtige Bezugspersonen im Verwandtschaftsnetz stellen die Großeltern der getrennt lebenden Kinder dar. Mit diesen Akteuren - v.a. mit der Mutter des Kindes - können Abstimmungserfordernisse bestehen, diese werden hier jedoch nicht als „Interaktion“ konzeptionalisiert. Es geht nicht primär um die Aushandlungsprozesse, sondern um das Handeln der Väter innerhalb dieses Rahmens. Deswegen werden die anderen Mitakteure primär als Ressourcen bzw. Restriktionen des väterlichen Agierens interpretiert.

Familien sind darüber hinaus mit zahlreichen anderen sozialen Gruppen verbunden und auf deren Unterstützung und Ressourcen angewiesen (Cheal 2002: 13). Neben dem familialen Umfeld zählen noch das weitere Verwandtschaftssystem, Freunde und Bekannte, Arbeitskollegen sowie gegebenenfalls nachbarschaftliche Beziehungen zur sozialen Umwelt des Vaters, die sein väterliches Sorgehandeln durch soziale Unterstützungsmechanismen oder Strukturen sozialer Kontrolle bestimmen (u.a. Matzner 2004: 25; Feldhaus/Huinink 2005: 199).

Am Beispiel der Kindesmutter wird verdeutlich, dass soziale Beziehungen sowohl Ressourcen als auch Restriktionen darstellen können. Häufig wird in der Literatur den Müttern eine Gate Keeper - Funktion zugeschrieben (Fthenakis et al. 2002: 142; NappPeters 1995: 40; Cabrera et al. 2000: 133). Das bedeutet die Ex-Partnerin „entscheidet“ über Quantität und Qualität der Vater-Kind-Beziehung. Wenn die Mutter eine intensive Vater-Kind-Beziehung nicht wünscht, kann sie sich der Umsetzung des väterlichen Wunsches in den Weg stellen. Der Vater wird größere Anstrengungen unternehmen müssen, seine Beziehung zum Kind trotz dieser Barriere aufrecht zu erhalten. Die Kindsmutter stellt auf diese Weise eine Restriktion für das väterliche Handeln dar. Ist der Mutter jedoch an einem guten Vater-Kind-Verhältnis gelegen und fördert dies, indem sie z.B. Besuche initiiert oder Besuchsregelungen forciert und einhält, dient sie dem Vater als Ressource.

Soziale Beziehungen stellen nicht nur eine Ressource für individuelles Handeln dar, sie erfordern auch „Investitionen“. Damit verursachen sie - im Sprachgebrauch der Rational Choice-Theorie - „Kosten“. Die Pflege sozialer Beziehungen, v.a. intensiver Nahbe- 
ziehungen, die über bloße Bekanntschaften hinaus reichen, ist sehr (zeit-) aufwendig (Esser 2000b: 243). ${ }^{49}$

Soziale Beziehungen können, wie auch schon bei der physischen Umwelt angenommen wurde, regionale Unterschiede im väterlichen Sorgehandeln aufweisen. So können beispielsweise in ländlichen Gegenden andere Normen und Werte Gültigkeit haben, die spezifische Verhaltens- und Beziehungsmuster begründen. Diese Unterschiede zeigen sich z.B. in den Scheidungs- und Geburtenraten in der Stadt und auf dem Land. Ferner ist zwischen verschiedenen Graden der Anonymität zu differenzieren. Das bedeutet, auf dem Land herrscht eine andere Form der sozialen Kontrolle, die sich auf das väterliche Sorgehandeln nach der elterlichen Trennung auswirken kann. Dies führt ferner zu einer besonderen sozialen Vernetzung, die gegebenenfalls spezifische Hilfs- und Kontrollmechanismen impliziert (Leip 2004: 126, 172).

Kühnel/Bamberg konzeptionalisieren individuelles Handeln als ein zweistufiges Modell. Nach der ausführlichen Darstellung des Framings wird im folgenden Abschnitt auf die Logik der Selektion, der Auswahl zwischen verschiedenen Handlungsoptionen eingegangen.

\subsection{Die Handlungsauswahl: Die Logik der Selektion}

Die Auswahl zwischen den vorher definierten Möglichkeiten väterlichen Sorgehandelns wird im folgenden Kapitel als rationale Wahl konzeptionalisiert. Zunächst werden die grundlegenden Annahmen der Rational Choice-Theorie und ihre Übertragung auf väterliches Sorgehandeln dargestellt. In einem daran anschließenden Abschnitt wird die Anwendbarkeit der Theorie der rationalen Wahl auf familiales Handeln kritisch diskutiert.

\subsubsection{Rational Choice und väterliches Sorgehandeln}

Es wurde bereits zu Beginn des Theoriekapitels darauf verwiesen, was eine rationale Handlung ausmacht. Rational ist danach eine Entscheidung zwischen Handlungsalternativen, bestehend aus dem Abwägen von Konsequenzen, die mit Kosten und Nutzen verbunden sind, und die basierend auf (subjektiven) Bewertungen (Präferenzen und Interessen) und Erwartungen evaluiert werden. Die Auswahl einer Handlung erfolgt nach dem

${ }^{49}$ Auf die besondere Bedeutung der Ressource Zeit wurde bereits im Abschnitt 4.2.2.1. Die physische Umwelt: Ressourcen und Restriktionen ausführlicher eingegangen. 
Prinzip der Nutzenmaximierung. Die nachstehenden Ausführungen werden die einzelnen Komponenten der „rationalen Wahl“ auf väterliches Sorgehandeln übertragen.

\subsubsection{Sorgehandeln als Entscheidung zwischen Alternativen}

Jeder Handlung geht nach der Rational Choice-Theorie - entsprechend ihrer Bezeichnung - eine Selektion (Choice) zwischen verschiedenen Handlungsoptionen voraus, die wie bereits beschrieben durch den Prozess des Framings in einem ersten Schritt eingegrenzt worden sind. Verschiedene Entscheidungsmöglichkeiten und die Intentionalität des Handelns werden dabei vorausgesetzt (Esser 1999: 248; Burkart 1994: 2f., 12; Miebach 2006: 395; Kunz 2004: 34, 160; Hill/Kopp 2004: 126; Schnabel 2006: 176).

\section{Abb. 4.2: Handlungsalternativen der Nachtrennungsväter.}

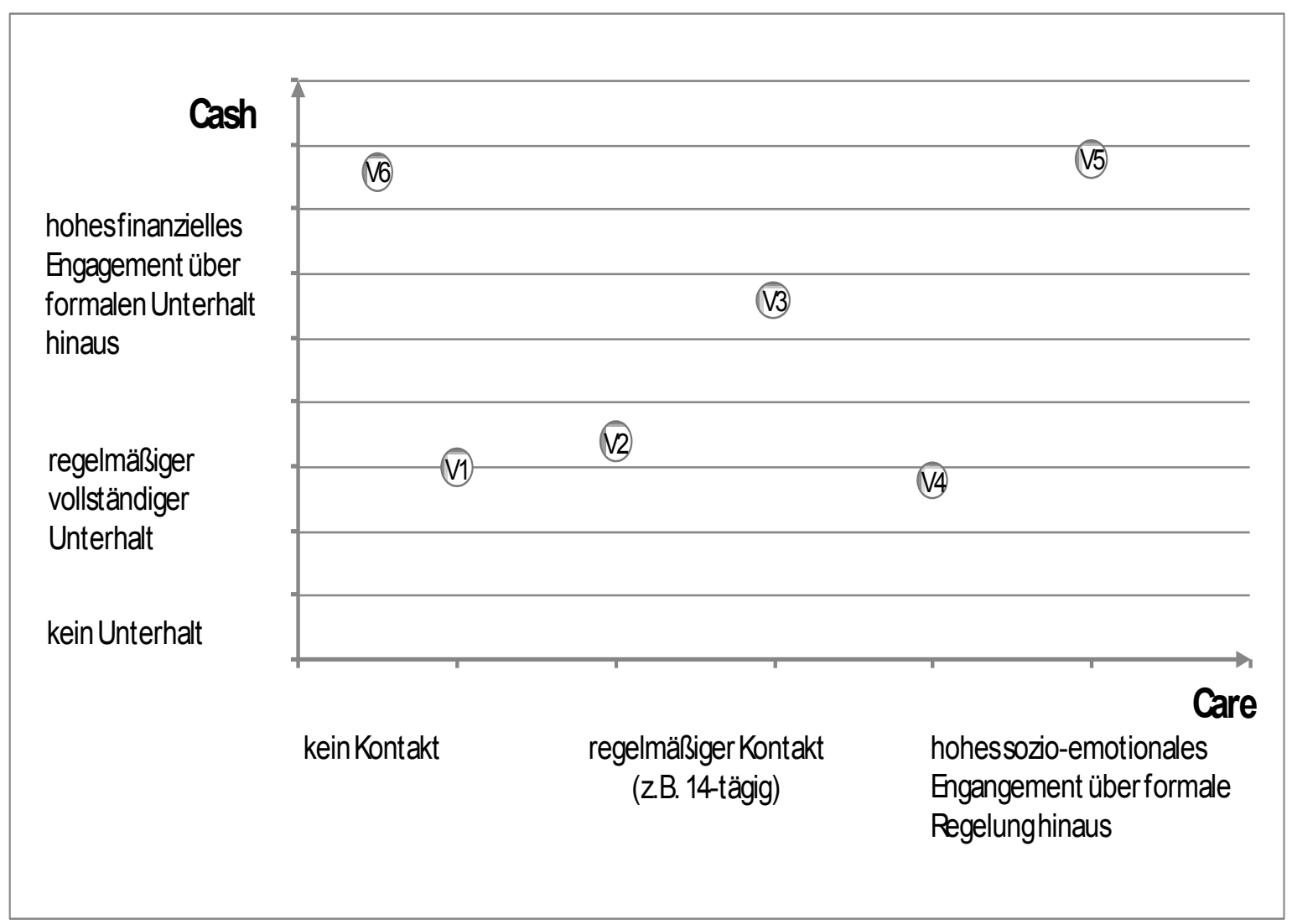

Quelle: eigene Darstellung.

Nachtrennungsväter wählen, entsprechend der Differenzierung wie sie in Kap. 2 Das Konzept väterlichen Sorgehandelns in Nachtrennungsfamilien entwickelt wurde, zwischen vielfältigen Kombinationsmöglichkeiten von Cash- und Care-Aspekten aus. Abbildung 4.2 stellt diese unterschiedlichen Kombinationsmöglichkeiten in vereinfachter Form graphisch dar. Aufgespannt in einem Koordinatensystem stehen dem Nachtrennungsvater unendlich viele verschiedene Kombinationsmöglichkeiten der beiden Dimensionen, Care und Cash, zur Verfügung. 
Je nach Ausgestaltung kann dem handelnden Vater eine Position im zweidimensionalen Raum zugesprochen werden. Zur Illustration sind 6 Beispielväter in die Matrix eingetragen. Die Eckpunkte bilden die Extreme. Ein besonders engagierter Vater leistet sowohl umfassende finanzielle als auch starke sozio-emotionale Unterstützung für seine getrennt lebenden Kinder (Vater 5 in der Abb. 4.2). Das andere Extrem stellt einen Vater dar, der sich weder sozio-emotional noch finanziell engagiert. Es besteht in diesem Fall gar kein Kontakt - auch kein finanzieller Transfer - zwischen Vater und Kind (unten links in Abb. 4.2). Die anderen beiden Eckpunkte stellen zwei Varianten dar, in denen eine Komponente sehr stark ausgeprägt ist, während die andere gar nicht gewährleistet wird. So zahlen die Väter entweder ohne jeglichen Kontakt zum Kind zu haben (Vater 6 in Abb. 4.2) oder sie kümmern sich ohne Unterhalt zu zahlen (unten rechts in Abb. 4.2). Die Extrempunkte sind empirisch, so ist zu vermuten, weniger interessant wie die zahlreichen Abstufungen dazwischen (Väter 1 bis 4 in Abb. 4.2). In den folgenden Ausführungen dient das dargestellte Handlungsmodell der Rational Choice-Theorie als Basis für die Erklärung väterlichen Engagements in Nachtrennungsfamilien. Ausgehend von rationalen Entscheidungen erklärt der Ansatz die Auswahl zwischen verschiedenen Niveaus väterlichen Kümmerns in Nachtrennungsfamilien. Dabei wird weder eine konkrete Handlung, wie z.B. ein Spaziergang mit dem Kind oder das Einrichten eines Dauerauftrages erklärt, sondern vielmehr geht es um generelle Handlungsmuster, d.h. zahlreiche Einzelhandlungen, die in ihrer Gesamtheit das väterliche Care bzw. Cash ausmachen und sich auf einem bestimmten Niveau einpendeln. ${ }^{50}$

Das Abwägen zwischen Alternativen ist notwendig, weil die jeweiligen Handlungsoptionen einander ausschließen (Esser 1999: 38ff.; Burkart 1994: 72). Des Weiteren veranlassen die Bedingungen universeller Knappheiten - nicht zuletzt bestimmt durch den situativen Kontext - die Akteure ihre Ressourcen mit Bedacht einzusetzen (Kunz 2004: 34). Dem Akteur stehen nur begrenzt Mittel zur Verfügung, um bestimmte Ziele und Wünsche umzusetzen (Esser 1999: 256). Die Zweck-Mittel-Rationalität, d.h. das Verhältnis zwischen Input und Output muss stimmen (Burkart 1994: 29). Im Ansatz der Rational Choice wird demnach davon ausgegangen, dass

„[...] actors are stopping before they act in order to count the likely cost. A calculation is being made“ (Glover 2002: 262).

50 Dabei werden zunächst vereinfachend Cash und Care getrennt voneinander betrachtet. In einem eigenständigen Kapitel wird dann auf das Verhältnis der beiden Dimensionen eingegangen (Kap. 9.4 Väterliches Sorgehandeln: Das Verhältnis von Care und Cash). 
Die Akteure wägen somit vor der Auswahl und Ausführung einer Handlung die Vor- und Nachteile aller Handlungsalternativen ab (Burkart 1994: 12). Dieser Kalkulation wird eine gewisse Konsistenz mit Blick auf spezifische Kontexte und Zeitpunkte, in denen die Wahl integriert ist, unterstellt (Kunz 2004: 38, 160; Becker 1992: 22). Das bedeutet, der Abwägungsprozess erfolgt nach angebbaren Kriterien (Leip 2004: 241). Es ist an dieser Stelle darauf zu verweisen, dass es sich dabei nicht zwingend um eine Abwägung auf der Bewusstseinsebene handeln muss. Die Annahme einer Entscheidung besagt lediglich, dass aus mehreren möglichen Alternativen schließlich nur eine zur Umsetzung kommen kann (Esser 1999: 248).

\subsubsection{Handlungskonsequenzen als Kosten und Nutzen}

Für die Entscheidung zwischen Alternativen müssen diese vergleichend betrachtet und bewertet werden (Burkart 1994: 85). Dies geschieht laut Kernthese des Rational ChoiceAnsatzes anhand der mit den jeweiligen Handlungen verbundenen Konsequenzen (Kühnel/Bamberg 1998: 257; Esser 2004: 249; Himmelweit 2002: 232). Dem Handeln unterliegt damit eine spezifische Zielorientierung (Kunz 2004: 160; Hill/Kopp 2004: 126).

Erwartete Handlungskonsequenzen werden als unterschiedlich zuträglich empfunden. Gewünschte Ziele und positive Konsequenzen stellen dabei den Nutzen einer Handlung dar, während unerwünschte, negativ bewertete Folgen einer Handlung auf der KostenSeite bilanziert werden, ein sog. negativer Nutzen (Esser 1999: 248ff.; Kunz 2004: 37).

Kosten verursachen Handlungen u.a. durch den Mitteleinsatz zur Zielerreichung. Neben dem direkten Mitteleinsatz ist jede Handlung immer auch mit Alternativ- oder Opportunitätskosten verbunden. ${ }^{51}$ Das bedeutet, jede Entscheidung für eine Handlung schließt die Ausführung aller anderen Optionen aus. Damit entgehen dem Akteur die mit diesen Alternativhandlungen verbundenen Erträge; sie werden zu Kosten (Kunz 2004: 37; Hill/Kopp 2004: 127). Insbesondere mit dem Steigen der Opportunitätskosten einer „Fehlentscheidung“ setzt ein intensives Abwägen der Kosten mit dem wahrscheinlichen Nutzen ein (Esser 1999: 358). Die Kosten einer Entscheidung können als materielle, zeitliche oder kognitive Ressourcen verstanden werden. Deren subjektiver Wert bemisst sich jedoch nicht im nominellen Wert der Geldbeträge oder verwendeten Zeitspanne, sondern in (Konsum-) Möglichkeiten, die nach der Entscheidung nicht mehr verwirklicht werden

\footnotetext{
${ }^{51}$ Darüber hinaus entstehen sog. Transaktionskosten, die unter den Bedingungen unvollständiger Informationen mit der Beschaffung und Verarbeitung neuer Informationen verbunden sind. Ferner können Kosten durch die Koordination mit anderen Akteuren oder durch die Kontrolle des Verhaltens anderer entstehen (Kunz 2004: 40).
} 
können (Schnabel 2006: 186). Nutzen - auf der anderen Seite - verschaffen Handlungen dem Akteur, wenn ihre Konsequenzen zu seiner Bedürfnisbefriedigung und Zielerreichung beitragen (Kunz 2004: 33; Esser 1999: 86; Hill/Kopp 2004: 125). Handeln stellt damit immer eine Produktion von Nutzen bzw. eine Vermeidung von Kosten dar. ${ }^{52}$

Von den restriktiven „harten“ ökonomischen Versionen der RC-Theorie unterscheiden sich neuere sozialwissenschaftliche v.a. darin, dass sie eine Vielzahl an Nutzenvarianten neben dem rein materiellen Nutzen zulassen (Opp 1999: 173ff.; Nauck 1989: 52; Kunz 2004: 33; Feldhaus/Huinink 2005: 198; Glover 2002: 263). Damit werden wesentliche Bereiche des sozialen Lebens, z.B. Handlungen aus Liebe, ein schlechtes Gewissen oder das befriedigende Gefühl, anderen eine Freude zu machen, nicht aus dem Erklärungsmodell ausgegrenzt (Kunz 2004: 40; Esser 1999: 253).

In der Literatur werden trotz ansonsten hoher gesellschaftlicher Variabilität von Zielen und Wünschen zwei generelle menschliche Bedürfnisse unterschieden, auf die sämtliche anderen Konkretisierungen langfristig ausgerichtet sind: das physische Wohlbefinden und die soziale Wertschätzung (Lindenberg 1981: 24; Hill/Kopp 2004: 125; Greshoff/Schimank 2003: 11f.). Diese Ziele sind allen Akteuren gemein. Beide können jedoch auf sehr vielfältige Weise und nach unterschiedlichen individuellen Vorlieben erreicht werden.

Neben der Ausgestaltung des Nutzens sind sich die ökonomischen und sozialwissenschaftlichen Rational Choice-Theoretiker des Weiteren uneinig über die Zielgerichtetheit des Nutzens, den die Akteure verfolgen. Fraglich ist, ob sich die Individuen im Rahmen ihrer Rationalität rein an ihrem eigenen Nutzen orientieren, oder auch die wahrgenommenen Bedürfnisse anderer Akteure berücksichtigen (Opp 1999: 173). Anhänger des homo oeconomicus-Modells konstatieren, dass die Wahl der Handlungen auf den Eigeninteressen der Akteure basiert und damit als „egoistisch“ zu charakterisieren ist. Die Bedürfnisse und das Wohlergehen anderer spielen dabei keine Rolle (Himmelweit 2002: 233; Esser 1999: 295). Neuere sozialwissenschaftliche Varianten der RC-Theorie gehen indessen davon aus, dass Rationalität nicht mit Egoismus gleichzusetzen ist (Glover 2002: 263; Kunz 2004: 11).

\footnotetext{
52 Die folgenden Ausführungen beziehen sich gleichermaßen auf das Konzept der Kosten, die einen negativen Nutzen ausmachen. Zur Vereinfachung wird hier generell von Nutzen gesprochen. Sobald eine analytische Differenzierung notwendig wird, wird darauf im Text explizit hingewiesen.
} 
„Die Analyse geht davon aus, dass der einzelne seine Wohlfahrt, so wie er sie sieht, maximiert - ob er nun egoistisch, altruistisch, loyal, boshaft oder masochistisch ist“ (Becker 1992: 22, Hervorhebungen im Original).

Diese Annahme scheint insbesondere mit Blick auf die Eltern-Kind-Beziehung nachvollziehbar. Die elterliche Rolle ist gerade bestimmt durch eine starke Verantwortung für das Wohlbefinden des Kindes. Deswegen weicht die vorliegende Theorie in Anbetracht der Fragestellung von einem reinen Eigennutzbegriff ab. Es wird zwar von Vätern, die rational entscheiden, ausgegangen, die väterliche Rationalität umfasst aber auch die Berücksichtigung der perzipierten Bedürfnisse relevanter Dritter, insbesondere der eigenen Kinder. ${ }^{53}$

Kosten und Nutzen väterlichen Sorgehandelns können entsprechend der vorangegangenen Ausführungen unterschiedliche Formen annehmen. Diese werden gegeneinander abgewogen, bevor der Vater sich für ein Handlungsmuster entscheidet und dies im Alltag immer wieder auf das Neue umsetzt. Im Folgenden werden zunächst Kosten und Nutzen, die von den Vätern mit den beiden Dimensionen väterlichen Sorgehandelns verbunden werden können, dargestellt. Danach wird auf die unterschiedlichen Determinanten eingegangen die die jeweiligen positiven bzw. negativen Konsequenzen bestimmen.

Die mit der Überweisung von Unterhalt aufgewendeten finanziellen Mittel stehen Trennungsvätern nicht mehr für andere Ausgaben zur Verfügung und verursachen somit Opportunitätskosten. Nach einem rein ökonomischen Verständnis der rationalen Handlungsauswahl scheint es zunächst keinen rationalen Grund zu geben, warum der Vater Unterhaltszahlungen leisten sollte. Sie stellen allein Kosten dar. Ein materiell-ökonomischer Nutzen scheint damit nicht verbunden zu sein. Dennoch gibt es nach der sozialwissenschaftlichen Variante der Theorie „gute Gründe“ Unterhalt zu zahlen. Hier ist zunächst an die Erfüllung der moralischen Erwartung für ihre Kinder finanziell zu sorgen, die an Eltern gestellt wird, zu denken. Diese Verpflichtung wird dann v.a. Vätern gegenüber formuliert, wenn sie als Ernährer der Familie konzeptionalisiert werden. Des Weiteren kann es für den Vater selbst einen Nutzen darstellen, seine Kinder materiell versorgt zu sehen.

Ein ähnliches Argument kann für das intensive Kümmern des Vaters nach der elterlichen Trennung angeführt werden. Innerhalb der Gesellschaft wird die elterliche Fürsorge für Kinder erwartet und sozial geschätzt. Eine starke Care-Norm verpflichtet Eltern sich um

${ }^{53}$ Siehe dazu ausführlich Kap. 4.3.2 Familiales Handeln als rationale Abwägung? - Eine kritische Betrachtung. 
ihre Kinder zu kümmern, auch über die elterliche Trennung hinaus (Himmelweit 2002: 247). Das bringt insbesondere Nachtrennungsvätern einen sozial-normativen Nutzen, mit dem ein „gutes Gefühl“, das Richtige zu tun, einhergeht (Nauck 1989: 53). In gleichem Maße wie unterlassene Unterhaltszahlungen vielleicht ein „schlechtes Gewissen“ auslösen, kann auch die Vernachlässigung der elterlichen Fürsorgepflicht Kosten verursachen. Wie bereits dargestellt, kann ebenso argumentiert werden, dass der Nutzen der Eltern vom wahrgenommenen Nutzen, sprich dem perzipierten Wohlbefinden, der Kinder abhängig ist (Becker 1985: 106).

Das Kümmern um und das Zusammensein mit dem Kind selbst können ferner einen Nutzen für den Elternteil darstellen. In modernen Gesellschaften sind Kinder zu kostspieligen Investitionen geworden, die im Alter kaum zusätzliche materielle Sicherheit bringen. Kinder als Altersvorsorge haben an Bedeutung verloren (Ostner 2007, May 2007). Nach einer ökonomischen Auslegung der Rational Choice-Theorie gäbe es danach heutzutage kaum noch Gründe überhaupt Kinder zu bekommen und in sie Zeit wie Geld zu investieren. Dass Akteure dennoch zu Eltern werden, lässt darauf schließen, dass Kinder weniger als „Produktionsgut“ sondern vielmehr als „Konsumgut“ verstanden werden (Leip 2004: 71; Himmelweit 2002: 235). Das Kümmern um und eine langfristige enge Beziehung zu Kindern bringt Eltern einen hohen intrinsischen Nutzen (Himmelweit 2002: 247; Nauck 1989: 57).

Dennoch können Nachtrennungsväter mit einem intensiven väterlichen Kümmern über Haushaltsgrenzen hinweg auch Kosten verbinden. Dazu zählen zunächst finanzielle und zeitliche Ressourcen, die aufgewendet werden müssen. Wie angemerkt wird väterliches Sorgehandeln in Nachtrennungsfamilien unter besonderen Bedingungen geleistet. ${ }^{54}$ Kosten entstehen dem Vater durch einen gewissen organisatorischen Aufwand sowie gegebenenfalls durch zu überbrückende Entfernungen zwischen den elterlichen Haushalten. Diese organisatorischen Anstrengungen umfassen auch den Kontakt mit der Ex-Partnerin, der gegebenenfalls mit negativen Emotionen belegt sein kann.

„Die Geburt eines Kindes macht optionsoptimierende Individuen [die Eltern, C.M.] zu wechselseitig aufeinander bezogenen, abhängigen und verpflichteten Familienpersonen“ (Knijn et al. 2007: 219).

Dieses Abhängigkeitsverhältnis der Eltern besteht nach dem Scheitern der elterlichen Partnerschaft fort. Eine Mutter, die den väterlichen Kontakt zum getrennt lebenden Kind erschwert, verursacht zusätzliche (emotionale) Kosten für den Vater. Soziale, finanzielle und zeitliche Belastungen bei der Aufrechterhaltung eines guten Verhältnisses zu externen

\footnotetext{
${ }^{54}$ Siehe dazu ausführlich Kap. 2.2 Nachtrennungsfamilien: das Phänomen abwesender Väter.
} 
Kindern können ferner im Zusammenhang mit einer potentiellen neuen Familie entstehen. Beide Familien - die getrennte wie die neue - konkurrieren um die knappen Ressourcen des Vaters.

Darüber hinaus müssen Kinder bzw. die Familie(n) mit anderen Lebensbereichen wie der Arbeitswelt oder Freizeitaktivitäten vereinbart werden. So verweist Becker (1981: 137) darauf,

„[...], dass es keinen 'guten’ Ersatz für Kinder gibt, allerdings Kinder durchaus in Konkurrenz zu anderen Konsumgütern zu sehen sind, [...].“

Kosten des Kümmerns von Nachtrennungsvätern umfassen im besonderen Maße die knappe Ressource Zeit. Auf die Besonderheiten wurde schon im Rahmen von Restriktionen in der Beschreibung der physischen Umwelt (Kap. 4.2.2.1.) hingewiesen. Zeitliche Ressourcen, die in die Betreuung der Kinder investiert werden, stehen nicht mehr für Erwerbsarbeit, für die Alltagsorganisation oder für die erwachsene Partnerbeziehung zur Verfügung (Leip 2004: 126).

Zusammenfassend kann festgehalten werden, dass Akteure Handlungen vermeiden, die ihnen wahrgenommene Kosten verursachen, während sie Handlungen suchen, von denen sie sich einen (maximalen) Nutzen versprechen. Vor der Auswahl einer Handlungsalternative werden die mit der jeweiligen Handlung verbundenen, perzipierten Vor- und Nachteile abgewogen. Diese Evaluation der Kosten und des Nutzens basiert auf individuellen Bewertungen und Erwartungen.

\subsubsection{Die Präferenzordnung als Bewertungen und Erwartungen}

Die Bewertungen von Handlungskonsequenzen basieren auf einem individuellen Präferenzsystem, das das Wünschenswerte von den indifferenten bzw. negativen Konsequenzen unterscheidet (Esser 1999: 42; Burkart 1994: 72; Schmid 2004: 149; Kunz 2004: 44). ${ }^{55}$ Mittels der unterschiedlichen Erwünschtheit und Dringlichkeit einzelner Ziele werden die Alternativen in eine Rangfolge gebracht, die als Präferenzordnung oder Nutzenfunktion bezeichnet wird (Esser 1999: 248; Schmid 2004: 150f.).

Wie angemerkt haben Akteure die grundlegenden Bedürfnisse der sozialen Wertschätzung und des physischen Wohlergehen gemein, unterscheiden sich jedoch in ihren Vorlieben

\footnotetext{
${ }^{55}$ Dies ist ein weiteres Merkmal eines breiten Verständnisses von Rationalität. Innerhalb der engen Version der RC-Theorie wird davon ausgegangen, dass lediglich Restriktionen handlungsleitend sind. Vertreter der weiten Variante gehen jedoch davon aus, dass neben den Restriktionen auch Präferenzen bei der Handlungsauswahl eine Rolle spielen (Opp 1999: 174).
} 
und Bestrebungen nach bestimmten Ressourcen, Objekten, Ereignissen und Leistungen, die dieses gewährleisten. Präferenzen können dabei unterschiedliche Formen annehmen: vom geliebten Lebenspartner oder Haustier, über alle möglichen Lebensgewohnheiten, der vertrauten Umgebung, dem Beruf, bis hin zu politischen oder religiösen Überzeugungen. Präferenzen sind zeitlich relativ stabil (Kunz 2004: 39; Esser 1999: 125ff.). ${ }^{56}$ Verhaltensänderungen stellen selten Präferenzänderungen dar. Vielmehr reagieren Akteure - so die Annahme der RC-Theorie - auf veränderte Sanktionen und Anreize innerhalb der Handlungssituation, die die Kosten-Nutzen-Relation beeinflussen (Kunz 2004: 39f.). Dies gilt aber nur dann und insofern, als sich dadurch für die Akteure die subjektive Nutzenbilanz ändert.

Präferenzordnungen basieren auf Vergleichbarkeit der Handlungskonsequenzen. Das bedeutet, der Vergleich verschiedener Handlungsfolgen muss zu einer „besser als“, „schlechter als“ oder „genauso gut wie“ Bewertung führen. Die Rangfolge verschiedener Konsequenzen dient als Grundlage für die Bewertung der Alternativen. Gegeben eine eindeutige Rangordnung der Zwecke nach ihrer Bedeutung für die Akteure und gegeben eine Rangfolge der Mittel nach ihrer perzipierten Effektivität zur Erfüllung dieser Zwecke, folgt daraus relativ eindeutig, welche Handlung der Akteur präferiert (Esser 1999: 298f.). Neben den Bewertungen werden somit ferner subjektive Erwartungen über die Eintretenswahrscheinlichkeit der Handlungskonsequenzen in die Kosten-Nutzen-Abwägung einbezogen (Esser 2004: 249; Himmelweit 2002: 232, Burkart 1994: 33). Die Erwartungen bestehen aus dem Produkt von Kontrolle und Effizienz eines Mittels zur Verwirklichung eines angestrebten Zieles. Es werden so Ziel-Mittel-Relationen hergestellt (Esser 1999: 199, 256).

Doch bei der Modellierung der Abwägungsprozesse bestehend aus Kosten und Nutzen, deren Bewertung und Eintretenswahrscheinlichkeit, wird nicht von „harter“ Rationalität ausgegangen, wie es in einigen ökonomischen Varianten der RC-Theorie der Fall ist. Das Individuum muss bzw. kann nicht vollständig über alle Bestandteile dieser Entscheidung informiert sein (Esser 1999: 254; Opp 1999: 174). Die Realität ist häufig komplexer als die menschliche Auffassungsgabe (Esser 1999: 297f.). Ferner sind die für eine „rationale“ Entscheidung nötigen Informationen knapp und gegebenenfalls nur unter hohem Aufwand zu erlangen (Esser 1999: 340; Schmid 2004: 150). Akteure sind lediglich - latent - mit „Modellen“ der wichtigsten typischen Situationen ausgestattet, die sie zur Bewältigung

${ }^{56}$ Die relative Stabilität der Präferenzen unterscheidet sich von Annahmen der ökonomischen Handlungstheorie, die von einer unbedingten Stabilität der Präferenzordnung ausgehen (Schmid 2004: 155; Kunz 2004: 36). 
des Alltags benötigen und die sie zu einer groben Abschätzung der möglichen Handlungsalternativen befähigen (Esser 2001: 333; Greshoff/Schimank 2003: 19). Rationalität in dem hier zugrunde liegenden Verständnis bezieht sich damit lediglich auf die Fähigkeit der Akteure, zwischen „besser“ und „schlechter“ zu unterscheiden und sich widerspruchsfrei danach zu verhalten (Kunz 2004: 38).

\subsubsection{Die Handlungswahl als Nutzenmaximierung}

Anhand der jeweiligen Bewertungen der Handlungskonsequenzen und der Erwartungen ihrer Eintretenswahrscheinlichkeit des Nutzens bzw. der Kosten bestimmen die Akteure das spezifische Gewicht jeder einzelnen Handlungsalternative (Esser 1999: 252; Greshoff/Schimank 2003: 13). Für die Auswahl einer Handlungsoption gilt:

„Bei der Wahl zwischen mehreren Handlungsalternativen bevorzugt der Akteur jene, bei der das Produkt von erzielbarem Wert [dem Nutzen, C.M.] mit der Wahrscheinlichkeit, ihn zu erzielen [der Erwartung, C.M.], maximal ist“(Nauck 1989: 51).

Oder anders ausgedrückt:

„Von mehreren Handlungsalternativen, die ein Akteur in Erwägung zieht, wählt er diejenige, für die die perzipierten Handlungskonsequenzen am positivsten bewertet und am sichersten erwartet werden“ (Kunz 2004: 45).

Dies entspricht dem Prinzip der kalkulierenden Nutzenmaximierung, die eine Kostenminimierung impliziert (Esser 2004: 249; Miebach 2006: 32; Himmelweit 2002: 232; Hill/Kopp 2004: 115; 125; Kunz 2004: 36; Brüderl 2004: 167; Leip 2004: 24).

Die Annahme der Nutzenmaximierung, wie sie v.a. von ökonomischen Vertretern des RCAnsatzes vertreten wird, ist jedoch in den Sozialwissenschaften umstritten (u.a. Schmid 2004: 155). Die einzelnen Rational Choice-Modelle unterscheiden sich danach, ob sie von einem Nutzen "maximizing" oder "satisficing" ausgehen (Kunz 2004: 155f.; Esser 1999: 309). Letzteres besagt, dass Akteure nicht immer so lange abwägen bis sie die „perfekte“ Lösung gefunden haben, sondern häufig schon früher zufrieden sind, d.h. wenn sie eine bestimmte Nutzenschwelle überschritten haben (Kunz 2004: 157). ${ }^{57}$

Theoretisch wird angenommen, dass der individuelle Akteur für jede sich ihm erschließende Handlungsalternative ein Wert-Erwartungs-Gewicht „berechnet“ und sich für das höchste entscheidet. Es kann jedoch davon ausgegangen werden, dass sich Individuen selten in der Realität die Mühe machen, umständliche Funktionen mit Nutzenwerten sowie

${ }^{57}$ Innerhalb der Literatur werden die vorangestellten Annahmen, die als Einschränkung der ökonomischen RC-Modelle gelten, mit Simons $(1955,1993)$ Konzept der begrenzten Rationalität, der bounded rationality, in Verbindung gebracht. 
deren Auftretenswahrscheinlichkeit aufzustellen. Dabei werden die meisten Entscheidungen nicht aufgrund von aufwendigen Abwägungsprozessen getroffen, sondern der Akteur hat vielmehr eine ganz generelle Vorstellung über eine grobe Richtung der Handlungskonsequenzen (Esser 1999: 353). Die Abwägungsprozesse verändern sich dadurch eventuell in ihrer Intensität und Dauer, nicht jedoch in ihrem Prinzip der Kosten-NutzenKalkulation. Daher ist es dennoch plausibel anzunehmen, dass ein Akteur vor Handlungen intuitiv nach Nutzen und Wahrscheinlichkeiten zwischen möglichen Alternativen abwägt (Miebach 2006: 403; Leip 2004: 63f.). Hintergrund ist dabei die Prämisse, dass Akteure i.d.R. situationsangemessen handeln. Die Situationsangemessenheit wird vom externen Beobachter, dem Forscher, festgelegt (Kunz 2004: 68). Einer rationalen Verhaltenserklärung kommt demnach die Aufgabe zu, die Logik der Situation zu definieren, an die der Akteur sein Handeln angepasst hat (Kunz 2004: 68; Miebach 2006: 431; Esser 1999: 249). Innerhalb der Thesengenerierung wird dementsprechend darauf verzichtet, die einzelnen Wert-Erwartungs-Gewichte (theoretisch) exakt in ihrer Höhe zu rekonstruieren. Stattdessen werden „Daumenregeln“ angenommen, die angeben, welche Handlung (abhängige Variable) unter welchen Umständen (die unabhängigen Variablen) wahrscheinlicher - da für das Individuum innerhalb der Rahmenbedingungen „rationaler“ - zu erwarten ist. Das Verständnis der begrenzten Rationalität als Fähigkeit der Akteure, zwischen besser und schlechter zu unterscheiden und sich widerspruchsfrei danach zu verhalten, wird in den Thesen aufgegriffen.

Zusammenfassend wird also theoretisch angenommen, dass ein Akteur mit gegebenen Präferenzen und aktuellen Bedürfnissen aus einem durch das Framing vorgegebenen Set an Handlungsalternativen auswählt. Jede Option verspricht jeweils einen gewissen Nutzen, ihre Umsetzung ist mit bestimmten Kosten verbunden und beides wird mit einer spezifischen Eintretenswahrscheinlichkeit assoziiert. Es wird die Handlungsmöglichkeit gewählt, die dem Handelnden subjektiv wahrscheinlich den maximalen Nutzen bei minimalen Kosten verspricht.

\subsubsection{Familiales Handeln als rationale Abwägung? - Eine kritische Betrachtung}

In der Literatur zeigt sich, dass der Ansatz der Rationalen Wahl als Modellierung von Handlungsentscheidungen ebenso vielfältig wie umstritten ist. Die Debatte über mögliche Grenzen aber auch Chancen der Theorie ist bei weitem nicht abgeschlossen. Es bestehen derzeit verschiedene Versionen und Interpretationen des Ansatzes, um den zahlreichen Kritikern zu begegnen. Grundlegende Einwände gegen die Rational Choice-Theorie beziehen sich zumeist auf ihre motivationale Eindimensionalität, ihre Tautologie, ihre Trivialität und Sinnleere, ihre mangelnde empirische Relevanz oder ihre fehlende Prognosetauglichkeit aufgrund fehlerhafter, gar „falscher“ Annahmen über das menschliche Handeln (u.a. Kunz 2004: 139; Leip 2004: 39). Mit dieser generellen Kritik sehen sich Ver- 
treter der Rational Choice -Theorie auch innerhalb der Familiensoziologie konfrontiert. Dennoch werden gesonderte Einwände gegen die Übertragung der Theorie auf familiales Handeln erhoben. Dieses scheint besonders „,irrational“ und daher mit dem theoretischen Ansatz nicht zu fassen. Die Charakteristika des familialen Handelns, die - so die Argumentation - im Widerspruch zur Rationalität stehen, drücken sich u.a. in seiner sozialen Dimension, seiner Normorientierung, seinem Bezug zu Emotionen und seiner generellen Unvereinbarkeit mit Begriffen wie „Kosten“ und „Rationalität“ aus. Im Folgenden wird auf wesentliche Aspekte der speziellen Kritik eingegangen.

\subsubsection{Familiales Handeln als soziales Handeln}

Kritiker, die davon ausgehen, dass rationales und familiales Handeln einander ausschließen, verweisen häufig auf die besonderen Charakteristika familialer Beziehungen.

„Es mag angemessen sein, Verhalten unter dem Aspekt von rationaler Wahl, Entscheidung und Nützlichkeitsorientierung zu sehen, wenn es um den Kauf von Konsumgütern geht. Aber im Bereich der Familie kann man ernsthafte Zweifel haben, ob das ökonomische Modell angemessen ist“ (Burkart 1994: 34).

Und an anderer Stelle:

„There is something special about family relationships as opposed to other kinds of social relationship [...]“ (Morgan 2002: 151, Hervorhebung C.M.).

Es wurde bereits darauf hingewiesen, dass ein wesentlicher Unterschied zwischen familialen und ökonomischen Interaktionen darin besteht, dass in letzteren situativ alle Beteiligten den größtmöglichen spezifischen Nutzen ohne Rücksicht auf die Kosten anderer erzielen. Diese Zielgerichtetheit auf den eigenen Nutzen muss besonders innerhalb der Familie kritisch reflektiert werden. Fraglich ist, ob sich die Familienmitglieder im Rahmen ihrer Rationalität rein an ihrem eigenen Nutzen orientieren, oder auch die perzipierten Bedürfnisse anderer Akteure berücksichtigen. Vertreter des homo oeconomicusModells konstatieren, dass die Wahl der Handlungen auf den Eigeninteressen der Akteure basiert und damit als „egoistisch“ zu charakterisieren ist. Die Verfolgung eigener Vorteile und Ziele steht im Vordergrund, während die Bedürfnisse und das Wohlergehen bzw. die Schädigung anderer Akteure keine Rolle spielt (siehe dazu u.a. Himmelweit 2002: 233; Kunz 2004: 36ff; Esser 1999: 295; Hill/Kopp 2004: 132f.). Altruistische Motivationen kommen in der Grundform der RC-Theorie zunächst nicht vor. Dies steht im Widerspruch zu familialen Handeln, denn Akteure beziehen neben eigenen zusätzlich auch die - von ihnen angenommenen - Interessen der anderen Familienmitglieder ein (Burkart 1994: 62; Duncan/Strell 2004: 42). Individueller Nutzen kann auf einer Skala zwischen den Extrempunkten des reinen Eigennutzes und des puren Altruismus zahlreiche Variationen annehmen. Dabei müssen rationale Handlungstheorien anerkennen, dass Individuen auch „vernünftig“ handeln (können), wenn sie den von ihnen wahrgenommenen Nutzen anderer berücksichtigen (Burkart 1994: 62f.). Dies gilt in besonderem Maße für Familien und 
speziell für Eltern. Gerade in der Eltern-Kind-Beziehung werden die vermuteten Interessen der anderen Akteure regelmäßig in die individuellen Handlungsentscheidungen einbezogen. Deswegen wird in der vorliegenden Analyse von einem weiteren Nutzenbegriff ausgegangen.

Neben der Berücksichtigung des Nutzens anderer müssen Handlungen innerhalb familialer Kontexte ferner als Aushandlungsprozesse und weniger als individuelle Entscheidungen konzeptionalisiert werden. Handeln im unmittelbaren Nahumfeld besteht im Wesentlichen aus Kooperation (Glover 2002: 262). So stellt die Arbeitsteilung innerhalb einer Lebensgemeinschaft das Ergebnis einer Aushandlung zwischen den Partnern dar. Individuen treffen Entscheidungen mit Bezug zu anderen Akteuren (Schnabel 2006: 179), wie es im hier zugrunde gelegten Modell mittels der sozialen Umwelt auch berücksichtigt wird. Die ursprüngliche Grundannahme eines autonomen Akteurs, der individuelle Entscheidungen nach Kosten-Nutzen-Abwägungen trifft, scheint angesichts der Kontaktmuster des familialen Alltags in einer Haushaltsgemeinschaft besonders realitätsfern. Eine Betrachtung des Vaters als „Einzelkämpfer“ scheint wenig angemessen. Nichtsdestotrotz werden mit Blick auf die Fragestellung der Arbeit seine Handlungsentscheidungen hier weniger innerhalb eines familialen Aushandlungsprozesses gesehen, sondern vielmehr als individuelle Handlungsauswahl unter „rationaler“ Berücksichtigung zwischenmenschlicher Ressourcen und Restriktionen verstanden. Das heißt, die anderen Akteure dienen dem Vater innerhalb seiner Entscheidung als Ressource, indem sie bestimmte Handlungen erleichtern oder ermöglichen. Sie können bestimmte Handlungsalternativen jedoch auch verhindern, wie die Ausführungen zur Gate Keeper-Funktion der Mutter deutlich gemacht haben. Des Weiteren kann argumentiert werden, dass, selbst wenn in Familien individuelle Entscheidungen seltener bzw. kollektiven untergeordnet sein sollten, dies nicht zwingend gegen rationale Entscheidungen sprechen muss. So werden Abwägungen im Sinne der Gemeinschaft, d.h. mit dem größtmöglichen Nutzen und den geringsten Kosten für die Familie oder die Haushaltsgemeinschaft getroffen. ${ }^{58}$

Unabhängig von der Erweiterung des Nutzenbegriffs über den reinen Eigennutz hinaus kann familiales Zusammenleben und die Investition in familiale Beziehungen selbst rational begründet werden. Familien unterscheiden sich von Arbeitsmarktbeziehungen in

\footnotetext{
${ }^{58}$ Dieses Argument kann im Übrigen auch gegen ökonomische Handlungen und Entscheidungen z.B. innerhalb eines Großunternehmens angeführt werden. Auch hier können individuelle Interessen gesamtökonomischen entgegenstehen oder untergeordnet werden. Die individuelle Entscheidung berücksichtigt daher auch die Kosten und Nutzen, die dem gesamten Unternehmen durch eine spezifische Handlung entstehen können und nicht primär die individuellen.
} 
ihrer Langfristigkeit. Diese veranlasst die einzelnen Akteure dazu, den wahrgenommenen Nutzen aller Beteiligten zu berücksichtigen. Von freundschaftlichen Beziehungen setzen sich Familien zumindest graduell ab, indem sowohl die Langfristigkeit als auch die Verbindlichkeit verwandtschaftlicher Relationen zu ungleich höheren Alternativkosten beim Verlassen der Familien führen als z.B. Bekannt- oder Freundschaften (Nauck 1989: 51). Es macht für den einzelnen Akteur durchaus „Sinn“ auf die Solidarität und Hilfe seiner Familienmitglieder zu vertrauen. Aufgrund dieser relationalen „Lebenslänglichkeit“ wäre eine rücksichtslose Ausbeutung der anderen Familienmitglieder irrational (Hill/Kopp 2004: 133).

„Wenn die Handlungen von Akteuren - wie dies für Partnerschaften, Ehen und Familien typisch - über längere Zeiträume wechselseitig eine ganz wichtige Quelle für die eigene Bedürfnisbefriedigung und Zielerreichung sind, dann muss ein rationaler Akteur ein Interesse daran haben, dass es seinen (familialen) Mitakteuren ,gut geht', dass sie also keinen Grund haben, ihn zu bestrafen bzw. ihm Anerkennung, Zuneigung oder Unterstützung zu verweigern“ (Hill/Kopp 2004: 133).

Durch das längerfristige Zusammenleben und Interagieren reduzieren sich ferner die Verhandlungs- und Sanktionskosten, die in anderen sozialen Gruppen zur Aufrechterhaltung der sozialen Ordnung notwendig sind. Insofern bietet die familiale Gemeinschaft mit ihrem Verpflichtungsgefühl und Hilfenetzwerk für alle Beteiligten eine Wohlstandssteigerung (Leip 2004: 197). Dennoch würden wahrscheinlich die wenigsten Akteure ihr familiales Engagement mit Argumenten des eigenen Nutzenvorteils begründen, wenn sie es denn überhaupt begründen oder nicht gar für „selbstverständlich“ halten. Dies deutet auf eine weitere Besonderheit hin, mit der familiale Beziehungen charakterisiert werden können:

„Beziehungen zwischen Familienmitgliedern unterscheiden sich grundlegend von solchen zwischen Arbeitskollegen in Unternehmen oder zwischen Mitgliedern in Organisationen. Die Interaktionen von Ehepartnern sowie von Eltern und Kindern sind mit größerer Wahrscheinlichkeit durch Liebe, Dankespflichten, Schuldbewusstsein und Pflichtgefühl motiviert als durch ein eng verstandenes Eigeninteresse“ (Becker 1992: 36).

Das Zitat verweist auf die enge Verbindung familialen Handelns zu Normen und Emotionen.

\subsubsection{Familiales Handeln als norm-orientiertes Handeln}

In der Literatur wird normkonformes und durch Emotionen geleitetes Handeln häufig als Gegensatz zu rationalem Entscheiden konzipiert (Burkart 1994: 11; Miebach 2006: 407). Normen werden als allgemeine Verhaltensvorschriften in Form von gesellschaftlichen Erwartungen an Akteure in bestimmten Situationen definiert (Opp 1986: 11; Esser 2000b: 51; Hill/Kopp 2004: 132). Sie legen ein typisches Handeln fest und entlasten Individuen damit von der Notwendigkeit, ständig aufs Neue situationsgerechte Handlungsmuster zu entwerfen (Bahrdt 1997: 53). Der Vorwurf der Unvereinbarkeit von Normen und 
rationalem Handeln findet sich über die Familiensoziologie hinaus. Dabei wird die Kritik häufig mit einem normativen Unterton formuliert:

„[...] wenn nur noch die kalkulierende Zweckrationalität die Welt beherrscht, dann ginge es langfristig den Menschen schlecht, und der Gesellschaft bzw. der Gattung drohten Krisen, vielleicht sogar der Untergang“ (Esser 1999: 245).

Es kann realistischer - und glücklicherweise, so die Aussage - davon ausgegangen werden, dass die soziale Welt nicht rein aus rationalen, ökonomisch kalkulierenden Akteuren besteht, die eifrig damit beschäftigt sind, ihre Eigeninteressen strategisch umzusetzen. Insbesondere Familienmitglieder hält mehr zusammen als Abwägungsprozesse, die Kosten und Nutzen gegenüber stellen.

RC-Theorien analysieren soziale Prozesse und Strukturen ausgehend vom Handeln der individuellen Akteure und ihrem Streben nach Bedürfnisbefriedigung. Das hat ihnen den Vorwurf eingehandelt, das Verständnis von Akteuren als Rollen- und Normenträger zu vernachlässigen bzw. soziale Ordnung insgesamt nicht erklären zu können (Kunz 2004: 8; Hill/Kopp 2004: 84). Die Annahmen eines homo oeconomicus, so das Argument, seien nicht mit denen des homo sociologicus vereinbar (Burkart 1994: 56). Dabei wird den verschiedenen Ansätzen der rationalen Wahl entweder Norm-Blindheit unterstellt oder vorgeworfen, dass sie Prämissen der rationalen Wahl allzu leichtfertig für die unbedingte Handlungsrelevanz der Normen aufgeben (Miebach 2006: 432). Im letzteren Fall kann die Theorie - laut ihren Kritikern - Diskrepanzen zwischen institutionellen Vorgaben und Handeln nicht erklären (Streeck/Thelen 2005: 11). Insgesamt werden dem Ansatz Schwierigkeiten konstatiert, Handeln als reguliertes Tun zu begründen. Dadurch entstehe der theoretische Zwang, Normen ausschließlich als situative Restriktionen zu interpretieren, ohne auf deren Genese und die Wirkungsweise einzugehen (Schmid 2004: 70; Coleman 1990: 292ff.). Insbesondere ist es schwierig, die Entwicklung eines inneren Sanktionsmechanismus des Akteurs mittels der Theorie zu erklären (Burkart 1994: 58). ${ }^{59}$

Ein wichtiges Argument der - scheinbaren - Unvereinbarkeit von Rationalität und Normen liegt demnach in der Unbedingtheit von Normen. Es ist fraglich, ob es möglich ist, sich für oder gegen normkonformes Handeln zu entscheiden. Normen erfordern ein bedingungsloses Folgen, während rationales Handeln das Ergebnis oft komplexer und unsicherer Kalkulationen ist (Esser 1999: 284; Leip 2004: 43). Normen wirken dagegen

\footnotetext{
${ }^{59}$ Dagegen ist an dieser Stelle anzumerken, dass die Entstehung von Normen und ihren Funktionsweisen als innere Sanktionierung - bei Non-Konformität - nicht im Vordergrund der hier behandelten Fragestellung stehen. Es geht weniger um die Frage, wie diese Mechanismen entstehen, sondern vielmehr um ihre Handlungsrelevanz.
} 
mechanisch und werden von Akteuren unbewusst befolgt (Burkart 1994: 59). Normkonformität dient dabei nicht primär der Nutzenoptimierung, von der die RC-Theorie ausgeht. Vielmehr wird den normativen Handlungsanleitungen Folge geleistet, „weil es sich so gehört“ - ohne tief greifende Reflexion möglicher Konsequenzen einer Devianz (Schimank 2004: 294f.; Esser 1999: 283). Damit können sie nur unabhängig von Selbstinteresse und Nutzenoptimierung betrachtet werden. Normen wie Rationalität stellen damit zwei gleichwertige, voneinander - zunächst - unabhängige Handlungsorientierungen dar (Burkart 1994: 60).

Dem ist entgegen zu halten, dass Normen und individuelle Rationalität sich nicht so unversöhnlich gegenüber stehen, wie dies in Teilen der Literatur vertreten wird. Die unbedingte Gültigkeit von Normen strukturiert den Entscheidungsraum der Akteure vor, in dem bestimmte Handlungsalternativen moralisch „undenkbar“ sind (Crow 2002: 294). Damit bestimmen sie den Prozess des Framings mit. Dies trifft v.a. auf die Norm der elterlichen Verpflichtung zu, die nur bedingt abwägbar oder gar an Dritte übertragbar ist. So können z.B. mütterliche wie väterliche Betreuungsaufgaben aus verschiedenen Gründen nur begrenzt durch andere Akteure substituiert werden (Lewis 2001: 163; Duncan/Strell 2004: 42). Kinderbetreuung basiert auf Verantwortung und einer liebevollen, emotionalen Beziehung zwischen Elternteil und Kind. Wie bereits verdeutlicht wurde, muss die besondere Qualität der Eltern-Kind-Beziehung innerhalb der rationalen Annahmen berücksichtigt werden (Marten 2007a: 218f.). Ferner besteht eine stark institutionalisierte elterliche Verpflichtung für Kinder materiell und sozio-emotional zu sorgen. Dies ist gar im Gesetz zum Schutz des Kindeswohls festgeschrieben. Auch der Gestaltungsspielraum zum Erfüllen dieser Pflicht ist gesetzlich durch die Vorgabe einer „Mindestversorgung“ von Kindern geregelt. Die Vernachlässigung von Kindern steht unter Strafe. Ein weiteres Beispiel für nicht verhandelbare elterliche Verpflichtungen stellen die Nachtrennungsväter dar. Sie besitzen

"[...] 'live-time responsibilities' that do not end with the termination of marriage" (Crow 2002: 290, Hervorhebungen C.M.).

Eine besondere normative Begrenzung des Handlungsspielraums besteht auch hier in der Gesetzgebung. Diese macht den Nachtrennungsvätern beispielsweise Vorgaben, wie viel Unterhalt sie ihren Kindern mindestens zahlen müssen, um ihrer Unterhaltsverpflichtung normativ - „angemessen“ nachzukommen. Die elterliche Verantwortung gegenüber den Kindern ist damit normativ stark geprägt und langfristig angelegt. Die Handlungserwartungen in Form von Normen definieren die Handlungssituation sowie die denkbaren Alternativen mit und lassen sich somit in das vorliegende Handlungsmodell nach Kühnel/Bamberg integrieren. 
Ferner lässt die Interpretation normativer Handlungsvorgaben immer einen mehr oder weniger großen Gestaltungsspielraum für die individuellen Akteure (Streeck/Thelen 2005: 11; Burkart 1994: 59). So erfordert nicht nur der Übergang zur Vaterschaft nach der Norm der „verantworteten Elternschaft“ eine bewusste und damit verantwortungsvolle Entscheidung, sondern auch die Erziehung und Betreuung der Kinder ist an diverse (normative) Standards gebunden. Dabei ist der „Preis“ eines Kindes, d.h. die in es investierten Kosten, weder als Zeit- noch als Geldaufwendung gänzlich frei wählbar (Leip 2004: 107). Dennoch gibt es über einen vorgegebenen Mindeststandard hinaus sehr viel Gestaltungs- und Handlungsspielraum innerhalb des elterlichen Handelns, der individuelle Entscheidungsprozesse zulässt, gar erfordert.

Entscheidungsspielraum in der Ausgestaltung väterlichen Sorgehandelns eröffnet sich ferner durch die Pluralisierung von Familienarrangements und damit verbunden durch die steigende Ausdifferenzierung an Vaterschaftsformen: der verheiratete Vater, der uneheliche Vater, der getrennt lebende geschiedene Vater, der getrennt lebende uneheliche Vater, der Erzeuger oder auch der soziale Vater, der mit seiner Partnerin und deren Kind eine Haushaltsgemeinschaft bildet (Clarke/Roberts 2002: 165; Marsiglio et al. 2000: 1173f.; Tölke 2005: 3). Dies wirkt sich ebenso auf die individuelle Ausgestaltung des väterlichen Bewusstseins und Handelns aus. Gleichzeitig leisten Vätern neben finanzieller zunehmend auch emotionale Unterstützung für ihre Familie. Die Norm des männlichen Ernährers verliert an Bedeutung (Knijn 1995: 171; O’Brien 2006: 3; Jensen 1999). Gleichzeitig wird die Erfüllung der Ernährernorm durch Veränderungen in den ökonomischen Rahmenbedingungen zunehmend erschwert (siehe dazu ausführlich Marten 2007b). Insgesamt entstehen neue Vaterformen und alte Modelle verblassen. Damit eröffnen sich „modernen“ Vätern zunehmend Handlungs- und Gestaltungsspielräume mit Blick auf ihre Beziehung zu ihren Kindern, was für einen bewussten Entscheidungsprozess über die Ausgestaltung der eigenen Vaterrolle spricht und eine Rational Choice-Erklärung nahe legt.

Dieses Argument wird gestützt dadurch, dass Vaterschaft kein biographisch zwingendes Ereignis (mehr) im Lebensverlauf darstellt: ${ }^{60}$

„But children are no longer to be taken for granted. Like most other things children have become optional under the conditions of modernity [...]” (Kaufmann/Schulze 2002: 6).

60 Zur (freiwilligen) Kinderlosigkeit von Männern in ländervergleichender Perspektive siehe Knijn et al. 2007. 
Die Geburt eines Kindes wird verstärkt als individuelle oder zumindest als eine partnerschaftliche Entscheidung und weniger als eine gesellschaftliche Verpflichtung wahrgenommen (Feldhaus/Huinink 2005: 193).

Neben dem Entscheidungsspielraum können Normen auch über die Kosten- und NutzenKonzepte in die Theorie der rationalen Wahl integriert werden. Neuere Ansätze der Rational Choice-Theorie erkennen die Wirksamkeit von Normen durchaus an und berücksichtigen diese in ihren Modellen (Burkart 1994: 56).

„Wenn wir verstehen wollen, warum Leute Normen folgen (oder warum eben nicht), kommen wir eher zu einem befriedigenden Ergebnis, [...], wenn wir annehmen, dass die Menschen dabei vernünftig handeln. Normen wirken bzw. üben einen starken Druck auf uns aus, weil es einen komplexen Bezug auf signifikante Andere und auf zukünftige Folgen gibt [...]“ (Burkart 1994: 61f.).

Es wird deutlich, dass jedes Handeln an seinen Konsequenzen orientiert ist, auch normgeleitetes. Die Geltung von Normen basiert - zumindest zu einem gewissen Teil - auf den mit der Befolgung bzw. Missachtung von Normen verbundenen negativen externen Folgen, den Sanktionen im Falle der Norm-Devianz (Esser 1999: 283). Dabei kann das Eigeninteresse auch durch moralische Vorgaben eingeschränkt werden (Burkart 1994: 56). Hier entstehen i.d.R. eher immaterielle Nachteile wie durch den Verlust des „guten Rufes“ oder durch soziale Missachtung. Zu den Devianzkosten können neben externen ebenso interne Sanktionsmechanismen, wie Schuld- oder Schamgefühle gezählt werden (Esser 2000c: 52, Burkart 1994: 62, Himmelweit 2002: 241).

Übertragen auf Nachtrennungsväter scheint das Leisten von Unterhaltszahlungen nach rein individuell nutzenorientierten - egoistischen - Überlegungen für den Vater nicht rational. Vereinfacht formuliert hätte er damit ausschließlich finanzielle Nachteile, jedoch kaum einen „materiellen“ Nutzen. Auch andere - emotionale oder soziale - Interessen würden damit nicht befriedigt, wenn man die Wirkung eines möglichen Pay-offs zwischen Cash und Care vernachlässigt. ${ }^{61}$ Es stellt sich damit die Frage, warum Väter trotzdem ihren finanziellen Verpflichtungen nachkommen. Dies kann u.a. mit der immer noch starken männlichen Ernährer-Norm erklärt werden. Männer sehen sich normativ verpflichtet, ihre Familie - getrennt oder zusammenlebend - materiell zu versorgen. Diese Verpflichtung wird nicht zuletzt in Gesetzen zusätzlich - mit Sanktionsmechanismen - manifestiert.

${ }^{61}$ Es liegt die Vermutung nahe, dass Väter, die Unterhalt zahlen, auch ein verstärktes Interesse an einer guten Beziehung zu ihrem Kind haben. Andersherum zahlen Väter, die den Kontakt aufrecht erhalten, auch eher Unterhalt. Einige empirische Untersuchungen können einen Zusammenhang zwischen den beiden hier betrachteten - Seiten des väterlichen Sorgehandelns nachweisen (u.a. Bradshaw et al. 1999). Unklar bleibt jedoch, in welche Richtung die Kausalbeziehung ausgestaltet ist. Auf das Verhältnis zwischen Care und Cash wird im Kapitel 9.4 Väterliches Sorgehandeln: Das Verhältnis von Care und Cash empirisch eingegangen. 
Die Kosten der Devianz müssen höher liegen als der Nutzen, der daraus erwartet wird. Sonst ließe sich rational nicht erklären, warum sich Individuen auch von Normen und Werten leiten lassen, die ihnen aufwendige Handlungen und Verzicht auf Reizvolles aufbürden (Esser 1999: 132).

Neben dem schlechten Gewissen kann Normkonformität auch zu einem Nutzen für den Akteur werden. Innerhalb normativer Frames gilt der Weg häufig schon als Ziel. Das Handeln und Streben ist in diesem Fall durchaus - wie es als Kritikpunkt gegen die Rational Choice-Theorie angeführt wird - unabhängig von den positiven aber auch negativen Konsequenzen. Bittet ein Freund z.B. um Hilfe, werden die Kosten für diese Hilfeleistung nicht zwingend rational kalkuliert. Die Norm „auf knappe Ressourcen zu achten“ wird zugunsten der Norm „Freunden in Not beizustehen“ in den Hintergrund gedrängt. Dennoch ist auch die normative Motivation von individuellen Handlungen mittels der Rational Choice-Theorie zu erklären. Ein relativ einfaches Argument liegt in der Tatsache, dass Akteure sich wohl fühlen, wenn sie Normen gerecht werden. Daraus ergibt sich der Nutzen des Handelns (Miebach 2006: 435f., Esser 1999: 133f.), genauso wie das Gute nicht tun ein schlechtes Gefühl auslöst. Damit haben Normen und ihre Befolgung einen Wert „an sich“ (Esser 2000c: 52).

Als ein Beispiel für ein mit hohem intrinsischen Wert verbundenen Handeln kann das väterliche Sorgehandeln insbesondere in Form von Care interpretiert werden. Unabhängig von ökonomischen Zwängen, genießen Frauen wie Männer die geteilte Intimität mit ihren Kindern (Ostner 2004: 176; Marten 2007a: 218f.). Eine enge Vater-Kind-Beziehung hat neben moralischen Vorgaben auch einen eigenen individuellen Nutzen. Dieser ist weniger in Geldeinheiten, sondern vielmehr als emotionaler und auch sozialer Gewinn zu sehen. Dieser Nutzen wird gegen andere - durch die objektiven Rahmenbedingungen gegebene Restriktionen durchgesetzt. So kann erklärt werden, warum ein Vater trotz des Widerstandes der Mutter, der hohen Kosten, die mit der Aufrechterhaltung des Kontaktes über weite Distanzen verbunden sind oder vielleicht auch trotz des Missmutes der neuen Partnerin versuchen wird, sich um sein Kind zu kümmern. Einerseits sieht der Vater Positives in einer intensiven Vater-Kind-Beziehung, und zum anderen gilt die Norm der elterlichen Verantwortung auch über die Existenz der elterlichen Beziehung hinaus. Es kann vermutet werden, dass sich generell die Mehrheit der getrennten Väter - u.a. weil sie gesamtgesellschaftlich sollen - sich um ihre Kinder kümmern „wollen“. Wenn sie es nicht tun, so die These, liegt dies v.a. an Restriktionen in Form von Handlungsbarrieren. Als besonders einflussreich werden hier die Beziehung zur Kindesmutter, die räumliche Entfernung, 
fehlende finanzielle Mittel und eine um knappe Ressourcen konkurrierende neue Familie des Vaters angenommen. ${ }^{62}$

Ein weiteres Argument für die Vereinbarkeit von Normen und der Theorie der rationalen Wahl besteht darin, dass die Träger der Werte und Normen - entsprechend dem methodologischen Individualismus - die Individuen selbst sind (Esser 2004: 250). Nur über die individuelle Manifestation als Wunschvorstellung oder Persönlichkeitsmerkmal von handelnden Akteuren können Normen Einfluss auf das soziale Handeln nehmen. Sie wirken sich im Präferenzsystem und innerhalb der Kosten-Nutzen-Abwägung aus. Normen kommt dabei ein indirekter Effekt bei der individuellen Nutzenproduktion zu. Veränderungen innerhalb gesellschaftlicher Normen und Werte wirken nach der Theorie der rationalen Wahl nicht unvermittelt, sondern lediglich über die individuellen Entscheidungen und Wahlhandlungen (Esser 1999: 132ff.; Hill/Kopp 2004: 132; Miebach 2006: 70). ${ }^{63}$ Die Basis von Normen besteht gerade in ihrer individuell wahrgenommenen Nützlichkeit für die jeweilige „Lebenssituation“.

Insgesamt kann festgehalten werden, dass einerseits Abwägungen moralischen Vorgaben folgen können. Normen sind v.a. innerhalb familialer Beziehungsstrukturen zur Erklärung väterlichen Handelns von Bedeutung (Crow 2002: 293; Barlow et al. 2002: 119; Morgan 2002: 154). Andererseits müssen auch moralische Entscheidungen abgewogen werden. Dies gilt insbesondere, wenn sich die Faktoren der Moral in ihrer Handlungsrelevanz widersprechen. Dann muss es zu einer Prioritätensetzung - sprich einer Handlungsentscheidung im Sinne des Abwägens - kommen (Opp 1986: 7; Leip 2004: 138). ${ }^{64}$ Die Ausführungen machen deutlich, das Normen und rationale Kosten-Nutzen-Abwägungen

62 Zur genaueren theoretischen Fundierung dieser Determinanten sowie der angenommenen Wirkungsrichtung siehe Kap. 5 Hypothesengenerierung.

63 Der indirekte Einfluss wird auch in Abb. 6.1 Mehrebenen-Modell der sozialen Situation im Kap. 6.1. Makro-soziale Rahmenbedingungen dargestellt.

${ }^{64}$ Es können noch zahlreiche andere Argumente zur Rationalität der Normkonformität angeführt werden. Mittels normkonformen Handelns wird die Existenz einer gesamtgesellschaftlichen „Ordnung“ gesichert, und damit eine verlässliche Umgebung für das eigene Tun (Esser 2001: 320). Es ist im eigenen Interesse und damit vernünftig Normen zu folgen, weil diese das soziale Zusammenleben regulieren (Burkart 1994: 62). Damit unterscheiden sich zweck- und wertrationales Handeln nur noch in der Form der Konsequenzen, die sie berücksichtigen. Im Falle einer zweckrationalen Entscheidung stehen individuelle Eigeninteressen im Vordergrund, während eine wertrationale Handlung auch „gesellschaftliche“ Folgen berücksichtigt. Darüber hinaus kann die Normorientierung kognitive Einsparungen hervorbringen. Dies kommt dem bereits beschriebenen as-Modus der Informationsverarbeitung gleich. Das bedeutet, es findet keine anstrengende und komplexe Berechnung der Folgekosten in Relation zum Nutzen der Konsequenzen statt, sondern die direkte und unreflektierte Ausführung der Handlung, welche die geltende Norm vorgibt. Die Allgemeingültigkeit einer Norm lässt den Akteur vermuten, dass es ratsam sei, sich daran zu halten. Die Norm wird schon ihren „guten“ Grund haben (Esser 1999: 283f., Esser 2001: 319). 
sich nicht konträr gegenüberstehen (müssen). Ferner wirken sie sowohl auf der ersten Stufen des Handlungsmodells von Kühnel/Bamberg, dem Framing, als auch innerhalb der Handlungsauswahl, der zweiten Modellstufe.

\subsubsection{Familiales Handeln als emotionales Handeln}

Neben der Normorientierung unterscheidet sich familiales Handeln ferner von z.B. ökonomischen Handlungen durch den besonderen Bezug zu Emotionen (Hill/Kopp 2004: 125). Der rationale Erklärungsansatz vertritt die Annahme, dass selbst intime Entscheidungen wie die Eheschließung, eine Scheidung oder die Anzahl gemeinsamer Kinder auf der Grundlage von Abwägungen der Für und Wider getroffen werden. Die Gewichte der einzelnen Nutzen- bzw. Kostenwerte werden durch Präferenzen determiniert, die zwischen Familienmitgliedern wesentlich von Altruismus, Pflichtgefühlen oder Schuldigkeiten abhängen (Becker 1992: 40).

„Die Präferenz für eigene Kinder spricht für besondere Gefühle gegenüber eigenen Kindern. Die Opfer von Eltern, die ihren Kindern helfen wollen, aber auch das umgekehrte Phänomen, sowie die Liebe, die Ehepartner in vielen Fällen verbindet, deuten auf die hochgradig persönlichen Beziehungen in Familien hin, die in anderen Organisationen üblicherweise nicht zu finden sind““ (Becker 1985: 106).

Entgegen der viel diskutierten gesellschaftlichen Veränderungen stellt die Familie weiterhin den primären Ort der Verantwortung für Kinder dar, in dem Eltern und Kinder über Gefühle u.a. der Zusammengehörigkeit - anstelle eines Vertrages - miteinander verbunden sind (Becker 1985: 114).

Auch die Entscheidung für ein Kind basiert im Wesentlichen auf emotionalen Aspekten, auch wenn mit der Geburt v.a. Wünsche und Bedürfnisse der Eltern erfüllt werden (Leip 2004: 153). Ähnliche Überlegungen gelten für die Eheschließung oder die Partnerwahl generell. Heutzutage werden Partnerschaften und Ehen weniger aus ökonomischen Beweggründen, wie der finanziellen Absicherung der Frau oder der geschlechtsspezifischen Arbeitsteilung auf dem Familienhof, geschlossen. Vielmehr basieren „moderne“ Partnerschaften auf tiefen Gefühlen. Gerade für die Befriedigung emotionaler Bedürfnisse ist die Partnerschaft und weiter gefasst die Familie von Bedeutung (Hill/Kopp 2004: 79).

Trotz ihrer weitreichenden Beteiligung an und Bedeutung für individuelle Handlungen werden Emotionen innerhalb der RC-Ansätze häufig vernachlässigt. Besonders psychologische und soziale Anreize sind innerhalb rationaler Wahltheorien trotz der empirischen Relevanz bisher nicht ausreichend berücksichtigt worden. Stattdessen wurden vornehmlich „harte“ - materielle - Anreize wie das Einkommen, Vermögen oder auch Strafen als Sanktionsmechanismen einbezogen (Kunz 2004: 139; Esser 2006: 151). 
Ein Grund der Vernachlässigung emotionaler Aspekte innerhalb der RC-Theorie könnte in der scheinbaren Unvereinbarkeit beider theoretischer Konzepte gesehen werden (Miebach 2006: 407; Burkart 1994: 74). Nicht nur nach

„,[d]em Alltagsverständnis [...] schließen rationale Entscheidungsfindung und von Emotionen geleitetes Handeln einander aus: Entweder man kalkuliert kaltherzig alle Optionen, die sich einem in einer Entscheidungssituation bieten, und entscheidet sich dann für die beste, oder man handelt spontan, von den Gefühlen des Augenblicks überwältigt““ (Schnabel 2006: 175).

Insgesamt scheinen sich rationale Entscheidungen mit ihren Charakteristika der Auswahl und Zielgerichtetheit und Emotionen, die Konsistenz und Vorhersagbarkeit gerade ausschließen, unversöhnlich gegenüberzustehen. Emotionen sind nicht wählbar; sie zeichnen sich durch ihren scheinbar zwangsläufigen und unwillkürlichen Charakter aus (Schnabel 2006: $176 f f.)^{65}$

Trotz der Widersprüche kann eine sinnvolle Integration von Emotionen innerhalb der rationalen Handlungstheorie deren Erklärungskraft erweitern (Schnabel 2006: 175). Dabei können Gefühle - wie Normen - über den Nutzenbegriff in die RC-Theorie integriert werden. Auch positive Emotionen, wie der perzipierte Nutzen anderer Akteure oder dem Folgeleisten wahrgenommener gültiger Normen, können als zusätzliche Handlungsanreize - als positive und negative Verstärker - interpretiert werden (Hill/Kopp 2004: 137). Emotionale Konsequenzen, wenn auch vom Akteur nur begrenzt kontrollierbar, können durchaus in die Bewertung der Für und Wider einer Handlung eingehen. Indem der Akteur Handlungen auswählt, die seiner Ansicht nach zu Situationen führen, die bestimmte „gute“ Gefühle wahrscheinlicher auslösen bzw. „schlechte“ Gefühle eher vermeiden, werden Emotionen selbst zum Objekt einer rationalen Wahl. Damit werden Gefühle genau wie andere materielle „Güter“ in die individuelle Nutzenfunktion integrierbar. Dies können der Spaß an einer gemeinsamen Aktivität sein, das Gefühl von Solidarität, Bestätigung oder „Nestwärme“, oder die Befriedigung darüber ,das Richtige’ zu tun. ${ }^{66}$ Durch die Integration von Gefühlen in die Erklärung können vordergründig „irrationale“ Entscheidungen durch ihren zusätzlichen emotionalen Nutzen erklärt werden. So lässt Liebe einmalige Seitensprünge verzeihen, Zuneigung über Peinlichkeiten hinwegsehen und politische Solidarität hilft über kleinere weltanschauliche Differenzen hinweg (Schnabel 2006: 180ff.). Innerhalb von (Inter-)Aktionen mit hoher emotionaler Signifikanz für den Akteur

${ }^{65}$ Die - scheinbare - Unvereinbarkeit rationaler und emotionaler Verhaltensweisen drückt sich in der Literatur in der klaren Differenzierung zweier Handlungsformen innerhalb der gängigen Typologien aus. So unterscheidet Weber - als einer der berühmtesten Vertreter - explizit zwischen affektionalem und zweckrationalem Handeln (Weber 1972: 13).

${ }^{66}$ Siehe dazu auch die Ausführungen zu Rationalität und Normen in Kap. 4.3.2.2 Familiales Handeln als norm-orientiertes Handeln. 
läuft das „komplette Programm“ emotionaler, kognitiver und intentionaler Prozesse zur Handlungsauswahl ab, während bei primärer kognitiver Bedeutung der Situation rein kognitiv-objektive Abwägungen getroffen werden. Es besteht somit ein deutlicher Unterschied zwischen kognitiv-objektivem Nutzen, wie er v.a. in den ökonomischen Varianten der RC-Theorie Verwendung findet und emotionalem Nutzen (Esser 2006: 161f.), der u.a. innerhalb familialer Interaktion zum Tragen kommt. Dies entspricht einer anderen Form der Rationalität, die Rationalität jedoch nicht ausschließt.

Neben dem emotionalen Nutzen selbst, können Emotionen - rein analytisch - die Evaluation verschiedener Kosten- und Nutzenaspekte sowie die Einschätzung der Eintrittswahrscheinlichkeit beeinflussen (Schnabel 2006: 185). Mit einem Hungergefühl im Magen wird der Nutzen einer Einladung zum Mittagessen anders bewertet als ohne dieses Gefühl. Damit hilft die theoretische Berücksichtigung von Emotionen, die intertemporalen Schwankungen in den individuellen Nutzenfunktionen zu erklären (ebd.: 179). Aber auch bewusst gewordene - längerfristige - Emotionen, wie Liebe, Begeisterung oder Stolz können in die Nutzenfunktion eingehen. Einer Optimierung des Nutzens durch eine rationale Abwägung steht dies nicht entgegen (Esser 2006: 160). Der Einfluss der Emotionen kann über die kurzfristigen situativen Effekte hinausreichen. Ein anhaltendes Gefühl der Angst vor einer nahenden Umweltkatastrophe oder vor dem Statusverlust kann über die Einstellung hinaus Werthaltungen oder gar Weltbilder prägen. Damit wirken sich Gefühle auch auf die Präferenzordnung der Akteure aus bzw. sind ein wesentlicher Bestandteil dieser (Hill/Kopp 2004: 137; Esser 2006: 146; Schnabel 2006: 186).

Darüber hinaus beeinflussen Emotionen die Definition der Situation. Anhaltende Gefühle verändern den Rahmen der Situation, so dass der Akteur andere Situationselemente in den Vordergrund rückt, mit diesen andere Ziele verbindet und sich mit anderen Handlungsalternativen konfrontiert sieht. Mit den Gefühlen variiert beispielsweise die Einschätzung der jeweiligen Interaktionspartner. Ein Treffen mit der aktuellen Lebenspartnerin wird sicherlich von anderen Emotionen begleitet, wie ein Zusammentreffen mit der ehemaligen Partnerin und Mutter des gemeinsamen Kindes. Die unterschiedliche Wertschätzung der Interaktionspartner führt gleichzeitig zu einer veränderten Eintretenswahrscheinlichkeit der jeweiligen Handlungsresultate (Schnabel 2006: 185ff.).

Auch die kurzfristigen Emotionen bestimmen den Prozess des Framings mit, indem sie eine Verbindung zwischen situativen Stimuli und einer bestimmten Reaktion unter Einfluss der Prozesse emotionaler Erregung herstellen (Esser 2006: 151). Der „Kurzschluss“ zwischen Situationsstimulus und emotionaler Reaktion kann als Argument gegen die Vereinbarkeit von Gefühlswelt und rationalen Abwägungsprozessen angeführt werden. Emotionen lösen spontane, unreflektierte Reaktionen auf Reize innerhalb einer Situation aus, ohne dass der Akteur auf umfassende Kosten-Nutzen-Kalkulationen zurückgreifen muss. 
Dabei ist zu bedenken, dass bestimmte Gefühle nicht mit klar abgrenzbaren, unbedingten Reaktionsmustern verbunden sind, sondern eine gewisse Variation in den Handlungsreaktionen erlauben (Hill/Kopp 2004: 139f.). Einige situative Reize können auch widersprüchliche Emotionen auslösen. Diese sog. „gemischten Gefühlen“ oder auch die wahrgenommene Diskrepanz zwischen „Kopf und Bauch“ (Esser 2006: 164) nötigt den Akteur, - ein weiteres Mal - eine Entscheidung zu treffen, welchem Körperteil er folgt. Damit heben Emotionen den Selektionszwang für die Akteure in doppelter Hinsicht (zwischen Handlungsalternativen als Reaktion auf ein Gefühl oder zwischen verschiedenen Gefühlen in einer Situation) nicht auf, vielmehr grenzen sie den Optionsraum ein (Hill/Kopp 2004: 139f.).

Es kann argumentiert werden, dass die Emotionalisierung einer Situation die Anwendung eines elaborierten rc-Modus behindert. Ein dominantes Gefühl - die emotionale Definition der Situation - verhindert die Berücksichtigung anderer Handlungsalternativen und kann sich damit gegenüber dem kognitiven Framing durchsetzen. Dies erklärt z.B. die Schwierigkeit trotz aller Vernunft und Einsicht mit einer zur emotionalisierten Sucht gewordenen Gewohnheit aufzuhören (Esser 2006: 164f.). Dessen ungeachtet kann eine situative Emotionalisierung aber auch das Problembewusstsein schärfen, wodurch die individuelle Motivation, eine optimale Lösung zu finden, steigt. Damit unterstützt die durch auf Emotionen basierende stärkere Nachhaltigkeit in der Zielverfolgung sogar die Rationalität der Entscheidung.

Die Ausführungen machen deutlich, dass Emotionen den Prämissen der rationalen Wahl nicht widersprechen müssen, sondern in diese sinnvoll integriert werden können. So unterstützen Emotionen Akteure bei der Einschätzung der Kosten- und Nutzenkomponenten, beeinflussen die Wahrnehmung der Eintrittswahrscheinlichkeit, verändern individuelle Präferenzordnungen oder bestimmen die Definition der Situation mit. Ähnlich wie Normen wirken sie auf beiden Stufen des Handlungsmodells. Emotionen stellen ferner eine wesentliche Motivationsquelle menschlichen Handelns dar. Sie helfen Entscheidungen zu erklären, die auf den ersten Blick der „harten“ Variante der Rational Choice-Theorie „irrational“ erscheinen.

\subsubsection{Familiales Handeln als rationales Handeln}

Die bisherigen Ausführungen haben die Konzepte „Kosten“ sowie „Nutzen“ unhinterfragt auf menschliche Beziehungen und den väterlichen Kontakt zu Kindern angewendet. Kin- 
der als Kosten und Nutzen zu konzeptionalisieren muss jedoch nicht nur moralisch kritisch reflektiert werden. ${ }^{67}$ Die Auswahl zwischen Handlungsalternativen setzt die Vergleichbarkeit der einzelnen Optionen voraus (Burkart 1994: 86; Esser 1999: 315). Fraglich ist, ob dies bei den Handlungsalternativen, zwischen denen Nachtrennungsväter wählen können, der Fall ist. Zunächst erscheint es wenig einleuchtend, dass z.B. Kinder aus verschiedenen Partnerschaften für den Vater mit unterschiedlichen Wertigkeiten belegt sein sollen, die eine Entscheidung für einen intensiveren Kontakt zu einem Kind im Unterschied zu einem anderen begründet. Doch ausgehend von einem einheitlichen Wert der Beziehungen zu allen Kindern können Differenzen im Aufwand, der nötig ist die Beziehung zu pflegen, ausgemacht werden. Der Kontakt zu Kindern außerhalb des eigenen Haushalts ist z.B. mit einem höheren organisatorischen, zeitlichen und auch finanziellen Aufwand verbunden als Aktivitäten mit Kindern, die mit dem Vater zusammenleben. Es entstehen u.U. Reisekosten und Anreisezeiten, es müssen Termine abgesprochen und Aktivitäten geplant werden. Ferner kann sich die gescheiterte Partnerschaft der Elternteile negativ auf den Kontakt zum Kind auswirken, denn dieser impliziert immer auch Kontakt zur Kindesmutter.

Die klassische Nutzentheorie unterstellt eine einheitliche Währung für alle Güter. Für das „Gut Kind“ ist diese Annahme sicherlich sehr begrenzt (Leip 2004: 63). Eine Eltern-KindBeziehung ist schwer in Geldeinheiten zu messen.

„Parents are, at best, guessing about the possible costs of children, weighting these against a set of preferences that are shaped by social norms. Their guesses have long-run implications. Unlike other investments children are not fungible; they cannot be bought or sold, or traded or exchanged” (Folbre 1994: 111).

Besonders wahrgenommene ökonomische Kosten von Kindern stehen häufig hinter der elterlichen Verantwortung zur Fürsorge zurück (Duncan/Strell 2004: 42), was nach strenger RC-Theorie wenig rational erscheint. Dennoch kann dieses Verhalten mittels RCAnnahmen erklärt werden, indem u.a. Emotionen und auch Normen wie dargestellt integriert werden. Es geht dabei weniger um die Erklärung der Beziehung zu Kindern als vielmehr um die väterliche Ausgestaltung in Form von Care und Cash. Erklärt werden väterliche Handlungsmuster und nicht väterliche Gefühle, die in der Tat schwer rational begründbar sind. Diese Emotionen dienen im theoretischen Modell primär der Erklärung väterlichen Handelns. Das Kosten-Nutzen-Konzept wird in der vorliegenden Untersuchung als theoretisches Instrument zur Erklärung väterlichen Handelns verstanden.

${ }^{67}$ Noch vor hundert Jahren galten Kinder als Produktionsgüter, die die alternden Eltern pflegten und versorgten sowie als Arbeitskräfte im Familienbetrieb mitarbeiteten. Heute stellen Kinder „Konsumgüter“ dar und die elterliche Beziehung zu ihnen ist hoch emotionalisiert (Leip 2004: 71; Ostner 2007: 239). 
Die Ausführungen weisen darauf hin, dass bei der Übertragung der RC-Theorie auf familiensoziologische Phänomene, wie dem väterlichen Sorgehandeln, ein relativ weiter Begriff des Nutzens bzw. der Kosten Verwendung finden muss, um den Besonderheiten des Feldes gerecht werden zu können. Die Erweiterung des Nutzensbegriffs z.B. um Emotionen hat innerhalb der Literatur vielfältige Kritik ausgelöst. Damit sei es

„[i]rgendwie [...] immer möglich, das Ergebnis individuellen Handelns als ,Nutzen’ für dieses Individuum zu interpretieren“ (Burkart 1994: 37).

Viele Autoren verweisen in diesem Zusammenhang auf die Gefahr der Tautologie:

„Denn unter diesen Voraussetzungen lässt sich für jedes Handeln ad hoc eine bestimmte Präferenz konstruieren, die zu diesem Handeln passt“ (Kunz 2004: 140, Hervorhebungen C.M.).

Bei der Theorie der Rationalen Wahl handelt es sich jedoch nicht um eine originalgetreue Abbildung der Wirklichkeit. Die zentralen Begriffe sind abstrakt definiert und beziehen sich nicht auf konkrete Ereignisse und Akteure. Sie dienen als formales Konzept zur Analyse menschlichen Verhaltens, wie es empirisch nachzuweisen ist (Kunz 2004: 141; Esser 2000b: 337). Damit ist der Ansatz tatsächlich inhaltsleer, wie ihm seine Kritiker vorwerfen und auch seine Vertreter einräumen. Aber gerade dies sichert seine vielseitige und vielschichtige Anwendbarkeit auf zahlreiche soziale Phänomene. Die generellen Vorteile der Theorie v.a. ihre Sparsamkeit und Einfachheit lassen sich somit auch für familiensoziologische Fragestellungen nutzen (Himmelweit 2002: 232; Morgan 2002: 151, Esser 2001: 329). Doch in dieser Vereinfachung sehen einige Wissenschaftler auch eine der zentralen Schwächen der Rational Choice-Theorie. Kritikpunkte

„[...] betreffen neben der individualistischen Perspektive und der Überbetonung formaler Aspekte insbesondere die oftmals sehr vereinfachende Vorgehensweise und die häufig unrealistischen Voraussetzungen“ (Kunz 2004: 135).

Durch die starke Abstraktion von der Realität, so das Argument, wird ihrer Komplexität nicht ausreichend Rechnung getragen und die theoretische Erklärungskraft vermindert sich (Kunz 2004: 142). Gern wird die Theorie kritisch auf mathematische Formeln beschränkt, abgeleitet aus unrealistischen Prämissen (Burkart 1994: 37). Dabei sollte bedacht werden, dass jedes theoretische Modell zur Erklärung der „sozialen Welt“ Vereinfachungen von der Realität vornimmt und vornehmen muss. Abstraktionen müssen jedoch nicht notwendiger Weise unrealistisch sein. Wesentlich ist, dass Nutzenmaximierung tatsächliches Handeln in einfacher Form beschreibt und zu empirisch überprüfbaren Thesen führt (Esser 1999: 21, 314ff.).

\subsubsection{Zwischenfazit I: Das Rationalitätsverständnis}

Die systematische Berücksichtigung von Normen und Emotionen verweist auf ein anderes Rationalitätsverständnis als das der Neo-Klassiker (Glover 2002: 262). Entscheidungen werden hier zwar immer noch anhand einer rationalen Abwägung der Pro und Contras 
vorgenommen, doch die Vor- und Nachteile betreffen neben ökonomischen, auch soziale, kognitive und emotionale Konsequenzen. Damit handelt es sich um ein weites RC-Verständnis (Opp 1999; Duncan/ Strell 2004: 41).

In den vorangegangenen Ausführungen wurde die Anwendbarkeit des RC-Ansatzes zur Analyse väterlichen Sorgehandelns diskutiert. Trotz der Eigenheiten des familiensoziologischen Gegenstandsbereiches, der es zunächst contraintuitiv erscheinen lässt rationale Entscheidungsprozesse auf familiale Beziehungen anzuwenden, die von starken Gefühlen, Normen und Alltagserfahrungen geprägt sind, kann der Ansatz in seiner weiten Version zur theoretischen Analyse fruchtbar gemacht werden.

"[...] analysts do not have to search around for new theories or modes of enquiry when they turn their attention to family life. Theoretical tools are already to hand" (Morgan 2002: 152, Hervorhebung C.M.).

Auch Becker (1981: 9) verweist darauf:

"The economic approach provides a framework applicable to all human behaviour - to all types of decisions and to persons from all walks of life. [...] [It] uses the assumptions of maximizing behavior, stable preferences, and equilibrium in implicit and explicit markets to provide a systematic analysis of the family" (Hervorhebungen C.M.).

Insgesamt lässt sich somit auch väterliches Sorgehandeln in Nachtrennungsfamilien als rationale Entscheidung theoretisch fassen. Im folgenden Abschnitt werden aus den bisher dargestellten theoretischen Annahmen empirisch prüfbare Hypothesen abgeleitet und begründet. Dabei beschränkt sich die Hypothesengenerierung auf solche Annahmen über Zusammenhänge, die am vorhandenen Material geprüft werden können. Hypothesen, die darüber hinaus mit Hilfe des theoretischen Modells entwickelt werden können, finden sich allein innerhalb der vorangegangenen Darstellung im Theorieteil. Diese Einschränkung gilt primär für den ersten Schritt des zweistufigen Modells. Elemente des Framings, die insbesondere innere Prozesse u.a. ausgedrückt in Einstellungen betreffen, können mit Hilfe des vorliegenden Datenmaterials nicht bzw. nur sehr eingeschränkt überprüft werden. Dennoch gilt das Modell von Kühnel/Bamberg in seiner Vollständigkeit - und nicht auf die Handlungsauswahl begrenzt - als theoretisch fruchtbar für die Analyse väterlichen Sorgehandelns in Nachtrennungsfamilien. 


\section{Hypothesengenerierung}

Im Theorieteil der Arbeit wurde bereits auf die Übertragung der theoretischen Annahmen auf väterliches Sorgehandeln im Speziellen eingegangen. Im Folgenden werden explizit Hypothesen entwickelt, die angeben, welche Faktoren väterliches Care bzw. Cash erleichtern bzw. behindern und damit die Wahrscheinlichkeit, dass sich ein Vater sozioemotional bzw. finanziell kümmert, erhöhen bzw. verringern. ${ }^{68}$ Die aufgestellten Annahmen werden probabilistisch formuliert. Des Weiteren wird immer von einer bestimmten Richtung des Wirkungszusammenhangs ausgegangen, der in der jeweiligen These explizit formuliert wird. Die einzelnen Determinanten werden entlang der Konzepte „soziale Umwelt“, „physische Umwelt“ und „Überzeugungssystem“, wie sie im Handlungsmodell von Kühnel/Bamberg entwickelt wurden, dargestellt. ${ }^{69}$

\subsection{Determinanten der physischen Umwelt}

Eine wichtige Handlungsbarriere der physischen Umwelt, die sich auf das väterliche Sorgehandeln in Form von Care auswirkt, stellt die Entfernung zwischen den elterlichen Wohnorten dar. Mit zunehmender Entfernung erfordern väterliche Besuche ein höheres Ausmaß an Organisation und Absprachen. Kurze Distanzen können eher spontan und kurzfristig überbrückt werden. Längere Anreisen sind darüber hinaus mit höheren finanziellen Belastungen verbunden wie den Reise- und gegebenenfalls auch Unterkunftskosten, wenn der Vater zum Kind reist, aber nicht im Haushalt der Mutter übernachtet. Neben finanziellen Kosten sind längere Reisen auch mit einem höheren zeitlichen Aufwand verbunden. Daher kann angenommen werden, dass gilt: Je höher all diese Kosten für einen Besuch insgesamt sind, desto niedriger ist die Wahrscheinlichkeit eines hohen Niveaus väterlichen Engagements. Es wird ein negativer Zusammenhang angenommen. Für die Überweisung von Unterhaltsraten dürfte die räumliche Entfernung dagegen nicht von Bedeutung sein. Es kann angenommen werden, dass der monetäre Transfer keinen

\footnotetext{
68 Thesen, die sich auf die Care-Komponente beziehen, werden mit einer „__“ versehen, Cash-Hypothesen werden mit „_2“ gekennzeichnet.

${ }^{69}$ Zum Teil können einzelne Determinanten inhaltlich mehreren der drei Dimensionen zugeordnet werden. Darauf wird an gegebener Stelle eingegangen. Das theoretische Modell dient vordergründig der Thesengenerierung, wird im folgenden Kapitel jedoch gleichzeitig für die strukturierte Darstellung von Thesen und im weiteren Verlauf der Analyse auch der Ergebnisse genutzt. Die Differenzierung in „soziale Umwelt“, „physische Umwelt“ und ,inneres Überzeugungssystems“ wird sich demnach durch die folgenden Darstellungen als roter Faden ziehen.
} 
persönlichen Kontakt zwischen Vater und Kind erforderlich macht. Hier wird kein Zusammenhang erwartet.

Tab. 5.1: Hypothesen zur Entfernung zwischen den Wohnorten.

\begin{tabular}{|c|l|}
\hline Dist_1 & $\begin{array}{l}\text { Mit steigender räumlicher Distanz zwischen den Wohnorten der Eltern sinkt die } \\
\text { Wahrscheinlichkeit eines hohen sozio-emotionalen Engagements des Vaters. }\end{array}$ \\
\hline Dist_2 & $\begin{array}{l}\text { Die räumliche Distanz zwischen den Wohnorten der Eltern hat keinen Einfluss auf } \\
\text { mögliche Unterhaltsprobleme. }\end{array}$ \\
\hline
\end{tabular}

Das väterliche Kümmern wird durch finanzielle, soziale, emotionale und zeitliche Ressourcen determiniert. ${ }^{71}$ Das Einkommen bestimmt danach die Verfügbarkeit ökonomischer Ressourcen und damit die Zahlungsfähigkeit des Vaters. Wenn der getrennt lebende Vater Zahlungsschwierigkeiten hat bzw. glaubt, die finanzielle Belastung der Unterhaltsraten nicht aufbringen zu können, wird er die Zahlungen einstellen (Braver et al. 1993: 99).

Tab. 5.2: Hypothesen zum Einkommen des Vaters.

\begin{tabular}{|c|l|}
\hline Ek_1 & $\begin{array}{l}\text { Mit steigendem Einkommen nimmt die Wahrscheinlichkeit eines hohen sozio-emo- } \\
\text { tionalen Engagements des Vaters zu. }\end{array}$ \\
\hline Ek_2 & Mit steigendem Einkommen sinkt die Wahrscheinlichkeit von Unterhaltsproblemen. \\
\hline
\end{tabular}

Doch auch väterliches Sorgehandeln im Sinne von Care kann mit finanziellen Aufwendungen verbunden sein, wie dies bereits bei der Entfernung skizziert wurde. Väter, die keine oder wenige finanzielle Mittel zur Verfügung haben, weisen größere Schwierigkeiten auf, einen regelmäßigen Kontakt aufrecht zu erhalten. Dies gilt insbesondere, wenn eine große räumliche Distanz zwischen den Wohnorten besteht. Skevik (2006: 121) spricht in diesem Zusammenhang von der "socioeconomic-advantaged parenting perspective“, nach der die sozio-ökonomische Position des Vaters das Ausmaß seines finanziellen wie sozio-emotionalen Kümmerns mitbestimmt. Kosten für väterliches Sorgehandeln sind somit immer „relativ“, d.h. sie sind in Relation zu den verfügbaren finanziellen Ressourcen des Vaters zu sehen. Für einen Vater mit wenig Geld stellen regelmäßige und vollständige Unterhaltsleistungen eine andere Herausforderung dar als für einen Vater, der keine finanziellen Sorgen hat. Hier ist von einem positiven Zusammenhang auszugehen: Mit steigendem Einkommen steigt auch die Wahrscheinlichkeit regelmäßiger und vollständiger Unterhaltsleistungen. Ein positiver Zusammenhang besteht

\footnotetext{
${ }^{70}$ Unterhaltsprobleme werden als ausbleibende Unterhaltszahlungen verstanden.

${ }^{71}$ Siehe dazu ausführlich Kap. 2.2 Nachtrennungsfamilien: das Phänomen abwesender Väter.
} 
auch für die Care-Komponente. Die Wahrscheinlichkeit einer intensiven Vater-Kind-Beziehung steigt mit dem Einkommen des Vaters.

Eine wichtige Determinante, die die Zahl und Form wahrgenommener Handlungsalternativen bestimmt, ist das Ausbildungsniveau des Vaters. Die Verfügbarkeit verschiedener Optionen ist nicht für alle Akteure gleich. So argumentiert auch Amendt (2003: 65f., 81f.), dass die soziale Lebenslage des Vaters Einfluss auf den Erhalt des Kontaktes zwischen Vater und Kind hat. Männer mit geringem Bildungsniveau erfahren innerhalb der Trennungskonflikte mit der Ex-Partnerin aufgrund der geringeren Verfügbarkeit sozialer Ressourcen, z.B. in Form von Konfliktlösestrategien, öfter Handlungsunfähigkeit und Machtlosigkeit. Eine überdurchschnittlich häufige Reaktion darauf ist der Kontaktabbruch zum Kind.

Tab. 5.3.: Hypothesen zum Ausbildungsniveau des Vaters.

\begin{tabular}{|c|l|}
\hline Ausb_1 & $\begin{array}{l}\text { Mit steigendem Bildungsniveau des Vaters nimmt die Wahrscheinlichkeit eines } \\
\text { hohen sozio-emotionalen Engagements des Vaters zu. }\end{array}$ \\
\hline Ausb_2 & $\begin{array}{l}\text { Mit steigendem Bildungsniveau des Vaters sinkt die Wahrscheinlichkeit von } \\
\text { Unterhaltsproblemen. }\end{array}$ \\
\hline
\end{tabular}

Handlungsalternativen zur Ausgestaltung des väterlichen Kümmerns hängen somit neben allgemeinen strukturellen Bedingungen wie z.B. dem Bildungssystem, dem Arbeitsmarkt oder den kulturellen geschlechtsspezifischen Rollenerwartungen, auch von der individuellen Milieu- und Schichtzugehörigkeit, insbesondere dem erworbenen Bildungskapital, den zugänglichen sozialen Unterstützungsnetzwerken sowie der biographischen Position ab (Burkart 1994: 249). Das Bildungsniveau des Vaters wirkt sich dabei nicht nur auf die Kontakthäufigkeit, sondern ferner auf die Qualität des väterlichen Engagements aus. Väter, die als „bildungsfern“ charakterisiert werden, werden z.B. Aktivitäten, die die schulische Ausbildung des Kindes betreffen, anders ausfüllen können, als Väter mit einem akademischen Hintergrund. Beide Vätergruppen werden diesem Bereich ferner unterschiedliche Bedeutung beimessen. Auch können sich die Art der Spielpraktiken zwischen diesen Vätergruppen unterscheiden. Insgesamt führen die theoretischen Überlegungen damit zu der Annahme, dass ein positiver Zusammenhang zwischen väterlichem Bildungsniveau und der Wahrscheinlichkeit eines intensiven Engagements des Vaters besteht.

Mit Blick auf die Zahlungsleistungen des Vaters kann der Ausbildung zunächst ein über das Einkommen vermittelter Einfluss zugesprochen werden. Ein höheres Ausbildungsniveau ist i.d.R. auch mit einem höheren Einkommen verbunden. Darüber hinaus kann angenommen werden, dass ein höheres Ausbildungsniveau zu einem anderen Bewusstsein und auch zu einer anderen Einsicht in die eigene Zahlungsverpflichtung führt. Männer mit 
langen Ausbildungszeiten werden zu einem späteren Zeitpunkt in ihrem Lebensverlauf zu Vätern; auch dies kann zu einem stärkeren - nicht nur finanziellen - Verantwortungsbewusstsein führen. Insgesamt wird hier davon ausgegangen, dass mit steigendem Niveau des väterlichen Bildungsstandes auch die Wahrscheinlichkeit steigt, dass der Vater seinen Unterhaltsverpflichtungen nachkommt und damit seltener Zahlungsprobleme vorliegen.

Als Teil der physischen Umwelt kann die Arbeitszeit als zeitliche Ressource bzw. Restriktion interpretiert werden. Zeit, die am Arbeitsplatz verbracht wird, steht nicht mehr für familiäre Aktivitäten zur Verfügung. Dies gilt insbesondere für extrem lange Arbeitszeiten des Vaters - die auch als Indikator für eine starke Karriereorientierung dienen können. ${ }^{72}$ Basierend auf der Bedeutung der Ressource Zeit, wie sie bereits im theoretischen Teil (Kap. 4.2.2.1) ausführlich dargestellt wurde, kann daher angenommen werden, dass mit zunehmendem Zeitanteil, den der Vater am Arbeitsplatz verbringt, die Wahrscheinlichkeit eines regelmäßigen und zeitaufwendigen Kontaktes zu seinem Kind sinkt.

\section{Tab. 5.4.: Hypothesen zur Arbeitszeit des Vaters.}

\begin{tabular}{|c|l|}
\hline ArbZ_1 & $\begin{array}{l}\text { Mit steigender Arbeitszeit sinkt die Wahrscheinlichkeit eines hohen sozio- } \\
\text { emotionalen Engagements des Vaters. }\end{array}$ \\
\hline ArbZ_2 & Mit steigender Arbeitszeit sinkt die Wahrscheinlichkeit von Unterhaltsproblemen. \\
\hline
\end{tabular}

Neben den zeitlichen Restriktionen, die mit längeren Arbeitszeiten verbunden sind, können sie jedoch auch zu zusätzlichen wirtschaftlichen Erträgen führen, vorausgesetzt es gilt die Beziehung, dass mehr Arbeitsstunden höher entlohnt werden, von der hier ausgegangen wird. Damit sollte sich die Arbeitszeit positiv auf die väterliche Zahlungsmoral auswirken, d.h. mit steigender Zahl an Arbeitsstunden sinkt die Wahrscheinlichkeit von Unterhaltsschwierigkeiten. ${ }^{73}$

\footnotetext{
72 Die berufliche Orientierung des Vaters verstanden als Karriereorientierung stellt einen wesentlichen Aspekt der inneren Bedingungen dar. Ein Vater, der sich in erster Linie als „Ernährer“ seiner Familie begreift, wird andere berufliche Ziele verfolgen, als ein Vater, der sein väterliches Handeln nicht auf finanzielle Dimensionen beschränkt bzw. konzentriert, sondern darüber hinaus auch Zeit mit seinem Kind verbringen möchte. Andersherum wird ein Karriere orientierter Mann seine Vaterschaft anders ausfüllen als ein „Familienmensch“. Hier wird die Arbeitszeit jedoch primär als Determinante der physischen Umwelt gesehen. Dies kann u.a. damit begründet werden, dass die Arbeitszeit nur bedingt vom Vater frei gewählt werden kann. Somit stellen die vorgegebenen Arbeits(markt)bedingungen den Kontext für väterliches Sorgehandeln dar.

${ }^{73}$ Dieser Effekt könnte jedoch durch die Determinante Einkommen selbst überlagert werden.
} 
Neben verschiedenen Determinanten der physischen Umwelt werden in der nachfolgenden Analyse auch Bestimmungsfaktoren berücksichtigt, die der sozialen Umwelt des Nachtrennungsvaters zuzuordnen sind.

\subsection{Determinanten der mikro-sozialen Umwelt}

Die mikro-soziale Umwelt des Vaters besteht zunächst aus seinem familialen Umfeld. Dazu zählen Mitglieder seiner getrennt lebenden Familie ebenso wie potentielle neue Familienmitglieder, sprich eine neue Partnerin oder andere Kinder im Haushalt. Neben anderen Lebensbereichen wie der Freizeit oder Berufstätigkeit konkurrieren die getrennt lebenden unterhaltsberechtigten Kinder dabei mit anderen Familienmitgliedern um die knappen Ressourcen des Vaters: Zeit und Geld. So argumentiert z.B. auch Becker (1985: 111):

„Die Zuwendung, die jedem Kind zu Teil wird, könnte abnehmen, wenn die Zahl der Kinder, die Zuwendung brauchen, steigt.“

Dies gilt für finanzielle, zeitliche und sozio-emotionale Zuwendung gleichermaßen. Eine wichtige Gruppe dieser „konkurrierenden“ Familienmitglieder sind dabei die Kinder im Haushalt des Vaters. Durch die Anwesenheit von Kindern im väterlichen Haushalt bekommt dieser die Möglichkeit, seine Vaterrolle innerhalb seines unmittelbaren Umfelds auszuleben. Kinder, die im alltäglichen Leben physisch nur zu bestimmten Besuchszeiten präsent sind, könnten in den Hintergrund geraten. Damit könnte der väterliche Wunsch nach einem engen Kontakt zu den externen Kindern eingeschränkt werden. Das Konkurrieren um Ressourcen ist dabei nicht auf die finanziellen beschränkt. Zeitliche Beanspruchungen verringern die Intensität des Kontaktes zum abwesenden Elternteil (Braver et al. 1993: 99; Palkovitz 1997: 213). Skevik (2006: 119) spricht in diesem Zusammenhang von der 'social parenting perspective'.

"We would expect contact to wane when the father establishes a new relationship, and most certainly if the new partner brings her own children into the household. This perspective emphasises social parenting over biological parenthood, and suggests that fathers involve in 'serial parenting' - that they parent the children who are at any point in time living in their household” (Skevik 2006a: 119).

\section{Tab. 5.5.: Hypothesen zur Zahl der Kinder im väterlichen Haushalt.}

\begin{tabular}{|c|l|}
\hline KiHH_1 & $\begin{array}{l}\text { Mit steigender Zahl unterhaltsberechtigter Kinder im väterlichen Haushalt sinkt } \\
\text { die Wahrscheinlichkeit eines hohen sozio-emotionalen Engagements des Vaters. }\end{array}$ \\
\hline KiHH_2 & $\begin{array}{l}\text { Mit steigender Zahl unterhaltsberechtigter Kinder im väterlichen Haushalt nimmt } \\
\text { die Wahrscheinlichkeit von Unterhaltsproblemen zu. }\end{array}$ \\
\hline
\end{tabular}

Auch „neue“ eigene Kinder, die der Vater gemeinsam mit einer neuen Partnerin hat, können ein höheres väterliches Engagement „zu Hause“ auslösen als über Haushaltsgrenzen hinweg. Mit zunehmender Zahl der Kinder im väterlichen Haushalt sinkt daher 
die Wahrscheinlichkeit eines intensiveren väterlichen Kontakts, ferner steigt die Gefahr von Zahlungsschwierigkeiten.

Ähnliche Überlegungen wie für die Kinder im Haushalt gelten auch für die vom Vater getrennt lebenden Kinder, die unterhaltsberechtigt sind. Je nach Anzahl der „externen“ unterhaltsberechtigten Kinder verändern sich die „Kosten“, die mit dem Aufrechterhalten eines intensiven Vater-Kind-Verhältnisses bzw. den Unterhaltszahlungen verbunden sind. Das bedeutet, die getrennt lebenden Kinder und gegebenenfalls die Kinder im väterlichen Haushalt konkurrieren um die knappen zeitlichen und finanziellen Ressourcen des Vaters. Es wird somit von folgendem Zusammenhang ausgegangen: Je höher die finanzielle Belastung aufgrund von verschiedenen Unterhaltsverpflichtungen ist, desto eher können Zahlungsschwierigkeiten auftreten. Ferner wird ein negativer Einfluss einer hohen Zahl getrennt lebender Kinder auf die Wahrscheinlichkeit einer hohen väterlichen Involviertheit angenommen.

Tab. 5.6.: Hypothesen zur Zahl der Kinder außerhalb des väterlichen Haushalts.

\begin{tabular}{|l|l|}
\hline \multirow{2}{*}{ ExtKi_1 } & $\begin{array}{l}\text { Mit steigender Zahl unterhaltsberechtigter Kinder außerhalb des väterlichen } \\
\text { Haushalts sinkt die Wahrscheinlichkeit eines hohen sozio-emotionalen } \\
\text { Engagements des Vaters. }\end{array}$ \\
\hline ExtKi_2 & $\begin{array}{l}\text { Mit steigender Zahl unterhaltsberechtigter Kinder außerhalb des väterlichen } \\
\text { Haushalts nimmt die Wahrscheinlichkeit von Unterhaltsproblemen zu. }\end{array}$ \\
\hline
\end{tabular}

Neben den an- und abwesenden Kindern im väterlichen Haushalt zählen die aktuellen Partnerschaften beider Elternteile als wichtiger Bestandteil der väterlichen sozialen Umwelt. Die Integration des abwesenden Elternteils in die Erziehung des Kindes ist abhängig von den aktuellen Beziehungssituationen (Depner/Bray 1993b: 192; Braver et al. 1993: 97). ${ }^{74}$

Eine neue Partnerschaft des Vaters erfordert emotionale, soziale und zeitliche Ressourcen, die ihm für sein unterhaltsberechtigtes Kind nicht mehr zur Verfügung stehen (Skevik 2006a: 119). Damit sinkt die Intensität des väterlichen Engagements. Neben der zusätzlichen Konkurrenz um väterliche Ressourcen kann die neue Partnerin das Sorgehandeln des getrennt lebenden Vaters selbst fördern aber auch behindern, indem sie dessen Notwendigkeit befürwortet oder ablehnt und damit den Vater motiviert bzw. hemmt. Es wird davon ausgegangen, dass eine neue Partnerschaft des Vaters eine hohe Intensität des

\footnotetext{
${ }^{74}$ Dabei heiraten bis zu 85\% der Männer nach der Scheidung erneut, während ihre Zahlungsverpflichtungen für frühere Familien weiter bestehen. Dieser Anteil ist höher als bei den Frauen (Bray/Berger 1993: 164).
} 
väterlichen Engagements unwahrscheinlicher werden lässt. Das Leisten von Unterhalt wird weniger von den emotionalen und sozialen Ressourcen, die in die neue Beziehung investiert werden, beeinflusst als von den aufgewendeten finanziellen Mitteln. Auch hier wirkt sich die Existenz einer neuen Partnerschaft negativ auf die Wahrscheinlichkeit verlässlicher Unterhaltsleistungen aus.

Tab. 5.7: Hypothesen zur Partnersituation des Vaters.

\begin{tabular}{|l|l|l|}
\hline BezVa_1 & $\begin{array}{l}\text { Eine neue Partnerin des Vaters senkt die Wahrscheinlichkeit eines hohen sozio- } \\
\text { emotionalen väterlichen Engagements gegenüber dem externen Kind. }\end{array}$ \\
\hline BezVa_2 & $\begin{array}{l}\text { Eine neue Partnerin des Vaters steigert die Wahrscheinlichkeit von } \\
\text { Unterhaltsproblemen. }\end{array}$ \\
\hline
\end{tabular}

Hat die Mutter einen neuen Partner, der gegebenenfalls im gemeinsamen Haushalt wohnt, kann für das Kind eine neue Vaterfigur im direkten sozialen Umfeld entstehen. Die Funktion, die vorher (im Idealfall) der getrennt lebende Vater innerhalb der familialen Strukturen eingenommen hat, kann u.U. verbunden mit physischer Anwesenheit vom neuen Partner der Mutter ausgefüllt werden (Bray/Berger 1993: 165). Der mögliche Rückgang des Engagements des abwesenden Vaters kann dabei unterschiedlich begründet sein. Zum einen kann der Vater sich aus eigenem Antrieb zurückziehen, um z.B. das Kind zu schonen. ${ }^{75}$ Zum anderen kann die Mutter ein Interesse daran haben, den kindlichen Kontakt zum leiblichen Vater zu unterbinden, um damit ihr „neues“ Familienleben zu schützen. ${ }^{76}$ Gleichzeitig kann jedoch auch vermutet werden, dass die Mutter den Kontakt zum Kindsvater unterstützt, um auf diese Weise zeitliche Freiräume für ihre neue Partnerschaft zu schaffen (Skevik 2006a: 120). Entscheidend ist in diesem Zusammenhang jedoch die Perspektive des Vaters. Für ihn kann die Anwesenheit eines „neuen Vaters“ im Haushalt „seiner alten Familie“ mit zusätzlichen emotionalen Kosten verbunden sein, die sich negativ auf die Wahrscheinlichkeit eines intensiven Kümmerns auswirken.

Gleichzeitig - und dies ist eher ein Argument mit Blick auf die inneren Bedingungen und Einstellungen des Vaters - kann der Vater die Einsicht in seine finanzielle Verpflichtung verlieren aufgrund eines - aus seiner Sicht - „neuen Ernährers“ in seiner getrennt leben-

\footnotetext{
75 Die durch die Trennung der Eltern und den Verlust der Familie erlittenen Schmerzen und Leiden werden mit Besuchen bei Kind und Vater aktualisiert (Braver et al. 1993: 93). Der Vater kann es für die Erziehung des Kindes als schädlich empfinden, wenn das Kind aus seiner alltäglichen Routine herausgerissen wird. Amendt (2004: 216ff.) spricht in diesem Zusammenhang vom „heroischen Verzicht dem Kind zuliebe“.

${ }^{76}$ Zur Gate Keeper-Funktion der Kindesmutter wird im folgenden Abschnitt detaillierter Stellung genommen.
} 
den Familie. ${ }^{77}$ Dies hat zur Folge, dass der Vater nicht mehr zahlen will, obwohl er dies möglicherweise kann.

Insgesamt wird daher theoretisch erwartet, dass sich ein neuer Partner im mütterlichen Haushalt negativ auf das Sorgehandeln des Nachtrennungsvaters in Form von Care auswirkt. Gleichzeitig erhöht sich damit die Wahrscheinlichkeit von Problemen im Zahlungsverhalten des abwesenden Vaters.

\section{Tab. 5.8: Hypothesen zur Partnersituation der Mutter.}

\begin{tabular}{|l|l|}
\hline BezMu_1 & $\begin{array}{l}\text { Eine aktuell bestehende Partnerschaft der Mutter senkt die Wahrscheinlichkeit } \\
\text { eines hohen sozio-emotionalen väterlichen Engagements gegenüber dem } \\
\text { externen Kind. }\end{array}$ \\
\hline BezMu_2 & $\begin{array}{l}\text { Eine aktuell bestehende Partnerschaft der Mutter erhöht die Wahrscheinlichkeit } \\
\text { von Unterhaltsproblemen. }\end{array}$ \\
\hline
\end{tabular}

Die vorangestellten Ausführen weisen bereits auf die Bedeutung des Verhältnisses zwischen den beiden Elternteilen für das väterliche Sorgehandeln hin. Dabei können unterschiedliche Zeitpunkte zur Evaluation der elterlichen Beziehung herangezogen werden. Wesentlich für das aktuelle Handeln des Vaters ist zunächst sein Verhältnis zur Kindesmutter im Zeitpunkt der Befragung, aber auch die elterliche Beziehung vor und während der Trennung ist von Bedeutung.

Die Familienkonstellation vor der Trennung umfasst das ehemalige Zusammenleben sowie den Familienstand der elterlichen Partnerschaft vor ihrem Scheitern. Sie stellt ein Indiz für die Qualität der damaligen Beziehung dar. Mit der veränderten Bedeutung der Institution Ehe wird heute kontrovers darüber diskutiert, ob eine nicht-eheliche Lebensgemeinschaft weniger gefestigt ist als eine Ehe. Im Zusammenhang mit der Realisierung von Kinderwünschen kommt der Ehe immer noch eine große Bedeutung zu (Vaskovics et al. 1997: 178). Familienplanung findet i.d.R. immer noch innerhalb institutionalisierter Beziehungen statt, die bereits von längerer Dauer und gefestigter Qualität sind. Die Entscheidung eine Ehe einzugehen ist eine bedeutende für den Lebensverlauf. Sie kann als Verbindlichkeitsindikator interpretiert werden.

Des Weiteren führt je nach Familienkonstellation der Trennungsprozess zu einem unterschiedlichen Aufwand und verschiedenen Konsequenzen für die Vater-Kind-Beziehung.

\footnotetext{
${ }^{77}$ Ein neuer Partner und eventuell (sozialer) Vater kann jedoch auch das Gegenteil beim Nachtrennungsvater auslösen. Aus Angst seine Position als Vater beim Kind zu verlieren, verstärkt er sein sozio-emotionales Engagement ebenso wie seine finanzielle Unterstützung. Die empirische Analyse wird zeigen in welche Richtung der angenommene Zusammenhang tatsächlich wirkt.
} 
Haben die Eltern keinen gemeinsamen Haushalt geführt, so bringt eine Trennung mit Blick auf die Wohnungssituation wenig Veränderungen mit sich. Der Scheidungsprozess, der mit hohem Regelungsbedarf verbunden ist, kann längerfristige - im Streitfall negative - Effekte auf die einzelnen Beziehungen innerhalb der Nachtrennungsfamilie haben (Bray/Berger 1993: 160). Der Faktor des Formalisierungsgrades der elterlichen Partnerschaft kann ferner als ein Aspekt der inneren Bedingungen interpretiert werden. Eine engere Bindung an die Mutter bzw. eine stärkere Verbindlichkeit der Partnerschaft kann zu einem höheren finanziellen wie auch sozio-emotionalen Verantwortungsgefühl führen und damit das väterliche Engagement stärken. So argumentiert auch Skevik (2006a: 118):

"It is frequently argued that fathers who have taken on the responsibilities of marriage - 'invested' in a wedding and promises of a joint future - will feel a deeper commitment to their families, including children, than men who have fathered children without such prior commitment."

Dahinter steht die Annahme, dass die Verbindlichkeit der elterlichen Beziehung bzw. das väterliche Verantwortungsbewusstsein vor der elterlichen Trennung sich auch danach durchsetzt. Dies impliziert ferner, dass weniger institutionalisierte Partnerschaften eine höhere Wahrscheinlichkeit eines geringeren väterlichen Kontakts aufweisen. Skevik (2006a: 118) spricht in diesem Zusammenhang von der “marital involvement hypothesis”, die sich auch im vorliegenden theoretischen Modell begründen lässt. Ähnliche Überlegungen können auf die väterliche Zahlungspraxis übertragen werden. Mit höherer Verbindlichkeit der elterlichen Partnerschaft sieht sich der Vater gegebenenfalls finanziell stärker in der Pflicht.

\section{Tab. 5.9: Hypothesen zur Familienform der Eltern vor der Trennung.}

\begin{tabular}{|c|l|}
\hline FamKon_1 & $\begin{array}{l}\text { Mit steigender Formalisierung der früheren Partnerschaft zwischen den Eltern } \\
\text { nimmt die Wahrscheinlichkeit eines hohen sozio-emotionalen Engagements } \\
\text { des Vaters zu. }\end{array}$ \\
\hline FamKon_2 & $\begin{array}{l}\text { Mit steigender Formalisierung der früheren Partnerschaft zwischen den Eltern } \\
\text { sinkt die Wahrscheinlichkeit von Unterhaltsproblemen. }\end{array}$ \\
\hline
\end{tabular}

Die vorangegangene Argumentation zum Institutionalisierungsgrad der elterlichen Partnerschaft vor der Trennung kann für die Determinante der Dauer der elterlichen Beziehung übernommen werden. Dabei gilt hier, dass die Verbindlichkeit der Beziehung mit zunehmender zeitlicher Dauer ansteigt. Des Weiteren kann angenommen werden, dass je länger die elterliche Partnerschaft - insbesondere in Form einer Haushaltsgemeinschaft besteht, desto mehr Zeit hatte der Vater eine Beziehung zum Kind aufzubauen und diese 
zu intensivieren. ${ }^{78}$ Eine starke Vater-Kind-Bindung vor der Trennung wirkt sich positiv auf die Wahrscheinlichkeit einer intensiven Beziehung nach der Trennung aus. So wird hier davon ausgegangen, dass mit zunehmender Beziehungsdauer die Wahrscheinlichkeit eines intensiven Kümmerns in Form von Care ansteigt. Der Einfluss auf die Zahlungspraxis wird mit den gleichen Argumenten begründet wie bei der elterlichen Familienkonstellation vor der Trennung. Es wird ein positiver Zusammenhang zwischen Dauer der Beziehung und vollständigen Unterhaltszahlungen angenommen.

Tab. 5.10.: Hypothesen zur Dauer der Beziehung vor der Trennung.

\begin{tabular}{|c|l|}
\hline DurBez_1 & $\begin{array}{l}\text { Mit steigender Beziehungsdauer der Eltern vor der Trennung steigt die } \\
\text { Wahrscheinlichkeit eines hohen sozio-emotionalen Engagements des Vaters. }\end{array}$ \\
\hline DurBez_2 & $\begin{array}{l}\text { Mit steigender Beziehungsdauer der Eltern vor der Trennung sinkt die } \\
\text { Wahrscheinlichkeit von Unterhaltsproblemen. }\end{array}$ \\
\hline
\end{tabular}

Neben dem Status der elterlichen Beziehung vor der Trennung zählt das Verhältnis der Eltern während des Trennungsprozesses als ein wichtiger Bestandteil der sozialen Umwelt des Vaters. Die Festlegung des Unterhalts und des Sorge- bzw. Umgangsrechts werden hier als Indikatoren für das elterliche Verhältnis während der Trennung interpretiert.

Der Entscheidungsprozess birgt aufgrund unterschiedlicher Interessen und einem i.d.R. ohnehin angespannten - emotionalen - Verhältnis zwischen den Elternteilen ein hohes Konfliktpotenzial in sich. Einvernehmlich getroffene Regelungen deuten darauf hin, dass die Eltern die partnerschaftlichen Konflikte entweder in Maßen halten oder die Ebene der Paarbeziehung von der Elternebene trennen können. Die zwischen Vater und Mutter geteilte Betreuung der Kinder ist weniger emotional belastet, wenn sich beide Elternteile gemeinsam auf diese Form des Sorgerechts geeinigt haben. Die konsensuale Festlegung von Unterhalt und Sorge- bzw. Umgangsrecht, so die Annahme hier, fördert die Wahrscheinlichkeit eines hohen Niveaus väterlichen Engagements. Auf die Leistung von Unterhalt hat die Sorgerechtsfestlegung keinen Einfluss. Dagegen deuten geringe Konflikte bei der Unterhaltsregelung darauf hin, dass der Vater seine finanzielle Verantwortung anerkennt sowie die Höhe des Unterhalts als gerechtfertigt betrachtet. Als ungerecht empfundene Unterhaltsforderungen führen eher zu Zahlungsschwierigkeiten.

\footnotetext{
${ }^{78}$ Hier ist einschränkend anzumerken, dass dies nur gilt, wenn die Trennung nicht kurz nach der Geburt des Kindes stattgefunden hat. Ein besserer Indikator wäre hier das Alter des Kindes. Dieses ist aufgrund methodischer Unsicherheiten jedoch nicht in allen Untersuchungen als Variable verfügbar. Des Weiteren kann angenommen werden, dass die Intensität des väterlichen Engagements mit steigendem Kindesalter ab einem gewissen Punkt aufgrund „normaler“ Abnabelungsprozesse wieder sinkt.
} 
Tab. 5.11.: Hypothesen zur Unterhalts- und Sorge- bzw. Umgangsfestlegung.

\begin{tabular}{|c|l|}
\hline FestUH_1 & $\begin{array}{l}\text { Mit steigendem Konfliktpotential im Rahmen der Unterhaltsfestlegung sinkt die } \\
\text { Wahrscheinlichkeit eines hohen sozio-emotionalen Engagements des Vaters. }\end{array}$ \\
\hline FestUH_2 & $\begin{array}{l}\text { Mit steigendem Konfliktpotential im Rahmen der Unterhaltsfestlegung nimmt } \\
\text { die Wahrscheinlichkeit von Unterhaltsproblemen zu. }\end{array}$ \\
\hline FestSR_1 & $\begin{array}{l}\text { Mit steigendem Konfliktpotential im Rahmen der Sorgerechtsfestlegung sinkt } \\
\text { die Wahrscheinlichkeit eines hohen sozio-emotionalen Engagements des } \\
\text { Vaters. }\end{array}$ \\
\hline FestSR_2 & $\begin{array}{l}\text { Das Konfliktpotential im Rahmen der Sorgerechtsfestlegung hat keinen } \\
\text { Einfluss auf mögliche Unterhaltsprobleme. }\end{array}$ \\
\hline
\end{tabular}

Neben dem Prozess der Festlegung bestimmter Regelungen während der Trennung spielt das Ergebnis dieser Aushandlungen eine wichtige Rolle für die sozio-emotionale Sorgepraxis des Vaters. Der Einfluss des tatsächlich festgelegten Sorge- und Umgangsrechts ist theoretisch sehr einsichtig. Ein gemeinsam ausgeübtes Sorgerecht gibt Vätern eine Legitimationsgrundlage für einen intensiven Kontakt zu ihrem Kind. Bei alleinigem Sorgerecht der Mutter ist der Vater darauf angewiesen, dass seine Ex-Partnerin ein väterliches Engagement über den festgelegten Umgang hinaus zulässt. ${ }^{79}$ Mit der Festlegung von Sorge- und Umgangsrecht wird manifestiert, wie viel der Vater sich (mindestens) kümmern kann. Die Unterhaltspraxis sollte dagegen nicht vom festgelegten Umgang beeinflusst werden. ${ }^{80}$

Tab. 5.12.: Hypothesen zur festgelegten Form des Sorge- bzw. Umgangsrechts.

\begin{tabular}{|c|l|}
\hline Sorge_1 & $\begin{array}{l}\text { Ein geteiltes Sorgerecht der Eltern erhöht die Wahrscheinlichkeit eines hohen } \\
\text { sozio-emotionalen Engagements des Vaters. }\end{array}$ \\
\hline Sorge_2 & $\begin{array}{l}\text { Ein geteiltes Sorgerecht der Eltern hat keinen Einfluss auf mögliche } \\
\text { Unterhaltsprobleme. }\end{array}$ \\
\hline
\end{tabular}

${ }^{79}$ Innerhalb einer deutschen Studie ergab sich, dass Probleme mit den Besuchsterminen seltener auftreten, wenn die Verantwortung gegenüber den Kindern nach der Trennung gemeinsam übernommen wird. Das gemeinsame Sorgerecht hat eine positive Auswirkung auf die Normalisierung der Situation innerhalb der Nachtrennungsfamilie (Amendt 2003: 86).

${ }^{80}$ An dieser Stelle kann eingewandt werden, dass das väterliche Care und Cash in einem engen Zusammenhang stehen (könnten). Ein Vater, der kaum Umgang zu seinem Kind aufgrund des festgelegten Sorgerechts hat, könnte wenig Einsicht in seine Zahlungsverpflichtung haben. Einige Autoren gehen von einem trade off zwischen Unterhaltszahlungen und einer hohen Kontakthäufigkeit aus (u.a. Bradshaw et al. 1999: 227). Bisher konnte die Richtung des angenommenen Zusammenhangs empirisch nicht eindeutig nachgewiesen werden. Die folgende Analyse betrachtet Cash separat von Care. Das Verhältnis zwischen beiden Dimensionen väterlichen Sorgehandelns wird in Kap. 9.4 Väterliches Sorgehandeln: Das Verhältnis von Care und Cash dargestellt. 
Als letzter Aspekt der elterlichen Beziehung erscheint das Verhältnis im Zeitpunkt der Befragung, also nach der Trennung, von Bedeutung. In der Literatur wird der Kindsmutter häufig eine "gate keeper"-Funktion zugesprochen: Sie ist es, die das väterliche Sorgehandeln - in welcher Form auch immer - zulassen muss. ${ }^{81}$ Dies kann sich besonders in Nachtrennungsfamilien aufgrund der emotional angespannten Situation schwierig gestalten. Die mütterliche Einflussnahme stellt damit eine wichtige Determinante väterlichen Sorgehandelns dar. Ein freundschaftliches Verhältnis zur Kindesmutter senkt die emotionalen Belastungen, die ein Vater gegebenenfalls mit der Aufrechterhaltung der Beziehung zu seinem Kind nach der elterlichen Trennung verbindet. Ein freundschaftliches Verhältnis zwischen den Eltern erleichtert ferner Absprachen und gemeinsame Entscheidungen, die das Kind betreffen. Insgesamt sollte eine gute Beziehung zwischen den Eltern die Wahrscheinlichkeit eines intensiven Kontakts des Vaters zum getrennt lebenden Kind erhöhen. So lange er annimmt, den Unterhalt zu Gunsten des Kindes zu zahlen und dieser aus seiner Perspektive nicht primär der Mutter zugute kommt, sollte das freundschaftliche Verhältnis der Eltern keine Auswirkungen auf das Leisten von Unterhaltszahlungen haben.

Tab. 5.13.: Hypothesen zum freundschaftlichen Verhältnis der Eltern.

\begin{tabular}{|l|l|}
\hline Freund_1 & $\begin{array}{l}\text { Ein freundschaftliches Verhältnis zwischen den Eltern nach der Trennung } \\
\text { erhöht die Wahrscheinlichkeit eines hohen sozio-emotionalen Engagements } \\
\text { des Vaters. }\end{array}$ \\
\hline Freund_2 & $\begin{array}{l}\text { Ein freundschaftliches Verhältnis zwischen den Eltern nach der Trennung hat } \\
\text { keinen Einfluss auf mögliche Unterhaltsprobleme. }\end{array}$ \\
\hline
\end{tabular}

Innerhalb des Handlungsmodells von Kühnel/Bamberg wirken sich neben der physischen und sozialen Umwelt des Vaters ferner das individuelle Überzeugungssystem auf den Framing- und Selektionsprozess aus.

\subsection{Determinanten innerhalb des Überzeugungssystems}

Neben der sozialen und physischen Umwelt und ihren Ressourcen sowie Restriktionen wird das väterliche Sorgehandeln ferner durch das akteursimmanente Überzeugungssystem bestimmt (u.a. Feldhaus/Huinink 2005: 199f.; Matzner 2004: 25). Dazu zählt die

${ }^{81}$ Diese Funktion ist in den meisten Scheidungsgesetzgebungen sogar institutionalisiert. So muss in vielen Ländern - auch in denen, die ein gemeinsames Sorgerecht in der Ehe und nach der elterlichen Trennung als Norm praktizieren - das gemeinsame Sorgerecht v.a. von den nicht verheirateten Eltern gemeinsam, also konsensuell, beantragt werden. Im deutschen und im britischen Fall können nicht verheiratete Väter nur mit dem Einverständnis der Mutter das gemeinsame Sorgerecht erhalten. Zum Teil gilt dies auch für die Umgangsrechte, so in Deutschland (Ostner 2007: 235). 
Persönlichkeit des Akteurs bestimmt durch Einstellungen, Wertorientierungen und Emotionen.

Tab. 5.14: Hypothesen zur ökonomischen Situation der Mutter.

\begin{tabular}{|c|l|}
\hline ÖkSitMu_1 & $\begin{array}{l}\text { Die wahrgenommene ökonomische Situation der Mutter hat keinen Einfluss } \\
\text { auf die Wahrscheinlichkeit eines hohen sozio-emotionalen Engagements des } \\
\text { Vaters. }\end{array}$ \\
\hline ÖkSitMu_2 & $\begin{array}{l}\text { Mit zunehmend als schlecht wahrgenommener ökonomischer Situation der } \\
\text { Mutter sinkt die Wahrscheinlichkeit von Unterhaltsproblemen. }\end{array}$ \\
\hline
\end{tabular}

Ein wichtiger Aspekt des inneren Überzeugungssystems ist die Einsicht des Vaters, dass er seiner getrennt lebenden Familie finanziell - aber auch sozio-emotional - verpflichtet ist. Diese Einsicht ist eher gegeben, wenn sich die Mutter in einer prekären ökonomischen Situation befindet, d.h. auf die Unterhaltszahlungen angewiesen ist. Dabei ist es weniger von Bedeutung, ob die Mutter tatsächlich finanziell schlecht gestellt ist. Wichtig ist vielmehr, wie der Vater ihre monetäre Situation wahrnimmt. Es kann jedoch nicht angenommen werden, dass ein Vater eher zahlt, weil die Mutter sich in einer finanziellen Notlage befindet, denn diese kann gerade durch ausbleibende Unterhaltszahlungen ausgelöst worden sein. Der Ursache-Wirkungs-Zusammenhang ist nicht eindeutig zu bestimmen. Es kann jedoch der umgekehrte Zusammenhang theoretisch fundiert werden: Ein Vater, der annimmt, dass seine Ex-Partnerin monetär gut gestellt ist, hat eine geringere Einsicht in seine finanzielle Verpflichtung als ein Vater, der davon ausgeht, dass die Mutter seine Unterstützung benötigt. Dies impliziert die Annahme, dass je schlechter der Vater die ökonomische Situation der Mutter wahrnimmt, desto eher kommt er seinen Zahlungsverpflichtungen nach. Auf das väterliche Sorgehandeln in Form von Care dürfte die wahrgenommene ökonomische Situation der Mutter keinen Einfluss haben.

\section{Tab. 5.15: Hypothesen zur Dauer seit der Trennung.}

\begin{tabular}{|c|l|}
\hline DurSep_1 & $\begin{array}{l}\text { Mit zunehmender zeitlicher Distanz zur elterlichen Trennung sinkt die } \\
\text { Wahrscheinlichkeit eines hohen sozio-emotionalen Engagements des Vaters. }\end{array}$ \\
\hline DurSep_2 & $\begin{array}{l}\text { Mit zunehmender zeitlicher Distanz zur elterlichen Trennung steigt die } \\
\text { Wahrscheinlichkeit von Unterhaltsproblemen. }\end{array}$ \\
\hline
\end{tabular}

Mit der wahrgenommen Fairness kann auch im Fall der Dauer seit der Trennung argumentiert werden, was ihre Einordnung im Rahmen des individuellen Überzeugungssystems rechtfertigt. Es gilt: Je länger die Trennung zurückliegt, desto eher treten Zahlungs- und Kontaktabbrüche auf. Mit der Zeit kann das finanzielle Verantwortungsgefühl des Vaters insbesondere für seine frühere Partnerin aber auch für gemeinsame Kinder abnehmen. Die Zeitkomponente führt ferner zu einer emotionalen Distanzierung und psychischen Ablösung, die durch die räumliche Trennung begünstigt wird (Amato 1998: 
256). Auch andere Studien belegen, dass die Gefahr des Kontaktabbruchs mit der zeitlichen Distanz zur Trennung steigt (Amendt 2003: 78, Bray/Berger 1993: 162).

Es wurde bereits bei den formulierten Hypothesen zur Anzahl der getrennt lebenden unterhaltsberechtigten Kinder mit dem väterlichen Commitment des Vaters gegenüber der Kindsmutter argumentiert. In eine ähnliche Richtung zielen die Annahmen mit Blick auf die Anzahl der gescheiterten Beziehungen, aus denen Kinder hervorgegangen sind. Diese Zahl der Elternschaften liefert erste Hinweise darauf, wie stark sich der Vater in der Vergangenheit an Partnerinnen gebunden hat. Eine hohe Anzahl an Elternschaften außerhalb seines Haushaltes kann als Indiz für einen unbeständigen Lebenswandel interpretiert werden. Ein geringes Verantwortungsgefühl und eine niedrige Verbindlichkeit der Partnerschaften dürfte sich negativ auf die Wahrscheinlichkeit eines intensiven väterlichen Sorgehandelns sowohl in Form von Care als auch von Cash auswirken. Hier gelten demnach ähnliche Argumente in umgekehrter Richtung, wie bei der Formalisierung der elterlichen Beziehung vor der Trennung. Mit Blick auf die finanziellen Unterstützungsleistungen kann angenommen werden, dass je mehr getrennt lebende Elternschaften für den Vater bestehen, desto eher wird er seinen Zahlungsverpflichtungen nicht nachkommen, weil diese eher höhere Kosten verursachen als Unterhaltsforderungen aus nur einer gescheiterten Beziehung.

Tab. 5.16: Hypothesen zur Anzahl der Beziehungen mit externen Kindern.

\begin{tabular}{|l|l}
\hline \multirow{2}{*}{ AnzBez_1 } & $\begin{array}{l}\text { Mit steigender Zahl gescheiterter Beziehungen, aus denen Kinder } \\
\text { hervorgegangen sind, sinkt die Wahrscheinlichkeit eines hohen sozio- } \\
\text { emotionalen Engagements des Vaters. }\end{array}$ \\
\hline \multirow{2}{*}{ AnzBez_2 } & $\begin{array}{l}\text { Mit steigender Zahl gescheiterter Beziehungen, aus denen Kinder } \\
\text { hervorgegangen sind, nimmt die Wahrscheinlichkeit von } \\
\text { Unterhaltsproblemen zu. }\end{array}$ \\
\hline
\end{tabular}

Basierend auf den dargestellten theoretischen Überlegungen können für insgesamt 17 Determinanten Hypothesen formuliert werden, die angeben, wie der jeweilige Prädiktor auf die Care- bzw. Cash-Komponente väterlichen Sorgehandelns wirkt. Unten stehende Tabelle 5.17 stellt die angenommenen Zusammenhänge im Überblick dar. 
Tab.: 5.17: Übersicht der theoretisch generierten Determinanten väterlichen Sorgehandelns und ihrer Wirkungsrichtung getrennt nach Cash und Care.

\begin{tabular}{|c|c|c|}
\hline THESE & DETERMINANTEN DER PHYSISCHEN UMWELT & $\mathbf{Z}$ \\
\hline Dist_1 & Distanz zwischen den Wohnorten steigt $\Rightarrow$ Care sinkt eher & - \\
\hline Dist_2 & Distanz zwischen den Wohnorten - ohne Einfluss auf Cash-Probleme & 0 \\
\hline Ek_1 & Einkommen steigt $\Rightarrow$ Care steigt eher & + \\
\hline Ek_2 & Einkommen steigt $\Rightarrow$ Cash-Probleme unwahrscheinlicher & - \\
\hline ArbZ_1 & Arbeitszeit steigt $\Rightarrow$ Care sinkt eher & - \\
\hline ArbZ_2 & Arbeitszeit steigt $\Rightarrow$ Cash-Probleme unwahrscheinlicher & - \\
\hline Ausb_1 & Bildungsniveau steigt $\Rightarrow$ Care steigt eher & + \\
\hline Ausb_2 & Bildungsniveau steigt $\Rightarrow$ Cash-Probleme unwahrscheinlicher & - \\
\hline THESE & DETERMINANTEN DER MIKRO-SOZIALEN UMWELT & $\mathbf{Z}$ \\
\hline KiHH_1 & Kinder im väterlichen Haushalt $\Rightarrow$ Care sinkt eher & - \\
\hline KiHH_2 & Kinder im väterlichen Haushalt $\Rightarrow$ Cash-Probleme wahrscheinlicher & + \\
\hline ExtKi_1 & Externe Kinderzahl steigt $\Rightarrow$ Care sinkt eher & - \\
\hline ExtKi_2 & Externe Kinderzahl steigt $\Rightarrow$ Cash-Probleme wahrscheinlicher & + \\
\hline BezVa_1 & Beziehung des Vaters formalisierter $\Rightarrow$ Care sinkt eher & - \\
\hline BezVa_2 & Beziehung des Vaters formalisierter $\Rightarrow$ Cash-Probleme wahrscheinlicher & + \\
\hline BezMu_1 & Beziehung der Mutter formalisierter $\Rightarrow$ Care sinkt eher & - \\
\hline BezMu_2 & Beziehung der Mutter formalisierter $\Rightarrow$ Cash-Probleme wahrscheinlicher & + \\
\hline FamKon_1 & ehemalige Beziehung formalisierter $\Rightarrow$ Care steigt eher & + \\
\hline FamKon_2 & ehemalige Beziehung formalisierter $\Rightarrow$ Cash-Probleme unwahrscheinlicher & - \\
\hline DurBez_1 & Längere elterliche Beziehung früher $\Rightarrow$ Care steigt eher & + \\
\hline DurBez_2 & Längere elterliche Beziehung früher $\Rightarrow$ Cash-Probleme unwahrscheinlicher & - \\
\hline FestUH_1 & Friedlichere Unterhaltsfestlegung $\Rightarrow$ Care steigt eher & - \\
\hline FestUH_2 & Friedlichere Unterhaltsfestlegung $\Rightarrow$ Cash-Probleme unwahrscheinlicher & + \\
\hline FestSR_1 & Friedlichere Sorgerechtssfestlegung $\Rightarrow$ Care steigt eher & - \\
\hline FestSR_2 & Friedlichere Sorgerechtsfestlegung - ohne Einfluss auf Cash-Probleme & 0 \\
\hline Sorge_1 & Gemeinsames Sorgerecht $\Rightarrow$ Care steigt eher & + \\
\hline Sorge_2 & Gemeinsames Sorgerecht - ohne Einfluss auf Cash-Probleme & 0 \\
\hline Freund_1 & Freundschaft der Eltern heute $\Rightarrow$ Care steigt eher & + \\
\hline Freund_2 & Freundschaft der Eltern heute - ohne Einfluss auf Cash-Probleme & 0 \\
\hline THESE & DETERMINANTEN DER INDIVIDUELLEN ÜBERZEUGUNG & $\mathbf{Z}$ \\
\hline ÖkSitMu_1 & Ökonomische Lage der Mutter - ohne Einfluss auf Care & 0 \\
\hline ÖkSitMu_2 & Schlechtere ökon. Lage der Mutter $\Rightarrow$ Cash-Probleme unwahrscheinlicher & - \\
\hline DurSep_1 & Längere Dauer seit der Trennung $\Rightarrow$ Care sinkt eher & - \\
\hline DurSep_2 & Längere Dauer seit der Trennung $\Rightarrow$ Cash-Probleme wahrscheinlicher & + \\
\hline AnzBez_1 & Anzahl Beziehungen mit Kindern steigt $\Rightarrow$ Care sinkt eher & - \\
\hline AnzBez_2 & Anzahl Beziehungen mit Kindern steigt $\Rightarrow$ Cash-Probleme wahrscheinlicher & + \\
\hline
\end{tabular}

$Z$ = Richtung des erwarteten Zusammenhangs

+ = positiver Zusammenhang; - = negativer Zusammenhang; 0 = kein Zusammenhang

Quelle: eigene Darstellung. 
Es wird deutlich, dass Cash und Care durch einzelne Determinante in gleicher Weise, d.h. mit der gleichen Wirkungsrichtung, bestimmt werden. ${ }^{82}$ Zum Teil werden jedoch auch gegenläufige Zusammenhänge erwartet. Aufgrund der unterschiedlichen Wirkungsrichtungen werden innerhalb der nachfolgenden Analyse die Care- und Cash-Komponente väterlichen Sorgehandelns zunächst getrennt voneinander erklärt. Es kann weiter davon ausgegangen werden, dass die einzelnen Determinanten auf verschiedenen Ebenen miteinander korrespondieren und sich wechselseitig beeinflussen (Matzner 2004: 25; Feldhaus/Huinink 2005: 199). Diesen Wechselwirkungen wird innerhalb einer multiplen Analyse Rechnung getragen. Zunächst werden jedoch die makro-sozialen Kontexte, in denen die Nachtrennungsväter eingebettet sind, beschrieben. Die drei Länder, Deutschland, Großbritannien und Norwegen, dienen dabei der parallelen Theorietestung.

${ }^{82}$ Es ist dabei darauf zu achten, dass Cash nicht als generelle Zahlungspraxis des Vaters verstanden werden darf, sondern als Zahlungsprobleme konzeptionalisiert wird (siehe dazu ausführlich Kap. 10.2. Die abhängige Variable: Cash). Damit käme einer „steigenden Cash-Variablen“ eine andere Bedeutung zu. Hier impliziert das Ansteigen der Cash-Variable die Zunahme von Zahlungsschwierigkeiten und nicht die verbesserte Leistung von Unterhalt z.B. in Form ihrer Höhe oder Regelmäßigkeit. Ein negativ angenommener Zusammenhang zu Cash ist damit als „gut“ zu bewerten für die Zahlungsmoral des Vaters, weil dies bedeutet, dass keine Zahlungsprobleme vorliegen. 


\section{Nationale Kontexte der Nachtrennungsväter}

Neben dem unmittelbaren, v.a. familialen Kontext ist der Vater als Akteur in weiter reichende soziale Rahmenbedingungen eingebettet. Das Framing einer Situation stellt keinen Prozess der freien Reflexion, der individuellen Interpretation und stetigen Neudefinition unterschiedlicher Handlungssituationen dar, sondern ist vielmehr von sozialen Institutionen umgeben und geprägt (Leip 2004: 25; Esser 2001: 157). So gilt:

"Despite their essentially personal and private nature, human sexual as well as otherwise passionate behaviour, in societies like ours, will regularly occur in highly regulated, often institutionalized frameworks of social relationships which for the individual have the character of a 'fait social' (Durkheim 1970) urging him to behave in certain ways rather than in others” (Strohmeier 2002: 322, Hervorhebungen C.M.).

Die Handlungsbeschränkungen bzw. -möglichkeiten der mikro-sozialen und physischen Umwelt werden durch Institutionen des makro-sozialen Kontextes bestimmt (Kunz 2004: 37). Abbildung 6.1 zeigt das hier zugrunde gelegte Verständnis der sozialen Situation graphisch als Mehrebenen-Modell.

\section{Abb. 6.1: Mehrebenen-Modell der sozialen Situation.}

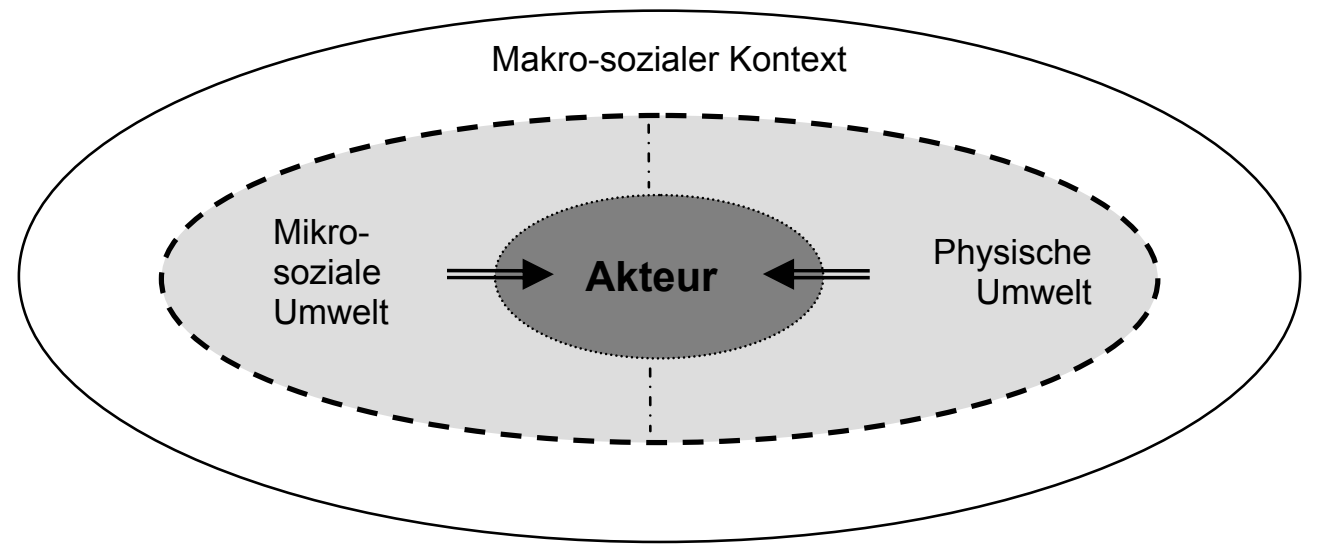

Quelle: eigene Darstellung.

Im Handlungsmodell in Anlehnung an Kühnel und Bamberg ${ }^{83}$ wird zwischen einer individuellen Akteursebene sowie dem sozialen Kontext differenziert. Letzterer kann je nach Abstraktionsgrad mit unterschiedlichen Inhalten gefüllt werden. Das bedeutet, der väterliche Handlungskontext umfasst sowohl (wohlfahrtsstaatliche) Institutionen, z.B. in

${ }^{83}$ Siehe dazu Kap 4.1 Das individuelle Handlungsmodell nach Kühnel/Bamberg, Abbildung 4.1. 
Form von Gesetzen oder Normen, die im Folgenden als makro-soziale Ebene verstanden werden, als auch das beschriebene soziale und physische Nahumfeld. Beide Dimensionen, so hier die Annahme, bestimmen das individuelle Handeln mit, jedoch in unterschiedlichen Wirkungsgraden. Im Zentrum des Modells steht entsprechend der bisherigen theoretischen Ausführungen der einzelne Nachtrennungsvater als Akteur. Er ist umgeben von einer physischen und mikro-sozialen Umwelt. Beide Dimensionen sind nicht unabhängig voneinander (gekennzeichnet durch eine gestrichelte Linie) und wirken sich „direkt“ auf das individuelle Handeln aus (ausgedrückt durch zwei Pfeile). Darüber hinaus ist der Vater in einen weiteren sozialen Kontext eingebettet, der als makro-soziale Rahmenbedingungen bezeichnet wird und im Wesentlichen die institutionelle Dimension umfasst. Es wird deutlich, dass makro-soziale Strukturen nicht direkt auf den Akteur wirken. Sie verändern den Rahmen des individuellen Handelns. Jeweils im Modell nebeneinander liegende Ebenen beeinflussen sich wechselseitig (dargestellt durch gestrichelte Linien). Dabei werden in der Analyse ausschließlich die Einflüsse, die sich von außen nach innen richten, berücksichtigt. Es wird an dieser Stelle explizit darauf hingewiesen, dass hier kein deterministisches Verständnis von Institutionen zu Grunde liegt. Institutionen sind keine vom Akteur unabhängige, bereits existierende Strukturen, denen Individuen machtlos gegenüber stehen. Auch wenn sie gerade ins alltägliche Handeln häufig als selbstverständlich und unveränderlich - damit unhinterfragt - einfließen, so unterliegen sie doch einem fortlaufenden Prozess der (Ent-) Institutionalisierung (Bühl 1994: 302). Das bedeutet, sie werden gerade über ihre Integration im Alltagshandeln reproduziert und sozial legitimiert (Esser 2000c: 3). Für die Erklärung väterlichen Sorgehandelns ist die zweiseitige Interdependenz jedoch von untergeordneter Bedeutung. Am Beispiel illustriert bedeutet das, dass für den Vater in erster Linie handlungsrelevant ist, welche Ressourcen z.B. in Form von Einkommen oder Zeit ihm konkret zur Verfügung stehen, welche Erwartungen die Kindsmutter an ihn heranträgt oder auch was sie an Sorgehandeln zulässt. Zwar geben z.B. gesetzliche Rahmenbedingungen formal vor, welche Rechte und Pflichten dem Vater je nach Familienform zustehen - dies bestimmt seine Möglichkeiten seine Ansprüche einzufordern - entscheidender ist jedoch, ob die Mutter ihm als Handlungsressource oder restriktion in der Umsetzung seines väterlichen Engagements dient.

\subsection{Makro-soziale Rahmenbedingungen}

Im nachstehenden Kapitel wird dargestellt, welche Dimensionen des makro-sozialen Kontexts von theoretischer Bedeutung sind und wie diese sich auf das Handeln von Nachtrennungsvätern auswirken. 


\subsubsection{Der makro-soziale Kontext von Nachtrennungsvätern}

Unter dem makro-sozialen Kontext werden im Folgenden primär institutionelle Rahmenbedingungen verstanden, die den (vor-)strukturierenden Bezugsrahmen des Handelns bilden (Esser 2000c: 45). Institutionen werden dabei definiert als die formalen wie informalen Spielregeln einer Gesellschaft inklusive des Instrumentariums ihrer Durchsetzung (Kunz 2004: 63; Miebach 2006: 67; Esser 1999: 53, Greshoff/Schimank 2003: 16).

„Institutions may be defined as building-blocks of social order: they represent sanctioned, that is, collectively enforced expectations with respect to the behaviour of specific categories of actors or the performance of certain activities. [...] They involve mutually related rights and obligations for actors, distinguishing between appropriate and inappropriate, 'right' and 'wrong', 'possible' and 'impossible' actions and thereby organizing behavior into predictable and reliable patterns” (Streeck/Thelen 2005: 9).

Institutionen sind in den Erwartungen der Akteure fixierte, kollektiv geteilte und sozial geltende Regeln des Handelns (Esser 2000c: 6). Durch Gewöhnungs-, Akkommodationsund Internalisierungseffekte führen die durch Institutionen gesetzten Handlungsbarrieren und normativen Erwartungen zu sozial geteilten Präferenzen (Mau 2002: 351; Knijn et al. 2007: 192). Institutionen stellen Grenzen des individuellen Handlungsspielraums innerhalb des Framings dar und wirken sich des Weiteren auf die Kosten-Nutzen-Abwägung, dem zweiten Schritt des Handlungsmodells, aus.

Regeln mit kollektivem Geltungsanspruch gibt es in vielen verschiedenen Varianten (Esser 2000c: 10). Grob kann zwischen formalen und informalen Institutionen - zwischen geschriebenen und ungeschriebenen Gesetzen - unterschieden werden (Esser 1999: 53; Greshoff/Schimank 2003: 16). Dabei zählen zu den formalisierten Institutionen einer Gesellschaft v.a. die Gesetzeslage und Verfassung, während die informalen - weniger formalisierten - institutionellen Regelungen sich in der Tradition und Kultur einer Gesellschaft widerspiegeln (Streeck/Thelen 2005: 9ff.). ${ }^{84}$

Mit der Vorgabe bestimmter Handlungsoptionen durch institutionelle Strukturen entsteht für die Individuen Handlungssicherheit derart, dass die einzelnen Akteure sich mehr oder weniger darauf verlassen können, dass die Regeln auch von den anderen Beteiligten befolgt werden (Esser 2000b: 232f.). Institutionen bestimmen die Erwartungen innerhalb einer Situation. Das bedeutet, das Handeln der einzelnen Akteure wird in einen im Prinzip nachvollziehbaren, für die Individuen bindenden weiteren sozialen Zusammenhang ge-

${ }^{84}$ Es wurde im Kap. 4.3.2.2 Familiales Handeln als norm-orientiertes Handeln bereits eine Form von Institutionen ausführlich dargestellt: Normen. Institutionen sind jedoch nicht synonym mit Normen zu verstehen. Vielmehr stellt das Konzept der Institution den Oberbegriff dar. Normen sind eine Variante von Institutionen. 
stellt. Institutionen konstituieren ferner soziale Ordnung (Streeck/Thelen 2005: 9, Esser 2000c: 14f.), indem sie zum einen das Geflecht sozialer und materieller (Austausch-) Beziehungen strukturieren (relationaler Aspekt). Des Weiteren werden Machtpositionen und soziale Belohnungen mittels Institutionen und der mit ihnen verbundenen Sanktionsmechanismen zugeordnet (regulativer Aspekt) (Bühl 1988: 345, Schimank 2004: 295). Ferner repräsentieren Institutionen über Ideologien und signifikante Symbole des kollektiven Sinnzusammenhangs einer Gesellschaft (kultureller Aspekt) (Bühl 1988: 345).

Regulativ legen die sanktionierenden Verhaltensregeln fest, wie bestimmte Handlungen ausgeführt werden müssen, damit sie Nutzen produzieren. Sie bestimmen Art und Effizienz des Mitteleinsatzes (Esser 2000b: 232f.) Sie weisen unterschiedlichen Handlungsalternativen verschiedene Kosten zu, normieren Standards bzw. konstituieren „Normalitätsmodelle“, das sind jene Optionen, die rechtlich und sozial privilegiert werden (Scheiwe 1999: 363). Damit legen sie die erwünschten und unerwünschten, die legitimen und die weniger zu empfehlenden Mittel, die sinnvollen und sinnlosen Zielen innerhalb einer Handlungssituation fest (Esser 1999: 53). Sie können somit als Ressource wie Restriktion individuellen Handelns zugleich fungieren. Als Restriktionen schließen sie die potentiellen Handlungsoptionen aus, die von den Regeln als illegitim festgelegt werden (Esser 2000c: 37). Die soziale Ordnung wird gestützt durch die Sanktionierung des Verstoßes gegen einzelne Verhaltensvorschriften; d.h. kollektiv forcierte Erwartungen werden mittels „Bestrafungen“ bei Abweichung durchgesetzt (Streeck/Thelen 2005: 9, Esser 2000c: 14). Als Sanktionen - die im Handlungsmodell als Kosten berücksichtigt werden - können dabei die Verhängung einer Strafe durch externe Instanzen oder intern wirksame Kontrollmechanismen z.B. innerhalb des familialen Systems wirken (Esser 2000c: 8). Auch Anreize stärken die Bedeutung bestimmter Institutionen.

Institutionen legen ferner den individuellen Zugang zu Ressourcen fest. Mit der Regulierung der Ressourcendistribution statten z.B. politische Bedingungen Akteure mit unterschiedlichen Zugängen zu bestimmten Lebens- und Vereinbarkeitsformen aus (Duncan/ Strell 2004: 43; Kuijsten/Strohmeier 1997: 421).

„In dem Wechselspiel zwischen individuellem Handeln und gesellschaftlicher Rahmenbedingungen kommt wohlfahrtsstaatlichen Systemen eine bedeutende Rolle zu: die Verteilung von und der Zugang zu gesellschaftlichen Ressourcen entscheiden über Lebenschancen und -bedingungen“ (Leitner 1999: 31).

Dadurch wirken sich institutionelle Rahmenbedingungen auf die Organisation des alltäglichen Zusammenlebens aus (Strohmeier 2002: 353) und nehmen Einfluss auf familiale (Beziehungs-) Strukturen, indem z.B. gegenseitige Verpflichtungsverhältnisse hergestellt werden (Hantrais 1999: 104).

Die bisher rein theoretische Darstellung des makro-sozialen Kontextes führt zu der Frage, was dies konkret für Nachtrennungsväter bedeutet. Auch nach der elterlichen Trennung 
werden an Väter soziale Erwartungen bezüglich der Ausgestaltung ihres Vaterseins in Form von Institutionen herangetragen. Eine wichtige Institution für väterliches Handeln generell stellen, wie dargestellt, soziale Normen dar. Die relationale Funktion sozialer Normen wird besonders innerhalb familialer Beziehungen deutlich. Es wurde bereits auf die moralische Verpflichtung hingewiesen, die Familienmitglieder einander gegenüber empfinden. ${ }^{85}$ Dieses Pflichtgefühl wird gestützt durch gesellschaftliche Erwartungen, am stärksten institutionalisiert in legalen Regelungen (Skinner 1999: 92). Das bedeutet, es muss berücksichtigt werden, inwiefern Institutionen bestimmte Verantwortlichkeiten gegenüber Kindern Vätern, Müttern oder anderen Parteien (z.B. dem Staat) zuschreiben und welche (Handlungs-) Erwartungen damit verbunden sind. Väter werden somit in ihrem Alltagshandeln durch die institutionellen Rahmenbedingungen beeinflusst, indem sie unterschiedliche Formen des Vaterseins in Recht und Praxis anerkennen (Knijn/Selten 2002: 171; Ribbens McCarthy/Edwards 2002: 200; Bergman/Hobson 2002: 92). Insgesamt werden Vätern in Form von Care und Cash unterschiedliche Rechte und Pflichten zugesprochen.

Entgegen der Variation von Normen - wie von Institutionen generell - zwischen und innerhalb von Gesellschaften, gilt in den meisten Gesellschaften eine primäre elterliche Verantwortung, sozial, emotional und finanziell für ihre Kinder zu sorgen, so auch in den hier untersuchten Ländern. Dennoch finden sich große Unterschiede in der erwarteten Ausgestaltung dieser Verantwortung, nicht zuletzt erkennbar an unterschiedlich stark ausgeprägten geschlechtsspezifischen Rollenerwartungen (Himmelweit 2002: 242). Die sozialen Erwartungen, die an Väter gestellt werden, sind eng verbunden mit der Diskussion um eine geschlechtsspezifische bzw. egalitäre Arbeitsteilung. Die Frage, wer sich sozioemotional und wer finanziell um die Kinder kümmern soll, wird in unterschiedlichen Gesellschaften verschieden diskutiert. Geschlechtsspezifische Rollenerwartungen bestimmen das institutionalisierte Verständnis von Eltern als Väter und Mütter entscheidend mit (Palkovitz 1997: 206ff.; Rollett/Werneck 2002: 329; Tölke/Hank 2005a: 15). Lange Zeit dominierte das männliche Ernährer- bzw. das weibliche Betreuungsmodell. Das bedeutet, das „Brot verdienen“ im Sinne der Lebensunterhaltssicherung wurde im Wesentlichen den Männern und Vätern auferlegt, während Frauen und Mütter primär die Verantwortung für Heim und Familie zugesprochen wurde (Ellingsæter 2003: 419; Leira 2006: 28f.; Lewis 2003: 62; Scheiwe 1999: 284; Crompton 1999: 2). Einige familienpolitische und wohlfahrtsstaatliche Leistungen, wie z.B. in Deutschland das Elterngeld, Mutterschutz-

${ }^{85}$ Zum Verhältnis von Normen und rationalem Handeln innerhalb familialer Strukturen siehe Kap. 4.3.2.2 Familiales Handeln als norm-orientiertes Handeln. 
regelungen, Kindergeld oder Steuererleichterungen für (verheiratete) Eltern, basierten auf der Annahme, dass in jeder familialen Haushaltsgemeinschaft ein (männlicher) Haupternährer zur Verfügung steht. Dieser hat es dem anderen Elternteil, i.d.R. der Mutter, ermöglicht, dem Arbeitsmarkt fern zu bleiben, um selbst die Kinderbetreuung zu übernehmen (Leira 2006: 29). Die Leistungen haben Einkommensausfälle häufig auf geringem Niveau kompensiert und damit eine traditionelle Teilung zwischen bezahlter und unbezahlter Arbeit gestützt (Ferrarini 2006: 59; Lewis 2003: 62). Zum Teil bestehen diese Regelungen heute fort.

Es wurde bereits dargestellt, dass durch demographische, ökonomische und soziale Veränderungen das Modell des männlichen Ernährers verstärkt unter Druck geraten ist, nicht zuletzt aufgrund der Annahmen auf denen es basierte. Es baute auf einem Arbeitsmarkt charakterisiert durch sichere Arbeitsplätze verbunden mit ausreichender Entlohnung sowie stabilen elterlichen Partnerschaften auf. Beide Prämissen sind im sozialen Wandel ins Wanken gekommen (Lewis 2003: 62; Hobson et al. 2006: 267f.). Darüber hinaus wird die Rolle der Väter in der Familie nicht mehr ausschließlich auf die finanzielle Versorgung begrenzt. Eine Re-Definition der Vaterrolle führt zu höheren sozialen Erwartungen an Väter wie auch zu einem stärkeren Wunsch der Väter selbst sich aktiv in die Erziehung und Betreuung ihrer Kinder einzubringen. ${ }^{86}$ Die Entwicklung - sowie die damit verbundene Diskussion - unterscheidet sich je nach nationalem Kontext in ihrem Ausmaß und ihrer Geschwindigkeit. So herrschen in den einzelnen Gesellschaften unterschiedliche Elternbilder vor, d.h. geschlechtspezifische Normen, die u.a. angeben, welche Eigenschaften und Fähigkeiten ein „guter“ Vater aufweisen sollte. Dem Vater werden dabei unterschiedliche Funktionen, wie Führung, Sorge, Pflege, Schutz, für sein Kind zugeschrieben. Erwartungen werden an ihn auf unterschiedlichen Ebenen gestellt: vom Kind, von der Kindesmutter, vom sozialen Umfeld. Darüber hinaus bestehen ebenso familienpolitisch oder -rechtlich konstituierte Erwartungen (Drinck 2005: 213f.). Je nach Rollenverständnis werden Männer durch Institutionalisierung und Sanktionierung unterschiedliche väterliche Rechten und Pflichten zugeschrieben, die sie in ihrem Handeln (mit)bestimmen (Hobson 2002; Leira 1999: xiii; Leira 1996: 6). Die Definition als Vater durch den Vater basiert dabei auf seinem eigenen Handeln, aber auch aus den ihm zugeschriebenen Rechten und Pflichten, d.h. daraus, was man ihn tun lässt und was von ihm erwartet wird (Drinck 2005: 214).

${ }^{86}$ Der Wandel der Definition der Vaterrolle und die damit verbundene wissenschaftliche Diskussion um die „Krise der Vaterschaft“ werden in Kap. 3 Die sozialwissenschaftliche Väterforschung - Eine kurze Bestandsaufnahme umfassend dargestellt. 
Das jeweils sozial geteilte Vaterleitbild spiegelt sich u.a. innerhalb der nationalen familienpolitischen Settings wieder, nicht zuletzt in den öffentlichen Diskursen (Lewis 2002: 125; Kolbe 2002: 12; Funder/Harrison 1993: 27).

"Family policy to a considerable extent is nothing less than the incorporation of social values into political institutions and social services" (Strohmeier 2002: 346, Hervorhebungen C.M.).

Als Familienpolitik können allgemein alle Maßnahmen verstanden werden, die dazu eingeführt werden und geeignet sind, nachhaltig Einfluss auf das familiale Zusammenleben zu nehmen (Strohmeier 2002: 325f.). Zu den politischen Bestimmungen, die Familien unterstützen, zählen u.a. Leistungen und Dienstleistungen für Familien mit Kindern z.B. öffentliche Kinderbetreuung, Steuererleichterungen, Maßnahmen zur verbesserten Vereinbarkeit von Familie und Beruf u.a. durch Elternzeitregelungen (Daly 2004: 136; Hantrais 1999a: 104). ${ }^{87}$

Neben einzelnen Verhaltensvorschriften können innerhalb der Nationalstaaten verschiedene ganzheitliche familienpolitische Profile ausgemacht werden, indem mittels (familien) politischer sowie gesetzlicher Diskurse und konkreter Maßnahmen spezifische Familienleitbilder gestützt werden. Staatliche (Familien-) Politik gestaltet Rahmenbedingungen des Alltagshandelns von Eltern und Kindern aktiv mit, indem sie Handlungsspielräume zur Verwirklichung bestimmter Formen des Familienlebens eröffnet bzw. versperrt. Außerdem reguliert sie Eltern-Kind- und Geschlechterbeziehungen, sowie soziale Praktiken, die mit diesen familialen Beziehungen verbunden sind, wie z.B. die Vereinbarkeit von Familie und Beruf (Kolbe 2002: 12; Kuijsten/Schulze 1997: 267; Strohmeier 2002: 321). Während einzelne politische Maßnahmen variable Handlungsmöglichkeiten eröffnen, wird das nationale Gesamtbild der Väter-Politik vom Akteur als relativ stabil wahrgenommen und wirkt als erste Orientierung für weitreichende (biographische) Entscheidungen (Strohmeier 2002: 321; Kuijsten/Strohmeier 1997: 421). So können Akteure z.B. mit Hilfe der familienpolitischen und -rechtlichen Vorgaben abschätzen, welche

\footnotetext{
${ }^{87}$ Neben den explizit auf Familien und ihre Lebensumstände abgestimmten Maßnahmen können sozial- und wirtschaftspolitische Regelungen Einfluss auf die Lebensbedingungen und sozialen Positionen von Familien(mitgliedern) nehmen, ohne diese als direkte Zielgruppe anzusprechen. Abgesehen von den rechtlichen haben somit auch die wirtschaftlichen Rahmenbedingungen Einfluss auf die Ausgestaltung väterlichen Sorgehandelns (Cheal 2002: 13ff.). Dazu zählen z.B. die Art der Erwerbstätigkeit, individuelle Ressourcen wie das Einkommen und das Bildungsniveau der Väter und Mütter (Marsiglio 1991: 975; Jaursch 2003: 51; Forste 2002: 586). Väterliches Engagement ist abhängig von Arbeitszeiten, Karriereabsichten und -aussichten; es wird aber auch beeinflusst durch die zunehmende Frauenerwerbstätigkeit (Marsiglio 1991: 975).
} 
staatliche Unterstützung oder „Widerstand“ sie bei der Umsetzung ihrer gewählten Lebensform erwarten können (Strohmeier 2002: 350). ${ }^{88}$

Als Ressourcen bzw. Restriktionen fungieren nicht nur materielle Gegebenheiten, wie sie z.B. durch monetäre Transfers innerhalb familienpolitischer Maßnahmen geleistet werden, sondern auch die Ausstattung mit spezifischen Rechten und Pflichten. Eine besondere Bedeutung kommt hier den jeweiligen Rechtssystemen zu. Das Familienrecht setzt Akteure über Verwandtschaftsverhältnisse in Beziehung zueinander, u.a. indem es bestimmte Unterhaltsverpflichtungen einzelner Familienmitglieder festschreibt (Hantrais/LohkampHimmighofen 1999: 15). Zumindest gesetzlich wird nach der Trennung des Elternpaares eine klare Differenzierung zwischen Care- und Cash-Aspekte beibehalten. Dabei ist die klassische Rollenverteilung im Gesetz z.T. stärker implementiert, als dies möglicherweise im alltäglichen (Zusammen-)Leben der Fall ist. Nicht nur in der deutschen Vergangenheit wurde bei der Scheidung in der Mehrzahl der Fälle das alleinige Sorgerecht der Mutter zugesprochen. Dem getrennt lebenden Vater oblag dabei rein rechtlich v.a. die finanzielle Funktion, die dem klassischen Versorgermodell entspricht. ${ }^{89}$ Im Rahmen der Diskussion von und um „neue“ Väter werden - auch deutschen - Nachtrennungsvätern über die gesetzlich festgeschriebene finanzielle Verpflichtung hinaus zunehmend Betreuungs- und Beziehungsaufgaben zugeschrieben. So sind mit elterlichen Trennungen häufig Besuchsund Unterhaltsregelungen verbunden, die - je nach nationalem Kontext - mehr oder weniger stark gesetzlich reglementiert sind.

Europäische Väter sind seit Ende der 1980er verstärkt auf der politischen Agenda vertreten. Das politische und öffentliche Interesse an Vätern und ihrem Sorgehandeln hat über die Zeit zugenommen (u.a.: Marsiglio et al. 2000; Hobson 2002, Tölke/Hank 2005b, Ostner/Schmitt 2007; Daly 2004: 138; Lammi-Taskula 2006: 79). Entsprechend der gewandelten sozialen Anforderungen an Väter werden ihnen in politischen Diskursen zunehmend Caring-Rechte zugesprochen (Hobson et al. 2006: 269; Skevik 2006a: 114). Verschiedene familienpolitische und -rechtliche Instrumente stehen dabei zur Verfügung, um die väterliche Sorge in Form von Care zu unterstützen oder gar zu forcieren (Daly 2004: 138; Ostner 2007: 230f.). Dazu zählt u.a. der Ausbau väterlicher Sorgerechte inner-

88 Darüber hinaus wirkt (Familien-) Politik meinungsbildend. Dies geschieht nicht nur durch konkrete familienpolitische Maßnahmen oder Gesetze, sondern auch schon im Vorfeld innerhalb des Entscheidungsfindungsprozesses, der politischen Maßnahmen vorgeschaltet ist. Der Einfluss auf die öffentliche Meinung erfolgt u.a. über politische Debatten und Kampagnen, die für die Akzeptanz politischer Entscheidungen werben (Kolbe 2002: 427ff.).

${ }^{89}$ Dem entspricht, dass z.B. 96\% der deutschen Stichprobe der Unterhaltspflichtigen Männer sind, wohingegen 94\% der Unterhaltsberechtigten Frauen darstellen (Forsa 2002: 33). Erst mit der Reform des Kindschaftsrechts 1998 lässt sich in Deutschland eine Entwicklung hin zum gemeinsamen Sorgerecht und damit einer Stärkung der Care-Komponente feststellen (Amendt 2004: 172). 
halb von Elterngeld-Regimen in Form von sog. "Daddy Months“. Auch Rechtsnormen, die sich auf die Familie beziehen, unterlagen einem weitreichenden Wandel in Europa (Kaufmann 2002: 458). In vielen Ländern hat sich ein - unterschiedlich ausgeprägter Prozess weg von der Institution Ehe vollzogen, so sie überhaupt institutionalisiert war. Elternschaft wird zunehmend auf biologische Abstammung und autonom von der Formalisierung der elterlichen Partnerschaft definiert (Hantrais 1999: 111). Dahinter steht die Intention die Rechte der Kinder unabhängig vom Familienstand der Eltern zu stärken. Des Weiteren werden Betreuungsrechte auch innerhalb der Familiengesetzgebung gestärkt, indem Sorge- und Umgangsrechte für Väter nach der elterlichen Trennung ausgebaut werden (Ostner 2002: 158; Leira 1999: x; Scheiwe 1999: 283; Björnberg 2006: 92, 107).

\subsubsection{Der Einfluss des makro-sozialen Kontextes}

Die theoretische Annahme, der makro-soziale Kontext wirke insbesondere vermittelt über die mikro-soziale und physische Umwelt auf das Handeln von Nachtrennungsvätern, ${ }^{90}$ wird im folgenden Abschnitt durch verschiedene Argumente gestützt.

Zunächst weisen empirische Untersuchungen darauf hin, dass die Akteure sich v.a. an ihren personalen Nah-Umwelten, ihrer direkten Lebenswelt, orientieren. Nach Esser (1999: 461) sind es

„[...] insbesondere die Bezugsgruppen der alltäglichen Lebenswelt, die dem Handeln der Menschen den nötigen Bezugsrahmen geben und somit - letztlich - die weiter gezogenen Vorgaben der Opportunitäten und der institutionellen Regeln, der geltenden gesellschaftlichen Interessen und kulturellen Ideen mit Sinn, Energie und Leben füllen.“

Auch Nauck (1989: 55) geht davon aus, dass ökonomisch-utilitaristische Nutzenerwartungen eher durch großräumige sozial-ökologische Kontexte wie der Gesamtgesellschaft oder regionale ökonomische Strukturen beeinflusst werden. Psychische Nutzenerwartungen, im vorliegenden Fall v.a. ausgedrückt in Gefühlen und Beziehungen zu anderen Akteuren, hängen dagegen primär von kleinräumigen Kontexten wie Wohnungsausstattungen oder dem familialen Netzwerk ab und wirken sich damit insbesondere auf das mikro-soziale (Interaktions-)Handeln aus, wie es hier erklärt wird. Dies kann u.a. mit der Kontakthäufigkeit im Alltag begründet werden. Familien leben zwar innerhalb nationaler Kontexte, sie nehmen jedoch zunächst v.a. ihr unmittelbares soziales Umfeld in Form der Verwandtschaft oder Nachbarschaft wahr. Sie leben weniger in Nationen als in Stadtvierteln, Vororten oder Dörfern. Primär die lokale Infrastruktur konfrontiert sie mit Handlungsbarrieren oder -chancen (Strohmeier 2002: 353).

${ }^{90}$ dargestellt in Abb. 6.1. Mehrebenen-Modell der sozialen Situation. 
In der Literatur finden sich zahlreiche Beispiele, die belegen, dass (familien-)politische und -rechtliche Maßnahmen nur begrenzt Einfluss auf das Handeln der Akteure nehmen.

"It is important to recognize that policies in themselves do not necessarily alter practice" (Ostner 2002: 163; Hervorhebung C.M.).

Das Zitat wurde im Zusammenhang mit der geschlechtsspezifischen Arbeitsteilung zwischen Männern und Frauen formuliert. Zunächst haben institutionelle Veränderungen auf die Erhöhung der Frauenerwerbstätigkeit und deren Vereinbarkeit mit familialen Betreuungspflichten abgezielt. In den letzten Jahren werden Vätern verstärkt Sorgerechte zugesprochen, um eine gleichberechtigtere Arbeitsteilung zwischen den Elternteilen zu ermöglichen. Doch die alltägliche Praxis von Elternpaaren erweist sich als relativ resistent gegen diese Veränderungen. Zwar haben sich die Einstellungen, insbesondere der Väter, die sich verstärkt über finanzielle Aspekte hinaus um ihre Kinder kümmern wollen, verändert, doch in der Realität besteht eine geschlechtsspezifischen Arbeitsteilung häufig fort. Neben familienpolitischen und -rechtlichen Maßnahmen müssen weitere Aspekte innerhalb des institutionellen Rahmens berücksichtigt werden. Hier ist v.a. an die geschlechtsspezifische Segregation des Arbeitsmarktes zu denken (Ostner 2002: 167). Auch innerhalb des skandinavischen Kontextes - dem eine Vorreiterrolle in der wohlfahrtsstaatlichen Gleichstellung der Geschlechter zugeschrieben wird - findet sich weiterhin eine hohe Diskrepanz zwischen gender-egalitären Ansprüchen und der alltäglichen Realität. Insgesamt zeigen die gewandelten Opportunitätsstrukturen auf makro-sozialer Ebene, die es Vätern ermöglichen, Erwerbs- und Familienpflichten besser zu vereinbaren, wenig Veränderungen im tatsächlichen Sorgehandeln der Väter. Nur wenige nutzen ihre Möglichkeiten (im vollen Umfang) (Skrede 2001: 8; Kitterød/ Kjeldstad 2003: 49; LammiTaskula 2006: 84f.; Ostner 2002: 164f.). Der Versuch der britischen New Labour-Partei, die Beschäftigungsfähigkeit allein erziehender Mütter mittels Aktivierungsreformen zu steigern, hat ebenso gezeigt, dass die Individuen Entscheidungen, die sich auf Elternschaft, Partnerschaft und Erwerbstätigkeit beziehen, unter anderen als ökonomisch „rationalen“ Aspekten fällen. Trotz verstärkter Anreize und auch Sanktionen in Form von Leistungskürzungen blieben die meisten allein erziehenden Mütter zu Hause, um sich um ihre Kinder zu kümmern (Marten 2007a). Damit greifen Gesetzesreformen und politische Kampagnen, die allein durch die richtigen Anreize von außen individuelles Handeln zu steuern versuchen, zu kurz (Barlow et al. 2002: 111).

Die Beispiele verdeutlichen die mögliche Diskrepanz zwischen familienpolitischen Zielen und den tatsächlichen Handlungsmustern der individuellen Akteure im Alltag. Es reicht nicht aus, die richtigen Anreize für erwünschtes bzw. entsprechende Restriktionen für unerwünschtes Handeln zu setzen. Institutionelle Rahmenbedingungen wirken nicht deterministisch auf Akteure. Dies kann ferner mit den besonderen Merkmalen des hier untersuchten Lebensbereichs begründet werden. Bei der Familie handelt es sich idealtypisch 
um einen wesentlichen Teil der Privatsphäre, der - anders wie z.B. der Arbeitsmarkt weitgehend unberührt von institutionellen Zwängen (geblieben) ist (Hill/Kopp 2004: 98). Familien stellen ungewöhnlich autonome, selbstbestimmte Systeme dar (Strohmeier 2002: 344). Innerhalb familialer Beziehungsgeflechte werden eigene Kontroll-, Interaktions- und Kommunikationsmechanismen entwickelt, die sich möglicherweise externen Einflüssen auf makro-sozialer Ebene entziehen. Zur Autonomie der Familie zählt z.B., dass das individuelle Fehlverhalten eines Familienmitglieds zunächst nicht Gegenstand rechtlicher Sanktionen, sondern vielmehr mittels informaler Sanktionsmechanismen innerhalb des sozialen Nahumfelds der Familie geregelt wird (Morgan 2002: 157).

Darüber hinaus geben gesellschaftliche Institutionen zwar Handlungs- und Rollenentwürfe vor, diese stellen aber zunächst leere Handlungsschablonen dar, die von den Individuen mit konkreten Interpretationen und Reaktionen gefüllt werden müssen (Hill/Kopp 2004: 99). Akteuren bleibt immer noch ein nicht zu vernachlässigender Entscheidungsspielraum innerhalb institutioneller Regeln, so dass nicht eine konkrete Handlung am Ende das Resultat sein muss, sondern vielfältige Handlungsmuster denkbar sind (Streeck/Thelen 2005: 11). Diese Handlungsmuster, so die Annahme, ähneln sich im sozialen Umfeld, u.a. weil die einzelnen Akteure sich gegenseitig im alltäglichen Kontakt als Handlungsorientierung dienen.

Die Diskrepanz zwischen familienpolitischen bzw. -rechtlichen Anreizstrukturen und dem tatsächlichen individuellen Handeln lässt vermuten, dass noch andere Determinanten als der makro-soziale Kontext das individuelle Handeln (in familialen Strukturen) bestimmen. Beispielsweise bekommen Menschen Kinder für sich selbst und ihre persönlichen Bedürfnisse. Sie berücksichtigen bei ihrer Entscheidung weniger, ob sie dem Staat oder der Gesellschaft als Ganzes damit einen Gefallen tun oder diesem gegenüber gar dazu verpflichtet sind (Feldhaus/Huinink 2005: 193). Vielmehr ist die Entscheidung für ein Kind eine Aushandlung zwischen potentiellen Elternteilen (Knijn et al. 2007: 219). Wesentliche biografische Entscheidungen einzelner Akteure werden nicht an mehr oder weniger kurzfristig ausgerichteten Maßnahmen der Familienpolitik oder des Familienrechts orientiert, die variierende Anreize setzen. Die Erhöhung des Kindergeldes wird daher nur denjenigen die Option eines Kindes eröffnen und sie zur Umsetzung bewegen, die bereits prinzipiell eine Entscheidung zur Elternschaft getroffen haben (Strohmeier 2002: 348)

Die Ausführungen haben bisher eine Wirkung in umgekehrter Richtung, vom Individuum zum Wohlfahrtsstaat, vernachlässigt. Doch die praktische Umsetzung familialer Lebensformen wie auch die politischen Maßnahmen sind Ausdruck gesellschaftlich dominierender Wertvorstellung und historisch gewachsener kultureller Normen (Kolbe 2002: 431). So können makro-soziale Vorgaben ihre Wirksamkeit nur entfalten, wenn sie durch individuelle Akteure in alltagspraktische Handlungen implementiert und damit „institutionali- 
siert“ werden (Streeck/Thelen 2005: 11). Individuelles Handeln wirkt auf politische Entscheidungen zurück (Hantrais/Lohkamp-Himmighofen 1999: 40). Wenn mehr und mehr erwachsene Bürger das Erwerbs- mit dem Familienleben kombinieren, sowohl ein Leben als Arbeitnehmer als auch Elternteil führen wollen, dann ist es zum einen sehr wahrscheinlich, dass der Missstand der Unvereinbarkeit sehr bald auf politisches Interesse stößt und dass zum anderen entsprechende Maßnahmen die erwartete Reaktion - gleichzeitige Erwerbstätigkeit und Elternschaft - bewirken. So sind familienpolitische bzw. - rechtliche Entscheidungen und ihre „Erfolge“ immer auch abhängig von der (gesellschaftlichen) „Nachfrage“ (Kuijsten/Schulze 1997: 267). Sie stellen nicht selten eine Reaktion auf Veränderungen der sozialen Realität dar (Hantrais/Lohkamp-Himmighofen 1999: 12). Die wechselseitige Beeinflussung institutioneller Rahmenbedingungen und individueller Akteure dient als ein weiteres Argument für die angenommene indirekte Wirkungsweise der makro-sozialen Strukturen auf das individuelle Handeln.

Theoretisch lassen sich vielfältige Gründe für und auch gegen einen angenommenen $\mathrm{Zu}$ sammenhang zwischen Institutionen und familialen Handeln auf der Mikroebene anführen. In der Forschung konnten jedoch bisher kaum direkte Zusammenhänge empirisch aufgedeckt oder gar quantifiziert werden (Fux 2002: 365). Dies scheint auf den ersten Blick auf die Nicht-Existenz eines Zusammenhangs hinzudeuten. Die erschwerte empirische Nachweisbarkeit einer solchen Korrelation liegt jedoch als Erklärung näher. Es ist nahezu unmöglich einen Effekt in individuellen Handlungen einem einzelnen politischen Instrument zuzuordnen bzw. seinen Anteil daran in konkreten Zahlen auszudrücken. Ferner gelingt es nicht zu bestimmen, wie eine Entwicklung verlaufen wäre, wenn die politischen Maßnahmen nicht in Kraft getreten wären (Kunz 2004: 151, Strohmeier 2002: 335; Fux 2002: 372; Hantrais/Lohkamp-Himmighofen 1999: 40).

Insgesamt machen die Ausführungen deutlich:

„[...] that there are limits how far the state can go in the direction of what Donzelot (1979) famously called 'policing of families'” (Crow 2002: 287, Hervorhebungen C.M.).

Dabei wird dem makro-sozialen Kontext nicht jegliche Bedeutung für individuelles Handeln abgesprochen, sondern vielmehr davon ausgegangen, dass dieser indirekt wirkt, wie dies auch im Modell dargestellt wird.

Die vorangegangenen Abschnitte haben theoretisch spezifiziert, welche Dimensionen der makro-sozialen Rahmenbedingungen väterliches Sorgehandeln mitbestimmen sowie ihre Wirkungsgrenzen aufgezeigt. Dazu zählen insbesondere geschlechtsspezifische Rollenbilder sowie familienpolitische und -rechtliche Kontexte. Im Folgenden werden die theoretischen Annahmen mit nationalen Begebenheiten aus den drei in der Analyse berücksichtigten Ländern gefüllt. Es wurde bereits darauf verwiesen, dass dies nicht dem aktuellen (Entwicklungs-)Stand der institutionellen Rahmenbedingungen väterlichen 
Sorgehandelns in den Ländern entspricht. Für West-Deutschland und Norwegen wird der Zeitraum bis 2002, in Großbritannien bis 1995 beschrieben. Die punktuelle Darstellung der makro-sozialen Dimensionen erfolgt dabei nicht für die einzelnen Länder nacheinander, sondern vielmehr werden die jeweiligen nationalen Besonderheiten einer Dimension in einem Abschnitt abgehandelt. ${ }^{91}$

\subsection{Der deutsche, britische und norwegische makro-soziale Kontext}

Es bestehen nationale Unterschiede in den institutionellen Rahmenbedingungen des familialen (Getrennt-)Lebens. Für die vorliegende Analyse reicht es aus, unterschiedliche makro-soziale Kontexte zu skizzieren, um die Allgemeingültigkeit der aufgestellten Theorie zu überprüfen. Im Folgenden werden die bisher rein theoretischen Überlegungen am Beispiel von drei Nationalstaaten, die sehr unterschiedliche institutionelle Settings für Väter repräsentieren, empirisch mit Inhalten gefüllt.

\subsubsection{Institutioneller Kontext deutscher, britischer und norwegischer (Nachtrennungs-)Väter}

Deutschland, Großbritannien und Norwegen repräsentieren unterschiedliche Wohlfahrtsstaatsregime. So bestehen z.B. in Norwegen und West-Deutschland sehr ausgeprägte sozialstaatliche Versicherungen einzelner Akteure gegen bestimmte soziale Risiken in unterschiedlicher Form, während in Großbritannien ein weniger extensiver Wohlfahrtsstaat die Akteure absichert. Das Ausmaß, d.h. Form und Umfang, staatlicher Intervention variiert somit in den nationalen Kontexten (Kolbe 2002: 427; Hantrais 1999: 105, Crow 2002: 290f.).

Deutschland wird allgemein als konservativer Wohlfahrtsstaat charakterisiert (Vogel 1999: 78). Dies ist auch auf die Familienpolitik und das Familienrecht um 2002 übertragbar (Ostner et al 2003: 5). Staatliche Intervention in der familialen Sphäre hat eine lange Tradition (Ostner 1997: 41; Hantrais 1999: 106). Dabei gilt das Prinzip der Subsidiarität und Solidarität. Zwar wird dem Staat eine wichtige Bedeutung als familienpolitischer Akteur eingeräumt, doch es besteht eine klare Grenze zwischen öffentlicher und privater Verantwortung (Ostner 2002: 154; Ostner et al 2003: 5; Hantrais/Lohkamp-Himmighofen 1999: 36). Daher wird der kleineren Handlungseinheit, der Familie oder den Eltern, Vor-

${ }^{91}$ Zur ausführlichen Begründung dieses methodischen Vorgehens siehe Kap. 1 Fragestellung und methodischer Aufbau der Arbeit. 
rang eingeräumt. Rechte und Pflichten werden an familiale Einheiten - nicht Individuen vergeben (Ostner 1997: 40; Hantrais 1999: 111; Fux 2002: 373).

Auch im britischen Kontext besteht 1995 eine klare Abgrenzung zwischen privater und öffentlicher Zuständigkeit. Doch anders als im deutschen Fall, wo das Prinzip der Subsidiarität durchaus Raum für staatliche Intervention lässt, findet in Großbritannien der staatliche Eingriff insbesondere in der Privatsphäre kaum Anwendung und Anerkennung (Lewis 2002: 126; Hantrais/Lohkamp-Himmighofen 1999: 36; Bennett 2006: 110). Insgesamt ist der britische Wohlfahrtsstaat als liberal zu bezeichnen, d.h. Marktlösungen werden staatlichen Interventionen vorgezogen. Dies trifft ebenso auf familienpolitische Themen, wie z.B. der Kinderbetreuung zu. Neben der hohen Marktorientierung zielt die wohlfahrtsstaatliche Intervention verstärkt auf private, d.h. individuelle bzw. familiale Lösungen ab (Ellingsæter 2003: 420; Fux 2002: 374f.; Finch 2003: 19). Dies beinhaltet auch die Prämisse, dass staatliche Interventionen im privaten Bereich der britischen Bürger auf einem Minimum gehalten werden (Lewis 2002: 126; Bennett 2006: 110; Hantrais 1999: 108; Clarke/Henwood 1997: 183; Fux 2002: 374). Diesem Ansatz entspricht sowohl die Abwesenheit eines Familienministeriums als auch einer familienpolitischen Tradition (Lewis 1997: 51; Meyer 2005: 291; Clarke/Henwood 1997: 183; Clasen 2005: 139). Es gibt keinen expliziten familienpolitischen Ansatz innerhalb der britischen Politik. ${ }^{92}$ Lediglich eine kategoriale Familienpolitik ist um 1995 auszumachen. Das bedeutet, dass insbesondere "families at risk" die Zielgruppe sozialpolitischer Maßnahmen darstellen (Scheiwe 1999: 373). Solange Eltern nicht auf Sozialleistungen angewiesen sind, schreitet der Staat nicht ein (Skevik 1998: 233). Insgesamt zeigen sich vor 1995 zwei Trends innerhalb des britischen Kontextes: zum einen werden wohlfahrtsstaatliche Transfers zunehmend eingegrenzt, und zum anderen werden sie auf die Bürger konzentriert, die sie am nötigsten brauchen (Clasen 2005: 166; Lewis 1997: 51).

92 Erst mit der Wahl der New Labour Partei 1998 ist der Familie verstärkt politische Aufmerksamkeit geschenkt worden. Im Rahmen der Aktivierungsprogramme haben familiale Verpflichtungen nicht länger als legitimer Grund gegolten, dem Arbeitsmarkt fern zu bleiben und von wohlfahrtsstaatlichen Leistungen abhängig zu sein (Meyer 2005: 295). Daraus resultierten u.a. aktive familienpolitische Strategien insbesondere Müttern aber auch Vätern, die Vereinbarkeit von Familie und Beruf zu erleichtern. Instrumente stellten die Expansion der Elternzeit, als Teil der sog. “Work-Life Balance Campaign” oder auch der Ausbau institutioneller Kinderbetreuung innerhalb der "National Childcare Strategy” dar (Kilkey 2004: 4; Meyer 2005: 292). Insgesamt kann die britische Familienpolitik heute als etwas interventionistischer, koordinierter und kohärenter charakterisiert werden. Wesentliche Ziele bestehen dabei v.a. darin, die elterliche Erwerbstätigkeit zu erhöhen sowie die Kinderarmut zu senken (Lewis 2003: 221; Clasen 2005: 177f.; Björnberg 2006: 93). Dabei ist jedoch kritisch anzumerken, dass mit der Steigerung der elterlichen Erwerbstätigkeit nicht die Anerkennung ihrer Betreuungsrechte einher ging. So werden Männer zwar zunehmend in die Elternzeitregelungen integriert, aber nicht so sehr um ihre Beteiligung an der Betreuung und Erziehung der Kinder an sich zu erhöhen, sondern vielmehr um die weibliche Erwerbsbeteiligung zu gewährleisten (Finch 2003: 25). 
Der norwegische Wohlfahrtsstaat hat zum Erhebungszeitraum 2002 im Gegensatz zu Deutschland und Großbritannien weitreichende Interventionsbefugnisse und -verpflichtungen innerhalb der Privatsphäre seiner Bürger. Leistungen richten sich dabei nicht an die Familie insgesamt, wie in Deutschland, sondern an einzelne Familienmitglieder. Leistungsansprüche werden i.d.R. universalistisch vergeben. Es besteht ein weitreichendes wohlfahrtsstaatliches Sicherheitsnetz, das auf dem Gleichberechtigungsprinzip aller Bürger unabhängig von Ethnie, Geschlecht und Schicht basiert. Insgesamt wird der norwegische Wohlfahrtsstaat als sozial-demokratisch charakterisiert (Leira 1996: 7f.; Leira 2006: 27; Dienel 2002: 244; Millar 1999: 34; Greve 2000: 101).

Neben der Interventionsreichweite unterscheiden sich die drei Wohlfahrtstaaten ferner in der Ausgestaltung ihrer Maßnahmen. Je nach nationalem Kontext schreiben die wohlfahrtsstaatlichen Institutionen den Familienmitgliedern unterschiedliche Verantwortlichkeiten zu. So werden in Deutschland wohlfahrtsstaatliche Leistungen wesentlich als Status erhaltend und relationierend konzeptionalisiert (Ostner 2002: 154f.; Ostner et al 2003: 5). Familienmitglieder werden über den Wohlfahrtsstaat in Beziehung zueinander gesetzt. Leistungsansprüche stehen z.B. Ehefrauen und Ehemännern oder Müttern und Vätern zu. Individuelle Rechte sind in Deutschland nur marginal ausgeprägt und werden ehelichen Rechten bzw. Pflichten untergeordnet (Ostner et al. 2003: 5; Millar 1999: 35; Ostner 2002: 155; Meyer 2005: 285). Des Weiteren haben - gemäß dem Subsidiaritätsprinzip - stark institutionalisierte Bindungen und Verpflichtungen zwischen einzelnen Familienmitgliedern über die finanzielle Unterstützung hinaus Bestand (Ostner 2002: 155; Ostner et al 2003: 17). Dies entspricht dem Konservatismus im deutschen System und wird insbesondere deutlich durch die starke Institutionalisierung der Ehe, die durch die Verfassung besonderen Schutz erfährt (Scheiwe 1999: 378; Yeandle 1999: 84, Clasen 2005: 142; Bennett 2006: 110; Federkeil 1997: 85). Ehebasierte Rechte werden nur bedingt an kohabitierende (Eltern-) Paare übertragen (Millar 1999: 35).

Entsprechend der wohlfahrtsstaatlichen Zurückhaltung eines liberalen Staatsverständnisses wird in Großbritannien der Eigenverantwortung große Bedeutung zugeschrieben (Ellingsæter 2003: 420; Kilkey 2004: 4; Clasen 2005: 166; Finch 2003: 2). Dies geht einher mit stark institutionalisierten familialen Verpflichtungen (Meyer 2005: 294) wie sie auch in Deutschland zu finden sind. Die Verlagerung wohlfahrtsstaatlicher Pflichten auf die Institution Familie entwickelte sich seit Ende der 1980er Jahre in Großbritannien. Mit Maßnahmen, wie der Abschaffung der Sozialhilfe für 16- und 17-Jährige, der Senkung von Leistungen für unter 25-Jährige und dem Wechsel von Stipendien zu Ausbildungsdarlehen für höhere Qualifikationen ist die finanzielle Abhängigkeit junger Menschen von ihren Familien verschärft worden. In die gleiche Richtung zielt die Bedürftigkeitsprüfung der Sozialhilfe, die verstärkt familiale statt individuelle Ressourcen berücksichtigt (Millar 
1999: 31; Yeandle 1999: 102). Die Organisation von Kinderbetreuung wird lange als private Angelegenheit verstanden. Es bestehen kaum Erleichterungen zur besseren Vereinbarung von familialen und beruflichen Verpflichtungen (Kilkey 2004: 4; Finch 2003: 19; Scheiwe 1999: 375).

Auch die Stärkung der finanziellen Verantwortung von Nachtrennungsvätern Ende der 1980er/Anfang der 1990er Jahre entspricht der Pflichtverschiebung vom britischen Staat hin zur Familie (Finch 2003: 6). Mit dem Anstieg Alleinerziehender und deren Abhängigkeit von Sozialleistungen aufgrund zunehmend ausbleibender Unterhaltszahlungen nimmt auch die Sorge der Regierung um das Verantwortungsbewusstsein getrennt lebender Väter zu (Lewis 2002: 125; Hantrais/Lohkamp-Himmighofen 1999: 15). Zur Entlastung der Staatsausgaben sollen diese verstärkt in die Pflicht genommen werden (Skevik 1998: 222; Knijn/Selten 2002: 169; Skinner 1999: 24). Die Betonung der finanziellen Verpflichtung abwesender Väter wird 1993 in der Trennungsgesetzgebung mit einer Reform - begleitet von einer hitzigen öffentlichen Diskussion - institutionalisiert. ${ }^{93}$ Die Veränderungen basieren auf der Annahme einer lebenslang bestehenden elterlichen Verpflichtung gegenüber den eigenen Kindern. Diese Verantwortung soll nicht beliebig auf den Staat übertragbar sein (Skevik 1998: 219; Skinner 1999: 35; Lowe 2004: 2). Die Unbedingtheit väterlicher Pflichten wird ferner durch die Tatsache unterstrichen, dass es in Großbritannien keinen Unterhaltsvorschuss gibt, der den Vater als Ernährer im Notfall substituiert.

Anders als in Deutschland stellt in Norwegen die Zielgruppe wohlfahrtsstaatlicher Leistungen nicht die Familie als Einheit, sondern einzelne Akteure dar. Familiale Verpflichtungen sind nur schwach institutionalisiert. Es bestehen z.B. kaum Unterhaltsansprüche zwischen vormals verheirateten Partnern nach der Scheidung (Skevik 1998: 234). Die Institution der Ehe spielt im norwegischen Kontext um 2002 eine untergeordnete Rolle. Rechte und Pflichten werden aus der Elternschaft abgeleitet. Dabei werden Kindern selbst individuelle Rechte zugesprochen (Millar 1999: 34). Starke Dienstleistungen und monetäre Transfers von Seiten des Staates sichern diese Rechte zur Entlastung der Individuen von familialen Verpflichtungen ab. So erfolgten seit den frühen 1990ern einige weitreichende Reformen innerhalb der norwegischen Familienpolitik, die die Elternschaft betrafen. Ausgaben für institutionelle Kinderbetreuung wurden erhöht, gleichzeitig wurden die elterlichen Betreuungsrechte von Müttern und Vätern innerhalb der Elternzeitregelung gestärkt (Skevik 2003b: 3; Ellingsæter/Leira 2006: 265). Insgesamt besteht in Norwegen eher eine öffentliche als eine private Abhängigkeit (Skrede 2001: 4).

\footnotetext{
${ }^{93}$ Siehe dazu ausführlich Kap. 6.2.2.1 Unterhaltsregelungen.
} 
Die unterschiedliche Ausgestaltung wohlfahrtsstaatlicher Instrumente zeigt sich ferner in den verschiedenen Annahmen zur geschlechtsspezifischen Arbeitsteilung, auf denen sie beruhen. Die deutsche Familienpolitik kann auch deswegen als konservativ beschrieben werden, weil sie lange die traditionelle Familienarrangements, wie die traditionelle Versorgerehe, in der dem Mann die Haupternährerrolle zukommt, unterstützt hat (Ostner 2002: 154f.; Yeandle 1999: 84; Clasen 2005: 141; Meyer 2005: 285; Dienel 2002: 244). Innerhalb des deutschen Wohlfahrtsstaates bestand und besteht z.T. weiterhin eine Institutionalisierung geschlechtsspezifischer Ungleichheiten in der Aufteilung bezahlter und unbezahlter Arbeit (Ostner et al. 2003: 5; Kolbe 2001: 184; Ellingsæter 2003: 419; Bennett 2006: 110; Scheiwe 1999: 380). Die weibliche Arbeitsmarktbeteiligung ist lange wenig gefördert worden. Im Gegenteil, deutschen Frauen und Müttern wird primär die familiale Betreuung und Hausarbeit zugeschrieben und durch wohlfahrtsstaatliche Transfers bzw. (fehlende) Dienstleistungen ermöglicht (Ostner 2002: 154f., Yeandle 1999: 85; Millar 1999: 35; Meyer 2005: 285; Hantrais 1999: 107). Insbesondere Mütter von Kindern unter drei Jahren werden aus der Erwerbspflicht entlassen, um ihre Kinder selbst zu betreuen (Ostner et al 2003: 13; Yeandle 1999: 85; Scheiwe 1999: 380; Federkeil 1997: 90; Ostner 1997: 28). Sozialversicherungsansprüche leiten sich für Mütter und Ehefrauen primär aus der Erwerbstätigkeit des (Ehe-)Mannes ab (Yeandle 1999: 84; Clasen 2005: 142). Gleichzeitig wird der männliche Ernährer durch ein ausreichendes Familieneinkommen und Ehe basierte Transfers und Steuererleichterungen, wie z.B. dem Ehegattensplitting, unterstützt (Ostner 2002: 155; Kolbe 2002: 407; Clasen 2005: 142). Die geschlechtsspezifische Rolleninstitutionalisierung wird jedoch nicht als individuelles Recht verstanden, sondern gilt vielmehr der Aufrechterhaltung der familialen Einheit basierend auf komplementären Rollenzuschreibungen (Ostner et al 2003: 5; Hantrais 1999: 107). Die Ungleichheit zwischen den Geschlechtern hat u.a. zur Folge, dass allein erziehenden Mütter in Deutschland stark vom Einkommen des getrennt lebenden Vaters abhängig sind (Andreß et al. 2003; Forsa 2002; Ostner 2002: 153), anders als z.B. in Norwegen.

Soziale, ökonomische und demographische Veränderungen, wie z.B. die sinkende Fertilitätsrate und die steigende Arbeitslosigkeit haben (auch) den deutschen Ernährer verstärkt herausgefordert (Ostner 1997: 23; Meyer 2005: 285). Für Väter ist es zunehmend schwerer geworden, der männlichen Ernährernorm gerecht zu werden. Begleitet wird die Entwicklung durch familienpolitische Maßnahmen zur Förderung der weiblichen Arbeitsmarktpartizipation (Björnberg 2006: 92f.). Familienpolitisch wird der Fokus verstärkt auf elterlich geteilte Betreuungspflichten gelenkt (Ostner 1997: 23). Dennoch bleiben Anreize für die mütterliche Betreuung insbesondere von Kleinstkindern bestehen (Ostner 2002: 156). Mutterschutz und Elternzeit, Pensionsleistungen und Kindergeld werden weiterhin - wenn auch abgeschwächt - auf die weibliche Betreuungsnorm ausgerichtet (Clasen 2005: 163; Meyer 2005: 285). Die Institutionalisierung und auch kontinuierliche 
Weiterentwicklung geschlechtsspezifischer Rollenerwartungen und damit eng verbundener Elternschaftskonzepte hat in Deutschland zwar zu einer Modifizierung des EinErnährer-Modells geführt, die grundsätzliche Arbeitsteilung zwischen den Geschlechtern wird bisher jedoch nur bedingt in Frage gestellt (Kolbe 2002: 413). So zeigt sich am Erwerbsverhalten von westdeutschen Eltern nach der Geburt eines Kindes, dass die Mehrheit der Frauen Teilzeit (oder gar nicht) arbeiten, während Väter sogar eine höhere Arbeitszeit als vor der Geburt angeben (Ostner 2002: 156). Deutsche Männer übernehmen häufig immer noch die Funktion des Haupternährers ihrer Familie. Dies kann im Übrigen auch in den männlichen Einstellungen zur Vaterschaft nachgewiesen werden, die für einen hohen Anteil Männer immer noch mit der Ernährerfunktion eng verbunden ist (Ostner 2002: 165). Insgesamt wird mit der Ausweitung der weiblichen Erwerbsbeteiligung zumindest auf einer Teilzeitbasis, zunehmend ein Anderthalb-Ernährer-Modell im deutschen Kontext erkennbar, in dem der Vollzeit beschäftigte männliche Ernährer durch eine Teilzeit tätige Frau unterstützt wird (Yeandle 1999: 100). Mit familienpolitischen Reformen nach 2001 unter der Rot-Grünen-Regierung wird dieser Trend weg von einer starken männlichen Versorgernorm weiter verfolgt. Individuelle Rentenansprüche für Eltern werden verbessert; finanzielle Anreize für kürzere Elternzeitperioden und eine bessere Vereinbarkeit der Elternzeit mit langer Teilzeitbeschäftigung geschaffen (Opielka 2004: 128; Meyer 2005: 288f.; Clasen 2005: 163). Die Instrumente zielen auf eine bessere Vereinbarkeit familialer und beruflicher Verpflichtungen - nicht nur für Mütter - ab (Clasen 2005: 163). Reformen des deutschen Elternzeitregimes integrieren verstärkt Väter in die Betreuung ihrer Kinder. ${ }^{94}$

Trotz des Mangels einer klar abgrenzbaren Familienpolitik werden auch im britischen Fall familialen Lebensbedingungen durch verschiedene sozial- und wirtschaftspolitische Maßnahmen mitbestimmt (Clarke/Henwood 1997: 183). Dabei kann der britische Wohlfahrtsstaat 1995 dem Modell des starken männlichen Ernährers zugeordnet werden (Finch 2003: 2; Millar 1999: 26; Yeandle 1999: 84, Clasen 2005: 142; Meyer 2005: 291). Doch während Deutschland den männlichen Ernährer explizit durch verschiedene familienpolitische Instrumente stützt, findet das Modell in Großbritannien seine Unterstützung durch die Abwesenheit einer expliziten Familienpolitik. So wird die Arbeitsteilung zwi-

94 So wurden 2007 in Deutschland individuelle Betreuungsrechte explizit für Väter implementiert. Des Weiteren sollten höhere finanzielle Kompensationsraten sowie die Kombinationsmöglichkeit der Elternzeit mit einer verlängerten Erwerbstätigkeit gezielt für Männer Anreize der Vereinbarkeit von Familie und Beruf setzen (Ostner et al. 2003: 20; Björnberg 2006: 97; Erler 2006: 124f.; Gerlach 2004: 320f.; Opielka 2004: 127f.). Die neueren Entwicklungen betreffen die in der Forsa-Untersuchung befragten Väter, die 2002 erhoben wurde, noch nicht. Wichtig ist an dieser Stelle, dass Deutschland sich (langsam) vom männlichen Ernährermodell entfernt und sich in einer Phase der Transition befindet. 
schen Männern und Frauen den Paaren selbst überlassen und nicht wohlfahrtsstaatlich reguliert (Lewis 2002: 126; Scheiwe 1999: 374). Marktorientierte und private Lösungen führen häufig zu einer traditionellen Arbeitsteilung, die passiv vom britischen Wohlfahrtsstaat toleriert wird (Kilkey 2004: 11; Meyer 2005: 291; Finch 2003: 25).

In Norwegen - dem Vertreter des skandinavischen Wohlfahrtsstaatsregimes - ist anders als in Deutschland und Großbritannien die Gleichberechtigung von Männern und Frauen schon früh(er) besonders gefördert worden (Eydal 2005: 154; Kjeldstad 2000: 79; Hantrais 1999: 106; Greve 2000: 101; Skrede 2001: 2; Skevik 2006b: 183; Ellingsæter 2003: 439; Kitterød/Kjeldstad 2003: 39). Es ist an dieser Stelle jedoch darauf zu verweisen, dass Norwegen im skandinavischen Cluster mit Blick lange eine Sonderstellung einnimmt. Ihm wird in der Literatur häufig die Rolle des "Latecomer" mit Blick auf die Entwicklung der Gleichberechtigung zwischen den Geschlechtern zugeschrieben (Skevik 1998: 220). Das bedeutet, die norwegische Familien- und Sozialpolitik ist länger als andere skandinavische Wohlfahrtsstaaten dem männlichen Ernährermodell verpflichtet geblieben. Darüber hinaus ist auch die Familienpolitik zum Erhebungszeitpunkt als “hybrid“, ,ambivalent“ oder „zweigleisig“ zu charakterisieren (Ellingsæter 2003: 421; Skrede 2001: 3). So finden sich neben familienpolitischen Instrumenten, die die gleichberechtigte Arbeitsteilung zwischen Männern und Frauen fördern und fordern, wie z.B. die Ausweitung der Elternzeitrechte sowie der steigenden Bereitstellung institutioneller Kinderbetreuung, auch Maßnahmen, die die traditionelle Arbeitsteilung begünstigen. Dazu zählt z.B. die sog. Cash for CareReform (konstanstøttereformen) von 1998, nach der Eltern von Kindern unter 3 Jahren finanzielle Transfers erhalten, sofern sie ihren Anspruch auf einen institutionellen Kinderbetreuungsplatz nicht geltend machen (Kitterød/Kjeldstad 2003: 40; Ellingsæter 2003: 421f.; Skevik 2003b: 24). Insgesamt wird eine gleichberechtigte Arbeitsteilung als Option geboten, weniger eine verbindliche Norm formuliert (Skrede 2001: 5). Nichtsdestotrotz insbesondere im Vergleich zu Großbritannien und Deutschland - kann in Norwegen 2002 ein Dual Earner/Dual Carer - Modell ausgemacht werden (Ellingsæter 1999: 43; Leira 2006: 29; Greve 2000: 101,). Die betonte Bedeutung der Beziehung des Kindes zu beiden Elternteilen - auch nach der Trennung - entspricht diesem Prinzip der gleichberechtigten Elternschaft (Skevik 2003b: 3; Kitterød/Kjeldstad 2003: 49). Die norwegische Familienpolitik räumt neben der Integration der Mütter am Arbeitsmarkt, auch Vätern weitreichende individuelle Betreuungsrechte ein (Skevik 2003b: 29; Ellingsæter 1999: 43; Leira 2006: 31; Hobson 2002, Brandth/Kvande 2003; Skrede 2001: 7; Kitterød/Kjeldstad 2003: 39; Eydal 2005: 154). Hobson und andere (2006: 279) sprechen von "proactive fathering policies”. Als eine direkt auf Väter zielende Maßnahme kann in diesem Zusammenhang die Implementierung von Zeitanteilen innerhalb der Elternzeitregelung genannt werden, die explizit für Väter reserviert werden, die sog. “daddy quota“ (Ellingsæter/Leira 2006: 265; Hobson et al. 2006: 268). Damit war 1993 Norwegen das erste Land, dass 
väterliche Betreuungsrechte institutionalisierte (Ellingsæter 2003: 439). Die Implementierung wurde mit der Annahme begründet, dass dies eine frühe Bindung und Beziehung zwischen Vater und Kind ermöglicht, sowie eine gleichberechtigte Arbeitsteilung zwischen Vater und Mutter erleichtert (Hobson et al. 2006: 268; Leira 2006: 34). ${ }^{95}$

Die unterschiedlichen Vaterschaftskonzepte mit implizierten geschlechtsspezifischen Ernährermodellen spiegeln sich ebenso in den jeweiligen politischen und öffentlichen Diskussionen wider. In Europa ist insgesamt ein ansteigendes politisches und soziales Interesse an Vätern zu erkennen. Dabei werden jedoch unterschiedliche Schwerpunkte gesetzt. Basierend auf dem männlichen Ernährermodell sind sich über finanzielle Aspekte hinaus kümmernde Väter erst kürzlich in Deutschland öffentlich thematisiert worden. Lange war die Diskussion über Väter negativ gefärbt. Sie wurden insbesondere in WestDeutschland von feministischen Wissenschaftlern mit Blick auf häusliche Gewalt, Kindesmisshandlung oder geschlechtsspezifische Machtkonstellationen untersucht (Ostner 2002: 150ff.). Ostner (2002: 150ff.) argumentiert, dass die öffentliche Vernachlässigung von Vaterschaftskonzepten mit der Abgrenzung zum Verständnis der väterlichen Rolle im nationalsozialistischen Deutschland sowie in der ehemaligen DDR zu begründen ist. Beide historischen Gesellschaften wurden durch eine besondere „Vaterlosigkeit“ und eine staatliche Substitution der Vaterrolle bestimmt. Ein starker „Vater Staat“ übernahm wesentliche Funktionen, die zuvor dem männlichen Ernährer zukamen. Die westdeutsche Nachkriegszeit war durch starke Vater-Sohn-Konflikte der 1968er Generation geprägt. Väter haben in dieser Zeit ein Stück ihrer starken Position innerhalb der Familie verloren. Die öffentlichen Debatten haben die symbolische Konfiguration der Vaterlosigkeit verbunden mit Diskussionen über die Sünden der Väter und Vorväter verdeutlicht. Konkrete väterliche Alltagspraktiken blieben darüber in der deutschen Öffentlichkeit verborgen. Dies wurde auch in der Ausgestaltung familienpolitischer Instrumente deutlich, die primär um den Ehemann als Ernährer und weniger um den Vater konstruiert wurden (Ostner 2002: 166). Väter in familienpolitischen Reformen wurden in Deutschland lange ausgeschlossen. Entweder wurden Notwendigkeiten des Arbeitsmarktes oder Bedürfnisse der Kinder als Argumente für bestimmte Instrumente angeführt. Mit der langsamen Veränderung geschlechtsspezifischer Rollenerwartungen und den bereits angedeuteten Wandel hin zum

\footnotetext{
${ }^{95}$ Kritisch ist in diesem Zusammenhang anzumerken, dass die veränderten Opportunitätsstrukturen für betreuende Väter nur moderate Veränderungen in der tatsächlichen Arbeitsteilung der Geschlechter im norwegischen Alltag bewirkt haben (Skrede 2001: 8; Kitterød/ Kjeldstad 2003: 49).
} 
Anderthalb-Ernährer-Modell finden jedoch langsam auch (betreuende) Väter in Deutschland zunehmend öffentliches Interesse. ${ }^{96}$

Auch in Großbritannien ist die Diskussion über Väter um 1993 nicht positiv gefärbt. Die politische Debatte Ende der 1980er wird geprägt von der steigenden väterlichen Abwesenheit. Mit der Zunahme des Anteils allein erziehender Mütter, die aufgrund ausbleibender Unterhaltszahlungen verstärkt von Sozialleistungen abhängig sind, steigt gleichzeitig die Belastung des britischen Staatshaushalts und bringt so besonders die ökonomischen Pflichten getrennter Väter auf die politische Agenda (Lewis 2002: 129; Finch 2003: 6). Die Diskussion über Väter rankt sich primär um ihr Unvermögen ihren finanziellen Pflichten nachzukommen ebenso wie um die fehlenden männlichen Vorbilder für die involvierten Kinder (Lewis 2002: 126; Skevik 1998: 219; Skinner 1999: 31). Der Rückgang der Zwei-Eltern-Familien und der Anstieg abwesender Väter wird verstärkt als Ursache für problematische Kindheiten, steigende Jugendkriminalität und anderen soziale Missstände gesehen (Clasen 2005: 170; Bradshaw 1999: 2; Lewis 2002: 131f.). So formulierte Margret Thatcher 1990 in einer Rede: ${ }^{97}$

"No father should be able to escape from his responsibility and that is why the Government is looking at ways of strengthening the system for tracing absent fathers and making the arrangements for recovering maintenance more effective.”

Das Zitat macht stellvertretend deutlich, dass die gesamte - sehr moralisierende - Debatte um väterliche Pflichten auch über die elterliche Partnerschaft hinaus in dieser Zeit sehr negativ geprägt ist (Ostner 2002: 166; Yeandle 1999: 98; Skinner 1999: 14). Britische Väter geraten unter Generalverdacht ihre finanzielle Verantwortung gegenüber ihren Familien nicht schultern zu wollen (Lewis 2002: 128). Das Ziel der britischen Bemühungen liegt weniger darin Vätern den Kontakt zu ermöglichen, sondern vielmehr sie an ihre Familien finanziell zu binden (Finch 2003: 6). ${ }^{98}$ Die Care-Dimension väterlichen

${ }^{96}$ So gibt es in Deutschland 2002 eine Kampagne "Mehr Spielraum für Väter” (online: www.vaeteraktuell.de/studien/Mehr_Spielraum_fuer_Vaeter.pdf) - in Skandinavien sind solche Medieninstrumente seit den 1980ern üblich gewesen (Kolbe 2001: 195).

${ }^{97}$ Rede vor National Children's Home, Inaugural Lecture of the George Thomas Society, 17.01.1990 zitiert nach Bradshaw et al. 1999: 124.

98 Ein anderes Bild wurde von verschiedenen Gruppierungen des Fathers' Rights Movement in der Öffentlichkeit gezeichnet, die z.T. aufgrund der Gesetzesreform gegründet wurden (Skinner 1999: 48). Sie prangerten an, dass das neue Gesetz die betreuende Funktion der Nachtrennungsväter vollends vernachlässige (Skinner 1999: 14f.). Dabei wurde auch diese Seite der Diskussion mit harten Argumenten geführt. So wurde der Selbstmord eines getrennt lebenden Vaters mit der angehobenen Unterhaltsforderung begründet, die im tags zuvor zugestellt wurde. Dies wurde medienwirksam inszeniert und eine Vaterrechtsgruppe zählt weiterhin die Suizide getrennt lebender Väter (Skinner 1999: 49). 
Kümmerns ist in der britischen Diskussion lange vernachlässigt worden (Lewis 2002: 125)..$^{99}$

In Norwegen hat die Diskussion um Väter eine andere Entwicklung genommen. Anders als in Deutschland und Großbritannien wird hier der Fokus auf Strukturen gelegt, die den Nachtrennungsvätern einen intensiven Kontakt mit ihren Kindern ermöglichen, anstatt ausbleibende Zahlungen zu bestrafen (Lewis 2002: 147). Insgesamt sind norwegische Väter und ihre Betreuungsrechte im politischen Diskurs stark präsent. So wird die tägliche Kinderbetreuung sowie die Vereinbarkeitsproblematik von Arbeit und Familie als Herausforderung für Väter, Mütter, Arbeitgeber und auch den Wohlfahrtsstaat generell früh thematisiert (Leira 2006: 47, Ellingsæter 1999: 45; Skevik 2003a: 12; Kitterød/ Kjeldstad 2003: 39). Anders als in Großbritannien wird die Vereinbarkeitsproblematik in Norwegen nicht allein als Problem der Eltern oder der Individuen verstanden. In Deutschland besteht die Herausforderung insbesondere für Mütter, die geringe wohlfahrtsstaatliche Unterstützung für die Ermöglichung ihrer eigenen Erwerbstätigkeit erfahren. In Norwegen wird seit den 1990ern der öffentliche Blick insbesondere auch auf die Betreuungsrechte bzw. pflichten von Vätern gerichtet (Skevik 2003b: 3). Es lässt sich ein diskursiver und konstruktiver Wandel der väterlichen Verantwortlichkeit von Cash zu Care ausmachen. Dieser wird u.a. begleitet von Medienkampagnen, die Männer zur sozio-emotionalen Fürsorge für ihre Kinder motivieren sollen (Hobson et al. 2006: 268f.). Die Entwicklung findet sich auch in der Scheidungsgesetzgebung. Seit den 1990ern hat der Kontakt zwischen Vater und Kind nach der elterlichen Trennung politische Priorität erfahren. Es soll vermieden werden, Nachtrennungsväter rechtlich zu diskriminieren. Anders als in Großbritannien, wo die öffentliche Sorge primär den ausbleibenden Unterhaltszahlungen gilt, wird in Norwegen die Care-Verantwortung der Väter betont (Skevik 2006a: 115; Leira 1996: 56). So wurde 1986 eine spezielle Regierungskommission eingesetzt, die sich speziell um die Belange der Väter kümmerte. Sie sorgte dafür, dass die Rechte und Pflichten von Vätern bezüglich der gemeinsam mit ihren Kindern verbrachten Zeit auf die politische Agenda gesetzt wurden (Kitterød/ Kjeldstad 2003: 39). Insgesamt sind „neue“ familiale Arrange-

\footnotetext{
${ }^{99}$ Neben der Familienpolitik hat sich das öffentliche Bild britischer Väter bis heute - ähnlich dem gesamteuropäischen Trend - stark verändert. Betreuende Väter werden zunehmend als Werbemittel eingesetzt, Sportikonen mit ihren Kindern abgebildet. Es wird auch im britischen Kontext von den „neuen“ Vätern ein stärkeres Engagement in der Betreuung und Erziehung neben der finanziellen Unterstützung ihrer Kinder erwartet (O’Brien 2006: 3; Lewis 2002: 131). Derzeit wird die Reform der Elternzeit durch die Implementierung individueller Betreuungsrechte für Väter geplant (Moss/O’Brien 2006: 221; Weldon 2006: 5f). All dies deutet eine ähnliche Entwicklung hin zum betreuenden Vater weg vom Ernährer-Modell an, wie sie in Deutschland und insbesondere in Norwegen bereits früher losgetreten wurde (zur Bedeutung von Vätern innerhalb nationaler Familienpolitiken siehe ausführlich Marten 2007b).
} 
ments mit einer aktiven Betreuungsfunktion der Väter seit den 1980ern in Norwegen zunehmend öffentlich diskutiert und unterstützt worden (Leira 2006: 27).

\subsubsection{Gesetzliche Nachtrennungsregelungen}

Das folgende Kapitel widmet sich der rechtlichen Institutionalisierung väterlichen Sorgehandelns. Dabei sei an dieser Stelle erneut darauf verwiesen, dass es nicht Ziel der Arbeit ist, einen systematischen Rechtsvergleich zu leisten bzw. konkrete Gesetzesregelungen im Einzelnen zu analysieren. Es geht vielmehr darum die legalen Rahmenbedingungen zu skizzieren, in denen das väterliche Sorgehandeln eingebettet ist.

\subsubsection{Unterhaltsregelungen}

Die bereits dargestellte starke männliche Ernährernorm findet sich auch innerhalb der deutschen Unterhaltsgesetzgebung von 2002 wieder. Innerhalb der Scheidungs- und Trennungsgesetzgebung sind elterliche Pflichten stark institutionalisiert. So müssen Eltern entweder die sozio-emotionale Betreuung im Alltag übernehmen oder die finanzielle Sorge, den sog. Barunterhalt, tragen. Der Elternteil, der mit dem Kind zusammenlebt, leistet, so die gesetzliche Annahme, seinen Teil der elterlichen Verantwortung im Alltag (Forsa 2002: 14; Scheiwe 1999: 132). ${ }^{100}$ Demgegenüber muss der getrennt lebende Elternteil, i.d.R. der Vater, finanziell für die Kinder und seine getrennt lebende Ehefrau aufkommen. ${ }^{101}$ Dabei wird innerhalb der deutschen Gesetzgebung lange stark auf eheliche Unterhaltspflichten abgestellt, aus denen sich der Unterhalt für die Kinder ableitet. Erst mit der Reform des Kindesunterhaltsgesetzes $1998^{102}$ wird nicht länger zwischen den Unterhaltsansprüchen ehelicher und nicht ehelicher Kinder unterschieden (Scheiwe 1999: 133f.; Forsa 2002: 23).

\footnotetext{
${ }^{100}$ Erst mit der Reform von 1998 gilt dies formal rechtlich auch für Väter, die die alltägliche Sorge für ihre Kinder übernommen haben. Vorher war die gültige Vorschrift nur geschlechtsneutral formuliert und wurde in der Rechtsprechung im Allgemeinen auch so ausgelegt, formal ist das Recht für Väter aber erst mit der Reform institutionalisiert worden (Scheiwe 1999: 132).

${ }^{101}$ Die Regelung basierte auf dem Rechtsgrundsatz der generellen Gleichwertigkeit von Betreuungs- und Barunterhalt. In der Praxis muss dies jedoch kritisch betrachtet werden, denn tatsächlich leisten alleinerziehende Elternteile i.d.R. nicht allein Betreuungsunterhalt, sondern es entstehen ihnen darüber hinaus Kosten in Form von Bar- oder Naturalunterhalt (Scheiwe 1999: 132f.). Ähnliche Überlegungen gelten jedoch auch umkehrt für den getrennt lebenden Vater, der Phasenweise die Betreuung übernimmt und während dieser Zeit seine Unterhaltszahlungen ebenso nicht aussetzt.

102 Gesetz zur Vereinheitlichung des Unterhaltsrechts minderjähriger Kinder (Kindesunterhaltsgesetz) vom 6.4.1998.
} 
In Norwegen sind beide Elternteile in Abhängigkeit von ihrer finanziellen Leistungsfähigkeit finanziell für ihre Kinder verantwortlich. Der abwesende Vater ist wie in Deutschland verpflichtet Unterhalt zu zahlen (NIA 2007: 26f.), dessen Berechnung jedoch u.a. am Einkommen der Kindesmutter orientiert wird. Dabei besteht seit 1981 eine gleichberechtigte Nachtrennungsregelung für Kinder, die innerhalb oder außerhalb der Institution Ehe geboren wurden (Skevik 2003b: 7f.; Corden 1999: 20). Unterhaltsverpflichtungen zwischen Ehepartnern haben kaum Bestand (Skevik 1998: 234; Millar 1999: 34).

In Großbritannien wird, wie bereits dargestellt, nicht zuletzt in der öffentlichen Diskussion ein starker Fokus auf die finanzielle Verpflichtung abwesender Väter gelegt. Dies stellt einen Wandel zur bisherigen - insbesondere gerichtlichen - Praxis dar. Lange wurde sozial, politisch und auch gerichtlich akzeptiert, dass Trennungsväter die Familie „ernährten“, mit der sie aktuell zusammenlebten. Damit wurden sie von Unterhaltspflichten gegenüber getrennt lebenden Familien weitestgehend entbunden, insbesondere dann, wenn nicht genügend finanzielle Mittel zur Verfügung standen. Gerichte setzten in diesen Fällen häufig sehr niedrige Unterhaltsniveaus fest, oder es wurden einmalige Abfindungen vereinbart. Dies war möglich, weil der britische Sozialstaat mit seiner kategorialen Familienpolitik insbesondere allein erziehende Mütter und ihre Kinder absicherte. Diese besaßen Sozialhilfeansprüche, wenn die väterlichen Zahlungen ausblieben (Lewis 2002: 137ff.; Finch 2003: 6; Scheiwe 1999: 139). Insgesamt waren die Unterhaltsansprüche getrennt lebender Kinder lange nur schwach institutionalisiert. Unterhaltszahlungen schienen in dieser Zeit optional und hingen stark vom guten Willen des Vaters ab (Scheiwe 1999: 373; Skinner 1999: 34).

Diese Philosophie des “clean break“ findet jedoch seit dem Child Support Act 1991, der reformierten Gesetzesgrundlage des Unterhalts keine Unterstützung mehr. Väter sollen, so die Argumentation dahinter, nur so viele Familien gründen, wie sie sich leisten können (Lewis 2002: 139). In der öffentlichen Diskussion wird dabei, wie dargestellt, auf die unkonditionalen elterlichen Pflichten hingewiesen. Bei Nachtrennungsvätern beinhaltet dies primär die Unterhaltszahlungen. Die neue Gesetzgebung stellt aufgrund der traditionell zurückhaltenden Staatsintervention sowie der bisherigen Vernachlässigung der Väter in der öffentlichen und politischen Debatte einen familienpolitischen Wandel dar (Lewis 2002: 141). Finanzielle Pflichten von Nachtrennungsvätern werden forciert, ausbleibende Zahlungen sanktioniert (Scheiwe 1999: 374). Zur konsequenten Feststellung und Durchsetzung von Unterhaltsansprüchen wird eine spezielle Verwaltungsbehörde geschaffen, die Child Support Agency (kurz: CSA) (Scheiwe 1999: 140, Clarke/Henwood 1997: 189). Zwar gibt es auch in Deutschland und Norwegen behördliche Institutionen zur Durchsetzung von Unterhaltspflichten, doch der CSA kommt im britischen Kontext eine besondere Bedeutung zu, da sie einen Paradigmenwechsel von der Clean Break-Praxis hin zur 
stärkeren Institutionalisierung väterlicher Zahlungspflichten implizierte. Des Weiteren ist sie bei ihrer Implementierung in der Öffentlichkeit stark umstritten, wird mit harten Argumenten eingeführt und auch verteidigt. ${ }^{103}$ Darüber hinaus wird im Child Support Act die väterliche Unterstützungspflicht auf alle biologischen Kinder unabhängig von ihrer Anwesenheit im gemeinsamen Haushalt oder dem Familienstand der Eltern ausgeweitet. Vorher bestanden Unterhaltsansprüche allein innerhalb der Institution Ehe, nun entsteht die Verpflichtung zu finanziellen Leistungen durch die Vaterschaft an sich (Lewis 2002: 139, Skevik 1998: 222; Skinner 1999: 81). Damit wird die männliche Ernährernorm über das Bestehen der Ehe hinaus festgeschrieben (Lewis 2002: 145; Finch 2003: 8).

Betreuungsrechte werden in Großbritannien innerhalb der neuen Unterhaltsregelung vernachlässigt. Dies zeigt sich u.a. in der Tatsache, dass die finanzielle Pflicht über den Bestand der sozialen Beziehung nach der Trennung z.B. zwischen den Eltern gestellt wird. Durch das Forcieren finanzieller Pflichten werden - so Kritiker der Reform - die Interessen von Vätern und Müttern, von ersten und neuen Familien gegeneinander ausgespielt. Die Auswirkungen auf die väterliche Sorgepraxis über finanzielle Transfers hinaus werden nicht berücksichtigt. Das ohnehin angespannte Verhältnis aller Beteiligten wird dadurch zusätzlich belastet (Skinner 1999: 82; Hantrais/Lohkamp-Himmighofen 1999: 15; Clarke/Henwood 1997: 189). Insgesamt stellt die Reform weniger ein familien- als vielmehr ein finanzpolitisches Instrument dar (Skinner 1999: 49).

Neben den unterschiedlich institutionalisierten finanziellen Verpflichtungen variieren die nationalen Kontexte ferner in den Instanzen, die diese durchsetzen. Die ökonomischen Transfers sind im deutschen Kontext stark reglementiert und standardisiert durch legale Prozeduren (Ostner 2002: 162; Hatland/Mayhew 2006: 91). Die Festlegung der Unterhaltshöhe erfolgt in 90\% der Trennungsfälle über Gerichte, denen im deutschen Kontext innerhalb von Nachtrennungsregelungen eine große Bedeutung zukommt. Zwar können

${ }^{103}$ Kritiker sehen die Situation der alleinerziehenden Mütter verschärft, indem ihnen eine stärkere ökonomische Abhängigkeit von ihren Ex-Partnern sowie eine stärkere soziale Kontrolle von Seiten des Staates zugemutet wird. Mütter verweigern die Kooperation mit der Behörde, u.a. weil der „eingetriebene“ Unterhalt auf die Sozialhilfeleistungen angerechnet wird und weil sie die Vater-Kind-Beziehung nicht zusätzlich belasten wollen (Skevik 1998: 235). Väter aus den mittleren und höheren Einkommensklassen empören sich, weil sie von Seiten der CSA als erstes in die Pflicht genommen werden (Scheiwe 1999: 141). Es werden innerhalb der Behörde strenge Erfolgsvorgaben gesetzt, so dass sich die Mitarbeiter aufgrund von höheren Erfolgschancen primär auf die Väter konzentrieren, die bereits zahlen (Lewis 2002: 140). Die betroffenen Väter protestieren gegen die neu festgelegte Höhe der Forderung ebenso wie gegen die Tatsache, dass in Fällen mit früheren Vereinbarungen über eine einmalige Abfindung sowie Unterhaltsverzichtserklärungen trotzdem Unterhaltszahlungen eingefordert werden. Die Akzeptanz wird weiter geschmälert durch die zentralisierte Ansiedlung der Behörde, die auf lokaler Ebene keine Ansprechpartner bereithält. Insgesamt ist damit die öffentliche Akzeptanz der Neuregelungen sowie die Kooperation sehr gering. Dies drückt sich auch in der negativen „Erfolgsbilanz“ aus (Scheiwe 1999: 141; Corden 1999: 9, 23). 
im Vorfeld Vereinbarungen zwischen den Eltern ausgehandelt werden; diese müssen jedoch in gerichtlichen Verfahren bestätigt werden. Die Institution des Jugendamtes kontrolliert Zahlungen zwischen Eltern und kann auf freiwilliger Basis und kostenlos auch im außergerichtlichen Aushandlungsprozess in Anspruch genommen werden (Ostner et al 2003: 6; Corden 1999: 18ff.).

Wie beschrieben, wird in Großbritannien mit dem Child Support Act 1991 eine Unterhaltsbehörde implementiert, die 1993 ihre Arbeit aufnimmt (Lewis 2002: 140; Finch 2003: 7; Clarke/Henwood 1997: 189; Skevik 1998: 222). Zuständig ist die Behörde für die Feststellung, Durchsetzung und Kontrolle von regelmäßigen und ausreichenden Unterhaltszahlungen. Mit der Einrichtung der Behörde wird die Bedeutung der Gerichte maßgeblich geschwächt (Skinner 1999: 49; Scheiwe 1999: 141; Finch 2003: 7; Corden 1999: 17). Gemäß der familienpolitischen Tradition insbesondere bei Familien in Notlage staatlich zu intervenieren werden Sozialleistungsansprüche an die Nutzung der CSA gebunden. Alleinerziehende, die wohlfahrtsstaatliche Transfers beantragen, müssen gleichzeitig mit der Behörde zusammenarbeiten. Doch die Anreize zur Kooperation werden eingeschränkt durch die Anrechnung jedes erhaltenen Cents Unterhalt auf die Sozialhilfe. Eltern, die keine staatliche Hilfe beanspruchen, steht die Nutzung der Behörde frei. Entsprechend den Prämissen eines individualistischen und liberalen Wohlfahrtsstaat gilt, dass so lange Eltern dem Staatshaushalt nicht zur Last fallen, sollen bzw. müssen sie eigene Vereinbarungen treffen (Skinner 1999: 81; Corden 1999: 18). Diese Regelung führt zu der widersprüchlichen Situation, dass in Großbritannien finanziell besser gestellte Väter eher ihren Verpflichtungen entgehen können als Väter, deren Ex-Partnerinnen nach der Trennung auf Sozialhilfe angewiesen ist und die sich höchstwahrscheinlich selbst in einer ökonomisch schlechteren Lage befinden (Skevik 1998: 234).

In Norwegen werden rund 90\% aller Unterhaltsregelungen auf dem Behördenweg entschieden. Dabei beanspruchen die sich trennenden Eltern die externe Hilfe im Aushandlungsprozess meist auf freiwilliger Basis. Nur in Fällen, in denen der unterhaltsberechtigte Elternteil Sozialleistungen beansprucht, ist die Nutzung der Behörde obligatorisch - wie auch in Großbritannien. Gerichten kommt dabei eine untergeordnete Bedeutung zu. Sie schreiten lediglich bei starken elterlichen Konflikten ein. Explizites Ziel der norwegischen Regelung ist es - anders als im britische Fall, wo die Eigenverantwortung durch NonIntervention gestärkt wird -, Eltern zu ermutigen eine eigene Vereinbarung zu finden und sie damit verstärkt in die Eigenverantwortung zu nehmen (Skevik 2003b: 8; Skevik 1998: 225f.; Leira 1996: 61; Corden 1999: 18). Es besteht die Annahme, dass diese eher eingehalten würden (Corden 1999: 45). Dem entspricht, dass Ehepaare zur Teilnahme an Mediationsgesprächen verpflichtet werden, wenn sie sich trennen wollen und gemeinsame Kinder haben (Skevik 2006a: 117; Leira 1996: 57). Es besteht dabei ein weitreichendes 
institutionalisiertes Hilfsangebot für Eltern, eigene Regelungen nach der Trennung auszuhandeln (Corden 1999: 18).

Die nationalen Gesetzgebungen unterscheiden sich ferner in der Berechnung der Unterhaltsleistungen. Die Höhe des Unterhalts hängt in Deutschland vom ökonomischen Leistungsvermögen des Vaters, seinem Lebensstandard sowie der Bedürftigkeit und dem Alter des Kindes ab (Ostner 2002: 162; Corden 1999: 26; Scheiwe 1999: 128). Dabei wird den Trennungskindern ein „angemessener“ Unterhalt zugesprochen. Das bedeutet, Unterhaltsleistungen werden am Lebensstandard des Vaters gemessen (Forsa 2002: 14; Ostner et al 2003: 10). Orientierung bietet für Westdeutschland die Düsseldorfer Tabelle. Die Vorgaben sind jedoch gesetzlich nicht verbindlich, sondern dienen lediglich zur richterlichen Orientierung, die jedoch in den meisten Fällen auch umgesetzt wird (Corden 1999: 24, 29; Forsa 2002: 14f.).

Anders als in Deutschland wird in Großbritannien mit der Reform 1993 eine komplizierte Formel zur Berechnung der Höhe von Unterhaltsforderungen eingeführt (Skinner 1999: 27; Lewis 2002: 140; Finch 2003: 7). ${ }^{104}$ Sie löst gerichtliche Ermessensentscheidungen ab (Corden 2001: 28; Smart/May 2004: 348). Die neue Kalkulation impliziert ferner eine Anhebung des Unterhaltsniveaus (Skinner 1999: 25). Wie in Deutschland kommt bei der Unterhaltsfestlegung der Höhe des väterlichen Einkommens und damit seiner Zahlungsfähigkeit große Bedeutung zu. Daneben wird die Anzahl und Bedürftigkeit der Kinder mit Unterhaltsansprüchen berücksichtigt (Corden 1999: 26ff.; Scheiwe 1999: 128; Skevik 1998: 222). ${ }^{105}$

Ähnlich wie Großbritannien hat auch Norwegen von einer Ermessensregelung zu einem standardisierten Regelungssystem gewechselt. Bis 1989 wurde der Unterhalt in Norwegen rein nach Ermessen der zuständige Mitarbeiter einer lokalen Behörde, den sog. fylkesmannen, oder der Richter im Rahmen der legalen Scheidungsprozesse je nach Einzelfall festgelegt. Aufgrund fehlender Ineffektivität der Ermessensfestlegung wurde mit einer Reform des Unterhaltsrechts 1989 eine - im britischen Vergleich weniger kompli-

${ }^{104}$ Das hatte zur Folge, dass die Behörde selbst im Durchschnitt ein halbes Jahre für die Feststellung der Höhe des Unterhalts benötigt (Scheiwe 1999: 140). Die Komplexität resultiert nicht zuletzt aus der Tatsache, dass in dem Kindesunterhalt ein Betrag für den Unterhalt der betreuenden Kindesmutter - unabhängig vom Familienstand der getrennten Eltern - integriert wurde (Skevik 1998: 222).

${ }^{105}$ Insgesamt ist die Reform von 1993 wenig erfolgreich und kontrovers diskutiert. 1999 werden die ersten Änderungen der Reform umgesetzt, u.a. wird die Berechnung der Unterhaltshöhe vereinfacht, indem wie in Norwegen ein Prozentanteil des väterlichen Einkommens als Unterhalt festgelegt wird. Ferner werden Teile der Unterhaltsleistungen nicht mehr auf die Sozialhilfeansprüche angerechnet (Finch 2003: 9). Diese Veränderungen betreffen die 1995 befragten Väter und ihre Nachtrennungsregelungen noch nicht. 
zierte - Formel zur Berechnung der Unterhaltshöhe implementiert. Die Zuständigkeit ging an die nationale Versicherungsbehörde über, um damit außergerichtliche Einigungen zwischen den Eltern zu erleichtern (Skevik 1998: 224f.; Corden 1999: 24ff.). Der Unterhalt wird seitdem als Anteil des väterlichen Bruttoeinkommens festgelegt: 11\% für ein unterhaltsberechtigtes Kind, 18\% für zwei, 24\% für drei und 28\% des Einkommens für vier und mehr Kinder (Skevik 2006b: 184; Skevik 2003a: 12; Leira 1996: 62). Die Berechnung basiert - ähnlich wie in den beiden anderen Ländern - auf dem Grundsatz, dass das Einkommen, die Anzahl der getrennt lebenden Kinder sowie die finanzielle Verpflichtung des Vaters gegenüber Kindern aus einer neuen Partnerschaft bei der Festlegung berücksichtigt werden müssen. Alle Kinder - vom Vater getrennt und mit ihm zusammen lebend - besitzen in Norwegen gleichberechtigte Unterhaltsansprüche (Skevik 2003b: 9; Skevik 2006b: 184).

Des Weiteren finden sich nationale Unterschiede in der Berücksichtigung des Einkommens der Kindsmutter. In Deutschland wird es nur berücksichtigt, wenn eine hohe Differenz zwischen den finanziellen Mitteln des anwesenden im Vergleich zum abwesenden Elternteil besteht. Mittels Bedarfskontrollbeträgen soll eine ausgewogene Verteilung des verfügbaren Einkommens zwischen Unterhaltspflichtigen und -berechtigten gewährleistet werden (Forsa 2002: 15; Corden 1999: 27) Aufgrund der immer noch traditionellen Aufgabenteilung deutscher Elternpaare in der alltäglichen Praxis stellt dies jedoch eher die Ausnahme als den Regelfall dar. Auch im britischen Kontext wird das Einkommen der Kindesmutter in die Berechnung des Unterhalts integriert (Finch 2003: 10; Corden 1999: 27).

Mit einer Reform 2001 (vollständig implementiert 2003), die gerade noch in den Erhebungszeitraum fällt und damit nur Väter betrifft, die seit Kurzem getrennt sind, wird die Institutionalisierung väterlicher Betreuungsrechte innerhalb der Trennungsgesetzgebung in Norwegen weiter vorangetrieben. Dahinter steht das Ziel, geschlechtsspezifische Gerechtigkeit in den Regelungen herzustellen und den Kontakt zwischen dem getrennten Vater und seinem Kind zu stärken. Interessanterweise wird diese Ambition über die Unterhalts- und nicht über die Sorgerechts- und Umgangsgesetzgebung verfolgt. Eine wesentliche Veränderung in der Berechnung der Unterhaltshöhe besteht in der Berücksichtigung der finanziellen Situation der Kindsmutter. Dazu werden die individuellen Anteile am vorherigen Haushaltseinkommen ermittelt. Wenn beide Eltern z.B. ungefähr das Gleiche zu den Gesamteinkünften beigetragen haben, dann werden nach der Trennung die erwarteten Kosten für das Kind halbiert (NIA 2007: 27; Skevik 2003a: 13; Skevik 2006b: 183ff.).

Die Anerkennung von Betreuungsrechten des Vaters drückt sich ferner in der Berücksichtigung von Betreuungskosten aus, die dem getrennt lebenden Elternteil mit regelmä- 
ßigen Besuchen entstehen. In Deutschland werden diese innerhalb der Düsseldorfer Tabelle nicht explizit bei der Berechnung des Unterhaltsniveaus berücksichtigt (Corden 1999: 53).

In Großbritannien bestand ein wesentlicher Kritikpunkt am Child Support Act 1991 in der fehlenden Berücksichtigung der Kontakthäufigkeit (Lewis 1997: 69). Diese wurden vormals berücksichtigt, wurden jedoch wegen Budgetrestriktionen gestrichen. Auch dies unterstreicht die Dominanz finanzieller Argumente gegenüber der Care-Dimension (Lewis 2002: 145).

Die norwegische Reform impliziert dagegen, dass ein regelmäßiger Umgang mit dem Kind den getrennten Vater von einem Teil seiner Unterhaltspflichten entbindet. Damit sollen die indirekten Kosten, die durch einen regelmäßigen Kontakt verursacht werden, Berücksichtigung finden. Die Vernachlässigung dieser Aufwendungen würde eine doppelte finanzielle Belastung für Trennungsväter bedeuten, so die dahinter stehende Argumentation. Der Unterhalt wird dabei tageweise reduziert (mindestens zwei, maximal 15 Nächte). Damit sollen Anreize geschaffen werden, dass Väter sich in der Betreuung ihrer Kinder über Haushaltsgrenzen hinweg engagieren (NIA 2007: 27; Skevik 2006a: 117; Hatland/Mayhew 2006: 91f.). Die Veränderungen der Reform werden wesentlich am Dual Earner/ Dual Carer-Modell orientiert. Ausgangspunkt ist die Erwerbstätigkeit beider Elternteile auch über die elterliche Trennung hinaus (Skevik 2003a: 13ff.). ${ }^{106}$

Die Institutionalisierung väterlicher Zahlungspflichten unterscheidet sich in den drei Ländern ferner in der Möglichkeit, Nachtrennungsväter gänzlich aus ihren finanziellen Verpflichtungen zu entlassen. In Deutschland besteht die Regelung des Selbstbehalts, der am Sozialhilfeniveau orientiert ist. Verdient der Vater weniger Einkommen, als er an Unterhalt leisten müsste, so entfällt seine Unterhaltspflicht. Von einem Einkommen, das minimal oberhalb dieser Schwelle liegt, wird anteilig Unterhalt abgeleitet (Corden 1999: 26; Forsa 2002: 15). In den Fällen, in denen der getrennt lebende Elternteil seinen Unterhaltsverpflichtungen nicht ausreichend nachkommt, garantiert der deutsche Wohlfahrtsstaat

\footnotetext{
${ }^{106}$ Seit 2002 müssen die getrennt lebenden Eltern ferner die Reisekosten untereinander teilen. Kritisch ist an dieser Stelle darauf zu verweisen, dass norwegischen Eltern in einer schweren Phase ihres Lebens - ihrer Trennung - eine hohe Disziplin und Anstrengung abverlangt wird. Es wird von ihnen eine aktive Kooperation sowie eine Arbeitsteilung zwischen bezahlter und unbezahlter Arbeit verlangt, die selbst verheiratete Elternpaare selten alltagspraktisch in Norwegen leben. Ex-Partner sollen sich unabhängig von ihren emotionalen Konflikten und Auseinandersetzungen gegenseitig in ihren Haushalten willkommen heißen und sozio-emotionale wie auch finanzielle Arrangements für das Leben nach der Trennung aushandeln. Getrennt lebende Familie befinden sich in einer Lebenskrise; dies ist vielleicht nicht der richtige Zeitpunkt neue „moderne“ Familienarrangements einzuführen (Skevik 2003a: 18).
} 
den sog. Unterhaltsvorschuss, einen minimalen finanziellen Transfer (Hatland/Mayhew 2006: 92). Die Anspruchsberechtigung entsteht mit dem ersten Ausbleiben einer Unterhaltszahlung. Der Bedarf wird nicht geprüft (Corden 1999: 42ff.; Forsa 2002: 22f.). Der Staat versucht den gezahlten Vorschuss vom unterhaltpflichtigen Vater zurück zu erhalten (Hantrais/Lohkamp-Himmighofen 1999: 17).

Auch innerhalb norwegischer Regelungen gibt es keinen Minimalbetrag für die Höhe des Unterhalts (Skevik 1998: 226; Corden 1999: 28). Das bedeutet, der Vater kann, anders als in Großbritannien und ähnlich wie in Deutschland, von seiner Unterhaltspflicht ganz entbunden werden, falls ihm die finanziellen Mittel fehlen (Skevik 2003a: 13; Skevik 2003b: 10). Ferner besteht in Norwegen wie in Deutschland die wohlfahrtsstaatliche Leistung des Unterhaltsvorschusses in Fällen, in denen der Vater unbekannt, zahlungsunfähig oder unwillig ist (Skevik 2003b: 26; Skevik 1998: 231f.; Leira 1996: 63). Anders als in Deutschland - aber entsprechend der Dual Earner/ Dual Carer-Norm - wird hier der Leistungsanspruch des anwesenden Elternteils jedoch nur bedarfsgeprüft gewährt. Diese Bedarfsprüfung berücksichtigt das Einkommen der Kindesmutter, den aktuellen Familienstand sowie die Anzahl eigener Kinder im Haushalt der Antragstellerin (NIA 2007: 27).

Die Regelungen in Großbritannien sehen im Gegensatz zu Deutschland und Norwegen dazu kein Nullniveau der Unterhaltspflicht vor. Zwar wird auch hier dem Vater ein Existenzminimum zugestanden, doch selbst Vätern, die kein zu veranlagendes Einkommen erwirtschaften, wird symbolisch ein Minimalbetrag an Unterhalt abverlangt (Skinner 1999: 26; Skevik 1998: 222f.). Dies unterstreicht die starke Betonung finanzieller Verpflichtungen britischer Nachtrennungsväter. Des Weiteren sieht der britische Wohlfahrtsstaat auch entgegen der Vergleichsländer keine Unterhaltsvorschussleistungen vor (Hatland/Mayhew 2006: 92; Finch 2003: 8; Corden 1999: 11). Dies unterstreicht das liberale Staatsverständnis, das die primäre (finanzielle) Verantwortung auf Seiten des getrennt lebenden Vaters sieht. Alleinerziehende Mütter sind bei ausbleibenden Unterhaltszahlungen auf Sozialhilfeleistungen angewiesen, die jedoch eine lange Tradition innerhalb der kategorialen Familienpolitik Großbritanniens haben. Hier haben insbesondere sozialpolitische Maßnahmen im Kampf gegen Kinderarmut gegriffen.

Neben der unterschiedlichen Unterhaltsregelungen in den nationalen Kontexten, die insbesondere die Cash-Komponente väterlichen Sorgehandelns betreffen, finden sich weitere Differenzen in der gesetzlichen Regelung von Sorgerecht und Umgang, die sich auf die Care-Dimension beziehen. 


\subsubsection{Sorge- und Umgangsrechte}

In Deutschland sind Sorgerechte geschiedener Väter im Vergleich zu denen von Müttern relativ schwach ausgeprägt. Noch bis 1980 galten Kinder von geschiedenen Eltern als Waisen mit Blick auf ihren Vater (Ostner 1997: 32). Lange wurde - und z.T. ist dies heute noch der Fall - den getrennten Müttern das Sorgerecht bevorzugt zugesprochen, auch wenn Gesetzestexte zunehmend geschlechtsneutral formuliert werden (Ostner 2002: 167; Hatland/Mayhew 2006: 89). Es liegt im Ermessen der Mutter, ob dem Vater ein regelmäßiger Kontakt zum Kind gewährt wird. Zwar besteht in Deutschland seit 1982 die Möglichkeit, ein gemeinsames Sorgerecht auszuüben, doch dies spielte in der gerichtlichen Praxis lange eine untergeordnete Rolle (Hantrais/Lohkamp-Himmighofen 1999: 11). Insgesamt haben legale und therapeutische Interventionen im Scheidungsfall häufig den sozio-emotionalen Ausschluss des Vaters unterstützt (Ostner 1997: 32). Ein aktives Engagement über die elterliche Trennung hinaus wird - insbesondere unehelichen Vätern erschwert oder gar unmöglich gemacht.

Ähnlich wie in Deutschland sind die Betreuungsrechte britischer Väter nach der elterlichen Trennung nicht sonderlich stark institutionalisiert (Skevik 2006b: 187; Finch 2003: 8). Zwar wird die elterliche Verantwortung - in Abgrenzung zur staatlichen Unterstützung - zunehmend betont, doch mit Blick auf Väter umfasst dies primär finanzielle Pflichten (Lewis 2002: 149; Lowe 2004: 1). Betreuungsrechte werden eher Müttern zugestanden (Hatland/Mayhew 2006: 89). Nichtsdestotrotz basiert das Konzept der elterlichen Verantwortung, wie es im Children Act von 1989 institutionalisiert wurde, auf der Annahme einer lebenslang geltenden elterlichen Verpflichtung. Dem entspricht, dass einem Vater nach der elterlichen Trennung die elterliche Verantwortung nicht ohne Weiteres entzogen werden kann, um sie der Mutter allein zuzusprechen (Lowe 2004: 2), wie dies z.B. im deutschen Kontext mit dem Konstrukt des alleinigen Sorgerechts möglich ist. Dies hat zunächst jedoch v.a. rechtliche Konsequenzen, die tägliche Sorge und Betreuung ist davon in Großbritannien nur formal betroffen.

Darüber hinaus ist die elterliche Eigenverantwortung innerhalb des Trennungsprozesses in den drei Ländern unterschiedlich ausgeprägt. So wird in Großbritannien die elterliche Verantwortung entsprechend einem liberalen Staatsverständnisses über die wohlfahrtstaatliche Zuständigkeit gestellt. Dem entsprechend kommt Eltern die primäre Verantwortung für die Aushandlung der Nachtrennungsregelungen zu. Ähnliches gilt auch für die Regelung des Sorgerechts und des Umgangs (Lowe 2004: 2, 35; Lewis 2002: 147). Der Staat spielt dabei eine untergeordnete Rolle. Nur bei starken Auseinandersetzungen werden gerichtliche Entscheidungen getroffen (Skinner 1999: 80; Lowe 2004: 2). Kontaktvereinbarungen finden außerhalb gerichtlicher Verfahren statt und werden auch nicht 
von Richtern überprüft (Lowe 2004: 36). Damit sind britische Nachtrennungsväter stark auf die Aushandlungsprozesse mit ihren Ex-Partnerinnen angewiesen.

Auch norwegische Eltern können - wie britische Eltern - eigenständig die elterliche Sorge festlegen. Es bestehen keine legislativen Kontrollmechanismen. Seit 1997 werden die Vereinbarungen im Bevölkerungsregister aufgenommen. Können die Eltern sich nicht einigen, besteht das gemeinsame Sorgerecht fort, bis ein Gericht auf Antrag eines Elternteils eine andere Entscheidung trifft. Eine Besonderheit im norwegischen System stellt die obligatorische Mediation für Eltern dar, die sich trennen wollen und gemeinsame Kinder unter 16 Jahren haben. Eine offizielle Anerkennung der Trennung bzw. die Scheidung wird nur ausgesprochen, wenn die Eltern eine institutionelle Vermittlungsstelle in Anspruch nehmen (Lødrup/Sverdrup 2004: 7, 19).

In Norwegen ist lange die Annahme vertreten worden, dass es im Interesse des Kindes sei, nach Trennung und Scheidung nur einem Elternteil, i.d.R. der Mutter, die Sorge zu übertragen. Die im Children Act von 1981 neu geregelten Sorge- und Umgangsrechte basieren nicht länger auf dieser Einschätzung. Seitdem ist es möglich die gemeinsame Sorge sogar gegen den Wunsch eines Elternteils oder gegen beide Eltern durchzusetzen, sofern dies dem Wohl des Kindes entspricht. Ein Elternteil kann nur unter besonderen Umständen das alleinige Sorgerecht zugesprochen werden (Lødrup/Sverdrup 2004: 2ff.; Eydal 2006: 6ff.). Diese Regelung wird in Deutschland mit der Kindschaftsrechtsreform 1998 deutlich später eingeführt.

Erst mit dieser Reform werden auch die Umgangs- und Sorgerechte der Väter in Deutschland gestärkt. Sie symbolisiert einen Wandel von einer unbedingten Privilegierung der Mutter-Kind-Dyade, bei der der Vater abwesend gilt, hin zur Anerkennung väterlicher Rechte und damit dem Recht des Kindes, Kontakt zu beiden leiblichen Elternteilen zu pflegen (Ostner 1997: 32). Mit den Gesetzesänderungen ist das gemeinsame Sorgerecht der Eltern zunehmend zur Norm geworden (Ostner et al 2003: 6; Dethloff/Martiny 2004: 7; Hantrais/Lohkamp-Himmighofen 1999: 11). Vor der Reform bestand für verheiratete Eltern die gesetzliche Notwendigkeit eine gerichtliche Entscheidung über das Sorgerecht zu fällen. Nach der Gesetzesänderung gilt das gemeinsame Sorgerecht - wie es auch schon in der Ehe Bestand hat - weiter, ohne dass eine Gerichtsentscheidung notwendig ist. Nur im Fall von Uneinigkeit zwischen den Eltern oder der konsensualen Festlegung des alleinigen Sorgerechts wird ein Gericht auf Antrag eines oder beider Eltern betraut (Forsa 2002: 17; Hatland/Mayhew 2006: 89; Dethloff/Martiny 2004: 16f.). Dabei soll die alleinige Sorge jedoch die Ausnahme bleiben (Dethloff/Martiny 2004: 7). Begründet wird die Entwicklung der Gesetzeslage hin zur Betonung des gemeinsamen Sorgerechts mit dem Kindeswohl (Ostner 2002: 160). Gleichzeitig besteht mit der Reform, wie in Norwegen, die Möglichkeit, ein gemeinsames Sorgerecht gegen den Willen der Eltern 
festzulegen, wenn dies dem besten Wohle des Kindes entspricht (Dethloff/Martiny 2004: 20).

Das britische Kindschaftsrecht wird mit dem Children Act 1989 - vollständig in Kraft getreten 1991 - weitreichend reformiert (Lowe 2004: 6). Dabei rückt das Kindeswohl in den Vordergrund und die elterliche Verantwortung wird gestärkt. Ferner wird beiden Elternteilen die Betreuungspflicht zugesprochen. Doch diese Pflicht erfährt nicht die gleiche Anerkennung im Vergleich zu der Unterhaltsverpflichtung, wie sie im Child Support Act festgeschrieben wird (Lewis 1997: 68f.; Clarke/Henwood 1997: 186). Erst kürzlich und damit für die Erhebung nur von untergeordneter Bedeutung - wird der Kontakt zwischen getrennt lebenden Vätern und ihren Kindern in der gerichtlichen Praxis verstärkt gefördert. Begründet wird diese Entwicklung mit psychologischen Studien, die die positiven Konsequenzen des väterlichen Umgangs für die kindliche Entwicklung herausstellen (Lewis 2002: 146). Dabei hat die Argumentation hinter der Stärkung väterlicher Rechte jedoch den finanziellen Fokus beibehalten: Mit dem Ziel, die Unterhaltsleistungen verlässlicher zu machen, werden Väter Betreuungsrechte eingeräumt. Dahinter steht die Annahme, dass Väter, denen der Zugang zu ihren Kindern gewährt wird, auch eher ihren ökonomischen Verpflichtungen nachkommen (Finch 2003: 8f.; Lewis 2002: 125).

Trotz der Betonung der gemeinsamen Sorge - primär ein legales Konstrukt - hat das Scheitern der elterlichen Beziehung eine räumliche Trennung zur Folge. Dabei wird das Kind in einem der beiden elterlichen Haushalte hauptsächlich leben (Dethloff/Martiny 2004: 18). Die tägliche Betreuung wird in Deutschland immer noch primär von der Mutter übernommen. Die deutsche Gesetzgebung hat explizit zwischen Sorgerecht und Umgang unterschieden. Umgang umfasst den Kontakt zwischen Vater und Kind nach der elterlichen Trennung. Dazu zählt persönlicher Kontakt in Form von Besuchen ebenso, wie Gespräche am Telefon oder ein schriftlicher Austausch. Das Umgangsrecht ist unabhängig vom Sorgerecht. Generell sollen Eltern ihre eigenen Umgangsregelungen aushandeln, doch ein gänzlicher Verzicht auf Kontakt nicht erlaubt. Dennoch werden die Vereinbarungen nicht durch das Familiengericht geprüft. In Deutschland gilt ein sog. Wohlverhaltensgebot, nachdem der anwesende Elternteil die Beziehung zwischen Kind und getrennt lebendem Elternteil nicht beeinträchtigen sollte. Im Falle des Boykotts des Verhältnisses oder eines regelmäßigen Kontaktes hängen die Konsequenzen jedoch vom Einzelfall ab. Der abwesende Vater kann sich an das Gericht wenden. Im Extremfall werden Sorgerechtsregelungen modifiziert (ebd.: 47ff.). Zur Stärkung väterlicher Besuchsrechte ist ferner das Mediationsangebot für uneinige Eltern im Trennungsprozess ausgeweitet worden (Ostner 2002: 163).

Auch das britische Recht verpflichtet den Elternteil, bei dem das Kind im Haushalt lebt, den Kontakt zum anderen Elternteil nicht zu verhindern. Das umfasst den physischen wie 
non-physischen Kontakt gleichermaßen. Es bestehen jedoch keine unmittelbaren Konsequenzen, wenn die Kindesmutter den Kontakt zum getrennt lebenden Vater boykottiert. Doch Gerichte können eingeschaltet und ein sog. court order bewirkt werden. Erst dann können sanktionierende Schritte bei wiederholtem Verstoß eingeleitet werden (Lowe 2004: 33ff.).

Seit den frühen 1990ern hat in Norwegen den Bedingungen, an die der Kontakt zwischen getrennt lebenden Vater und seinen Kindern geknüpft wird, besondere Aufmerksamkeit gegolten. Dabei sollte jegliche (gesetzliche) Diskriminierung der Väter nach der elterlichen Trennung und Scheidung vermieden werden. Anders als in Großbritannien, wo finanzielle Pflichten im Vordergrund stehen, hat sich die norwegische Debatte auf die väterlichen Betreuungs- und Kontaktpflichten konzentriert (Skevik 2006a: 115; Leira 1996: 56). Der väterliche Kontakt nach der Trennung wird institutionell gefördert, indem z.B. 1956 das Recht auf Kontakt zu beiden Eltern nach der Trennung in Kraft trat (Eydal 2006: 8; Lødrup/Sverdrup 2004: 14). In der Regel legen die Eltern im gegenseitigen Einvernehmen die Umgangsregelung fest, ohne dass eine gerichtliche Prüfung erfolgt. Nur in dem Fall, dass keine Einigung gefunden werden kann, entscheidet das Gericht. Kontaktrechte werden unabhängig von den Sorgerechten gewährt. Wird dem getrennt lebenden Elternteil der Kontakt durch die anwesende Mutter verwehrt, droht ihr in Norwegen eine Geldstrafe, deren Höhe sich an der Anzahl der vereinbarten Besuchstage richtet, an denen kein Kontakt möglich war (Lødrup/Sverdrup 2004: 15f.). Die gesetzliche Durchsetzung der Besuchsrechte ist jedoch nur möglich, wenn der Kontakt vorher formal vereinbart wurde (Leira 1996: 60).

Die nationalen Kontexte unterscheiden sich ferner mit Blick auf die Differenzierung zwischen ehelicher und nicht ehelicher Elternschaft. Mit der Reform 1998 werden väterliche Rechte in Deutschland gestärkt, da sie nicht länger an den Familienstand der getrennten Eltern gebunden sind (Federkeil 1997: 90; Hatland/Mayhew 2006: 85). Die Privilegierung verheirateter Väter basierte bis dahin auf der Annahme, dass Väter, die sich ihrer Verantwortung stellen wollten, dies mit der Heirat der Kindesmutter ausdrückten. Unverheiratete Väter erkannten - so das Argument - weder die Mutter noch die gemeinsamen Kinder ausreichend an. Erst die Ehe ermöglichte den Anspruch auf alle Rechte, die mit einer Vaterschaft verbunden waren (Ostner et al 2003: 5). Mit der Reform werden eheliche und uneheliche Väter mit Blick auf den Umgang und das Sorgerecht gleichberechtigter behandelt. Bei nicht ehelichen Kindern wurde bis 1998 der Mutter das alleinige Sorgerecht nach der Trennung zugesprochen. Auch mit der Reform ist dies noch der Fall, doch ein gemeinsames Sorgerecht wird inzwischen auch bei nicht verheiratet gewesenen Eltern ermöglicht. Ein alleiniges Sorgerecht der Mutter vor der Trennung besteht danach fort. Dies gilt auch für die Vereinbarung eines gemeinsamen Sorgerechts vor dem Scheitern der elter- 
lichen Beziehung; auch dieses bleibt erhalten. Es werden die gleichen Regelungen wie bei geschiedenen Eltern angewandt (Forsa 2002: 18; Ostner et al. 2003: 6; Dethloff/Martiny 2004: 7, 26). Die Neuregelungen haben die Rechte unehelicher Väter gestärkt, doch die Notwendigkeit des mütterlichen Einverständnisses stellt sie nicht vollends mit verheirateten Vätern gleich. Unterhaltspflichten hatten dagegen für beide Vätergruppen gleichermaßen schon länger Bestand (Forsa 2002: 23; Scheiwe 1999: 133f.). Bereits 1969 wurden unverheiratete Männer als Väter anerkannt. Dies implizierte jedoch nur finanzielle Verpflichtungen. Der Kontakt zwischen Vater und Kind hing lange Zeit im Wesentlichen vom Willen der Mutter ab (Ostner 2002: 161f.; Ostner 1997: 45). ${ }^{107}$

Auch innerhalb der britischen Gesetzgebung bestehen im Erhebungszeitraum Unterschiede zwischen Rechten ehelicher bzw. unehelicher Väter nach der elterlichen Trennung. Dabei wird der Kindesmutter in jedem Fall die elterliche Verantwortung zugesprochen. Verheiratete Väter bekommen diese mit der Eheschließung zugeschrieben. Unverheiratete Väter können sich auf der Geburtsurkunde des Kindes registrieren lassen, um Sorgerechte zu erhalten. Dies erfordert jedoch das Einverständnis der Mutter (Lowe 2004: 19ff.; Lewis 2002: 126; Finch 2003: 6; Clarke/Henwood 1997: 186; Hatland/Mayhew 2006: 85; Skinner 1999: 80). Hat der Vater die elterliche Verantwortung zugesprochen bekommen, kann diese ihm nach der Trennung nur mit einer gerichtlichen Anordnung entzogen werden (Lowe 2004: 21). Anders als die elterlichen Rechte, die ein Vater nicht mit der Geburt des Kindes automatisch zugesprochen bekommt, bestehen finanzielle Verpflichtungen bedingungslos (Finch 2003: 8).

Wie bereits angedeutet, wird in Norwegen beiden Elternteilen die Sorge zuteil, wenn diese verheiratet waren (Eydal 2006: 5; Lødrup/Sverdrup 2004: 2). Mit der Scheidung - so die generelle Regelung - sollte sich an dieser gemeinsamen Sorge nichts ändern. Nur in „Notfällen“ wird einem Elternteil allein das Sorgerecht zugesprochen. Mit der Geburt unehelicher Kinder erhält die Mutter zunächst allein die Sorge, wenn die Eltern nichts anderes vereinbart haben. Die norwegische Regierung legt jedoch nahe, dass generell die Eltern die Sorge teilen sollten, wenn sie unehelich zusammenleben. Bestand die Vereinbarung

\footnotetext{
${ }^{107}$ Die unterschiedliche Anerkennung väterlicher Rechte in und außerhalb der Ehe wurde ferner durch die automatische Amtspflegschaft deutlich, die bei der Geburt eines unehelichen Kindes in Kraft trat. Dabei übernahm das Jugendamt in Westdeutschland die Feststellung der Vaterschaft, das Geltendmachen von Unterhaltsansprüchen sowie die Regelung von Erb- und Pflichtrechtsteilen im Falle des Todes des Vaters. Mit der Reform gibt es nur noch die freiwillige Beistandschaft, die von ehelichen wie nicht verheirateten Elternpaaren unabhängig von der vereinbarten Sorgerechtsform beansprucht werden kann (Forsa 2002: 18; Dethloff/Martiny 2004: 6).
} 
über die gemeinsame Sorge, änderte daran die Trennung zunächst nichts. Es gelten die gleichen Regelungen, wie bei verheirateten Paaren (Lødrup/Sverdrup 2004: 7ff.). ${ }^{108}$

\subsubsection{Väterliche Rechte und Pflichten - ein zusammenfassender Vergleich}

Insgesamt zeigt die Darstellung der nationalstaatlichen Rahmenbedingungen eine unterschiedliche Institutionalisierung väterlicher Rechte und Pflichten. So weist Norwegen zum Zeitpunkt der Erhebung eine starke Betonung väterlicher Rechte auf. Dies kann u.a. mit der schwach ausgeprägten männlichen Ernährernorm, der positiven Debatte über Caring Fathers, individuellen Betreuungsrechten von Vätern innerhalb der Elternzeitregelung oder auch der Implementierung einer Kommission, die sich explizit mit den Rechten von Vätern auseinandersetzt, begründet werden. Nach der elterlichen Trennung müssen Väter nicht „um jeden Preis“ - wie etwa in Großbritannien - Unterhalt zahlen. Eine Leistungsunfähigkeit wird durch staatliche Transfers - wie in Deutschland - ausgeglichen. Das gemeinsame Sorgerecht hat in Norwegen als Rechtsnorm eine lange Tradition.

In Deutschland bietet sich Vätern traditionell eine Asymmetrie institutionalisierter Rechte und Pflichten nach der Trennung (Ostner et al 2003: 6). Das Ungleichgewicht hat mit den jüngsten Entwicklungen jedoch zunehmend abgenommen, indem zwar nicht die Unterhaltspflicht für die Kinder in ihrer Bedeutung geschwächt, aber die Sorgerechte der Väter gestärkt wurden. Diese Entwicklung kann u.a. an der schwindenden Bedeutung des männlichen Alleinernährers generell, der Stärkung des gemeinsamen Sorgerechts oder der jüngsten Implementierung von Betreuungsrechten für Väter innerhalb der Elternzeitregelungen deutlich gemacht werden. Dennoch sind traditionelle Rollenzuschreibungen weiterhin erkennbar geblieben. So werden getrennt lebende Väter schon immer in die finanzielle Pflicht genommen, Sorgerechte werden ihnen nur zögerlich und erst in jüngster Zeit zugesprochen; dabei sind diese weiterhin stark abhängig vom Konsens der Mutter. Auch nach der Trennung verbleiben Kinder meist im mütterlichen Haushalt, so dass dem getrennt lebenden Elternteil im Wesentlichen die finanzielle Unterstützungsleistung bleibt.

Der britische Kontext wird im Erhebungszeitraum um 1995 geprägt von einer stark negativ gefärbten Diskussion über die väterliche Abwesenheit. Dabei stehen ausbleibende Unterhaltszahlungen im Vordergrund. Wesentliche familienpolitische Instrumente, wie die Einführung einer Child Support Agency, die Betonung der biologischen statt ehelichen Vaterschaft und selbst die Ermöglichung väterlichen Kontakts nach der elterlichen Tren-

108 Die automatische Sorge beider Eltern mit der Geburt unabhängig vom elterlichen Familienstand ist in Norwegen diskutiert worden; bislang ist dies jedoch nicht in die Gesetzeslage eingegangen (Eydal 2006: 6). 
nung, sind darauf ausgerichtet, väterliche Unterhaltszahlungen verlässlicher zu machen. Die starke männliche Ernährernorm, wie sie im britischen Wohlfahrtsstaat implementiert ist, findet sich somit auch in den gesetzlichen Trennungsregelungen. Nachtrennungsväter müssen z.B. immer einen geringen symbolischen Anteil Unterhalt zahlen, auch wenn sie finanziell schlecht gestellt sind. Ausbleibende Unterhaltszahlungen werden nicht staatlich substituiert. Die Verantwortung wird allein beim Vater gesehen. Einschränkend ist jedoch auf die bis 1993 gängige Praxis des "clean breaks“ zu verweisen. Väter, denen nicht ausreichend Mittel zur Versorgung mehrere Familien zur Verfügung stehen, wurden bis dato angehalten, die Familie finanziell zu unterstützen, mit der sie zusammenlebten. Die allein erziehenden Mütter und ihre Kinder wurden mittels einer ausgeprägten Armutspolitik aufgefangen. Darüber hinaus ist die CSA nur für einen Teil der Unterhaltspflichtigen zuständig. Getrennt lebende Väter, deren Ex-Partnerin nicht auf Sozialleistungen angewiesen sind, können ihren Zahlungspflichten eher entgehen.

Stark vereinfachend und in Anlehnung an die Ausführungen von Hobson und Morgen (2002: 13) können unterschiedliche Institutionalisierungsgrade väterlicher Rechte - primär Care-Aspekte des väterlichen Sorgehandelns - und Pflichten - v.a. auf Cash bezogen nach der elterlichen Trennung in Deutschland, Großbritannien und Norwegen ausgemacht werden. So werden die institutionellen Kontexte zum spezifischen Erhebungszeitraum grob - und aufgrund des mangelnden systematischen Ländervergleichs mit aller Vorsicht - in die folgende Matrix (Tab. 6.1.) eingeordnet. Tab. 6.1: Väterliche Rechte und Pflichten nach der elterlichen Trennung in
unterschiedlichen nationalstaatlichen Kontexten.

\begin{tabular}{|l|c|c|}
\hline & Care-Rechte & Cash-Pflichten \\
\hline Norwegen (2002) & ++ & $(+)$ \\
\hline Deutschland (2002) & + & ++ \\
\hline Großbritannien (1995) & $(+)$ & + \\
\hline
\end{tabular}

+ + = stark ausgeprägt; + = moderat ausgeprägt; $(+)$ = schwächer ausgeprägt

Quelle: eigene Darstellung.

Die Abbildung zeigt die unterschiedliche Ausprägung väterlicher Rechte und Pflichten im Ländervergleich. Dabei wird mit den verschiedenen Pluszeichen ausgedrückt, dass in allen drei Ländern väterliche Rechte und Pflichten bestehen. Selbst wenn in Norwegen finanzielle Verpflichtungen insbesondere der Nachtrennungsväter eine untergeordnete Rolle 
zukommt - zum einen im Vergleich zu den Betreuungsrechten im norwegischen Regime selbst und zum anderen im Vergleich zu den institutionalisierten Väterrechten in Großbritannien und Deutschland - bestehen auch für norwegische Väter institutionalisierte Unterhaltspflichten. Insgesamt wird die Klassifizierung nicht als klassische Typologisierung verstanden. ${ }^{109}$ Vielmehr wird gezeigt, dass väterliche Rechte und Pflichten unterschiedlich in den einzelnen nationalstaatlichen Kontexten institutionalisiert werden.

In die dargestellten nationalen Settings ist väterliches Sorgehandeln in Nachtrennungsfamilien, wie im Mehrebenen-Modell der sozialen Situation (Abb. 6.1) dargestellt, eingebettet. Die befragten deutschen, britischen und norwegischen Väter, die getrennt von ihren Kindern leben, dürften damit unterschiedliche Randbedingungen ihres Handelns vorfinden. Der zwei-stufige Prozess der Situationsdefinition und der Handlungsauswahl bleibt jedoch der Gleiche. Die Rahmung der Situation könnte sich unterschiedlich gestalten. Es können erste vorsichtige länderspezifische Annahmen aus den Ausführungen abgeleitet werden.

Eine Schlussfolgerung aus den nationalen Charakteristika besteht darin, dass an die Nachtrennungsväter unterschiedliche Erwartungen herangetragen werden. So wird von norwegischen Vätern angenommen, dass sie sich über die finanzielle Unterstützung hinaus sozio-emotional um ihre getrennt lebenden Kinder kümmern sollten. Dazu werden ihnen bessere Gelegenheitsstrukturen, z.B. in Form von ausgeprägten Care-Rechten bereitgestellt. Dies könnte eine Erklärung für ein höheres sozio-emotionales Engagement in Norwegen im Vergleich zu Deutschland und Großbritannien sein, wo Care-Rechte im Zeitpunkt der Erhebung weniger stark institutionalisiert waren.

Die britische Philosophie des “clean break“ war 1995 zum Zeitpunkt der Erhebung zwar gesetzlich nicht mehr institutionalisiert, in den inneren Überzeugungssystemen - insbesondere der bereits länger getrennt lebenden Väter - könnte dieses Verständnis des Vaterseins jedoch weiterhin eine Rolle spielen. Das bedeutet, britische Väter fühlen sich verstärkt für die Familie verantwortlich, mit der sie gerade zusammenleben. Dies könnte sich auf die Zahl ausbleibender Unterhaltszahlungen ebenso auswirken, wie auf den Anteil der Kontaktabbrüche. Gleichzeitig stärkt es die Bedeutung einer neuen Familie.

${ }^{109}$ Eine klare Abgrenzung zwischen den Ländern würde eine systematisierten Ländervergleich erforderlich machen. So zeigen Deutschland und Großbritannien z.B. in einigen Dimensionen starke Ähnlichkeiten. Einige Instrumente in Großbritannien weisen auf starke väterliche Rechte hin, wie z.B. die schwer entziehbare elterliche Verantwortung. Des Weiteren können Unstimmigkeiten in der norwegischen Familienpolitik, wie z.B. die erwähnte Cash for Care-Reform, auch in Richtung einer institutionalisierten männlichen Ernährernorm und damit starken väterlichen Pflichten interpretiert werden. 
Des Weiteren kann argumentiert werden, dass die Art der Festlegung von Unterhaltspflichten und auch von Sorge- bzw. Umgangsrechten, die sich in den nationalen Settings unterscheidet, auf das väterliche Sorgehandeln Einfluss nimmt. Sowohl im britischen wie auch im norwegischen Kontext wird die elterliche Eigenverantwortung für diese Aushandlungsprozesse betont. In Großbritannien werden die Eltern damit aber eher allein gelassen. So lange keine Sozialhilfeansprüche geltend gemacht oder Beschwerden vor Gericht eingelegt werden, können die Eltern aushandeln, was sie für richtig halten. Damit sind britische Väter sehr vom Verhältnis zu ihrer Ex-Partnerin abhängig. In Norwegen sollen Eltern auch ihre eigenen Lösungen finden, doch hier wird ihnen ein institutionalisiertes Hilfsangebot zur Verfügung gestellt. Eltern müssen eine Mediation in Anspruch nehmen, wenn sie sich scheiden lassen wollen. Damit handeln sie zwar auch eigenständig die Nachtrennungsregelung aus, werden dabei jedoch durch professionelle Instanzen begleitet.

Die nationalen Kontexte legen ferner die Vermutung nahe, dass dem Unterhalt eine unterschiedliche Bedeutung zukommt. Im deutschen Fall wird der Kindesunterhalt lange an den Betreuungsunterhalt gebunden, der der geschiedenen Kindesmutter zustand. Zahlungen dienen der Versorgung der gesamten getrennt lebenden Familie. Dies impliziert eine andere Bedeutung des Unterhalts - nicht nur für den Vater. In Norwegen sind dagegen eheliche Unterhaltspflichten marginal ausgeprägt. Es wird davon ausgegangen, dass sich allein erziehende Mütter (weiterhin) durch eigene Erwerbstätigkeit selber versorgen. Der Vater trägt allein seinen Anteil an den Kosten, die mit dem Lebensunterhalt des Kindes verbunden werden. Diese Argumentation findet sich ferner in der Tatsache, dass norwegische Mütter genauso ihren finanziellen Teil zur Versorgung der Kinder beitragen sollen wie der getrennt lebende Elternteil. Es stellt sich die Frage, wie sich dies auf das finanzielle Sorgehandeln der Väter auswirkt. Deutsche Väter könnten eher keinen Unterhalt zahlen wollen, weil sie damit der Kindsmutter schaden können oder keine Einsicht in die Versorgung der Ex-Partnerin zeigen. Norwegische Väter können bei den Unterhaltstransfers die Paarebene von der Elternebene formal besser trennen. Sie sind nur dem Kind gegenüber verpflichtet. Sie zahlen daher vielleicht eher. Gleichzeitig kann argumentiert werden, dass deutsche Väter sich weiterhin als Ernährer ihrer getrennt lebenden Familie betrachten und die Notwendigkeit ihrer Zahlungen so lange betonen bis ein neuer Partner der Mutter diese Funktion übernimmt.

Die starke Institutionalisierung der Ehe und damit verbundener Rechte und Pflichten könnte sich insbesondere auf das Sorgehandeln deutscher Väter auswirken. Mit der Ehelichung der Kindsmutter wird institutionell angenommen, dass der Vater seine (finanzielle) Verantwortung für Frau und Kinder anerkannt hat. Unehelichen Vätern wurde dieses Verantwortungsbewusstsein lange abgesprochen. In Norwegen dürfte der Institutionalisierungsgrad der elterlichen Partnerschaft dagegen weniger Einfluss darauf haben, wie 
intensiv sich der Trennungsvater kümmert. Die norwegische Elternschaft ist weniger mit einer ehelichen Partnerschaft verbunden wie z.B. in Deutschland.

Die formulierten theoretischen Annahmen beziehen sich auf das Niveau des väterlichen Sorgehandelns sowie die möglicherweise unterschiedliche Bedeutung einzelner Determinanten im Ländervergleich. Die aufgestellten Vermutungen über die Einflüsse der nationalen Kontexte auf das väterliche Sorgehandeln können innerhalb der folgenden Sekundäranalyse aufgrund der Datenlage jedoch nicht empirisch geprüft werden. An den aufgestellten individuellen Hypothesen, wie sie im Kapitel 5 formuliert worden sind, sollten die nationalstaatlichen Unterschiede jedoch nichts ändern. Die Annahmen sind auf individueller Ebene formuliert, so dass sie Raum und zeitlich ungebunden in allen drei Kontexten Gültigkeit besitzen sollten. Darüber hinaus wird, wie theoretisch begründet, dem direkten Nahumfeld eine größere Bedeutung für die Erklärung väterlichen Sorgehandelns eingeräumt. Die folgende Sekundäranalyse konzentriert sich auf die Überprüfung der individuellen Hypothesen. Auch wenn eine strenge empirische Testung der Auswirkungen der länderspezifischen Besonderheiten am vorhandenen Material nicht möglich ist, werden sie dennoch in der folgenden Untersuchung insbesondere in der inhaltlichen Interpretation der Ergebnisse im Hinterkopf behalten. 


\section{TEIL III: EMPIRISCHE ANALYSE}

\section{Datengrundlage der Sekundäranalyse}

\subsection{Die deutsche Untersuchung von Forsa}

Im Rahmen eines Forsa-Projektes „Unterhaltszahlungen für Kinder in Deutschland“ “110 wird in der Zeit von Juli 2001 bis Juni 2002 eine bundesweite Repräsentativerhebung durchgeführt. In deren Mittelpunkt stehen verschiedene Aspekte der Unterhaltsfestlegung und -praxis bei minderjährigen, unterhaltsberechtigten Kindern, deren Eltern nicht mehr in einer Partnerschaft leben (Forsa 2002: 11). Dabei wird primär der Frage nachgegangen, welche Fälle von ausbleibenden oder nicht in festgelegter Weise erfolgenden Unterhaltszahlungen betroffen sind, und welche Bestimmungsgründe sich dahinter verbergen. Erarbeitet wird ein Querschnittsdatensatz basierend auf standardisierten Telefoninterviews einer repräsentativen Stichprobe von Unterhaltsberechtigten und Unterhaltsverpflichteten. Ergänzt wird die Untersuchung durch Interpretationen teilstrukturierter Interviews zur Vertiefung einzelner inhaltlicher Aspekte. Dabei sind die Zielpersonen alle telefonisch erreichbaren Personen über 14 Jahre, die innerhalb eines Haushalts entweder mit einem unterhaltspflichtigen Kind ohne den dazugehörigen anderen Elternteil zusammenleben oder die gegenüber einem Kind außerhalb ihres Haushalts unterhaltspflichtig sind (ebd.: 34f.). Es bestehen damit zwei Stichproben: einerseits die der insgesamt 2.000 Unterhaltsberechtigten, andererseits die der 1.303 Unterhaltspflichtigen (ebd.: 162). Die nachfolgende Sekundäranalyse bezieht lediglich die Väter ein, die 95,7\% der ursprünglichen Stichprobe alle Unterhaltspflichtigen ausmachen. Des Weiteren werden zur besseren Vergleichbarkeit mit den anderen beiden Studien nur diejenigen Väter berücksichtigt, bei denen Unterhaltspflichten gegenüber bis zu 18-jährigen Kindern bestehen. Innerhalb des methodischen Vorgehens der Arbeit wurde bereits auf die Besonderheiten des deutschen Falls und die Differenzierung in Ost und West eingegangen. ${ }^{111}$ Aufgrund der immer noch bestehenden Unterschiede zwischen ost- bzw. westdeutschen Rahmenbedingungen und Vätern stellt die folgende Analyse lediglich die Ergebnisse der Nachtrennungsväter dar,

\footnotetext{
${ }^{110}$ Durchgeführt wurde die Untersuchung im Auftrag des Bundesministeriums für Familie, Senioren, Frauen und Jugend vom Forschungsinstitut Forsa. Die vorliegende Sekundäranalyse der Daten unterliegt allein der Verantwortung der Autorin Carina Marten.

${ }^{111}$ Vgl. dazu ausführlich Kap. 1 Fragestellung und methodischer Aufbau der Arbeit.
} 
die angeben im Westen Deutschlands zu leben. Insgesamt können somit 947 Männer in den Berechnungen berücksichtigt werden. ${ }^{112}$

Im Vordergrund des standardisierten Fragebogens, der sich auf die Unterhaltspflichtigen bezieht, steht der sog. Barunterhalt, dessen Festlegung und Zahlungspraxis. Darüber hinaus werden Informationen auf der Ebene der Befragten eingeholt, und es werden Fragen zur Kindesmutter gestellt. Auf beiden Ebenen werden neben soziodemographischen Aspekten wie dem Alter, dem Schulabschluss, der Erwerbstätigkeit oder der Staatsangehörigkeit auch Informationen zur aktuellen Lebenslage, wie z.B. der momentanen Partnerschaftssituation erfasst. Im Zusammenhang mit der vergangenen gemeinsamen Beziehung werden unterschiedliche Sachverhalte wie z.B. die ehemalige Familienkonstellation, die damaligen Lebensumstände oder auch die zeitliche Dauer seit der Trennung erhoben. Ferner werden genauere Angaben zu den unterhaltsberechtigten Kindern erfragt. Im deutschen Datensatz beginnen die Auskünfte des Vaters mit dem jüngsten Kind und damit mit den Angaben zur zeitlich letzten Beziehung. ${ }^{113}$

\subsection{Die britische Untersuchung von Bradshaw und Mitarbeitern}

Unter dem Titel “Absent Fathers?“ führen Bradshaw und seine Mitarbeiter 1995/1996 eine Untersuchung zu den Lebensumständen getrennt lebender Väter in Großbritannien durch. ${ }^{114}$ Ziel ist es dabei u.a., die Einflussfaktoren zu benennen, die die Leistung von Unterhaltszahlungen bzw. die Aufrechterhaltung einer intensiven Vater-Kind-Beziehung begünstigen. Mittels persönlichen Face-to-Face-Interviews werden sowohl getrennt lebende Väter wie auch unterhaltsberechtigte Mütter von leiblichen und biologischen Kindern befragt. Darüber hinaus wurden, wie im deutschen Fall, qualitative Interviews geführt. Im Folgenden werden nur die quantitativen Angaben der Trennungsväter berücksichtigt. Mittels Filterführung wird die Sekundäranalyse nur Angaben zu Kindern im Alter von bis zu 16 Jahren sowie Kindern zwischen 17 und 18 Jahren, die sich in Vollzeitausbildung befinden, berücksichtigen. Damit können 616 Fälle analysiert werden.

\footnotetext{
${ }^{112}$ Zur genauen Zusammensetzung der einzelnen Stichproben findet sich im Anhang eine Übersicht (Tab. B.1 bis B.3).

${ }^{113}$ Die Angaben zu weiteren Beziehungen können jedoch nicht ausgewertet werden, weil diese im Datensatz nicht mehr enthalten sind. So werden von 44 Vätern, die angeben, Kinder aus mehr als einer gescheiterten Beziehung zu haben, nur die Informationen zur letzten aufgeführt.

${ }^{114}$ Die erforderlichen Vor- und Nacharbeiten zur Erhebung der Originaldaten wurden von Julie Williams, Jonathan Bradshaw, Carol Stimson und Christine Skinner durchgeführt; der Datensatz durch NOP Market Research Limited sowie dem Office of Population Censuses and Surveys erhoben. Finanziert wurde die Untersuchung vom Economic and Social Research Council sowie dem Department of Social Security. Die nachfolgende Sekundäranalyse liegt allein im Verantwortungsbereich der Autorin der vorliegenden Arbeit.
} 
Anders als in der deutschen und auch in der norwegischen Studie wird in der britischen Untersuchung versucht, Angaben zu allen getrennten Elternschaften des Vaters zu erfassen. Maximal werden Informationen zu drei gescheiterten Beziehungen, aus denen Kinder hervorgegangen sind, erhoben. Zunächst wird erfragt, aus wie vielen Partnerschaften, der Vater getrennt lebende Kinder hat, die dann in verschiedenen Themenblöcken des Fragebogens abgearbeitet werden. Die Erhebung beginnt dabei mit den Angaben zur ersten getrennten Beziehung des Vaters, aus der Kinder entstanden sind. Hier liegen die Angaben fast aller Väter vor, im Gegensatz zu den Informationen zu weiteren Elternschaften. Die Häufigkeitsverteilung der Variablen zur Anzahl der getrennt lebenden Beziehungen mit Kindern legt eine höhere Anzahl an Fällen nahe, als Informationen zur zweiten und dritten Elternschaft tatsächlich vorliegen. So müssten insgesamt 175 Väter Angaben zu mehr als einer Elternschaft machen, je nach Variable sind es jedoch nur 30 bis 40 Fälle, die Auskünfte zu weiteren Beziehungen geben. Diese Besonderheit des britischen Datensatzes legt nahe, die Analyse auf die erste Partnerschaft des Vaters zu beziehen, aus der Kinder entstanden sind. Leider sind die Angaben zum Unterhalt nur für die letzte Beziehung erfragt worden. Damit stellt sich die Schwierigkeit, die sozio-emotionalen Angaben von Mutter und Kind den richtigen Angaben zum Unterhalt, zur finanziellen Unterstützung und den Merkmalen der Mutter zuzuordnen, d.h. die Merkmale einer Mutter $\mathrm{X}_{\mathrm{m}}$ und ihren Kindern $\mathrm{x}_{1}$ und $\mathrm{x}_{2}$ müssen den Unterhaltszahlungen des Vaters an diese Mutter $\mathrm{X}_{\mathrm{m}}$ zugeordnet werden. ${ }^{115}$ Aufgrund dieser Unsicherheit im britischen Datensatz berücksichtigt die folgende Sekundäranalyse nur diejenigen Väter, die entweder nur eine Beziehung mit Kindern hatten, die inzwischen gescheitert ist, oder die nur eine Antwort bezüglich der Beziehung zur Kindesmutter und zum Kind geben. Dieses Vorgehen basiert auf der Annahme, dass Väter, die im ersten Teil Angaben machen, der sich primär auf Care-Aspekte

115 Diese Inkonsistenz im Datensatz kann mehrere Ursachen haben und nicht abschließend geklärt werden. Zunächst kann die Variable (c4), die angibt wie viele Beziehungen der Vater in der Vergangenheit hatte, die gescheitert sind und Kinder hervorgebracht haben, fehlerhaft sein. Die hohen Ausfälle könnten vorsichtig als Hinweis gedeutet werden. Insgesamt liegen nur 498 Angaben von insgesamt 616 Vätern vor. 114 Vätern werden mit der Ziffer 5 kodiert. Dem Codebook ist jedoch nicht zu entnehmen, wofür diese Kodierung steht. Vielleicht gibt es tatsächlich nur rund 30 Väter, die eine zweite und dritte Beziehung haben und zu dieser Angaben machen, anstatt der 173, wie es die Häufigkeitstabelle der Variablen c4 nahelegt. Es gibt jedoch keinerlei weitere Informationen in dem Datensatz, die diese Vermutung bestätigen oder entkräften. Eine andere naheliegende Annahme ist, dass der Fragebogen zu umfangreich konzipiert worden ist, so dass die Väter immer nur zu einer Beziehung Angaben gemacht haben und der Interviewer nicht mehr zu den Fragen in den Blöcken F bis I (2. und 3. bzw. letzte Beziehung) gekommen ist. In der Dokumentation des Datensatzes sind auch für diese Vermutung keinerlei Anzeichen zu finden. In der Veröffentlichung der Ergebnisse von Bradshaw und MitarbeiterInnen (1999) scheinen die Angaben zu der ersten gescheiterten Beziehung mit Kindern herangezogen worden zu sein. Doch auch dies wird nicht expliziert, sondern folgt aus eigenen Nachrechnungen, die ähnliche Ergebnisse erzeugten wie die im Buch dargestellten. Es kann jedoch nicht mit abschließender Sicherheit gesagt werden, ob diese sich auf dieselbe Beziehung beziehen, wie die Blöcke J, K und T, die v.a. finanzielle Aspekte des väterlichen Sorgehandelns abbilden. 
bezieht, im zweiten Teil zur Cash-Dimension die Perspektive nicht wechseln, d.h. wenn sie mit einer Beziehung anfangen, dann bleiben sie bei dieser Elternschaft auch im späteren Teil des Fragebogens, der sich auf den Unterhalt bezieht und laut Intervieweranweisung eine andere Beziehung betreffen sollte. Insgesamt 39 Väter, die Angaben zu mehr als einer Elternschaft machen, werden aufgrund der Unsicherheiten aus der Analyse ausgeschlossen. ${ }^{116}$ Es bleiben damit Informationen zu 576 britischen Nachtrennungsvätern erhalten. $^{117}$

Insgesamt sind von den drei vorliegenden Untersuchungen die britischen Daten inhaltlich am umfangreichsten. Es können 22 unterschiedliche Themenblöcke im gesamten Fragebogen ausgemacht werden. Erhoben werden dabei u.a. ausführliche Informationen zur Haushaltszusammensetzung des Vaters, zu seiner aktuellen und vergangenen Partner-

${ }^{116}$ Dabei ist darauf zu verweisen, dass die hier ausgeschlossenen Väter mehr als eine Angabe zu gescheiterten Beziehungen mit Kindern gemacht haben. Es kann angenommen werden, dass Väter mit mehreren getrennt lebenden Elternschaften besondere sozio-ökonomische Charakteristika aufweisen. Dies zeigt auch die nähere Betrachtung dieser ausgeschlossenen Gruppe. Unterschiede zwischen Vätern, die in die Berechnungen eingehen zu denen, die ausgeschlossen werden, ergeben sich für die Variable des Einkommens. Hier zeigen die Ausfälle ein geringeres Einkommensniveau. Ähnliches gilt für die Angaben zur ökonomischen Situation der Väter. Nicht berücksichtigte Fälle schätzen ihre ökonomische Situation häufiger schlecht ein, als Väter, die in die Untersuchung eingehen. Darüber hinaus sind sie häufiger erwerbslos bzw. geben eine geringere Arbeitszeit und ein niedrigeres Ausbildungsniveau an. Die Väter können weniger Auskunft über die Beziehungssituation der Kindsmutter geben, waren deutlich seltener mit dieser vor der Trennung verheiratet sowie auch kürzer liiert und zeigen häufiger Streitigkeiten bei der Festlegung des Unterhalts. Weniger der ausgeschlossenen Väter machen Angaben zum Verhältnis zur Kindsmutter im Zeitpunkt der Befragung. Wenn sie eine Antwort geben, charakterisieren sie dieses seltener freundschaftlich als Väter, die in die Analyse eingehen. Auch die Informationen zur ökonomischen Situation der Mutter liegen den nicht berücksichtigten Vätern - wie schon bei der mütterlichen Partnersituation - seltener vor. Die Väter, die antworten können, beschreiben die Finanzlage der ehemaligen Partnerin häufiger als schlecht. Desweiteren weisen die nicht berücksichtigten Väter ein geringeres Durchschnittsalter auf. Kaum nennenswerte Unterschiede in den Angaben ergeben sich jedoch zur Distanz zwischen den Wohnorten, zur Kinderzahl im Haushalt des Vaters und zur väterlichen Partnersituation. Die Zusammensetzung dieser Gruppe Väter weist auf einen höheren Anteil an „Problemfällen“ hin. Das bestätigt auch die Betrachtung der Care- bzw. Cash-Variable - unabhängig voneinander - in dieser Gruppe. Die ausgeschlossenen Väter zeigen deutlich mehr Unterhaltsausfälle und Kontaktabbrüche. Insgesamt wird durch den Ausschluss dieser bestimmten Vätergruppe das väterliche Sorgehandeln in Großbritannien insgesamt (etwas) unterschätzt.

117 Es kann abschließend jedoch nicht geklärt werden, ob innerhalb dieses Vorgehens die Informationen einer Beziehung richtig zugeordnet werden. Der Vergleich der Väter, deren Angaben als gesichert gelten können, weil sie nur eine Elternschaft angeben und zu dieser auch Informationen liefern, mit Vätern, deren Antworten Unsicherheit in sich bergen, weil sie mehr Elternschaften angeben als sie Antworten geben, scheint wenig hilfreich. Väter, die mehr als eine getrennte Beziehung aufweisen, aus der Kinder hervorgegangen sind, zeigen andere Charakteristika wie auch eine andere Ausgestaltung ihres väterlichen Sorgehandelns als Väter, die nur eine Elternschaft nennen. Ein Vergleich würde zwangsläufig zu Unterschieden zwischen den beiden Gruppen führen und keinen Aufschluss über die Zuordnung der Beziehungen in der unsicheren Gruppe liefern. Desweiteren könnte ein Vergleich mit den „richtigen“ Mehrfachantworten sinnvoll erscheinen. Das bedeutet, Väter, die eine zweite oder dritte Elternschaft angeben und gleichzeitig eine zweite oder dritte Antwort geben, könnten mit den Vätern verglichen werden, die nur eine Antwort liefern trotz der Angabe mehrerer Elternschaften. Die Zahl der richtigen Mehrfachnennungen ist mit knapp 20 Fällen jedoch zu klein, um daraus sinnvolle Schlüsse ziehen zu können. 
schaftssituation generell, zu Einzelheiten der jeweiligen Partnerschaften und den jeweils zugehörigen Kindern, zu seiner Unterhaltspraxis differenziert in formal festgelegten sowie eigenständigen (informalen) finanziellen Leistungen, zur sozio-ökonomischen Lage der beiden getrennten Partner, Erfahrungen mit der Unterhaltsbehörde sowie dem weiteren Verwandtschaftssystem.

\subsection{Die norwegische Untersuchung von NOVA}

Die norwegische Untersuchung „Samværfedrenes situasjon: økonomiske og normative problemstillinger“ wird 2001/2002 durchgeführt vom Forschungsinstitut NOVA. ${ }^{118}$ Erfasst werden Väter, die aufgrund der elterlichen Trennung im Alltag nicht mit ihren Kindern in einem Haushalt zusammenleben. Es wurden 1035 aus dem Register der nationalen Versicherung, dass 90\% aller unterhaltsberechtigten Eltern erfasst, zufällig postalisch angeschrieben. Insgesamt liegen die Antworten von 584 Vätern vor (Skevik 2006a: 122; Skevik/Hyggen 2002: 125). Die 10\% der nicht registrierten Nachtrennungseltern haben entweder keine Regelung des Unterhalts über eine externe Instanz in Anspruch genommen oder der allein erziehende Elternteil ist nicht auf Sozialleistungen angewiesen. Damit fehlen insbesondere Information über Elternpaare, die der Mittelklasse angehören und ein geringes Konfliktpotential aufweisen. Wie in Deutschland und Großbritannien werden Väter mit Kindern über 18 Jahren nicht in der Analyse berücksichtigt. Es werden, ähnlich wie in Deutschland, nur Angaben zum jüngsten getrennt lebenden Kind erhoben. Es bleiben insgesamt Informationen zu 565 Fällen erhalten. Der standardisierte Fragebogen umfasst dabei wie in den anderen beiden Datensätzen verschiedene Aspekte. So werden Einzelheiten zum Kontakt zwischen getrennt lebendem Vater und Kind, zur Unterhaltspraxis und damit verbundene Einstellungen, zum Verhältnis zur Kindsmutter sowie zur aktuellen Lebenssituation des Vaters erfasst.

\footnotetext{
${ }^{118}$ Die norwegischen Daten, die in der vorliegenden Arbeit verwendet wurden, basieren auf dem Datensatz „Samværsfedrenes situasjon - økonomiske og normative problemstillinger 2002““. Der anonymisierte Datensatz wurde durch Norwegian Social Science Data Service (NSD) zugänglich gemacht. Statistics Norway (SSB) hat das Sampling und Interviewen zu verantworten. Die folgenden Analysen und Interpretationen liegen jedoch allein im Verantwortungsbereich der Autorin der vorliegenden Arbeit.
} 


\section{Operationalisierung und Ausgestaltung väterlichen Sorgehandelns}

Im folgenden Kapitel wird die jeweilige Operationalisierung der abhängigen Variablen in den einzelnen Datensätzen dargestellt. Es wurde bereits darauf verwiesen, dass die Untersuchungen unabhängig voneinander konzeptionalisiert und durchgeführt wurden. Deswegen müssen z.T. sehr unterschiedliche Operationalisierungen für die theoretischen Konzepte Care und Cash - aber auch bei den erklärenden Faktoren -verwendet werden. Trotz der Unsicherheiten, die daraus für den direkten Vergleich der Ergebnisse resultieren, berücksichtigt die vorgenommene Operationalisierung aller Variablen die Vergleichsperspektive soweit dies möglich und methodisch vertretbar erscheint. Das bedeutet, Antwortkategorien werden angeglichen und harmonisiert. So werden z.B. Angaben in Meilen auf Kilometer umgerechnet. Des Weiteren werden Variablen gruppiert, wenn sie nicht in allen Datensätzen als metrische Angaben vorliegen. Dabei orientiert sich die Antwortkategorisierung der neuen Variable an den Vorgaben der anderen Datensätze. Für die bessere Vergleichbarkeit wird damit ein Verlust an Detailinformationen in Kauf genommen. ${ }^{119}$

\subsection{Die abhängige Variable: Care}

Die Theoretisierung väterlichen Sorgehandelns in Form von Care in Kapitel 2.1 Väterliches Sorgehandeln in Form von Care und Cash hat bereits deutlich gemacht, dass die sozio-emotionale Dimension väterlichen Sorgehandelns unterschiedliche Formen und Ausprägungen annehmen kann. Einen Überblick über die sehr unterschiedlichen Aspekte, die in den nationalen Datensätzen diesbezüglich erhoben wurden, gibt Tabelle 8.1. Aufgrund der Uneinheitlichkeit wird die abhängige Variable Care als Index verschiedener Variablen, die in dem jeweiligen Datensatz zur Verfügung stehen, operationalisiert. Das bedeutet, in jedem Datensatz umfasst der Care-Index ähnliche Gesichtspunkte väterlichen Kümmerns. Die folgenden Analysen und Interpretationen basieren auf der Annahme, das trotz der unterschiedlichen Zusammensetzung des Kriteriums ein ähnliches Konstrukt ge-

119 Auch wenn die einzelnen Datensätze nicht in einem großen Datensatz kumuliert wurden, werden die Variablen zur besseren Differenzierung mit spezifischen Länderendungen versehen: Variablen des britischen Datensatzes enden auf_B, norwegische Variablen auf_N, während Variablen in der Forsa-Studie die Endung _G erhalten. Im Folgenden werden alle Ausführungen zu den Ländern alphabetisch dargestellt. Das bedeutet, Ausführungen, die sich auf den deutschen Fall beziehen, werden zuerst dargestellt, dann folgen die Angaben zu Großbritannien und Norwegen. Inhaltlich hat die Reihenfolge der Darstellung keine Bedeutung. 
messen wird: Das väterliche Kümmern in Form von Care, das über die reine quantitative Kontakthäufigkeit hinausreicht.

Tab. 8.1: Care-Dimensionen erfasst in den einzelnen Studien.

\begin{tabular}{|c|c|c|c|c|}
\hline Dimensionen & Variablen & 芯 & $\begin{array}{l}\frac{3}{0} \\
\frac{\pi}{0} \\
\frac{0}{0} \\
\frac{\pi}{0} \\
\frac{\pi}{0}\end{array}$ & $\stackrel{\nwarrow}{\text { O }}$ \\
\hline \multirow{5}{*}{ Kontakthäufigkeit } & Letzter Kontakt & - & + & + \\
\hline & Kontakthäufigkeit & + & + & - \\
\hline & Anzahl der Tage im letzten Monat & - & - & + \\
\hline & Anzahl der Nächte im letzten Monat & - & - & + \\
\hline & Gemeinsame Urlaube/Ferien & + & + & - \\
\hline \multirow{5}{*}{ Art des Kontaktes } & Telefonischer Kontakt & - & + & + \\
\hline & Schriftlicher Kontakt & - & + & + \\
\hline & Übernachtungen & - & $(+)$ & + \\
\hline & Ort des Kontakts & - & + & - \\
\hline & Dauer der Besuche & - & + & - \\
\hline \multirow{3}{*}{ Gestaltung } & Aktivitäten & + & + & + \\
\hline & Anwesenheit dritter Personen & - & - & + \\
\hline & Harmonie während Besuchen & + & - & - \\
\hline \multirow{2}{*}{$\begin{array}{l}\text { Andere } \\
\text { Beteiligungsformen } \\
\text { an Kindererziehung }\end{array}$} & $\begin{array}{l}\text { Elterliche Aktivitäten } \\
\text { (Chauffeurdienste, Schulaktivitäten, ...) }\end{array}$ & - & + & - \\
\hline & Entscheidungspartizipation & + & + & - \\
\hline
\end{tabular}

Quelle: eigene Darstellung.

Kontakt, verstanden als direkte Interaktion zwischen Vater und Kind, ist eine grundlegende Voraussetzung für väterliches Sorgehandeln. Kontakt allein ist aber nicht gleichzusetzen mit väterlichem Kümmern. Es umfasst rein quantitative Aspekte des väterlichen Engagements. Eine höhere Zahl väterlicher Besuche führt nicht automatisch zu einer höheren Qualität der Vater-Kind-Beziehung, dennoch ist für den Erhalt der Beziehung ein regelmäßiger Umgang von entscheidender Bedeutung (Amendt 2003: 77). Kümmern kann unabhängig von der Kontakthäufigkeit eine unterschiedlich stark ausgeprägte Intensität annehmen. Daher bezieht sich die folgende Sekundäranalyse nicht auf die Kontakthäufigkeit zwischen abwesendem Vater und seinem Kind, sondern vielmehr auf die Intensität seines Kümmerns über die rein zahlenmäßige Erfassung seiner Besuche hinaus. ${ }^{120}$ Es gilt ein komplexes Handlungsgeflecht zu erklären, das neben der Quantität auch die Qualität

${ }^{120}$ Statistische Berechnungen in allen drei Datensätzen ergeben, dass die Kontakthäufigkeit z.T. durch andere Determinanten bestimmt wird als die Care-Variable. Dies unterstützt die Argumentation, dass väterliches Sorgehandeln nach der elterlichen Trennung mehr ist als der rein quantitative Aspekt der Häufigkeit von Besuchen. 
väterlichen Sorgehandelns berücksichtigt. Im Folgenden wird ein Index konstruiert, der das unterschiedliche Niveau väterlichen Engagements erfasst.

Innerhalb der Forsa-Studie werden verschiedene Variablen zur Besuchsregelung und praxis erhoben, die Indikatoren für das Ausmaß väterlichen Kümmerns darstellen. Dazu zählen neben der Kontakthäufigkeit die Zeitgestaltung während der Besuche, das Verbringen gemeinsamer Ferien sowie die Partizipation an wichtigen, das Kind betreffenden Entscheidungen. ${ }^{121}$ Zur Konstruktion des Care-Indexes werden zunächst die verschiedenen Dimensionen väterlichen Kümmerns differenziert und gewichtet. ${ }^{122}$

Zur Beantwortung der Frage, wie die Väter die gemeinsame Zeit mit ihren Kindern normalerweise gestalten, wird ihnen eine Auswahl von Aktivitäten zur Verfügung gestellt. Mehrfachnennungen sind in der Beantwortung möglich. Die einzelnen Formen väterlichen Kümmerns sind von unterschiedlicher Qualität für die Intensität der Vater-Kind-Beziehung. ${ }^{123}$ Diese wird im Folgenden anhand verschiedener Dimensionen väterlichen Engagements festgemacht. Im Vordergrund des Interesses steht dabei das väterliche Sorgen auf Handlungsebene, das m.E. in erster Linie aus der direkten Auseinandersetzung mit dem Kind und erzieherischen Aspekten besteht. ${ }^{124}$

Die erste Dimension anhand derer die Aktivitäten in ihrer Bedeutung für das väterliche Kümmern differenziert werden können, stellt der mit ihnen verbundene Aufwand dar. Dieser wird unterschieden in zeitliche und finanzielle Ressourcen. Finanzielle Ressourcen sind dabei abhängig vom verfügbaren Einkommen. Da die Qualität des Vaterseins nicht in erster Linie von den verfügbaren, finanziellen Mitteln abhängt, geht der finanzielle Aufwand einer Aktivität einfach in die Berechnung ein. Die zeitlichen Ressourcen sind nicht

\footnotetext{
${ }^{121}$ Eine Variable erfasst zusätzlich die Harmonie der Besuche bei bzw. vom Vater. Aufgrund der geringen Aussagekraft der Antwortvorgaben („,harmonische Stunden oder Tage“, „,angespannte Stunden und Tage mit Stress und Streit“ und „mal so oder mal so“), der hohen Subjektivität der Einschätzung und der Tatsache, dass nicht einmal zwei Prozent der Befragten angaben, dass die gemeinsamen Stunden angespannt verlaufen, wird diese Variable nicht weiter berücksichtigt.

${ }^{122}$ Zur genauen Konstruktion der einzelnen Indizes siehe Tab. A.1 bis A.3 im Anhang.

${ }^{123}$ Die folgenden Überlegungen basieren im Wesentlichen auf den Ausführungen von Palkovitz (1997).

${ }^{124}$ Es wird darauf hingewiesen, dass die Ausführungen keine konkreten Handlungen berücksichtigen, sondern vielmehr generelle Überlegungen bzgl. der vorgegebenen Antwortkategorien darstellen. So kann das Betreiben eines gemeinsamen Hobbys sehr verschiedene Ausmaße väterlichen Kümmerns umfassen. So ist z.B. das Wandern in den Alpen mit einem anderen Aufwand verbunden und von anderer Qualität für die Vater-Kind-Beziehung wie das Sammeln von Briefmarken. Insgesamt ist aber das Betreiben eines Hobbys von anderer Bedeutung für die Intensität väterlichen Sorgehandelns als z.B. das alltägliche Spielen. Ferner sind die einzelnen Aktivitäten nicht trennscharf zu differenzieren. Das gemeinsame Hobby kann zum Beispiel das Betreiben einer Sportart sein, und fällt damit auch in die Aktivität „Sport treiben“. Trotzdem erscheint eine generelle Gewichtung der einzelnen Aktivitäten innerhalb des Indexes inhaltlich sinnvoll.
} 
eindeutig zu beurteilen. So wurde der Zeitanteil, den die einzelne Aktivität an der gemeinsam verbrachten Zeit ausmacht, nicht erfasst. Dabei macht es durchaus einen qualitativen Unterschied, ob ein Vater zwei Stunden mit seinem Kind Spaziergänge unternimmt und eine halbe Stunde fernsieht, oder ob die zeitlichen Anteile der beiden Aktivitäten umgekehrt sind. Da nähere Angaben fehlen, wird im Folgenden ein alltägliches Verständnis bzgl. der generellen Dauer einzelner Aktivitäten zugrunde gelegt. Ein durchschnittlicher Spaziergang beansprucht z.B. ein bis zwei Stunden. Entscheidender ist in diesem Zusammenhang die Relation zu den anderen berücksichtigten Aktivitäten. Hobbys und Ausflüge sind z.B. üblicherweise zeitlich aufwendiger als das gemeinsame Spielen, Gespräche führen oder Lernen. Wie die finanziellen Aufwendungen abhängig sind vom Einkommen des Vaters, ist die mit dem Kind verbrachte Zeit u.a. abhängig von der insgesamt verfügbaren Zeit und den formalen Vereinbarungen zum väterlichen Umgangsrechts zwischen den Eltern. Der zeitliche Aspekt wird mit dem Faktor zwei in die Index-Konstruktion aufgenommen.

Unter der Dimension Planung wird der mit den einzelnen Aktivitäten verbundene organisatorische Aufwand verstanden. Dazu zählen z.B. die Anreise oder Absprachen mit der Mutter. Eine Aktivität mit relativ hohem organisatorischen Aufwand stellt z.B. das Unternehmen von gemeinsamen Ausflügen dar, während ein gemeinsamer Spaziergang nicht viel Organisation im Vorfeld erfordert. Organisatorisch aufwendige Aktivitäten sind nicht automatisch die intensivsten Besuche für das Kind, entscheidender sind die Kommunikation oder direkte Interaktion mit dem Vater. Daher wird die Planung einfach gewichtet.

Zentrale entwicklungsrelevante Dimensionen elterlichen Handelns ist das Unterstützungsund Kontrollverhalten (Herlth 2002: 589), das unter dem Konzept Monitoring/Kontrolle erfasst wird. Kontrolle umfasst dabei die Formulierung von Regeln, Vermittlung von Disziplin, Überwachung und Beaufsichtigung (Amato 1998: 245). Erziehung impliziert das elterliche Lenken. Dazu zählt u.a. das Erlernen von sozialen Fähigkeiten, das Essen gesunder Nahrungsmittel oder die Fertigkeit, sich sicher zu bewegen. So ist z.B. das gemeinsame Fernsehen mit weniger elterlicher Kontrolle verbunden, wohingegen das Lernen oder die Erledigung von Hausaufgaben mehr Überwachung erfordern. Monitoring hat dabei sehr viel mit elterlicher Verantwortung zu tun. Eng in Zusammenhang mit der Kontrolle stehen die mit einer Unternehmung verbundenen vom Vater ausgehenden Lehr- und Lerneffekte. Dazu zählen z.B. das Geben von Ratschlägen, das Bereitstellen von Handlungs- und Problemlösungsstrategien oder das Fördern von Interessen. Alles in allem stellt der Vater ein wichtiges Rollenmodell innerhalb der kindlichen Entwicklung dar, deswegen werden beide Dimensionen mit einem Faktor von drei einbezogen.

Die direkte Auseinandersetzung mit dem Kind in einer Face-to-Face-Interaktion erfordert eine hohe Fokussierung auf das Kind. So beschäftigt sich der Vater innerhalb einer per- 
sönlichen Unterhaltung anders mit einem Kind als beim Computerspielen oder Fernsehen. Diese Form der direkten Auseinandersetzung ist für die kindliche Entwicklung von besonderer Bedeutung, daher wird sie hier dreifach gewichtet. Damit einher geht die Möglichkeit der Kommunikation. Grundsätzlich geben viele Aktivitäten die Möglichkeit zur verbalen Auseinandersetzung, aber bei der simultanen Beschäftigung mit anderen Tätigkeiten sinkt die Intensität der Kommunikation. Die Möglichkeit zur Unterhaltung ist für die Vater-Kind-Beziehung von großer Wichtigkeit. Da die Inhalte der Gespräche für die Qualität der Kommunikation eine wichtige Bedeutung haben, hier aber nicht erfasst wurden, wird hier nur der Faktor zwei eingesetzt. ${ }^{125}$

In den vorangegangenen Ausführungen wurde bereits auf die Notwendigkeit der Integration der Aktivität in den kindlichen Alltag hingewiesen. ${ }^{126}$ Durch die räumliche Trennung wird der Vater v.a. aus alltäglichen Aktivitäten ausgeschlossen. Handlungen, wie die Versorgung oder Verpflegung des Kindes, können aufgrund der neuen Familien- und Wohnsituation nur bedingt durch den Vater ausgeführt werden, während spannende, für das Kind außergewöhnliche Unternehmungen zunehmend in den Mittelpunkt der Interaktion rücken. ${ }^{127}$ Diese besonderen Unternehmungen, wie z.B. das Verbringen gemeinsamer Urlaube, bewirkt eine andere Form der Kommunikation und Interaktion zwischen Vater und Kind. Diese Dimension wird mit dem Faktor zwei gewichtet.

Jeder Aktivität wird entlang der dargestellten Dimensionen ein Wert zugeordnet, der die Intensität dieser Ebene innerhalb der Aktivität widerspiegelt. ${ }^{128}$ Die Bewertung erfolgt relativ zu den anderen Aktivitäten. So ist gemeinsames Fernschauen weniger kommunikativ als z.B. Spazieren gehen. Gleichzeitig ist hier nicht die subjektive Einschätzung der Höhe des finanziellen oder zeitlichen Aufwands aus der Perspektive des Vaters abgebildet, sondern vielmehr eine generelle Bewertung, die weitestgehend unabhängig von der Verfügbarkeit der jeweiligen Ressourcen ist. Die Werte, die den einzelnen Aktivitäten zugeordnet werden, werden entsprechend der einzelnen Gewichte aufsummiert. Damit ergibt sich für jede Aktivität ein Zahlenwert, der die Intensität des väterlichen Kümmerns ausdrückt.

\footnotetext{
${ }^{125}$ In diesem Zusammenhang ist darauf zu verweisen, dass die Intensität der Kommunikation stark altersabhängig ist. Dieser Aspekt gilt auch für die anderen Faktoren und Dimensionen, wird hier aber zur Vereinfachung außer Acht gelassen.

${ }^{126}$ Siehe Kap. 2.2 Nachtrennungsfamilien: das Phänomen abwesender Väter.

${ }^{127}$ Amendt (2004: 174) spricht in diesem Zusammenhang vom Vorurteil gegen die sog. sugar-daddys.

${ }^{128}$ Bewertet werden die einzelnen Dimensionen nach einer Skala von 0=“none“, 1=“low", 2=“moderat" bis 3="high".
} 
Die Aufsummierung der Werte für die jeweilige Aktivität ergibt eine Rangfolge, die der mit den Aktivitäten verbundenen Intensität väterlichen Engagements entspricht. Aus dieser Rangordnung wird die Gewichtung der einzelnen Variablen innerhalb der CareVariable abgeleitet. Am Ende kann jedem Befragten anhand seiner Antworten ein Wert zugeordnet werden, der das Niveau seines väterlichen Kümmerns ausdrückt. Insgesamt wurden innerhalb des deutschen Datensatzes die 12 Aktivitäten, die am häufigsten genannt wurden, und gemeinsam verbrachte Ferien bzw. Urlaube nach diesem System bewertet.

Daraus resultiert eine Rangfolge der Aktivitäten, die den Gewichten in der Konstruktion des Care-Indexes entspricht. Das bedeutet: Je nachdem, welche Aktivität der Vater angibt, erhält er dafür eine bestimmte Punktzahl. Gibt ein deutscher Vater z.B. an, dass er mit seinem Kind gemeinsame Urlaube verbringt oder normalerweise mit ihm Hausaufgaben für die Schule erledigt, wird dies in seinem Punktescore jeweils mit dem Faktor sechs berücksichtigt. „Unterhaltungen“, „gemeinsames Spielen“ und „gemeinsame Mahlzeiten“ werden fünffach gewichtet. Mit dem Faktor vier gehen die Aktivitäten ,gemeinsame Hobbys“, „Sport“ und „Spaziergänge bzw. Ausflüge“ ein. Die Variablen „gemeinsames Fernsehen“, „PC spielen/arbeiten“, „Freunde/Verwandte besuchen“, „Musik hören“ und „Kinobesuche“ werden nach dem erläuterten Punktevergabesystem mit der geringsten Intensität väterlichen Engagements bewertet, so dass sie jeweils nur einfach im Care-Index berücksichtigt werden. ${ }^{129}$

Aufgrund der Möglichkeit, mehrere Antworten anzukreuzen, gilt: Je mehr Aktivitäten der Vater angibt, desto höher wird seine Punktzahl insgesamt. Das bedeutet inhaltlich, dass ein differenziertes väterliches Sorgehandeln - die Nennung vieler Aktivitäten - qualitativ höher eingeschätzt wird als das Handeln eines Vaters, der mit seinem Kind nur wenige der genannten Tätigkeiten ausübt. Dies ist sicherlich ein analytisch vereinfachtes Verständnis väterlichen Sorgehandelns. Ein wesentliches Entscheidungskriterium über die Qualität des väterlichen Kümmerns besteht im Wohlbefinden des Kindes. ${ }^{130}$

${ }^{129}$ Das genaue Punktevergabesystem und Rangfolgen der Aktivitäten der drei Datensätze ist den Tabellen A.1 bis A.3 im Anhang zu entnehmen. Auf die detaillierten Argumente hinter den einzelnen Werten wird an dieser Stelle aus Platzgründen verzichtet. Statistische Berechnungen haben gezeigt, dass sich der Care-Index sehr robust gegenüber anderen Gewichtungen verhält. So führt ein Index, der die Aktivitäten ungewichtet berücksichtigt, zu ähnlichen Ergebnissen wie der hier verwendete Care-Index. Inhaltlich scheint die Gewichtung der einzelnen Komponenten gut begründet.

${ }^{130}$ Insgesamt ist darauf zu verweisen, dass die Konstruktion des Care-Indexes eine sehr vereinfachte Erfassung väterlichen Sorgehandelns darstellt. So werden beispielsweise emotionale Aspekte vollends vernachlässigt. Eine ausführliche kritische Auseinandersetzung mit der Operationalisierung findet sich im Kap. 11.2 Charakteristika der Modellvariablen. 
Neben den Aktivitäten während der väterlichen Besuche wird innerhalb des deutschen Care-Indexes ferner die väterliche Entscheidungspartizipation berücksichtigt. Zur Erfassung wurden die Väter gefragt, ob wichtige Entscheidungen, die das Kind betreffen, normalerweise von den Eltern gemeinsam getroffen oder diese eher von der Mutter allein gefällt werden. Die mehrheitlich gemeinsam getroffenen Entscheidungen werden im Index mit dem Faktor vier gewichtet. Auch wenn die aktive Teilnahme des Vaters an Entscheidungsprozessen im Wesentlichen ein kognitives Auseinandersetzen mit entscheidenden, das Leben seines Kindes betreffenden Aspekte erfordert und dieses nicht primär der hier fokussierten Handlungsebene zuzurechnen ist, stellt die Partizipation des Vaters an wesentlichen Entscheidungen einen wichtigen Teil väterlichen Kümmerns dar.

Antwortet ein Vater auf die Frage nach den Aktivitäten oder der Entscheidungspartizipation nicht, verweigert er die Angabe oder wird fälschlicherweise kein Wert für den Fall eingetragen, bekommt der Vater innerhalb seines Care-Scores den Wert 0 zugeordnet. Damit verringert sich sein Wert, der sein väterliches Kümmern ausdrückt. Väter mit unvollständigen Angaben fallen damit nicht gänzlich aus der Analyse heraus. Diese Vorgehensweise basiert auf der Annahme, dass ein Vater, der keine Angabe macht, die vorgegebene Aktivität auch nicht ausübt. Dennoch kann damit das väterliche Sorgehandeln in seiner Komplexität unterschätzt werden.

Neben den Aktivitäten und der Entscheidungspartizipation, die die qualitative Ebene väterlichen Sorgehandelns widerspiegeln, werden bei der Care-Komponente die Häufigkeit der Besuche und damit die Quantität des väterlichen Handelns als Basis väterlichen Sorgehandelns berücksichtigt. Die Kontakthäufigkeit ist aufsteigend kodiert und wird aufgrund ihrer hohen Bedeutung für die Vater-Kind-Beziehung fünffach gewichtet. ${ }^{131}$ Dementsprechend wird dem Vater mit einer höheren Kontakthäufigkeit äquivalent ein höherer Wert im Care-Index angerechnet. Besteht zwischen dem Vater und seinem Kind kein Kontakt, wird dies mit dem Gewicht Null berücksichtigt. Diese Väter weisen insgesamt eine Punktzahl von Null auf, da aufgrund des Kontaktabbruchs auch gemeinsame Aktivitäten ausgeschlossen sind.

Nach diesem System wird innerhalb der Forsa-Studie für insgesamt 872 westdeutsche Väter eine Punktzahl errechnet, die auf einer Skala von 1=“gar kein Kümmern“ bis 6=“sehr intensives Kümmern“ eingeordnet wird und die jeweilige Intensität väterlichen

${ }^{131}$ Väter, die hier fehlende Werte im Datensatz aufweisen, werden anders als bei den Aktivitäten und der Entscheidungspartizipation gänzlich in der Analyse vernachlässigt. Die Interpretation des Care-Indexes ohne die verfügbaren Informationen über die Kontakthäufigkeit macht inhaltlich keinen Sinn. 
Engagements des Befragten innerhalb der Nachtrennungsfamilie angibt. Ihre Verteilung wird in Tab. 8.3 am Ende dieses Kapitels wiedergegeben. ${ }^{132}$

Ähnlich wie im deutschen Datensatz werden auch im britischen verschiedene CareAspekte erhoben. Anders als in der deutschen Studie, in der nach der Kontakthäufigkeit gefragt wird, und auch entgegen der norwegischen Untersuchung, die nur den letzten Kontakt erfasst, erhebt die Bradshaw-Studie beides, den letzten Kontakt und die Kontakthäufigkeit. Auf die Frage nach dem letzten face-to-face Kontakt, den die Väter mit dem Kind hatten, können sie mit „nie gesehen“; ,,vor über 3 Jahren“; ,,vor 1 bis 2 Jahren“; „,vor 6 bis 12 Monaten“; „im letzten halben Jahr“; „,im letzten Monat“; „in den letzten 2 Wochen“; „letzte Woche“; „diese Woche“ oder „heute“ antworten. ${ }^{133}$ Hier nimmt mit steigender Kodierung der zeitliche Abstand zum letzten Besuch ab. Väter, die auf die Frage angeben, ihr Kind länger als 12 Monate nicht gesehen zu haben, werden in den folgenden Variablen gemäß der Filterführung nicht mehr erfasst. Sie machen innerhalb der kategorisierten Care-Variablen die Ausprägung „kein Kontakt“ aus. Im britischen Care-Index zählt die dargestellte Variable des letzten Kontakts als Basis der Care-Werte der einzelnen Väter und geht, wie im deutschen Fall, fünffach gewichtet in den Index ein.

\footnotetext{
${ }^{132}$ Kontrollberechnungen haben erhebliche Unterschiede in den Ergebnissen von ost- und westdeutschen Nachtrennungsvätern ergeben. Die Variable, die die mit Blick auf familienorientierte Normen immer noch vorhandene Teilung Deutschlands in Ost und West abbildet, erwies sich zur Erklärung väterlichen Sorgehandelns sowohl in Form von Care als auch von Cash als signifikant. Im Folgenden finden in der Darstellung der Ergebnisse lediglich die westdeutschen Väter Berücksichtigung (zur theoretischen Begründung der Vernachlässigung ostdeutscher Väter siehe ausführlich Kap. 1 Fragestellung und methodischer Aufbau der Arbeit).

${ }^{133}$ Kritisch ist hier anzumerken, dass der letzte Kontakt nur bedingt Auskunft über die generelle Häufigkeit des Kontaktes gibt. Väter, die nach einer längeren Kontaktpause zufällig ihr Kind in der letzten Woche gesehen haben, werden mit Vätern, die wöchentlich Kontakt zu ihren Kindern halten, in eine Kategorie gruppiert. Innerhalb der generellen Kontakthäufigkeit wäre dies nicht der Fall. Es kann vermutet werden, dass sich diese Sonderfälle auf univariater Ebene ausgleichen. Für die Zusammenhangsanalyse auf individueller Ebene können sie jedoch zu Verzerrungen führen. Aufgrund mangelnder Alternativen - insbesondere in der norwegischen Studie - wird dennoch die Variable des letzten Kontakts in den Care-Index als Basis integriert. Es wird die Annahme getroffen, dass sie Ähnliches, wenn auch nicht Gleiches, misst wie die generelle Kontakthäufigkeit. Ein Vorteil gegenüber der Frage nach der generellen Kontakthäufigkeit könnte hier in den unterschiedlichen Erinnerungseffekten vermutet werden. Bei der Frage nach dem letzten Kontakt ist die Erinnerungsleistung eventuell geringer, als wenn der Vater abschätzen muss, wie häufig er im Durchschnitt sein Kind sieht. Dazu muss er zunächst den Zeitraum bestimmen und diesen dann mit der Häufigkeit des Kontakts in Relation setzen. Die subjektive Färbung der Antwort könnte hier u.U. stärker ausgeprägt sein als bei der Frage nach dem letzten Kontakt, der je nach zeitlicher Distanz relativ leicht erinnert werden kann. In der britischen Studie finden sich in der Variable des letzten Kontakts weniger Ausfälle als in der Variablen zur Kontakthäufigkeit.
} 
Tab. 8.2: Komponenten der nationalen Care-Indizes.

\begin{tabular}{|c|c|c|}
\hline Forsa & Bradshaw & NOVA \\
\hline Kontakthäufigkeit & - & - \\
\hline - & Letzter Kontakt & Letzter Kontakt \\
\hline- & $\begin{array}{l}\text { Schriftlicher/telefonischer } \\
\text { Kontakt }\end{array}$ & Telefonischer Kontakt \\
\hline- & - & Schriftlicher Kontakt \\
\hline Gemeinsamer Urlaub & Ferien & - \\
\hline $\begin{array}{ll}\text { Lernen } & \text { für } \\
\text { Schule/Hausaufgaben } & \end{array}$ & Hausaufgabenhilfe & Hausaufgaben machen \\
\hline Spielen & Spielen & Spielen \\
\hline Gemeinsame Mahlzeiten & - & $\begin{array}{l}\text { Gemeinsame } \\
\text { Mahlzeiten/Hausarbeit }\end{array}$ \\
\hline Ausflüge & Ausflüge & Ausflüge \\
\hline Fernsehen & Fernsehen & Fernsehen \\
\hline Kinogänge & Kinogänge & Kinogänge \\
\hline PC spielen/arbeiten & - & PC spielen/ Internet \\
\hline Sport treiben & Sport treiben & Sport \\
\hline $\begin{array}{l}\text { Freunde/Verwandte } \\
\text { besuchen }\end{array}$ & $\begin{array}{l}\text { Freunde/Verwandte } \\
\text { besuchen }\end{array}$ & - \\
\hline Unterhaltungen & - & - \\
\hline Gemeinsames Hobby & - & - \\
\hline Unterhalten & - & - \\
\hline Musik hören & - & - \\
\hline- & - & Vorlesen \\
\hline- & Einkaufen & Einkaufen \\
\hline- & Besondere Freude gemacht & - \\
\hline- & Freunde zu Besuch & - \\
\hline- & Partizipation Schule & - \\
\hline- & $\begin{array}{l}\text { Partizipation Abholen von } \\
\text { Schule }\end{array}$ & - \\
\hline- & $\begin{array}{l}\text { Partizipation } \\
\text { Chauffeurdienste }\end{array}$ & - \\
\hline- & Partizipation Baby Sitting & - \\
\hline Entscheidungspartizipation & Entscheidungspartizipation & - \\
\hline
\end{tabular}

Quelle: eigene Darstellung.

Des Weiteren werden im Index neben dem letzten Kontakt die Variablen berücksichtigt, die die Häufigkeit des indirekten telefonischen oder schriftlichen Kontakts, die Aktivitäten, die Vater und Kind gemeinsam unternehmen, gemeinsam verbrachte Ferien sowie die Partizipation des Vaters an elterlichen Aufgaben und Entscheidungen erfassen. Die einzelnen Komponenten werden in unterschiedlichem Maße innerhalb des Indexes nach dem Schema des deutschen Index gewichtet. 
Wie im norwegischen Datensatz werden auch die britischen Väter gefragt, wie häufig sie zwischen den Besuchen postalischen oder telefonischen Kontakt mit ihren Kindern haben. ${ }^{134}$ Die Befragten können dabei mit „nie“, „seltener“ als jährlich, „ein bis zweimal im Jahr“, „mindestens einmal in 3 Monaten“, „mindestens einmal pro Monat“, „mindestens vierzehntägig“ oder „mindestens einmal pro Woche“ antworten. Hier wird mit steigender Kodierung von null bis sechs häufiger indirekter Kontakt gehalten und auf eine weitere Gewichtung verzichtet.

Wie im deutschen Fall werden die britischen Väter gebeten anzugeben, was sie innerhalb der gemeinsam verbrachten Zeit mit den Kindern unternehmen. Dabei wird, wie in der norwegischen Studie, explizit auf den letzten Kontakt zwischen Vater und Kind Bezug genommen. Aktivitäten, die auch in den anderen Fragebögen vorkommen, werden gleiche Gewichtungen zugewiesen. Dazu zählen „Spielen“(Gewicht: 5), „Ausflüge/Aktivitäten draußen“ (Gewicht: 4), „Einkaufen“(Gewicht: 4), „Sport“ (Gewicht: 4), „Fern/ Video gesehen“ (Gewicht: 1), „Kino“ (Gewicht: 1) und „Verwandtenbesuche“(Gewicht: 1). Neu hinzugekommen ist die Aktivität „Spielfreunde zu Besuch“, die nach der Klassifizierung mit einem einfachen Gewicht versehen wird, sowie die „Treats“, die man grob mit „eine Freude machen/besondere Belohnung“ übersetzen kann. Diese Kategorie ist derart weit fassbar, dass sie aufgrund ihrer Unbestimmtheit zweifach in den Care-Index eingeht. ${ }^{135}$ Wie auch schon in der deutschen Studie gehen die gemeinsam verbrachten Ferien sechsfach in die Berechnungen ein. ${ }^{136}$

\footnotetext{
${ }^{134}$ Zum Erhalt einer ausreichenden Fallzahl wurden auch hier die Väter ohne Angaben nicht aus der Analyse herausgenommen, sondern vielmehr eine fehlende Antwort als „kein indirekter Kontakt“ interpretiert. Damit können mehr Väter in die Berechnungen integriert werden. Kritisch muss jedoch gesehen werden, dass dieses Vorgehen die Gefahr birgt, das väterliche Sorgehandeln zu unterschätzen, falls die Väter doch zwischen den Besuchen mit ihren Kindern telefonieren oder mailen, diese Angaben jedoch - aus welchen Gründen auch immer - verloren gegangen sind.

${ }^{135}$ Um dem Kind eine Freude machen zu können, muss man dessen Vorlieben und Interessen kennen. Ferner ist diese Aktivität gegebenenfalls mit einer gewissen Organisation im Vorfeld verbunden.

${ }^{136}$ Dabei werden in der britischen und norwegischen Studie nach den Aktivitäten des letzten Kontakts gefragt, während innerhalb des Datensatzes von Forsa die „üblicherweise“ unternommenen Aktivitäten erfasst werden. Ein Vater, der nach dem letzten Kontakt gefragt wird, wird je nach Dauer des Besuchs wahrscheinlich weniger unterschiedliche Aktivitäten angeben. Während ein Vater, der gebeten wird, die Aktivitäten zu nennen, die er „normalerweise“ mit seinem Kind unternimmt, an all die Unternehmungen denken wird, die häufiger vorkommen und eben nicht nur beim letzten Mal. Ferner können äußere Umstände, wie z.B. schlechtes Wetter bestimmte Aktivitäten wie Ausflüge oder das Spielen draußen verhindern, die der Vater unter normalen Umständen unternehmen hätte. Es kann vermutet werden, dass damit deutsche Väter mehr Aktivitäten nennen, als im norwegischen und britischen Fall. Tatsächlich nennen britische Väter ca. drei Aktivitäten, norwegische im Durchschnitt vier und deutsche Väter geben fünf verschiedene Aktivitäten an.
} 
Neben den genannten Freizeitaktivitäten wird im britischen Datensatz eine weitere wichtige Dimension väterlichen Sorgehandelns berücksichtigt: die Partizipation des Vaters an bestimmten Kind bezogenen Alltagsaktivitäten. Die Frage lautet: „Sind Sie in eine der folgenden Aktivitäten involviert?“. Mehrfachnennungen sind möglich. Die beiden nach der Klassifizierung wichtigsten Aktivitäten sind dabei die Partizipation an Schulaktivitäten sowie die Hilfe bei den Hausaufgaben. Letztere wurde auch in den anderen beiden Datensätzen als Aktivität genannt und klassifiziert. Beide werden im britischen Care-Score mit dem Gewicht sechs berücksichtigt, da sie in eine ähnliche Richtung interpretierbar sind. Die Bringdienste zur Schule, generelle Chauffeurdienste für das Kind sowie die Beteili-

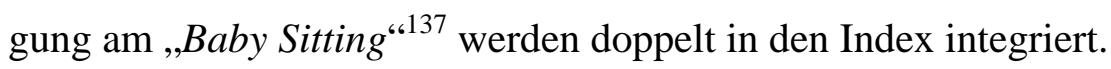

Ähnlich wie im deutschen Fall geben britische Väter an, inwieweit sie an Entscheidungen, die das Kind betreffen, beteiligt sind. Dabei wird zwischen verschiedenen Lebensbereichen, die die Entscheidungen umfassen, unterschieden. ${ }^{138}$ Je häufiger der Vater in den genannten Lebensbereichen Entscheidungen gemeinsam mit der Kindsmutter trifft, desto stärker wird dies innerhalb seines Care-Wertes berücksichtigt. ${ }^{139}$ Die väterliche Partizipation an Entscheidungen wird wie im deutschen Fall mit vier gewichtet.

Nach dem beschriebenen System kann innerhalb der Bradshaw-Studie insgesamt 576 Befragten ein Wert zugewiesen werden, der ihr väterliches Engagement mit Blick auf Care angibt. $^{140}$

Innerhalb der norwegischen Untersuchung wird wie im britischen Datensatz nach der letzten Begegnung zwischen Vater und Kind gefragt. Als Antwortkategorien werden „nie gesehen“, „länger als 1 Jahr“, „zwischen 6 und 12 Monaten“, „zwischen 3 und 6 Monaten“, „zwischen 1 und 3 Monaten“, „weniger als 1 Monat“, „weniger als ein Monat, aber länger als 1 Woche“ sowie „weniger als eine Woche“ aufsteigend kodiert. Mit zunehmen-

\footnotetext{
137 Ohne dass es innerhalb des Datensatzes näher spezifiziert wurde, wird darunter die Übernahme von Betreuungsphasen v.a. am Abend verstanden, die weniger die direkte Auseinandersetzung mit dem Kind erfordern, sondern vielmehr das zu Bett bringen, Abendrituale sowie die väterliche Erreichbarkeit im Notfall.

${ }^{138}$ Im Einzelnen zählen dazu Entscheidungen zur Schulwahl, der Ferienplanung, der Gesundheitsversorgung und einem eventuellen Umzug. Aufgrund der geringen Antwortzahl und der Spezifität der Entscheidung, sie setzt voraus, dass die Mutter umgezogen ist oder dies wollte, wird die letzte Entscheidungspartizipation nicht berücksichtigt.

139 Dabei wurden die gegebenen positiven Antworten auf die Fragen durchgezählt und daraus eine neue Variable konstruiert, die angibt, in wie vielen Bereichen der Vater Entscheidungsbefugnis besitzt. Je mehr Bereiche dies umfasst, desto höher ist die Kodierung.

${ }^{140}$ Mit der Kodierung: 1="kein Kümmern“ (0 Punkte), 2="sehr geringe Intensität“ (unter 33 Punkten), 3=“geringe Intensität“ (34 bis 52 Punkte), 4=“moderate Intensität“ (53 bis 71 Punkte), 5=“intensives Kümmern“ (72 bis 90 Punkte) und 6=“sehr intensives Kümmern“ (über 91 Punkte).
} 
der Kodierung sinkt damit die zeitliche Distanz zum letzten Kontakt zwischen Vater und Kind. Dementsprechend wird dem Vater mit einem kürzeren zeitlichen Abstand zum letzten Kontakt äquivalent ein höherer Wert im Care-Index angerechnet. Zur Vergleichbarkeit insbesondere mit dem britischen Fall werden auch hier Väter, die länger als ein Jahr keinen Kontakt zu ihrem Kind gepflegt haben, zusammen mit der Kategorie „nie gesehen“ als „kein Kontakt“ in der untersten Antwortausprägung des Care-Indexes zusammengefasst. Der letzte Kontakt gilt als Basis des gesamten Care-Indexes und geht fünffach in die Berechnung ein.

Wie im deutschen und britischen Fall werden auch hier zur Konstruktion des CareIndexes zunächst die verschiedenen Dimensionen väterlichen Kümmerns differenziert und gewichtet. Diese Gewichtung entspricht denen im deutschen bzw. britischen Care-Index. So werden innerhalb des norwegischen Datensatzes die gemeinsam unternommenen Aktivitäten mit Hilfe von Mehrfachnennungen erfasst. $\mathrm{Zu}$ den aufgezählten Aktivitäten, die auch im deutschen Datensatz zu finden sind, zählen „Hausaufgaben machen“ (Gewicht: 6), „Spielen“ (Gewicht: 5), „gemeinsame Ausflüge“ (Gewicht: 4), „Sport“ (Gewicht: 1), „Fernsehen“ (Gewicht: 1), „PC spielen/Internet“ (Gewicht: 1) und „Kino“ (Gewicht: 1). Diese werden mit den gleichen Gewichten versehen, wie im deutschen Care-Index. Darüber hinaus werden die Aktivitäten „Einkaufen“ (das nach dem oben beschriebenen Schema die Gewichtung 4 erhält), „Vorlesen“ (gewichtet mit 5) sowie „gemeinsame Mahlzeit/ sonstige Hausarbeit“ (mit 4 gewichtet) genannt und in den Care-Index integriert.

Des Weiteren wird im norwegischen Datensatz mittels einer Itembatterie die Art des Kontaktes zwischen Vater und Kind erhoben. Jeweils eine Variable erhebt dabei die Häufigkeit des telefonischen und schriftlichen Kontakts, wie er auch schon bei den britischen Vätern Berücksichtigung findet. ${ }^{141}$ Die beiden Variablen zur Erfassung der Art des Kontakts gehen ohne Gewichtung in den Index ein. Eine aufsteigende Kodierung sorgt dafür, dass ein Vater, der höhere Häufigkeiten bei den einzelnen Kontaktformen angibt, auch eine höhere Care-Punktzahl aufweist.

\footnotetext{
${ }^{141}$ Die Grundfrage lautet „Welche Art von Kontakt haben Sie mit dem Kind?“ mit den Anhängen „Ich spreche mit dem Kind am Telefon.“ und „Ich schreibe dem Kind Briefe oder E-Mails.“ und der Kodierung $0=$ ="nie“; 1=“selten“; 2=“ab und zu“; 3=“oft.
} 
Nach dem beschriebenen System wird innerhalb der NOVA-Studie für insgesamt 565 Fälle eine Punktzahl errechnet. ${ }^{142}$

Insgesamt wird das väterliche Sorgehandeln wie beschrieben in den einzelnen Studien mit unterschiedlichen Komponenten in einem Care-Index operationalisiert. Dabei werden nicht alle erhobenen Aspekte, wie sie in Tabelle 8.1 abgebildet sind, berücksichtigt, sondern versucht eine vergleichbare Variable für jeden Datensatz zu konstruieren. Tabelle 8.2 zeigt zusammenfassend die einzelnen Aspekte, die in den nationalen Indizes berücksichtigt werden im Überblick. ${ }^{143}$

Trotz der unterschiedlichen Operationalisierung wird innerhalb der Analyse davon ausgegangen, dass die Konstrukte das Gleiche oder theoretisch zumindest sehr ähnliche Phänomene messen. Tabelle 8.3 bzw. Abbildung 8.1 beinhalten die Häufigkeitsverteilung des jeweiligen Care-Indexes nach Datensatz.

Tab. 8.3: Häufigkeitsverteilungen der nationalen Care-Indizes.

\begin{tabular}{|l|r|r|r|r|r|r|}
\hline & \multicolumn{2}{|c|}{ Forsa } & \multicolumn{2}{c|}{ Bradshaw } & \multicolumn{2}{c|}{ NOVA } \\
\cline { 2 - 7 } & $\mathrm{N}$ & in $\%$ & $\mathrm{~N}$ & in $\%$ & $\mathrm{~N}$ & \multicolumn{1}{c|}{ in $\%$} \\
\hline kein/kaum Kontakt & 93 & $10,7 \%$ & 84 & $14,58 \%$ & 44 & $7,8 \%$ \\
\hline sehr wenig intensiv & 19 & $2,2 \%$ & 46 & $7,99 \%$ & 18 & $3,2 \%$ \\
\hline wenig intensiv & 83 & $9,5 \%$ & 132 & $22,92 \%$ & 73 & $12,9 \%$ \\
\hline moderat & 242 & $27,8 \%$ & 162 & $28,13 \%$ & 197 & $34,9 \%$ \\
\hline intensiv & 280 & $32,1 \%$ & 104 & $18,06 \%$ & 185 & $32,7 \%$ \\
\hline sehr intensiv & 155 & $17,8 \%$ & 48 & $8,33 \%$ & 48 & $8,5 \%$ \\
\hline Total & 872 & $100,0 \%$ & 576 & $100,0 \%$ & 565 & $100,0 \%$ \\
\hline
\end{tabular}

Quelle: eigene Berechnung.

142 Mit der Kodierung: 1=“kein Kümmern“ (0 Punkte), 2=“sehr geringe Intensität“" (unter 23 Punkte), 3=“geringe Intensität“ (23 bis 33 Punkte), 4=“moderate Intensität“ (34 bis 44 Punkte), 5=“intensives Kümmern“ (45 bis 55 Punkte) und 6="sehr intensives Kümmern“ (über 55 Punkte).

143 Aspekte, die nur in einzelnen Datensätzen vorkommen, werden vernachlässigt. Zum Erhalt eines Minimums an Vergleichbarkeit müssen die Aspekte mindestens in zwei Datensätzen ähnlich erfasst sein. 


\section{Abb. 8.1: Graphische Häufigkeitsverteilung der abhängigen Variable Care.}

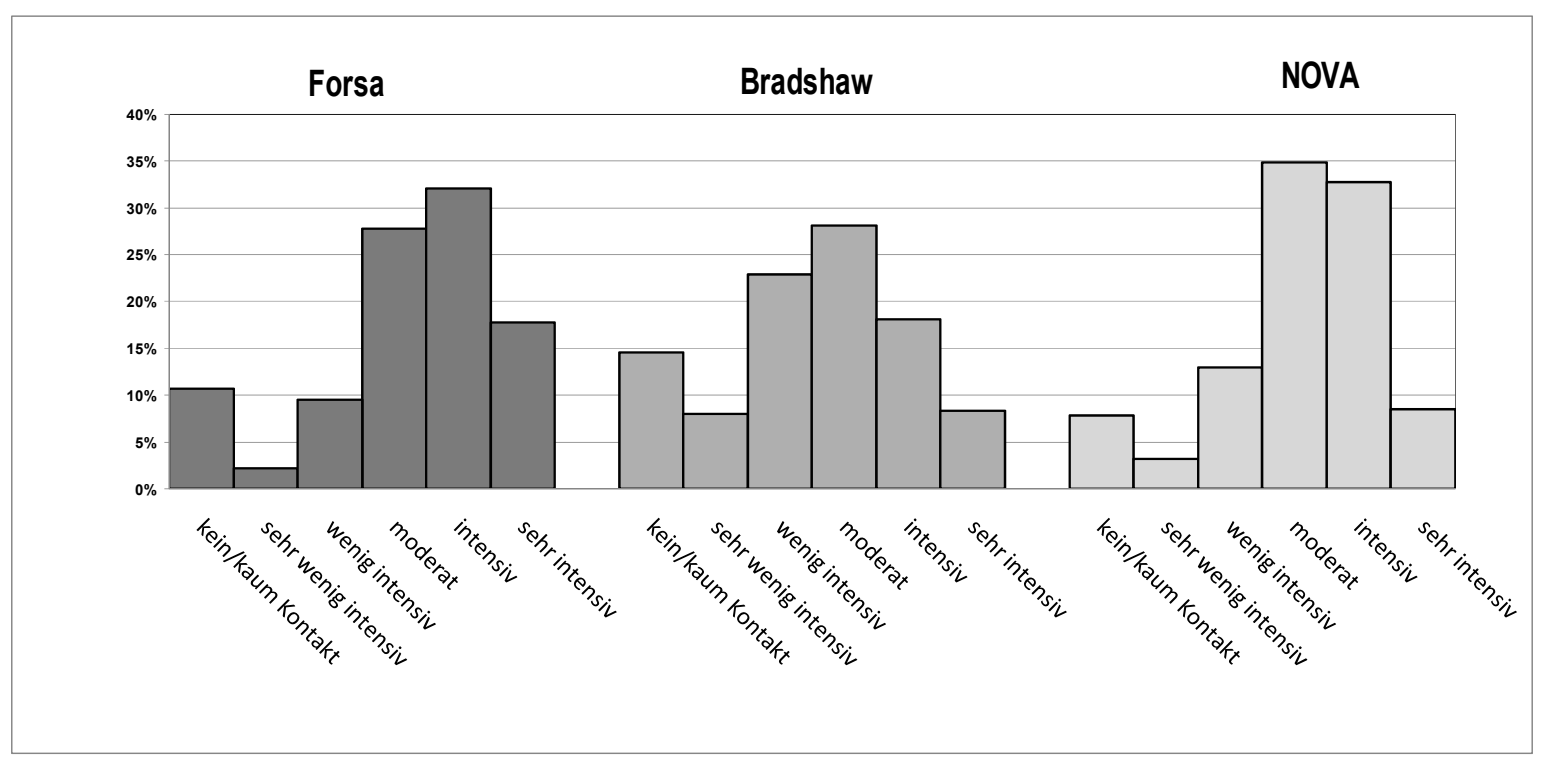

Quelle: eigene Darstellung.

Dennoch können die Ausprägungen der einzelnen Care-Variablen in ihrem Niveau zwischen den Ländern nicht verglichen werden. Aussagen über die deutlich stärkere Ausprägung väterlichen Sorgehandelns norwegischer Väter im Vergleich zu britischen oder deutschen Vätern sind damit nicht möglich. Lediglich Gruppenvergleiche innerhalb eines Landes im Vergleich zu Gruppenunterschieden in den anderen Ländern können inhaltlich sinnvoll interpretiert werden. Das bedeutet beispielsweise der Anteil der obersten Kategorie im Vergleich zu zweithöchsten Kategorie väterlichen Cares. Die Niveaus in den jeweiligen Ländern können jedoch - nicht zuletzt aufgrund der unterschiedlichen Anzahl berücksichtigter Dimensionen im Index - ganz unterschiedliche sein. Lediglich die Angabe zu keinem Kontakt gibt in allen drei Ländern weitgehend das Gleiche an. Dieser ist im britischen Fall am deutlichsten ausgeprägt.

\subsection{Die abhängige Variable: Cash}

Die theoretischen Ausführungen zum Cash-Konzept väterlichen Sorgehandelns haben bereits auf die unterschiedlichen Dimensionen hingewiesen. ${ }^{144}$ Die drei unabhängig voneinander durchgeführten Studien weisen jeweils sehr spezifische Operationalisierungen zur Erfassung der finanziellen Seite väterlichen Sorgehandelns auf. Tabelle 8.4 zeigt in

${ }^{144}$ Siehe dazu ausführlich Kap. 2.1 Väterliches Sorgehandeln in Form von Care und Cash. 
einem ersten Überblick, welche finanziellen Aspekte jeweils in den Datensätzen berücksichtigt werden.

Tab. 8.4:Cash-Dimensionen erfasst in den einzelnen Studien.

\begin{tabular}{|c|c|c|c|c|}
\hline Dimension & Variablen & 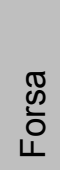 & $\begin{array}{l}3 \\
\frac{3}{0} \\
\frac{5}{0} \\
\frac{0}{0} \\
\frac{\pi}{0} \\
0\end{array}$ & $\stackrel{\S}{\text { o }}$ \\
\hline \multirow{2}{*}{ Zahlung generell } & Je Zahlung von Unterhalt (ja/nein) & - & + & - \\
\hline & Derzeit Zahlung von Unterhalt (ja/nein) & - & + & - \\
\hline Höhe & Höhe des Unterhalts & + & + & + \\
\hline \multirow{4}{*}{ Probleme } & Rechtzeitigkeit der Unterhaltszahlung & + & - & - \\
\hline & Regelmäßigkeit der Unterhaltszahlung & - & + & - \\
\hline & Rückzahlung von Unterhaltsvorschuss & + & (n.r.) & + \\
\hline & Höhe der Unterhaltsschulden & + & (n.r.) & + \\
\hline \multirow{3}{*}{$\begin{array}{l}\text { „Freiwillige“/ } \\
\text { andere Leistungen }\end{array}$} & $\begin{array}{l}\text { „Informale“ finanzielle Unterstützung } \\
\text { (Höhe) }\end{array}$ & - & + & - \\
\hline & $\begin{array}{l}\text { Verwendungszweck informeller } \\
\text { Unterstützung }\end{array}$ & - & + & - \\
\hline & Übernahme von Reisekosten & - & - & + \\
\hline
\end{tabular}

+ = erhoben; - = nicht erhoben; n.r. = nicht relevant

Quelle: eigene Darstellung.

Ein genereller Aspekt der Cash-Komponente besteht in der Tatsache, ob überhaupt Unterhalt gezahlt wird. Im britischen Datensatz wird zwischen den Fragen, ob jemals Unterhalt gezahlt wurde und ob dies derzeit der Fall ist, differenziert. Auf diese Weise können zahlende, nicht-zahlende und nie-zahlende Väter (current, past und never payers) ausgemacht werden. ${ }^{145}$ Die Angabe, dass ein Vater nie für seine getrennt lebenden Kinder finanziell aufgekommen ist, fehlt in den beiden anderen Datensätzen. Hier wird lediglich nach der Höhe des derzeitigen Unterhalts gefragt, die auch im britischen Fall erfasst wird. Die Höhe des Unterhalts wird in allen drei Datensätzen erhoben. ${ }^{146}$ Ein weiterer Aspekt finan-

145 Die Unterscheidung findet sich auch in der Filterführung des Fragebogens wieder, d.h. jeder Gruppe werden gesondert Fragen gestellt, die nur z.T. die gleichen Inhalte abdecken.

${ }^{146}$ Die Art der Fragestellung ist jeweils jedoch sehr unterschiedlich. So werden in Großbritannien die Höhe des Unterhalts für die Kinder der letzten Beziehung, der Zeitraum, den die Zahlung umfasst sowie die Zahl der Kinder, die diese Zahlung betrifft, getrennt voneinander erfasst. In Norwegen wird nach dem monatlichen Unterhalt für alle Kinder gefragt. Es bleibt unklar, wie viele Kinder dies umfasst. Im deutschen Da- 
zieller Unterstützung durch Nachtrennungsväter betrifft Zahlungsprobleme, die in Deutschland und Großbritannien mittels der Rechtzeitigkeit sowie Regelmäßigkeit der Zahlungen erfasst werden. In Norwegen wird, wie auch in Deutschland, nach der Rückzahlung von Unterhaltsschulden und deren Höhe gefragt. ${ }^{147}$ Der britische Datensatz erfasst über den formal geregelten Unterhalt hinaus Aspekte „freiwilliger“ finanzieller Unterstützung, den sog. informal support, während im norwegischen Survey die Väter zusätzlich angeben, wer die gegebenenfalls anfallenden Reisekosten übernimmt. ${ }^{148}$

Die unterschiedliche Art der Berechnung der Höhe des Unterhalts sowie die national verschiedene Berücksichtigung von Unterhaltsleistungen im Steuersystem machen die finanzielle Belastung der Väter in den nationalen Kontexten wenig vergleichbar. Vor allem die Höhe des Unterhalts ist durch institutionelle Vorgaben, in Deutschland durch Tabellen, in Großbritannien und Norwegen durch mehr oder weniger komplexe Formeln, festgelegt. Der Handlungsspielraum ist für die individuellen Väter gering; das Leisten von Unterhalt stellt nur bedingt eine freiwillige Entscheidung dar. Des Weiteren ist die Angabe, wie viel Unterhalt vereinbart wurde, wie sie z.B. in der deutschen Studie erfasst wird, nicht zwingend identisch mit der tatsächlich gezahlten Summe. Für Aussagen über die Motivation des väterlichen Sorgehandelns in Form von Cash ist die Höhe des formal festgelegten Unterhalts wenig erkenntnisreich. Es ist zu vermuten, dass die Väter sich bei der Begründung der Höhe ihrer Leistungen primär auf gesetzliche Regelungen und deren Umsetzung berufen. ${ }^{149}$ Interessanter im Sinne der Fragestellung ist vielmehr, warum Väter freiwillig

tensatz wird dagegen nach dem Unterhalt pro Monat und Kind sowie die Gesamtbelastung durch Unterhaltsverpflichtungen für alle Kinder mit und ohne Betreuungsunterhalt für die Mutter gefragt. Des Weiteren wird der Unterhalt in der jeweiligen Landeswährung (Euro, Norwegische Kronen und Britische Pfund) erhoben.

147 Diese Frage ist im britischen Kontext ohne Bedeutung, da hier kein staatliches Unterhaltsvorschusssystem existiert.

148 Der britische Datensatz ist die einzige der drei Studien, die finanzielle Unterstützung über den formal festgelegten Unterhalt hinaus konzeptionalisiert und erfasst. Alle Väter, unabhängig von ihrer Zahlungspraxis bezüglich des Unterhalts, werden nach so genannten informalen Unterstützungsleistungen für bestimmte Items gefragt. Dazu zählen im Einzelnen als Antwortvorgaben die finanzielle Unterstützung für Kinderkleidung, Schuhen, Haushaltsgütern, Ferien und Ausflüge, Kosten für Haus oder Wohnung, andere Rechnungen, Schulgebühren oder -ausflüge, Ausgaben für das Auto, Spielzeug oder Geschenke, Kreditkosten, Unterstützung beim Sparen sowie Taschengeld. Hier sind Mehrfachnennungen möglich. In einer zweiten Frage wurden die Väter um eine Einschätzung des Gesamtbetrages ihrer informellen Unterstützung gebeten. Im Folgenden kann diese Form der finanziellen Unterstützung in der Analyse nicht weiter berücksichtigt werden.

${ }^{149}$ Es kann argumentiert werden, dass der Anteil des Unterhalts am Einkommen des Vaters die relative Belastung des Vaters ausdrückt und als Indikator seiner Zahlungsmoral interpretiert werden kann. Dieses Vorgehen würde ferner die Vergleichbarkeit der jeweiligen nationalen Leistungsniveaus ermöglichen. Verschiedene Gründe sprechen jedoch gegen diese Form der Operationalisierung. Zunächst weist die Erfassung des individuellen Einkommens besonders im britischen Datensatz hohe Ausfälle auf. Gleichzeitig werden 
mehr geben, als es der Gesetzgeber von ihnen offiziell verlangt. Lediglich der britische Datensatz stellt jedoch diese Informationen zur Verfügung.

Des Weiteren ist das Leisten von Unterhaltszahlungen eingebettet in eine starke gesellschaftliche Norm väterlicher Verpflichtung. ${ }^{150}$ Insbesondere im Rahmen eines mehr oder weniger starken männlichen Ernährermodells wird von Vätern erwartet, sich um ihre Kinder zumindest finanziell nach der elterlichen Trennung zu kümmern, wenn diese im Haushalt der Mutter verbleiben. Die Zahlungsverpflichtung ist in allen drei Ländern mehr oder weniger stark institutionalisiert. ${ }^{151}$ Interessant ist es daher weniger, warum Väter in Nachtrennungsfamilien dieser Norm - gestärkt durch gesetzliche Durchsetzungsmechanismen wie Gerichte und Sanktionen - gerecht werden, sondern vielmehr aus welchen Gründen sie sich dazu entscheiden, dies gerade nicht zu tun. Was bewegt Nachtrennungsväter dazu, finanziell nicht für ihre Kinder aufzukommen? Zur Erklärung des finanziellen Sorgehandelns der Nachtrennungsväter wird daher im Folgenden der Indikator „Zahlungsprobleme“ herangezogen. Damit wird Cash innerhalb der folgenden Analyse als relativ eng gefasster Begriff verstanden: die Nicht-Erfüllung der Zahlungsverpflichtung aus Perspektive des befragten Nachtrennungsvater.

Der deutsche und norwegische Datensatz liefern, anders als der britische, keine Informationen über Väter, die nie und gar keinen Unterhalt gezahlt haben. Hier können lediglich unterschiedliche Abstufungen in den Zahlungsproblemen ausgemacht werden. Innerhalb der Forsa-Studie wird gefragt, ob es dem Befragten in der Vergangenheit schon einmal oder häufiger passiert sei, dass der Unterhalt nicht rechtzeitig gezahlt werden konnte. ${ }^{152}$ Die Antwortkategorien sind mit 1=“"nein, nie“, 2=“ja, einmal“ und 3=“ja, häufiger“ kodiert. Das bedeutet mit steigender Kodierung nehmen die Zahlungsprobleme zu. Die

die Angaben in allen drei Ländern für unterschiedliche Zeiträume und entweder als Netto- oder als Bruttobetrag erfasst. Dies schränkt die Vergleichbarkeit zwischen den Ländern weiter ein. Auch die jeweilige Erhebung der Höhe des Unterhalts weist in den nationalen Studien wesentliche Unterschiede auf. So wird z.B. in Norwegen nach den gesamten Unterhaltsverpflichtungen für alle Kinder und der Kindesmutter zusammen gefragt. In Großbritannien müsste der Unterhalt pro Kind mit der zusätzlichen Information zur Anzahl der externen Kinder berechnet werden. Ferner würden im britischen Fall bei dieser Form der Operationalisierung all diejenigen Väter aus der Analyse ausgeschlossen, die nie für ihre Kinder gezahlt haben.

${ }^{150}$ Siehe dazu ausführlich Kap. 4.3.2.2 Familiales Handeln als norm-orientiertes Handeln.

${ }^{151}$ Siehe dazu ausführlich Kap. 6 Nationale Kontexte der Nachtrennungsväter.

152 Darüber hinaus werden Zahlungsprobleme mit der Frage „Müssen oder mussten Sie schon einmal Unterhalt, der von der Unterhaltsvorschusskasse des Jugendamtes gezahlt wurde, zurück bezahlen?“ mit den Antwortvorgaben „ja, aktuell“, „ja, früher“ und „,nein“ erfasst. Diese Variable stellt einen Indikator für eine spezielle Form von Unterhaltsproblemen dar. Das Ausbleiben von Zahlungen führte zum Einschalten einer externen Institution. 
Häufigkeitsverteilung der deutschen Cash-Variablen in Tabelle 8.5 (weiter unten im Text) macht deutlich, dass die Kategorie für keine Zahlungsschwierigkeiten relativ stark besetzt ist. Demgegenüber finden sich innerhalb der mittleren und untersten Kategorie, die das einmalige bzw. häufigere Ausbleiben von Unterhaltszahlungen erfassen, nur wenige Fälle. ${ }^{153}$ Aufgrund der mangelnden Zellenbesetzung wird die Cash-Variable dichotomisiert, so dass sie angibt, ob der Vater jemals eine Zahlung verpasst hat oder nicht. ${ }^{154}$ Innerhalb des deutschen Datensatzes können bei der Cash-Variable insgesamt 900 (westdeutsche) Fälle berücksichtigt werden.

Britische Väter werden zunächst gefragt, ob sie jemals Kindesunterhalt an frühere Partnerinnen gezahlt haben. ${ }^{155}$ Danach werden sämtliche Angaben für derzeit zahlende (current payers oder Zahler), für derzeit nicht zahlende (past payers oder Nicht-Zahler) sowie für nie zahlende Väter (never payers oder Nie-Zahler) unterschieden. Angaben zur Zahlungspraxis werden dabei - entsprechend - nur für Väter erhoben, die jemals Unterhalt gezahlt haben, jedoch getrennt nach Vätern, die zum Zeitpunkt der Befragung immer noch ihren finanziellen Verpflichtungen nachkommen und Vätern, die die Unterhaltszahlungen eingestellt haben. Väter, die angeben jemals Unterhaltszahlungen geleistet zu haben (heute und früher Zahlende), werden gefragt, wie regelmäßig sie ihre finanziellen Verpflichtungen erfüllt haben. Als Antwortvorgaben stehen „immer gezahlt“, „meistens gezahlt, ab und zu verpasst“ und „unregelmäßige Zahlungen, häufig verpasst“ zur Verfügung. Zusammen mit der Angabe, ob die Väter jemals und derzeit Unterhalt zahlen, kann eine Variable konstruiert werden, die das Ausmaß der Zahlungsprobleme in fünf Abstufungen angibt. ${ }^{156}$ Mit der Kodierung steigen auch die Unterhaltsprobleme an. Zur besseren Vergleichbarkeit der Ergebnisse in den einzelnen Datensätzen werden auch im britischen die

\footnotetext{
153 Die nur schwach ausgeprägte Varianz der Antworten könnte mit dem Phänomen sozialer Erwünschtheit zusammenhängen (siehe dazu ausführlich Kap. 11.2 Charakteristika der Modellvariablen sowie Kap. 11.3 Die Zusammensetzung der Stichproben).

${ }^{154}$ Mit der Kodierung: 0 = „keine Probleme“, 1 = „Zahlungsprobleme“,

${ }^{155}$ Die Angaben zur Unterhaltspraxis werden nur für die letzte Beziehung erfasst, die gescheitert ist und aus der Kinder hervorgegangen sind.

${ }^{156}$ Mit „1“ werden alle Fälle kodiert, die zu den aktuell zahlenden Vätern zählen und angeben, dass sie immer ihrer finanziellen Pflicht nachgekommen sind. Die „2“ wird den Vätern zugewiesen, die zu den derzeit zahlenden Vätern gehören, die Zahlungsprobleme aufweisen, d.h. meistens oder unregelmäßig zahlen. Kategorie „3“ umfasst die früher Zahlenden, die damals immer ihren Zahlungsverpflichtungen nachgekommen sind. Hier bestehen die Zahlungsprobleme primär in der Einstellung der Zahlung. Die Zahlungsprobleme bei den derzeit Unterhaltszahlenden bestehen demgegenüber rein aus der Unregelmäßigkeit. Dies wird als weniger schwerwiegend eingeschätzt, als die generelle Einstellung von Unterhaltszahlungen zum Zeitpunkt der Befragung. Früher zahlende Väter, deren Zahlungsmoral durch unregelmäßige Zahlungen geprägt ist, werden mit „4“ kodiert; während die Väter, die angeben, nie Unterhalt gezahlt zu haben, mit einer „5“ kodiert werden.
} 
Zahlungsprobleme dichotomisiert. Väter ohne Zahlungsprobleme zahlen derzeit und immer. Alle anderen Väter werden unabhängig von der Schwere dieser Probleme als Väter mit Zahlungsproblemen ausgewiesen.

Tab. 8.5: Kategorien und Häufigkeiten der Cash-Variablen.

\begin{tabular}{|c|c|c|c|c|c|c|c|c|}
\hline \multicolumn{3}{|c|}{ Forsa } & \multicolumn{3}{|c|}{ Bradshaw } & \multicolumn{3}{|c|}{ NOVA } \\
\hline Cash1_G & $\mathrm{n}$ & in $\%$ & Cash1_B & $\mathrm{n}$ & in $\%$ & Cash1_N & $\mathrm{n}$ & in $\%$ \\
\hline nein, nie & 738 & $82,0 \%$ & derzeit, immer gez. & 317 & $56,6 \%$ & keine Schulden & 393 & $72,4 \%$ \\
\hline ja, einmal & 51 & $5,7 \%$ & derzeit, unregelm. gez. & 33 & $5,9 \%$ & ja, unter $€ 1.232$ & 74 & $13,6 \%$ \\
\hline ja, häufiger & 111 & $12,3 \%$ & früher, immer gez. & 80 & $14,3 \%$ & ja, zw. €1.233-6.160 & 49 & $9,0 \%$ \\
\hline-- & -- & - & früher, unregelm. gez. & 24 & $4,3 \%$ & ja, mehr als $€ 6.160$ & 27 & $5,0 \%$ \\
\hline-- & -- & - & nie gezahlt & 106 & $18,9 \%$ & -- & - & -- \\
\hline Total & 900 & $100 \%$ & Total & 560 & $100 \%$ & Total & 543 & $100 \%$ \\
\hline Cash2_G & $n$ & in $\%$ & Cash2_N & $\mathrm{n}$ & in $\%$ & Cash2_N & $\mathrm{n}$ & in $\%$ \\
\hline keine Probleme & 738 & $82,0 \%$ & keine Probleme & 317 & $55,5 \%$ & keine UH-Schulden & 393 & $72,4 \%$ \\
\hline Zahlungsprobl. & 162 & $18,0 \%$ & Zahlungsprobleme & 254 & $44,5 \%$ & UH-Schulden & 150 & $27,6 \%$ \\
\hline Total & 900 & $100 \%$ & Total & 571 & $100 \%$ & Total & 543 & $100 \%$ \\
\hline
\end{tabular}

n=absolute Zahl; Zahlungsprobl. = Zahlungsprobleme; gez. = gezahlt; unregelm. = unregelmäßig; zw. = zwischen; UH = Unterhalt

Quelle: eigene Berechnung.

Innerhalb der NOVA-Studie werden Zahlungsprobleme mit Hilfe der Frage erfasst, ob derzeit Unterhaltsschulden bestehen. Als Antworten stehen „,nein“ (=1), „ja, unter 1.232 Euro“, „,ja, zwischen 1.232 und 6.161 Euro“ (=2) und „,ja, über 6.161 Euro“ (=3) zur Verfügung. ${ }^{157}$ Auch hier steigen mit der Kodierung die Zahlungsprobleme an, wenn man annimmt, dass höhere Schulden mit stärkeren Problemen der Unterhaltsleistung gleichzusetzen sind.

Die Höhe der Unterhaltsschulden kann zwar als ein Indikator für das Ausmaß väterlicher Zahlungsprobleme verstanden werden. Es sind jedoch weitere Informationen notwendig, um die hier vorgegebenen Antworten sinnvoll interpretieren zu können. So bleibt ungewiss, über welchen Zeitraum die Schulden entstanden sind. Auch ist unklar, für wie viele Kinder die Verbindlichkeiten entstanden sind. Damit würde ein Vater, der für fünf Kinder dementsprechend höhere Forderungen zu befriedigen hat und dem beim einmaligen Ausbleiben höhere Schulden entstehen, in die gleiche Antwortkategorie sortiert werden müssen, wie ein Vater, dem für ein einzelnes Kind Unterhaltsschulden entstehen, wenn er über

157 Die etwas merkwürdigen Werte sind durch die Umrechnung der ursprünglichen Antwortvorgaben in Euro entstanden. Sie lauteten „,nein“, „ja, mehr als 10.000 NOK“, „,ja, zwischen 10.000 und 50.000 NOK“ und „ja, mehr als 50.000 Kronen“. ${ }^{157}$ Laut Umrechnungskurs vom 07.02.2007 unter www.bdb.de/html/reisekasse/waehrungsrechner.asp entspricht 1 Norwegische Krone 0,12321 Euro. Im Zeitpunkt der Erhebungen galten gegebenenfalls andere Kurse. Zur ungefähren Einschätzung der Werte bietet eine Umrechnung nach derzeitigem Kurs ausreichende Orientierung. 
einen längeren Zeitraum seinen Verpflichtungen nicht nachkommt. Beides stellen jedoch unterschiedliche Intensitäten von Unterhaltsproblemen dar.

Anders als im deutschen und britischen Datensatz werden innerhalb der norwegischen Variablen nur Zahlungsprobleme erfasst, die die Beteiligung einer dritten Instanz aufweisen. Die Unterhaltsschulden entstehen bei der Unterhaltsvorschusskasse, die die ausbleibenden finanziellen Leistungen des Vaters bei getrennt lebender Mutter und Kindern ausgleichen. Private Lösungen ausbleibender Zahlungen, die Unterhaltsschulden nur bei der Mutter persönlich verursachen, werden hier nicht gesondert erfasst. Das kann zu einer Überschätzung der Zahlungsleistung abwesender Väter in Norwegen führen. ${ }^{158}$

Wie im deutschen und britischen Datensatz werden auch im norwegischen Fall die Angaben zu Zahlungsproblemen in einer dichotomen Variablen zusammengefasst, in der Vätern, die Unterhaltsschulden - unabhängig von der Höhe - angeben, Unterhaltsprobleme zugeschrieben werden.

\section{Abb. 8.2: Graphische Häufigkeitsverteilung der abhängigen Variable Cash nach Datensatz.}

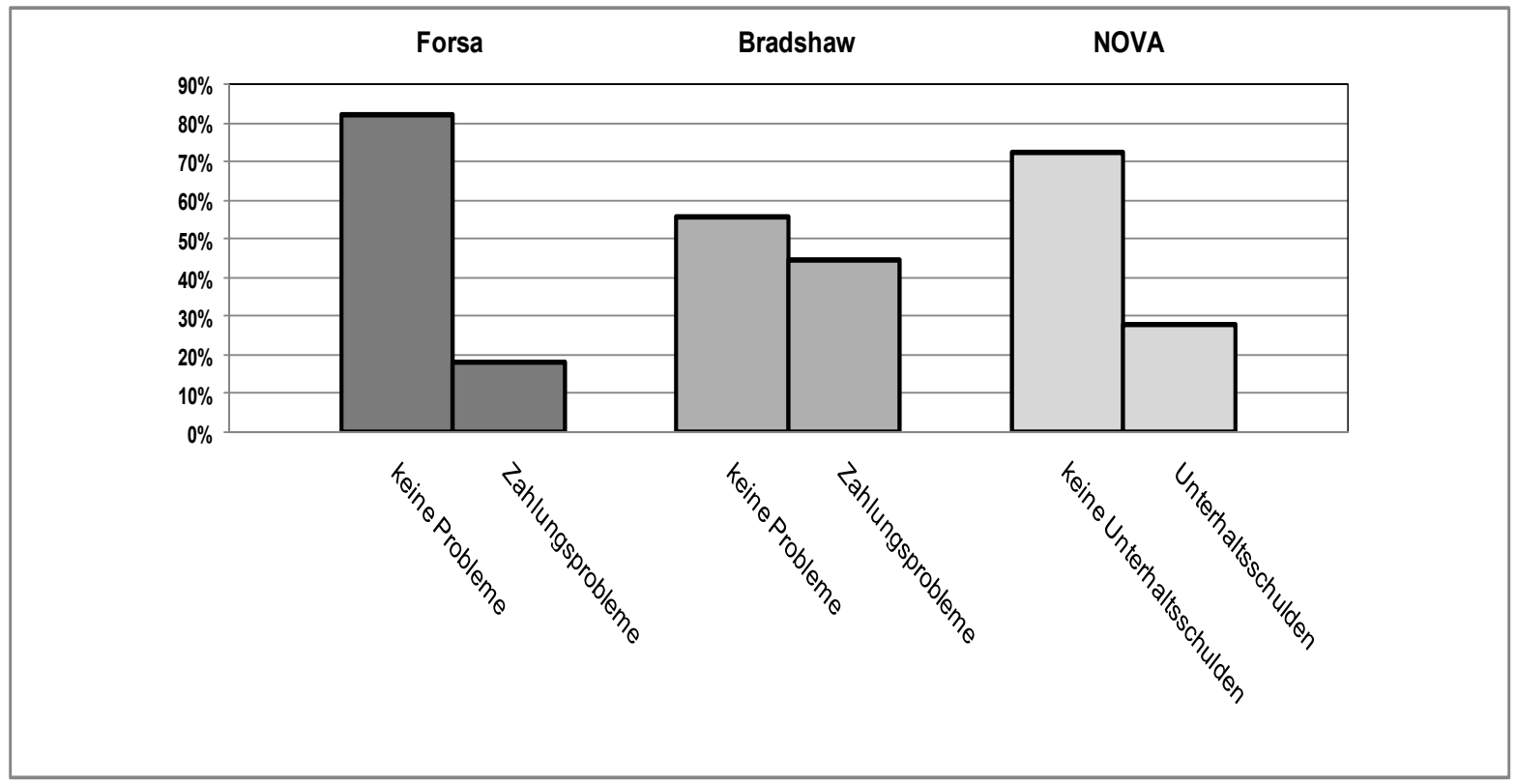

Quelle: eigene Darstellung.

158 Dagegen ist jedoch einzuwenden, dass die überwiegende Mehrheit norwegischer Eltern Unterhaltszahlungen freiwillig über diese Behörde regeln. Damit wird wahrscheinlich nur ein geringer Anteil an Zahlungsschwierigkeiten hier nicht berücksichtigt (siehe ausführlicher Kap. 11.2 Charakteristika der Modellvariablen). 
Tabelle 8.5 zeigt im Überblick die Häufigkeiten und Kodierungen der beschriebenen Cash-Variablen. Dabei wird zunächst die Variable mit mehreren Kategorien mit zunehmenden Zahlungsschwierigkeiten und im unteren Teil die dichotome Cash-Variable, die daraus resultiert, dargestellt. Letztere geht in die Berechnungen ein. Ihre Häufigkeitsverteilung ist in Abbildung 8.2 dargestellt.

Die Operationalisierungen der Cash-Variablen weisen, ähnlich wie Care, in den jeweiligen Datensätzen nationale Unterschiede auf. Dennoch kann - hier leichter als beim sozioemotionalen Kümmern - davon ausgegangen werden, dass die Konstrukte das gleiche Phänomen - sprich Zahlungsprobleme - abbilden. Da die Unterhaltsprobleme in allen drei Datensätzen sehr grob gemessen werden, sind vergleichende Aussagen ferner mit Vorsicht zu formulieren. Dennoch zeigen die Häufigkeitsverteilungen in Deutschland und Norwegen im Vergleich zu Großbritannien deutlichere Unterschiede zwischen den beiden Antwortkategorien. Die Variation innerhalb der britischen Variable ist stärker ausgeprägt, während die norwegische insbesondere die deutsche Cash-Variable deutlich schiefer verteilt sind. Inhaltlich bedeutet dies, dass britische Väter häufiger Zahlungsschwierigkeiten aufweisen als deutsche oder norwegische Nachtrennungsväter, die in den Untersuchungen befragt wurden. ${ }^{159}$

\subsection{Statistisches Vorgehen: Modelle und Maßzahlen}

In Kapitel 5 Hypothesengenerierung wurde die theoretische Fundierung der einzelnen Thesen ausführlich dargestellt. Die folgenden Kapitel widmen sich primär der empirischen Überprüfung der angenommenen Zusammenhänge. ${ }^{160}$

Die Hypothesen formulieren Annahmen zu den Zusammenhängen zwischen einer abhängigen Variablen, Care bzw. Cash, und jeweils einer unabhängigen Variablen auf bivariater Ebene. ${ }^{161}$ Dem entsprechend werden zunächst die Daten mit Hilfe bivariater Analyseverfahren untersucht. Dazu wird in Anlehnung an die Darstellungsform innerhalb der

\footnotetext{
159 Die möglichen methodischen Ursachen der geringeren Varianz, eine umfassende Interpretation sowie eine kritischen Einordnung aller Ergebnisse findet sich im Kap. 9.3 Interpretation der bivariaten und multiplen Ergebnisse sowie im abschließenden Teil IV dieser Arbeit.

${ }^{160}$ Alle vorliegenden Ergebnisse basieren auf eigenen Berechnungen mit Hilfe des Programms SPSS 13 und fallen allein in den Verantwortungsbereich der Verfasserin der vorliegenden Dissertationsschrift.

${ }^{161}$ Es gibt in der Literatur verschiedene Begriffe für die abhängige und unabhängige Variable. Im Folgenden werden die Bezeichnungen abhängige, endogene, erklärte bzw. zu erklärende, Ziel-, Prognosevariable, Kriterium(svariable) und Regressand synonym verwendet. Das Gleiche gilt für die Begriffe der unabhängigen, exogenen, erklärenden, Prädiktorvariablen, des Faktors und Regressors (siehe auch Kühnel/Krebs 2001: 317; Backhaus et al. 2006: 50, 428).
} 
Veröffentlichung der Studienergebnisse aller drei Untersuchungen für jede erklärende Variable zunächst eine Kreuztabelle konstruiert, der die relativen Häufigkeiten der abhängigen Variablen bezüglich der entsprechenden erklärenden Variablen zu entnehmen sind. Ziel der Kreuztabellierung und der Kontingenzanalyse ist es Zusammenhänge zwischen zwei Variablen aufzudecken. Dabei können die Variablen unterschiedliche Skalenniveaus aufweisen. Des Weiteren ist die Assoziationstabelle eine geeignete Darstellungsform, die einen ersten Überblick über die Datenlage verschafft (Baur 2004: 157). Metrisch skalierte Prädiktoren werden dazu in überschaubare Kategorien zusammengefasst. ${ }^{162}$ Dies birgt die Gefahr eines Informationsverlustes in sich. Deswegen werden die weiteren Analyseschritte, die über die tabellarische Darstellung hinausweisen, immer mit der Variablen vorgenommen, die das höhere Skalenniveau aufweist. Dies gilt auch für die Zielvariable Care, die als Punktescore metrisch skaliert ist. Sie wird zur besseren Veranschaulichung in den Kreuztabellen in Kategorien dargestellt, in die Berechnung der Zusammenhangsmaße geht sie als Punktzahl metrisch ein. Wird in einem Land eine Variable metrisch in den anderen jedoch kategorial erfasst, wird immer die niedrigere Skalierung verwendet, um ein Mindestmaß an Vergleichbarkeit zu erhalten, auch wenn dies mit Informationsverlusten verbunden ist.

Der zweite bivariate Analyseschritt dient der Verdichtung der aufgrund der Kreuztabellen vermuteten Zusammenhänge auf ein Zusammenhangsmaß. Statistische Maßzahlen sichern die Ergebnisse der Kreuztabellierung ab und machen sie mit anderen Kreuztabellen vergleichbar, indem sie Auskunft über Art und Ausmaß des Zusammenhangs geben (Baur 2004: 173). Dabei stehen verschiedene Maße in der Statistik zur Verfügung, die je nach Erkenntnisinteresse und Datenmaterial Anwendung finden. Im Folgenden werden v.a. asymmetrische Maße zur Messung des Zusammenhangs zwischen Variablen herangezogen, da die Hypothesen explizit zwischen einer abhängigen und einer unabhängigen Variablen differenzieren (Kühnel/Krebs 2001: 341f.).

Zwar unterscheiden sich die anzuwendenden Zusammenhangsmaße nach dem jeweiligen Skalenniveau der vorliegenden Daten. Die abhängigen Variablen Care bzw. Cash liegen als metrische bzw. dichotome Variable vor. Die erklärenden Variablen weisen verschiedene Skalenniveaus auf. Dennoch werden sie in der nachfolgenden Analyse als metrisch

\footnotetext{
162 Nicht für alle Prädiktoren ist die Präsentation der Ergebnisse in kreuztabellierter Form gleichermaßen geeignet. An entsprechender Stelle wird auf den begrenzten Informationsgehalt der Darstellungsweise hingewiesen. Der Vollständigkeit halber wird für alle erklärenden Variablen eine Assoziationstabelle aufgeführt.
} 
behandelt. ${ }^{163}$ Dieses Vorgehen ermöglicht auf bivariater Ebene den Vergleich der einzelnen Effekte untereinander. Das verwendete Maß wird der einfachen Regressionsanalyse als bivariates Modell entnommen (Wittenberg/Cramer 2003: 180). Dabei kann mit Hilfe einer Regression - anders als mit der Korrelation - angegeben werden, welcher Art der Zusammenhang in Stärke und v.a. in Richtung ist.

Um zusätzlich zur bivariaten Analyse Scheinkausalitäten der Determinanten ausschließen zu können, wird in einem weiteren Schritt eine multifaktorielle Untersuchung mit allen Prädiktoren durchgeführt, die bivariat signifikante Effekte gezeigt haben. So können gemeinsame Wirkungseffekte der einzelnen erklärenden Variablen aufgedeckt werden (Bühl/Zöfel 2005: 342). Der Einfluss einer unabhängigen Variablen wird unter Berücksichtigung der anderen Faktoren untersucht. Neben Scheineffekten schafft dieses Vorgehen ein Gesamtbild der Determinanten väterlichen Sorgehandelns. Es werden Aussagen über die Bedeutung der einzelnen Determinanten in einem Gesamtmodell, das im Folgenden als Modell All bezeichnet wird, ermöglicht.

Dieses Modell liefert zwar eine multiple Absicherung bivariater Zusammenhänge, aber es integriert einige Variablen, die keinen Beitrag zur Erklärungskraft leisten. In einem abschließenden Analyseschritt wird daher ein multiples Modell berechnet, indem ausschließlich signifikante Determinanten enthalten sind. Ausgangspunkt stellt das Modell All dar. Gemäß dem Rückwärts-Verfahren beginnt eine schrittweise Merkmalsentfernung mit der Entfernung der Variable, deren Ausschluss zum geringsten Rückgang des Bestimmtheitsmaßes führen, d.h. mit der Variable mit dem höchsten Signifikanzniveau. Wenn sich das multiple Bestimmtheitsmaß der Regression beim Weglassen dieser Variablen nicht signifikant verkleinert, wird diese Prädiktorvariable aus dem Merkmalssatz entfernt und das Verfahren entsprechend fortgesetzt. Das Verfahren bricht ab, wenn sich durch das Entfernen der nächsten Variablen das Bestimmtheitsmaß signifikant verkleinern würde, d.h. wenn alle zur Erklärung des Kriteriums irrelevanten Variablen entfernt wurden (Rudolf/Müller 2004: 48; Bühl/Zöfel 2005: 358f.). Am Ende des Verfahrens steht ein

163 Dieses methodische Vorgehen kann kritisch gewürdigt werden, ist in der empirischen Sozialforschung jedoch nicht unüblich, insbesondere wenn die ordinalskalierten Variablen mehr als drei Ausprägungen aufweisen. Ferner sind die hier betroffenen ordinalen Variablen häufig inhaltlich zumindest annähernd als metrisch zu interpretieren, dazu zählen z.B. die Prädiktoren der Entfernung, des Einkommens oder die ökonomische Situation der Mutter. Darüber hinaus kann angeführt werden, dass theoretisch begründet von streng monotonen Zusammenhängen ausgegangen wird, die anhand von Regressionsmodellen überprüft werden. Bestehende Zusammenhänge können auf diese Weise gegebenenfalls unterschätzt werden und nicht lineare Beziehungen zwischen Variablen unentdeckt bleiben. Primäres Ziel der Analyse ist es die aufgestellten Hypothesen empirisch zu testen. Die theoretischen Annahmen gehen von monotonen Zusammenhängen aus, so dass das strenge statistische Vorgehen gerechtfertigt erscheint. 
zweites multiples Modell, hier als Modell Best Fit bezeichnet, das nur Faktoren beinhaltet, die signifikant zur Erklärung des Regressanden beitragen.

Aufgrund der sehr unterschiedlichen Daten, die die einzelnen Studien erhoben haben, muss an dieser Stelle auf die eingeschränkten Interpretationsmöglichkeiten der multiplen Modelle hingewiesen werden. Zwar können die multiplen Analysen länderweise interpretiert werden, eine vergleichende Perspektive ist jedoch restringiert. Es können keine Aussagen über das Zusammenwirken zweier Prädiktoren getroffen werden, von denen in einem Land nur einer erhoben wurde. Am Ende der multiplen Analyse stehen daher drei unabhängige Modelle, die den Einfluss aller Prädiktoren auf die abhängigen Variablen Care und Cash jeweils für ein Land abbilden.

Zunächst werden wie beschrieben die beiden multiple Regressionsgleichungen - jeweils zwei pro abhängige Variable - ermittelt. In der Interpretation der Modelle wird die Güte der Funktion geprüft, d.h. es wird untersucht, wie gut das Modell die soziale Realität abzubilden vermag. Dabei wird zwischen einer globalen Prüfung der Regressionsfunktion und der Prüfung der einzelnen Koeffizienten unterschieden. Die globale Güte gibt an, wie gut das Modell die abhängige Variable erklärt. Die Überprüfung der einzelnen Koeffizienten beschäftigt sich mit der Frage, ob und wie gut die einzelnen Prädiktoren zur Erklärung der Zielvariablen beitragen (Backhaus et al. 2006: 63). Bei jedem Assoziationsmaß - innerhalb der bivariaten Regression und im multiplen Modell - wird sein Wert, seine Signifikanz und die ausgewiesene Wirkungsrichtung des Zusammenhangs aufgeführt, interpretiert und verglichen. Dabei liegt die Signifikanzgrenze aller Angaben entsprechend wissenschaftlicher Standards bei 0,05.

Die vorangegangenen Ausführungen haben sich generell auf Regressionsanalysen und auf das statistische Vorgehen, das beiden abhängigen Variablen gemein ist, bezogen. Aufgrund der unterschiedlichen Konstruktion der Kriteriumsvariablen - Care als metrische Variable und Cash dichotom - werden unterschiedliche Regressionsmodelle berechnet, selbst wenn dies die Vergleichbarkeit der Ergebnisse für Care und Cash wesentlich einschränkt. Damit können keine Aussagen über die unterschiedlichen Effektstärken in Relation zueinander, d.h. der beiden Prognosevariablen im Vergleich, getroffen werden. Vergleichende Interpretationen in Richtung und Signifikanz der Wirkungszusammenhänge sind weiterhin möglich.

Zur Aufdeckung der gemeinsamen und einzelnen Wirkungseffekte der erklärenden Variablen auf die Care-Variable wird eine lineare Regression berechnet (Bühl/Zöfel 2005: 334). Im einfachsten Fall besteht die Regressionsgleichung nur aus der Konstanten und einem Faktor. Die einfache Regression gibt im Folgenden den Zusammenhang auf bivariater Ebene an. Unabhängig von der Anzahl der aufgenommenen Regressoren wird für 
jede unabhängige Variable ein Regressionskoeffizient $\beta$ als Regressionsgewicht die Steigung der Geraden angegeben und interpretiert. Inhaltlich bedeutet dabei ein positives Vorzeichen des Koeffizienten, dass mit dem Anstieg des Regressors sich der Vorhersagewert des Kriteriums um ß erhöht (Backhaus et al. 2006: 55). Bei einem negativen Regressionsgewicht besteht ein negativer Zusammenhang zwischen den Variablen. Der Koeffizient gibt damit Aufschluss über die Wirkungsrichtung des Zusammenhangs. Des Weiteren kann er als Maß für die Stärke des Effekts interpretiert werden. Bei einem Wert von Null liegt kein Einfluss der unabhängigen Variablen vor. Dies kann zum einen bedeuten, dass tatsächlich kein Zusammenhang besteht. Zum anderen kann ein Gewicht von Null darauf hinweisen, dass keine lineare Beziehung zwischen den Variablen besteht (Kühnel/Krebs 2001: 395f.).

Im Regressionsgewicht finden die Maßeinheiten Berücksichtigung, in denen die jeweiligen Variablen gemessen werden. Dies erschwert die inhaltliche Interpretation der Effekte in vergleichender Perspektive. Deswegen wird innerhalb der folgenden Analyse das standardisierte Regressionsgewicht $ß$ verwendet, das von den ursprünglichen Einheiten unabhängig die Stärke des Wirkungszusammenhangs angibt (Kühnel/Krebs 2001: 395; Backhaus et al. 2006: 61f.). Innerhalb der multiplen Regression gibt der standardisierte Partialregressionskoeffizient, das sog. Beta-Gewicht, Hinweise über die relative Wichtigkeit der einzelnen Prädiktorvariablen (Bühl/Zöfel 2005: 345; Backhaus et al. 2006: 62). Zur Beurteilung der Stärke des ausgewiesenen positiven oder negativen Zusammenhangs werden im Folgenden die in der Literatur üblichen Faustregeln angewandt: Ein Koeffizient unter 0,05 ist in seiner Bedeutung zu vernachlässigen. Werte zwischen 0,05 und 0,2 weisen auf einen eher geringen Zusammenhang hin, während Gewichte zwischen 0,2 und 0,5 einen mittleren Zusammenhang repräsentieren. Ein Koeffizient, der größer als 0,5 ist, zeigt einen starken, ein Wert über 0,7 sogar einen sehr hohen Effekt an (Kühnel/Krebs 2001: 404f.).

Neben der bivariaten Zusammenhangsanalyse bzw. der Prüfung der Effekte einzelner Prädiktoren innerhalb eines multiplen Modells wird dieses ferner einer globalen Prüfung unterzogen. Das bedeutet, mittels des Bestimmtheitsmaßes $R^{2}$ wird die Güte der Anpassung des linearen Regressionsmodells mit mehreren Faktoren gemessen. Der Determinationskoeffizient gibt das Verhältnis zwischen erklärter zur gesamten Streuung an und drückt damit die Erklärungskraft des Modells aus (Backhaus 2006: 64ff., Wittenberg/Cramer 2003: 187, Brosius 1998: 535). Das Bestimmtheitsmaß als eine normierte Größe nimmt Werte zwischen null und eins an (Kühnel/Krebs 2001: 415f.; Backhaus et al. 2006: 66). Innerhalb einer multiplen Regression wird der Determinationskoeffizient durch die Anzahl der berücksichtigten unabhängigen Variablen bestimmt. Daher wird hier das korrigierte 
Bestimmtheitsmaß adjusted $R^{2}$ berücksichtigt (Backhaus et al. 2006: 68; Bühl/Zöfel 2005: 337).

Zur Analyse der Cash-Variablen wird eine binär logistische Regressionsanalyse herangezogen, die die Abhängigkeit einer dichotomen Variablen von anderen Variablen prüft (Bühl/Zöfel 2005: 334; Rudolf/Müller 2004: 237). Das logistische Modell gibt mit Hilfe einer nicht-linearen Regression an, welche Wahrscheinlichkeit das Eintreten eines bestimmten Ereignisses in Abhängigkeit verschiedener Einflussgrößen hat (Kühnel/Krebs 2001: 606; Backhaus et al. 2006: 426; Bühl/Zöfel 2005: 352).

Wie innerhalb der linearen Regression werden auch hier verschiedene bivariate und multiple Modelle berechnet. Zunächst wird für jede Erklärungsgröße ein Modell formuliert und die logistische bivariate Regressionsfunktion geschätzt. In einem zweiten Schritt werden die multiplen Modelle erstellt. In beiden Fällen werden die geschätzten Koeffizienten der logistischen Regression für Cash interpretiert und auf ihre Güte überprüft.

Bei der logistischen Regression ist die Interpretation der Regressionskoeffizienten insofern ähnlich wie in der linearen Regression, als das gilt: Je größer der vorzeichenbereinigte Wert eines Regressionsgewichts ist, desto steiler verläuft die Regressionskurve. Positive Koeffizienten weisen einen positiven Zusammenhang, negative Vorzeichen eine entgegengesetzte Wirkungsrichtung aus. Ein Regressionskoeffizient von null bedeutet, dass die Werte der abhängigen nicht mit den Werten der unabhängigen Variablen variieren (Kühnel/Krebs 2001: 608). Im Gegensatz zur linearen Regression können jedoch keine Aussagen über den Betrag des Anstiegs bzw. des Absinkens des Kriteriums getroffen werden, wenn der Prädiktor sich um eine Einheit erhöht (Kühnel/Krebs 2001: 608). Die gleichen Veränderungen in den Beobachtungswerten des Prädiktors können in verschiedenen Bereichen der logistischen Funktion unterschiedlich auf die Eintrittswahrscheinlichkeit des zu erklärenden Ereignisses wirken. Auf den ersten Blick gibt der Regressionskoeffizient nur Aufschluss über die Richtung des Einflusses. Zu einer Interpretationserleichterung führt die Analyse der Verhältnisse zwischen der Eintrittswahrscheinlichkeit und ihrer Gegenwahrscheinlichkeit, d.h. der Chance (im Englischen: odd), dass das Ereignis ausbleibt. Die logarithmierten Odds, die sog. Logits, drücken die Einflussstärke des Faktors aus. Sie erlauben eine Interpretation analog zur linearen Regressionsanalyse. Die sog. Odds Ratio, auch als Effektkoeffizienten bezeichnet, geben mittels einer Tendenzaussage, über die die Regressionskoeffizienten nicht hinausreichen können, die genaue Höhe der Einflussstärken der jeweiligen Regressoren auf die Eintrittswahrscheinlichkeit an (Backhaus et al. 2006: 439ff.). Da das Logitmodell ein multiplikatives Modell ist, gelten hier andere Regeln für die Interpretation der Koeffizienten: Ein Wert um eins weist auf keinen Zusammenhang hin, während Werte größer eins einen positiven und Maße kleiner eins einen negativen Effekt ausdrücken (Andreß et 
al. 1997: 271; Rudolf/Müller 2004: 240ff.). Wie im linearen Modell werden auch hier die standardisierten Effektkoeffizienten ausgegeben, das bedeutet die Effektstärke wird in der Standardabweichung der unabhängigen Variable - nicht des Kriteriums - ausgedrückt, um damit unterschiedliche Messeinheiten zu einheitlich vergleichbar zu machen. ${ }^{164}$

Abschließend werden die multiplen Cash-Modelle einer Güteprüfung des Gesamtmodells unterzogen. Die Gesamterklärungskraft wird u.a. in den Pseudo-R-Quadrat-Statistiken ausgegeben (Backhaus et al. 2006: 448). Dazu zählt auch das für die Aufklärung der Gesamtstreuung von SPSS ausgegebene Nagelkerkes $R^{2}$. In der empirischen Praxis wird im Falle von Werten größer 0,2 von einer akzeptablen Gesamtgüte des Modells gesprochen; gute Erklärungen werden durch Werte zwischen 0,2 und 0,4 abgebildet und Werte über 0,5 stehen für eine sehr gute Erklärungskraft des Modells (Backhaus et al. 2006: 456).

Für die Testung der bivariaten Zusammenhänge folgt die Darstellung im Folgenden für jede zu überprüfende These der gleichen Struktur: Zunächst werden Operationalisierungen der entsprechenden erklärenden Variablen in den einzelnen Datensätzen gegenübergestellt. ${ }^{165}$ Erfasst eine Studie zur entsprechenden These keine Angaben, die eine sinnvolle Operationalisierung zulassen, werden lediglich die verbleibenden anderen Studien aufgeführt, so dass nicht alle Thesen an allen Datensätzen überprüft werden. In einem zweiten Schritt werden die statistischen Ergebnisse in Form von Kreuztabellen und den erläuterten Zusammenhangsmaßen kurz dargestellt. Es folgt abschließend eine Einschätzung, ob die bivariaten Ergebnisse die aufgestellte These stützen. In einem späteren Kapitel erfolgt eine Gesamtschau der Ergebnisse und eine abschließende Interpretation. ${ }^{166}$

164 Diese Effektkoeffizienten werden in SPSS unstandardisiert als Exp (B) ausgegeben und so auch in den Tabellen der Ergebnisdarstellung genannt. Es wird gesondert darauf verwiesen, dass sie standardisiert worden sind.

165 Dies dient der Übersichtlichkeit der Darstellung. Die z.T. sehr unterschiedlichen Ausprägungen der zahlreichen erklärenden Variablen finden sich in den Kreuztabellen wieder, so dass eine gemeinsame Darstellung der Konstruktion der Variablen und ihrer Kreuztabellierung zum besseren Verständnis der nationalen Unterschiede sinnvoll erscheint.

${ }^{166}$ Die Ergebnisse der einzelnen Analyseschritte, deskriptiv, bi- und multivariat variieren mit den Resultaten der jeweiligen Publikationen zu den einzelnen Datensätzen. Das hat je nach Datensatz unterschiedliche Ursachen. So wurden beispielsweise innerhalb der Forsa-Studie die Unterhaltspflichtigen insgesamt betrachtet, während hier nur die unterhaltspflichtigen Väter berücksichtigt werden, die insgesamt 96\% der befragten Unterhaltspflichtigen in der deutschen Studie ausmachen (Forsa 2002: 33). Ferner werden die einzelnen erklärenden Variablen aller Datensätze harmonisiert, so dass sie soweit dies möglich ist, ähnliche Antwortkategorien aufweisen. Trotz aller Schwierigkeiten, die der internationale Vergleich unabhängig voneinander entstandener Datensätze mit sich bringt, wird die vergleichende Perspektive weitgehend aufrecht erhalten. Dies führt zu unterschiedlichen Kategorisierungen und Konzeptionalisierungen einzelner Variablen im Vergleich zum ursprünglichen Datenmaterial. Auch dies kann andere Ergebnisse zur Folge haben. 


\section{Ergebnisse: Darstellung und Interpretation}

Im folgenden Kapitel werden die aufgestellten Thesen am vorliegenden Datenmaterial zunächst auf bivariater Ebene getestet. Dazu werden der theoretisch erwartete Zusammenhang zwischen den dargestellten abhängigen Variablen Care bzw. Cash und dem betrachteten Prädiktor kurz wiederholt, die Operationalisierung der erklärenden Variable in den einzelnen Datensätzen dargestellt ${ }^{167}$ und abschließend die bivariaten Zusammenhänge in Form einer Kreuztabelle analysiert.

\subsection{Operationalisierung der Prädiktoren und bivariate Zusammenhänge}

Der erste Teil der Analyse widmet sich zunächst der Präsentation der Operationalisierungen der Prädiktoren in den jeweiligen Datensätzen. Die gleichzeitige Darstellung von These, Operationalisierung und Kreuztabellierung pro Prädiktor dient der besseren Les- und Vergleichbarkeit. Sie verdeutlicht ferner die nationalen Unterschiede in den Daten und die damit verbundenen Schwierigkeiten der vergleichenden Perspektive. Da dieselben Determinanten für Care und Cash getestet werden, berücksichtigt die Darstellung der bivariaten Ergebnisse zunächst beide Kriteriumsvariablen gleichermaßen. Zusammenhänge werden nacheinander für jede erklärende Variable analysiert. Die Darstellung folgt der Reihenfolge der aufgestellten Hypothesen.

\subsubsection{Der Einfluss der Prädiktoren der physischen Umwelt}

Mit Blick auf die Entfernung zwischen den elterlichen Wohnungen wird vermutet, dass mit steigender Distanz zwischen den Wohnorten zwar das sozio-emotionale Kümmern abnimmt, sich aber nicht die Zahlungsmoral ändert.

Anders als in den norwegischen und deutschen Untersuchungen wird die Entfernung zwischen den elterlichen Wohnorten im britischen Fall nicht in Kategorien gemessen. Die

\footnotetext{
${ }^{167}$ Es ist an dieser Stelle darauf zu verweisen, dass die Fragen in der jeweiligen Landessprache gestellt wurden. Im Folgenden werden eigene und im norwegischen Fall Übersetzungen Dritter aufgeführt. Es ist nicht auszuschließen, dass aufgrund der Übersetzung und auch aufgrund sprachlicher Unterschiede, Unsicherheiten in der Analyse und der Interpretation der Ergebnisse bestehen. Es wird jedoch grundsätzlich davon ausgegangen, dass trotz unterschiedlicher nationaler Kontexte die Fragen ähnliche, wenn nicht gar gleiche Dimensionen abbilden.
} 
befragten Väter geben den Abstand in Meilen an. ${ }^{168}$ Wie Tabelle 9.1 zeigt, werden die britischen Antworten in Kategorien zusammengefasst, die denen der beiden anderen Datensätze ähneln. So können in Bezug auf die Entfernungen im britischen Datensatz Väter ausgemacht werden, deren Kinder im Ausland leben, wohingegen der norwegische Datensatz nur solche erfasst, deren Kinder mehr als fünf Stunden entfernt wohnen. Diese Distanzen werden im deutschen Fall nicht erfragt. Demzufolge besteht im deutschen Datensatz eine Antwortkategorie weniger. ${ }^{169}$

Tab. 9.1: Operationalisierungen der Entfernung zwischen den Wohnorten.

\begin{tabular}{|c|c|c|c|}
\hline \multirow[b]{2}{*}{ Frage } & Forsa & Bradshaw & NOVA \\
\hline & $\begin{array}{l}\text { Wie weit wohnt die } \\
\text { Mutter heute von Ihnen } \\
\text { entfernt? }\end{array}$ & $\begin{array}{l}\text { Wie weit wohnen } \\
\text { sie von (Name der } \\
\text { Ex-Partnerin) weg } \\
\text { in Meilen? }\end{array}$ & $\begin{array}{l}\text { Wie groß ist die Entfernung } \\
\text { zwischen dem Zuhause, wo } \\
\text { das Kind gewöhnlich wohnt } \\
\text { und ihrem Zuhause }\end{array}$ \\
\hline Kategorien & $\begin{aligned} 1= & \text { im selben Ort } / \text { der } \\
& \text { selben Stadt; } \\
2= & \text { in der näheren } \\
& \text { Umgebung }(<50 \mathrm{~km}) \\
3= & 51 \text { bis } 200 \mathrm{~km} \\
& \text { entfernt; } \\
4= & \text { weiter weg als } \\
& 200 \mathrm{~km} ;\end{aligned}$ & $\begin{array}{l}1=\text { unter } 5 \mathrm{~km} \\
2=5 \text { bis } 50 \mathrm{~km} ; \\
3=51 \text { bis } 200 \mathrm{~km} ; \\
4=\text { über } 200 \mathrm{~km} ; \\
5=\text { im Ausland }\end{array}$ & $\begin{aligned} 1= & \text { in Nachbarschaft, zu Fuß } \\
& \text { erreichbar } \\
2= & \text { mit Auto/Bus unter } 30 \\
& \text { Minuten } \\
3= & \text { halbe bis } 2 \text { Stunden weg } \\
4= & 2 \text { bis } 5 \text { Stunden weg } \\
5= & \text { mehr als } 5 \text { Stunden } \\
& \text { entfernt }\end{aligned}$ \\
\hline
\end{tabular}

Quelle: eigene Darstellung.

Die nachstehende Kreuztabelle (Tab. 9.2) gibt die Häufigkeiten der einzelnen Antwortkategorien für Care bzw. Cash auf bivariater Ebene wieder.

Die Kreuztabellierung der abhängigen Variablen Care mit den Kategorien der erklärenden Variablen Entfernung zwischen den elterlichen Wohnorten zeigt im deutschen Fall einen Trend in Richtung eines negativen Zusammenhangs. Die Zellen, die für ein höheres väterliches Engagement bei gleichzeitiger räumlicher Nähe unter $50 \mathrm{~km}$ stehen, weisen eine höhere Besetzung auf, als z.B. die Zellen, in denen die Väter zusammengefasst sind, die mehr als 200km weit weg leben und trotzdem mindestens einen intensiven Kontakt zu ihrem Kind haben. Gleichzeitig ist der Anteil der Kontaktabbrüche bei den Vätern, die in weiterer Entfernung als $50 \mathrm{~km}$ wohnen, höher. Ein ähnlicher Trend kann im britischen Datensatz ausgemacht werden. Väter, die in der Nähe ihrer Kinder leben, weisen häufiger moderates, intensives oder sehr intensives Sorgehandeln auf. Mit steigender Entfernung

\footnotetext{
${ }^{168}$ Zum besseren Verständnis für den deutschen Leser wurden die Angaben in Kilometer umgerechnet. Eine Meile entspricht demnach $1,61 \mathrm{~km}$.

${ }^{169}$ Die jeweiligen Häufigkeitsverteilungen der erklärenden Variablen und damit die jeweilige Zusammensetzung der Stichproben finden sich im Anhang (Tab. B.1 bis B.3).
} 
zwischen den Wohnorten nehmen die Häufigkeiten in den Zellen für „keinen Kontakt“ und „sehr wenig intensives“ Kümmern zu, was auch durch die norwegischen Daten unterstützt wird. Insbesondere in den beiden höchsten Kategorien des Kriteriums nimmt die Zellenbesetzung mit steigender räumlicher Distanz ab. Die Betrachtung der unteren drei Ausprägungen der Care-Variablen zeigt, dass hier der Anteil der Kontaktabbrüche bzw. das Auftreten von (sehr) wenig intensivem Kümmern deutlich zunimmt. In allen drei Datensätzen kann ein negativer Zusammenhang mit der Variablen Care ausgemacht und damit die aufgestellte Hypothese gestützt werden.

Auch mit Blick auf die Cash-Variable weisen die drei Datensätze ähnliche Ergebnisse auf. Jedoch kann ein eindeutiger Zusammenhang anhand der Kreuztabellen nicht ausgemacht werden. Deutsche Väter, die in einer Entfernung zwischen 50 und 200km von ihrem Kind wohnen, weisen etwas häufiger Zahlungsprobleme. In Großbritannien trifft dies für die Kategorien „unter 5km“, „51 bis 200km“ sowie für die im Ausland lebenden Väter zu. In den norwegischen Daten kann anhand der Kreuztabelle am ehesten von einem Effekt ausgegangen werden. Väter, die in unmittelbarer Nähe zu ihren Kindern leben, weisen im Vergleich zu den höheren Entfernungskategorien häufiger keine Zahlungsschwierigkeiten auf. Dies ließe auf einen positiven Zusammenhang schließen: Mit steigender Entfernung nehmen auch die Probleme der Unterhaltsleistung zu. Die Differenzen zwischen den einzelnen Spalten sind jedoch als eher gering einzustufen, wodurch die theoretische Annahme, dass zwischen der Entfernung und der Zahlungsmoral des Vaters kein Zusammenhang besteht, in Deutschland und Großbritannien bestätigt wird. Im norwegischen Fall kann von einer „,tendenziellen“ Stützung der These ausgegangen werden. ${ }^{170}$

${ }^{170}$ Die Berechnung des Zusammenhangsmaßes wird zeigen, inwieweit eine Beziehung zwischen den Variablen besteht. 


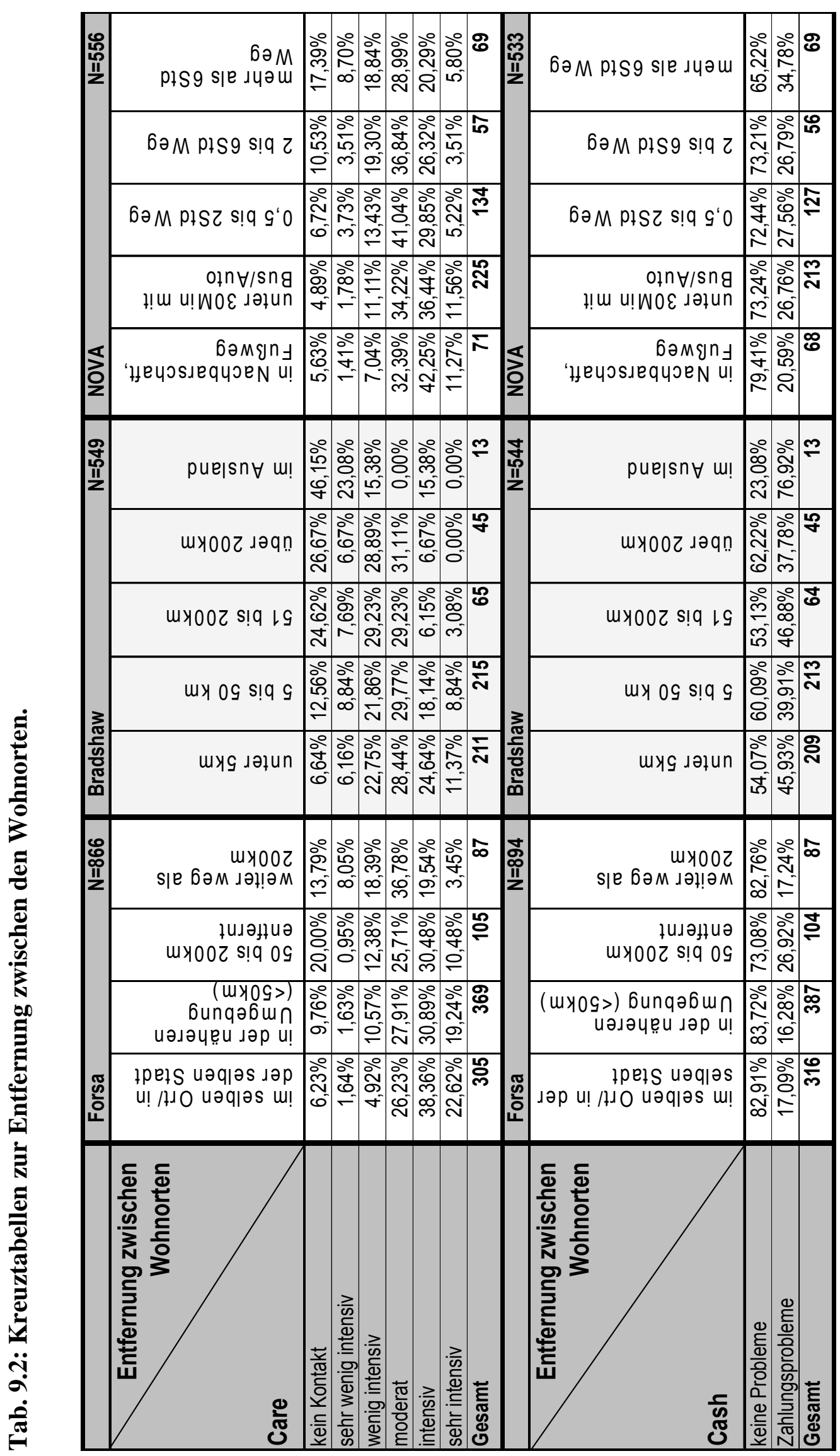


Eine wichtige Komponente der physischen Umwelt des Vaters stellt sein Einkommen dar. Mit dem Einkommen steigt theoretisch die Wahrscheinlichkeit eines intensiven Kontakts zwischen Vater und Kind, wobei gleichzeitig die Wahrscheinlichkeit von Zahlungsproblemen sinkt.

Tab. 9.3: Operationalisierungen des Einkommens des Vaters.

\begin{tabular}{|c|c|c|c|}
\hline \multirow{4}{*}{ Frage } & Forsa & Bradshaw & NOVA \\
\hline & \multirow{3}{*}{$\begin{array}{l}\text { Wie hoch ist Ihr } \\
\text { persönliches } \\
\text { Nettoeinkommen im } \\
\text { letzten Monat? Ich meine } \\
\text { damit die Summe, die } \\
\text { sich aus Lohn, Gehalt, } \\
\text { Einkommen aus } \\
\text { selbstständiger Tätigkeit, } \\
\text { Rente oder Pension; } \\
\text { jeweils nach Abzug der } \\
\text { Steuern und } \\
\text { Sozialversicherungs- } \\
\text { beiträge. }\end{array}$} & $\begin{array}{l}\text { Zahlungsscheck } \\
\text { vorgelegt }\end{array}$ & \multirow{3}{*}{$\begin{array}{l}\text { Wie hoch war ungefähr } \\
\text { Ihr Bruttojahres- } \\
\text { einkommen im Jahr } \\
2000 ?\end{array}$} \\
\hline & & $\begin{array}{l}\text { Wie hoch ist Ihr } \\
\text { gewöhnlicher } \\
\text { Bruttoverdienst, d.h. mit } \\
\text { Überstunden, Bonussen, } \\
\text { Trinkgeldern, und } \\
\text { ähnliches, aber vor } \\
\text { Steuern und Abzügen? }\end{array}$ & \\
\hline & & $\begin{array}{l}\text { Wieviel nehmen sie } \\
\text { normalerweise mit nach } \\
\text { Hause, d.h. nach } \\
\text { Abzügen für Steuern, } \\
\text { Versicherungen, } \\
\text { Gewerkschaftsbeiträgen } \\
\text { und Rentenzahlungen? }\end{array}$ & \\
\hline Metrisch & - & In Pfund & \\
\hline Dimension & $\begin{array}{l}\text { Nettoeinkommen im } \\
\text { Monat und in Euro }\end{array}$ & $\begin{array}{l}\text { Einzelinformationen } \\
\text { Einkommen netto und } \\
\text { brutto in Pfund } \\
\text { umgerechnet in } \\
\text { Euro,sowie Zeitraumder } \\
\text { Bezahlung gegeben }\end{array}$ & $\begin{array}{l}\text { Jahreseinkommen brutto } \\
\text { in NOK umgerechnet in } \\
\text { Euro }\end{array}$ \\
\hline & 1 = kein Einkommen & 1 = kein Einkommen & $\begin{array}{c}1=\text { unter 12.321 Euro } \\
\text { [unter } 100.000 \mathrm{NOK} \text { ] }\end{array}$ \\
\hline & 2 = unter500 Euro & $2=$ unter500 Euro & $\begin{aligned} 2= & 12.321-18.481 \text { Euro } \\
& {[100.000-150.000 \text { NOK }] }\end{aligned}$ \\
\hline & $3=500-900$ Euro & $3=500-900$ Euro & $\begin{aligned} 3= & 18.482-24.642 \text { Euro } \\
& {[150.000-200.000 \text { NOK }] }\end{aligned}$ \\
\hline & $4=900-1250$ Euro & $4=900-1250$ Euro & $\begin{aligned} 4= & 24.643-30.802 \text { Euro } \\
& {[200.000-250.000 \mathrm{NOK}] }\end{aligned}$ \\
\hline Kategorien & $5=1250-1750$ Euro & $5=1250-1750$ Euro & $\begin{aligned} 5= & 30.803-36.963 \text { Euro } \\
& {[250.000-300.000 \text { NOK }] }\end{aligned}$ \\
\hline & $6=1750-2250$ Euro & $6=1750-2250$ Euro & $\begin{aligned} 6= & 36.964-43.123 \text { Euro } \\
& {[300.000-350.000 \mathrm{NOK}] }\end{aligned}$ \\
\hline & $7=2250-2750$ Euro & $7=2250-2750$ Euro & $\begin{aligned} 7= & 43.124-55.444 \text { Euro } \\
& {[350.000-450.000 \mathrm{NOK}] }\end{aligned}$ \\
\hline & $8=2740-3250$ Euro & $8=2740-3250$ Euro & $\begin{aligned} 8= & \text { über } 55.444 \text { Euro } \\
& \text { [über } 450.000 \text { NOK] }\end{aligned}$ \\
\hline & $9=3250-3750$ Euro & $9=3250-3750$ Euro & --- \\
\hline & $10=$ über 3750 Euro & 10 = über 3750 Euro & --- \\
\hline
\end{tabular}

Quelle: eigene Darstellung. 
Anders als in der deutschen oder norwegischen Untersuchung geben die britischen Väter das Einkommen als Betrag in Britischen Pfund und nicht in Kategorien an. Dabei liegen sowohl Angaben zum Brutto- wie zum Nettowert vor, während in Deutschland das Nettoeinkommen pro Monat und in Norwegen das Jahresbruttoeinkommen erfragt werden. Die Vergleichbarkeit der Angaben wird zusätzlich durch die unterschiedliche Kategorisierung innerhalb der norwegischen und deutschen Daten erschwert. Zum besseren Verständnis orientieren sich die Kategorien der konstruierten Einkommensvariable im britischen Datensatz an den deutschen Vorgaben. Weder im norwegischen noch im britischen Datenmaterial ist die Ausprägung „kein Einkommen“ vorhanden. Zwar weisen die britischen Väter z.T. Null-Kodierungen auf, doch es ist nicht eindeutig zu interpretieren, ob sie fehlende Angaben oder fehlendes Einkommen repräsentieren. Deswegen wird unter Hinzunahme der Information einer vorliegenden Erwerbstätigkeit die Kategorie „kein Einkommen“ konstruiert. Väter, die kein Einkommen angeben und ferner antworten erwerbslos zu sein, werden als einkommenslos eingestuft. ${ }^{171}$ In Norwegen ist dieses Vorgehen nicht ohne Weiteres möglich. Hier wird nach dem Jahreseinkommen gefragt. Väter, die im Zeitpunkt der Erhebung ohne Erwerbstätigkeit sind, können im Verlauf des Jahres bereits Einkommen generiert haben. ${ }^{172}$ Die Kategorie „kein Einkommen“ fehlt somit im norwegischen Datensatz. Das Einkommen wird zum besseren Verständnis für alle drei Datensätze in Euro angegeben. ${ }^{173}$

Aus der Kreuztabelle der Variablen des väterlichen Einkommens können auf den ersten Blick keine eindeutigen Zusammenhänge für die Care-Variablen ausgemacht werden. Dies ist mit der bedingten Eignung der Darstellungsform einer Kreuztabelle für Variablen mit vielen Ausprägungen zu begründen. Denn beide aufeinander bezogenen Variablen weisen zu viele Kategorien auf. Deshalb wird auf eine genauere Darstellung der Zellenbesetzung an dieser Stelle verzichtet und auf das einfache Regressionsmodell im zusammenfassenden Abschnitt am Ende dieses Kapitels verwiesen. ${ }^{174}$

\footnotetext{
${ }^{171}$ In der Variablen, die das Einkommen der britischen Väter erfasst, wird das Bruttowocheneinkommen in Pfund ausgewiesen. Dieses wird auf ein Monatseinkommen mit dem Faktor 4,3 hochgerechnet.

${ }^{172}$ Die Kreuztabellierung der Variablen Erwerbstätigkeit und Einkommen ergibt, dass von den 102 Vätern, die nicht erwerbstätig sind, 85 ein Jahreseinkommen angeben.

${ }^{173}$ Laut Umrechnungskurs vom 07.02.2007 unter www.bdb.de/html/reisekasse/waehrungsrechner.asp entspricht 1 Britisches Pfund 1,51798 Euro und 1 Norwegische Krone entspricht 0,12321 Euro. Zum Zeitpunkt der Erhebungen galten gegebenenfalls andere Kurse, aber zur ungefähren Einschätzung der Werte bietet eine Umrechnung nach derzeitigem Kurs eine ausreichende Orientierung.

${ }^{174}$ Siehe Kap. 9.1.4.3 Zwischenfazit II: Die bivariaten Ergebnisse.
} 


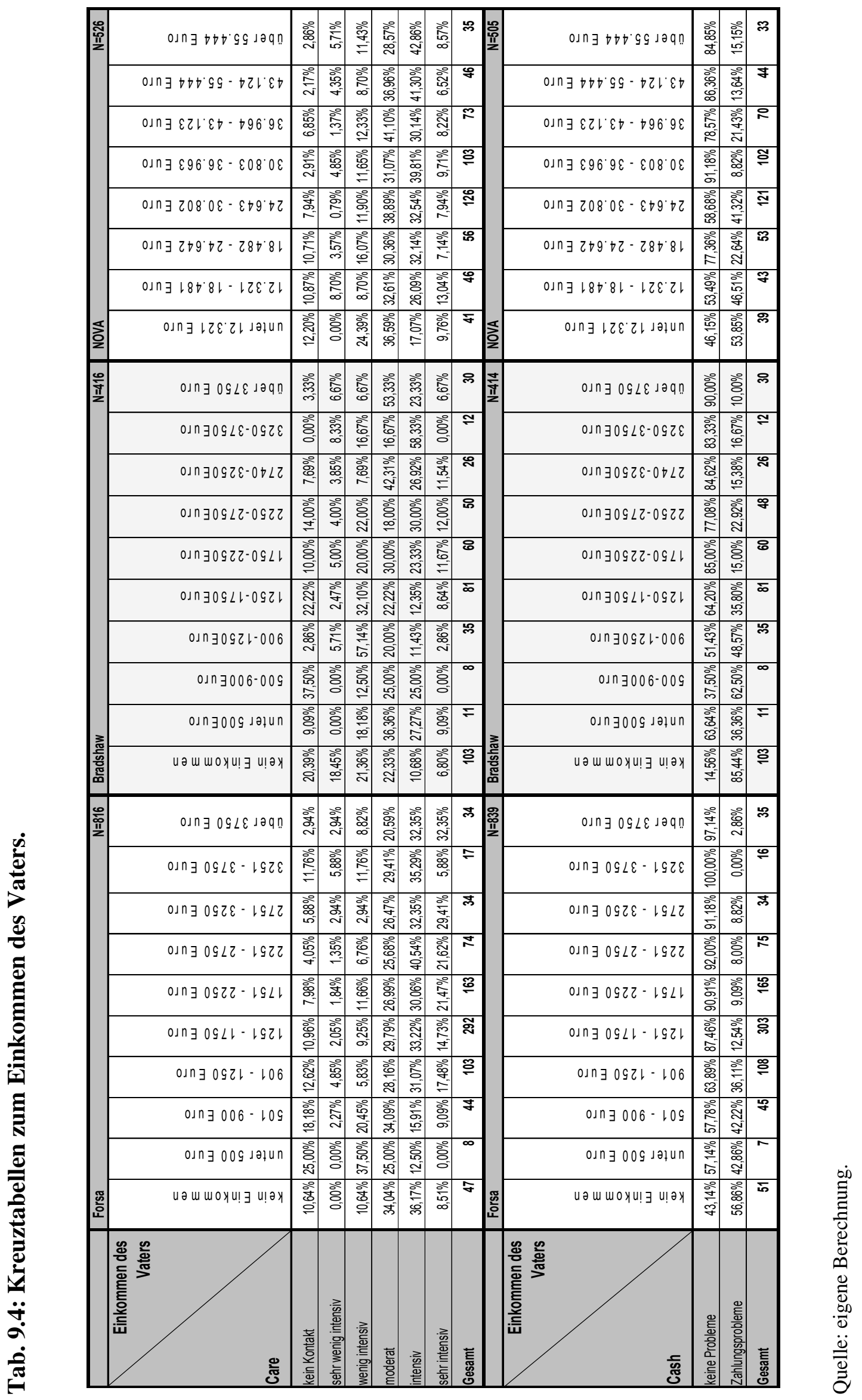


Die Ergebnisse der Cash-Variablen sind aufgrund ihrer Dichotomisierung leichter zu erfassen. Dabei zeigen die deutschen Daten deutlich, dass der Anteil der Väter mit Zahlungsproblemen mit steigendem Einkommen sinkt. Diese Tendenz weisen auch die britischen Ergebnisse auf, wenn auch nicht in der gleichen Deutlichkeit. Höhere Einkommenskategorien haben deutlich größere Anteile an Väter, die keine Schwierigkeiten mit dem Leisten von Unterhalt zeigen, als die unteren Einkommensklassen. Am deutlichsten sind die umgedrehten Verhältnisse zwischen Einkommen und Zahlungsproblemen an den Extrempunkten der Kategorisierung zu erkennen: Rund 15\% der britischen Väter ohne Einkommen leisten Unterhalt, während die anderen 85\% Zahlungsschwierigkeiten haben. Auf der anderen Seite können rund 85\% der drei obersten Einkommensklassen ihren Zahlungsverpflichtungen nachkommen, während rund 15\% Probleme mit der finanziellen Unterstützung ihrer getrennt lebenden Kinder angeben. Dies deutet auf den vermuteten negativen Zusammenhang hin. Auch im norwegischen Datenmaterial findet sich das Verhältnis von ca. 85\% ohne und 15\% mit Zahlungsproblemen in den oberen beiden Einkommensklassen. Am unteren Ende der Skalierung zeigt sich auch hier ein umgekehrtes Verhältnis, wenn auch nicht mit so starker Eindeutigkeit wie im britischen Fall. Insgesamt stützen alle drei Datensätze die These eines negativen Zusammenhangs zwischen steigendem Einkommen des Vaters und dem Auftreten von Zahlungsschwierigkeiten.

Als eine weitere Ressource der physischen Umwelt zählt das Ausbildungsniveau des Vaters. Dabei bewirkt ein steigendes Ausbildungsniveau ein stärkeres sozio-emotionales Engagement des Vaters und geringere Zahlungsausfälle.

Deutsche und norwegische Väter geben ihren höchsten Bildungsabschluss an. Mit zunehmender Kodierung steigt dabei auch das Ausbildungsniveau. Im britischen Datenmaterial liegt die Frage nach der beruflichen Qualifikation als Mehrfachnennung vor. Damit muss das endgültige Bildungsniveau zunächst noch konstruiert werden, da ein Vater potentiell alle Qualifikationen aufweisen kann. Es wird immer die höchste Nennung des einzelnen Vaters als sein Ausbildungsstand gewertet. Die Verwendung dieser Konstruktion ist mit einigen methodischen Schwierigkeiten verbunden, die u.a. zu starken Ausfällen führen. ${ }^{175}$ Wie in Deutschland und Norwegen steigt die Kodierung mit der Bildung an.

${ }^{175}$ Die Originalfrage der Variablen lautet: „Which, if any, of these qualifications do you have?“. Die Kategorie ,andere“ ist inhaltlich nur schwer zu interpretieren. Sie sagt nichts über die Höhe des Ausbildungsniveaus aus und wird hier daher generell vernachlässigt. Alle anderen Antwortvorgaben weisen mit steigender Kodierung ein höheres Bildungsniveau aus. Jede Ausprägung wird im britischen Datensatz als Dummy-Variable konstruiert, d.h. es wird eine Variable gebildet, die angibt, ob der individuelle Vater diesen Abschluss hat oder nicht. Diese Dummies sind mit den Namen v4m1 bis v4m9 versehen. Das bedeutet, die 0 für „none“ gibt es nicht mehr in der Variablenbezeichnung. Die Häufigkeitsverteilungen der einzelnen 
Die deutschen Ergebnisse neigen in Richtung eines positiven Zusammenhangs zwischen dem väterlichen Bildungsniveau und der Ausgestaltung der Care-Komponente väterlichen Sorgehandelns. Ein steigender Anteil an Vätern berichtet ein sehr intensives Care mit zunehmendem Ausbildungslevel, während Väter häufiger keinen Kontakt zu ihren Kindern haben, wenn sie geringer qualifiziert sind. Ein ähnlicher Hang findet sich auch im britischen Datensatz. Auch hier weisen Väter mit höheren Bildungsabschlüssen häufiger ein sehr intensives Care auf, während der Anteil der hochqualifizierten Väter in den unteren Care-Kategorien abnimmt. Sowohl im deutschen wie auch im britischen Datensatz lassen sich jedoch in den mittleren Bildungs- und Care-Kategorien keine auffälligen Zusammenhangsmuster erkennen. Innerhalb des norwegischen Teils der Kreuztabelle können keine eindeutigen Richtungen ausgemacht werden. Somit muss insgesamt festgehalten werden, dass in zwei der drei Datensätze eine sehr schwache Tendenz in den Extrem-

Dummies werden in SPSS immer mit der ersten Qualifizierung „CSE/GCE/GCSE“ überschrieben. Dies lässt die Vermutung zu, dass bei der Kodierung mit dieser begonnen wurde. Gestützt wird diese Annahme durch die Existenz einer Dummyvariablen, die explizit mit „none“ überschrieben ist. Doch es gibt eine Dummy mehr als Antworten vorgegeben werden. Die Variablen v4m1 bis v4m7 weisen jeweils alle Fälle auf (616). Die Variable v4m8 ist mit „any other“ überschrieben und umfasst 398 Fälle, während Variable v4m9 mit „none“ im Ausgabeviewer betitelt ist und 435 Fälle aufweist. Wenn man davon ausgeht, dass mit der ersten Qualifikation „CSE/GCE/GCSE“ begonnen wurde, dann würden die Variablen v4m1 bis v4m7 den Antwortvorgaben im Fragebogen entsprechen. Dann bliebe jedoch offen, wofür die Variable v4m8 „any other“ steht, da v4m7 die Antwortkategorie „any other“ umfassen müsste. Beide Variablen weisen unterschiedliche Häufigkeitsverteilungen auf, d.h. Väter, die bei der einen Variablen keine anderen Qualifikationen angeben, werden in Variable v4m8 mit anderen Variablen geführt und auch umgekehrt. Mit dieser Inkonsistenz in den Daten wird wie folgt umgegangen: Es wird zunächst der Annahme gefolgt, dass mit der ersten Qualifikation „CSE/GCE/GCSE“ innerhalb der Kodierung begonnen wurde. Das bedeutet, die Variablen v4m1 bis v4m6 stehen jeweils für eine Qualifikation: v4m1 = „CSE/GCE/GCSE“, v4m2 = „ONC or diploma“, v4m3 = „BTECH/A level“, v4m4 = „HNC or diploma“, v4m5 = „,degree“ und v4m6 = „postgraduate“. Die Variable v4m7 erfasst 19 Väter mit einer Angabe, diese werden als mit einer anderen Qualifikation ausgestattet gewertet und aus der Analyse ausgeschlossen, solange sie keinen anderen Abschluss nennen. Fälle, die noch eine der ersten Qualifikationen neben dieser ,anderen“ angeben, werden mit dieser in die Analyse eingehen. Das kann zur Folge haben, dass ihr Ausbildungsniveau unterschätzt wird, weil die ,andere“ nicht spezifizierte Qualifikation höherwertig ist. Aus Mangel an Alternativen muss dies methodisch jedoch zugunsten einer höheren Fallzahl in Kauf genommen werden. Variable v4m9 wird als „keine Qualifizierung“ verstanden, wie sie auch überschrieben sein könnte. 188 Väter geben an, keine Qualifizierung zu haben und weisen auch keine Nennungen in den anderen Qualifikation (v4m1 bis v4m6) auf. Dies scheint mit den inhaltlichen Annahmen konsistent. Bei allen anderen Vätern wurde die höchste Nennung in den Dummies als ihr Ausbildungsniveau verstanden. Entsprechend der beschriebenen Unsicherheiten weist die Variable „Qualifizierung des Vaters“ hohe Ausfälle auf und muss mit großer Vorsicht behandelt werden. Da das Ausbildungsniveau stark mit dem Einkommen korreliert, wurde eine Kreuztabellierung der beiden Variablen vorgenommen. Es bestehen vorsichtige Hinweise, dass das Vorgehen richtig ist. Gegen systematische Ausfälle spricht ferner, dass die britischen Ausfälle mit Blick auf das Ausbildungsniveau keine wesentlichen Unterschiede im väterlichen Sorgehandeln zu Vätern zeigen, bei denen Informationen vorliegen. Dies gilt für das Sorge- ebenso wie für das Unterhaltshandeln gleichermaßen. Auch im norwegischen Datensatz bestehen kaum Differenzen zwischen den beiden Vätergruppen mit und ohne Angaben zu ihrem Bildungsniveau. Lediglich im Zahlungsverhalten treten bei norwegischen Ausfällen etwas häufiger Probleme auf, als bei Vätern, die Angaben machen. Damit könnten diese im norwegischen Fall leicht unterschätzt werden. 
kategorien festzustellen ist, während der norwegische Datensatz auf keinen eindeutigen Zusammenhang hinweist.

Tab. 9.5: Operationalisierungen des Ausbildungsniveaus des Vaters.

\begin{tabular}{|c|c|c|c|}
\hline \multirow[b]{2}{*}{ Frage } & Forsa & Bradshaw & NOVA \\
\hline & $\begin{array}{l}\text { Welchen höchsten } \\
\text { Schulabschluss } \\
\text { haben Sie? }\end{array}$ & $\begin{array}{l}\text { Welche dieser } \\
\text { Qualifizierungen } \\
\text { haben Sie? } \\
\text { Mehrfachnennung } \\
\text { möglich. }\end{array}$ & $\begin{array}{l}\text { Welche } \\
\text { (abgeschlossene) } \\
\text { Ausbildung besitzen } \\
\text { Sie? }\end{array}$ \\
\hline Kategorien & $\begin{aligned} 1= & \text { ohne Abschluss; } \\
2= & \text { Haupt-/Volks- } \\
& \text { schulabschluss } \\
3= & \text { Realschule } \\
& \text { (mittlere Reife) } \\
4= & \text { Abschluss } \\
& \text { Polytechn. } \\
& \text { Oberschule } \\
5= & \text { Fachhochschule; } \\
6= & \text { Hochschulreife } \\
7= & \text { Fach- } / \text { Hoch- } \\
& \text { schulstudium }\end{aligned}$ & $\begin{aligned} 0= & \text { keine } \\
1= & \text { CSE } / \text { GCE } / \\
& \text { GCSE }^{176} ; \\
2= & \text { ONC } / \text { Diplom; } \\
3= & \text { BTECH } / \text { A level } ; \\
4= & \text { HNC } / \text { Diplom; } \\
5= & \text { degree } \\
6= & \text { Postgraduate }\end{aligned}$ & $\begin{aligned} 1= & \text { Oberschule } \\
& \text { (inkl. 9.KI); } \\
2= & \text { Berufs-, Fach-, } \\
& \text { Handelsschule o.ä } \\
3= & \text { Abitur, } \\
& \text { Wirtschafts- } \\
& \text { gymnasium; } \\
4= & \text { Uni unter 4Jahren; } \\
5= & \text { Uni über 4Jahre; }\end{aligned}$ \\
\hline
\end{tabular}

Quelle: eigene Darstellung.

Mit Blick auf Cash weisen die Angaben der deutschen Väter in keine klare Richtung. Der Anteil der Zahlungsprobleme je nach Ausbildungsniveau schwankt zwischen rund 8\% bei den Vätern mit Fachhochschulreife und gut 20\% bei Vätern ohne Abschluss und denen mit Abitur, so dass sich kein eindeutiges Zusammenhangsmuster erschließt. Anders in den britischen und norwegischen Daten, hier nimmt der Anteil der Väter, die ihrer Unterhaltspflicht mit Einschränkungen nachkommen, mit steigendem Bildungsniveau ab, wobei dieser Trend in den norwegischen Ergebnissen am deutlichsten zu erkennen ist. Dadurch werden in zwei der drei Kreuztabellen die theoretischen Annahmen für Cash bestätigt.

176 Die Abkürzungen stehen für: $(\mathrm{G}) \mathrm{C}(\mathrm{S}) \mathrm{E}$ = (General) Certificate of (Secondary) Education; ONC = Ordinary National Certificate; A Level = Advanced Level, B Tech = technische Version des Advanced Level; HNC = Higher National Certificate; degree = Bachelor-Abschluss; Postgraduate = Universitätsabschluss höher als Bachelor, z.B. Master- oder Doktor-Titel. 


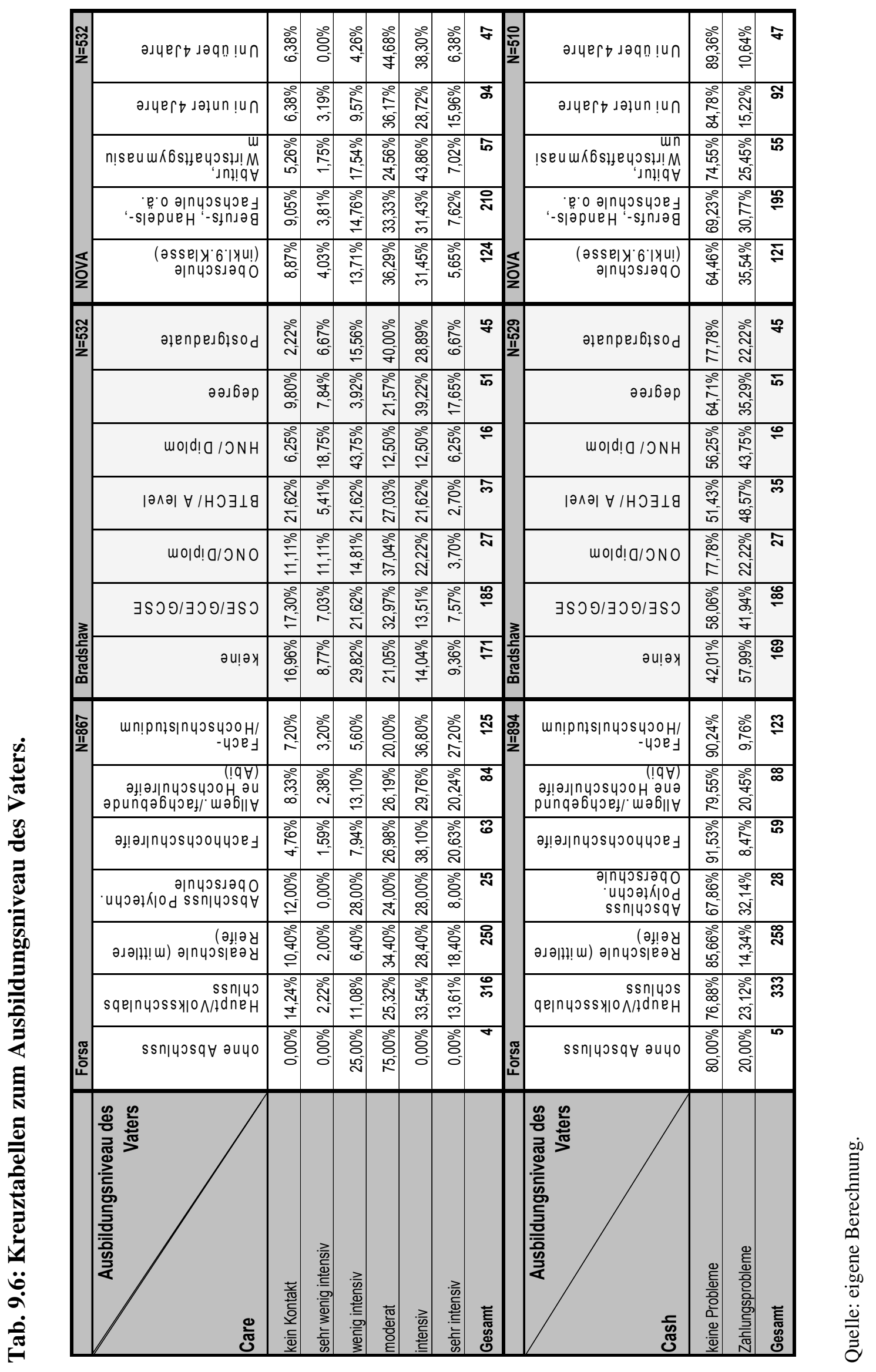


Neben finanziellen Mitteln ist die Ressource Zeit ein wichtiger Bestandteil der physischen Umwelt, der restriktiv auf das Ausmaß väterlichen Sorgehandelns wirken kann. Einen wichtigen Indikator für die Verfügbarkeit zeitlicher Ressourcen stellt die Arbeitszeit des Vaters dar. Es wird angenommen, dass mit steigender Arbeitszeit die mit dem Kind verbrachten Zeitanteile sinken. Längere Arbeitszeiten sollten sich dagegen positiv auf die Zahlungspraxis des Vaters auswirken.

Tab. 9.7: Operationalisierungen der Arbeitszeit des Vaters.

\begin{tabular}{|l|l|l|l|}
\hline \multirow{2}{*}{ Frage } & \multicolumn{1}{|c|}{ Forsa } & \multicolumn{1}{|c|}{ Bradshaw } & \multicolumn{1}{c|}{ NOVA } \\
\cline { 2 - 4 } & - & $\begin{array}{l}\text { Wie viele Stunden arbeiten Sie } \\
\text { normalerweise in der Woche? } \\
\text { Das heißt inklusive der } \\
\text { Überstunden, aber ohne Pausen } \\
\text { in all Ihren bezahlten Jobs. }\end{array}$ & $\begin{array}{l}\text { Wie viele Stunden arbeiten sie } \\
\text { normalerweise wöchentlich? } \\
\text { Rechnen Sie auch bezahlte } \\
\text { Überstunden und Extraarbeit zu } \\
\text { Hause mit ein. }\end{array}$ \\
\hline Metrisch & - & in Stunden pro Woche & in Stunden \\
\hline Kategorien & - & $\begin{array}{l}0=\text { nicht erwerbstätig, } \\
1=\text { unter 30 Stunden; } \\
2=30 \text { bis 40 Stunden; }\end{array}$ & $\begin{array}{l}0=\text { nicht erwerbstätig; } \\
1=\text { unter 30 Stunden; } \\
2=30 \text { bis 40 Stunden; } \\
3=\text { über 40 Stunden }\end{array}$ \\
\hline
\end{tabular}

Quelle: eigene Darstellung.

Wie im norwegischen Datensatz wird die Arbeitszeit des Vaters auch in der britischen Studie in Stunden pro Woche gemessen. Zur besseren Vergleichbarkeit werden die beiden Variablen kategorisiert. Die Angabe der väterlichen Arbeitszeit fehlt in der deutschen Untersuchung gänzlich.

Die Ergebnisse der Kreuztabelle zwischen der väterlichen Arbeitszeit und Care weisen auf kein eindeutiges Bild hin. So brechen erwerbslose britische Väter eher den Kontakt als Väter, die eine gewisse Stundenzahl an Erwerbstätigkeit angeben. Die Anzahl der Stunden scheint darüber hinaus - anders als vermutet - keinen wesentlichen Einfluss auf das väterliche Kümmern zu haben. Väter mit einer Arbeitszeit unter 30 Stunden neigen häufiger dazu, „intensiven“ Kontakt zu ihren Kindern zu haben, als Väter mit höherer Arbeitsintensität. Gleichzeitig weisen diese Väter einen deutlich höheren Anteil an wenig intensivem Care auf. Eine eindeutige Richtung eines Wirkungszusammenhangs kann anhand des britischen Materials nicht ausgemacht werden. Ähnliches trifft für die Ergebnisse der norwegischen Studie zu. Auch hier haben Väter ohne Erwerbstätigkeit häufiger keinen Kontakt zu ihren getrennt lebenden Kindern, als Väter die eine Arbeitszeit angeben kön- 
nen. Innerhalb der jeweiligen Stundenkategorisierungen lassen sich keine augenfälligen Tendenzen ausmachen. Die aufgestellte Hypothese mit Blick auf die Arbeitszeit kann damit in beiden Datensätzen, die die nötigen Informationen dazu liefern, nicht gestützt werden.

Tab. 9.8: Kreuztabellen zur Arbeitszeit des Vaters.

\begin{tabular}{|c|c|c|c|c|c|c|c|c|c|}
\hline & Forsa & \multicolumn{3}{|c|}{ Bradshaw } & $\mathrm{N}=574$ & \multicolumn{3}{|l|}{ NOVA } & \multirow{2}{*}{ 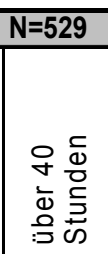 } \\
\hline Care & \multirow{8}{*}{ 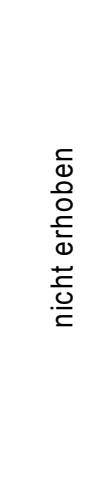 } & 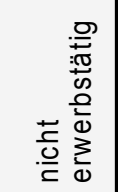 & 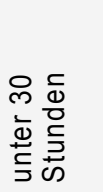 & 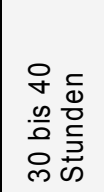 & 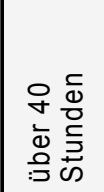 & 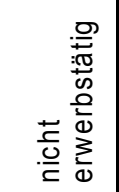 & 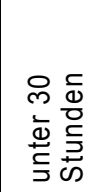 & 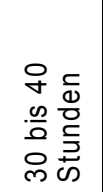 & \\
\hline kein Kontakt & & $20,83 \%$ & $15,79 \%$ & $11,46 \%$ & $11,74 \%$ & $13,73 \%$ & $8,33 \%$ & $6,64 \%$ & $5,44 \%$ \\
\hline sehr wenig intensiv & & $14,88 \%$ & $0,00 \%$ & $4,46 \%$ & $6,09 \%$ & $3,92 \%$ & $4,17 \%$ & $3,13 \%$ & $2,04 \%$ \\
\hline wenig intensiv & & $20,24 \%$ & $36,84 \%$ & $25,48 \%$ & $21,74 \%$ & $13,73 \%$ & $0,00 \%$ & $14,06 \%$ & $12,93 \%$ \\
\hline moderat & & $25,00 \%$ & $15,79 \%$ & $32,48 \%$ & $28,70 \%$ & $31,37 \%$ & $45,83 \%$ & $37,50 \%$ & $26,53 \%$ \\
\hline intensiv & & $10,71 \%$ & $26,32 \%$ & $19,11 \%$ & $22,17 \%$ & $26,47 \%$ & $33,33 \%$ & $30,47 \%$ & $44,22 \%$ \\
\hline sehr intensiv & & $8,33 \%$ & $5,26 \%$ & $7,01 \%$ & $9,57 \%$ & $10,78 \%$ & $8,33 \%$ & $8,20 \%$ & $8,84 \%$ \\
\hline \multirow[t]{2}{*}{ Gesamt } & & 168 & 19 & 157 & 230 & 102 & 24 & 256 & 147 \\
\hline & Forsa & \multicolumn{3}{|c|}{ Bradshaw } & $\mathrm{N}=570$ & \multicolumn{3}{|l|}{ NOVA } & $\mathrm{N}=509$ \\
\hline Arbeitszeit & \multirow{4}{*}{ 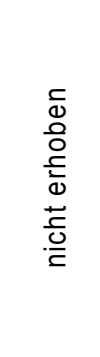 } & 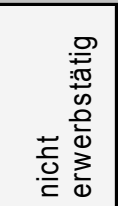 & 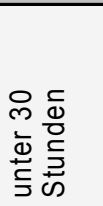 & 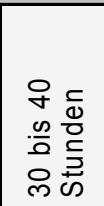 & 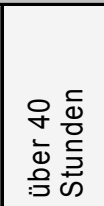 & 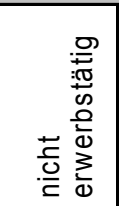 & 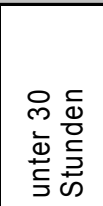 & 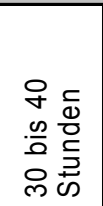 & 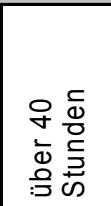 \\
\hline keine Probleme & & $18,56 \%$ & $42,11 \%$ & $70,51 \%$ & $73,68 \%$ & $61,22 \%$ & $68,18 \%$ & $75,30 \%$ & $76,76 \%$ \\
\hline Zahlungsprobleme & & $81,44 \%$ & $57,89 \%$ & $29,49 \%$ & $26,32 \%$ & $38,78 \%$ & $31,82 \%$ & $24,70 \%$ & $23,24 \%$ \\
\hline Gesamt & & 167 & 19 & 156 & 228 & 98 & & 247 & 142 \\
\hline
\end{tabular}

Quelle: eigene Darstellung.

Mit Blick auf die Zahlungspraxis der Väter lassen sich dagegen besser Aussagen über mögliche Zusammenhänge treffen. Die Ergebnisse des britischen Datenmaterials zeigen einen zunehmenden Anteil an Vätern mit steigender Arbeitszeit, die keine Zahlungsschwierigkeiten aufweisen. Während knapp 81\% der erwerbslosen Väter ihren Unterhaltspflichten nicht (vollständig) nachkommen (können), nimmt dieser Anteil sukzessive mit wachsender Zahl der Arbeitsstunden ab. Väter, die mehr als 40 Stunden in der Woche am Arbeitsplatz verbringen, weisen nur in rund 26\% der Fälle Zahlungsschwierigkeiten auf. In eine ähnliche Richtung zeigen die norwegischen Ergebnisse, wenn auch mit weniger deutlichen Differenzen. Väter ohne Erwerbstätigkeit und ohne Zahlungsprobleme machen immer noch die Mehrheit mit etwa 61\% aus, während bei den Vätern mit einer Arbeitswoche von über 40 Stunden mit gut 77\% angeben, keine Zahlungsausfälle zu haben. Damit kann der angenommene negative Zusammenhang zwischen Arbeitszeit und Zahlungsproblemen als gestützt angesehen werden. 


\subsubsection{Der Einfluss der Prädiktoren der mikro-sozialen Umwelt}

Neben den Determinanten der physischen Umwelt kommt ferner der mikro-sozialen Umwelt Bedeutung zu, die u.a. aus der Zusammensetzung des väterlichen Haushalts besteht. Es wird theoretisch angenommen, dass die Anwesenheit weiterer minderjähriger Kinder im Haushalt das väterliche Sorgehandeln gegenüber getrennt lebenden Kindern einschränkt und auch Zahlungsprobleme wahrscheinlicher macht.

Tab. 9.9: Operationalisierungen der Zahl der Kinder im väterlichen Haushalt.

\begin{tabular}{|l|l|l|l|}
\hline \multirow{2}{*}{ Frage } & \multicolumn{1}{|c|}{ Forsa } & \multicolumn{1}{c|}{ Bradshaw } & NOVA \\
\cline { 2 - 4 } & $\begin{array}{l}\text { Wie viele Kinder unter } \\
18 \text { leben bei lhnen? }\end{array}$ & $\begin{array}{l}\text { Konstruiert aus der Zusammensetzung } \\
\text { des Haushalts (alle Kinder unabhängig } \\
\text { von ihrem Status) }\end{array}$ & - \\
\hline Metrisch & - & Anzahl & - \\
\hline Kategorien & $1=$ keins; & $\begin{array}{l}1=\text { kein Kind; } \\
2=1 \text { Kind; }\end{array}$ & - \\
& $2=1$ Kind; & $\begin{array}{l}2=2 \text { Kinder; } \\
3=2 \text { Kinder; }\end{array}$ & $\begin{array}{l}4 \text { Kinder; } \\
5=3 \text { Kinder; }\end{array}$ \\
& $5=4$ und mehr Kinder & 5 und mehr Kinder & \\
\hline
\end{tabular}

Quelle: eigene Darstellung.

Die Angabe über Kinder im väterlichen Haushalt wird im britischen Datensatz - anders als im deutschen - nicht direkt erfasst. Aus den Angaben der Väter zu den einzelnen Mitgliedern ihres Haushalts kann dennoch die Information gewonnen werden, wie viele Kinder - unabhängig von ihrem Status - im väterlichen Haushalt leben. ${ }^{177}$ Die Kategorisierung folgt der Variablen im deutschen Datensatz. Deutsche Väter werden explizit nach der Zahl der unterhaltsberechtigten Kinder in ihrem Haushalt gefragt. Innerhalb der norwegischen Studien sind diese Informationen nicht verfügbar. ${ }^{178}$

177 Das können Kinder des Vaters und seiner aktuellen Partnerin sein, ebenso wie Kinder von vergangenen Partnerschaften, Stief- und Adoptivkinder jeweils vom Befragten selbst oder seiner Partnerin. Ältere Kinder, die bereits in einem eigenen Haushalt leben, wurden durch einen Filter aus der Analyse generell ausgeschlossen.

${ }^{178}$ In der ursprünglichen Fassung des Fragebogens wurde eine Frage formuliert, die nach der derzeitigen Haushaltszusammensetzung des Vaters fragt. Die Frage wurde als Mehrfachnennung mit den Antwortmöglichkeiten „Ehepartnerin“, „Partnerin“, „eigene Kinder aus früheren Beziehungen“, „Kinder der (Ehe)Partnerin“; „(Ehe)Partnerin und gemeinsame Kinder“ und „,andere Erwachsene“ konstruiert. Leider gingen die Mehrfachnennungen aufgrund eines Kodierfehlers verloren. Innerhalb des Datensatzes wurde die Variable mit jeweils nur einer Antwort pro Fall berücksichtigt, wobei unklar bleibt, welche der gegebenen Antworten kodiert wurde. Die Variable ist inhaltlich nicht zu interpretieren. Lediglich die dichotomisierte Frage, ob der Vater derzeit allein lebt, die der Erfassung der Haushaltszusammensetzung vorausgeschaltet wurde, ist verwendbar. Diese liefert jedoch nur sehr geringen interpretativen Spielraum, weil bei einem Vater, der nicht allein lebt, viele Möglichkeiten der Haushaltszusammensetzung denkbar wären, wie die 
Tab. 9.10: Kreuztabellen zur Zahl der Kinder im väterlichen Haushalt.

\begin{tabular}{|c|c|c|c|c|c|c|c|c|c|c|c|}
\hline & Forsa & & & & $\mathrm{N}=872$ & Bradsha & & & & $\mathrm{N}=576$ & NOVA \\
\hline are Kinder im väterl. & 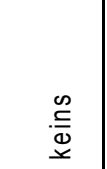 & $\begin{array}{l}\stackrel{0}{\subseteq} \\
\stackrel{x}{\leftarrow}\end{array}$ & 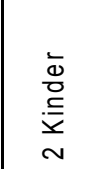 & 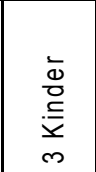 & 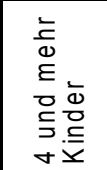 & 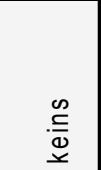 & 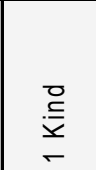 & 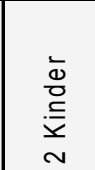 & 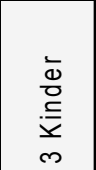 & 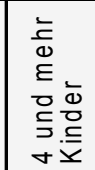 & \multirow{8}{*}{ 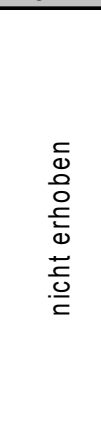 } \\
\hline kein Kontakt & $9,75 \%$ & $13,33 \%$ & $20,00 \%$ & $27,27 \%$ & $0,00 \%$ & $11,86 \%$ & $11,70 \%$ & $34,43 \%$ & \begin{tabular}{|l|}
$19,05 \%$ \\
\end{tabular} & $16,67 \%$ & \\
\hline sehr wenig intensiv & $1,87 \%$ & $1,33 \%$ & $5,71 \%$ & $9,09 \%$ & $50,00 \%$ & $6,96 \%$ & $8,51 \%$ & $9,84 \%$ & $14,29 \%$ & $16,67 \%$ & \\
\hline wenig intensiv & $9,35 \%$ & $12,00 \%$ & $5,71 \%$ & $9,09 \%$ & $50,00 \%$ & $21,39 \%$ & $24,47 \%$ & $29,51 \%$ & $23,81 \%$ & $25,00 \%$ & \\
\hline moderat & $27,50 \%$ & $29,33 \%$ & $31,43 \%$ & $27,27 \%$ & $0,00 \%$ & $30,93 \%$ & $26,60 \%$ & $16,39 \%$ & $23,81 \%$ & $16,67 \%$ & \\
\hline intensiv & $33,11 \%$ & $30,67 \%$ & $20,00 \%$ & $18,18 \%$ & $0,00 \%$ & $19,07 \%$ & $21,28 \%$ & $8,20 \%$ & $14,29 \%$ & $16,67 \%$ & \\
\hline sehr intensiv & $18,42 \%$ & $13,33 \%$ & $17,14 \%$ & $9,09 \%$ & $0,00 \%$ & $9,79 \%$ & $7,45 \%$ & $1,64 \%$ & $4,76 \%$ & $8,33 \%$ & \\
\hline \multirow[t]{2}{*}{ Gesamt } & 749 & 75 & 35 & 11 & 2 & 388 & 94 & 61 & 21 & 12 & \\
\hline & Forsa & & & & $\mathrm{N}=900$ & Bradsha & & & & $\mathrm{N}=571$ & NOVA \\
\hline Kinder im väterl. & 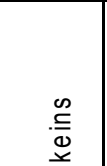 & $\begin{array}{l}\text { 듣 } \\
\text { - }\end{array}$ & 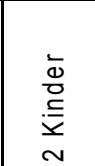 & 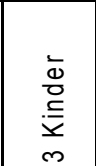 & 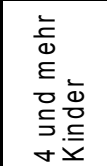 & 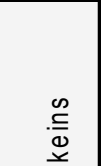 & 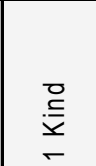 & 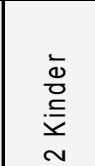 & 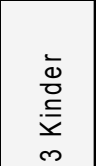 & 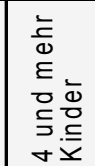 & \multirow{4}{*}{ 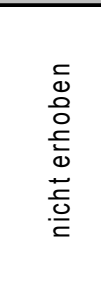 } \\
\hline keine Probleme & $82,13 \%$ & $82,05 \%$ & $83,87 \%$ & $63,64 \%$ & $100,00 \%$ & $57,92 \%$ & $53,76 \%$ & $50,00 \%$ & $38,10 \%$ & $50,00 \%$ & \\
\hline Zahlungsprobleme & $17,87 \%$ & $17,95 \%$ & $16,13 \%$ & $36,36 \%$ & $0,00 \%$ & $42,08 \%$ & $46,24 \%$ & $50,00 \%$ & $61,90 \%$ & $50,00 \%$ & \\
\hline Gesamt & 778 & 78 & 31 & & 2 & 385 & 93 & 60 & 21 & & \\
\hline
\end{tabular}

Quelle: eigene Darstellung.

Die Ergebnisse der Kreuztabelle 9.10 weisen im deutschen Fall auf eine negative Wirkungsrichtung hin. Väter, die nicht mit Kindern in einem Haushalt leben, haben häufiger (sehr) intensiven Kontakt zu ihren getrennt lebenden Kindern, wohingegen Väter ohne Kontakt eher mit weiteren Kindern zusammenwohnen. Dabei steigt der Anteil der Kontaktlosen mit der Zahl der Kinder in der eigenen Lebensgemeinschaft an. Die britischen Daten weisen ähnliche Kontaktmuster der Väter auf, wenn auch nicht in der gleichen Eindeutigkeit wie im deutschen Fall. Auch hier ist der Anteil der Kontaktabbrüche unter den Vätern höher, die mit zwei oder mehr Kindern zusammenleben. Der Anteil steigt jedoch nicht eindeutig mit der Kinderzahl an. Dies kann mit der geringen Fallzahl in den höheren Kategorien der Kinderanzahl begründet werden. ${ }^{179}$ Damit wird der formulierte Zusammenhang zwischen Care und der Anzahl externer Kinder im deutschen Fall gestützt, während die britischen Daten tendenziell in die gleiche Richtung weisen.

Antwortvorgaben vermuten lassen. Dennoch wird die Variable aufgrund von mangelnden Alternativen im Zusammenhang mit der Partnersituation des Vaters Verwendung finden.

${ }^{179}$ Die deutschen Daten weisen in der Variablen zur Anzahl der Kinder im väterlichen Haushalt nur eine geringe Variation auf. Knapp 86\% der befragten Väter in Deutschland gibt an, mit keinem Kind zusammenzuleben. In Großbritannien sind dies mit 67\% deutlich weniger. Die britische Variable weist jedoch starke Ausfälle auf. Die britischen Väter, die keine Angaben zur Anzahl minderjähriger Kinder in ihrem Haushalt machen, zeigen tendenziell etwas mehr sozio-emotionales und finanzielles Engagement, so dass das Niveau der Care-Variable etwas höher liegen könnte, als hier abgebildet. 
Auf die Zahlungspraxis scheint gemäß der deutschen Kreuztabelle die Anzahl der Kinder im Haushalt des Vaters dagegen keinen Einfluss zu haben. Der Anteil der Väter ohne Zahlungsschwierigkeiten ist bei den Vätern mit ein bis zwei Kindern genauso hoch wie bei den Vätern, die nicht mit Kindern zusammenwohnen. Lediglich die Zellen, die die Fälle integrieren, die mit drei und mehr Kindern zusammenleben und die keine Probleme mit den Unterhaltsleistungen aufweisen, sind etwas schwächer besetzt. Dies ist allerdings aufgrund der geringen Fallzahl an Vätern insgesamt, die mit Kindern leben, nicht sonderlich aussagekräftig. Der angenommene Zusammenhang wird somit im deutschen Fall nicht bestätigt. Anders im britischen Material, hier scheinen Väter, die mit Kindern in einem Haushalt leben, tendenziell eher Zahlungsprobleme zu haben als Väter, bei denen keine Kinder wohnen. Dabei steigt mit der Zahl der Kinder im väterlichen Haushalt auch der Anteil der Väter mit Zahlungsproblemen an. Dies lässt auf den erwarteten Zusammenhang schließen.

Neben den Kindern im väterlichen Haushalt wird weiterhin vermutet, dass die Wahrscheinlichkeit eines hohen väterlichen sozio-emotionalen Kümmerns negativ mit der Zahl der unterhaltsberechtigten Kinder außerhalb des väterlichen Haushalts zusammenhängt. Ferner ist anzunehmen, dass gilt: Je höher die Unterhaltsforderungen, desto eher treten Zahlungsschwierigkeiten auf.

Tab. 9.11: Operationalisierungen der Zahl der Kinder außerhalb des väterlichen Haushalts.

\begin{tabular}{|l|l|l|l|}
\hline \multirow{2}{*}{ Frage } & \multicolumn{1}{|c|}{ Forsa } & \multicolumn{1}{c|}{ Bradshaw } & \multicolumn{1}{c|}{ NOVA } \\
\cline { 2 - 4 } & $\begin{array}{l}\text { Und wie viele Kinder } \\
\text { sind das, die bei ihrer } \\
\text { Mutter leben? }\end{array}$ & $\begin{array}{l}\text { Wie viele Ihrer } \\
\text { leiblichen oder } \\
\text { adoptierten Kinder } \\
\text { leben nicht dauerhaft } \\
\text { bei Ihnen? }\end{array}$ & $\begin{array}{l}\text { (konstruiert aus } \\
\text { Häufigkeit der } \\
\text { Altersangaben, max. } \\
\text { zu vier Kindern } \\
\text { vorhanden) }\end{array}$ \\
\hline Metrisch & - & Anzahl & - \\
\hline Kategorien & $1=1$ Kind; & $1=1$ Kind; & $1=1$ Kind; \\
& $2=2$ Kinder; & $2=2$ Kinder; \\
& $3=3$ Kinder; & $3=3$ Kinder; & 2 Kinder; \\
& $4=4$ und mehr Kinder & $4=4$ und mehr Kinder & 4 Kinder; \\
& & &
\end{tabular}

Quelle: eigene Darstellung.

In allen drei Studien finden sich Angaben zu der Zahl der externen unterhaltsberechtigten Kinder. Mit zunehmender Kodierung steigt dabei die Zahl der externen Kinder an. Im britischen Fall wird die entsprechende Variable aus den Einzelinformationen zu den jeweiligen Kindern konstruiert. Dabei werden in der vorliegenden Analyse Kinder unter 17 Jahren, die bei der Mutter leben, sowie 17- und 18-Jährige, die bei der Mutter leben und sich in der Ausbildung befinden, als unterhaltsberechtigte Kinder gezählt. Auch im norwegischen Fall wird nicht direkt nach der Zahl der Unterhaltsverpflichtungen gefragt, die 
Information lässt sich jedoch aus den Angaben zu den einzelnen Kindern rekonstruieren. $^{180}$

Die Kreuztabellierung der abhängigen Variable Care und der Anzahl unterhaltsberechtigter, getrennt lebender Kinder weist in allen drei Datensätzen kein eindeutiges Zusammenhangsmuster auf. Der Vergleich der ersten drei Kategorien - der Anteil an Vätern mit mehr als vier externen Kindern ist in allen drei Studien sehr gering - deutet im britischen und norwegischen Datensatz gar auf einen positiven Zusammenhang hin, der den hier getroffenen theoretischen Annahmen widersprüchlich gegenübersteht. So haben Väter mit einer Unterhaltsverpflichtung deutlich häufiger gar keinen Kontakt als Väter von zwei und mehr externen Kindern. Gleichzeitig weisen Väter mit zwei und drei Kindern eher intensive Kontaktmuster auf als Väter von externen Einzelkindern. Dies gilt für den britischen ebenso wie für den norwegischen Datensatz. Die Angaben der deutschen Väter lassen auf kein einheitliches Ergebnis schließen. Der angenommene Zusammenhang für Care kann somit nicht gestützt werden, die Kreuztabellierung deutet gar die entgegengesetzte Wirkungsrichtung an.

\footnotetext{
${ }^{180}$ Dies setzt jedoch voraus, dass die Väter zu all ihren unterhaltsberechtigten Kindern außerhalb ihres Haushaltes Angaben machen. Des Weiteren werden Väter mit mehr als vier Kindern nur mit vieren in die Analyse eingehen, weil nach weiteren Kindern nicht gefragt wurde. Dennoch gibt die auf diese Weise konstruierte Variable erste Anhaltspunkte über die Anzahl der externen Kinder und ihren Einfluss auf das väterliche Sorgen.
} 


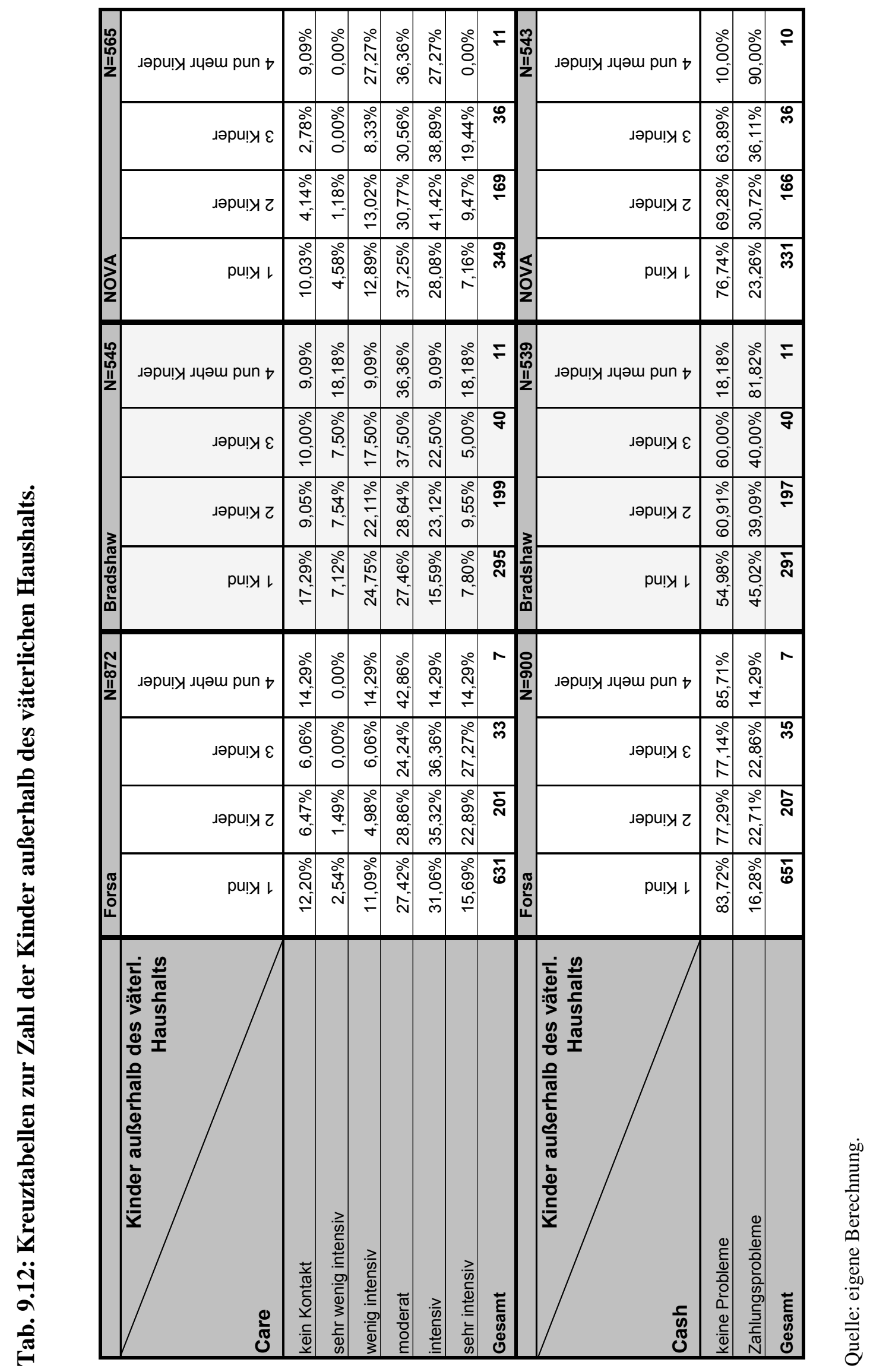


Die Ergebnisse für Cash sind im deutschen und britischen Fall ähnlich uneindeutig wie für Care. Zwar ist der Anteil der deutschen Väter, die nur einem Kind gegenüber unterhaltspflichtig sind, im Fall ausbleibender Unterhaltszahlungen geringer als bei mehreren Kindern, doch mit der steigenden Anzahl getrennt lebender Kinder lassen sich keine weiteren Wirkungszusammenhänge beobachten. Ähnliches gilt für die Ergebnisse der britischen Untersuchung. Hier ist das Verhältnis von unterhaltszahlenden Vätern und Vätern mit Zahlungsproblemen über die ersten drei Kategorien des Prädiktors relativ gleich. Die Kategorie der Väter mit mehr als 3 Kindern weicht deutlich davon ab. Das Verhältnis ist mit deutlich mehr Zahlungsproblemen umgekehrt zu den anderen Ausprägungen. Auf einen eindeutigen Zusammenhang lässt dies nicht schließen, da nur elf Väter angeben, Unterhaltspflichten gegenüber mehr als drei Kinder zu haben. ${ }^{181}$ Dagegen weisen die norwegischen Daten auf einen positiven Zusammenhang zwischen steigenden Zahlungsproblemen und der Anzahl externer Kinder hin. Damit stützen lediglich die Angaben der norwegischen Väter, den angeführten theoretischen Zusammenhang.

Tab. 9.13: Operationalisierungen der Partnersituation des Vaters.

\begin{tabular}{|c|c|c|c|}
\hline & Forsa & Bradshaw & NOVA \\
\hline Frage & $\begin{array}{l}\text { Konstruiert aus s3: } \\
\text { „Welchen } \\
\text { Familienstand haben } \\
\text { Sie derzeit?“; s4: } \\
\text { „Haben Sie derzeit } \\
\text { eine feste } \\
\text { Partnerschaft?“; s5: } \\
\text { Wohnt die Partnerin } \\
\text { mit Ihnen in einem } \\
\text { Haushalt? }\end{array}$ & $\begin{array}{l}\text { (konstruiert aus b1: „Wie } \\
\text { ist Ihr heutiger } \\
\text { Familienstand?“; b4: „Ist } \\
\text { Ihre aktuelle Partnerin } \\
\text { mit Ihnen verheiratet?“ } \\
\text { a1b2 und a1b3: } \\
\text { Haushaltszusammen- } \\
\text { setzung) }\end{array}$ & $\begin{array}{l}\text { Wenn Ihr Kind nicht } \\
\text { bei Ihnen ist, } \\
\text { wohnen Sie allein } \\
\text { oder mit anderen? }\end{array}$ \\
\hline Kategorien & $\begin{aligned} 1= & \text { keine neue } \\
& \text { Partnerin; } \\
2= & \text { neue Partnerin; } \\
3= & \text { neue Partnerin } \\
& \text { im Haushalt; } \\
4= & \text { neue Ehefrau im } \\
& \text { Haushalt }\end{aligned}$ & $\begin{aligned} 1= & \text { keine neue Partnerin } \\
& \text { im Haushalt; } \\
2= & \text { neue Partnerin im } \\
& \text { Haushalt; } \\
3= & \text { neue Ehefrau im } \\
& \text { Haushalt }\end{aligned}$ & $\begin{array}{l}0=\text { mit anderen; } \\
1=\text { allein }\end{array}$ \\
\hline
\end{tabular}

Quelle: eigene Darstellung.

Ein weiterer Aspekt der mikro-sozialen Umwelt stellt die derzeitige Partnersituation des Vaters dar. Es wird angenommen, dass eine neue Partnerin sich hemmend auf das väter-

\footnotetext{
${ }^{181}$ Insgesamt weist der Prädiktor im britischen Datensatz hohe Ausfälle auf, die deutlich weniger sozio-emotionales Engagement und häufiger Zahlungsschwierigkeiten zeigen als Väter, die die Zahl ihrer Unterhaltsverpflichtungen benennen. Damit werden Probleme im Kontakt und im Leisten von Unterhaltszahlungen hier etwas unterschätzt.
} 
liche Sorgehandeln auswirkt und beeinflusst die Wahrscheinlichkeit von Zahlungsschwierigkeiten positiv.

Innerhalb der deutschen Studie liegen die Angaben zur väterlichen Beziehungssituation am differenziertesten vor. Hier werden auch Partnerinnen außerhalb des eigenen Haushalts erfasst, während innerhalb der Bradshaw-Studie die väterliche Partnerschaftssituation mit verschiedenen Variablen erhoben wird, die aber keinerlei Informationen über eine Beziehung ohne gemeinsamen Wohnsitz liefern. Zwei geben in dichotomer Weise an, ob der Vater mit einer neuen Partnerin zusammenlebt bzw. diese geheiratet hat. Die dritte Variable integriert beide Informationen zu einer Variablen, die die Partnerschaftssituation des Vaters insgesamt beschreibt. Als Antwortkategorien liegen vor „keine neue Partnerin im Haushalt“, „neue Partnerin im Haushalt“ und „,neue Ehefrau im Haushalt“. In Norwegen liegen nur begrenzt Informationen zur väterlichen Partnersituation vor. ${ }^{182}$ Lediglich die Frage, ob der Vater den Haushalt mit jemandem teilt, kann als Indikator verwendet werden. Dieser ist aber nur sehr vorsichtig und mit viel Unsicherheit zu interpretieren, da Väter, die mit jemandem in einer Haushaltsgemeinschaft leben, nicht notwendigerweise mit einer neuen Partnerin zusammenwohnen müssen. Neben der Möglichkeit einer Wohngemeinschaft wäre es ferner denkbar, dass getrennt lebende Väter in ihre Herkunftsfamilie zurückkehren. Es bleibt damit offen, mit wem der Vater zusammenlebt. Inhaltlich kann nur die Information, dass der Vater alleine lebt sinnvoll interpretiert werden. Sie gibt an, dass der Vater mit keiner neuen Partnerin und auch keinen weiteren Kindern in seinem Haushalt zusammenwohnt.

Unter den deutschen Vätern kann kein eindeutiger Zusammenhang zwischen der väterlichen Beziehungslage und dem Sorgehandeln ausgemacht werden; die Kreuztabelle weist kein eindeutiges Bild auf. Aus den Ergebnissen des britischen Datensatzes geht dagegen ein negativer Zusammenhang zwischen väterlichem Care und der aktuellen Partnersituation des Vaters hervor. Väter, die mit einer neuen Partnerin zusammenleben, weisen einen höheren Anteil an Kontaktabbrüchen auf als Väter, die keine neue Partnerin im Haushalt haben. Dabei ist der Anteil der Väter ganz ohne Kontakt am höchsten, wenn die neue Beziehung als Ehe formalisiert wurde. Innerhalb der drei höchsten Care-Ausprägungen zeigt sich, dass der Anteil der Väter mit steigender Formalisierung der neuen Partnerschaft abnimmt. Auch die norwegischen Angaben deuten einen Zusammenhang zwischen der väterlichen Beziehungssituation und dem väterlichen Kümmern in Form von Care an. Alleinlebende Väter weisen ein höheres Niveau sozio-emotionalen Engagements auf.

${ }^{182}$ Es handelt sich hierbei, wie dargestellt, um einen Kodierfehler. 
Zwei von drei Datensätzen liefern somit Hinweise, dass die aufgestellte Hypothese empirisch stimmt.

Tab. 9.14: Kreuztabellen zur Partnersituation des Vaters.

\begin{tabular}{|c|c|c|c|c|c|c|c|c|c|}
\hline & Forsa & & & $\mathrm{N}=869$ & Bradsha & $\overline{a w}$ & $\mathrm{~N}=416$ & NOVA & $\mathrm{N}=554$ \\
\hline $\begin{array}{r}\text { Partnersituation } \\
\text { des Vaters }\end{array}$ & 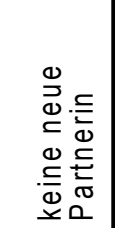 & 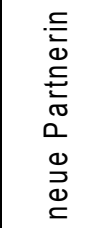 & 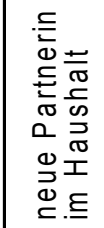 & 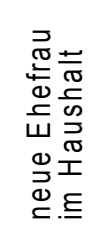 & 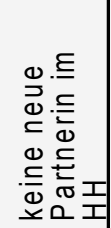 & 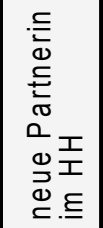 & 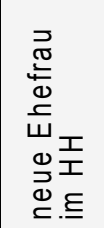 & 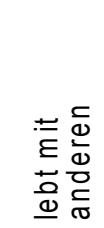 & 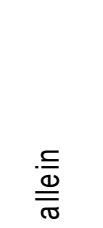 \\
\hline kein Kontakt & $8,09 \%$ & $6,04 \%$ & $20,00 \%$ & $18,03 \%$ & $10,69 \%$ & $11,86 \%$ & $25,74 \%$ & $8,92 \%$ & $5,00 \%$ \\
\hline sehr wenig intensiv & $2,02 \%$ & $1,65 \%$ & $1,67 \%$ & $4,10 \%$ & $7,55 \%$ & \begin{tabular}{|l|}
$6,78 \%$ \\
\end{tabular} & $8,82 \%$ & $4,14 \%$ & $2,08 \%$ \\
\hline wenig intensiv & $8,09 \%$ & $12,64 \%$ & $5,83 \%$ & $13,93 \%$ & $21,07 \%$ & $28,81 \%$ & $22,06 \%$ & $14,97 \%$ & $9,58 \%$ \\
\hline moderat & $29,21 \%$ & $23,08 \%$ & $25,00 \%$ & $30,33 \%$ & $29,87 \%$ & $27,97 \%$ & $25,00 \%$ & $36,62 \%$ & $33,33 \%$ \\
\hline intensiv & $35,96 \%$ & $30,77 \%$ & $30,83 \%$ & $22,13 \%$ & $19,18 \%$ & $17,80 \%$ & $16,18 \%$ & $29,62 \%$ & $37,50 \%$ \\
\hline sehr intensiv & $16,63 \%$ & $25,82 \%$ & $16,67 \%$ & $11,48 \%$ & $11,64 \%$ & \begin{tabular}{|l|}
$6,78 \%$ \\
\end{tabular} & $2,21 \%$ & $5,73 \%$ & $12,50 \%$ \\
\hline \multirow[t]{2}{*}{ Gesamt } & 445 & 182 & 120 & 122 & 318 & 118 & 136 & 314 & 240 \\
\hline & Forsa & & & $\mathrm{N}=897$ & Bradsha & & $\mathrm{N}=414$ & NOVA & $\mathrm{N}=534$ \\
\hline $\begin{array}{r}\text { Partnersituation } \\
\text { des Vaters }\end{array}$ & 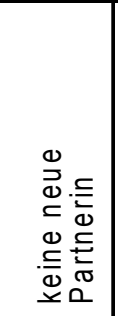 & 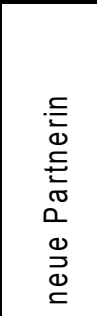 & 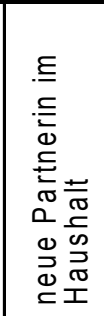 & 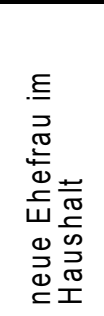 & 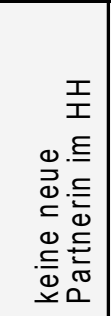 & 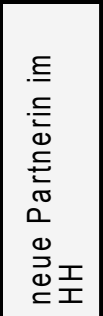 & 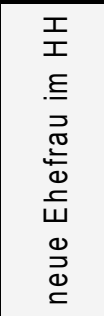 & 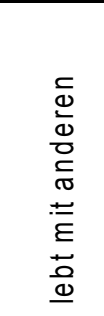 & 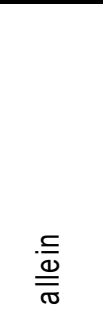 \\
\hline keine Probleme & $81,34 \%$ & $83,78 \%$ & $82,26 \%$ & $81,10 \%$ & $50,48 \%$ & $60,68 \%$ & $62,96 \%$ & $73,58 \%$ & $71,49 \%$ \\
\hline Zahlungsprobleme & $18,66 \%$ & $16,22 \%$ & $17,74 \%$ & $18,90 \%$ & $49,52 \%$ & $39,32 \%$ & $37,04 \%$ & $26,42 \%$ & $28,51 \%$ \\
\hline Gesamt & 461 & 185 & 124 & 127 & 315 & 117 & 135 & 299 & 235 \\
\hline
\end{tabular}

Quelle: eigene Darstellung.

Unterhaltsprobleme scheinen im deutschen Datensatz unabhängig von der Partnersituation des Vaters zu sein. Die Anteile der Väter, die Unterhalt leisten, und derer, die Zahlungsprobleme aufweisen, ist über alle Kategorien des Prädiktors annähernd gleich. Im Fall der britischen Nachtrennungsväter steigt der Anteil der Väter ohne Unterhaltsschwierigkeiten sogar mit zunehmender Formalisierung der väterlichen Beziehung an. Dies deutet auf einen entgegengesetzten Zusammenhang hin, wie er theoretisch postuliert wurde. Die norwegischen Daten weisen wie die deutschen keinen Zusammenhang aus. Damit kann die angenommene Korrelation anhand der Kreuztabellierung nicht bestätigt werden.

Neben der väterlichen Partnerschaftssituation wird theoretisch ein Einfluss der aktuellen Beziehungssituation der Mutter vermutet. Hierbei steht ein neuer Partner im Haushalt von Mutter und Kind einem intensiven Care des getrennt lebenden Vaters möglicherweise entgegen. Ebenso können Zahlungsschwierigkeiten wahrscheinlicher werden. 
Tab. 9.15: Operationalisierungen zur Partnersituation der Mutter.

\begin{tabular}{|c|c|c|c|}
\hline & Forsa & Bradshaw & NOVA \\
\hline Frage & $\begin{array}{l}\text { (konstruiert aus } \\
\text { f51_1: „Lebt die } \\
\text { Mutter des Kindes } \\
\text { heute mit einem } \\
\text { Partner } \\
\text { zusammen?"; f52_1: } \\
\text { „Und ist die Mutter } \\
\text { des Kindes } \\
\text { verheiratet?") }\end{array}$ & $\begin{array}{l}\text { Lebt Ihre letzte Ex- } \\
\text { Partnerin mit einem } \\
\text { neuen Ehemann } \\
\text { oder Partner? }\end{array}$ & $\begin{array}{l}\text { Ist die Kindesmutter } \\
\text { eine Ehe oder } \\
\text { zusammenwohnende } \\
\text { Partnerschaft } \\
\text { eingegangen, seit Ihr } \\
\text { Verhältnis endete? }\end{array}$ \\
\hline Kategorien & $\begin{aligned} 1= & \text { kein neuer } \\
& \text { Partner im } \\
& \text { Haushalt; } \\
2= & \text { neuer Partner im } \\
& \text { Haushalt; } \\
3= & \text { neuer Ehemann } \\
& \text { im Haushalt }\end{aligned}$ & $\begin{aligned} 1= & \text { keine neue } \\
& \text { Partnerschaft } \\
2= & \text { neue } \\
& \text { Partnerschaft }\end{aligned}$ & $\begin{array}{l}1=\text { kein neuer Partner; } \\
2=\text { neuer Partner }\end{array}$ \\
\hline
\end{tabular}

Quelle: eigene Darstellung.

Mit Blick auf die Partnerschaftssituation der Mutter wird im britischen Datensatz wie in Norwegen aber anders als in Deutschland nicht zwischen Kohabitation und Ehe unterschieden. Die dichotomisierte Frage lautet, ob die Ex-Partnerin mit einem neuen Ehemann oder Partner lebt. Dabei wird hier - entgegen der norwegischen Variable - die derzeitige Beziehungssituation der Mutter abgebildet. Weniger institutionalisierte Beziehungen, d.h. Partner, die nicht im Haushalt der Mutter leben, können auf diese Weise in den beiden Datensätzen nicht erfasst werden. Die Informationen zur Partnersituation der Mutter im norwegischen Datensatz sind darüber hinaus aufgrund der Frageformulierung begrenzt interpretierbar. Die Frage, ob die Mutter jemals seit der Trennung eine Partnerschaft kohabitierend oder gar ehelich - eingegangen ist, sagt nichts über die aktuelle Beziehungssituation der Mutter aus. Partnerschaften können inzwischen wieder beendet sein, was jedoch einen längeren Zeitraum seit der Trennung voraussetzt. Ferner kann vermutet werden, dass Beziehungen, die in einem gemeinsamen Haushalt bzw. als Ehe gelebt werden prinzipiell länger andauern. Aus Mangel an Alternativen wird die Variable als Indikator für die Partnerschaftssituation der Mutter für den norwegischen Fall verwendet. Am ausdifferenziertesten gibt die deutsche Variable Auskunft über eine mögliche neue Partnerschaft der Mutter. Doch auch hier kann neben einem Partner bzw. Ehemann im Haushalt keine Partnerschaft ohne gemeinsamen Wohnort ausgemacht werden. Für alle Daten- 
sätze gilt, dass die Variable aufgrund der Unkenntnis der Väter über die neue Beziehungssituation der Mütter mit hohen Ausfällen belastet ist. ${ }^{183}$

Tab. 9.16: Kreuztabellen zur Partnersituation der Mutter.

\begin{tabular}{|c|c|c|c|c|c|c|c|}
\hline & Forsa & & $\mathrm{N}=750$ & Bradshaw & $\mathrm{N}=506$ & $\overline{\text { NOVA }}$ & $\mathrm{N}=507$ \\
\hline $\begin{array}{r}\text { Partnersituation der } \\
\text { Mutter }\end{array}$ & 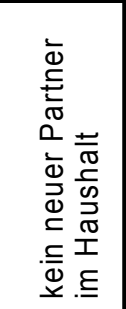 & 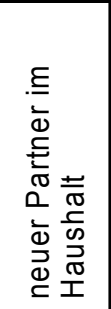 & 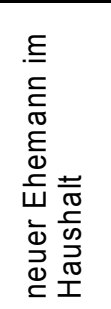 & 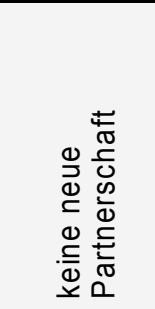 & 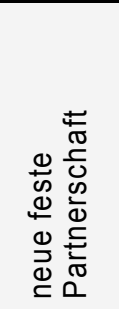 & 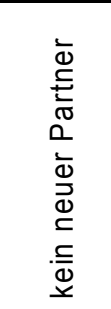 & 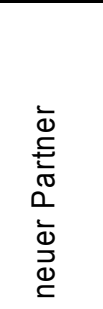 \\
\hline kein Kontakt & $3,25 \%$ & $6,49 \%$ & $12,78 \%$ & $3,29 \%$ & $16,04 \%$ & $1,06 \%$ & $8,46 \%$ \\
\hline sehr wenig intensiv & $1,62 \%$ & $1,15 \%$ & $3,89 \%$ & $2,82 \%$ & $9,22 \%$ & $2,13 \%$ & $3,13 \%$ \\
\hline wenig intensiv & $6,82 \%$ & $10,69 \%$ & $10,56 \%$ & $24,88 \%$ & $23,55 \%$ & $10,64 \%$ & $14,42 \%$ \\
\hline moderat & $29,55 \%$ & $26,72 \%$ & $32,78 \%$ & $31,92 \%$ & $29,35 \%$ & $34,04 \%$ & $35,74 \%$ \\
\hline intensiv & $38,31 \%$ & $36,26 \%$ & $22,22 \%$ & $24,41 \%$ & $15,36 \%$ & $40,96 \%$ & $30,72 \%$ \\
\hline sehr intensiv & $20,45 \%$ & $18,70 \%$ & $17,78 \%$ & $12,68 \%$ & $6,48 \%$ & $11,17 \%$ & $7,52 \%$ \\
\hline Gesamt & 308 & 262 & 180 & 213 & 293 & 188 & 319 \\
\hline & \multicolumn{2}{|l|}{ Forsa } & $\mathrm{N}=775$ & Bradshaw & $\mathrm{N}=502$ & NOVA & $\mathrm{N}=488$ \\
\hline $\begin{array}{r}\text { Partnersituation d } \\
\text { Mutt }\end{array}$ & 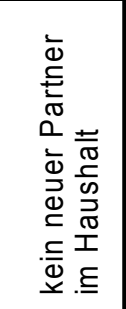 & 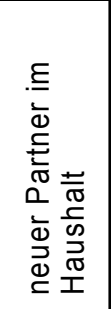 & 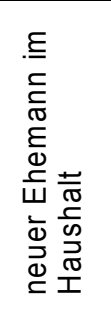 & 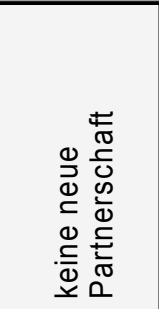 & 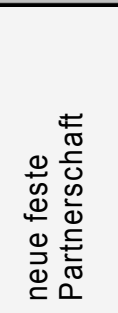 & 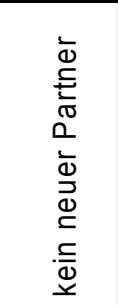 & 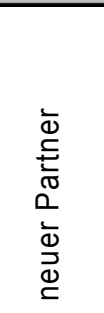 \\
\hline keine Probleme & $83,39 \%$ & $83,33 \%$ & $83,33 \%$ & $61,61 \%$ & $56,70 \%$ & $76,54 \%$ & $72,82 \%$ \\
\hline Zahlungsprobleme & $16,61 \%$ & $16,67 \%$ & $16,67 \%$ & $38,39 \%$ & $43,30 \%$ & $23,46 \%$ & $27,18 \%$ \\
\hline Gesamt & 319 & 270 & 186 & 211 & 291 & 179 & 309 \\
\hline
\end{tabular}

Quelle: eigene Berechnung.

Die Ergebnisse der Kreuztabelle zeigen im deutschen Fall einen negativen Zusammenhang zwischen der Intensität des väterlichen Cares und der aktuellen Beziehungssituation der Kindsmutter. Der Anteil der Väter, die keinen, kaum oder wenig Kontakt zu ihren Kindern haben, steigt mit der Formalisierung der mütterlichen Partnerschaft an. Die ExPartnerin von Vätern, die häufigen und intensiven Kontakt zu ihren getrennt lebenden Kindern pflegen, sind häufiger nicht in einer neuen Lebensgemeinschaft. In die gleiche

183 Getrennt lebende Mütter informieren nicht zwingend ihren Ex-Partner über ihre neue Beziehungssituation. Deutsche und norwegische Väter, die nicht über die neue Partnersituation der Kindsmutter informiert sind, zeigen dabei deutlich weniger Intensität in ihrem sozio-emotionalen Sorgehandeln, als Väter, die über die Beziehungslage ihrer Ex-Partnerin Auskunft geben können. Unter britischen Vätern ist die Differenz zwischen Befragten, die Angaben machen und denen die keine Kenntnis besitzen, sogar noch ausgeprägter. Deutsche, britische und norwegische Väter, die keine Angaben zur Beziehungssituation der ExPartnerin machen können, zeigen ferner häufiger Zahlungsschwierigkeiten. 
Richtung weisen die britischen und norwegischen Daten, wobei aufgrund der empirischen Grundlage in beiden Fällen das (Nicht-)Vorliegen einer neuen Partnerschaft lediglich dichotom differenziert werden kann. Sowohl in Norwegen als auch in Großbritannien zeigen Väter ein höheres Care-Niveau, wenn die ehemalige Partnerin keine neue Beziehung eingegangen ist. Damit lässt sich die theoretische Annahme eines negativen Zusammenhangs mit Hilfe aller drei Datensätze stützen.

Mit Blick auf finanzielle Unterstützungsleistungen des Vaters scheinen in allen drei Studien keine Zusammenhänge mit der Beziehungssituation der Mutter vorzuliegen. Das Verhältnis unterhaltsleistender und nicht zahlender Väter ist über alle Kategorien der erklärenden Variablen annähernd gleich.

Als eine weitere Determinante väterlichen Sorgehandelns wird die Beziehung der Eltern vor, während und nach der Trennung betrachtet. So wird beispielsweise der Formalisierungsgrad vor der elterlichen Trennung als ein Indikator für das väterliche Commitment gegenüber Mutter und Kind verstanden. Mit zunehmender Verbindlichkeit der elterlichen Beziehung vor der Trennung steigt - so die Annahme - die Wahrscheinlichkeit eines intensiven väterlichen Engagements sowie zuverlässiger Unterhaltsleistungen.

Tab. 9.17: Operationalisierungen der Familienform der Eltern vor der Trennung

\begin{tabular}{|c|c|c|c|}
\hline & Forsa & Bradshaw & NOVA \\
\hline Frage & $\begin{array}{l}\text { (konstruiert aus f13_1: } \\
\text { "Sind Sie mit der } \\
\text { Mutter des Kindes } \\
\text { noch verheiratet und } \\
\text { leben getrennt, sind } \\
\text { Sie von ihr } \\
\text { geschieden oder } \\
\text { waren Sie nie mit ihr } \\
\text { verheiratet?; f14d: } \\
\text { "Und seit welchem } \\
\text { Jahr wohnen sie nicht } \\
\text { mehr zusammen?") }\end{array}$ & $\begin{array}{l}\text { (konstruiert aus d1: } \\
\text { „Haben Sie und (Name } \\
\text { der Ex-Partnerin) } \\
\text { jemals zusammen- } \\
\text { gewohnt (verheiratet } \\
\text { oder nicht)?“; d3: } \\
\text { „Waren Sie } \\
\text { miteinander } \\
\text { verheiratet?“ }\end{array}$ & $\begin{array}{l}\text { In welchem } \\
\text { Verhältnis standen } \\
\text { sie zur Kindes- } \\
\text { mutter? }\end{array}$ \\
\hline Kategorien & $\begin{aligned} 1 \text { = } & \text { nicht zus.gelebt; } \\
2= & \text { zus.gelebt, } \\
& \text { unverheiratet; } \\
3= & \text { verheiratet, } \\
& \text { zus.gelebt }\end{aligned}$ & $\begin{aligned} 1= & \text { nicht zus.gelebt; } \\
2= & \text { zus.gelebt, } \\
& \text { unverheiratet } \\
3= & \text { verheiratet, } \\
& \text { zus.gelebt }\end{aligned}$ & $\begin{aligned} 1= & \text { nicht zus.gelebt; } \\
2= & \text { zus.gelebt, } \\
& \text { unverheiratet } \\
3= & \text { verheiratet, } \\
& \text { zus.gelebt }\end{aligned}$ \\
\hline
\end{tabular}

Quelle: eigene Darstellung.

Innerhalb der deutschen Untersuchung werden die Informationen zum Familienstand vor der elterlichen Trennung mit Hilfe zweier Variablen rekonstruiert. Zum einen werden die deutschen Väter gefragt, ob sie mit der Kindsmutter verheiratet waren. Zum anderen geben sie an, seit wann sie nicht mehr mit ihr zusammenleben. Hier gibt es die Antwortvorgabe, dass die Eltern nie zusammen gelebt haben. Beide Informationen zusammen ergeben die hier Verwendung findende Variable zur elterlichen Beziehungssituation vor der 
Trennung. Im britischen Datensatz wird die Partnersituation der Eltern vor der Trennung wie die aktuelle Partnersituation des Vaters mittels dreier Variablen erfasst. Dabei wird zunächst nach Kohabitation und Ehe differenziert. Beide Dimensionen werden in einer dritten Variablen zum damaligen Familienstand integriert. Norwegische Väter werden direkt danach gefragt, in welchem Verhältnis sie zur Kindsmutter standen. In allen drei Studien kann zwischen „nicht zusammengelebt“, „unverheiratet, zusammengelebt“ sowie „verheiratet, zusammengelebt“ differenziert werden. Aufgrund der zunehmenden Formalisierung wird die Kodierung als ansteigende Verbindlichkeit der elterlichen Beziehung interpretiert.

Tab. 9.18: Kreuztabellen zur Familienform der Eltern vor der Trennung.

\begin{tabular}{|c|c|c|c|c|c|c|c|c|c|}
\hline & Forsa & & $\mathrm{N}=872$ & Bradsh & & $\mathrm{N}=574$ & NOVA & & $\mathrm{N}=557$ \\
\hline $\begin{array}{r}\text { Familienform der } \\
\text { Eltern }\end{array}$ & 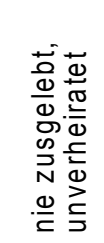 & 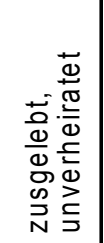 & 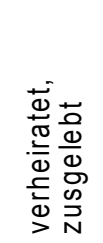 & 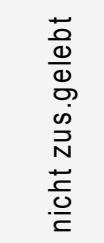 & 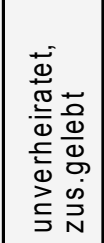 & 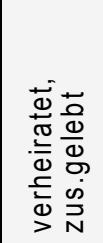 & 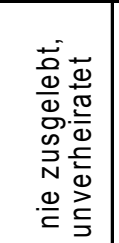 & 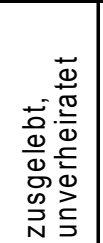 & 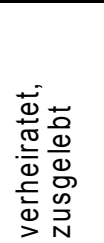 \\
\hline kein Kontakt & $20,78 \%$ & $17,44 \%$ & $7,54 \%$ & $26,83 \%$ & $17,31 \%$ & $12,35 \%$ & $27,40 \%$ & $5,70 \%$ & $3,91 \%$ \\
\hline sehr wenig intensiv & $5,19 \%$ & $5,23 \%$ & $0,96 \%$ & $7,32 \%$ & $10,58 \%$ & $7,46 \%$ & $4,11 \%$ & $2,63 \%$ & $3,52 \%$ \\
\hline wenig intensiv & $16,88 \%$ & $11,63 \%$ & $8,03 \%$ & $24,39 \%$ & $22,12 \%$ & $23,08 \%$ & $6,85 \%$ & $13,16 \%$ & $14,84 \%$ \\
\hline moderat & $19,48 \%$ & $30,81 \%$ & $27,93 \%$ & $17,07 \%$ & $27,88 \%$ & $29,37 \%$ & $34,25 \%$ & $34,21 \%$ & $35,16 \%$ \\
\hline intensiv & $31,17 \%$ & $24,42 \%$ & $34,35 \%$ & $12,20 \%$ & $15,38 \%$ & $19,35 \%$ & $21,92 \%$ & $35,09 \%$ & $34,38 \%$ \\
\hline sehr intensiv & $6,49 \%$ & $10,47 \%$ & $21,19 \%$ & $12,20 \%$ & $6,73 \%$ & $8,39 \%$ & $5,48 \%$ & $9,21 \%$ & $8,20 \%$ \\
\hline Gesamt & 77 & 172 & 623 & 41 & 104 & 429 & 73 & 228 & 256 \\
\hline & Forsa & & $\mathrm{N}=900$ & Bradsh & & $\mathrm{N}=569$ & NOVA & & $\mathrm{N}=488$ \\
\hline $\begin{array}{r}\text { milienform der } \\
\text { Eltern }\end{array}$ & 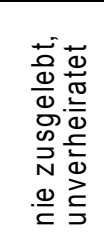 & 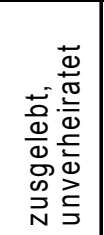 & 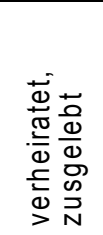 & 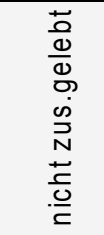 & 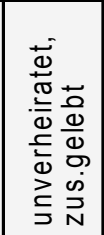 & 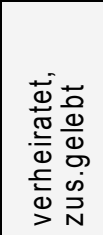 & 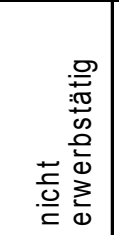 & 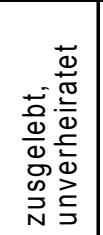 & 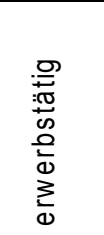 \\
\hline keine Probleme & $84,62 \%$ & $82,76 \%$ & $81,48 \%$ & $51,22 \%$ & $33,01 \%$ & $61,65 \%$ & $70,00 \%$ & $70,70 \%$ & $74,40 \%$ \\
\hline Zahlungsprobleme & $15,38 \%$ & $17,24 \%$ & $18,52 \%$ & $48,78 \%$ & $66,99 \%$ & $38,35 \%$ & $30,00 \%$ & $29,30 \%$ & $25,60 \%$ \\
\hline Gesamt & 78 & 174 & 648 & 41 & 103 & 425 & 70 & 215 & 250 \\
\hline
\end{tabular}

Quelle: eigene Berechnung.

Sowohl die Ergebnisse der Kreuztabellierung im deutschen Datensatz wie auch im britischen Survey weisen auf einen positiven Zusammenhang zwischen der steigenden Formalisierung der elterlichen Beziehung vor der Trennung und dem Ausmaß väterlichen Sorgehandelns in Form von Care hin. Väter, die unverheiratet mit der Mutter in einer Partnerschaft lebten, ohne einen gemeinsamen Haushalt zu führen, weisen in beiden Studien einen deutlich höheren Anteil an Kontaktabbrüchen auf als Väter, die die Kindsmutter geheiratet haben und mit ihr zusammenlebten. Gleichzeitig findet sich in den beiden höchsten Kategorien des väterlichen Cares sowohl im deutschen als auch im britischen Datensatz ein Anstieg der Zellenbesetzung mit zunehmender Kodierung der 
Formalisierung der elterlichen Beziehung. Die Ergebnisse in Deutschland und Großbritannien stützen damit den prognostizierten Zusammenhang. Anders dagegen die Angaben der norwegischen Väter. Hier lässt sich nicht ohne Weiteres eine eindeutige Zusammenhangsrichtung erkennen. Zwar weisen Väter, die keine formalisierte Beziehung zur Kindesmutter aufweisen, d.h. weder mit ihr gelebt noch verheiratet waren, deutlich häufiger Kontaktabbrüche zu ihren getrennt lebenden Kinder auf als Väter, die mit der Mutter gelebt oder und mit ihr verheiratet waren, doch die Unterschiede zwischen den Kategorien „nicht eheliche Lebensgemeinschaft“ und „Ehe“ sind als marginal zu charakterisieren. Damit stützen die norwegischen Ergebnisse - sehr vorsichtig formuliert - tendenziell die aufgestellte Hypothese.

Sowohl im norwegischen als auch im deutschen Datensatz lässt sich kein Zusammenhang zwischen der Cash-Variablen und dem früheren Familienstand ausmachen. Das Verhältnis zwischen Unterhaltsleistungen und Zahlungsproblemen ist in beiden Datensätzen über die Kategorien gleich ausgestaltet. Daher kann der negativ postulierte Zusammenhang als nicht bestätigt angesehen werden. Auch die britischen Ergebnisse weisen keinerlei Korrelationstendenzen auf. Es ist an dieser Stelle jedoch auf die Merkwürdigkeit hinzuweisen, dass sich in den beiden Kategorien „nicht zusammengelebt“ und „verheiratet, zusammengelebt“ ein höherer Anteil an Vätern findet, die keine Unterhaltsprobleme aufweisen. In der ersten Kategorie liegt dieser leicht über 50\%, während die ehemals verheirateten Väter mit 60\% Unterhaltszahlern einen deutlich höheren Anteil ohne Schwierigkeiten stellen. Erstaunlicherweise dreht sich dieses Verhältnis in der mittleren Kategorie der nicht-ehelichen Lebensgemeinschaft um. Knapp 33\% der Väter, die mit der Kindesmutter zusammengelebt haben, kommen ihren Zahlungsverpflichtungen nach, während rund 67\% Schwierigkeiten in den Unterhaltsleistungen angeben. Auch hier kann wie in den anderen beiden Datensätzen kein eindeutiger Zusammenhang ausgemacht werden, aber die Verhältnisse über die Ausprägungen der erklärenden Variablen variieren erheblich. Insgesamt kann somit die formulierte Hypothese für den Zusammenhang zwischen der früheren Partnerschaft der Eltern und dem heutigen Zahlungsverhalten des Vaters empirisch in allen drei Datensätzen mittels der Kreuztabellierung nicht gestützt werden.

Einen weiteren Indikator für die Verbindlichkeit der elterlichen Beziehung vor der Trennung stellt die Dauer der Beziehung dar. Mit zunehmender Dauer der früheren Partnerschaft steigt auch die Wahrscheinlichkeit eines intensiven Vater-Kind-Kontakts nach der elterlichen Trennung. Mit einem längeren Fortbestand der elterlichen Partnerschaft steigt ebenso die Einsicht in finanzielle Verpflichtungen.

Der deutsche Datensatz liefert keinerlei Informationen zur Dauer der elterlichen Beziehung. Der norwegische Datensatz fragt nach der Dauer der Ehe bzw. des Zusammenlebens. Beide Angaben müssen nicht notwendigerweise die gleiche Information bein- 
halten. Bei einer partnerschaftlichen Trennung wird zuerst der gemeinsame Haushalt aufgelöst und dann die Scheidung vollzogen, wohingegen in Großbritannien nur nach der Dauer des Zusammenlebens gefragt wird. Beide Variablen gehen als Indikatoren für die Dauer der elterlichen Beziehung metrisch in die Regressionen ein. Für die Kreuztabellen werden gleiche Kategorien verwendet.

Tab. 9.19: Operationalisierungen der Dauer der Beziehung vor der Trennung.

\begin{tabular}{|c|c|c|c|}
\hline & Forsa & Bradshaw & NOVA \\
\hline Frage & - & $\begin{array}{l}\text { Wie lange haben Sie im Ganzen } \\
\text { (verheiratet oder nicht) } \\
\text { zusammengelebt? }\end{array}$ & $\begin{array}{l}\text { Wie lange dauerte die Ehe/ } \\
\text { das Zusammenwohnen? }\end{array}$ \\
\hline Metrisch & - & In Monaten & In Jahren \\
\hline Kategorien & - & $\begin{array}{l}0=\text { nie zusammengelebt; } \\
1=\text { unter } 5 \text { Jahren; } \\
2=5 \text { bis } 10 \text { Jahre; } \\
3=11 \text { bis } 15 \text { Jahre; } \\
4=\text { über } 16 \text { Jahre }\end{array}$ & $\begin{array}{l}0=\text { nie zusammengelebt; } \\
1=\text { unter } 5 \text { Jahre; } \\
2=5 \text { bis } 10 \text { Jahre; } \\
3=11 \text { bis } 16 \text { Jahre; } \\
4=\text { über } 16 \text { Jahre }\end{array}$ \\
\hline
\end{tabular}

Quelle: eigene Darstellung.

Tab. 9.20: Kreuztabellen zur Dauer der Beziehung vor der Trennung.

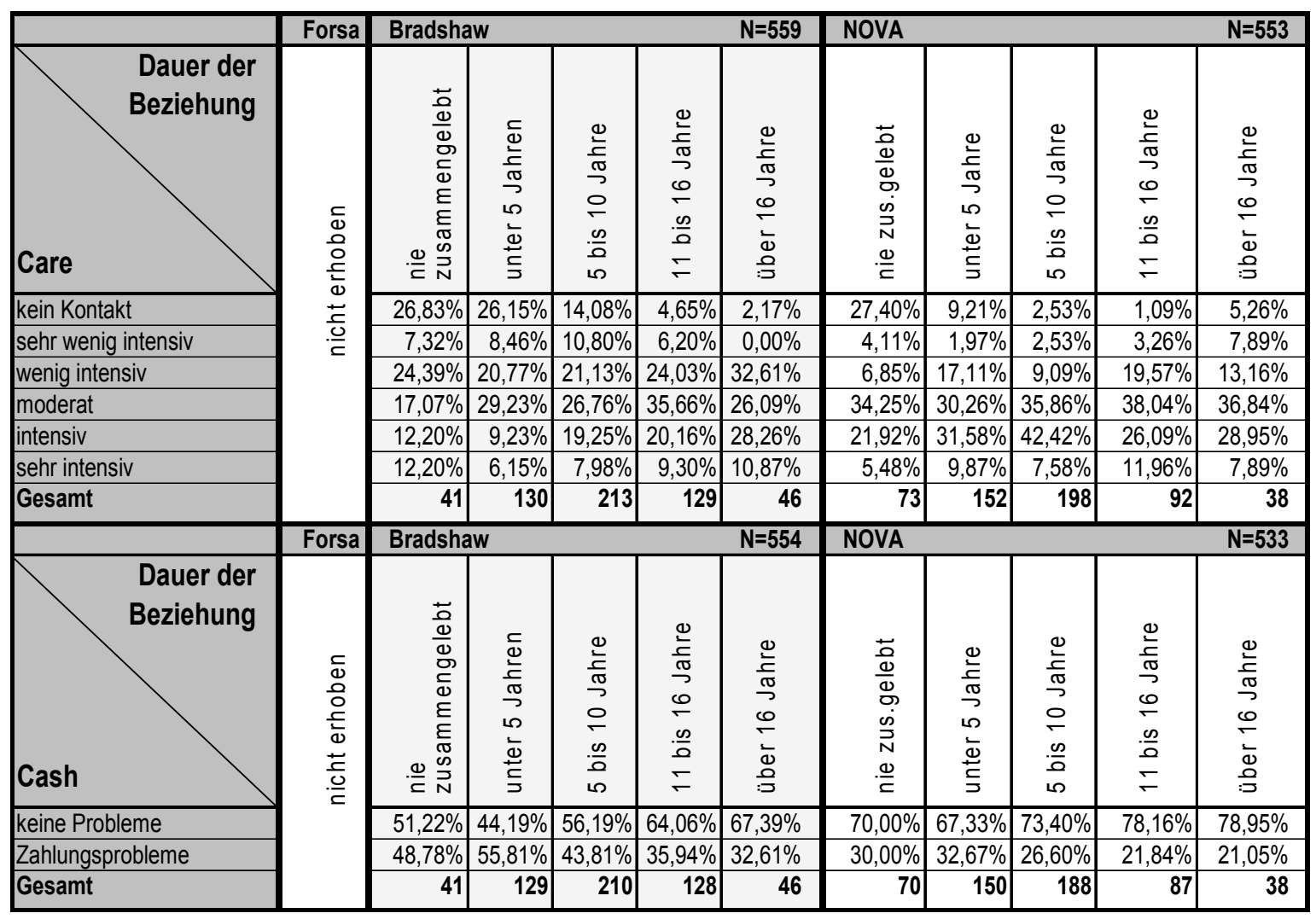

Quelle: eigene Berechnung.

Die Angaben der britischen Väter deuten einen positiven Zusammenhang zwischen der Dauer der Beziehung vor der Trennung und dem väterlichen Sorgehandeln in Form von Care nach dem Scheitern der elterlichen Partnerschaft an. Mit zunehmender Dauer der 
Beziehung nimmt der Anteil der Väter ohne Kontakt ab. Gleichzeitig steigt der Anteil der Väter, die angeben sich mindestens intensiv sozio-emotional zu engagieren, mit der Länge der Partnerschaft an. Dieses Bild lässt sich in den norwegischen Daten nicht eindeutig ausmachen. Zwar ist auch hier der Anteil der Kontaktabbrüche unter den Väter am stärksten, die keinen Haushalt mit der Kindesmutter geführt haben und die damit das kürzeste Zusammenleben - nämlich gar keins - aufweisen, doch Väter, die kohabitiert haben, lassen keinen eindeutigen Zusammenhang erkennen. Damit wird die aufgestellte Hypothese durch einen von zwei möglichen Datensätzen gestützt.

Für das finanzielle Handeln der britischen Väter neigen die Daten in Richtung eines schwachen negativen Zusammenhangs. Das bedeutet, dass der Anteil der Väter, die keine Zahlungsprobleme aufweisen, mit der Dauer der elterlichen Partnerschaften etwas abnimmt. Ähnliches zeigt sich auch in den norwegischen Ergebnissen, wenn auch mit noch geringerer Deutlichkeit. Damit kann der erwartete Zusammenhang wenn überhaupt als tendenziell bestätigt gesehen werden.

Tab. 9.21: Operationalisierungen der Unterhaltsfestlegung.

\begin{tabular}{|c|c|c|c|}
\hline & Forsa & Bradshaw & NOVA \\
\hline Frage & $\begin{array}{l}\text { Und wie wurde die } \\
\text { Höhe des Unterhalts } \\
\text { für das Kind } \\
\text { festgelegt? }\end{array}$ & $\begin{array}{l}\text { (konstruiert aus j3m1 } \\
\text { bis j3m9: „Wurden } \\
\text { jemals eine der } \\
\text { folgenden Instrumente } \\
\text { zur Festlegung des } \\
\text { Unterhalts genutzt?“ } \\
\text { Mehrfachnennungen } \\
\text { möglich) }\end{array}$ & $\begin{array}{l}\text { Wie wurde der Betrag } \\
\text { in ihrem Fall } \\
\text { festgelegt? }\end{array}$ \\
\hline Original & $\begin{aligned} 1 & =\text { allein mit Mutter; } \\
2= & \text { durch das } \\
& \text { Jugendamt; } \\
3= & \text { mit Hilfe eines } \\
& \text { Anwalts } / \text { Notars } \\
& \text { ohne Gericht; } \\
4= & \text { vor Gericht durch } \\
& \text { Vergleich; } \\
5= & \text { per Gerichtsurteil }\end{aligned}$ & $\begin{aligned} 1 & =\text { per Gericht } \\
2 & =\text { per DSS } \\
3= & \text { per CSA; } \\
4= & \text { per Family } \\
& \text { Mediation Service; } \\
5= & \text { informal durch } \\
& \text { Eltern } \\
6= & \text { durch Befragten }\end{aligned}$ & $\begin{aligned} 1 & =\text { private } \\
& \text { Vereinbarung; } \\
2= & \text { eigene Verein- } \\
& \text { barung, durch } \\
& \text { Unterhaltsstelle } \\
& \text { geändert; } \\
3= & \text { durch Unterhalts- } \\
& \text { stelle }\end{aligned}$ \\
\hline Kategorien & $\begin{aligned} 1 & =\text { mit Mutter allein; } \\
2= & \text { mit Hilfe Experten; } \\
3= & \text { mit gerichtlicher } \\
& \text { Hilfe }\end{aligned}$ & $\begin{array}{l}1=\text { zwischen Eltern; } \\
2=\text { mit Hilfe Dritter }\end{array}$ & $\begin{aligned} 1 & =\text { private } \\
& \text { Vereinbarung; } \\
2= & \text { eigene Verein- } \\
& \text { barung, durch } \\
& \text { Unterhaltsstelle } \\
& \text { geändert; } \\
3= & \text { durch Unterhalts- } \\
& \text { stelle }\end{aligned}$ \\
\hline
\end{tabular}

Quelle: eigene Darstellung.

Ein weiterer Einflussfaktor für das väterliche Sorgehandeln wird in der Aushandlung des Unterhalts gesehen. Eine einvernehmliche Einigung der Eltern fördert das väterliche Sorgehandeln in Form von Care und verhindert das Ausbleiben von Unterhaltszahlungen. 
Tab. 9.22: Kreuztabellen zur Unterhaltsfestlegung.

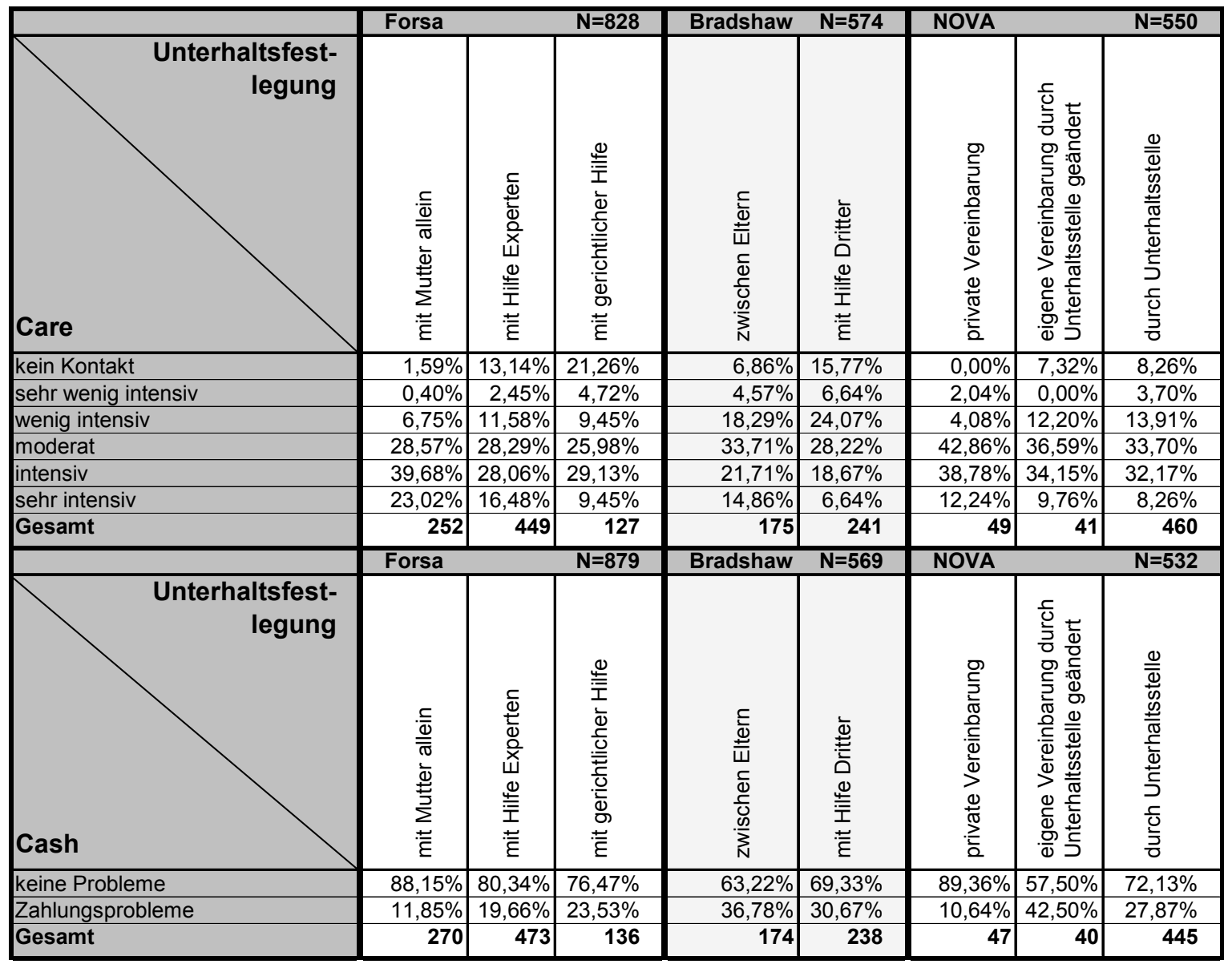

Quelle: eigene Berechnung.

Merkmale, die den Trennungsprozess beschreiben, werden im britischen Datensatz nur marginal erhoben. Die einzige Frage, die in diesem Zusammenhang gestellt wird, bezieht sich auf die Festlegung des Unterhalts, die derzeit, früher und nie zahlenden Vätern in gleicher Form gestellt wurde. Die Frage für getrennt lebende Männer lautet: „Wenn Sie an die aktuellen Unterhaltszahlungen denken, wie haben Sie sich darauf geeinigt, wie wurden sie festgelegt?“. Dabei können sie mehrfach zwischen verschiedenen Festlegungsarten wie

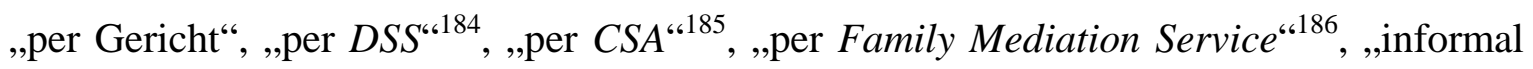
zwischen den Eltern“ und „durch den Befragten selbst“ wählen. Die Antwortvorgaben werden zur leichteren Interpretation auf zwei Kategorien reduziert. Dabei liefert die Vari-

\footnotetext{
${ }^{184}$ DSS steht für das Departement of Social Service.

${ }^{185}$ CSA steht für Child Support Agency, siehe dazu ausführlich Kap. 6.2.2.1 Unterhaltsregelungen.

${ }^{186}$ Diese Antwortvorgabe gibt es zwar im Fragebogen, aber keiner der Befragten nutzt sie.
} 
able Informationen darüber, ob die Festlegung „zwischen den Eltern“ oder „mit Hilfe Dritter“, d.h. mittels dem Einschalten einer Institution erfolgt. ${ }^{187}$ Mit steigender Kodierung nimmt im deutschen wie norwegischen Fall die Nutzung externer Institutionen und damit die Fremdbestimmtheit der elterlichen Entscheidung zu. Aufgrund unterschiedlicher institutioneller Rahmenbedingungen in Deutschland und Norwegen weisen jedoch die beiden Variablen jeweils drei unterschiedliche Ausprägungen auf. ${ }^{188}$ Diese werden in der Analyse beibehalten. Beide Variablen können inhaltlich ähnlich interpretiert werden: Mit steigender Kodierung nimmt die Einigkeit zwischen den Eltern bei der Festlegung ab, bzw. steigt die Einbeziehung externer Instanzen an. Es kann angenommen werden, dass bei der Einschaltung von Gerichten in Deutschland ebenso wie bei der Festlegung des Unterhalts durch die norwegische Unterhaltsstelle ein höheres Konfliktpotential zwischen den Eltern vorliegt, als wenn sie gemeinsam zu einer Einigung gekommen sind. ${ }^{189}$

\footnotetext{
${ }^{187}$ Zunächst wurde auch die Kategorie „durch den Befragten selbst“ in die Analyse aufgenommen. Im Verlauf der Arbeit ergab sich jedoch, dass die Ausprägung nicht ohne weiteres mit den inhaltlichen Annahmen der Variablen in den beiden anderen Datensätzen harmoniert. Zwar kann davon ausgegangen werden, dass ein Vater, der die Höhe des Unterhalts, die er als angemessen ansieht, selbst bestimmt, diese auch am ehesten zahlen wird. Fremdbestimmte Unterhaltsbeträge werden wahrscheinlicher als ungerecht empfunden. Doch in den anderen beiden Studien basiert die Interpretation primär auf der Einigkeit in der elterlichen Entscheidung. Eine eigenmächtige Festlegung des Vaters muss nicht notwendigerweise auf elterlichen Konsens beruhen. Der elterliche Konsens stellt eine andere inhaltliche Dimension dar wie die individuelle Kontrollwahrnehmung des Vaters. Zur besseren Vergleichbarkeit wird daher die britische Variable auf zwei Antwortvorgaben, „zwischen Eltern“ und „mit Hilfe Dritter“ festgelegter Unterhalt, reduziert. Ferner bestehen Unsicherheiten in der Konstruktion der Variablen im britischen Datensatz generell. Auf die Frage, wie der vereinbarte Unterhalt festgelegt wurde, d.h. ob und welche Hilfe externer Instanzen in Anspruch genommen wurde, können britische Väter mehr als eine Antwort geben. Unklar bleibt jedoch, was Mehrfachnennungen inhaltlich bedeuten. Es muss offen bleiben, ob ein Vater verschiedene Festlegungsarten für Unterhaltsverpflichtungen unterschiedlicher Kinder genutzt hat oder ob bei einem Kind unterschiedliche Formen der Festlegung in Anspruch genommen wurden. Fraglich bleibt dann, warum ein zweites oder drittes Mal der Unterhalt geregelt werden musste. Aufgrund dieser Unsicherheiten in der Interpretation werden Fälle, die auf diese Fragen mehr als eine Antwort gegeben haben aus der Analyse ausgeschlossen. Die genauere Betrachtung der Ausfälle zeigt, dass Väter, die nicht in der Analyse berücksichtigt werden, häufiger Zahlungsschwierigkeiten zeigen.

${ }^{188}$ In Norwegen laufen unterschiedlich ausgeprägte Konflikte über ein und dieselbe staatliche Instanz, während in Deutschland verschiedene Institutionen je nach Konfliktgrad in Anspruch genommen werden.

${ }^{189}$ Die Variable zur Unterhaltsfestlegung zeigt in allen Datensätzen einige Ausfälle. Innerhalb der deutschen Studie weisen Fälle, die keine Angaben zur Unterhaltsfestlegung machen (können), auch häufiger Ausfälle im sozio-emotionalen Sorgehandeln auf. Im Niveau der Care-Komponenten zeigen sich in Norwegen nur leichte Unterschiede. Dabei ergibt sich für die Ausfälle ein etwas höheres Engagement. Leichte Differenzen finden sich auch in den britischen Ausfällen. Hier ergibt sich für die Väter ohne Angaben zur Unterhaltsfestlegung jedoch ein niedrigeres sozio-emotionales Engagement. Die Ergebnisse liefern keine eindeutigen Hinweise auf die systematischen Auswirkungen der Ausfälle in der Erklärung von Care. Deutsche Väter, die keine Angaben zur Unterhaltsfestlegung machen (können), fallen häufig auch bei der Angabe zu ihrer Unterhaltspraxis aus. Das liegt u.a. daran, dass bei einem Teil der Ausfälle, der Unterhalt noch nicht geregelt ist. Bei den Vätern, bei denen Informationen zum finanziellen Sorgehandeln vorliegen und die keine Angaben zur Festlegung machen, zeigen sich kaum Unterschiede zur Zahlungspraxis von Vätern, die sowohl Cash als auch die Unterhaltsfestlegung angeben. Anders dagegen unter britischen Befragten, hier treten Zahlungsprobleme unter Vätern, die keine Angaben zur Unterhaltsfestlegung machen deutlich häufiger auf,
} 
Die Ergebnisse der Kreuztabelle zeigen über alle drei Länder hinweg einen negativen Zusammenhang zwischen der Art der Unterhaltsfestlegung und dem väterlichen Care. Dabei weisen im deutschen und norwegischen Fall Väter, die den Kontakt abgebrochen haben, höhere Anteile an gerichtlichen bzw. institutionellen Regelungen und damit an Streitigkeiten auf als Väter, die sich mit der Mutter im Privaten geeinigt haben. In dieser Gruppe ist der Anteil stark engagierter Väter höher. Auch im britischen Datensatz wird der vermutete Effekt der Unterhaltsfestlegung durch das empirische Material gestützt. Väter, die die Hilfe außerfamilialer Akteure in Anspruch genommen haben, weisen höhere Anteile in den unteren drei Abstufungen des Kümmerns in Form von Care auf als Väter, die den Konsens mit der Kindsmutter finden konnten. Das umgekehrte Verhältnis findet sich in den oberen Ausprägungen der Care-Variable. Damit wird der vermutete Zusammenhang in allen drei Datensätzen durch die empirischen Ergebnisse gestützt.

Deutsche Väter mit Zahlungsproblemen weisen einen höheren Anteil an gerichtlichen Unterhaltsfestlegungen auf. Dagegen geben Väter, die sich mit der Mutter einigen konnten, seltener an, Unterhaltsleistungen nicht gezahlt zu haben. Im norwegischen und britischen Fall findet sich dagegen keine Unterstützung der aufgestellten Hypothese. Es ergibt sich in Norwegen kein einheitliches Bild. Dabei ist darauf zu verweisen, dass eine deutliche Mehrheit der norwegischen Elternpaare in diesem Datensatz den Unterhalt durch die Unterhaltsstelle hat festlegen lassen. Nur rund 90 Väter geben an, sich privat geeinigt zu haben und davon hat in 41 Fällen die Unterhaltsstelle die elterliche Vereinbarung geändert. ${ }^{190}$ Im britischen Fall kommen sogar die Väter, die eine konsensuelle Einigung mit der Mutter angeben, häufiger ihren Unterhaltsverpflichtungen nicht nach, was im Widerspruch zum angenommenen Verhältnis des Prädiktors zum Kriterium Cash steht. Der Unterschied zwischen den beiden Gruppen „zwischen Eltern“ und „mit Hilfe Dritter“ festgelegter Unterhalt ist jedoch nicht sonderlich groß. Insgesamt kann somit in nur einer Studie der angenommene Zusammenhang nachgewiesen werden.

Ähnliche Überlegungen wie für die Festlegung des Unterhalts gelten auch für die Festlegung des Sorge- bzw. Umgangsrechts. Bei einer einvernehmlichen Einigung dürfte der Vater eher einen regelmäßigen Kontakt zu seinem Kind pflegen, als wenn die Festlegung einen elterlichen Konflikt geschürt hat. Auf seine Zahlungsmoral dürfte die Sorgerechtsfestsetzung keinen Einfluss haben.

als bei antwortenden Vätern. Dies kann jedoch auch der Konstruktion der unabhängigen Variablen geschuldet sein.

${ }^{190}$ Zur Bedeutung der Unterhaltsstelle in Norwegen siehe Kap. 6.2.2.1 Unterhaltsregelungen. 
Tab. 9.23: Operationalisierungen der Sorgerechts- bzw. Umgangsfestlegung.

\begin{tabular}{|l|l|l|l|}
\hline \multirow{3}{*}{ Frage } & \multicolumn{1}{|c|}{ Forsa } & Bradshaw & \multicolumn{1}{c|}{ NOVA } \\
\cline { 2 - 4 } & $\begin{array}{l}\text { Ist die Entscheidung, bei wem } \\
\text { das Kind lebt und wer für das } \\
\text { Kind sorgen soll, } \\
\text { einvernehmlich mit der Mutter } \\
\text { gefallen, haben Sie sich erst } \\
\text { nach Auseinandersetzungen } \\
\text { geeinigt oder wurde die Frage } \\
\text { erst durch ein Gerichtsurteil } \\
\text { entschieden? }\end{array}$ & - & $\begin{array}{l}\text { Wie kam es zu der heutigen } \\
\text { Umgangsvereinbarung } \\
\text { zwischen Ihnen und der }\end{array}$ \\
Kindesmutter?
\end{tabular}

Quelle: eigene Darstellung.

Regelungen des Sorgerechts und des Umgangs werden innerhalb der britischen Untersuchung gar nicht erfasst. Im deutschen Datensatz kann auf eine Variable zur Erfassung der Sorgerechtsfestlegung direkt zurückgegriffen werden. Als Antworten werden eine „einvernehmlich“, „nach Auseinandersetzung“ und „durch Gerichtsurteil“ festgelegte Regelung differenziert. ${ }^{191}$ Auch im norwegischen Fall werden drei Ausprägungen vorgegeben, zu denen die „private Vereinbarung“, „durch Behörde“ und „per Gericht“ getroffene Einigungen zählen. Innerhalb der vorliegenden Analyse werden zusätzlich die Väter aufgenommen, die angeben keinerlei Vereinbarungen mit der Mutter getroffen zu haben. In diesen Fällen scheint es mit Blick auf die Umgangsregelung keinen Aushandlungsbedarf zu geben, so dass von einem elterlichen Konsens ausgegangen werden kann. Im norwegischen Datensatz handelt es sich um die Vereinbarung einer Umgangsregelung und nicht wie in der deutschen Studie um die Festlegung des Sorgerechts. Beides sind unterschiedliche Sachverhalte, die hier jedoch inhaltlich ähnlich zu interpretieren sind, wenn es um ihre Festlegung geht. Sie werden beide als Indikatoren für die Beziehung der Eltern während der Trennung interpretiert.

\footnotetext{
191 Ausfälle zur Sorgerechtsfestlegung weisen in der deutschen Studie - ähnlich wie bei den Angaben zur Unterhaltsfestlegung - gleichzeitig auch hohe Informationsverluste in den Variablen Care und Cash auf. Die Väter, die Angaben zum Prozess der Sorgerechtsentscheidung machen, zeigen kaum Unterschiede in ihrem Sorgehandeln, weder sozio-emotional noch finanziell.
} 
Tab. 9.24: Kreuztabellen zur Sorgerechts- bzw. Umgangsfestlegung.

\begin{tabular}{|c|c|c|c|c|c|c|c|c|}
\hline & Forsa & & $\mathrm{N}=808$ & Bradshaw & NOVA & & & $\mathrm{N}=550$ \\
\hline $\begin{array}{r}\text { Sorgerechts- } \\
\text { festlegung } \\
\text { (Umgang) }\end{array}$ & 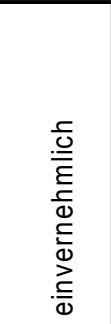 & 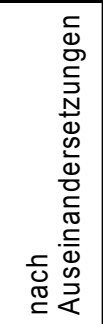 & 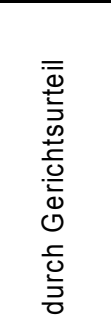 & \multirow{8}{*}{ 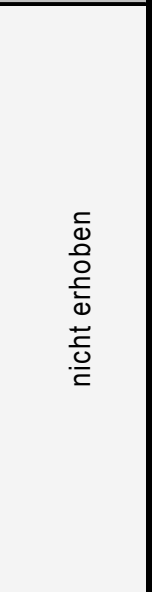 } & 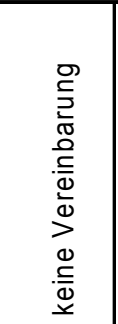 & 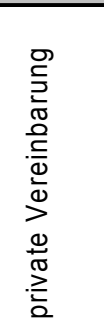 & 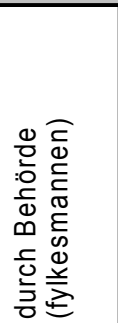 & 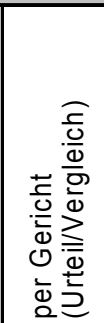 \\
\hline kein Kontakt & $7,05 \%$ & $13,79 \%$ & $23,81 \%$ & & $17,68 \%$ & $0,65 \%$ & $0,00 \%$ & $16,67 \%$ \\
\hline sehr wenig intensiv & $1,44 \%$ & $5,17 \%$ & $3,17 \%$ & & $7,18 \%$ & $0,33 \%$ & $5,00 \%$ & $2,38 \%$ \\
\hline wenig intensiv & $9,29 \%$ & $10,34 \%$ & $13,49 \%$ & & $16,57 \%$ & $9,77 \%$ & $15,00 \%$ & $16,67 \%$ \\
\hline moderat & $26,60 \%$ & $31,03 \%$ & $29,37 \%$ & & $32,04 \%$ & $36,48 \%$ & $40,00 \%$ & $33,33 \%$ \\
\hline intensiv & $34,29 \%$ & $31,03 \%$ & $21,43 \%$ & & $23,76 \%$ & $40,39 \%$ & $30,00 \%$ & $26,19 \%$ \\
\hline sehr intensiv & $21,31 \%$ & $8,62 \%$ & $8,73 \%$ & & $2,76 \%$ & $12,38 \%$ & $10,00 \%$ & $4,76 \%$ \\
\hline \multirow[t]{2}{*}{ Gesamt } & 624 & 58 & 126 & & 181 & 307 & 20 & 42 \\
\hline & Forsa & & $\mathrm{N}=841$ & Bradshaw & NOVA & & & $\mathrm{N}=533$ \\
\hline $\begin{array}{r}\text { Sorgerechts- } \\
\text { festlegung } \\
\text { (Umgang) }\end{array}$ & 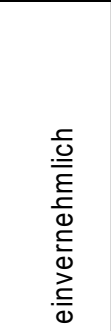 & 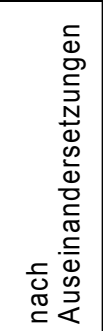 & 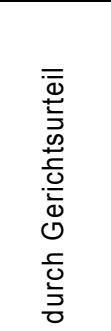 & \multirow[t]{4}{*}{ 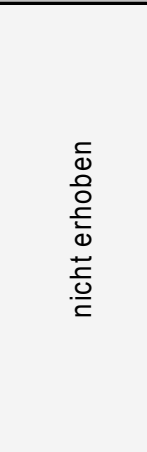 } & 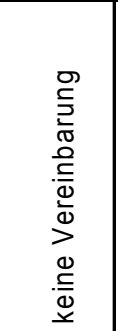 & 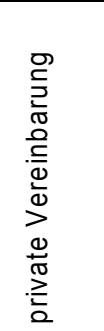 & 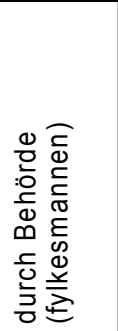 & 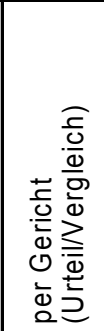 \\
\hline keine Probleme & $82,75 \%$ & $80,70 \%$ & $78,86 \%$ & & $66,09 \%$ & $76,51 \%$ & $73,68 \%$ & $64,29 \%$ \\
\hline Zahlungsprobleme & $17,25 \%$ & $19,30 \%$ & $21,14 \%$ & & $33,91 \%$ & $23,49 \%$ & $26,32 \%$ & $35,71 \%$ \\
\hline Gesamt & 661 & 57 & 123 & & 174 & & & \\
\hline
\end{tabular}

Quelle: eigene Berechnung.

Die deutschen Ergebnisse zur Festlegung des Sorgerechts weisen in die gleiche Richtung wie die Ergebnisse zur Unterhaltseinigung. Dabei geben Väter, die den Kontakt zu ihren getrennt lebenden Kindern abgebrochen haben oder ein (sehr) wenig intensives Verhältnis pflegen, deutlich öfter an, durch ein Gerichtsurteil mit der Mutter die Form des Sorgerechts festgelegt zu haben. Väter, die sich dagegen (sehr) intensiv um ihre Kinder kümmern, haben sich häufiger mit der Mutter einvernehmlich geeinigt. Die deutschen Väter stützen damit die angenommene Hypothese. Anders im norwegischen Datensatz, hier können keine eindeutigen Zusammenhangsmuster anhand der Kreuztabelle ausgemacht werden. ${ }^{192}$

192 Dabei muss die geringe Variation in der norwegischen Variablen berücksichtigt werden. Eine große Mehrheit der Väter einigt sich mit der Kindsmutter einvernehmlich. Darüber hinaus haben viele Eltern keine Vereinbarung getroffen. 
Tab. 9.25: Operationalisierungen der festgelegten Form des Sorgerechts.

\begin{tabular}{|c|c|c|c|}
\hline & Forsa & Bradshaw & NOVA \\
\hline Frage & $\begin{array}{l}\text { Hat die Mutter das alleinige } \\
\text { Sorgerecht für das Kind } \\
\text { oder haben Sie zusammen } \\
\text { ein gemeinsames } \\
\text { Sorgerecht? }\end{array}$ & - & $\begin{array}{l}\text { Welche Besuchsregelung für } \\
\text { das Kind ist zur Zeit } \\
\text { abgesprochen? }\end{array}$ \\
\hline Kategorien & $\begin{array}{c}1=\text { alleiniges Sorgerecht } \\
\text { der Mutter; } \\
2=\text { gemeinsames } \\
\text { Sorgerecht }\end{array}$ & - & $\begin{aligned} 0= & \text { keine Vereinbarung } \\
1= & \text { weniger als üblicher } \\
& \text { Umgang; } \\
2= & \text { üblicher Umgang; } \\
3= & \text { mehr als üblicher Umgang }\end{aligned}$ \\
\hline
\end{tabular}

Quelle: eigene Darstellung.

Die Unterhaltsleistungen scheinen in Deutschland negativ durch eine formalisierte Festlegung des Sorgerechts beeinflusst zu werden. Die Kreuztabelle zeigt, dass Zahlungsprobleme häufiger auftreten, wenn sich die Eltern vor Gericht über das Sorgerecht geeinigt haben. Der Anteil der Väter, die ihren Unterhaltspflichten nicht nachkommen, ist am geringsten unter den Elternpaaren, die sich einvernehmlich geeinigt haben. Die Verhältnisse zwischen Unterhaltszahlern und Vätern mit Zahlungsschwierigkeiten sind jedoch über alle drei Ausprägungen der erklärenden Variable sehr ähnlich. Damit scheint es sich - wenn überhaupt - um einen sehr schwachen Zusammenhang zwischen Sorgerechtsfestlegung und Unterhaltszahlungen zu handeln. Die norwegischen Daten weisen auf keinen Zusammenhang hin, wie dies auch theoretisch postuliert wird.

Es wird ferner angenommen, dass die Form des festgelegten Sorgerechts bzw. Umgangs wesentlich die Ausgestaltung des väterlichen Kontakts determiniert. Ein gemeinsames Sorgerecht bzw. ein hohes Umgangsrecht wirkt sich positiv auf die Care-Praxis des Vaters aus, während für die Zahlungsmoral des Vaters theoretisch kein Zusammenhang angenommen wird.

Bei der Form des festgelegten Sorgerechts bzw. des Umgangs gelten ähnliche Überlegungen wie beim Prozess der Festlegung von Sorgerecht bzw. Umgang. Beide Konzepte bilden unterschiedliche Sachverhalte ab. Das deutsche Sorgerecht umfasst insbesondere die rechtliche Verantwortung und Vertretung des Kindes durch die Eltern, während sich die Umgangsregelung auf den tatsächlich vereinbarten persönlichen Kontakt zwischen Vater und Kind bezieht. Dennoch wirkt sich beides ähnlich auf das väterliche Care-Handeln aus. Beide Variablen sind aufsteigend kodiert, d.h. mit zunehmender Kodierung steigt das Niveau des vereinbarten Sorgerechts bzw. Umgangs. Im britischen Datensatz fehlen derartige Informationen. 
Tab. 9.26: Kreuztabellen zur festgelegten Form des Sorgerechts.

\begin{tabular}{|c|c|c|c|c|c|c|c|}
\hline & Forsa & $\mathrm{N}=842$ & Bradshaw & NOVA & & & $\mathrm{N}=549$ \\
\hline $\begin{array}{r}\text { Form des } \\
\text { Sorgerechts } \\
\text { (Umgang) }\end{array}$ & 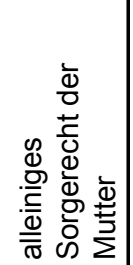 & 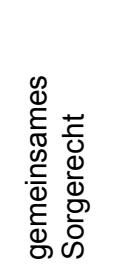 & \multirow{8}{*}{ 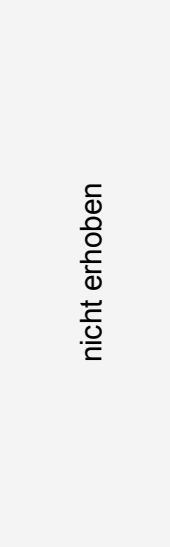 } & 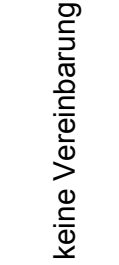 & 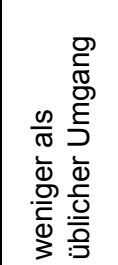 & 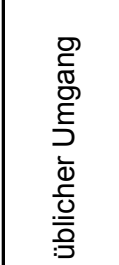 & 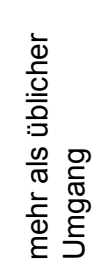 \\
\hline kein Kontakt & $18,88 \%$ & $2,02 \%$ & & $19,08 \%$ & $4,17 \%$ & $1,84 \%$ & $0,00 \%$ \\
\hline sehr wenig intensiv & $4,04 \%$ & $0,25 \%$ & & $7,51 \%$ & $0,00 \%$ & $1,84 \%$ & $0,00 \%$ \\
\hline wenig intensiv & $14,16 \%$ & $4,53 \%$ & & $17,34 \%$ & $12,50 \%$ & $13,36 \%$ & $4,50 \%$ \\
\hline moderat & $27,87 \%$ & $27,20 \%$ & & $30,06 \%$ & $50,00 \%$ & $38,25 \%$ & $28,83 \%$ \\
\hline intensiv & $25,62 \%$ & $39,04 \%$ & & $23,70 \%$ & $29,17 \%$ & $35,02 \%$ & $48,65 \%$ \\
\hline sehr intensiv & $9,44 \%$ & $26,95 \%$ & & $2,31 \%$ & $4,17 \%$ & $9,68 \%$ & $18,02 \%$ \\
\hline \multirow[t]{2}{*}{ Gesamt } & 445 & 397 & & 173 & 48 & 217 & 111 \\
\hline & Forsa & $\mathrm{N}=876$ & Bradshaw & NOVA & & & $\mathrm{N}=531$ \\
\hline $\begin{array}{r}\text { Form des } \\
\text { Sorgerechts } \\
\text { (Umgang) }\end{array}$ & 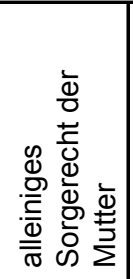 & 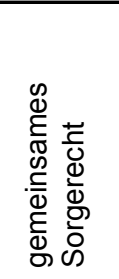 & \multirow[t]{4}{*}{ 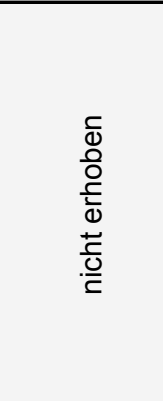 } & 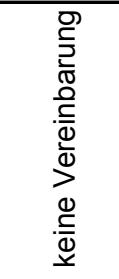 & 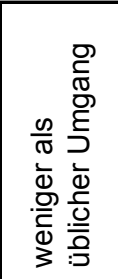 & 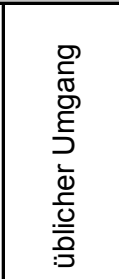 & 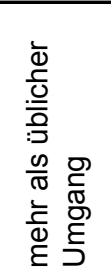 \\
\hline keine Probleme & $80,43 \%$ & $83,17 \%$ & & $66,07 \%$ & $71,74 \%$ & $72,12 \%$ & $82,57 \%$ \\
\hline Zahlungsprobleme & $19,57 \%$ & $16,83 \%$ & & $33,93 \%$ & $28,26 \%$ & $27,88 \%$ & $17,43 \%$ \\
\hline Gesamt & 460 & 416 & & 168 & 46 & & 109 \\
\hline
\end{tabular}

Quelle: eigene Berechnung.

Die Form des festgelegten Sorgerechts weist im deutschen Datenmaterial eindeutig auf einen positiven Zusammenhang mit der Care-Variablen hin. Väter, die sich das Sorgerecht mit der Mutter teilen, zeigen ein höheres Niveau an sozio-emotionalem Sorgehandeln. Dagegen findet sich ein höherer Anteil an Kontaktabbrüchen sowie (sehr) wenig väterlichem Engagement unter Vätern, die nicht das gemeinsame Sorgerecht haben. Auch die norwegischen Ergebnisse stützen die aufgestellte Hypothese. Hier ist der Anteil der Väter, die weder Kontakt noch eine Vereinbarung über die Regelung des Umgangs haben, deutlich höher als es Väter ohne Kontakt unter denjenigen gibt, die mit der Kindesmutter generell eine Umgangsregelung getroffen haben. Demgegenüber geben Väter, die den üblichen oder gar mehr als den üblichen Umgang festgelegt haben, häufiger ein (sehr) intensives Niveau väterlichen Kümmerns an. Damit bestätigen beide Datensätzen, in denen Informationen zur Sorgerechts- bzw. Umgangsfestlegung vorhanden sind, den theoretisch erwarteten Zusammenhang.

Mit Blick auf das Zahlungsverhalten weisen die deutschen Väter keinen Zusammenhang mit der Form des festgelegten Sorgerechts auf. Der Anteil der auftretenden Unterhaltsprobleme ist in beiden Gruppen, mit alleinigem Sorgerecht der Mutter und mit gemeinsamem Sorgerecht der Eltern, relativ gleich. Innerhalb der norwegischen Ergebnisse zeigt 
sich eine Tendenz in Richtung eines negativen Zusammenhangs. Väter, die mehr als den üblichen Umgang mit der Mutter vereinbart haben, zeigen deutlich seltener Schwierigkeiten ihren Unterhaltsverpflichtungen nachzukommen als Väter ohne Vereinbarung. Die norwegischen Daten deuten somit einen Zusammenhang an, der so nicht erwartet wurde.

Auf die in der Literatur häufig genannte Funktion der Mutter als "gate keeper", die den Zugang zum Kind erschweren oder auch erleichtern kann, wurde bereits verwiesen. Ein wichtiger Aspekt in diesem Zusammenhang ist das elterliche Verhältnis zum Zeitpunkt der Befragung. Es kann angenommen werden, dass sich ein freundschaftliches Verhältnis zwischen den Eltern nach der Trennung positiv auf die sozio-emotionale Vater-Kind-Beziehung auswirkt, auf das finanzielle Handeln des Vaters jedoch keinen Einfluss hat.

Tab. 9.27: Operationalisierungen des freundschaftlichen Verhältnisses der Eltern.

\begin{tabular}{|c|c|c|c|}
\hline & Forsa & Bradshaw & NOVA \\
\hline Frage & - & $\begin{array}{l}\text { Würden Sie die heutige } \\
\text { Beziehung zu (Name der Ex- } \\
\text { Partnerin) als ... bezeichnen? }\end{array}$ & $\begin{array}{l}\text { Würden Sie sagen, dass sie } \\
\text { jetzt ein freundschaftliches } \\
\text { Verhältnis zur Kindesmutter } \\
\text { haben? }\end{array}$ \\
\hline Original & - & $\begin{aligned} 1 & =\text { freundschaftlich; } \\
2 & =\text { freundschaftlich } \\
& \quad \text { distanziert; } \\
3 & =\text { distanziert; } \\
4 & =\text { distanziert; feindselig; } \\
5 & =\text { feindselig; } \\
6 & =\text { keine Beziehung }\end{aligned}$ & $\begin{array}{l}1 \text { = nicht freundschaftlich; } \\
2 \text { = freundschaftlich }\end{array}$ \\
\hline Kategorien & - & $\begin{array}{l}1 \text { = nicht freundschaftlich; } \\
2 \text { = freundschaftlich }\end{array}$ & $\begin{array}{l}1 \text { = nicht freundschaftlich; } \\
2 \text { = freundschaftlich }\end{array}$ \\
\hline
\end{tabular}

Quelle: eigene Darstellung.

Im deutschen Datensatz fehlen die Angaben zum freundschaftlichen Verhältnis, anders als in der norwegischen und britischen Erhebung. Während im norwegischen Fragebogen lediglich eine dichotome Erfassung vorliegt, die angibt, ob das Verhältnis zwischen den Eltern als freundschaftlich zu beschreiben sei oder nicht, liefern die britischen Befragten ein differenzierteres Bild. Sie können ihr heutiges Verhältnis zur Ex-Partnerin als „freundschaftlich“, „freundschaftlich, distanziert“, „distanziert“, „distanziert; feindselig“ oder „feindselig“ beschreiben, oder gar angeben, dass keine Beziehung besteht. Zur Vergleichbarkeit der Ergebnisse wird auch hier eine Dichotomie gebildet. Dabei umfasst die Kategorie „nicht freundschaftlich“ die die letzten vier Antwortvorgaben („distanziert“ bis „keine Beziehung“), während die Kategorie „freundschaftlich“ die beiden „freundschaftlichen“ Dimensionen integriert. 
Tab. 9.28: Kreuztabellen zum freundschaftlichen Verhältnis der Eltern.

\begin{tabular}{|c|c|c|c|c|c|}
\hline & Forsa & Bradshaw & $\mathrm{N}=569$ & NOVA & $\mathrm{N}=547$ \\
\hline $\begin{array}{l}\text { Freundschaft } \\
\text { zwischen Eltern }\end{array}$ & \multirow{8}{*}{$\begin{array}{l}\frac{5}{\Phi} \\
\frac{8}{0} \\
\frac{0}{\Phi} \\
\frac{\vec{t}}{0}\end{array}$} & 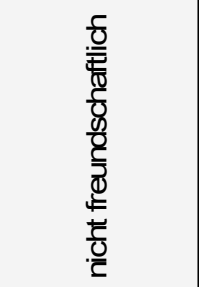 & 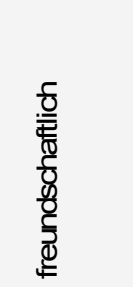 & 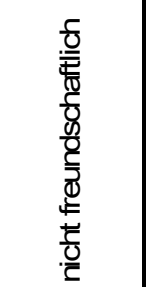 & 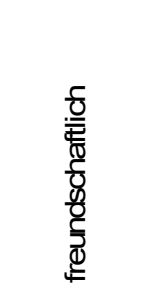 \\
\hline kein Kontakt & & $30,08 \%$ & $2,79 \%$ & $17,19 \%$ & $2,25 \%$ \\
\hline sehr wenig intensiv & & $13,82 \%$ & $3,72 \%$ & $4,69 \%$ & $2,54 \%$ \\
\hline wenig intensiv & & $25,61 \%$ & $21,05 \%$ & $17,19 \%$ & $10,70 \%$ \\
\hline moderat & & $19,11 \%$ & $34,98 \%$ & $31,77 \%$ & $36,90 \%$ \\
\hline intensiv & & $7,32 \%$ & $26,01 \%$ & $25,00 \%$ & $37,18 \%$ \\
\hline sehr intensiv & & $4,07 \%$ & $11,46 \%$ & $4,17 \%$ & $10,42 \%$ \\
\hline \multirow[t]{2}{*}{ Gesamt } & & 246 & 323 & 192 & 355 \\
\hline & Forsa & Bradshaw & $\mathrm{N}=564$ & NOVA & \\
\hline $\begin{array}{l}\text { Freundschaft } \\
\text { zwischen Eltern }\end{array}$ & \multirow[t]{3}{*}{$\begin{array}{l}\frac{5}{8} \\
\frac{0}{0} \\
\frac{0}{0} \\
\frac{0}{2}\end{array}$} & 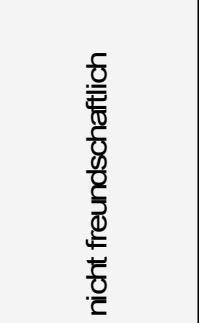 & 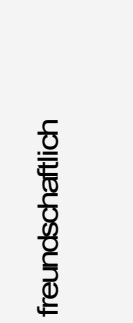 & 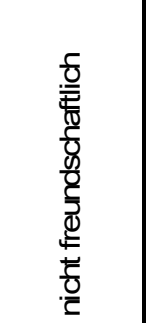 & 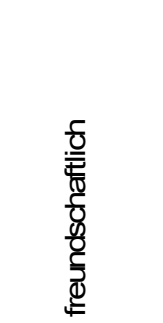 \\
\hline \begin{tabular}{|l|} 
keine Probleme \\
Zahlungsprobleme
\end{tabular} & & $\begin{array}{l}45,68 \% \\
54,32 \%\end{array}$ & $\begin{array}{l}63,24 \% \\
36,76 \%\end{array}$ & \begin{tabular}{|l|}
$69,35 \%$ \\
$30,65 \%$ \\
\end{tabular} & $\begin{array}{l}74,78 \% \\
25,22 \%\end{array}$ \\
\hline $\begin{array}{l}\text { Zahlungsprobleme } \\
\text { Gesamt }\end{array}$ & & 243 & 321 & 186 & 341 \\
\hline
\end{tabular}

Quelle: eigene Berechnung.

Die Ergebnisse zwischen einem freundschaftlichen Verhältnis der Eltern und dem väterlichen Sorgehandeln weisen in beiden Datensätzen auf einen Zusammenhang zwischen Prädiktor und dem Kriterium Care hin. So zeigen sowohl britische wie auch norwegische Väter, die eine freundschaftliche Beziehung zur Kindsmutter angeben, einen größeren Anteil an hohem väterlichem Engagement als Väter, die das Verhältnis zur Ex-Partnerin als nicht freundschaftlich charakterisieren. Diese haben häufiger den Kontakt zu ihren Kindern abgebrochen oder sie geben öfter geringere Kontaktniveaus an. Damit stützen die Ergebnisse insgesamt den aufgestellten Zusammenhang.

Auf das Zahlungsverhalten sollte das elterliche Verhältnis im Zeitpunkt der Befragung indes keinen Effekt haben. Die Ergebnisse bestätigen diese theoretische Annahme jedoch nicht. In beiden Datensätzen weisen Väter, die ein freundschaftliches Verhältnis zur Kindsmutter pflegen, deutlich seltener Unterhaltsprobleme im Vergleich zu Vätern, die das Verhältnis als wenig freundschaftlich beschreiben. Dabei ist die Differenz der Anteile zwischen den Ausprägungen der Prädiktorvariable innerhalb der britischen Ergebnisse deutlicher ausgeprägt als in Norwegen. Insgesamt weist das britische und norwegische 
Datenmaterial auf einen nicht vermuteten negativen Zusammenhang zwischen einer freundschaftlichen Beziehung der Ex-Partner und dem väterlichen Zahlungsverhalten hin.

\subsubsection{Der Einfluss der Prädiktoren des individuellen Überzeugungssystems}

Eine Determinante, die innerhalb der theoretischen Überlegungen den inneren Bedingungen des Vaters zugeordnet wird, stellt die ökonomische Situation der Mutter dar. Dabei, so die Annahme, hat die ökonomische Situation keinen Einfluss auf das väterliche Sorgen in Form von Care. Auf die Zahlungsprobleme wirkt sie sich jedoch aus, insofern dass Väter, die ihre Ex-Partnerinnen in einer guten finanziellen Lage sehen, eher Zahlungsprobleme aufweisen, als Väter, die glauben, die Frau benötige das Geld dringend.

Tab. 9.29: Operationalisierungen der ökonomischen Situation der Mutter.

\begin{tabular}{|c|c|c|c|}
\hline & Forsa & Bradshaw & NOVA \\
\hline Frage & - & $\begin{array}{l}\text { Wie würden Sie die } \\
\text { finanzielle Situation } \\
\text { beschreiben, in der Ihre } \\
\text { letzte Ex-Partnerin heute } \\
\text { lebt? }\end{array}$ & $\begin{array}{l}\text { Im Großen und Ganzen: glauben } \\
\text { Sie, dass Ihre frühere Partnerin } \\
\text { jetzt ökonomisch schlechter oder } \\
\text { besser gestellt ist als zu der Zeit, in } \\
\text { der sie zusammen wohnten? }\end{array}$ \\
\hline Kategorien & - & $\begin{array}{l}1=\text { komfortabler Standard; } \\
2=\text { mittlerer Standard } \\
3 \text { = kommt zurecht; } \\
4=\text { Situation schwierig; } \\
5=\text { Situation sehr schwierig }\end{array}$ & $\begin{aligned} 1= & \text { sie kann sich mehr leisten; } \\
2= & \text { sie kann sich genauso viel } \\
& \text { leisten wie vor der Trennung; } \\
3= & \text { sie kann sich weniger leisten }\end{aligned}$ \\
\hline
\end{tabular}

Quelle: eigene Darstellung.

Innerhalb der deutschen Studie gibt es keine Angaben zur finanziellen Situation der Mutter. Britische Väter werden nach ihrer subjektiven Einschätzung der ökonomischen Situation der Mutter gefragt. Die Antwortvorgaben auf die Frage „Wie würden Sie die finanzielle Situation beschreiben, in der Ihre letzte Ex-Partnerin heute lebt?“ lauten „komfortabler Standard“, „mittlerer Standard“ (im Original: „doing alright“), „kommt zurecht“, „Situation schwierig“ und „Situation sehr schwierig“. Mit zunehmender Kodierung wird die derzeitige finanzielle Situation der Mutter problematischer wahrgenommen. Im norwegischen Datensatz wird die aktuelle mütterliche Finanzlage mit der vor der Trennung verglichen. Obwohl dies nicht notwendigerweise die gleiche Information bereitstellt, wie die Frage nach der Einschätzung der aktuellen finanziellen Lage, kann sie dennoch als Indikator dafür Verwendung finden. Auch in der norwegischen Variablen verschlechtert sich die ökonomische Situation mit steigender Kodierung. ${ }^{193}$

193 Ähnliche Überlegungen wie bei der aktuellen Partnersituation der Mutter können auch für die Beurteilung ihrer ökonomischen Lage angeführt werden. Hier haben die getrennt lebenden Väter ebenso keinen 
Tab. 9.30: Kreuztabellen zur ökonomischen Situation der Mutter.

\begin{tabular}{|c|c|c|c|c|c|c|c|c|c|}
\hline & Forsa & Bradsha & & & & $\mathrm{N}=457$ & NOVA & & $\mathrm{N}=486$ \\
\hline $\begin{array}{r}\text { Okonomische } \\
\text { Situation der } \\
\text { Mutter }\end{array}$ & \multirow{8}{*}{ 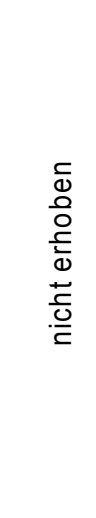 } & 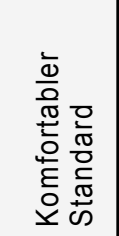 & 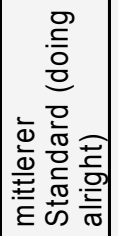 & 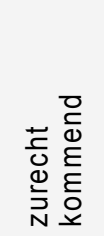 & 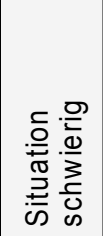 & 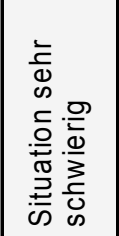 & 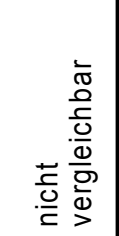 & 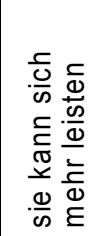 & 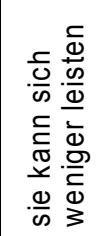 \\
\hline kein Kontakt & & $10,43 \%$ & $7,14 \%$ & $4,08 \%$ & $5,26 \%$ & $0,00 \%$ & 9,92\% & $4,35 \%$ & $2,93 \%$ \\
\hline sehr wenig intensiv & & $6,13 \%$ & $9,29 \%$ & $6,12 \%$ & $2,63 \%$ & $5,56 \%$ & $5,79 \%$ & $4,35 \%$ & $1,10 \%$ \\
\hline wenig intensiv & & $25,15 \%$ & $25,71 \%$ & $22,45 \%$ & $23,68 \%$ & $33,33 \%$ & $18,18 \%$ & $13,04 \%$ & $12,09 \%$ \\
\hline moderat & & $28,22 \%$ & $32,14 \%$ & $37,76 \%$ & $36,84 \%$ & $27,78 \%$ & $33,88 \%$ & $28,26 \%$ & $37,73 \%$ \\
\hline intensiv & & $20,86 \%$ & \begin{tabular}{|l|}
$17,14 \%$ \\
\end{tabular} & $21,43 \%$ & $26,32 \%$ & $33,33 \%$ & $27,27 \%$ & $38,04 \%$ & $36,26 \%$ \\
\hline sehr intensiv & & $9,20 \%$ & $8,57 \%$ & $8,16 \%$ & $5,26 \%$ & $0,00 \%$ & $4,96 \%$ & $11,96 \%$ & $9,89 \%$ \\
\hline \multirow[t]{2}{*}{ Gesamt } & & 163 & 140 & 98 & 38 & 18 & 121 & 92 & 273 \\
\hline & Forsa & \multicolumn{3}{|c|}{ Bradshaw } & & $\mathrm{N}=454$ & NOVA & & $\mathrm{N}=533$ \\
\hline $\begin{array}{l}\text { Ökonomische } \\
\text { Situation der }\end{array}$ & \multirow[t]{4}{*}{ 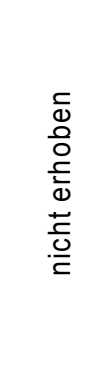 } & 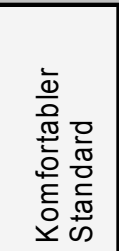 & 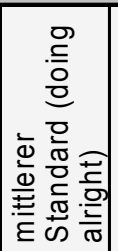 & 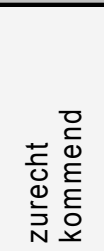 & 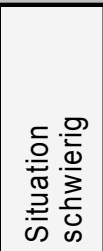 & 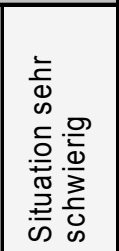 & 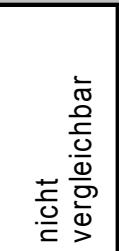 & 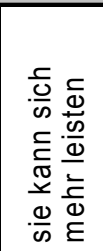 & 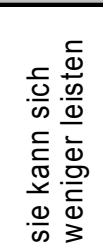 \\
\hline keine Probleme & & $67,08 \%$ & $60,71 \%$ & $60,82 \%$ & $39,47 \%$ & $44,44 \%$ & $74,34 \%$ & $80,90 \%$ & $68,05 \%$ \\
\hline Zahlungsprobleme & & $32,92 \%$ & $39,29 \%$ & $39,18 \%$ & $60,53 \%$ & $55,56 \%$ & $25,66 \%$ & $19,10 \%$ & $31,95 \%$ \\
\hline Gesamt & & 161 & & & 38 & 18 & 113 & 89 & 266 \\
\hline
\end{tabular}

Quelle: eigene Berechnung.

Die britischen Daten weisen darauf hin, dass das väterliche Sorgehandeln in Form von Care in keinem eindeutigen Zusammenhang mit der ökonomischen Situation der Mutter steht, da die kreuztabellierten Ergebnisse kein eindeutiges Zusammenhangsmuster aufweisen. Das gilt auch für die Angaben der norwegischen Väter. Beide Datensätze unterstützen somit die theoretische Annahme, dass kein Effekt zwischen den beiden Variablen besteht.

Die Zahlungspraxis britischer Väter deutet demgegenüber tendenziell einen Zusammenhang zur mütterlichen Finanzlage an. Dieser verläuft entgegen der theoretischen Annahme. Väter, die angeben, die Kindsmutter sei heute ökonomisch (sehr) schlecht gestellt, weisen eher Zahlungsausfälle auf als Väter, deren ehemalige Partnerin sich in einer von

direkten Einblick (mehr). So zeigt die Analyse der Ausfälle, dass norwegische wie britische Väter, die Angaben zur finanziellen Situation ihrer ehemaligen Partnerin machen können, ein höheres väterliches Engagement in Form von Care aufweisen als Väter, die dies nicht können. Innerhalb des britischen Datensatzes weisen die Ausfälle der Variablen zur finanziellen Lage der Mutter deutlich häufiger Zahlungsprobleme auf. Anders unter norwegischen Vätern, hier hat die Kenntnis bzw. Beurteilung der ökonomischen Situation der Mutter keinen Einfluss auf die Zahlungspraxis. Väter mit und ohne Angaben weisen ähnliche Anteile an Unterhaltsproblemen auf. 
den Akteuren wahrgenommenen (sehr) guten finanziellen Lage befindet. Im norwegischen Fall findet sich der höchste Anteil an Zahlungsschwierigkeiten unter den Vätern, die angeben ihrer Partnerin ginge es heute finanziell besser als noch vor der Trennung. Dieses Ergebnis entspricht der formulierten Hypothese. Väter, deren Ex-Partnerin sich heute weniger leisten kann, stellen jedoch nicht den geringsten Anteil in der Gruppe mit Zahlungsproblemen. Die Verhältnisse zwischen dem Anteil der unterhaltszahlenden und der nicht zahlenden Väter sind sehr ähnlich. Der erwartete Zusammenhang kann in beiden Datensätzen daher nicht ausgemacht werden.

Einen weiteren Indikator für das empfundene Verantwortungsbewusstsein des Vaters wird in der Dauer seit der Trennung gesehen. Es wird angenommen, dass mit zunehmendem zeitlichem Abstand zur elterlichen Trennung der Kontakt zwischen Vater und Kind sinkt und die Wahrscheinlichkeit von Zahlungsschwierigkeiten steigt.

Tab. 9.31: Operationalisierungen der Dauer seit der Trennung.

\begin{tabular}{|c|c|c|c|}
\hline & Forsa & Bradshaw & NOVA \\
\hline Frage & $\begin{array}{l}\text { Und seit welchem } \\
\text { Jahr wohnen sie nicht } \\
\text { mehr zusammen? }\end{array}$ & $\begin{array}{l}\text { Wann endete die } \\
\text { Beziehung? }\end{array}$ & $\begin{array}{l}\text { Wie viel Zeit ist } \\
\text { vergangen seit die } \\
\text { Ehe/ das } \\
\text { Zusammenwohnen } \\
\text { beendet wurde? }\end{array}$ \\
\hline Metrisch & In Jahren & In Monaten & - \\
\hline Kategorien & $\begin{array}{l}1=\text { weniger als } 1 \text { Jahr; } \\
2=1-2 \text { Jahre; } \\
3=2-3 \text { Jahre; } \\
4=3 \text { Jahre u. länger } \\
5=\text { nie zus.gelebt }\end{array}$ & $\begin{array}{l}1=\text { weniger als } 1 \text { Jahr; } \\
2=1 \text { bis } 2 \text { Jahre; } \\
3=2 \text { bis } 3 \text { Jahre; } \\
4=3 \text { Jahre u. länger } \\
5=\text { nie zus.gelebt }\end{array}$ & $\begin{array}{l}1=\text { weniger als } 1 \text { Jahr; } \\
2=1-2 \text { Jahre; } \\
3=2-3 \text { Jahre; } \\
4=3 \text { Jahre u. länger } \\
5=\text { nie zus.gelebt }\end{array}$ \\
\hline
\end{tabular}

Quelle: eigene Darstellung.

Die Dauer seit der elterlichen Trennung wird im britischen Datensatz in Monaten gemessen. Die Frage wird als „Wann endete die Beziehung?“ formuliert und nicht an solche Partnerschaften gestellt, die nie zusammengewohnt haben. Diese fallen wie in der deutschen und norwegischen Studie aus der Erfassung. Mit Hilfe der Angabe, ob die Eltern früher einmal zusammengelebt haben, wird die letzte Kategorie in allen drei Datensätzen zusätzlich konstruiert. ${ }^{194}$ In der norwegischen Untersuchung liegen die Angaben zur Dauer

${ }^{194}$ Damit die Väter, die nie mit der ehemaligen Partnerin zusammengelebt haben - die in allen drei Studien eine kleine Minderheit ausmachen - , nicht gänzlich aus der multiplen Analyse herausfallen, werden sie hier als höchste Kategorie kodiert. Inhaltlich ist dies nicht ganz konsistent, denn nur weil die Paare nie zusammengezogen sind, bedeutet dies nicht, dass ihre Trennung am längsten her ist. Wird jedoch vom Auszug wenn auch als Indikator für die Trennung - ausgegangen, liegt der Punkt des Nicht-mehr-Zusammenwohnens bei diesen Paaren am längsten zurück. 
seit der Trennung lediglich in Kategorien vor. Zur besseren Vergleichbarkeit werden diese auf die anderen beiden Datensätze übertragen.

Tab. 9.32: Kreuztabellen zur Dauer seit der Trennung.

\begin{tabular}{|c|c|c|c|c|c|c|c|c|c|c|c|c|}
\hline & Forsa & & & $\mathrm{N}=790$ & Bradsha & & & $\mathrm{N}=479$ & NOVA & & & $\mathrm{N}=498$ \\
\hline $\begin{array}{r}\text { Dauer seit } \\
\text { Trennung }\end{array}$ & 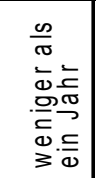 & 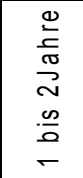 & 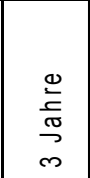 & 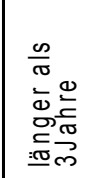 & 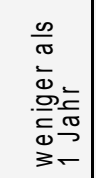 & 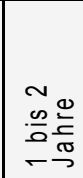 & 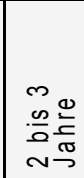 & 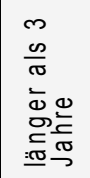 & 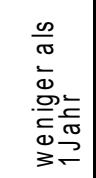 & 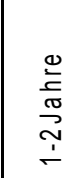 & 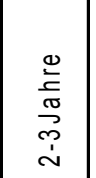 & 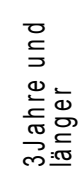 \\
\hline kein Kontakt & $0,00 \%$ & $4,32 \%$ & $8,00 \%$ & $11,74 \%$ & $2,00 \%$ & $4,08 \%$ & $9,09 \%$ & $15,77 \%$ & $0,00 \%$ & $4,17 \%$ & $4,26 \%$ & $6,23 \%$ \\
\hline sehr wenig intensiv & $0,00 \%$ & $0,00 \%$ & $2,67 \%$ & $2,39 \%$ & $10,00 \%$ & $6,12 \%$ & $4,55 \%$ & $8,63 \%$ & $2,94 \%$ & $2,08 \%$ & $0,00 \%$ & $3,52 \%$ \\
\hline wenig intensiv & $12,50 \%$ & $4,94 \%$ & $5,33 \%$ & $10,28 \%$ & $20,00 \%$ & $28,57 \%$ & $15,91 \%$ & $24,70 \%$ & $5,88 \%$ & $6,25 \%$ & $12,77 \%$ & $15,45 \%$ \\
\hline moderat & $50,00 \%$ & $34,57 \%$ & $25,33 \%$ & $26,97 \%$ & $30,00 \%$ & $34,69 \%$ & $47,73 \%$ & $25,30 \%$ & $32,35 \%$ & $35,42 \%$ & $25,53 \%$ & $36,31 \%$ \\
\hline intensiv & $12,50 \%$ & $39,51 \%$ & $34,67 \%$ & $29,91 \%$ & $26,00 \%$ & $14,29 \%$ & $15,91 \%$ & $18,15 \%$ & $44,12 \%$ & $41,67 \%$ & $46,81 \%$ & $30,62 \%$ \\
\hline sehr intensiv & $25,00 \%$ & $16,67 \%$ & $24,00 \%$ & $18,72 \%$ & $12,00 \%$ & $12,24 \%$ & $6,82 \%$ & $7,44 \%$ & $14,71 \%$ & $10,42 \%$ & $10,64 \%$ & $7,86 \%$ \\
\hline Gesamt & 8 & 162 & 75 & 545 & 50 & 49 & 44 & 336 & 34 & 48 & 47 & 369 \\
\hline & Forsa & & & $\mathrm{N}=817$ & Bradsha & & & $\mathrm{N}=474$ & NOVA & & & $\mathrm{N}=478$ \\
\hline $\begin{array}{l}\text { Dauer seit } \\
\text { Trennuna }\end{array}$ & 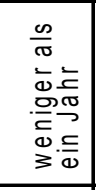 & 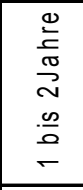 & $\begin{array}{l}\frac{\Phi}{2} \\
\stackrel{\frac{\pi}{\sigma}}{D} \\
m\end{array}$ & 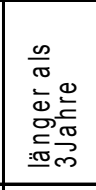 & 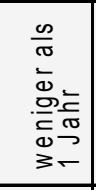 & 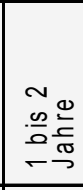 & 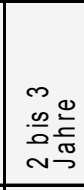 & 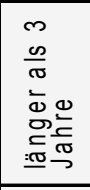 & 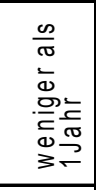 & 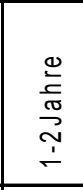 & 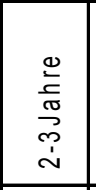 & 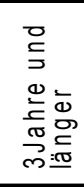 \\
\hline keine Probleme & $85,71 \%$ & $84,24 \%$ & $82,43 \%$ & $80,91 \%$ & $50,00 \%$ & $64,58 \%$ & $65,91 \%$ & $55,09 \%$ & $63,64 \%$ & $64,58 \%$ & $62,79 \%$ & $74,58 \%$ \\
\hline Zahlungsprobleme & $14,29 \%$ & $15,76 \%$ & $17,57 \%$ & $19,09 \%$ & $50,00 \%$ & $35,42 \%$ & $34,09 \%$ & $44,91 \%$ & $36,36 \%$ & $35,42 \%$ & $37,21 \%$ & $25,42 \%$ \\
\hline Gesamt & 7 & 165 & 74 & 571 & 48 & 48 & 44 & 334 & 33 & 48 & 43 & 354 \\
\hline
\end{tabular}

Quelle: eigene Berechnung.

Tabelle 9.32 zeigt bei den deutschen Vätern kein eindeutiges Zusammenhangsmuster zwischen der Dauer seit der elterlichen Trennung und dem sozio-emotionalen Kümmern des Vaters zum Zeitpunkt der Befragung. Zwar ist der Anteil der Väter mit Kontaktabbrüchen am höchsten unter den Befragten, bei denen die Trennung länger als drei Jahre zurück liegt, doch innerhalb der anderen drei Kategorien, d.h. für Väter, die kürzer als drei Jahre getrennt leben, können keine empirischen Auffälligkeiten ausgemacht werden, die auf einen Zusammenhang hindeuten. Der Anteil der Väter, die den Kontakt abgebrochen haben, ist unter den länger getrennt Lebenden etwas höher als in den anderen Gruppen. Gleichzeitig sind die Anteile der höheren Care-Niveaus unter den Vätern größer, bei denen die Trennung noch kein Jahr zurückliegt. Im britischen Datensatz weisen die Ergebnisse tendenziell auf eine negative Beziehung hin. Väter, die länger als zwei Jahre getrennt leben, haben deutlich häufiger den Kontakt abgebrochen im Vergleich zu Vätern, bei de- 
nen die Trennung kürzer zurückliegt. Gleichzeitig weisen sie geringere Anteile an den oberen Sorgekategorien auf. Damit kann die aufgestellte These anhand von zwei Datensätzen bestätigt werden. ${ }^{195}$

Innerhalb der deutschen und britischen Daten lässt sich kein Zusammenhang zwischen der Dauer seit der Trennung und der väterlichen Unterhaltsleistung erkennen. Im deutschen Fall ist eine leichte Zunahme des Anteils der Zahlungsprobleme mit steigender zeitlicher Distanz zur elterlichen Trennung zu erkennen. Doch die Unterschiede über die Ausprägungen der erklärenden Variablen sind marginal. Im britischen Datensatz schwanken die Anteile über die Kategorien, so dass gar keine Tendenz erkennbar ist. Ähnliches gilt auch für die norwegischen Angaben. Hier weisen Väter, die länger als drei Jahre getrennt von ihren Kindern leben, sogar einen geringeren Anteil an Unterhaltsschwierigkeiten auf, was gegen den theoretisch vermuteten Zusammenhang spricht. Aber auch hier lassen die Ergebnisse der anderen Kategorien keinen fundierten Schluss auf einen Zusammenhang zu. Alle drei Studien stützen damit die angenommene These zunächst nicht.

Tab. 9.33: Operationalisierung der Anzahl der Beziehungen mit externen Kindern.

\begin{tabular}{|c|c|c|c|}
\hline \multirow{2}{*}{ Frage } & Forsa & Bradshaw & NOVA \\
\hline & $\begin{array}{l}\text { Wie viele } \\
\text { verschiedene Mütter } \\
\text { gibt es denn? }\end{array}$ & $\begin{array}{l}\text { Wie viele } \\
\text { Beziehungen hatten } \\
\text { Sie, aus denen Kinder } \\
\text { hervorgegangen sind? }\end{array}$ & $\begin{array}{l}\text { Haben diese Kinder } \\
\text { dieselbe Mutter? }\end{array}$ \\
\hline Original & $\begin{array}{l}1 \text { = } 1 \text { Mutter; } \\
2 \text { = } 2 \text { Mütter; } \\
3=3 \text { Mütter; } \\
4=4 \text { und mehr Mütter }\end{array}$ & $\begin{aligned} 1= & \text { aus } 1 \text { Beziehung; } \\
2= & \text { aus } 2 \\
& \text { Beziehungen; } \\
3= & \text { aus } 3 \text { und mehr } \\
& \text { Beziehungen }\end{aligned}$ & $\begin{aligned} 1= & \text { ja, alle haben } \\
& \text { dieselbe Mutter; } \\
2= & \text { nein, sie haben } 2 \\
& \text { verschiedene } \\
& \text { Mütter; } \\
3= & \text { nein, sie haben } 3 \\
& \text { oder mehr versch. } \\
& \text { Mütter }\end{aligned}$ \\
\hline Kategorien & $\begin{array}{l}1 \text { = } 1 \text { Mutter; } \\
2=2 \text { Mütter; } \\
3=3 \text { und mehr Mütter }\end{array}$ & $\begin{aligned} 1= & \text { aus } 1 \text { Beziehung; } \\
2= & \text { aus } 2 \\
& \text { Beziehungen; } \\
3= & \text { aus } 3 \text { und mehr } \\
& \text { Beziehungen }\end{aligned}$ & $\begin{array}{l}1 \text { = } 1 \text { Mutter; } \\
2=2 \text { Mütter; } \\
3=3 \text { und mehr Mütter }\end{array}$ \\
\hline
\end{tabular}

Quelle: eigene Darstellung.

\footnotetext{
195 Kritisch ist an dieser Stelle ferner auf die unterschiedlichen Zellenbesetzungen in den drei Studien zu verweisen. In allen drei Untersuchungen die überwiegende Mehrheit der befragten Väter länger als drei Jahre getrennt lebend. Die geringe Variation kann die Ergebnisse verzerren. Darüber hinaus fehlen bei vielen deutschen und britischen Vätern die Angaben zur Dauer seit der Trennung. Dabei zeigen die deutschen Ausfälle etwas weniger väterliches Sorgehandeln in Form von Care, während sie gleichzeitig seltener Zahlungsschwierigkeiten haben. Mit Blick auf das sozio-emotionale Handeln gilt im britischen Fall das Gleiche. Auch hier zeigen Väter ohne Angaben etwas weniger Engagement. In ihrer Unterhaltspraxis unterscheiden sie sich dagegen nicht.
} 
Die Anzahl der Beziehungen des Vaters, aus denen Kinder hervorgegangen sind, stellt den letzten berücksichtigten Einflussfaktor dar. Es wird davon ausgegangen, dass mit steigender Zahl gescheiterter Beziehungen in Verbindung mit Elternschaften die Wahrscheinlichkeit eines intensiven Kontakts ebenso abnimmt wie die Wahrscheinlichkeit verlässlicher Unterhaltszahlungen.

Innerhalb des britischen Datensatzes wird die Information, wie viele Beziehungen der Vater gehabt hat, die gescheitert sind und aus denen Kinder hervorgegangen sind, in den Kategorien „aus einer Beziehung“, „,aus zwei Beziehungen“ und „aus drei und mehr Beziehungen“ erfasst. ${ }^{196}$ Norwegische Väter können auf die Frage, ob die getrennt lebenden Kinder dieselbe Mutter haben, ebenso mit drei Kategorien antworten. In Deutschland werden vier Ausprägungen ursprünglich vorgegeben, welche in drei Antworten den anderen beiden Datensätzen angepasst werden.

In allen drei Datensätzen ist der Anteil der Väter, die aus mehr als einer Beziehung Elternschaften aufweisen, im Vergleich zu den Vätern, die nur aus einer Beziehung getrennt lebende unterhaltsberechtigte Kinder haben, deutlich geringer. Dabei zeigt die Kreuztabellierung des deutschen empirischen Materials, dass diese Mehrfach-Väter häufiger den Kontakt abgebrochen haben und seltener (sehr) intensives väterliches Engagement aufweisen. Diese Ergebnisse lassen vorsichtig auf einen negativen Zusammenhang schließen. Deutlicher wird dieser in den britischen Daten sichtbar. Die Angaben norwegischer Väter lassen dagegen keinen eindeutigen Schluss zu. Insgesamt kann damit die theoretisch begründete Hypothese von zwei der drei Datensätze gestützt werden.

Deutsche Väter, die mehr als eine Elternschaft angeben, weisen einen deutlich höheren Anteil an Zahlungsschwierigkeiten auf als Väter mit nur einer getrennt lebenden Vaterschaft. Der damit angedeutete positive Zusammenhang zwischen der Anzahl der Elternschaften und den Zahlungsproblemen ist noch deutlicher unter den britischen Nachtrennungsvätern erkennbar. Auch in Norwegen kommen die Väter, die von mehr als einer Mutter getrennt leben, häufiger ihren Unterhaltsverpflichtungen nicht nach. Die Ergebnisse aller drei Studien rechtfertigen damit den angenommenen Zusammenhang.

\footnotetext{
${ }^{196}$ Diese Variable weist eine hohe Ausfallrate auf. Es könnte ein Effekt sozialer Erwünschtheit vermutet werden. Vielleicht ist es den Vätern unangenehm diese Frage zu beantworten, wenn sie mehr als ein oder zwei Elternschaften aus ihrer Vergangenheit angeben müssten. Dies bleibt jedoch reine Spekulation. Die Ausfälle in der Variablen zur Anzahl der getrennt lebenden Kinder zeigen im britischen Datensatz deutlich weniger sozio-emotionales Engagement und häufiger Zahlungsschwierigkeiten im Vergleich zu Vätern, die die Zahl ihrer Unterhaltsverpflichtungen benennen.
} 
Tab. 9.34: Kreuztabellen zur Anzahl der Beziehungen mit externen Kindern.

\begin{tabular}{|c|c|c|c|c|c|c|c|c|c|}
\hline & Forsa & & $\mathrm{N}=872$ & Bradsha & & $\mathrm{N}=460$ & NOVA & & $\mathrm{N}=551$ \\
\hline sehr wenig intensiv & $2,00 \%$ & $9,09 \%$ & $0,00 \%$ & $6,48 \%$ & $14,05 \%$ & $13,33 \%$ & $3,44 \%$ & $0,00 \%$ & $0,00 \%$ \\
\hline wenig intensiv & $9,43 \%$ & $9,09 \%$ & $50,00 \%$ & $24,38 \%$ & $17,36 \%$ & $20,00 \%$ & $13,16 \%$ & $9,80 \%$ & $16,67 \%$ \\
\hline moderat & $27,83 \%$ & $27,27 \%$ & $0,00 \%$ & $29,32 \%$ & $27,27 \%$ & $20,00 \%$ & $35,83 \%$ & $27,45 \%$ & $0,00 \%$ \\
\hline intensiv & $32,31 \%$ & $27,27 \%$ & $0,00 \%$ & $21,60 \%$ & $13,22 \%$ & $13,33 \%$ & $32,59 \%$ & $37,25 \%$ & $50,00 \%$ \\
\hline sehr intensiv & $18,16 \%$ & $0,00 \%$ & $50,00 \%$ & $9,57 \%$ & $4,96 \%$ & $0,00 \%$ & $8,70 \%$ & $7,84 \%$ & $16,67 \%$ \\
\hline Gesamt & 848 & 22 & 2 & 324 & 121 & 15 & 494 & 51 & 6 \\
\hline keine Probleme & $82,51 \%$ & $60,87 \%$ & $100,00 \%$ & $60,94 \%$ & $50,41 \%$ & $13,33 \%$ & $73,84 \%$ & $58,82 \%$ & $0,00 \%$ \\
\hline Zahlungsprobleme & $17,49 \%$ & $39,13 \%$ & $0,00 \%$ & $39,06 \%$ & $49,59 \%$ & $86,67 \%$ & $26,16 \%$ & $41,18 \%$ & $100,00 \%$ \\
\hline Gesamt & 875 & 23 & 2 & 320 & 121 & 15 & 474 & 51 & 5 \\
\hline
\end{tabular}

Quelle: eigene Berechnung.

Insgesamt ergeben sich anhand der Kreuztabellen sehr heterogene Ergebnisse zwischen den einzelnen Datensätzen aber auch mit Blick auf die einzelnen Hypothesen. In einigen Tabellen lassen sich augenscheinlich Zusammenhänge erkennen, die auch theoretisch begründet werden können. Andere Tabellen lassen dagegen keine eindeutigen Schlüsse über generelle Wirkungsrichtungen oder Zusammenhänge zu. Im folgenden Abschnitt werden daher die Ergebnisse der Kreuztabellen noch einmal zusammenfassend mit einem Zusammenhangsmaß für jede erklärende Variable überprüft und interpretiert. Abschließend wird ein Überblick über die (nicht) bestätigten Zusammenhänge auf bivariater Ebene gegeben.

\subsubsection{Ergebnisse der bivariaten Zusammenhangsanalyse}

In den vorangegangenen Abschnitten wurde die jeweilige Operationalisierung sowie Kreuztabellierung der einzelnen Prädiktoren mit Care bzw. Cash dargestellt. Im Folgenden werden die bivariaten Zusammenhänge, die in den Kreuztabellen (nicht) zu beobachten waren, in einem Zusammenhangsmaß verdichtet und interpretiert. 


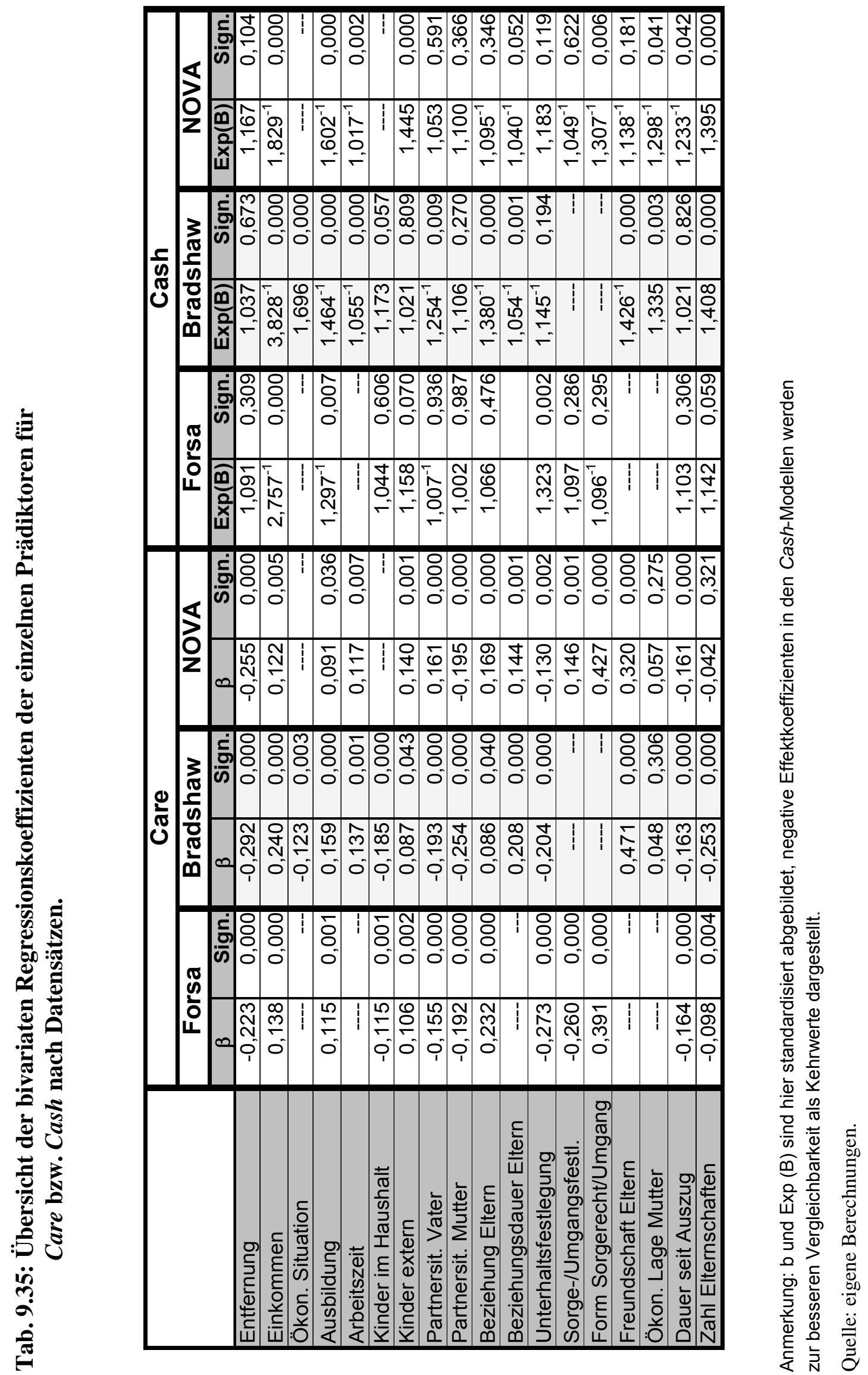


Die hier präsentierten Ergebnisse können von denen der Kreuztabellierung, die einen ersten Eindruck geliefert haben, abweichen. Dies liegt u.a. darin begründet, dass im nachstehenden Analyseschritt immer jener Prädiktor Verwendung findet, der das höchste Skalenniveau aufweist. Das bedeutet, dass - soweit vorhanden - nun metrische Variablen beibehalten und nicht kategorisiert werden. Dies gilt auch für die abhängige Variable Care. Die Darstellung folgt den theoretischen Annahmen in ihrer aufgestellten Reihenfolge anhand der jeweiligen Koeffizienten, die in ihrer Richtung, Stärke und Signifikanz interpretiert werden. ${ }^{197}$ Sie beginnt mit den Effekten, die die Prädiktoren auf das Kriterium Care haben. Es folgen die Ergebnisse für die Cash-Variable. Das Kapitel der bivariaten Analyse schließt mit einer Übersichtstabelle der gefundenen Zusammenhänge und bestätigten Hypothesen. Inhaltliche Interpretationen finden sich gebündelt im Anschluss an die Präsentation der multiplen Regressionsmodelle.

Tabelle 9.35 stellt die einzelnen Regressionsgewichte der erklärenden Variablen je Datensatz für Care in metrischer Form als Score und Cash wie bisher als dichotome Variable getrennt dar. ${ }^{198}$

\subsubsection{Bivariate Zusammenhänge: Care}

Die Entfernung zwischen den Wohnorten der Eltern, so die theoretische Annahme, hat einen negativen Effekt auf die Intensität des väterlichen Engagements in Form von Care. Innerhalb der drei untersuchten Datensätze kann dieser Zusammenhang auf bivariater Ebene signifikant nachgewiesen werden. Dabei sind die Regressionskoeffizienten mit einer Stärke von rund -0,25 ähnlich groß und weisen einen moderaten Zusammenhang aus. Inhaltlich bedeutet dies, dass sich in Deutschland, Großbritannien und Norwegen Nachtrennungsväter weniger intensiv um ihre getrennt lebenden Kinder kümmern, je weiter sie von diesen entfernt wohnen. Damit kann die aufgestellte These in allen drei Datensätzen empirische Unterstützung finden.

Dem Einkommen des Vaters wird demgegenüber ein positiver Zusammenhang mit dem Niveau väterlichen Sorgens in Form von Care unterstellt. Auch diese Korrelation kann im

\footnotetext{
197 Zur Begründung der einzelnen Maßzahlen siehe ausführlich Kap. 8.3 Statistisches Vorgehen: Modelle und Maßzahlen.

198 Es wurde bereits darauf verwiesen, dass für alle - auch ordinalen - Prädiktoren metrische Zusammenhangsmaße ausgegeben werden. Zwar kann bei einigen Variablen kritisch eingewandt werden, dass ihr Skalenniveau nicht als metrisch zu interpretieren ist. Zur besseren Vergleichbarkeit der einzelnen Zusammenhänge wird hier jedoch ein gemeinsames Skalenniveau theoretisch angenommen (siehe dazu ausführlicher Kap. 8.3 Statistisches Vorgehen: Modelle und Maßzahlen).
} 
Datenmaterial der drei Länder signifikant nachgewiesen werden. Dabei ist das Assoziationsmaß im britischen Fall mit 0,24 am stärksten, gefolgt vom deutschen mit 0,138 und dem norwegischen mit 0,122. ${ }^{199}$ Die ausgewiesenen Zusammenhänge nehmen eine moderate Stärke an. Wie theoretisch angenommen, steigt damit das Niveau des väterlichen Sorgehandelns mit dem Einkommen des Vaters an.

Das Ausbildungsniveau zeigt in allen drei Datensätzen, wie theoretisch angenommen, einen positiven Zusammenhang mit dem väterlichen Care. Mit steigendem Bildungsniveau des Vaters nimmt das väterliche Engagement in seiner Intensität zu. Dabei weisen die Koeffizienten in den drei Datensätzen unterschiedliche Stärken auf. Ein eher schwacher - gar zu vernachlässigter - Zusammenhang findet sich in den norwegischen Daten mit einem Wert von 0,091. Hier liegt das Signifikanzniveau bei 0,036 noch im Rahmen der angenommenen Signifikanzgrenze. Sowohl im deutschen wie auch im britischen Datenmaterial ist der Effekt deutlich signifikant. Die größere Maßzahl findet sich hierbei in Großbritannien mit 0,159. Sie lässt - unter Berücksichtigung der Unsicherheiten in der Operationalisierung $^{200}$ - vorsichtig auf einen moderaten Zusammenhang zwischen dem Bildungsstand des Vaters und seinem Sorgehandeln schließen. Im deutschen Datensatz beträgt das Maß 0,115. Insgesamt stützen zwei der drei Studien den erwarteten Zusammenhang empirisch ab, während die norwegischen Daten mit einer schwachen Korrelation zumindest in die angenommene Richtung weisen.

Die Arbeitszeit wird theoretisch als Restriktion der väterlichen Involviertheit auf sozioemotionaler Ebene verstanden. Damit sollte ein negativer Zusammenhang zwischen steigender Arbeitszeit und Care bestehen. Die empirischen Ergebnisse widerlegen diese Annahme jedoch in beiden Studien, in denen die erklärende Variable vorliegt. In Großbritannien wie in Norwegen weisen die Regressionskoeffizienten mit 0,137 und 0,117 signifikant einen moderaten positiven Zusammenhang aus. Damit steigt die sozio-emotionale Involviertheit des Vaters entgegen der Hypothese mit seiner Arbeitszeit an.

\footnotetext{
${ }^{199}$ Das britische Ergebnis muss dabei aufgrund hoher Ausfälle in der Erhebung der Einkommensvariablen mit Vorsicht interpretiert werden. Hier gehen nur wenige Fälle in die Analyse ein, und zwar die, die bereit waren Auskunft über ihre Einkünfte zu geben. Dies kann die Ergebnisse gegebenenfalls verzerren. Die Betrachtung der britischen Ausfälle in der Einkommensvariablen zeigt jedoch, dass Väter ohne Angaben auf univariater Ebene im Vergleich zu Vätern, die ihr Einkommen angeben, keine nennenswerten Unterschiede im väterlichen Sorgehandeln - weder sozio-emotional noch finanziell - aufweisen.

${ }^{200}$ Siehe dazu die Ausführungen zu den Mängeln in der Operationalisierung des väterlichen Bildungsstandes im britischen Datensatz im Kap. 9.1.1. Der Einfluss der Prädiktoren der physischen Umwelt.
} 
Die Zahl der Kinder im Haushalt des Vaters soll laut Hypothese einen negativen Effekt auf das väterliche Kümmern um getrennt lebende Kinder haben. Diese Annahme kann empirisch bestätigt werden. Sowohl im deutschen wie auch im britischen Datensatz liegen die notwendigen Informationen zur Thesenprüfung vor und der angenommene Zusammenhang wird signifikant gestützt. Dabei ist der Effekt unter britischen Vätern mit -0,185 nur leicht stärker als in Deutschland ausgeprägt. In beiden Ländern weisen Väter mit steigender Anzahl an Kindern in ihrem Haushalt ein geringeres Sorgehandeln in Form von Care auf.

Anders dagegen die Berechnungen der Regressionsmaße im Fall der Anzahl der externen Kinder; diese weisen eine entgegengesetzte Wirkungsrichtung aus, als innerhalb der Hypothese formuliert wird. Zwar kann ein empirischer Zusammenhang nachgewiesen werden, doch entgegen der theoretischen Erwartung gibt das Maß eine positive Relation an. Bei Regressionsgewichten um 0,1 ist der Zusammenhang jedoch nicht sonderlich stark ausgeprägt. Dennoch bedeutet das Ergebnis inhaltlich, dass mit steigender Zahl an Unterhaltsverpflichtungen sich deutsche, britische und norwegische Väter signifikant intensiver kümmern, auch wenn mehr Kinder um die begrenzten Ressourcen des Vaters konkurrieren.

Die aktuelle Partnersituation des Vaters hängt, wie erwartet, negativ mit dem Niveau väterlichen Engagements zusammen. In Deutschland und Großbritannien weisen die Daten mit einem Koeffizienten von -0,155 und -0,193 auf einen mäßigen Zusammenhang mit negativer Wirkungsrichtung hin. Das bedeutet, je stärker die väterliche Beziehung formalisiert ist, desto eher leidet das väterliche Care darunter. Im norwegischen Fall wird entgegen den beiden anderen Datensätzen ein positiver Zusammenhang errechnet. Dies erklärt sich mit der Kodierung im norwegischen Datenmaterial, da hier, wie dargestellt, nur zwischen „der Vater lebt mit anderen“ bzw. „der Vater lebt allein“ differenziert wird. ${ }^{201}$ Ein positiver Zusammenhang bedeutet demnach, dass allein lebende Väter eine höhere Wahrscheinlichkeit für ein intensives väterliches Sorgehandeln aufweisen. Der Zusammenhang ist mit einem Wert von 0,161 ebenso wie in Deutschland und Großbritannien als moderat aber signifikant zu charakterisieren. Damit stützen die Daten in allen drei Ländern die theoretische Annahme auf bivariater Ebene.

Auch der Partnersituation der Mutter wird theoretisch ein negativer Effekt auf das sozioemotionale Handeln des Vaters unterstellt. Innerhalb des empirischen Materials findet sich

${ }^{201}$ Siehe dazu ausführlich Kap. 9.1.2 Einfluss der Prädiktoren der mikro-sozialen Umwelt. 
dabei mit einem Koeffizienten von -0,192 in Deutschland, -0,195 in Norwegen und -0,254 in Großbritannien ein moderater negativer Zusammenhang. Somit bestätigt sich empirisch die Annahme, dass sich ein neuer Partner im Leben der Mutter negativ auf das Sorgehandeln des Vaters in Form von Care auswirkt.

Ferner wird theoretisch angenommen, dass die Familienform der Eltern vor der Trennung mit steigender Formalisierung einen positiven Effekt auf die Intensität des sozio-emotionalen Engagements des Vaters hat. Diese Annahme kann von allen drei Datensätzen gestützt werden. Dabei weisen die deutschen Daten auf einen moderaten, signifikanten Zusammenhang hin $(0,232)$, in Norwegen ist das Regressionsgewicht mit 0,169 geringfügig und in Großbritannien mit 0,086 deutlich schwächer ausgeprägt, aber auch (noch) signifikant. Inhaltlich kann damit geschlussfolgert werden, dass sich eine stärkere Formalisierung der elterlichen Beziehung vor ihrem Scheitern positiv auf die Intensität des väterlichen Sorgehandelns nach der Trennung auswirkt.

Ein ähnlicher Zusammenhang wird für die Dauer der elterlichen Beziehung erwartet. Auch hier weisen die beiden Datensätze, die die notwendigen Informationen bereitstellen, den theoretisch angenommenen positiven Zusammenhang signifikant aus. Dabei ist der Effekt im britischen Datenmaterial mit 0,208 als moderater Zusammenhang zu interpretieren und etwas stärker ausgeprägt als bei den norwegischen Vätern $(0,144)$. Mit einem längeren Fortbestehen der elterlichen Beziehung vor ihrem Scheitern steigt damit die Wahrscheinlichkeit eines intensiveren Kümmerns auf sozio-emotionaler Ebene.

Mit Blick auf den Prozess der Unterhaltsfestlegung stützen die empirischen Ergebnisse in allen drei Fällen signifikant die theoretische Annahme, dass sich der Vater intensiver um seine getrennt lebenden Kinder kümmert, je höher der Konsens zwischen den Eltern bei der Festlegung ist. Dabei ist das Regressionsgewicht in Deutschland mit -0,273 und in Großbritannien mit -0,204 stärker ausgeprägt als in Norwegen mit -0,13. Die empirischen Ergebnisse bestätigen in Richtung und Signifikanz die aufgestellte Hypothese. Das bedeutet, dass es eher zu Kontaktabbrüchen bzw. zu einem geringeren Niveau väterlichen Sorgehandelns in Form von Care kommt, je „höher“ die in den Konflikt der Festlegung eingeschaltete Instanz jeweils ist.

Der gleiche Einfluss wird auch der Sorgerechts- bzw. Umgangsfestlegung zwischen den Eltern als Indikator für das elterliche Verhältnis während der Trennung unterstellt. Doch das empirische Material liefert widersprüchliche Ergebnisse. In Deutschland kann ein guter negativer Effekt des Prädiktors auf die zu erklärende Variable Care $(-0,260)$ auf signifikantem Niveau nachgewiesen werden. Auch in Norwegen besteht ein signifikanter Zusammenhang, doch dieser weist die entgegengesetzte Wirkungsrichtung aus als theoretisch angenommen. Dies bedeutet inhaltlich, dass in Deutschland der Kontakt zwischen 
Vater und Kind weniger intensiv ist, wenn die Festlegung des Sorgerechts mit starken Auseinandersetzungen zwischen den Eltern verbunden war. Im norwegischen Fall dagegen führt eine Festlegung des Umgangsrechts mit Hilfe externer Instanzen, Behörden oder Gerichten, zu einem höheren sozio-emotionalen Engagement unter Nachtrennungsvätern.

Eindeutige Ergebnisse zur Unterstützung des theoretisch begründeten Zusammenhangs liefern wiederum die Daten mit Blick auf die Form des festgelegten Sorge- bzw. Umgangsrechts. In beiden Studien ergeben sich mit einem Beta-Gewicht von rund 0,4 im Vergleich zu den anderen Regressionskoeffizienten starke Zusammenhänge auf signifikantem Niveau. Damit kommt der formal festgelegten Form, wie erwartet, eine besondere Bedeutung für die Ausgestaltung der väterlichen Kontaktpraxis zu. Deutsche Väter mit gemeinsamem Sorgerecht kümmern sich signifikant stärker als Väter, die sich nicht die Sorge mit der Mutter teilen. Für norwegische Väter gilt, je mehr Umgang in Relation zum „normalen Umgang“ sie festgelegt haben, desto intensiver kümmern sie sich um ihre Kinder. Die bivariaten Ergebnisse sowohl in Norwegen wie auch in Deutschland bestätigen damit die aufgestellte Hypothese. An dieser Stelle ist die Richtung zwischen Ursache und Wirkung jedoch kritisch zu reflektieren. Denn auf den ersten Blick scheint das vorliegende Ergebnis auf bivariater Ebene trivial, es bleibt aber unklar, was zuerst da ist: ob ein Vater, der sich auch vor der Trennung schon viel um sein Kind kümmert, einen hohen Umgang „aushandelt“ oder ob ein Vater, der auf einer hohen Kontakthäufigkeit bei der Festlegung des Umgangs beharrt, diese auch ausfüllt.

Eine weitere Determinante väterlichen Cares wird im freundschaftlichen Verhältnis der Eltern im Zeitpunkt der Befragung gesehen. Auch hier bestätigt sich die theoretische Annahme empirisch. Das bedeutet, dass Väter sich intensiver kümmern, wenn sie das Verhältnis zur Kindsmutter als freundschaftlich charakterisieren. Dabei ist der Effekt in beiden Datensätzen, in denen die Information verfügbar ist, mit einem Koeffizienten von 0,471 in Großbritannien und 0,32 in Norwegen (signifikant) stark ausgeprägt.

Der ökonomischen Situation der Mutter wird theoretisch keine Bedeutung zur Erklärung von Care zugesprochen. Diese Annahme findet durch die empirischen Ergebnisse in Großbritannien und Norwegen Bestätigung, da die Koeffizienten sowohl in Großbritannien als auch in Norwegen sehr schwach ausgeprägt und nicht signifikant sind. Damit wird die Hypothese durch beide Studien gestützt.

In allen drei Datensätzen findet sich ein mäßiger Zusammenhang zwischen der Dauer seit der Trennung und Care. Mit Koeffizienten von -0,164 in Deutschland, -0,163 in Großbritannien und -0,161 in Norwegen sind die Effekte dabei in ihrer Stärke ähnlich ausgeprägt. Inhaltlich bedeutet dies, dass mit fortschreitender zeitlicher Distanz zur Trennung der El- 
tern die Intensität des sozio-emotionalen Kontakts zwischen Vater und Kind abnimmt. Der erwartete Zusammenhang wird empirisch gestärkt.

Als letzter Prädiktor wird die Anzahl der gescheiterten Partnerschaften, aus denen Kinder hervorgegangen sind, in die Analyse von Care einbezogen. Hierbei besteht die theoretische Annahme, dass mit steigender Zahl der Elternschaften von unterschiedlichen Müttern das Sorgehandeln in seiner Intensität abnimmt. Der erwartete negative Zusammenhang wird nur im britischen Datensatz mit einem Koeffizienten von -0,253 eindeutig bestätigt. Auch im deutschen Fall wird ein negativer Zusammenhang ausgewiesen, der mit -0,098 aber als eher schwach wenn auch signifikant zu charakterisieren ist. Die norwegischen Angaben lassen auf keinen signifikanten Zusammenhang schließen. Somit wird die angenommene Beziehung zwischen der Anzahl der externen Elternschaften und dem Niveau des sozio-emotionalen Engagements des Vaters nur in zwei Studien ansatzweise gestützt.

Insgesamt zeigen die Ergebnisse mit Blick auf die Care-Variable kaum länderspezifische Unterschiede in der Wirkungsrichtung der angenommenen Zusammenhänge. Lediglich die Sorge- bzw. Umgangsrechtsfestlegung weisen unterschiedliche Vorzeichen im gefundenen Zusammenhang auf. Die Stärke der Effekte variiert geringfügig zwischen den Ländern.

\subsubsection{Bivariate Zusammenhänge: Cash}

Als Indikator für das finanzielle Sorgehandeln des Vaters werden die Zahlungsprobleme herangezogen. Dabei werden theoretisch andere Zusammenhänge zwischen den einzelnen Prädiktoren und der abhängigen Variable Cash angenommen als noch bei Care. So soll die Entfernung zwischen den elterlichen Wohnorten keinen Einfluss auf das Leisten von Unterhaltszahlungen haben. Diese Annahme wird durch die empirischen Ergebnisse gestützt. Keiner der ausgegebenen Regressionskoeffizienten ist mit entsprechender Signifikanz relevant.

Anders sehen dagegen die Maßzahlen aus, die den Zusammenhang zwischen der Zahlungsmoral und dem väterlichen Einkommen quantifizieren. Es wird theoretisch angenommen, dass mit steigendem Erwerbseinkommen die Wahrscheinlichkeit von Zahlungsschwierigkeiten abnimmt. In allen drei Datensätzen wird diese These empirisch signifikant belegt. Dabei ist der negative Effekt in Großbritannien mit rund 3,8 ${ }^{-1}$ am stärksten, während in Deutschland das Gewicht mit $2,8^{-1}$ und Norwegen mit $1,8^{-1}$ zwar schwächer ausgeprägt sind, aber immer noch einen starken Zusammenhang ausweisen. Gemäß dieser Ergebnisse nimmt die Wahrscheinlichkeit, dass ein Vater nicht zuverlässig seinen Zahlungspflichten nachkommt, mit dem Anstieg des Einkommens ab. 
Sich überlagernde Effekte können auch für das Ausbildungsniveau des Vaters vermutet werden. ${ }^{202}$ Hier wird ebenso ein negativer Zusammenhang postuliert. Dieser wird empirisch in allen drei Datensätzen gestützt. In Norwegen drückt die Maßzahl von 1,602 ${ }^{-1}$ einen moderaten Einfluss aus, ebenso die Regressionsgewichte in Großbritannien mit $1,464^{-1}$ und Deutschland mit $1,297^{-1}$. Mit steigendem Ausbildungsniveau des Vaters sinkt damit die Auftretenswahrscheinlichkeit von Zahlungsproblemen.

Ein negativer Zusammenhang wird weiterhin zwischen Unterhaltsproblemen und der Arbeitszeit des Vaters vermutet. Die Ergebnisse bestätigen die Hypothese mit einem Effektkoeffizienten von $1,055^{-1}$ in Großbritannien und $1,017^{-1}$ in Norwegen zwar signifikant, aber weisen nur einen sehr schwachen Zusammenhang aus.

Die Zahl der Kinder im Haushalt des Vaters soll laut Hypothese einen Effekt auf die Zahlungspraxis ausüben: mit steigender Anzahl an Kindern innerhalb des väterlichen Haushalt nimmt die Wahrscheinlichkeit von Zahlungsschwierigkeiten für Kinder außerhalb des Haushalts zu. Diese Annahme findet sich durch die empirischen Daten nur bedingt gestützt. So wird in Großbritannien mit einem Niveau von 0,057 die Signifikanzgrenze für einen positiven Zusammenhang von 1,173 nur knapp überschritten; während sich im deutschen Datensatz keinerlei signifikante Anhaltspunkte für den erwarteten Zusammenhang finden. Die Anzahl der Kinder innerhalb der Wohnung des Vaters scheint daher keinen Einfluss auf seine Zahlungspraxis für getrennt lebende Kinder zu haben.

Auch die Berechnungen der Effektkoeffizienten im Fall der Anzahl der externen Kinder stützen nur bedingt die theoretischen Erwartungen. In Norwegen kann ein positiver Zusammenhang zwischen Anzahl der getrennt lebenden Kinder und dem Auftreten von Zahlungsschwierigkeiten gefunden werden. Mit einem signifikanten Koeffizienten von 1,445 ist dieser als moderat zu beschreiben. Ähnliches gilt für den deutschen Fall mit einem Effektkoeffizienten von 1,158. Dieser liegt mit 0,07 jedoch knapp oberhalb der Signifikanzgrenze. Keinen Zusammenhang lassen die britischen Daten erkennen. Streng genommen wird die aufgestellte These damit nur in einem Datensatz empirisch bestätigt. Der zweite Datensatz weist Tendenzen in die angenommene Wirkungsrichtung auf, während in der dritten Studie gar kein Effekt des Prädiktors zu finden ist.

Die Partnersituation des Vaters determiniert, so die postulierte Annahme, negativ die Zahlungspraxis des Vaters. Diese Annahme findet sich in den Ergebnissen der drei Stu-

202 Diese werden im zweiten Schritt, der multiplen Analyse, genauer betrachtet (siehe Kap. 9.2 Ergebnisse der multiplen Zusammenhangsanalyse). 
dien nicht bestätigt. In Norwegen und Deutschland bestehen keine signifikanten Einflussnahmen, während in Großbritannien gar ein signifikanter negativer Effekt mit einem Koeffizienten von $1,254^{-1}$ ausgemacht wird. Das bedeutet, wenn ein Vater keine neue Partnerin hat, kommt er seinen Zahlungspflichten eher nicht nach, als wenn er aktuell in einer Beziehung lebt. Dies steht im Widerspruch zur aufgestellten Hypothese, die einen positiven Zusammenhang mit dem Auftreten von Zahlungsproblemen aufgrund knapper finanzieller Ressourcen erwartet, um die verschiedene Akteure innerhalb der väterlichen sozialen Umwelt konkurrieren. Eine neue Partnerin scheint jedoch in Großbritannien einen positiven Einfluss auf die väterliche Zahlungsmoral zu haben.

Auch für die Variable der Partnersituation der Mutter werden die erwarteten Zusammenhänge nicht bestätigt. Hier wird theoretisch ein positiver Zusammenhang zwischen einem neuen Partner im Leben der Mutter und der Wahrscheinlichkeit ausbleibender Unterhaltszahlungen angenommen. Zwar finden sich in allen drei Datensätzen positive Koeffizienten, doch keiner von diesen erweist sich als signifikant. Die formulierte These ist damit widerlegt. Die Beziehungssituation der Mutter hat auf das Zahlungsverhalten von Nachtrennungsvätern in Norwegen, Deutschland und Großbritannien keinen Einfluss.

Die Berücksichtigung der früheren Familienform der Eltern auf bivariater Ebene führt ebenso zu heterogenen Ergebnissen in ländervergleichender Perspektive. In Deutschland und Norwegen hat die Familienkonstellation vor der elterlichen Trennung keinen Einfluss auf das Zahlungsverhalten des Vaters danach. In Großbritannien kann der erwartete negative Effekt jedoch empirisch abgebildet werden. Hier zahlen Väter mit höherer Wahrscheinlichkeit nicht, wenn der Vater vor der Trennung nicht mit der Mutter verheiratet war oder nicht mit ihr zusammengelebt hat. Die britischen Daten stützen damit die formulierte Hypothese, die anderen beiden Datensätze nicht.

Laut den theoretischen Ausführungen wird der Dauer der elterlichen Beziehung ein negativer Einfluss auf die Zahlungsprobleme zugesprochen. Das bedeutet, Väter, die länger mit der Kindesmutter in einer Partnerschaft gelebt haben, kommen eher ihren Zahlungsverpflichtungen nach als Väter mit kürzeren Beziehungen zur Ex-Partnerin. Dieser Zusammenhang findet sich in den beiden Datensätzen, in denen die Informationen vorhanden sind, empirisch Unterstützung. Doch mit Effektkoeffizienten von $1,054^{-1}$ und $1,040^{-1}$ ist die Stärke der gefundenen Zusammenhänge zu vernachlässigen. Insofern lässt sich die aufgestellte Hypothese nur sehr bedingt empirisch gesichert stützen.

Ein Anstieg des mit der Festlegung des Unterhalts verbundenen Konfliktpotentials wirkt sich laut Hypothese auf das verstärkte Auftreten von Zahlungsschwierigkeiten aus. Das bedeutet, eine Übereinkunft über den zu zahlenden Unterhalt, die auf Konsens basiert, führt zu weniger Unterhaltsausfällen. Diesen Zusammenhang bestätigen die deutschen 
Daten mit einem Koeffizienten von 1,323 signifikant, wohingegen sich in Norwegen und Großbritannien dagegen keine empirischen Belege für die Annahme finden lassen.

Es wird ferner angenommen, dass die Sorgerechts- bzw. Umgangsfestlegung keinen Zusammenhang mit dem finanziellen Unterstützungshandeln des Vaters aufweist. Diese These findet in Deutschland und in Norwegen empirische Bestätigung. Es können keine Effekte ausgemacht werden. Damit wird die These bestmöglich gestützt.

Dies trifft nur zum Teil auf die Form des festgelegten Sorgerechts bzw. Umgangs zu, der auch kein Effekt auf das Zahlungsverhalten unterstellt wird. Deutsche Väter, deren ExPartnerinnen das alleinige Sorgerecht haben, weisen keine höhere Wahrscheinlichkeit für das Auftreten von Zahlungsschwierigkeiten auf als Väter, die sich die Sorge teilen. In Norwegen besteht dagegen ein negativer Zusammenhang zwischen Umgangsform und ausbleibenden Zahlungen. Das bedeutet, je weniger Umgang zwischen den Eltern festgelegt wird, desto eher treten Unterhaltsprobleme auf.

Es wird theoretisch angenommen, dass zwischen dem freundschaftlichen Verhältnis der Eltern zum Zeitpunkt der Befragung und dem Zahlungsverhalten des Vaters kein Zusammenhang bestehen dürfte. Innerhalb der norwegischen Daten findet diese Annahme empirische Unterstützung. Väter, die das Verhältnis zu ihrer Ex-Partnerin als freundschaftlich beschreiben, weisen keine größere oder kleinere Wahrscheinlichkeit von Unterhaltsunregelmäßigkeiten auf wie z.B. Väter, die keine freundschaftliche Beziehung angeben. Anders bei den britischen Vätern, hier ergibt sich empirisch ein signifikanter relativ starker, negativer Zusammenhang zwischen der Variablen Freundschaft zwischen den Eltern und Cash. Väter, die ihre Beziehung zur Kindsmutter als Freundschaft beschreiben, weisen mit geringerer Wahrscheinlichkeit Zahlungsprobleme auf als Väter, die das Verhältnis als nicht freundschaftlich charakterisieren.

Der ökonomischen Situation der Mutter wird ein negativer Zusammenhang mit Zahlungsschwierigkeiten unterstellt. Diesen Zusammenhang weisen die norwegischen Ergebnisse nicht aus. Der Koeffizient liegt knapp oberhalb der Signifikanzgrenze. Die Wirkungsrichtung der angenommenen Korrelation wird jedoch im norwegischen Fall bestätigt. Im britischen Datensatz ist der Wirkungszusammenhang dagegen andersherum gerichtet als theoretisch angenommen. Mit schlechterer ökonomischer Lage der Mutter nimmt auch die Wahrscheinlichkeit ausbleibender Unterhaltszahlungen zu. Der Koeffizient erweist sich als signifikant.

Es wird theoretisch begründet, dass mit fortschreitender Dauer seit der elterlichen Trennung die Wahrscheinlichkeit auftretender Zahlungsprobleme steigt. Diese Annahme kann mit keiner der Studien gestützt werden. In Deutschland und Großbritannien zeigt sich em- 
pirisch kein signifikanter Zusammenhang. Norwegische Väter dagegen weisen gar einen (knapp signifikanten) negativen Effekt auf, der konträr zur aufgestellten Hypothese steht. Inhaltlich bedeutet das, je kürzer die Trennung zurückliegt, desto eher treten Zahlungsprobleme auf.

Als letzte Determinante wird auch in der Erklärung der Cash-Variable die Anzahl der Beziehungen des Vaters, aus denen Kinder hervorgegangen sind, berücksichtigt. Die Theorie führt zu der Annahme, dass mit steigender Anzahl an Elternschaften auch die Wahrscheinlichkeit ausbleibender Zahlungen zunimmt. Dieser Effekt findet sich in (fast) allen Studien wieder. Mit jeweils einem Koeffizienten von 1,142 in Deutschland, 1,408 in Großbritannien und 1,395 in Norwegen weisen die Ergebnisse auf einen deutlichen positiven Zusammenhang hin. Einschränkend ist anzumerken, dass dieser innerhalb der deutschen Studie mit 0,059 nur knapp die angenommene Signifikanzgrenze übersteigt. In zwei der drei Untersuchungen wird die Hypothese damit deutlich bestätigt, während die deutschen Ergebnisse in die gleiche Richtung tendieren.

Insgesamt zeigt sich bei der Erklärung der Zahlungsvariablen ein differenzierteres Bild als noch beim väterlichen Sorgehandeln in Form von Care. Hier werden längst nicht so viele erwartete Zusammenhänge durch das empirische Material bestätigt. Häufig bestehen die angenommenen Effekte gar nicht (signifikant) und z.T. weisen die Zusammenhänge in eine andere Wirkungsrichtung. Des Weiteren sind die Unterschiede zwischen den Ländern deutlich größer als beim Kriterium des sozio-emotionalen Handelns. Im folgenden Abschnitt wird ein zusammenfassendes Zwischenfazit aus den dargestellten bivariaten Ergebnissen gezogen.

\subsubsection{Zwischenfazit II: Die bivariaten Ergebnisse}

Die dargestellten bivariaten Ergebnisse zeigen, dass sich die Thesen mit Blick auf die Care-Komponente väterlichen Sorgehandelns weitestgehend bestätigt haben. Lediglich der Effekt der Arbeitszeit und der Anzahl der externen Kinder muss in die entgegengesetzte Wirkungsrichtung korrigiert werden. Darüber hinaus bestätigte sich der Zusammenhang der Anzahl der getrennt lebenden Vaterschaften nicht. Alle übrigen Ergebnisse weisen in die erwartete Richtung und sind im empirischen Material signifikant. Die Ergebnisse nach formulierten Hypothesen zusammengefasst bildet Tabelle 9.36 ab. 
Tab.: 9.36: Übersicht der Ergebnisse der bivariaten Thesentestung getrennt nach Care und Cash

\begin{tabular}{|c|c|c|c|c|c|c|}
\hline \multirow{2}{*}{$\begin{array}{l}\text { Unabhängige Variable } \\
\text { Physische Umwelt }\end{array}$} & \multicolumn{3}{|c|}{ Care } & \multicolumn{3}{|c|}{ Cash } \\
\hline & & $Z_{t}$ & $Z_{\text {be. }}$ & & $\mathbf{Z}_{\mathrm{t}}$ & $\mathbf{Z}_{\text {be }}$ \\
\hline Distanz steigt & $\Rightarrow$ Care sinkt & - & $3 / 3$ & ohne Einfluss & 0 & $3 / 3$ \\
\hline Einkommen steigt & $\Rightarrow$ Care steigt & + & $3 / 3$ & $\Rightarrow$ Cash $^{203}$ sinkt & - & $3 / 3$ \\
\hline Bildungsniveau steigt & $\Rightarrow$ Care steigt & + & $2-3 / 3$ & $\Rightarrow$ Cash sinkt & - & $3 / 3$ \\
\hline Arbeitszeit steigt & $\Rightarrow$ Care sinkt & - & $0 / 2$ & $\Rightarrow$ Cash sinkt & - & $2 / 2$ \\
\hline Soziale Umwelt & & $\mathbf{Z}_{\mathrm{t}}$ & $Z_{\text {be }}$ & & $\mathbf{Z}_{\mathrm{t}}$ & $\mathbf{Z}_{\mathrm{be}}$ \\
\hline Zahl Kinder im Haushalt steigt & $\Rightarrow$ Care sinkt & - & $2 / 2$ & $\Rightarrow$ Cash steigt & + & $0 / 2$ \\
\hline Externe Kinderzahl steigt & $\Rightarrow$ Care sinkt & - & $0 / 3$ & $\Rightarrow$ Cash sinkt & + & $1-2 / 3$ \\
\hline Partnersit. Vater formalisierter & $\Rightarrow$ Care sinkt & - & $3 / 3$ & $\Rightarrow$ Cash sinkt & + & $0 / 3$ \\
\hline Partnersit. Mutter formalisierter & $\Rightarrow$ Care sinkt & - & $3 / 3$ & $\Rightarrow$ Cash steigt & + & $0 / 3$ \\
\hline Partnersit. Eltern formalisierter & $\Rightarrow$ Care steigt & + & $3 / 3$ & $\Rightarrow$ Cash sinkt & - & $1 / 3$ \\
\hline Beziehungsdauer steigt & $\Rightarrow$ Care steigt & + & $2 / 2$ & $\Rightarrow$ Cash sinkt & - & $1-2 / 2$ \\
\hline Friedl. Unterhaltsfestlegung & $\Rightarrow$ Care steigt & - & $3 / 3$ & $\Rightarrow$ Cash sinkt & + & $1 / 3$ \\
\hline Friedl. Sorgerechtsfestlegung & $\Rightarrow$ Care sinkt & - & $1 / 2$ & ohne Einfluss & 0 & $2 / 2$ \\
\hline Gemeinsames Sorgerecht & $\Rightarrow$ Care steigt & + & $2 / 2$ & ohne Einfluss & 0 & $1 / 2$ \\
\hline Freundschaft & $\Rightarrow$ Care steigt & + & $2 / 2$ & ohne Einfluss & 0 & $1 / 2$ \\
\hline Individuelle Überzeugung & & $\mathbf{Z}_{\mathrm{t}}$ & $Z_{\text {be. }}$ & & $\mathbf{Z}_{\mathrm{t}}$ & $Z_{\text {be }}$ \\
\hline Ökon. Sit. der Mutter schlechter & ohne Einfluss & 0 & $2 / 2$ & $\Rightarrow$ Cash steigt & - & $0 / 2$ \\
\hline Dauer seit Trennung steigt & $\Rightarrow$ Care sinkt & - & $3 / 3$ & $\Rightarrow$ Cash steigt & + & $0 / 3$ \\
\hline Anzahl Elternschaften steigt & $\Rightarrow$ Care sinkt & - & $1-2 / 2$ & $\Rightarrow$ Cash steigt & + & $2-3 / 3$ \\
\hline
\end{tabular}

$Z_{\mathrm{t}} \quad$ = Richtung des theoretisch erwarteten Zusammenhangs

$Z_{\text {be. }} \quad=$ Bestätigung der These durch bivariaten empirischen Zusammenhang

$+\quad=$ positiver Zusammenhang erwartet;

- $\quad$ = negativer Zusammenhang erwartet;

$0 \quad=$ kein Zusammenhang erwartet

$3 / 3=$ drei der drei Datensätze, die die nötigen Informationen zur Testung bereitstellen, bestätigen die aufgestellte These.

$1-2 / 3$ = ein bis zwei Studien stützen den angenommenen Zusammenhang, $d . h$. in einem Datensatz wird die Annahme bestätigt, im anderen tendenziell aufgrund eines schwachen Zusammenhangs oder einer geringeren Signifikanz gestützt.

Quelle: Eigene Darstellung.

${ }^{203}$ Zur Erinnerung ist an dieser Stelle darauf verwiesen, dass Cash für Unterhaltsprobleme steht, nicht für geleistete Zahlungen, was die umgekehrte Wirkungsrichtung implizieren würde. 
Ein anderes Bild liefern die Resultate zur Erklärung der Cash-Variablen. Die Ergebnisse sind insgesamt als sehr heterogen zu beschreiben. Zum einen werden die erwarteten Zusammenhänge nur z.T. durch die Studien bestätigt; zum anderen liefern die jeweiligen Untersuchungen mit Blick auf einzelne Prädiktoren keine einheitlichen Wirkungszusammenhänge. So stützen die Ergebnisse zu den Faktoren der physischen Umwelt gänzlich die theoretischen Annahmen. Anders dagegen die empirischen Zusammenhänge für die Faktoren der sozialen Umwelt und des Überzeugungssystems; hier zeigen sich häufiger nicht bestätigte Thesen. Lediglich die Annahme, dass kein Zusammenhang zwischen finanziellen Transfers und der Festlegung des Sorgerechts bzw. Umgangs wird gestützt. Alle anderen Determinanten werden entweder vollständig widerlegt, wie z.B. im Fall der Anzahl der Kinder im Haushalt oder der aktuellen Partnersituation beider Elternteile oder sie finden sich nur in einzelnen Untersuchungen im empirischen Material wieder, wie z.B. bei der elterlichen Beziehungsform vor der Trennung oder dem freundschaftlichen Verhältnis zwischen den Eltern.

\subsection{Ergebnisse der multiplen Zusammenhangsanalyse}

Im folgenden Abschnitt werden die bivariaten Ergebnisse zunächst anhand jeweils eines multiplen Regressionsmodells für Care und Cash überprüft. An dieser Stelle ist noch einmal auf die eingeschränkte vergleichende Perspektive zwischen den Datensätzen auf multipler Ebene hinzuweisen. Die Studien haben nicht die exakt gleichen Operationalisierungen verwendet und v.a. nicht dieselbe Anzahl an Variablen erhoben. Nicht erfasste Informationen können - selbstverständlich - nicht in die Berechnungen eingehen. Ein strenger internationaler Vergleich des Einflusses einer Variablen unter Berücksichtigung anderer Variablen würde voraussetzen, dass in allen Modellen die gleichen Variablen vorhanden sind. Dies ist jedoch aufgrund der Datenlage nicht gewährleistet. Die Ergebnisdarstellung wird sich daher auf nationale Interpretationen konzentrieren, um damit einerseits die bivariaten Resultate abzusichern und zum anderen Aussagen über die relative Bedeutung der Determinanten auf nationaler Ebene zu treffen. Dementsprechend erfolgt die Darstellung nach Ländern differenziert. ${ }^{204}$ Vorsichtige Vergleiche über die generelle Bedeu-

\footnotetext{
${ }^{204}$ In einer frühen Phase der Sekundäranalyse wurde noch davon ausgegangen, dass ein „Minimal“-Modell aufgestellt werden könnte, das nur Variablen aufnimmt, die in allen drei Datensätzen gleichermaßen vertreten sind. Damit wäre nicht nur ein „minimales“ Modell zu berechnen gewesen, sondern dieses hätte auch als „minimalistisch“ charakterisiert werden müssen, da nur sehr wenige Variablen in allen drei Untersuchungen gleichermaßen zu finden sind. Die Aussagekraft wurde inhaltlich zu gering eingeschätzt und deswegen eine Vorgehensweise nach einzelnen Datensätzen gewählt.
} 
tung einzelner Einflussfaktoren über die Länder hinweg finden sich in der Interpretation der Ergebnisse im Anschluss an die multiple Analyse.

\subsubsection{Determinanten im multiplen Regressionsmodell für Care}

Die bivariaten Ergebnisse haben die aufgestellten Hypothesen, welche Determinanten das väterliche Sorgehandeln in Form von Care wie beeinflussen, weitestgehend bestätigt. In Tabelle. 11.37 werden die gefundenen bivariaten Regressionskoeffizienten den Ergebnissen der beiden multiplen Modelle getrennt nach Datensätzen gegenübergestellt.

Im deutschen Datensatz zeigen sich die einzelnen Determinanten auch im multiplen Modell unter Berücksichtigung aller anderen Einflussgrößen weitestgehend signifikant. Lediglich die Variablen des väterlichen Einkommens, des Ausbildungsniveaus, der Kinder im Haushalt des Vaters und der Dauer seit der Trennung verlieren ihre signifikante Bedeutung im multiplen Modell All. Im Modell Best Fit, in dem nur noch signifikante Faktoren berücksichtigt werden, gewinnt die Variable des Einkommens wieder Signifikanz. Der Regressionskoeffizient weist jedoch auf einen eher schwachen Zusammenhang hin.

Zur Bestimmung des sozio-emotionalen Sorgehandelns deutscher Väter trägt wesentlich die formale Regelung der Trennung bei, d.h. die Form des festgelegten Sorgerechts sowie der Festlegungsprozess von Sorgerecht und Unterhalt. Damit kommt dem Verhältnis während der Trennung in Deutschland eine wichtige Bedeutung zu. Neben den Trennungsregelungen bestimmt die elterliche Beziehung vor der Trennung auch im multiplen Modell väterliches Sorgen. Je formalisierter die elterliche Partnerschaft war, desto höher das sozio-emotionale Engagement des Vaters nach der Trennung. Mit der Familiensituation vor der Trennung eng verbunden ist die Anzahl der unterhaltsberechtigten, externen Kinder. Bereits auf bivariater Ebene ergibt sich hier ein positiver Zusammenhang, der theoretisch in die andere Richtung angenommen wurde. Die entgegengesetzte Wirkungsrichtung wird multiple bestätigt. Das bedeutet, mit steigender Zahl getrennt lebender Kinder nimmt auch das väterliche Engagement zu. Es wirkt sich auf multipler Ebene ferner die aktuelle Partnersituation beider Elternteile aus. Dabei bestimmt eine neue Partnerin des Vater sein Sorgehandeln ebenso negativ wie ein neuer Partner an der Seite der Mutter. Beide Regressionseffekte sind ähnlich stark ausgeprägt. Darüber hinaus hat auch die frühere Beziehungsgeschichte des Vaters Bedeutung, denn mit steigender Anzahl an Partnerschaften, aus denen Kinder hervorgegangen sind, nimmt sein väterliches Engagement in Form von Care ab. Dieser Zusammenhang zeigt sich bereits bivariat und wird im multiplen Modell bestätigt. Mit Blick auf die physischen Ressourcen bestätigen sich der Effekt der Entfernung zwischen den Wohnorten ebenso wie der des väterlichen Einkommens. Beide sind mit Koeffizienten knapp unter 0,1 jedoch eher schwach ausgeprägt. 


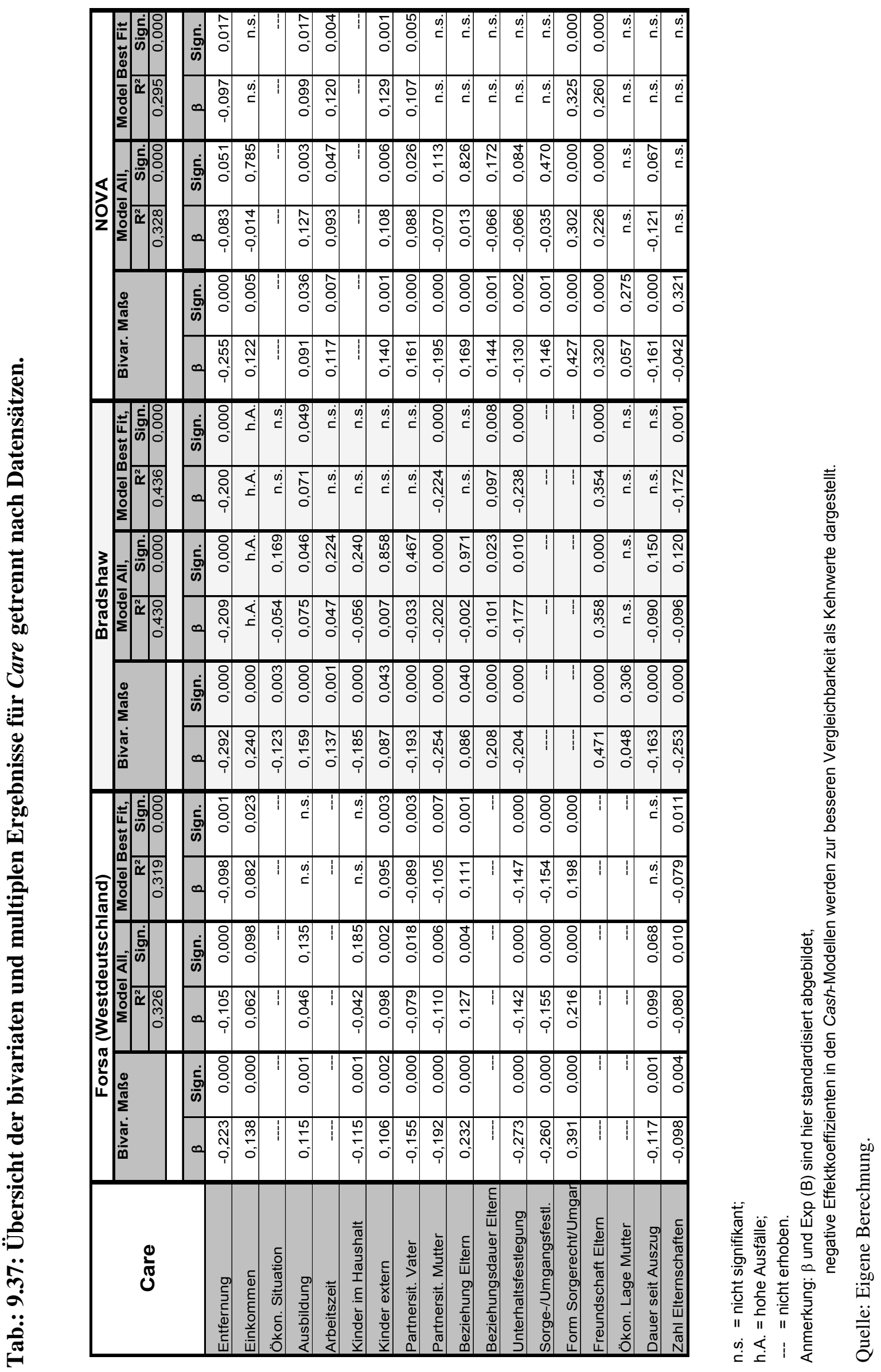


Insgesamt ergeben sich im deutschen Fall für fast alle Determinanten signifikante Zusammenhänge innerhalb eines multiplen Modells, die in ihrer Richtung mit denen auf bivariater Ebene übereinstimmen. Eine Ausnahme bilden die drei Variablen Ausbildungsniveau, Zahl der Kinder im Haushalt des Vaters und Dauer seit der Trennung, die nicht mehr signifikant Einfluss nehmen. Das multiple Regressionsmodell weist mit einer erklärten Varianz von insgesamt über 30\% auf signifikantem Niveau eine zufrieden stellende Güte auf. Wenn auch die einzelnen Beta-Gewichte alle mit Werten von um die 0,1 bzw. 0,2 eher schwache bis moderate Zusammenhänge andeuten, trägt die Kombination der einzelnen Faktoren insgesamt gut zur Erklärung der Variation in der abhängigen Variablen Care bei.

In Großbritannien zeigt sich in Bezug auf die Bestätigung der bivariaten Zusammenhänge ein etwas differenzierteres Bild. Es können nur noch sieben der ursprünglich 14 bivariat bedeutenden Variablen auch multiple signifikante Effekte zeigen. Zu den bestätigten Zusammenhängen zählt dabei der positive Einfluss der freundschaftlichen Beziehung der Eltern nach der Trennung. Die Variable ergibt mit 0,354 das mit Abstand stärkste Gewicht. Die anderen Determinanten zeigen geringere Effekte auf Care. Dazu zählen u.a. die Beziehungssituation der Mutter, die Entfernung zwischen den Wohnorten sowie die Festlegung des Unterhalts, die mit je einem Regressionsgewicht von -0,2 die nächst wichtigsten Variablen im Erklärungsmodell darstellen. Zwei weitere Variablen, die im Modell All die Bestätigung ihrer bivariaten Wirkungszusammenhänge finden, sind die Dauer der Beziehung zwischen den Eltern und das Bildungsniveau des Vaters. Werden nur die Variablen mit Signifikanz im multiplen Modell Best Fit berücksichtigt, weist auch die Anzahl der Beziehungen, aus denen Kinder hervorgegangen sind, einen signifikanten Koeffizienten auf.

Ohne Bedeutung für die multiple Erklärung des sozio-emotionalen Sorgehandelns in Großbritannien zeigen sich die Variablen zur Beschreibung der väterlichen Familiensituation (aktuelle Partnersituation und Kinder im Haushalt), der ökonomischen Situation des Vaters, ${ }^{205}$ der Arbeitszeit, der Anzahl getrennt lebender, unterhaltsberechtigter Kinder,

${ }^{205}$ Im britischen Fall weist die Variable Einkommen aufgrund methodischer Mängel, wie dargestellt, hohe
Ausfälle und damit ein hohes Maß an Unsicherheit in den Ergebnissen auf. Innerhalb der folgenden
multiplen Analyse wird daher zugunsten einer höheren Fallzahl die selbsteingeschätzte ökonomische
Situation als Substitut des persönlichen Einkommens verwendet. So wurden britische Väter innerhalb der
Studie gefragt, wie sie ihre aktuelle finanzielle Situation beschreiben würden. Als Antwortvorgaben dienten
„sehr gut“, „gut“, „ok“, „,schlecht“ und „,sehr schlecht“. Damit wird mit ansteigender Kodierung die eigene
ökonomische Lage schlechter eingeschätzt. Daraus folgt, dass ein umgekehrter Zusammenhang zwischen
Cash und der monetären Selbsteinschätzung angenommen wird, wie er mit dem aufsteigendem Einkommen
und damit gegenläufiger Kodierung erwartet wird. Zwar ist das Erfragen des Einkommens als „objektiver“ 
der Familienform der Eltern und der Dauer seit der Trennung. Insgesamt können mit dem multiplen Modell dennoch insgesamt über $40 \%$ der Varianz erklärt werden, wodurch dem Modell eine hohe Erklärungskraft zukommt.

In Norwegen zeigen sieben der insgesamt 14 bivariat signifikanten Ergebnisse auch im multiplen Modell Regressionsgewichte, die innerhalb der Signifikanzgrenzen liegen. Dabei sind die beiden stärksten Einflussfaktoren auf das sozio-emotionale Kümmern norwegischer Väter das als freundschaftlich bezeichnete Verhältnis zur Kindsmutter und das festgelegte Umgangsrecht. ${ }^{206}$ Eher moderate Effekte weisen die Koeffizienten zur Arbeitszeit des Vaters sowie zur Anzahl der getrennt lebenden Kinder in beiden multiplen Modellen auf. Während für die Variablen zur Entfernung zwischen den Wohnorten, zum Ausbildungsniveau des Vaters sowie seiner Partnersituation die bivariaten Zusammenhänge zwar auch auf multipler Ebene signifikant bestätigt werden können, weisen diese jedoch auf letzterer nur schwache Effekte auf.

Von keiner signifikanten Bedeutung im multiplen Modell Norwegens sind die Effekte des Einkommens, der Partnersituation der Mutter, der Charakteristika der elterlichen Beziehung vor der Trennung in Form und Dauer, der Unterhalts- und Sorgerechtsfestlegung sowie der Dauer seit dem Auszug. Insgesamt kann dem norwegischen Modell mit einer Güte von rund 30\% erklärter Varianz auf signifikantem Niveau wie in den anderen Datensätzen eine gute empirische Erklärungskraft zugesprochen werden.

Zusammenfassend bestätigen die multiplen Modelle v.a. in Norwegen und Großbritannien nur bedingt die bivariaten Effekte. Im folgenden Abschnitt werden die Ergebnisse der multiplen Analyse der Cash-Variablen dargestellt. Eine Gesamtschau der Resultate sowie eine generellere und umfangreiche Interpretation aller Ergebnisse erfolgt in einem den Analyseteil abschließenden Kapitel.

\subsubsection{Determinanten im multiplen Regressionsmodell für Cash}

Die multiplen Ergebnisse der Cash-Variablen werden in Tabelle 9.38 nach Datensätzen getrennt dargestellt. Innerhalb der deutschen Untersuchung haben sich auf bivariater Ebene nur wenige der angenommenen Zusammenhänge für die Cash-Variable empirisch

und im vorliegenden Fall v.a. als vergleichbarer mit den anderen Datensätzen einzustufen, aber dennoch wird hier die Annahme getroffen, dass beide Konstrukte ähnliche Inhalte abbilden.

${ }^{206}$ Beide Determinanten zeigen jeweils auch in den anderen beiden Datensätzen einen größeren Einfluss, auch wenn jeweils nur einer der Faktoren - in Deutschland das festgelegte Sorgerecht und in Großbritannien die freundschaftliche Beziehung zwischen den Eltern - erhoben wurde. 
bestätigt. Die multiplen Modelle schränken die Ergebnisse weiter ein. Nur zwei der fünf bivariat signifikanten Effekte finden sich auch im Gesamtmodell wieder. Den stärksten Einfluss auf die väterliche Zahlungspraxis hat dabei das Einkommen, was inhaltlich sehr plausibel ist. Des Weiteren wirkt sich die Art der Unterhaltsfestlegung zwischen den Eltern aus. Mit zunehmendem Konflikt während des Festlegungsprozesses steigt die Wahrscheinlichkeit ausbleibender Unterhaltsleistungen an. Mit einem Signifikanzniveau von 0,027 ist dieses Ergebnis jedoch nicht so gut empirisch abgesichert wie der Effekt des Einkommens. Über diese beiden Determinanten hinaus gibt es keine weiteren Einflussfaktoren, die Zahlungsprobleme begünstigen. Dabei sind die beiden Determinanten ausreichend um rund 19\% der Varianz im finanziellen Handeln deutscher Nachtrennungsväter auf signifikantem Niveau zu erklären. Insgesamt liefern die Ergebnisse der Forsa-Studie damit für zwei theoretisch begründete Effekte die empirische Grundlage und bestätigen die nicht angenommenen Zusammenhänge der Variablen Entfernung zwischen den Wohnorten sowie Festlegung und Form des Sorgerechts.

Anders in Großbritannien und Norwegen, in beiden Datensätzen finden sich - wie auch schon auf bivariater Ebene - eine höhere Zahl an Einflussgrößen, die das finanzielle Sorgehandeln der Väter determinieren. Jedoch sind diese in jedem Land unterschiedlich. So wird in Großbitannien das Auftreten von Unterhaltsschwierigkeiten - ebenso wie schon ein hohes sozio-emotionales Engagement des Vaters - durch das (nicht vorhandene) freundschaftliche Verhältnis zwischen den Eltern geprägt. Auch auf multipler Ebene zeigt sich demnach ein Zusammenhang zwischen einer freundschaftlichen Beziehung der Eltern und dem Ausbleiben von Unterhaltsproblemen. Neben dem elterlichen Verhältnis zum Zeitpunkt der Befragung ist ferner die aktuelle Familiensituation des Vaters für sein Zahlungsverhalten von Bedeutung. Anders als beim Care, auf das weder die aktuelle Partnersituation des Vaters noch Kinder in seinem Haushalt einen Effekt zeigen, wird die Cash-Variable von beiden signifikant und mit deutlicher Stärke bestimmt. Doch während mit steigender Anzahl an Kindern im väterlichen Haushalt erwartungsgemäß die Wahrscheinlichkeit von Zahlungsproblemen steigt, wirkt sich die stärkere Formalisierung und Verbindlichkeit einer neuen Beziehung entgegen der theoretischen Annahme positiv auf die Zahlungsmoral aus. Das bedeutet, dass Väter, die mit einer neuen Partnerin oder gar einer neuen Ehefrau zusammenleben, eher ihren finanziellen Verpflichtungen nachkommen als Väter, die nach der elterlichen Trennung allein stehend sind. Damit werden die bereits bivariat aufgetretenen Ergebnisse bestätigt und die Hypothese widerlegt. Neben den genannten Faktoren wird das Zahlungsverhalten britischer Trennungsväter ferner erwartungsgemäß - durch sozio-ökonomische Einflussgrößen der physischen Umwelt bestimmt. Dazu zählen in erster Linie die ökonomische Situation, die auf multipler Ebene einen moderaten bis starken Zusammenhang mit der Cash-Variablen aufweist. Weniger stark ausgeprägt aber dennoch signifikant zeigen sich die Effekte der Variablen Aus- 
bildungsniveau und Arbeitszeit. Letzterer ist jedoch mit einem Effektkoeffizienten von 1,055 als sehr schwach zu charakterisieren.

In Großbritannien bestehen diejenigen Zusammenhänge, die die elterliche Beziehung vor der Trennung in Form und Dauer noch auf bivariater Ebene gezeigt haben, im multiplen Modell nicht fort. Gleiches gilt für die ökonomische Situation der Mutter und die Anzahl der gescheiterten Beziehungen, aus denen Kinder hervorgegangen sind. Damit können die mit ihnen verbundenen Thesen nicht weiter als empirisch gestützt betrachtet werden. Insgesamt erklären die multiple signifikanten Variablen rund 35\% der Varianz; damit weist das Modell für empirische Analysen eine recht hohe Güte auf, die sich deutlich von den anderen beiden Datensätzen abhebt.

Zusammenfassend kann für den britischen Fall festgestellt werden, dass sechs der zehn bereits bivariat gefundenen Effekte auch auf multipler Ebene bestehen bleiben. Diese repräsentieren drei bestätigte theoretische Annahmen. Die anderen beiden Variablen weisen Zusammenhänge auf bivariater und multipler Ebene auf, die eine umgekehrte Wirkungsrichtung anzeigen, wie sie theoretisch vermutet wurde. Dazu zählen die Arbeitszeit ebenso wie die bereits angesprochene Partnersituation des Vaters. Damit können die zwei diesbezüglichen Hypothesen zwar eine Unterstützung des generell angenommenen Effekts erfahren, jedoch muss die Richtung korrigiert werden. Der Einfluss der Variablen Kinder im väterlichen Haushalt ist bivariat knapp oberhalb des angenommenen Signifikanzniveaus, im multiplen Modell besteht dagegen ein signifikanter Zusammenhang. Dies stützt eine weitere Hypothese. Die britischen Ergebnisse bestätigen ferner die Annahme, dass die Entfernung zwischen den Wohnorten keinen Einfluss auf das Zahlungsverhalten der Väter hat. 


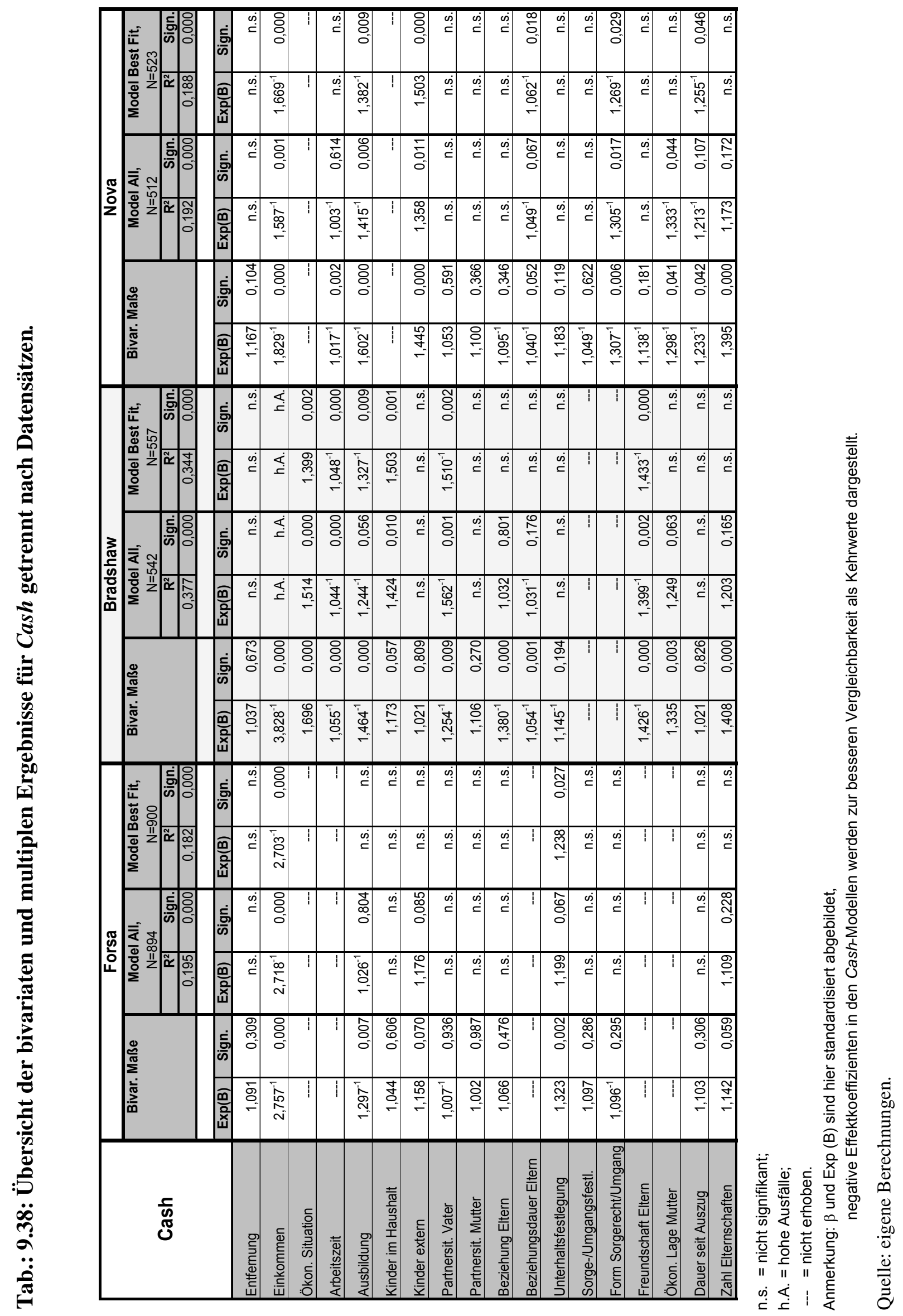


Auch im norwegischen Fall konnten auf bivariater Ebene verschiedene Einflussgrößen auf signifikantem Niveau ausgemacht werden, die im multiplen Modell nur teilweise Bestätigung finden. Am stärksten wirkt sich erwartungsgemäß das Einkommen auf die Zahlungspraxis des Vaters aus. Den zweitstärksten Effekt zeigt die Anzahl der externen Kinder. Beide Variablen stellen wesentliche Bestandteile der Berechnungsformel des Unterhaltsniveaus dar. Des Weiteren wirkt sich das väterliche Bildungsniveau auf die Auftretenswahrscheinlichkeit von Unterhaltsproblemen aus. Dieser Effekt bestätigt die erwartete Wirkungsrichtung, wie sie bereits bivariat ausgemacht werden konnte. Etwas überraschend bestimmt der festgelegte Umgang in Norwegen die Zahlungspraxis der Nachtrennungsväter mit. Ein Koeffizient von $1,269^{-1}$ weist dabei signifikant auf einen moderaten negativen Effekt hin. Das bedeutet, dass Väter, denen ein geringerer Umgang mit ihren Kindern zugesprochen worden ist, häufiger Zahlungsunregelmäßigkeiten aufweisen. Darüber hinaus wird das Auftreten von Unterhaltsproblemen durch die Dauer seit der Trennung beeinflusst. Hier wurde schon bei den bivariaten Ergebnissen auf die entgegengesetzte Wirkungsrichtung hingewiesen. Ein negativer Effektkoeffizient bedeutet inhaltlich, dass mit kürzerer zeitlicher Distanz zur elterlichen Trennung häufiger Zahlungsschwierigkeiten auftreten. Deutlich schwächer ausgeprägt ist die positive Bedeutung der Beziehungsdauer, wobei dieser Zusammenhang mit einem Gewicht von 1,062 ${ }^{-1}$ vernachlässigt werden kann. Die bivariat angezeigte Bedeutung der Arbeitszeit und der Zahl der gescheiterten Partnerschaften, aus denen Kinder hervorgegangen sind, findet innerhalb des multiplem Modells keine Unterstützung mehr. Insgesamt erklären die genannten signifikanten Variablen rund 19\% der Varianz, was für eine respektable Gesamtgüte des Modells spricht. Die norwegischen Ergebnisse stützen insgesamt die zwei Hypothesen zu den Effekten des Einkommens und der Ausbildung des Vaters sowie die zwei Annahmen, dass kein Zusammenhang zur Sorgerechtsfestlegung und zur Entfernung zwischen den Wohnorten besteht.

Die multiple Analyse zur Absicherung der bivariat aufgezeigten Zusammenhänge hat heterogene Ergebnisse hervorgebracht. Nur ein Teil der bivariaten Effekte konnte auch unter Berücksichtigung anderer Determinanten bestätigt werden. Im folgenden Abschnitt werden die wesentlichen Resultate zusammenfassend dargestellt und ausführlich v.a. in ihrer inhaltlichen Bedeutung diskutiert.

\subsection{Interpretation der bivariaten und multiplen Ergebnisse}

Insgesamt zeigen sich viele der bivariaten Ergebnisse auf multipler Ebene nicht bestätigt. Damit können einige der Hypothesen, die auf bivariater Ebene empirische Unterstützung gefunden haben, durch ein multiples Modell nicht länger aufrecht erhalten werden. Tabelle 9.39. stellt die Ergebnisse der Thesentestung aus der bivariaten und multiplen Zusammenhangsanalyse je Datensatz für Care und Cash getrennt gegenüber. Dabei ist dar- 
auf hinzuweisen, dass dies nicht der Darstellung der Zusammenhänge, sondern der Wiedergabe der bestätigten bzw. der widerlegten Hypothesen dient.

Tab.: 9.39: Zusammenfassende Gegenüberstellung der durch die bivariaten bzw. multiplen Ergebnisse bestätigten Hypothesen nach Datensatz und Care bzw. Cash getrennt.

\begin{tabular}{|c|c|c|c|c|c|c|c|c|c|c|c|c|c|c|}
\hline \multirow{3}{*}{\begin{tabular}{|l|} 
Determinante \\
Physische Umwelt \\
\end{tabular}} & \multicolumn{7}{|c|}{ CARE } & \multicolumn{7}{|c|}{ CASH } \\
\hline & \multirow[b]{2}{*}{$\mathrm{Z}_{\mathrm{t}}$} & \multicolumn{2}{|c|}{ FS } & \multicolumn{2}{|c|}{ BS } & \multicolumn{2}{|c|}{ NV } & \multirow[b]{2}{*}{$Z_{t}$} & \multicolumn{2}{|c|}{ FS } & \multicolumn{2}{|c|}{ BS } & \multicolumn{2}{|c|}{ NV } \\
\hline & & $Z_{\text {be }}$ & $\mathbf{Z}_{\mathrm{me}}$ & $Z_{\text {be }}$ & $\mathbf{Z}_{\mathrm{me}}$ & $Z_{\text {be }}$ & $Z_{\text {me }}$ & & $Z_{\text {be }}$ & $Z_{m e}$ & $Z_{\text {be }}$ & $Z_{\text {me }}$ & $Z_{\text {be }}$ & $\mathbf{Z}_{\mathrm{me}}$ \\
\hline Entfernung & - & $\checkmark$ & $\checkmark$ & $\checkmark$ & $\checkmark$ & $\checkmark$ & $\checkmark$ & 0 & $\checkmark$ & $\checkmark$ & $\checkmark$ & $\checkmark$ & $\checkmark$ & $\checkmark$ \\
\hline Einkommen & + & $\checkmark$ & $\checkmark$ & $\checkmark$ & $x$ & $\checkmark$ & $x$ & - & $\checkmark$ & $\checkmark$ & $\checkmark$ & $\checkmark$ & $\checkmark$ & $\checkmark$ \\
\hline Bildungsniveau & + & $\checkmark$ & $x$ & $\checkmark$ & $\checkmark$ & $\checkmark$ & $\checkmark$ & - & $\checkmark$ & $x$ & $\checkmark$ & $\checkmark$ & $\checkmark$ & $\checkmark$ \\
\hline Arbeitszeit & - & --- & --- & $x$ & $x$ & $x$ & $x$ & - & --- & --- & $\checkmark$ & $x$ & $\checkmark$ & $x$ \\
\hline Soziale Umwelt & $\mathrm{Z}_{\mathrm{t}}$ & $Z_{\text {be }}$ & $\mathrm{Z}_{\mathrm{me}}$ & $Z_{\text {be }}$ & $Z_{\text {me }}$ & $Z_{b e}$ & $Z_{\text {me }}$ & $\mathrm{Z}_{\mathrm{t}}$ & $Z_{\text {be }}$ & $Z_{\text {me }}$ & $Z_{\text {be }}$ & $Z_{\mathrm{me}}$ & $z_{\text {be }}$ & $\mathbf{Z}_{\mathrm{me}}$ \\
\hline Kinder im Haushalt & - & $\checkmark$ & $x$ & $\checkmark$ & $x$ & --- & --- & + & $x$ & $x$ & $x$ & $\checkmark$ & --- & --- \\
\hline Externe Kinderzahl & - & $x$ & $x$ & $x$ & $x$ & $x$ & $x$ & + & $x$ & $x$ & $x$ & $x$ & $\checkmark$ & $\checkmark$ \\
\hline Partnersit. Vater & - & $\checkmark$ & $\checkmark$ & $\checkmark$ & $x$ & $\checkmark$ & $\checkmark$ & + & $x$ & $x$ & $x$ & $x$ & $x$ & $x$ \\
\hline Partnersit. Mutter & - & $\checkmark$ & $\checkmark$ & $\checkmark$ & $\checkmark$ & $\checkmark$ & $x$ & + & $x$ & $x$ & $x$ & $x$ & $x$ & $x$ \\
\hline Elterl. Beziehung & + & $\checkmark$ & $\checkmark$ & $\checkmark$ & $x$ & $\checkmark$ & $x$ & - & $x$ & $x$ & $\checkmark$ & $x$ & $x$ & $x$ \\
\hline Beziehungsdauer & + & --- & --- & $\checkmark$ & $\checkmark$ & $\checkmark$ & $x$ & - & --- & --- & $\checkmark$ & $x$ & $\checkmark$ & $x$ \\
\hline Unterhaltsfestlegung & - & $\checkmark$ & $\checkmark$ & $\checkmark$ & $\checkmark$ & $\checkmark$ & $x$ & + & $\checkmark$ & $\checkmark$ & $x$ & $x$ & $x$ & $x$ \\
\hline Festl. Sorge/Umg. & - & $\checkmark$ & $\checkmark$ & -- & --- & $\checkmark$ & $x$ & 0 & $\checkmark$ & $\checkmark$ & --- & --- & $\checkmark$ & $\checkmark$ \\
\hline Sorgerecht/Umgang & + & $\checkmark$ & $\checkmark$ & --- & --- & $\checkmark$ & $\checkmark$ & 0 & $\checkmark$ & $\checkmark$ & --- & --- & $x$ & $x$ \\
\hline Freundschaft & + & --- & --- & $\checkmark$ & $\checkmark$ & $\checkmark$ & $\checkmark$ & 0 & --- & --- & $x$ & $x$ & $\checkmark$ & $\checkmark$ \\
\hline Indiv. Überzeugung & $\mathrm{z}_{\mathrm{t}}$ & $\mathbf{Z}_{\mathrm{be}}$ & $\mathbf{Z}_{\mathrm{me}}$ & $Z_{\text {be }}$ & $\mathbf{Z}_{\mathrm{me}}$ & $Z_{b e}$ & $\mathbf{Z}_{\mathrm{me}}$ & $\mathbf{Z}_{\mathrm{t}}$ & $\mathbf{Z}_{\text {be }}$ & $\mathbf{Z}_{\mathrm{me}}$ & $\mathbf{z}_{\mathrm{be}}$ & $\mathbf{Z}_{\mathrm{me}}$ & $\mathbf{Z}_{\mathrm{be}}$ & $\mathbf{Z}_{\mathrm{me}}$ \\
\hline Finanzen d. Mutter & 0 & --- & --- & $\checkmark$ & $\checkmark$ & $\checkmark$ & $\checkmark$ & - & $x$ & $x$ & $x$ & $x$ & $x$ & $x$ \\
\hline Dauer seit Trennung & - & $\checkmark$ & $\checkmark$ & $\checkmark$ & $x$ & $\checkmark$ & $x$ & + & $x$ & $x$ & $x$ & $x$ & $x$ & $x$ \\
\hline Zahl Elternschaften & - & $\checkmark$ & $\checkmark$ & $\checkmark$ & $\checkmark$ & $\mathbf{x}$ & $\mathbf{x}$ & + & $\mathbf{x}$ & $x$ & $\checkmark$ & $x$ & $\checkmark$ & $x$ \\
\hline \multicolumn{15}{|c|}{$Z_{t}=$ Richtung theoretisch erwarteter Zusammenhang } \\
\hline \multicolumn{15}{|c|}{$Z_{\text {be }}=$ empirische Bestätigung durch bivariaten Koeffizienten } \\
\hline \multicolumn{15}{|l|}{$Z_{m e}=$} \\
\hline \multicolumn{15}{|c|}{$\checkmark$} \\
\hline \multicolumn{15}{|l|}{$x$} \\
\hline \multicolumn{15}{|l|}{$=$ positiver Zusar } \\
\hline \multicolumn{15}{|l|}{ - Meganivel Lusan } \\
\hline \multirow{2}{*}{\multicolumn{15}{|c|}{0 = kein Zusammenhang erwartet }} \\
\hline --- = nicht erhoben & & & & & & & & & & & & & & \\
\hline
\end{tabular}

Quelle: eigene Darstellung. 
Es wurde bereits deutlich auf die Schwierigkeiten der vergleichenden Perspektive auf die vorliegenden Daten und ihre empirischen Resultate hingewiesen. Im Folgenden wird es daher nicht darum gehen, strenge Vergleiche zwischen nationalen Regressionsmodellen zu ziehen. Vielmehr werden nationale Auffälligkeiten und generelle Tendenzen - mit all der Vorsicht, die in der Interpretation geboten sein muss - getrennt für Care und Cash zusammengefasst und interpretiert.

\subsubsection{Die Ergebnisse für Care}

Die Ergebnisse für Care zeigen einige generelle Trends auf, die in allen drei Datensätzen mit Blick auf die jeweilige Hypothesentestung zu ähnlichen Schlussfolgerungen führen. So besteht in den deutschen, britischen und norwegischen Daten gleichermaßen ein negativer Zusammenhang zwischen dem Niveau sozio-emotionalen Engagements und der ansteigenden räumlichen Distanz zwischen den elterlichen Wohnorten. Dies entspricht ebenso den theoretischen Annahmen wie die These, dass ein freundschaftliches Verhältnis zwischen den Eltern den Kontakt zwischen Vater und Kind erleichtert, gar fördert. Auch diese Annahme findet in den beiden Datensätzen, die diese Variable bereithalten, empirische Unterstützung. Ferner kann die Hypothese über den Einfluss, den die Form des festgelegten Sorgerechts bzw. Umgangs auf das väterliche Kümmern ausübt, empirisch bestätigt werden. Ein gemeinsames Sorgerecht bzw. eine Vereinbarung über häufigen Umgang wirkt sich positiv auf das Engagement des Vaters aus. Des Weiteren legen die Ergebnisse der beiden Datensätzen, die diese Angaben erfassen, nahe, dass die ökonomische Situation der Mutter in keinem Zusammenhang mit der Ausprägung der CareVariablen steht.

Zwei Hypothesen können anhand des vorliegenden empirischen Materials eindeutig als widerlegt gelten. Dazu zählt zunächst der angenommene Zusammenhang, den die Anzahl getrennt lebender Kinder auf das väterliche Sorgehandeln ausüben soll. Postuliert wurde, dass dieses mit steigender Anzahl externer Kinder aufgrund begrenzter zeitlicher Ressourcen des Vaters nachlässt. Dieses Ergebnis findet sich im Datenmaterial nicht. In Deutschland und Norwegen besteht zwar ein Zusammenhang zwischen der Anzahl der externen Kinder und Care sowohl auf bivariater als auch auf multipler Ebene, dieser ist jedoch entgegen der Annahme positiv. Das bedeutet, mit steigender Zahl an Kindern, die außerhalb des väterlichen Haushaltes leben, nimmt das väterliche Sorgehandeln zu. Dieses Ergebnis kann verschieden interpretiert werden. Zunächst kann ein Kontaktabbruch zu mehreren externen Kindern höhere emotionale Kosten verursachen als das fehlende Verhältnis zu einem einzelnen Kind. Ferner kann eventuell von einem Zusammenhang zwischen der Dauer der Beziehung, aus der die Zahl der getrennt lebenden Kinder entstanden ist, ausgegangen werden. Mehrere Kinder - aus einer Beziehung, die die Väter in allen drei Datensätzen mehrheitlich angeben - setzen eine längerfristige Verbindung zwischen 
den Eltern voraus. Diese kann zum einen eine bewusste Familienplanung und damit auch eine bewusste Entscheidung zur Vaterschaft implizieren, was eventuell für eine starke Familienorientierung des Befragten sprechen könnte. Ferner können damit die Argumente zum Einfluss der Dauer der elterlichen Partnerschaft und der damit verbundenen Verfestigung dieser Beziehung angeführt werden. Danach kann ein höheres Verpflichtungsempfinden des Vaters mit einer längeren Partnerschaftsdauer einhergehen. Der fehlende Einfluss der Variable der Beziehungsdauer auf multipler Ebene - zumindest in Norwegen - suggeriert jedoch, dass es weniger um die Verbindlichkeit der elterlichen Partnerschaft als vielmehr um das Vater-Kind-Verhältnis selbst geht. Mehrere Kinder aus einer Beziehung und der dafür notwendige dauerhaftere Bestand der familialen Gemeinschaft in einem Haushalt ermöglichen die Entwicklung einer intensiven Beziehung zwischen Vater und Kind vor der elterlichen Trennung. Diese wirkt sich möglicherweise positiv auf das väterliche Sorgehandeln nach der elterlichen Trennung aus. Ferner können die Beziehungen zu mehreren Kindern aus einer Elternschaft, die zusammen bei ihrer Mutter leben, leichter in einem gemeinsamen Besuch des Vaters integriert werden, als wenn der Vater verschiedene Haushalte aufsuchen muss. Damit konkurrieren weniger die Kinder einer Haushaltsgemeinschaft um die knappen väterlichen Ressourcen, sondern eher Kinder aus verschiedenen Elternschaften. Dies bedeutet inhaltlich, dass es nicht so sehr die Höhe der - in diesem Fall v.a. der zeitlichen - Verpflichtungen als vielmehr die Verbindlichkeit der Partnerschaften bzw. der Lebenswandel des Vaters ist, die sein sozio-emotionales Sorgehandeln bestimmen. ${ }^{207}$

Die Ausführungen deuten an, dass es eventuell nicht die Zahl der externen Kinder ist, die das väterliche Sorgehandeln primär bestimmen, sondern der Information, aus wie vielen Beziehungen diese Kinder entstanden sind, eine wichtige Bedeutung zukommt. Sicherlich gibt es Unterschiede im väterlichen Sorgehandeln je nachdem, ob eine Vaterschaft zu mehreren Kindern aus einer Partnerschaft entstanden ist oder ob mehrere Vaterschaften aus unterschiedlichen Beziehungen bestehen. Dies sagt nicht zuletzt etwas über den väterlichen Lebenswandel bzw. die Verbindlichkeit seiner Partnerschaften aus. Der Einfluss der Anzahl der Elternschaften im multiplen Modell zeigt sich allerdings allein in Großbritannien und Deutschland. Innerhalb der deutschen Daten ist der Effekt jedoch nur sehr schwach ausgeprägt. Dieser ist ferner mit Blick auf die Dominanz der Ein-Elternschaften

\footnotetext{
${ }^{207}$ Die Ergebnisse für Cash zeigen, dass hier die Höhe der finanziellen Aufwendungen wichtiger ist. Dies macht inhaltlich für beide Variablen aufgrund ihrer unterschiedlichen Charakteristika durchaus Sinn: sozioemotionales Handeln ist eng verbunden mit dem Verhältnis zwischen Vater und Kind, insbesondere der emotionalen Bindung, während Unterhaltsaufwendungen v.a. von finanziellen Ressourcen und weniger dem sozialen Kapital abhängen.
} 
unter den deutschen Befragten (97\% des Samples) nicht sonderlich aussagekräftig. Der Einwand gilt ebenso für den nicht nachgewiesenen Zusammenhang im norwegischen Datenmaterial. Auch hier geben fast 90\% der Väter an, nur aus einer getrennten Partnerschaft als Vater hervorgegangen zu sein.

Ähnliche Einwände gibt es gegen die Ergebnisse mit Blick auf die Variablen der Anzahl externer Kinder. So weist die Variable im deutschen und norwegischen Datensatz wenig Variation auf. Das bedeutet, fast drei Viertel der befragten Trennungsväter in Deutschland geben an, dass sie Unterhaltsverpflichtungen gegenüber einem getrennt lebenden Kind haben. In Norwegen sind dies mit knapp zwei Dritteln etwas weniger, aber immer noch die Mehrheit, während im britischen Sample etwas über 50\% nur einem getrennt lebenden Kind gegenüber unterhaltspflichtig sind. ${ }^{208}$ Gleichzeitig weist die Variable im britischen Fall stärkere Ausfälle auf. Diese zeigen deutlich weniger sozio-emotionales Engagement und häufiger Zahlungsschwierigkeiten als Väter, die die Zahl ihrer Unterhaltsverpflichtungen benennen.

Neben dem Zusammenhang zwischen Care und der Anzahl getrennt lebender Kinder zeigt auch die Wirkung der väterlichen Arbeitszeit in eine andere Richtung als ursprünglich theoretisch angenommen. So zeigt sich auf bivariater Ebene sowohl in Großbritannien wie auch in Norwegen ein positiver Zusammenhang, der im norwegischen Fall auch auf multipler Ebene fortbesteht. Dieses Ergebnis kann damit erklärt werden, dass mit längeren Arbeitszeiten häufig höhere finanzielle Kompensationen verbunden sind. Wie die Ergebnisse zum Einkommen zeigen, besteht ein positiver Zusammenhang zwischen einem hohen Erwerbseinkommen und dem väterlichen Care. Längere Arbeitszeiten können ferner mit der Karriereorientierung und auch mit dem Bildungsniveau des Vaters in Verbindung gebracht werden. Damit begrenzt eine hohe Arbeitszeit zwar die verfügbare Zeit, gleichzeitig eröffnet sie jedoch (zusätzliche) finanzielle Ressourcen. Ferner muss kritisch mit Blick auf die aufgestellte Hypothese eingewandt werden, dass die Arbeitszeit als Restriktion zeitlicher Ressourcen sich primär auf die Quantität der Vater-Kind-Beziehung

\footnotetext{
${ }^{208}$ Damit leben die britischen Väter in der vorliegenden Stichprobe deutlich häufiger von mehreren Kindern getrennt als z.B. die norwegischen oder deutschen. Gleichzeitig sind diese getrennt lebenden Kinder auch öfter von mehr als einer Kindsmutter. Dies wirft die interessante Frage nach nationalen Lebensverläufen auf. Ist dies ein zufälliges Ergebnis, dass durch die Stichprobenerhebung zustande gekommen ist, oder kriegen britische Väter generell mehr Kinder von verschiedenen Frauen? Lange wurde es Vätern mit begrenzten finanziellen Mitteln in Großbritannien ermöglicht, ihren finanziellen Pflichten nicht nachzukommen, damit sie ihre aktuelle Familie versorgen (siehe dazu ausführlich Kap. 6.2.2.1 Unterhaltsregelungen). Diese Regelung besteht heute nicht mehr, aber einige der befragten Väter wurden zu dieser Zeit getrennt. Führt das zu dem Umstand das britische Männer häufiger mehr Kinder bzw. Familien haben, als sie sich „leisten“ können?
} 
auswirkt, d.h. die Kontakthäufigkeit. Die Qualität des väterlichen Engagements ist davon nur sekundär betroffen. ${ }^{209}$ Weniger Kontakte können durchaus intensiver ausgestaltet werden, als sehr viele Besuche, die z.B. mit Fernsehen verbracht werden. Es kann somit theoretisch argumentiert werden, dass ein Vater mit einer höheren Arbeitszeit generell im Leben wie auch im Beruf engagierter ist und sich dies positiv auf die Intensität seines Kümmerns um getrennt lebende Kinder auswirkt. Die zunächst theoretisch begründete These wurde anhand der empirischen Ergebnisse widerlegt. Die entgegengesetzte Wirkungsrichtung kann jedoch theoretisch plausibilisiert werden.

Über die Daten ähnliche, wenn auch nicht identische Resultate ergeben sich für die Effekte, die den Determinanten der physischen Umwelt unterstellt wurden. Dabei weisen die einzelnen Studien jedoch verschiedenen sozio-ökonomischen Aspekten Bedeutung zu. In Deutschland ist es das Einkommen des Vaters, dass sich positiv auf die Intensität seines Sorgehandelns auswirkt. In Norwegen und Großbritannien wird die Care-Variable eher durch die Ausbildung des Vaters bestimmt. Neben Einflussgrößen der sozialen Umwelt müssen daher zur Erklärung des sozio-emotionalen Engagements auch sozio-ökonomische Determinanten wie das Einkommen und das Bildungsniveau des Vaters herangezogen werden. Damit werden zwei weitere Thesen tendenziell durch die empirischen Ergebnisse gestützt.

Deutlichere nationale Unterschiede ergeben sich für den Zusammenhang sozio-emotionalen Kümmerns des Vater und der aktuellen Partnersituation beider Elternteile. In allen Datensätzen kann diesen eine Bedeutung zugesprochen werden, jedoch mit national unterschiedlichen Schwerpunkten. So kümmern sich in Deutschland Nachtrennungsväter verstärkt um ihre Kinder, wenn weder sie selbst noch die Kindsmutter in einer neuen Partnerschaft leben. Dies entspricht den theoretischen Annahmen. In Großbritannien ist nur die Partnersituation der Mutter für das väterliche Handeln von Bedeutung, während es in Norwegen die Beziehungssituation der Vaters ist, die seine Sorgepraxis determiniert. Inhaltlich lassen sich die nationalen Unterschiede nur schwer erklären. Die Effekte können jedoch auch methodische Ursachen haben. Im norwegischen Fall ist für die Partnersituation des Vaters aufgrund der Mängel in der empirischen Erhebung lediglich eine Unterscheidung zwischen allein lebenden Vätern und Vätern möglich, die mit anderen Personen unabhängig von ihrem Verwandtschafts- und Beziehungsstatus in einer Haushaltsgemeinschaft leben. Die Variable gibt sehr restringierte Auskunft über die Haushalts-

${ }^{209}$ Auf das Verhältnis zwischen Care und Kontakthäufigkeit wurde bereits hingewiesen (siehe Kap. 8.1 Die abhängige Variable: Care). 
zusammensetzung des Vaters und weniger über seine Partnersituation. Dennoch wirkt es sich positiv auf das Sorgehandeln in Norwegen aus, wenn der Vater allein lebt.

Der Einfluss der mütterlichen Partnersituation ist vor dem Hintergrund hoher Ausfälle zu sehen, da die väterlichen Angaben zur aktuellen Lebenssituation der Mutter in allen drei Datensätzen starke Lücken aufgrund von Unwissenheit der Väter aufweisen. Getrennt lebende Partner informieren sich nicht zwingend gegenseitig über ihre neue Beziehungssituation. Neue Partner bleiben den Trennungsvätern insbesondere dann verborgen, wenn diese nicht im Haushalt der Kindsmutter leben. Deutsche und norwegische Väter, die nicht über die neue Partnersituation der Kindsmutter informiert sind, zeigen dabei deutlich weniger Intensität in ihrem sozio-emotionalen Sorgehandeln als Väter, die über die Beziehungslage ihrer Ex-Partnerin Auskunft geben können. Unter britischen Vätern ist die Differenz zwischen Befragten, die Angaben zur Partnersituation der Kindsmutter machen und denen die keine Kenntnis besitzen, sogar noch ausgeprägter. Deutsche, britische und norwegische Väter, die keine Angaben zur Beziehungssituation der Ex-Partnerin machen können, zeigen des Weiteren häufiger Zahlungsschwierigkeiten. Der nachgewiesene Effekt kann vermittelt sein über das aktuelle Verhältnis zwischen den Eltern, denn ein freundschaftliches Verhältnis impliziert eine stärkere - private - Kommunikation und Interaktion, die die Kenntnis über die aktuelle Partnersituation erhöht.

Neben dem Einfluss der aktuellen Partnersituationen der Elternteile weisen alle drei Untersuchungen ferner auf den Einfluss des Verhältnisses der Eltern zueinander hin. Dabei unterstreichen die norwegischen und britischen Ergebnisse, wie dargestellt, die Bedeutung einer freundschaftlichen Beziehung zum Zeitpunkt der Befragung. Auffällig ist darüber hinaus, dass sowohl in Deutschland wie auch in Großbritannien der Beziehung zwischen den Eltern vor und während der Trennung eine wichtige Rolle in der Erklärung des väterlichen Sorgehandelns zukommt; diese in Norwegen jedoch keine Bedeutung hat. Das Handeln norwegischer Väter lässt sich primär - mit Blick auf die elterliche Beziehung mit dem aktuellen Verhältnis der Eltern erklären. In Deutschland liegen nur Informationen zum elterlichen Verhältnis vor bzw. während der Trennung vor. Aussagen über die Beziehung nach der Trennung sind hier nicht möglich. Dabei ist für das Handeln deutscher Nachtrennungsväter wichtig, in welcher Familienform sie mit der Kindsmutter gelebt haben, während bei englischen Väter von größerer Bedeutung ist, wie lange die elterliche Beziehung andauerte. Beide Variablen stellen Indikatoren für die Verbindlichkeit der Partnerschaft dar. Mit zunehmendem partnerschaftlichen Commitment des Vaters verstärkt sich auch die Intensität seines Engagements. Die Stärke der elterlichen Bindung scheint im norwegischen Fall von untergeordneter Bedeutung für das Verhältnis zwischen Vater und Kind nach der elterlichen Trennung zu sein. Das Ergebnis deutet insofern auf eine unterschiedliche Bedeutung der Familienform in ihrer institutionellen Formalisierung in den 
Ländern hin. So bekommen in Deutschland immer noch mehrheitlich verheiratete Elternpaare Kinder, während die Elternschaft in Norwegen nicht an die Institution Ehe gebunden ist, was sich ebenso in der Zusammensetzung der einzelnen Stichproben zeigt. Die in Norwegen befragten Väter weisen einen deutlich höheren Anteil an vormals unehelichen Lebensgemeinschaften auf als dies im deutschen und britischen Sample der Fall ist. Auch die rechtliche Gleichstellung ehelicher und nichtehelicher Väter mit Blick auf ihre Pflichten nach der elterlichen Trennung weist in Norwegen ferner auf eine stärkere Differenzierung zwischen den Ebenen der Elternschaft und Partnerschaft hin. ${ }^{210}$ Dies kann zusammengefasst ein möglicher Erklärungsansatz für die fehlende Bedeutung der elterlichen Beziehungsform vor und auch während der Trennung für das väterliche Sorgehandeln in Norwegen darstellen.

Als Indikator für das Verhältnis zwischen den Eltern während der Trennungsphase dient die Festlegung des Sorge- und Umgangsrechts sowie des Unterhalts. Dabei weist die Unterhaltsfestlegung in den einzelnen Datensätzen unterschiedliche Effektstärken in den nationalen Regressionsmodellen auf, die eventuell mit den verschiedenen institutionellen Rahmenbedingungen der Unterhaltsfestlegung begründet werden können. In Norwegen erfolgen die Regelungen zum Unterhalt fast ausschließlich über eine behördliche Instanz, wohingegen in Großbritannien die CSA nur in stark konfliktären Fällen oder wenn die Mutter staatliche Leistungen beantragt eingeschaltet wird. Ähnliches gilt in Deutschland, hier ist die Festlegung des Unterhalts in den Scheidungsprozess integriert und damit stark standardisiert sowie formalisiert. Starke Konflikte drücken sich in einer höheren Instanz aus, die in das Einigungsverfahren eingeschaltet wird. Damit müsste in Deutschland und Großbritannien ein ähnlich starker Effekt der Variablen Unterhaltsfestlegung auf Care zu erwarten sein, weil institutionelle Hilfe im Fall starker Uneinigkeit zwischen den Eltern in Anspruch genommen wird. Dies zeigt sich auch in den Ergebnissen. In Norwegen besitzt die Variable zur Unterhaltsfestlegung kaum Informationsgehalt mit Blick auf die Stärke elterlicher Konflikte. Die überwiegende Mehrheit der Eltern (80\% der Stichprobe) nimmt diese externe Instanz in Anspruch, was den fehlenden Effekt im norwegischen Regressionsmodell erklären kann.

Kritisch ist an dieser Stelle jedoch anzumerken, dass die Zusammenhänge ebenso aufgrund von Mängeln in den Daten entstanden sein könnten, da die entsprechenden Variablen in allen drei Datensätzen von hohen Ausfällen betroffen sind. Innerhalb der deutschen Studie weisen Fälle, die keine Angaben zur Unterhaltsfestlegung machen (können),

${ }^{210}$ Siehe dazu ausführlich Kap. 6.2 Der deutsche, britische und norwegische makro-soziale Kontext. 
auch häufiger Ausfälle im sozio-emotionalen Sorgehandeln auf. Im Niveau der CareKomponenten zeigen sich in Norwegen nur leichte Unterschiede, wobei sich für die Ausfälle ein etwas höheres Engagement ergibt. Leichte Differenzen finden sich auch in den britischen Ausfällen. Hier zeigen Väter ohne Angaben zur Unterhaltsfestlegung jedoch ein niedrigeres sozio-emotionales Engagement. Insofern liefern die Ergebnisse keine eindeutigen Hinweise auf die systematischen Auswirkungen der Ausfälle bei der Erklärung von Care.

Widersprüchliche Ergebnisse liefert die Berücksichtigung der Sorgerechtsfestlegung in Deutschland im Vergleich zur Umgangsregelung in Norwegen. In Deutschland wird der erwartete negative Zusammenhang empirisch belegt. Das bedeutet, dass mit steigendem elterlichen Konflikt um das Sorgerecht das sozio-emotionale Engagement des Trennungsvaters sinkt. In Norwegen ergibt sich dagegen auf bivariater Ebene ein signifikant positiver Effekt. Das bedeutet inhaltlich, mit steigender Uneinigkeit bei der Festlegung des Umgangs nimmt das väterliche Sorgehandeln in Form von Care nach der Trennung zu. Diese beiden - zunächst widersprüchlich erscheinenden - Ergebnisse können unterschiedliche Ursachen haben und lassen ferner unterschiedliche Interpretationen zu. Zunächst wurde bereits auf die jeweils andere Operationalisierung der Sorgerechts- bzw. Umgangsvereinbarung verwiesen. ${ }^{211}$ In der deutschen Studie wird nach dem Sorgerecht, der rechtlichen Formalisierung des väterlichen Sorgehandelns in Form von Care gefragt, während im norwegischen Datensatz die Festlegung des Umgangs, nach deutschem Verständnis die Vereinbarung des tatsächlichen Kontakts, erfasst wird. ${ }^{212}$ Die Einigung auf die Form des Sorgerechts sagt noch nichts darüber aus, wie häufig der Vater sein Kind sehen darf. Die Einigung auf den Umgang hingegen repräsentiert die formale Legitimation des alltäglichen Kontakts zwischen Vater und Kind. Somit beinhaltet die Einigung in Norwegen, die hier als Indikator für das Konfliktpotential zwischen den Eltern verstanden wird, die tatsächliche Ausgestaltung des Kümmerns, während die deutsche Festlegung primär das elterliche Entscheidungsrecht betrifft. Des Weiteren muss das Sorgerecht von Rechts wegen in Deutschland entschieden werden, wohingegen eine Umgangsübereinkunft in Norwegen nicht zwingend erforderlich ist. Der Kontakt zwischen Vater und Kind kann sich nach der elterlichen Trennung im Rahmen der alltäglichen Praxis einspielen, ohne auf eine konkrete Absprache basieren zu müssen. Dies stützen auch die empirischen Daten im

\footnotetext{
${ }^{211}$ Siehe dazu ausführlich Kap. 9.1.2. Der Einfluss der Prädiktoren der mikro-sozialen Umwelt.

${ }^{212}$ An dieser Stelle kommt es auf die exakte Übersetzung der Frage im norwegischen Originaldatensatz an. Es kann nicht ausgeschlossen werden, dass es sich um eine Übersetzungsungenauigkeit aus dem Norwegischen handelt.
} 
norwegischen Fall. Ein hoher Anteil Väter gibt an, keine Vereinbarung über den Kontakt mit der Kindsmutter getroffen zu haben. Dort, wo eine Festlegung statt gefunden hat, wird diese von der Mehrheit als „privat getroffen“ charakterisiert. Daher kann auch der Mangel an Variation innerhalb der Prädiktorvariable für das widersprüchliche Zusammenhangsmaß im norwegischen Fall verantwortlich sein. Es liegt die Vermutung nahe, dass v.a in den Fällen Absprachen zum Umgang- formalisiert - getroffen werden, in denen die Eltern sich nicht einig sind. Aufgrund der geringen Einklagbarkeit väterlichen Engagements, wenn sich der Vater dem Kontakt zu seinem Kind verschließt, werden sich v.a. jene Eltern vor Gericht treffen, bei denen der Vater mehr Umgang einfordert, was sein höheres väterliches Engagement erklären könnte. Des Weiteren kann angeführt werden, dass ein erstrittener Umgang auch genutzt wird. Es scheint daher theoretisch plausibel anzunehmen, dass die beiden Variablen in den jeweiligen Datensätzen inhaltlich doch nicht - wie zunächst angenommen - das Gleiche messen und deswegen andere Wirkungszusammenhänge in den empirischen Ergebnissen entstehen. Auf multipler Ebene wird der Zusammenhang in Norwegen darüber hinaus nicht mehr bestätigt.

In Norwegen zeigt sich mit Blick auf die Trennungsregelung primär das Ergebnis des Aushandlungsprozesses - die Form des festgelegten Umgangs - von Bedeutung, während der Weg dahin weniger wichtig ist. Dies kann u.U. mit der hohen Eigenverantwortung der Eltern innerhalb der Regelung und der gleichzeitig stark institutionalisierten Hilfestellung erklärt werden. Anders als im britischen Kontext werden Eltern gar zur Mediation im Scheidungsprozess „gezwungen“, wenn Kinder involviert sind. Damit ist die elterliche Eigenverantwortung nicht nur institutionalisiert - wie in Großbritannien - sondern findet darüber hinaus in institutionalisierten Bahnen statt, d.h. Eltern werden mit ihrer Eigenverantwortung nicht allein gelassen. ${ }^{213}$ Britische Eltern werden in ihrer eigenverantwortlichen Aushandlung nicht im gleichen Maße staatlich unterstützt. Dies kann gegebenenfalls zu einer schnelleren Eskalation elterlicher Auseinandersetzungen führen, da Konflikte seltener durch externe Instanzen geschlichtet werden. Starke Auseinandersetzungen während der Trennung wirken sich negativ auf das Sorgehandeln danach aus.

In allen drei Datensätzen kommt, wie dargestellt, der Trennungsregelung eine Bedeutung in der Erklärung sozio-emotionalen Handelns von Nachtrennungsvätern zu. In Deutschland weisen dabei sowohl die Festlegung wie auch das Ergebnis der Aushandlung einen

\footnotetext{
${ }^{213}$ Interessant wäre an dieser Stelle zu untersuchen, ob sich andere Aushandlungsergebnisse einstellen, wenn der Prozess der Übereinkunft „staatlich überwacht“ wird. Einigen sich britische Eltern auf weniger hohe Unterhaltszahlungen, wenn sich keine dritte Instanz einmischt? Verweigern norwegische Mütter eher den Kontakt, wenn staatliche Institutionen sie nicht daran hindern?
} 
Effekt auf. In Großbritannien ist es nur der Festlegungsprozess, weil Angaben zum Ergebnis nicht erfasst wurden, während es in Norwegen insbesondere das Aushandlungsergebnis ist. Es muss jedoch offen bleiben, inwiefern die Variablen das Verhältnis zwischen den Eltern im Trennungsprozess abbilden oder tatsächlich institutionelle Rahmungen der Situation repräsentieren. Das bedeutet, Eltern, die in ihrer Aushandlung der Trennungsregelung institutionell stark geleitet werden, indem wie z.B. in Deutschland die Unterhaltsfestlegung z.B. im formalen Scheidungsverfahren integriert ist oder wie in Norwegen Mediation im Scheidungsverfahren obligatorisch ist, weisen eventuell ein anderes Konfliktpotential auf als Eltern, die vollkommen in Eigenverantwortung diese Verhandlungen führen. In einer Phase starker negativer Emotionen und Belastungen gelingt es den Paaren eigenständig vielleicht nicht immer, die Eltern- von der Paarebene zu trennen. Das erklärt aber noch nicht, warum in Deutschland trotz starker Institutionalisierung der Scheidungsund Trennungsprozeduren von Eltern, die Trennungsregelung trotzdem so eine große Bedeutung zukommt.

Insgesamt zeigen die Ergebnisse mit Blick auf die Care-Variable kaum länderspezifische Unterschiede in der Wirkungsrichtung der angenommenen Zusammenhänge. Die Bedeutung der Effekte innerhalb der multiplen Modelle variiert jedoch zwischen den Ländern, was z.T. auf methodischen Gegebenheiten beruht, aber auch inhaltlich vorsichtig interpretiert werden kann.

Im deutschen Modell der multiplen Regression weisen die drei Variablen zur Trennungsregelung - die Festlegung von Unterhalt und Sorgerecht sowie die Form des Sorgerechts die stärkste Bedeutung für die Erklärung väterlichen Cares auf. Dieses Ergebnis lässt zwei Schlussfolgerungen zu: zum einen ist das Verhältnis und der Umgang zwischen den Eltern zum Zeitpunkt der Trennung entscheidend für die spätere Ausgestaltung des väterlichen Kümmerns. ${ }^{214}$ Des Weiteren scheinen institutionelle Regelungen des Trennungsprozesses - wie z.B. die Rolle der Gerichte oder die Bedeutung außergerichtlicher Einigungen mit institutioneller Hilfe - dieses Verhältnis beeinflussen zu können. Hier wäre an eine verstärkte Mediation von behördlicher Seite zu denken, die in Deutschland auch zunehmend in Anspruch genommen wird. Die Überlegungen zur Bedeutung institutioneller Rahmenbedingungen widersprechen auf den ersten Blick der Schlussfolgerung aus den bivariaten

\footnotetext{
${ }^{214}$ Es kann angenommen werden, dass sich das Verhältnis während des Trennungsprozesses auch auf die Ausgestaltung der elterlichen Beziehung längerfristig auswirkt. Es muss dabei Spekulation bleiben, dass auch im deutschen Fall die Freundschaft der Eltern zum Zeitpunkt der Befragung von Bedeutung für das väterliche Sorgehandeln sein könnte. Die Information wurde nicht erhoben, so dass eine empirische Prüfung dieser Annahme nicht möglich ist.
} 
Ergebnissen, das väterliche Care sei eher durch das mikro- als das makro-soziale Umfeld bestimmt. Da in das multiple Modell jedoch noch einige andere Determinanten eingehen, die nicht primär dem makro-sozialen Kontext zuzurechnen sind, und die Effekte der anderen Faktoren darüber hinaus nicht so stark von denen der drei genannten Variablen abweichen, kann weiterhin davon ausgegangen werden, dass Care durch eine vielfältige Kombination v.a. mikro-sozialer Einflussfaktoren bestimmt wird. Zumal der Einfluss der Festlegung von Sorgerecht und Unterhalt primär als Verhältnis zwischen den Eltern im Zeitpunkt der Trennung interpretiert wird und weniger als institutionelle Einflussnahme.

Innerhalb des britischen Modells fällt die besondere Bedeutung der elterlichen Freundschaft nach der Trennung auf, da sie den stärksten Effekt auf die Care-Variable hat. Danach folgt die Partnersituation der Mutter. Ein neuer Partner an der Seite der Ex-Partnerin hindert den Trennungsvater offensichtlich, sich um seine getrennt lebenden Kinder intensiver zu kümmern. Es kann spekuliert werden, ob auch das freundschaftliche Verhältnis zwischen den Eltern von der Anwesenheit eines neuen Partners der Mutter beeinträchtigt wird. Im Vergleich zu den anderen Modellen fällt ferner im britischen Fall auf, dass die Anzahl der Elternschaften innerhalb der britischen Regression eine nicht zu vernachlässigende Bedeutung zukommt, während sie in Deutschland und Norwegen keine Rolle spielt. Es wurde bereits darauf hingewiesen, dass dieses Ergebnis auch mit der mangelnden Variation in den Variablen zur Zahl der Elternschaften in Norwegen und Deutschland zu erklären sein könnte. Im britischen Fall zeigt sich zwar eine ausreichende Varianz, die Variable ist jedoch von starken Ausfällen betroffen. Mit Blick auf die Anzahl der Elternschaften des getrennt lebenden Vaters zeigen die britischen Daten kaum Unterschiede in der Häufigkeitsverteilung der abhängigen Variablen Care und Cash zwischen den Vätern mit und ohne Angaben.

Ähnlich wie in Deutschland ist auch in Norwegen die Form des Umgangs - bzw. des Sorgerechts im deutschen Fall - ein wichtiger Prädiktor für das väterliche Sorgehandeln in Form von Care. Vergleichbar mit Großbritannien ist der starke Zusammenhang mit dem freundschaftlichen Verhältnis zwischen den Eltern. Ein interessantes Ergebnis im norwegischen Fall stellt die Bedeutung der räumlichen Distanz zwischen den elterlichen Wohnsitzen dar. Trotz der großen Entfernungen, die durch die geographischen Gegebenheiten in Norwegen eine besondere Herausforderung darstellen, spielen diese eine untergeordnete Rolle für die väterliche Fürsorge in Form von Care. Aufgrund der spezifischen weil dünnen Besiedelung Norwegens ist ein Umzug in eine andere Stadt häufig mit einer größeren Entfernung zwischen den elterlichen Wohnorten verbunden. Diese Entfernungen sind dabei meist nur mit dem Flugzeug zu überbrücken, Zugfahrten implizieren oft lange Reisezeiten. Dennoch spielt die räumliche Distanz in Norwegen nur eine kleine (signifikante) Rolle für das väterliche Kümmern, anders als beispielsweise in Großbritannien. Das be- 
deutet, dass norwegische Väter entweder auch weite Entfernungen nicht scheuen, um ein hohes Engagement zu zeigen, oder dass sie seltener in eine andere Stadt bzw. Gegend ziehen. Die Häufigkeitsverteilungen der einzelnen Datensätze stützen letzteres jedoch nicht. Rund 20\% der befragten norwegischen Väter leben weiter als zwei Stunden Reiseweg von ihren Kindern entfernt, während in Deutschland und Großbritannien nur rund $10 \%$ angeben weiter als $200 \mathrm{~km}$ auseinander zu wohnen.

\subsubsection{Die Ergebnisse für Cash}

In der Darstellung der bivariaten Ergebnisse wurde bereits auf deren Heterogenität mit Blick auf die Cash-Variable verwiesen. Diese wird durch die multiplen Analyse weiter verschärft. Dabei fällt zunächst ins Auge, dass in Deutschland lediglich zwei Variablen einen relativ großen Anteil der Varianz aufklären, während sowohl im britischen wie auch im norwegischen Fall eine höhere Anzahl an Determinanten Einfluss hat. Für die Bestätigung der einzelnen Hypothesen findet sich allerdings in allen drei Studien nur sehr mäßige empirische Unterstützung.

In allen drei Datensätzen zeigen sich dabei insbesondere Zusammenhänge für die Faktoren der physischen Umwelt. Wie theoretisch zu erwarten war, spielen sozio-ökonomische Aspekte, insbesondere das Einkommen, eine wichtige Rolle für die Erklärung des finanziellen Unterstützungsverhaltens der Nachtrennungsväter. Der postulierte Zusammenhang des Einkommens findet sich in allen drei Studien empirisch wieder. In Großbritannien und Norwegen wirkt sich darüber hinaus das Bildungsniveau des Vaters, bei britischen Vätern zusätzlich die Arbeitszeit auf die finanziellen Transfers aus. Die Ergebnisse stützen damit die Einkommensthese insgesamt sowie die Annahmen zum Bildungsniveau tendenziell.

Der Entfernung zwischen den elterlichen Wohnorten sowie dem Einigungsprozess auf das Sorgerecht bzw. den Umgang wurde theoretisch kein Einfluss unterstellt. Diese Annahmen werden durch die empirischen Ergebnisse gestützt. In allen Untersuchungen, in denen die Variablen vorliegen, konnten keine Zusammenhänge auf signifikantem Niveau nachgewiesen werden. Dies spricht für die Gültigkeit der aufgestellten Hypothesen.

Wie angedeutet werden einige der postulierten Zusammenhänge durch die vorliegenden Daten nicht gestützt. So zeigen die Ergebnisse, dass entgegen der Annahme kein Zusammenhang zwischen der Partnersituation der Mutter und dem finanziellen Sorgehandeln des Vaters besteht. In keinem der drei Datensätze sind die Regressionsgewichte von signifikanter Bedeutung. Des Weiteren kann kein Effekt des Prädiktors, der den früheren Familienstand der Eltern angibt, auf die Cash-Variable ausgemacht werden. Dies gilt ebenso für die Anzahl der getrennten Beziehungen, aus denen Kinder hervorgegangen 
sind. ${ }^{215}$ Die mit den Variablen verbundenen Hypothesen können damit nicht aufrecht erhalten werden.

Die anderen, bisher nicht genannten Variablen weisen demgegenüber starke Variationen in den Ergebnissen zwischen den Untersuchungen auf, die vorsichtig auf einzelne nationale Charakteristika hindeuten. Auf die Sparsamkeit des deutschen Modells wurde bereits hingewiesen. Die Bedeutung des Einkommens ist inhaltlich sehr plausibel, da die Verfügbarkeit der finanziellen Mittel entscheidend für die Gewährleistung von Unterhaltszahlungen ist. Gleichzeitig ist sie ein Bestandteil der Berechnung der Leistungshöhe in allen drei Ländern, so dass ihre herausragende Bedeutung nicht weiter verwundert. Interessant ist, dass in Deutschland keine weiteren Bestandteile der Berechnungsformel, wie die Anzahl der Unterhaltsverpflichtungen oder die Anwesenheit von Kindern im väterlichen Haushalt, Einfluss zu haben scheinen. ${ }^{216}$

Der Zusammenhang mit der Unterhaltsfestlegung in den deutschen Ergebnissen ist ähnlich plausibel wie der des Einkommens. Uneinigkeit in der Höhe der Unterhaltsverpflichtung kann aufgrund von Uneinsichtigkeit eher zu Zahlungsverweigerung führen. Es ist davon auszugehen, dass Unterhaltspflichtige eher ein Interesse daran haben, die Zahlungen gering zu halten bzw. eine andere Vorstellung von der Angemessenheit der Beträge haben als der unterhaltsberechtigte Elternteil. Fraglich ist jedoch, warum die Variable der Unterhaltsfestlegung in den anderen beiden Datensätzen keinen Effekt auf die Zahlungspraxis hat. Dies kann in beiden Fällen methodisch begründet werden. Mit der britischen Variablen der Unterhaltsfestlegung sind hohe Unsicherheiten verbunden, da lediglich für 416 Fälle die notwendigen Informationen aus verschiedenen Variablen konstruiert werden können. Gleichzeitig ist die Variation in den beiden Ausprägungen nicht sonderlich stark ausgeprägt. Im norwegischen Fall ist auch die fehlende Variation in den Ausprägungen zu bemängeln. Die meisten Väter haben den Unterhalt mit der Unterhaltsstelle festgelegt (83\%). Inhaltliche Argumente für die unterschiedlichen Ergebnisse wurden bereits mit Blick auf die Care-Variable angeführt. Diese gelten auch hier. In Deutschland wird die Einigung auf den Unterhalt gerichtlich i.d.R. nur in Konfliktfällen gelöst. In Norwegen dagegen ist die Integration einer behördlichen Instanz im Festlegungsprozess die Norm.

\footnotetext{
${ }^{215}$ Es gelten die gleichen methodischen Bedenken wie schon bei der Darstellung der Care-Ergebnisse genannt wurden. Es gibt kaum Variation über die Ausprägungen der Variablen und im britischen Fall weist sie darüber hinaus hohe Ausfälle auf.

${ }^{216}$ Was jedoch auch mit der fehlenden Varianz in beiden Variablen begründet werden kann. Deutsche Väter geben mehrheitlich an, mit keinem Kind nach der Trennung zusammenzuleben und nur einem Kind gegenüber unterhaltspflichtig zu sein.
} 
Dies könnte begründen, warum im norwegischen Fall kein Zusammenhang zwischen der Unterhaltsfestlegung und Cash besteht, in Deutschland dagegen schon. Die formulierte These wird damit nur bedingt gestützt. Des Weiteren könnte dem Unterhalt in Deutschland, wie angedeutet, eine andere Bedeutung zukommen, als z.B. in Norwegen. Deutsche Väter zahlen Unterhalt für Kind und Kindsmutter, während zwischen norwegischen Eltern kaum finanzielle Ansprüche nach der Trennung bestehen. Es wird im Sinne des Dual Earner/Dual Carer-Modells davon ausgegangen, dass die Mütter sich selbst versorgen können. Es kann vermutet werden, dass getrennte Väter lieber für ihre Kinder als für ihre Ex-Partnerinnen zahlen. Dies könnte ein weitere Anhaltspunkt für die Erklärung des deutschen Ergebnisses sein.

In Großbritannien stechen im multiplen Modell zur Erklärung ausbleibender Unterhaltszahlungen insbesondere zwei Besonderheiten heraus: zum einen kommt dem aktuellen freundschaftlichen Verhältnis zwischen den Eltern - wie auch schon bei der Care-Variablen - eine hohe Erklärungskraft zu. Verstehen sich die Eltern im Befragungszeitpunkt gut, so nimmt die Wahrscheinlichkeit von Zahlungsproblemen ab. Dieser Zusammenhang wurde theoretisch nicht angenommen und findet sich nicht im norwegischen Datensatz. Hier kann zunächst argumentiert werden, dass die Ursache-Wirkungsrichtung nicht eindeutig zu bestimmen ist. Das Verhältnis zwischen den Eltern wird sicherlich durch ausbleibende Unterhaltsleistungen belastet. Offen bleibt jedoch, warum sich dieser Zusammenhang nicht in Norwegen findet. Ein Erklärungsansatz könnte sich in der institutionellen Regelung der Zahlungstransfers finden. In Großbritannien besteht eine größere Autonomie der Eltern in der Festlegung des Unterhalts - zumindest vor der Einführung der Child Support Agency und solange genügend finanzielle Mittel bereitstehen. Gleichzeitig erfolgt der monetäre Transfer in elterlicher Eigenverantwortung. Britische Eltern legen Unterhaltszahlungen häufiger nach eigenen Aushandlungsprozessen fest, genau wie der Transfer der Leistungen nicht über behördliche Instanzen läuft. Nur im starken Konfliktfall wird die Hilfe Dritter beansprucht. Dies ist in Norwegen anders: Hier werden die Zahlungen mehrheitlich (freiwillig) über eine Behörde geregelt, so dass die persönliche Beziehung der Eltern dadurch nicht so stark belastet wird. Des Weiteren wird ein ausbleibender Unterhalt in Norwegen durch finanzielle Transfers des Wohlfahrtsstaates ausgeglichen, was zusätzlich das Konfliktpotential zwischen den Eltern abschwächt. Die freundschaftliche Beziehung ist in Norwegen ohne Bedeutung für das Leisten von Unterhalt. Inhaltlich können die Ergebnisse mit der unterschiedlichen Differenzierung zwischen persönlicher Ebene der ehemaligen Partner und institutionalisierter Trennungsregelung in den nationalen Kontexten plausibel erklärt werden.

Neben dem freundschaftlichen Verhältnis kommt in Großbritannien zum anderen der neuen Familie in der Erklärung väterlicher Unterhaltsleistungen eine besondere Bedeutung 
zu. Dabei senkt eine neue Partnerschaft des Vaters, entgegen der aufgestellten Hypothese, die Wahrscheinlichkeit von Zahlungsproblemen, während neue Kinder im väterlichen Haushalt Unterhaltsschwierigkeiten begünstigen. Letzteres stützt die theoretische Annahme, wenn auch nur im britischen Fall. Dass die Anzahl der Kinder im väterlichen Haushalt sich nur in Großbritannien signifikant auswirkt, kann u.a. durch die fehlende Varianz in der Variablen im deutschen Datensatz begründet sein. ${ }^{217}$ Britische Väter geben häufiger an, mit Kindern zusammen zu leben, während in Deutschland 85\% der Väter ohne Kinder wohnen. Der positive Zusammenhang zwischen einer neuen Partnerschaft des Vaters und dem Ausbleiben von Zahlungsproblemen verwundert zunächst. Inhaltlich bedeutet dies entgegen der theoretischen Annahme, dass eine neue Frau an der Seite des Vaters das Leisten von Unterhaltszahlungen unterstützt. Dies kann theoretisch nur bedingt erklärt werden. Die neue Frau im Leben des Vaters kann ihn an seine vergangene Familie und an damit verbundene Pflichten erinnern. Eine Zahlungsverweigerung wäre demzufolge mit höheren emotionalen Kosten verbunden, da eine neue Partnerin ihn gegebenenfalls für seine fehlende Zahlungsmoral sozial missachten könnte. Denkbar wäre auch der Einfluss einer dritten Variablen, wie etwa dem Einkommen. Männer mit geringerem sozialen Status und damit verbundenen mit geringerem Einkommen sind eventuell häufiger partnerlos nach der Trennung. Auch könnten Männer, die ihren Zahlungspflichten einer ersten getrennt lebenden Familie nicht gerecht werden, keine neue Partnerschaft eingehen aus Furcht vor neuen finanziellen Belastungen. Diese Annahmen bleiben jedoch reine Spekulation und können am vorliegenden Material aufgrund der hohen Ausfälle in der Einkommensvariablen nicht gesichert geprüft werden.

Neben der großen Bedeutung der neuen Familie für die Zahlungspraxis britischer Trennungsväter zeigt sich in diesem Zusammenhang ein weiterer interessanter Aspekt: Die Bedeutung der neuen Familie ist mit Blick auf das sozio-emotionale Sorgehandeln zu vernachlässigen, während es für die finanzielle Unterstützung der getrennt lebenden Familie besonders wichtig ist. Damit findet sich die angenommene Clean Break-Philosophie, wie sie lange in Großbritannien praktiziert wurde, nur mit Blick auf Unterhaltsvariable. Ebenso ist in diesem Zusammenhang auffällig, dass sich hier zwar die Partnersituation der Mutter auf die Ausgestaltung des Care auswirkt, nicht jedoch auf das Zahlungsverhalten.

Auf bivariater Ebene ergab sich im britischen Material ein signifikant positiver Zusammenhang zwischen der ökonomischen Situation der Mutter und dem Auftreten von Zahlungsschwierigkeiten des Vaters. Das bedeutet, dass eine schlechtere finanzielle Lage

${ }^{217}$ Für Norwegen liegen keine Informationen zur Kinderzahl im Haushalt des Vaters vor. 
der Kindsmutter vermehrt zu Unterhaltsproblemen führt. Obwohl theoretisch die umgekehrte Wirkungsrichtung angenommen wurde, kann eine eindeutige Wirkungsrichtung des Effekts angezweifelt werden, insofern die ausbleibende finanzielle Unterstützung des Vaters gerade die schlechte monetäre Situation der Mutter ausgelöst haben kann. Auch kann aufgrund von sozialen Partnerschaftsmustern vermutet werden, dass eine schlechte ökonomische Lage der Mutter mit einer schlechten finanziellen Lage des Vaters einhergeht. Offen bleibt jedoch, warum dieser Zusammenhang allein für Großbritannien zu finden ist und nicht in Norwegen. Dies kann mit Hilfe operationalisierungstechnischer Mängel begründet werden. Die norwegischen Väter wurden um eine Einschätzung der finanziellen Lage der Kindsmutter mit den Antwortvorgaben „sie kann sich weniger leisten“ „sie kann sich genauso viel leisten“ und „sie kann sich weniger leisten“ gebeten. Die Bewertung der ökonomischen Situation zum Zeitpunkt der Befragung ist daher auf ihre Relation zu „früher“ ausgerichtet. Damit sind aber nur Aussagen möglich, die die Richtung der Entwicklung angeben, d.h. ob sich die Lage verbessert oder verschlechtert hat oder gleich geblieben ist. Die Ausprägungen geben keine Auskunft über den Status Quo während der elterlichen Beziehung. Es bleibt unklar, ob sich die Mutter von einem luxuriösen auf einen komfortablen Lebensstandard oder von einer mittleren auf eine schwierige Finanzlage verschlechtert hat, was inhaltlich einen bedeutsamen Unterschied macht. Die Ergebnisse in der norwegischen Studie sind deshalb nur mit großer Vorsicht zu interpretieren. Eventuell besteht damit in Norwegen doch ein Zusammenhang zwischen ökonomischer Situation der Mutter und der Zahlungspraxis des Vaters, der durch das vorliegende Material jedoch nicht nachgewiesen werden kann.

Darüber hinaus weist die Variable in beiden Datensätzen hohe Ausfälle auf. Ähnliche Überlegungen wie bei der aktuellen Partnersituation der Mutter können ebenso für die Beurteilung ihrer ökonomischen Lage angeführt werden. Auch hier haben die getrennt lebenden Väter keinen direkten Einblick mehr. Norwegische wie britische Väter, die Angaben zur finanziellen Situation ihrer ehemaligen Partnerin machen können, zeigen ein höheres väterliches Engagement in Form von Care als Väter, die dies nicht können. Innerhalb des britischen Datensatzes weisen die Ausfälle der Variablen zur finanziellen Lage der Mutter deutlich häufiger Zahlungsprobleme auf. Anders unter norwegischen Vätern, hier hat die Kenntnis bzw. Beurteilung der ökonomischen Situation der Mutter keinen Einfluss auf ihre Zahlungspraxis. Väter mit und ohne Angaben weisen ähnliche Anteile an Unterhaltsproblemen auf. Die Unsicherheiten in der norwegischen Variablen und die hohen Ausfälle in der britischen Variable schwächen das Ergebnis. Gestützt wird dies durch die Tatsache, dass der Zusammenhang im multiplen Modell in Großbritannien nicht weiter nachgewiesen werden kann. 
In Norwegen sticht innerhalb des nationalen multiplen Modells die Bedeutung der Form des festgelegten Umgangs heraus. Dem Umgang wurde, wie dem Sorgerecht, kein Einfluss auf die Cash-Variable unterstellt. Im deutschen Datenmaterial findet diese Annahme Unterstützung, im norwegischen demgegenüber beeinflusst die Form des festgelegten Umgangs das Zahlungsverhalten der Väter mit. Die unterschiedlichen Ergebnisse können mit den gleichen Argumenten erklärt werden, wie sie bereits bei der Care-Variablen angeführt wurden. Da der festgelegte Umgang in Norwegen nicht das Gleiche abbildet wie das festgelegte Sorgerecht in Deutschland, können beide Ergebnisse inhaltlich plausibilisiert werden. Die Festlegung eines gemeinsamen Sorgerechts hat für den deutschen Vater konkret primär rechtliche Konsequenzen. Sie legt seine Entscheidungsbefugnis über wichtige Belange, die sein Kind betreffen, fest und sagt noch nichts über seine Umgangsrechte aus. Diese Befugnis hat somit auf seine Zahlungsverpflichtung und sein Verpflichtungsempfinden keinerlei Auswirkungen. Demgegenüber ist für die Ausübung eines gemeinsamen Sorgerechts ein intensives sozio-emotionales Engagement erforderlich, wodurch der Effekt auf Care im Unterschied zu Cash sinnvoll erscheint. Der festgelegte Umgang in Norwegen drückt dagegen das väterliche Engagement in erster Linie in Form der „erlaubten“ Kontakthäufigkeit aus. Ein Vater, der ein starkes Umgangsrecht mit der Kindsmutter vereinbart hat, zeigt ein hohes Interesse und Engagement. Doch die inhaltliche Differenzierung zwischen Umgangs- und Sorgerecht lässt theoretisch nicht auf einen Effekt auf finanzielle Unterstützungshandlungen schließen. Inhaltlich ist der festgestellte Zusammenhang durchaus plausibel in seiner Wirkungsrichtung. Denn je mehr Umgang vereinbart wird, desto eher fließen auch finanzielle Transfers in vereinbarter Regelmäßigkeit. Das Ergebnis lässt vermuten, dass in Norwegen ein stärkerer Zusammenhang zwischen Care und Cash besteht, als beispielsweise in Deutschland und Großbritannien. ${ }^{218}$ Offen bleibt jedoch zunächst, warum dieser Effekt allein in Norwegen auftritt. Eventuell ließen sich hierfür methodische Gründe anführen. Die Variation zwischen den Ausprägungen der erklärenden Variable ist eher gering und die Kategorisierung inhaltlich nicht sonderlich aufschlussreich. Nur ein geringer Anteil der Väter gibt an, weniger als den „üblichen“ Unterhalt vereinbart zu haben. Ein großer Teil hat gar keine formale Vereinbarung über den Umgang getroffen, was nicht zwingend bedeutet, dass der Vater keinen Kontakt hat. Im Gegenteil, es bedeutet, dass die Eltern keine Vereinbarung benötigen, weil sie sich bezüglich der Umgangsintensität einig sind. Damit kann also etwas über das Verhältnis

${ }^{218}$ Zur Relation von Care und Cash siehe ausführlich Kap. 9.4 Väterliches Sorgehandeln: Das Verhältnis von Care und Cash. 
der Eltern ausgesagt werden, jedoch nichts über die Form des festgelegten Umgangs. ${ }^{219}$ Auch dies könnte den nicht erwarteten Zusammenhang verursacht haben.

Eine weitere Besonderheit des norwegischen Cash-Modells stellt der ausgewiesene negative Effekt der Dauer seit der Trennung dar. Das bedeutet, mit kürzerer zeitlicher Distanz zur Trennung treten eher Zahlungsschwierigkeiten auf. Theoretisch wurde die umgekehrte Wirkungsrichtung angenommen. Dieses Ergebnis ist theoretisch sinnvoll schwer zu interpretieren. Es könnte vermutet werden, dass mit kürzerer Dauer nach der Trennung noch keine Unterhaltsregelung vereinbart wurde, die zu ausbleibenden Zahlungen und damit zum Anhäufen von Unterhaltsschulden führt. Doch bei Zeitspannen von bis zu einem, zwei und drei Jahren nach der Trennung scheint diese Annahme eher ungerechtfertigt. Methodisch kann das irritierende Ergebnis dagegen mit der schiefen Verteilung über die Ausprägungen des Prädiktors begründet werden. Eine überwiegende Mehrheit der befragten Väter lebt länger als drei Jahre von ihren Kindern getrennt, wobei der größte Teil dieser Väter auch seinen Unterhaltsverpflichtungen nachkommt. Mit einer Signifikanz von 0,046 ist das Ergebnis gerade noch im Rahmen der akzeptierten Signifikanzgrenze, was den Zusammenhang in seiner Bedeutung schwächt.

Insgesamt weisen trotz der unterschiedlichen Wirkungsgrade der einzelnen Faktoren die nationalen Modellen hohe Erklärungskraft auf. So werden in Norwegen und Deutschland knapp 30\% der Varianz erklärt, während in Großbritannien sogar um die 40\% der Variation der abhängigen Variablen Care aufgeklärt werden können. Weiterhin zeigen auch die empirischen Modelle zur Erklärung der Cash-Variablen in allen drei Ländern eine akzeptable Güte. In Norwegen und Deutschland können knapp 20\% der Varianz aufgeklärt werden, in Großbritannien sind es sogar um die 35\%. Dies stützt die Ergebnisse, die bereits auf bivariater Ebene angedeutet haben, dass im britischen Fall auch Faktoren der sozialen Umwelt zur Erklärung des finanziellen Unterstützungshandelns eine hohe Relevanz zukommt. Diese sind in Norwegen und insbesondere in Deutschland von untergeordneter Bedeutung. Das Ergebnis legt die Vermutung nahe, dass monetäre Transfers in Deutschland und Norwegen stärker von anderen Determinanten abhängen, die im Modell keine Berücksichtigung finden, als in Großbritannien. In diesem Zusammenhang wäre auf die institutionellen Rahmenbedingungen zu verweisen. Der britische Kontext wurde zum Zeitpunkt der Erhebung durch eine familienpolitische Non-Intervention im privaten Bereich der Familie charakterisiert. Dies könnte eine Erklärung dafür sein, dass finanzielle Unterstützung in Nachtrennungsfamilien verstärkt auf Aushandlungsprozessen innerhalb

${ }^{219}$ Diese Einschränkung muss auch für den Zusammenhang mit der Care-Variablen angeführt werden. 
der sozialen Umwelt beruhen. Dafür spricht auch die Bedeutung der neuen Familie und der freundschaftlichen Beziehung zwischen britischen Eltern, die im multiplen Modell einen wichtigen Erklärungsbeitrag leisten.

Von den theoretischen Annahmen, die die Unterhaltsschwierigkeiten erklären sollten, haben sich nur wenige im Fall der Cash-Komponente bestätigt. So gilt es als empirisch abgesichert, dass mit steigendem Einkommen seltener Probleme mit den Unterhaltszahlungen auftreten. Ferner konnte belegt werden, dass die räumliche Distanz zwischen den Eltern sowie die Festlegung des Sorgerechts ohne signifikante Bedeutung für die Zahlungspraxis sind, wie dies auch theoretisch begründet wurde. Insgesamt können so für die Cash-Dimension weniger Thesen bestätigt werden als für die Care-Komponente. Darüber hinaus verweisen die Ergebnisse zur Zahlungspraxis stärkere nationale Unterschiede auf.

Dieses Ergebnis lässt verschiedene Schlüsse zu. Zunächst kann aufgrund der nicht bestätigten theoretischen Annahmen vermutet werden, dass entweder nur wenige Einflussfaktoren die Zahlungspraxis der Nachtrennungsväter bestimmen oder in der vorliegenden Analyse nicht die entscheidenden Prädiktoren Berücksichtigung gefunden haben. Aufgrund der üblicherweise hohen Komplexität sozialer Phänomene wird im Folgenden von letzterem ausgegangen. Diese Annahme unterstützt auch die unterschiedliche Güte der multiplen Modelle für Care und Cash. So kann zumindest in Norwegen und Deutschland ein höherer Anteil an Variation durch das Gesamtmodell des sozio-emotionalen Handelns erklärt werden, als dies das Modell für die finanzielle Unterstützung kann; dagegen lässt sich in Großbritannien durch beide Modelle ähnlich viel Variation aufklären. Doch auch im britischen Fall ist Potential für eine höhere Güte des Modells gegeben. Wenn die hier untersuchten Determinanten also nicht ausreichen, um das väterliche Sorgehandeln in Form finanzieller Unterstützungsleistungen zu erklären, stellt sich die Frage nach anderen Bestimmungsfaktoren.

Dies führt gleichzeitig zur zweiten Schlussfolgerung. Die Ergebnisse - zumindest in Deutschland und Norwegen - zeigen, dass die väterliche Zahlungspraxis wesentlich durch Determinanten der physischen Umwelt bestimmt wird. Die soziale Umwelt in Form von Beziehungen weist weniger signifikante Zusammenhänge auf, weshalb Bestimmungsfaktoren daher v.a. innerhalb der physischen Rahmenbedingungen zu suchen wären.

Physische Kontexte väterlichen Sorgehandelns - so die mit der ersten und zweiten eng verbundene dritte Konsequenz, die die Ergebnisse vorsichtig zulassen - werden wesentlich durch institutionelle Vorgaben bestimmt. Das Zahlungsverhalten kann eher durch staatliche Intervention gefördert, gar forciert werden als z.B. das sozio-emotionale Engagement. Kümmert sich ein Vater nicht um seine getrennt lebenden Kinder, hat der 
Wohlfahrtsstaat kaum Möglichkeiten dieses Verhalten zu unterbinden. Ein Vater kann nicht gezwungen, sondern nur ermutigt werden ein hohes sozio-emotionales Engagement zu zeigen.

Die These der besonderen Bedeutung institutioneller Vorgaben wird gestützt durch die vierte Folgerung aus den vorliegenden Ergebnissen: Auch die nationalen Unterschiede in den Determinanten weisen darauf hin, dass die Zahlungspraxis von Nachtrennungsvätern durch Besonderheiten im nationalen Kontext (mit)bestimmt werden. Insbesondere die Ergebnisse der britischen Studie setzen sich hinsichtlich der Cash-Komponente von den beiden anderen Datensätzen ab. In Großbritannien wirken vermehrt auch Prädiktoren aus der sozialen Umwelt auf das väterliche Unterhaltshandeln, während diese Effekte in Deutschland und Norwegen nicht bzw. kaum Einfluss zeigen. Die Ergebnisse weisen einerseits darauf hin, dass in Deutschland und Norwegen das väterliche Engagement in Form von Care durch Determinanten sowohl der physischen als auch der sozialen Umwelt und aus dem inneren Überzeugungssystem bestimmt werden, während andererseits die Zahlungsprobleme v.a. durch Komponenten der physischen Umwelt determiniert werden. Weitere Determinanten könnten in den institutionellen Rahmenbedingungen gesucht werden. In Großbritannien zeigen sich im Vergleich dazu ähnliche Ergebnisse für Care, Cash wird im britischen Kontext zusätzlich jedoch durch die sozialen Beziehungen im Nahumfeld determiniert. Wenn die Annahme stimmt, dass das väterliche Cash außerdem durch institutionelle Determinanten erklärt werden muss - die hier aufgrund von fehlenden Informationen in den Datensätzen nicht aufgenommen werden können -, wird auch die Sonderstellung des britischen Kontextes anschaulich. Wie in Kap. 6.2. Der deutsche, britische und norwegische makro-soziale Kontext ausführlich dargestellt, zeichnet sich die britische Familienpolitik durch ein hohes Maß an Nicht-Intervention innerhalb der familialen Privatsphäre aus. Das bedeutet, dass die Eltern in der Regelung ihrer Trennung weitestgehend auf eigenverantwortliche Aushandlungen angewiesen sind. Lediglich in Fällen, in denen die Mutter staatliche Leistungen beantragt, greift der Staat ein. Dies erklärt, warum die Zahlungspraxis unter britischen Vätern stärker vom direkten sozialen Umfeld, insbesondere vom Verhältnis zur Kindsmutter, abhängt als z.B. in Norwegen und Deutschland, wo die Regelung des Unterhalts stark standardisiert und institutionalisiert ist.

Die Berechnungen ergeben andere Determinanten - in Zahl und Wirkung - für Care und Cash. Zusammen mit dem in Norwegen nachgewiesenen Einfluss des festgelegten Umgangs auf das Zahlungsverhalten der Nachtrennungsväter lassen die Ergebnisse die Frage zum Verhältnis der beiden Dimensionen väterlichen Sorgehandelns aufkommen. Im folgenden Kapitel wird die Beziehung zwischen den beiden abhängigen Variablen genauer betrachtet. 


\subsection{Väterliches Sorgehandeln: Das Verhältnis von Care und Cash}

Bisher folgten die Ausführungen und Analysen theoretisch begründet einer strikten Differenzierung zwischen Care und Cash. Im theoretischen Teil wurde darauf verwiesen, dass Väter verschiedene Handlungsalternativen haben, in denen Care und Cash in unterschiedlicher Weise kombiniert werden können. Der folgende Abschnitt nähert sich dem Verhältnis der beiden Dimensionen auf verschiedene Weise. Zunächst werden die einzelnen Determinanten, die Care im Vergleich zu Cash bestimmen, gegenübergestellt. In einem zweiten Schritt wird dann die Relation der beiden Zielvariablen zueinander betrachtet. Es wird der Frage nachgegangen, ob ein statistischer Zusammenhang zwischen den beiden Kriterien auf signifikantem Niveau besteht. Darauf folgt die Typisierung des väterlichen Sorgehandelns in vier Formen anhand der Dichotomisierung und Kreuztabellierung der beiden abhängigen Variablen.

In Tabelle 9.40 werden die Einflussgrößen und ihre Relevanz für die Erklärung je nach Kriterium gegenübergestellt. ${ }^{220}$ Der Vergleich der Determinanten, die jeweils auf das sozio-emotionale bzw. finanzielle Unterstützungshandeln des Vaters wirken, zeigt zum einen, dass die Cash-Variable durch weniger der vorliegenden Faktoren beeinflusst wird als die Care-Variable. Zum anderen wird deutlich, dass es einige Schnittstellen zwischen den Faktoren gibt.

Innerhalb des deutschen Datensatzes bestimmen alle Determinanten der Cash-Variablen auch die Care-Komponente. Dabei handelt es sich aber nur um zwei Einflussgrößen: das Einkommen sowie die Festlegung des Unterhalts. Alle anderen Erklärungsfaktoren, die auf das sozio-emotionale Handeln des Vaters wirken, haben für sein finanzielles Unterstützungshandeln keine Bedeutung.

Die britischen Ergebnisse weisen in eine ähnliche Richtung. Auch hier haben weniger Variablen Einfluss auf beide Kriterien, als es Determinanten mit unterschiedlicher Bedeutung gibt. Zu den Erklärungsfaktoren beider Dimensionen zählen das Bildungsniveau des Vaters und das freundschaftliche Verhältnis zwischen den Eltern. Damit wird die besondere Bedeutung der elterlichen Beziehung zum Zeitpunkt der Befragung einmal mehr herausgestellt. In Großbritannien scheint ein gutes Vater-Kind-Verhältnis nach der Trennung - finanziell wie sozio-emotional - bedingt durch ein gutes Verhältnis zur Ex-Partnerin zu sein. Gleiche Ergebnisse für die Zusammenhänge mit den beiden zu erklärenden

${ }^{220}$ An dieser Stelle ist darauf zu verweisen, dass in der Tabelle nicht die Bestätigung der Hypothesen betrachtet wird, sondern überprüft wird, welche Determinanten Care im Vergleich zu Cash bestimmen. 
Variablen ergeben sich in Großbritannien darüber hinaus für die Variablen der externen Kinderzahl, der elterlichen Beziehungsform, der finanziellen Situation der Mutter sowie der Dauer seit der Trennung. Alle genannten Variablen haben weder Einfluss auf die Ausgestaltung von Care noch auf die von Cash. Anders verhält es sich mit den Determinanten, die die Distanz zwischen den elterlichen Wohnsitzen, das Einkommen, die Arbeitszeit, die Anzahl Kinder im Haushalt des Vaters, die aktuelle Partnersituation des Vaters und der Mutter, die Beziehungsdauer, die Unterhaltsfestlegung und die Anzahl der Elternschaften angeben. Diese weisen jeweils unterschiedliche Effekte auf Care im Vergleich zu Cash auf, was z.T. den jeweils aufgestellten Hypothesen entspricht.

Tab.: 9.40: Relevante Determinanten für Care und Cash im Vergleich nach Datensätzen.

\begin{tabular}{|c|c|c|c|c|c|c|}
\hline & \multicolumn{2}{|c|}{ Forsa } & \multicolumn{2}{|c|}{ Bradshaw } & \multicolumn{2}{|c|}{ NOVA } \\
\hline Physische Umwelt & Care & Cash & Care & Cash & Care & Cash \\
\hline Distanz & $\checkmark$ & $x$ & $\checkmark$ & $x$ & $\checkmark$ & $x$ \\
\hline Einkommen & $\checkmark$ & $\checkmark$ & $x$ & $\checkmark$ & $x$ & $\checkmark$ \\
\hline Bildungsniveau & $x$ & $x$ & $\checkmark$ & $\checkmark$ & $\checkmark$ & $\checkmark$ \\
\hline Arbeitszeit & --- & --- & $x$ & $\checkmark$ & $\checkmark$ & $x$ \\
\hline Soziale Umwelt & Care & Cash & Care & Cash & Care & Cash \\
\hline Kinder im Haushalt & $x$ & $x$ & $x$ & $\checkmark$ & --- & --- \\
\hline Externe Kinderzahl & $\checkmark$ & $x$ & $x$ & $x$ & $\checkmark$ & $\checkmark$ \\
\hline Partnersit. des Vaters & $\checkmark$ & $x$ & $x$ & $\checkmark$ & $\checkmark$ & $x$ \\
\hline Partnersit. der Mutter & $\checkmark$ & $x$ & $\checkmark$ & $x$ & $x$ & $x$ \\
\hline Elterl. Beziehungsform & $\checkmark$ & $x$ & $x$ & $x$ & $x$ & $x$ \\
\hline Beziehungsdauer & --- & --- & $\checkmark$ & $x$ & $x$ & $\checkmark$ \\
\hline Unterhaltsfestlegung & $\checkmark$ & $\checkmark$ & $\checkmark$ & $x$ & $x$ & $x$ \\
\hline Festl. Sorgerecht/Umgang & $\checkmark$ & $x$ & --- & --- & $x$ & $x$ \\
\hline Sorgerecht/Umgang & $\checkmark$ & $x$ & --- & --- & $\checkmark$ & $\checkmark$ \\
\hline Freundschaft & --- & --- & $\checkmark$ & $\checkmark$ & $\checkmark$ & $x$ \\
\hline Indiv. Überzeugung & Care & Cash & Care & Cash & Care & Cash \\
\hline Finanzen d. Mutter & --- & --- & $x$ & $x$ & $x$ & $x$ \\
\hline Dauer seit Trennung & $\checkmark$ & $x$ & $x$ & $x$ & $x$ & $\checkmark$ \\
\hline Anzahl Elternschaften & $\checkmark$ & $x$ & $\checkmark$ & $x$ & $x$ & $x$ \\
\hline
\end{tabular}

Quelle: eigene Darstellung. 
Die norwegischen Daten geben Hinweise darauf, dass sozio-emotionales und finanzielles Unterstützungshandeln von Nachtrennungsvätern durch mehr gemeinsame Faktoren bestimmt werden, als sie Unterschiede aufweisen. So können Zusammenhänge sowohl mit der Care- als auch der Cash-Variable für die Determinanten der Bildung, der Anzahl getrennt lebender Kinder und der Form des festgelegten Umgangs ausgemacht werden. Nicht von Bedeutung für die Erklärung der Ausprägungen beider abhängiger Variablen sind die Partnersituation der Mutter, die Ausgestaltung der elterlichen Beziehung, die Unterhaltssowie Umgangsfestlegung, die finanzielle Situation der Mutter und die Anzahl der getrennten Beziehungen, aus denen Kinder hervorgegangen sind. All diese Variablen zeigen keinen signifikanten Einfluss auf die Kriterien. Die Distanz zwischen den elterlichen Wohnorten, die Arbeitszeit, die Tatsache, dass der Vater allein lebt, die Dauer der elterlichen Beziehung, die ökonomische Situation der Mutter, das freundschaftliche Verhältnis zwischen den Eltern nach der Trennung sowie die zeitliche Distanz zum Scheitern der elterlichen Beziehung wirken sich demgegenüber alle unterschiedlich auf die beiden abhängigen Variablen aus. Die aufgeführten Determinanten zeigen entweder Effekte auf Care oder auf Cash, nicht jedoch auf beide.

Insgesamt werden die beiden abhängigen Variablen zu einem großen Teil von unterschiedlichen Determinanten bestimmt; sie weisen jedoch auch gemeinsame Einflussgrößen auf. Im Folgenden wird die Perspektive auf die statistische Relation zwischen den beiden Dimensionen väterlichen Sorgehandelns gelenkt. Tabelle 9.41 bildet die Koeffizienten der einfachen Regressionen zwischen den abhängigen Variablen mit Care als Kriteriumsvariable ab.

Tab.: 9.41: Regressionskoeffizienten für jeweils Care und Cash als abhängige Variable.

\begin{tabular}{|l|c|c|c|c|}
\hline & & Forsa & Bradshaw & NOVA \\
\hline Care als Kriterium & $\boldsymbol{\beta}$ (Sign.) & $-0,116(0,001)$ & $-0,288(0,000)$ & $-0,059(0,171)$ \\
\hline
\end{tabular}

Quelle: eigene Berechnungen.

Es zeigt sich, dass in Großbritannien ein deutlicher Zusammenhang zwischen der sozioemotionalen und finanziellen Handlungspraxis des Vaters besteht. Auch in Deutschland ist ein Effekt der Zahlungspraxis auf die Care-Variable nachzuweisen, der jedoch etwas schwächer ausgeprägt ist. Beide Koeffizienten weisen auf einen negativen Zusammenhang hin. Das bedeutet, dass Väter, die ihren Zahlungsverpflichtungen nicht oder nicht vollständig nachkommen, deutlich häufiger ein geringeres Niveau sozio-emotionalen Engagements aufweisen als Väter, die regelmäßig Unterhaltszahlungen leisten. In Norwegen besteht dagegen kein signifikanter Einfluss der Cash- auf die Care-Variable. Letzteres ist auch insofern erstaunlich, weil die Form des festgelegten Umgangs auf die Zahlungs- 
moral norwegischer Trennungsväter wirkt. Die zunächst getroffene Annahme, dass dies auf einen Zusammenhang zwischen Care und Cash hinweist, findet hier keine Bestätigung. Damit ist der Einfluss der Variablen der Umgangsform weniger ein Ausdruck für den Zusammenhang von sozio-emotionalen und finanziellen Aspekten väterlichen Sorgehandelns, sondern bildet etwas anderes, wie z.B. das Verhältnis der Eltern, ab. Viele der norwegischen Väter geben an, keine formale Vereinbarung mit der Kindsmutter getroffen zu haben, was auf eine relative Einigkeit schließen lässt. Somit drückt sich in der Variablen die Konfliktstärke in der Festlegung aus. Der Effekt kann daher durch die steigende Uneinigkeit der Eltern entstanden sein und weniger durch das sinkende Umgangsrecht.

Es stellt sich die Frage, warum in Norwegen kein Zusammenhang zwischen Care und Cash besteht, während dies in Deutschland und Großbritannien der Fall ist. Ein Erklärungsansatz ist vielleicht in der bereits erwähnten starken Standardisierung finanzieller Regelungen nach der elterlichen Trennung zu sehen. Norwegische Eltern nutzen mehrheitlich freiwillig eine externe Instanz, die Unterhaltszahlungen zwischen den Eltern transferiert und zudem ausbleibende Zahlungen ausgleicht. Insgesamt müssten sich damit Streitigkeiten zwischen den getrennt lebenden Eltern abmildern. Sie wirken sich nicht so stark auf die sozio-emotionale Beziehung aller Beteiligten aus. Dies könnte eine Erklärung für den fehlenden Zusammenhang darstellen.

In Großbritannien dagegen basiert die Nutzung der Unterhaltsbehörde weniger auf Freiwilligkeit. Wie dargestellt sind britische Mütter, die Sozialleistungen beanspruchen wollen, zum Zeitpunkt der Erhebung verpflichtet, den Namen des Kindsvaters zu nennen. Die Behörde relationiert die beiden Parteien als „Gegner“ und weniger als „Partner“ in der gemeinsamen Nachtrennungsregelung zum Wohle des Kindes, insbesondere mit Blick auf Unterhaltsleistungen. Einige unterhaltsberechtigte Mütter verzichten gar auf Sozialhilfeansprüche, um damit die fragile Beziehung zwischen ihren Kindern und den dazu gehörigen Vätern nicht zu gefährden. ${ }^{221}$ Ohne die Inanspruchnahme der externen Instanz der CSA sind die Eltern bei der Regelung der Unterhaltsleistungen weitestgehend auf sich selbst gestellt geblieben. Sowohl die Aushandlung der Höhe als auch die tatsächlichen Transfers werden primär eigenverantwortlich geregelt. Es wäre denkbar, dass darin ein gewisses Konfliktpotential besteht, das sich negativ auf das sozio-emotionale Sorgehandeln auswirkt. Ausbleibenden Unterhaltszahlungen kann mit verwehrtem Zugang zum Kind begegnet werden. Diese Reaktion ist auch im umgekehrten Fall denkbar: Verwehrter Umgang resultiert in ausbleibenden Unterhaltszahlungen. Andersherum können geleistete

${ }^{221}$ Siehe dazu ausführlich Kap. 6.2.2 Gesetzliche Nachtrennungsregelungen. 
Zahlungen zu einer gewissen Anspruchshaltung beim Trennungsvater führen, ein stärkeres Recht auf Umgang zu haben. Bradshaw und Mitarbeiter (1999: 227) vermuten, dass für die getrennt lebenden Väter ein 'trade-off' zwischen Umgang mit dem Kind und Unterhaltszahlung bestehen muss - viele Väter sehen nicht ein, weshalb sie Unterhalt zahlen sollen, wenn sie keinen (einfachen) Zugang zum Kind haben können. Insgesamt können finanzielle Transfers zu einem Druckmittel zwischen den Eltern werden. Dies gilt möglicherweise verstärkt in Kontexten, in denen getrennt lebende Eltern in der Regelung gegenseitiger finanzieller Unterstützung auf sich gestellt sind. ${ }^{222}$

Im Theoriekapitel wurde bereits auf die verschiedenen Handlungsalternativen der Nachtrennungsväter verwiesen. Dabei wird von einem durch die beiden Dimensionen Care und Cash aufgespannten Raum ausgegangen, in dem sich die Väter durch ihr Sorgehandeln in einer Kombinationsmöglichkeit theoretisch als Punkt positionieren. Zur Analyse des Verhältnisses zwischen Care und Cash wird abschließend eine Typologie väterlichen Sorgehandelns entwickelt. Dazu wird die kategorisierte Care-Variable dichotomisiert, indem jeweils die letzten bzw. ersten drei Ausprägungen in den Differenzierungen „kein/kaum Care“ bzw. „regelmäßiges/intensives Care“ zusammengefasst werden. Die durch eine Vier-Felder-Tafel aufgespannten Zellen der dichotomen Care- und Cash-Variablen werden als Typen väterlichen Sorgehandelns dargestellt und in ihren jeweiligen Häufigkeiten untersucht. ${ }^{223}$ Dabei wird differenziert zwischen Vätern, die weder sozioemotionales noch finanzielles Unterstützungshandeln zeigen, Vätern, die sich über finanzielle Transfers hinaus kaum bzw. gar nicht um ihre Kinder nach der elterlichen Trennung kümmern, Vätern, die nur sozio-emotionales Sorgehandeln aufweisen, jedoch keinen Unterhalt zahlen und Vätern, die ihren finanziellen und sozio-emotionalen Pflichten gegenüber ihren getrennt lebenden Kindern nachkommen. Tabelle 9.42 stellt die Häufigkeiten der einzelnen Vätergruppen in absoluten Zahlen und gültigen Prozenten nach Datensätzen gegenüber.

\footnotetext{
${ }^{222}$ Finanzielle Transfers zwischen getrennten Elternteilen stehen ferner im engen Zusammenhang mit der symbolischen Bedeutung von Geld und von monetären Unterstützungsleistungen zwischen Familienmitgliedern (Funder 1993b: 191). Hier wäre an unterschiedlich wahrgenommene Abhängigkeiten zu denken. Auch dies könnte nationale Unterschiede begründen. So bestehen in Norwegen beispielsweise kaum Unterhaltsverpflichtungen zwischen geschiedenen Partnern, während in Deutschland lange auch der getrennten Ehefrau Leistungsansprüche zukommen.

${ }^{223}$ Die vier Kombinationsmöglichkeiten repräsentieren die vier Ecken innerhalb der Matrix in Abb. 4.2 (vgl. Kap. 4.3.1.1 Sorgehandeln als Entscheidung zwischen Alternativen).
} 
Tab.: 9.42: Häufigkeitsverteilung der Typologie väterlichen Sorgehandelns nach Datensätzen getrennt.

\begin{tabular}{|l|r|r|r|r|r|r|}
\hline & \multicolumn{2}{|c|}{ Forsa } & \multicolumn{2}{c|}{ Bradshaw } & \multicolumn{2}{c|}{ NOVA } \\
\cline { 2 - 7 } & Häufigk. & gültige $\%$ & Häufigk. & gültige \% & Häufigk. & gültige \% \\
\hline kein Sorgehandeln & 43 & $5,07 \%$ & 158 & $27,72 \%$ & 44 & $8,13 \%$ \\
\hline nur Cash & 148 & $17,45 \%$ & 100 & $17,54 \%$ & 87 & $16,08 \%$ \\
\hline nur Care & 106 & $12,50 \%$ & 95 & $16,67 \%$ & 106 & $19,59 \%$ \\
\hline Cash\&Care & 551 & $64,98 \%$ & 217 & $38,07 \%$ & 304 & $56,19 \%$ \\
\hline Gesamt & $\mathbf{8 4 8}$ & $\mathbf{1 0 0} \%$ & $\mathbf{5 7 0}$ & $\mathbf{1 0 0} \%$ & $\mathbf{5 4 1}$ & $\mathbf{1 0 0 \%}$ \\
\hline
\end{tabular}

Quelle: eigene Berechnung.

Die gültigen Prozente der einzelnen Datensätze lassen sich anschaulich, wie in Abbildung 9.1 dargestellt, in einem Balkendiagramm zusammenfassen.

Die Häufigkeitsverteilung der einzelnen Kombinationsmöglichkeiten von Care und Cash, die hier zu vier Typen zusammengefasst werden, zeigt verschiedene Auffälligkeiten. Zunächst wird deutlich, dass sich in allen drei Ländern eine Mehrheit der befragten Nachtrennungsväter sowohl sozio-emotional wie auch finanziell um ihre Kinder kümmert. Im Gegensatz dazu gibt nur eine Minderheit der Väter an, den Kontakt zur getrennt lebenden Familie gänzlich, d.h. in Form von Besuchen wie auch Zahlungen, eingestellt zu haben. ${ }^{224}$ Die beiden Ausprägungen zeigen jedoch auch, dass die Väter in der Bradshaw-Studie deutlich seltener beide Dimensionen väterlichen Sorgehandelns ausfüllen und sich gleichzeitig häufiger gar nicht kümmern. In den beiden Ausprägungen, in denen der Vater sich entweder nur sozio-emotional oder nur finanziell engagiert, sind in allen drei Ländern relativ ähnliche Anteile zu finden.

${ }^{224}$ Dies kann u.a. damit erklärt werden, dass sich Väter, die sich gar nicht kümmern, aus Gründen sozialer Erwünschtheit entweder insgesamt seltener auf die Teilnahme an Untersuchungen, wie die drei vorliegenden, einlassen. Arme und nicht zahlende Väter verweigern dabei häufiger die Auskunft (Harrison 1993: 127). Ferner besteht die Gefahr, dass Trennungsväter ihre Antworten „schönen“, d.h. (un-)wissentlich ein höheres Engagement angeben als sie tatsächlich aufweisen. Vergleiche mit den Angaben unterhaltsberechtigter, anwesender Mütter ergeben, dass Mütter dazu tendieren, die Bemühungen der Trennungsväter in ihren Antworten zu schmälern, während Väter eher „,übertreiben“ (siehe dazu u.a. Forsa 2002; Bradshaw 1999). Das Niveau des „wahren“ Sorgehandelns liegt irgendwo dazwischen. 
Abb.9.1: Graphische Häufigkeitsverteilung der Typologie väterlichen Sorgehandelns nach Datensätzen getrennt.

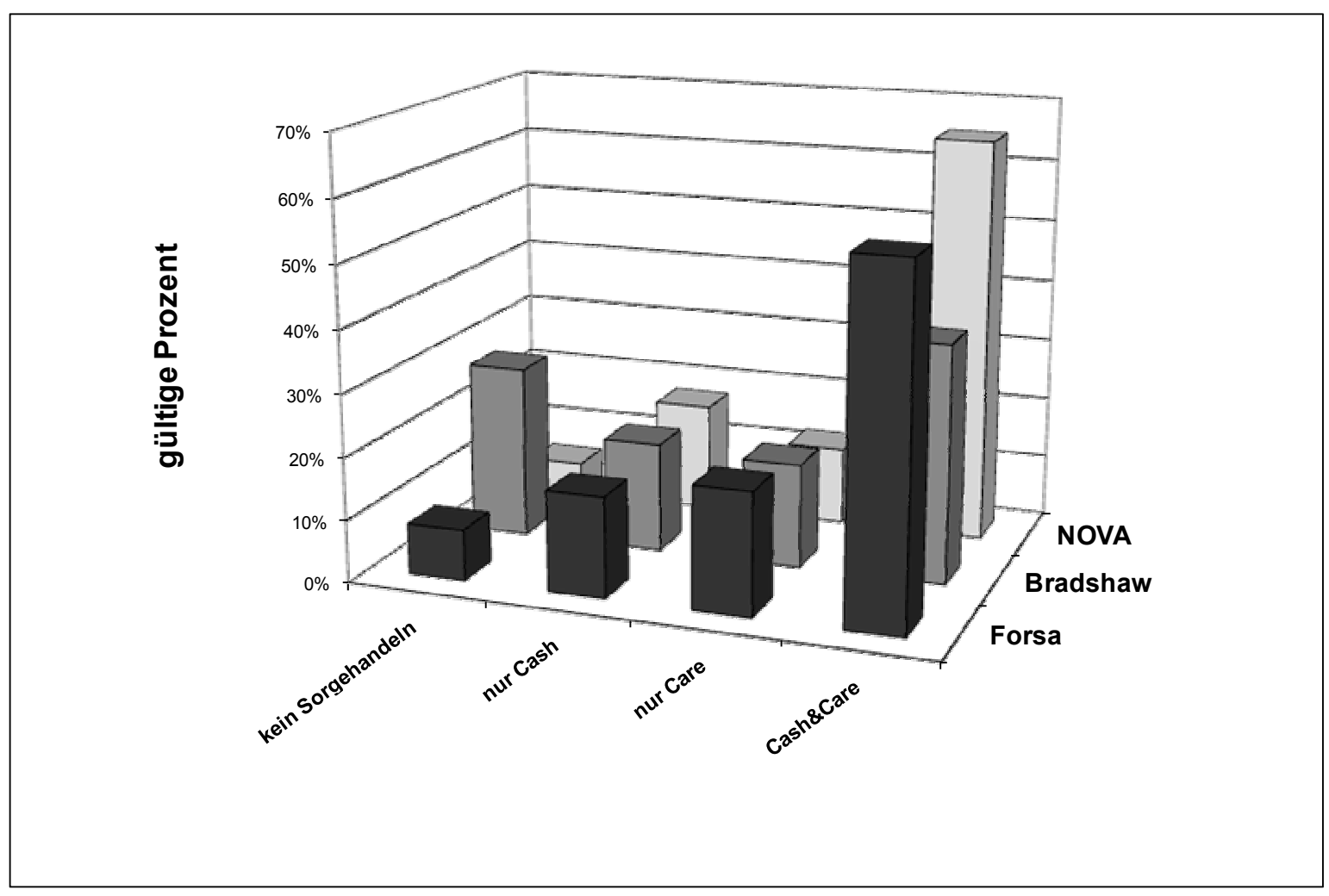

Quelle: eigene Darstellung.

Zum Teil werden widersprüchliche oder national unterschiedliche Resultate mit Unzulänglichkeiten in den Daten begründet. Im Anschluss an die Darstellung der empirischen Analyse werden die wesentlichen Ergebnisse im Folgenden zusammengefasst und abschließend kritisch reflektiert. 


\section{TEIL IV: ABSCHLIESSENDE BETRACHTUNG}

\section{Was bestimmt väterliches Sorgehandeln? - Die Ergebnisse im Überblick}

Sozio-emotionales wie auch finanzielles Sorgehandeln von Nachtrennungsvätern wird durch unterschiedliche Faktoren der sozialen und physischen Umwelt sowie Determinanten des individuellen Überzeugungssystems bestimmt. Die Annäherung zur Erklärung des sozio-emotionalen Engagements des Vaters auf bivariater Ebene deutet zunächst an, dass die meisten der theoretischen Annahmen empirisch Bestätigung finden. Die multiple Regression modifiziert diesen Eindruck jedoch. Zwar bestätigen sich einige der erwarteten Zusammenhänge, andere Thesen müssen jedoch als widerlegt betrachtet werden und viele Annahmen weisen länderspezifische Unterstützung bzw. Widerlegung auf. Konkret hat die Analyse der Care-Dimension die Hypothesen zum Einfluss der Entfernung zwischen den Wohnorten und der Form des Sorgerechts bzw. des Umgangs, den die Eltern vereinbart haben, in allen Untersuchungen bestätigt. Ebenso hat sich das freundschaftliche Verhältnis der Eltern als relevant erwiesen. Dabei kommt ihm in beiden Studien, in denen es Angaben dazu gibt, eine herausragende Bedeutung zu. Väter kümmern sich nach der elterlichen Trennung eher um ihre Kinder, wenn den Eltern es gelingt, eine freundschaftliche Beziehung aufrechtzuerhalten bzw. aufzubauen. Auch den Variablen der Arbeitszeit und der Anzahl der getrennt lebenden Kinder kommt, wie erwartet, Erklärungskraft in den Regressionsmodellen zu. Doch beide wirken in entgegengesetzter Richtung als theoretisch angenommen. Mit steigender Arbeitszeit sowie mit zunehmender Zahl unterhaltsberechtigter Kinder außerhalb des väterlichen Haushalts nimmt auch die Intensität des väterlichen Engagements zu.

Alle anderen Determinanten, die in der Analyse berücksichtigt werden, führen zu länderspezifischen Ergebnissen, d.h. die Hypothesen werden in einigen Ländern bestätigt, während sie gleichzeitig in anderen keine empirische Unterstützung erfahren. So weisen die Ergebnisse in Deutschland darauf hin, dass das väterliche Engagement in Form von Care stark von der Form und dem Ergebnis der Trennungsregelung abhängt. Hier spielen insbesondere die Festlegung von Unterhalt und Sorgerecht sowie die Form der rechtlich vereinbarten Sorge eine Rolle. Hinzu kommt die bereits erwähnte Entfernung zwischen den Wohnorten. In Großbritannien bestimmt v.a. das freundschaftliche Verhältnis zwischen den Eltern das väterliche Sorgehandeln nach der Trennung. Darüber hinaus wirkt sich auch die aktuelle Beziehungssituation der Mutter aus. Beides, die Freundschaft der Eltern und ein neuer Partner im Leben der Mutter, sind dabei vermutlich nicht unabhängig voneinander. Ferner spielt auch in Großbritannien die Entfernung zwischen den Wohnorten 
eine Rolle. In Norwegen wird die Care-Variable, wie in Deutschland, durch die Form des festgelegten Umgangs bestimmt. Wie in Großbritannien wirkt sich ferner das freundschaftliche Verhältnis zwischen den Eltern aus. ${ }^{225}$ Der Entfernung kommt dagegen eine geringere Bedeutung zu.

Insgesamt zeigen alle nationalen Regressionsmodelle eine Bedeutung der berücksichtigten Determinanten aus der physischen Umwelt. Dabei finden sich in den Ergebnissen jedoch unterschiedliche Schwerpunkte auf der Ausbildung, dem Einkommen und/oder der Arbeitszeit des Vaters. Sicher sind diese Faktoren inhaltlich nicht identisch, aber dennoch verbunden. Des Weiteren spielt in den drei Untersuchungen einheitlich die Beziehung zwischen den Eltern eine Rolle. Dabei werden nationale Differenzen in der Bedeutung des elterlichen Verhältnisses zu unterschiedlichen Zeitpunkten - vor, während und nach der Trennung - deutlich. In Norwegen erklärt, wie dargestellt, die elterliche Beziehung im Zeitpunkt der Befragung das väterliche Engagement. In Großbritannien ist darüber hinaus und in Deutschland mangels an Daten ausschließlich die Beziehung zwischen den Eltern vor und während der Trennung relevant.

Mit Blick auf das finanzielle Unterstützungshandeln der Nachtrennungsväter deuten bereits die bivariaten Ergebnisse auf eine hohe Heterogenität in den jeweiligen nationalen Erklärungen hin. In Deutschland sind im Wesentlichen zwei Determinanten relevant: das väterliche Einkommen sowie die Festlegung des Unterhalts. In Großbritannien und Norwegen zeigen sich neben anderen Faktoren der physischen Umwelt, die die Ressourcen des Vaters mitbestimmen, auch Einflussgrößen der sozialen Umwelt von Bedeutung. In Norwegen zählen dazu die Form des festgelegten Umgangs, die Dauer der Beziehung zur Kindsmutter sowie die zeitliche Distanz zur Trennung, die die Auftretenswahrscheinlichkeit von Zahlungsschwierigkeiten beeinflussen. In Großbritannien dagegen sind es das freundschaftliche Verhältnis zur Kindsmutter sowie die neue Familiensituation des Vaters, die sein Zahlungsverhalten erklären.

Von den theoretischen Annahmen zu den Determinanten, die die Unterhaltsschwierigkeiten erklären sollten, haben sich nur wenige bestätigt. So gilt als empirisch abgesichert, dass mit steigendem Einkommen seltener Probleme mit den Unterhaltszahlungen auftreten. Ferner konnte belegt werden, dass die räumliche Distanz zwischen den Eltern sowie

\footnotetext{
${ }^{225}$ Interessant wäre an dieser Stelle zu untersuchen, ob sich andere Aushandlungsergebnisse einstellen, wenn der Prozess der Übereinkunft „staatlich überwacht“ wird. Einigen sich britische Eltern auf weniger hohe Unterhaltszahlungen, wenn sich keine dritte Instanz einschaltet? Verweigern norwegische Mütter eher den Kontakt, wenn staatliche Institutionen sie nicht daran hindern?
} 
die Festlegung des Sorgerechts ohne signifikante Bedeutung für die Zahlungspraxis sind, wie dies auch theoretisch begründet wurde. Insgesamt können so für die Cash-Dimension weniger Thesen bestätigt werden als für die Care-Komponente.

Zusammenfassend führt die Untersuchung zu zwei zentralen Ergebnissen: zum einen lassen sich die theoretischen Annahmen nur bedingt am empirischen Material bestätigen. Die Theorie zur Erklärung väterlichen Sorgehandelns insbesondere für die finanzielle Unterstützungspraxis in Nachtrennungsfamilien hat sich nur begrenzt empirisch bewährt. Nur wenige Annahmen haben in allen Untersuchungen Unterstützung gefunden, aber auch nur wenige wurden gänzlich widerlegt. Dies weist auf die zweite wesentliche Schlussfolgerung der vorliegenden Ergebnisse hin: Die einzelnen Länder zeigen sehr unterschiedliche Erklärungsmuster väterlichen Sorgehandelns insbesondere für das finanzielle Unterstützungshandeln in Nachtrennungsfamilien. Es stellt sich die Frage, was die Ergebnisse für die aufgestellte Theorie bedeuten und wie sie methodisch zu bewerten sind. Damit setzt sich das folgende Kapitel kritisch auseinander. 


\section{Einordnung der Ergebnisse: Eine kritische Würdigung}

Gemäß der ausgemachten Desiderate der (Nachtrennungs-)Väter-Forschung in den Sozialwissenschaften hat die vorliegende Untersuchung explizit die Perspektive der individuellen Väter eingenommen. Orientiert an der forschungsleitenden Fragestellung, was väterliches Sorgehandeln in Nachtrennungsfamilien bestimmt, und basierend auf der bisherigen Vernachlässigung theoretisch fundierter Annahmen zur Benennung der Determinanten basiert die vorliegende Analyse auf der empirischen Thesentestung. Ziel war es, einen theoretischen wie (quantitativ) empirischen Beitrag zur Erklärung väterlichen Sorgehandelns in Nachtrennungsfamilien zu leisten. Im Folgenden werden die theoretischen wie empirischen Konsequenzen aus den vorliegenden Ergebnissen gezogen. Dazu werden zunächst die theoretischen Erweiterungen spezifiziert und in einem zweiten Schritt wird auf die Daten und ihre Güte kritisch eingegangen.

\subsection{Theoretische Implikationen der Ergebnisse}

Die empirischen Resultate weisen auf länderspezifische Unterschiede hin, die zunächst theoretisch so nicht erwartet wurden. Es wurde davon ausgegangen, dass die aufgestellte Theorie Raum und zeitlich ungebunden in den drei Ländern gleichermaßen Gültigkeit besitzt. Sie diente dabei als Instrument und erster Anhaltspunkt zur Auswahl von Einflussgrößen aus der Vielzahl von möglichen und in den verschiedenen Datensätzen erhobenen Determinanten. Zur Strukturierung erwiesen sich die theoretischen Annahmen als sehr hilfreich.

Die Ergebnisse zeigen, dass sich einige der Hypothesen am empirischen Material bewährt haben, während andere nur in einigen Datensätzen Gültigkeit besitzen und wieder andere widerlegt werden konnten. Es fällt z.B. auf, dass sich für die Care-Dimension im deutschen Fall fast alle aufgestellten theoretischen Annahmen sowohl auf bivariater wie auch auf multipler Ebene empirisch stützen lassen. Dies legt die Vermutung nahe, dass die theoretischen Annahmen aus einer eher „deutschen“ Perspektive gelenkt worden sind. Die Determinanten weisen in den verschiedenen Kontexten unterschiedliche Wirkungen auf. Die Resultate geben an, welche Determinanten in den jeweiligen makro-sozialen Zusammenhängen wirken, jedoch tragen sie nur marginal zur Erklärung der Länderunterschiede bei. Dies spricht jedoch aus verschiedenen Gründen nicht generell gegen die aufgestellte Theorie, sondern eher für eine Erweiterung des theoretischen Modells sowie der empirischen Untersuchung. Die Ausweitung bezieht sich im Wesentlichen auf die zu berücksichtigenden Determinanten. Es müssen Lücken in den einzelnen Datensätzen geschlossen, neue Variablen aus dem individuellen Nahumfeld zusätzlich aufgenommen und der makro-soziale Kontext stärker berücksichtigt werden. 
Es wurde theoretisch angenommen, dass väterliches Sorgehandeln primär mit Faktoren aus dem unmittelbaren Umfeld des Vaters zu erklären ist, welches durch den makro-sozialen Kontext vorstrukturiert wird. Die makro-soziale Dimension wirkt sich jedoch nicht allein auf die soziale Situation aus, in die die Handlung eingebettet ist, sondern bestimmt ferner den Akteur selbst in seiner individuellen Überzeugung, seiner Wahrnehmung und Handlungsauswahl mit. Auf die Bedeutung familialer Normen für das väterliche Sorgehandeln wurde ausführlich eingegangen. ${ }^{226}$ Auf empirischer Ebene konnten diese jedoch aufgrund der jeweiligen Datenlage nicht direkt integriert werden. So bleibt zunächst offen, welche Erwartungen an den Nachtrennungsvater aus seiner sozialen Umwelt herangetragen werden; ebenso wie unklar bleibt, welche Erwartungen der Vater selbst an sein Handeln stellt. Letzteres wird im Modell mit Hilfe des inneren Überzeugungssystems theoretisch erfasst, welches durch nationale Kontexte mitbestimmt wird. So zeigen die Ausführungen zu den nationalen Rahmenbedingungen unterschiedliche Vaterleitbilder, die im jeweiligen politischen und öffentlichen Kontext diskutiert und institutionalisiert werden. ${ }^{227}$ Wie diese sich in den Erwartungen sozialer Akteure widerspiegeln, muss anhand der vorliegenden Datensätze ungeprüft bleiben. Hier könnte jedoch ein - wenn nicht der - (theoretische) Schlüssel zur Erklärung nationaler Unterschiede in den empirischen Modellen liegen. Es kann, wie dargestellt, argumentiert werden, dass sich norwegische Väter aufgrund der stark ausgeprägten Betonung der geschlechtsspezifischen Gleichstellung eher mit Erwartungen zur Betreuungs- und Erziehungsbeteiligung konfrontiert sehen. Finanzielle Unterstützungsleistungen können von untergeordneter Bedeutung sein, weil Mütter in den Arbeitsmarkt integriert sind und damit den Lebensunterhalt ihrer Kinder mit eigener Erwerbstätigkeit sichern (sollen) - dies gilt verstärkt auch für Alleinerziehende. Erwerbstätige Mütter sind somit (idealerweise) eher auf Betreuungsunterstützung der getrennt lebenden Väter angewiesen und weniger auf ihre finanziellen Transfers. In Deutschland und insbesondere Großbritannien können dagegen stärker Unterhaltsstatt Betreuungsansprüche an die Trennungsväter gestellt werden - wie dies auf makrosozialer Ebene der Fall ist. Es wird erwartet, dass sie sich zumindest finanziell nach der elterlichen Trennung an den „Kosten“ der Kinder beteiligen. Dies wird gestützt durch die jeweiligen Ernährermodelle, wie sie in der deutschen und britischen Gesellschaft im Erhebungszeitraum zu finden sind.

Die theoretischen Annahmen bleiben rein spekulativ, weil sie anhand der vorliegenden Datensätze nicht geprüft werden können. Des Weiteren tragen sie lediglich zur Erklärung

\footnotetext{
${ }^{226}$ Siehe Kap. 4.3.2.2 Familiales Handeln als norm-orientiertes Handeln.

${ }^{227}$ Siehe Kap. 6.2 Der deutschen, britische und norwegische Kontext.
} 
der nationalen Unterschiede in den väterlichen Sorgeniveaus bei und liefern weniger die Begründung, warum in den Ländern den jeweiligen Determinanten unterschiedliche Bedeutung zukommt. Die Interpretation der einzelnen länderspezifischen Ergebnisse hat bereits ansatzweise versucht diese mit nationalen Eigenheiten zu erklären. Diese Erklärungsversuche basieren jedoch auf keiner analytischen Systematik und können auf der Grundlage der vorliegenden Untersuchung lediglich als theoretische Spekulationen eingeordnet werden. Wie stark die nationalen Kontexte und in welche Richtung sie auf das väterliche Sorgehandeln wirken, könnte nur ein einheitlicher Datensatz mit expliziter Berücksichtung der institutioneller Vorgaben klären. ${ }^{228}$ Die vorliegende Untersuchung leistet zwar eine theoretisch systematische Studie väterlichen Sorgehandelns auf mikro-sozialer Ebene, die makro-soziale Umwelt muss jedoch stärker in den Erklärungsansatz integriert werden.

Darüber hinaus ist mit Blick auf die bestätigten Hypothesen kritisch anzumerken, dass wenn auch theoretisch begründet - von streng monotonen Zusammenhängen ausgegangen worden ist, die anhand von Regressionsmodellen überprüft werden. Nur einige Thesen haben sich über alle Datensätze hinweg auf diese Weise bestätigen lassen. Dies legt den Schluss nahe, dass die Theorie so keinen empirischen Bestand hat. Dem kann entgegnet werden, dass die vorliegende Analyse rein Thesen testend vorgeht. Dies impliziert streng monoton angenommene Zusammenhänge, durch die bestehende - nicht-lineare - Zusammenhänge eventuell unterschätzt oder gar nicht entdeckt werden. Doch die Ergebnisse zeigen, dass es weniger die nicht bestätigten Hypothesen über alle Datensätze sind, die die wissenschaftliche Aufmerksamkeit wecken sollten, sondern vielmehr die länderspezifischen Unterschiede.

\subsection{Charakteristika der Modellvariablen}

Neben einer theoretischen Erweiterung des Modells um eine stärkere Berücksichtigung makro-sozialer Bestimmungsfaktoren können die länderspezifischen Variationen ferner methodische Ursachen haben. Ein zentraler Ansatzpunkt besteht dabei in der Unabhängigkeit der Datensätze und ihrer recht unterschiedlichen Untersuchungsdesigns. Die vergleichende Wohlfahrtsstaatsforschung geht üblicherweise von quantitativem Daten-

\footnotetext{
${ }^{228}$ Ein aus den Lücken der vorliegenden Datensätze, den empirischen Ergebnissen und theoretischen Erweiterungen abgeleiteter Idealdatensatz und dessen ausführliche Darstellung findet sich im nachfolgenden Kap. 12 Ausblick: Weiterführende Forschungsansätze. Dort wird ferner diskutiert, wie die Integration makrosozialer Determinanten gelingen kann.
} 
material aus, das in allen berücksichtigten Ländern die gleichen Variablen umfasst. ${ }^{229}$ Aus Mangel an Alternativen musste die vorliegende Untersuchung auf drei unabhängig voneinander entwickelte Datensätze zurückgreifen. Dabei zeigt sich insgesamt, dass der Vergleich von Datensätzen, die in unterschiedlichen Sozial-, Zeit- und Forschungskontexten eigenständig entstanden sind, sich schwierig gestaltet (siehe auch Kaufmann/Schulze 2002: 9). Zwar nimmt die Untersuchung keine strenge wohlfahrtsstaatliche Vergleichsperspektive ein, dennoch kann sich die Unabhängigkeit der Studien negativ auf die Ergebnisse auswirken. Zunächst erheben die einzelnen Studien, wie dargestellt, nicht alle die gleichen Inhalte. Des Weiteren werden diese in sehr unterschiedlichem Maße operationalisiert. So liegt z.B. in einer Studie die Entfernung zwischen den elterlichen Wohnorten gemessen in Meilen vor, während in der anderen Kategorien in Kilometerangaben als Antwortmöglichkeiten vorgegeben werden und in der dritten Untersuchung die Wegstrecke in Zeiteinheiten kategorial abgebildet wird. Dies ist nur ein Beispiel für die Herausforderung des Vergleichs unabhängiger Studien. Die Angleichung der Antwortvorgaben - in diesem Fall die Kategorisierung der räumlichen Distanz gemessen in Meilen - führt zu einer Änderung und bei einigen Items gar zu einem Verlust an Informationsdichte in den Daten. Dieses Vorgehen kann ferner zur Folge haben, dass von Variablen fälschlicherweise angenommen wird, sie würden ein ähnliches gar gleiches Konzept messen; dies aber nur bedingt gegeben ist. Hinzu kommen sprachliche und kulturelle Unterschiede in Frageformulierungen und Operationalisierungen. Es kann nur eingeschränkt davon ausgegangen werden, dass die Konstrukte empirisch gleiche Sachverhalte abbilden. Entscheidend ist hier ein zumindest vergleichbares Verständnis der gestellten Fragen, d.h. Begriffe besitzen eine gleichwertige Bedeutung und sind bei den Befragten mit ähnlichen Assoziationen verbunden (Pickel 2003: 156; Niedermayer/Widmaier 2003: 85). In den jeweiligen Ländern reflektiert die Sprache historisch gewachsene Institutionen. Terminologien sind keine rein technischen Label, sondern in den nationalen Konnotationen sind Werte und Einstellungen der jeweiligen gesellschaftlichen Tradition impliziert (Corden 2001: 296; Hantrais 1999: 102). Es ist nicht klar zu differenzieren zwischen Differenzen, die durch die kulturell unterschiedlichen Auffassungen der Frage entstanden sind und tatsächlich vorhandenen nationalen Variationen (Pickel 2003: 157). Inwieweit dies tatsächlich bei den vorliegenden Datensätzen der Fall ist, kann nicht abschließend geklärt werden.

Neben sprachlichen Herausforderungen hat die vergleichende Perspektive auf das Datenmaterial gezeigt, dass die einzelnen Studien z.T. unterschiedliche inhaltliche Schwer-

${ }^{229}$ Als Beispiele solcher Datensätze für den europäischen Raum können z.B. das European Value Survey oder das Family and Fertility Survey genannt werden. 
punkte gesetzt haben. Innerhalb der deutschen Untersuchung wird dies z.B. mit der forschungsleitenden Fragestellung begründet. Ziel der Forsa-Studie ist es primär, ausbleibende Unterhaltszahlungen zu erklären. Aspekte der Care-Dimension werden dabei v.a. als Erklärungsfaktoren der Unterhaltspraxis genutzt. So fehlen im deutschen Datensatz z.B. die Angaben zum letzten Kontakt oder zu indirekten Umgangsformen wie dem postalischen oder telefonischen Kontakt. Auch im norwegischen Datensatz weist die abhängige Variable Care Lücken auf. So wird beispielsweise nicht nach der Kontakthäufigkeit generell gefragt. Es gibt Angaben zur Anzahl der gemeinsam verbrachten Tage (und Nächte) im letzten Monat sowie zum letzten Kontakt. Der letzte Monat muss jedoch nicht repräsentativ für das generelle Kontaktmuster des Vaters sein, sondern ist u.a. abhängig vom Erhebungszeitpunkt. So kann eine Befragung über die Ferienzeit eine höhere Kontakthäufigkeit aufgrund gemeinsam verbrachter Urlaube erfassen. Diese Information wird im norwegischen Datensatz jedoch nicht gesondert erhoben. Es ist zu vermuten, dass zur Beschreibung väterlichen Sorgehandelns auf univariater Ebene dieses Vorgehen keine Auswirkungen auf die Ergebnisse hat. Fälle, die aufgrund eines günstigen Befragungszeitpunkts besser in ihrem Engagement abgebildet werden, werden voraussichtlich durch die schlechter gestellten Befragten ausgeglichen. Doch auf bivariater Ebene, der Herstellung von individuellen Zusammenhängen zwischen Variablen, kann die Berücksichtigung des letzten Kontaktes zu anderen Ergebnissen führen, wie z.B. die Integration der generellen Kontakthäufigkeit. Sowohl im deutschen wie auch im norwegischen Material fehlen darüber hinaus Angaben zur Dauer des Kontakts, zur Veränderung des Kontaktmusters über die Zeit und zur Partizipation an elterlichen Alltagshandlungen, wie Chauffeurdiensten. Der britische Datensatz beinhaltet die ausführlichsten Informationen zum väterlichen Sorgehandeln in Form von Care. Die unterschiedliche Zusammensetzung der nationalen Care-Indizes kann zu unterschiedlichen Erklärungsgrößen führen. Die Unterschiede erscheinen theoretisch wie methodisch jedoch eher gering. So erwiesen sich die Indizes innerhalb von Kontrollrechnungen mit unterschiedlichen Gewichtungen der einzelnen Komponenten als relativ robust. Dennoch kann nur eine identische Erhebung in allen drei Ländern genauere Auskunft über diese mögliche Unsicherheit in den Daten liefern.

Neben den bisherigen kritischen Einwänden mit Blick auf nationale Unterschiede in den Ergebnissen und deren Erklärung, müssen generelle Aspekte der Untersuchung bzw. der Datenerhebung angeführt werden. Dazu zählt zunächst die Tatsache, dass die Erfassung und Erklärung väterlichen Sorgehandelns durch die Tatsache erschwert wird, dass es nicht das väterliche Sorgehandeln an sich gibt. Dies machen die einleitenden Ausführungen zur 
Care-Dimension väterlichen Sorgehandelns deutlich. ${ }^{230}$ Väter können in vielfältiger Weise, in unterschiedlich starkem Ausmaß und zu verschiedenen Zeitpunkten ihre Rolle als Elternteil ausfüllen. So müssen z.B. interindividuelle Unterschiede der beteiligten Akteure, d.h. sowohl auf Seiten der Eltern als auch der Kinder Berücksichtigung finden. Selbst wenn einige Tätigkeiten und Handlungen mehr Zeit in Anspruch nehmen, so können andere trotz geringerem zeitlichen Aufwands eine größere sozio-emotionale Bedeutung für den Elternteil oder das Kind haben (Palkovitz 1997: 212ff.). Es gibt unterschiedliche Auffassungen von „gutem“ väterlichen Engagement. Einige Väter verstehen darunter, möglichst viele Aktivitäten mit ihrem Kind zu unternehmen, die gleichzeitig aber ein Minimum z.B. an Kommunikation oder physischem Kontakt erfordern. Faktoren, die die individuelle Wahl der Unternehmungen und damit das Ausmaß väterlicher Sorge wesentlich mitbestimmen, sind u.a. Persönlichkeitsmerkmale sowie unterschiedliche kindliche bzw. väterliche Prioritäten und Ressourcen. Die jeweiligen Relevanzstrukturen variieren darüber hinaus lebensphasenspezifisch (Kilkey 2000: 99; Lowe 2004: 7). Damit einher geht die subjektive Evaluation der gemeinsam verbrachten Zeit, d.h. diese Faktoren bestimmen die Beurteilung, was in der spezifischen Situation als erforderlich oder angemessen gilt (Palkovitz 1997: 214f.; Kudera 2002: 153, 182). Die genannten Aspekte betreffen das individuelle Überzeugungssystem des Vaters, welches in die vorliegende Untersuchung empirisch nicht ausreichend eingegangen ist.

Die Ausführungen deuten ebenso auf die besondere Bedeutung des Zeitverlaufs hin, der in den einzelnen Untersuchungen nicht berücksichtigt wird. Es wird keine dynamische Betrachtung väterlichen Sorgens vorgenommen, d.h. der Faktor "Zeit“ und die damit einhergehenden Veränderungen der einzelnen Determinanten und ihrer Einflussstärke werden nicht erfasst. So ist es z.B. möglich, dass der väterliche Kontakt nach einem zeitweiligen Abbruch wieder aufgenommen wird. Elterliche Fürsorge variiert kurz- wie langfristig über den Zeitverlauf und über die jeweiligen Entwicklungsphasen der Eltern, der Kinder sowie in Beziehung zum sozialen Umfeld und anderen Lebensumständen (Palkovitz 1997: 206, 213).

Die Ausführungen zur Konstruktion der Care-Indizes haben verdeutlicht, dass die Aktivitäten nach Quantität und Qualität für die kindliche Entwicklung zu erfassen sind. In allen drei Untersuchungen können zwar einzelne Aktivitäten differenziert werden, ihr zeitlicher Anteil am väterlichen Engagement wird dagegen nicht erfasst. Hier könnten ausdifferenzierte Zeitverwendungsstudien weitere Einblicke liefern. Die Ausführungen zeigen, dass

${ }^{230}$ Siehe Kap. 2.1 Väterliches Sorgehandeln in Form von Care und Cash. 
väterliches Sorgen theoretisch und empirisch schwer zu fassen ist. Obwohl die biologische Elternschaft dichotom charakterisiert wird, kann das Elternsein an sich nur schwer in wenige Kategorien gepresst werden. Insgesamt muss der Komplexität väterlichen Sorgehandelns in der Operationalisierung Rechnung getragen werden, ohne den Index zu überfrachten.

In der Konstruktion der jeweiligen Indizes dient in der vorliegenden Analyse die Kontakthäufigkeit als Basiswert. Väter, die hier keine Angaben aufweisen, werden aus der Analyse ausgeschlossen. Der Vergleich der Ausfälle zeigt zunächst, dass in Norwegen und Großbritannien kaum Informationslücken bestehen. Nur ein britischer und zwei norwegische Väter haben nicht angegeben, wann sie ihr Kind das letzte Mal gesehen haben. In Deutschland sind es dagegen 75 Väter, für die keine Angaben vorliegen. Die genauere Betrachtung der deutschen Ausfälle zeigt, dass mit Blick auf die räumliche Entfernung zwischen den Eltern, die Anzahl der Kinder innerhalb und außerhalb des väterlichen Haushalts, die jeweilige Partnersituation beider Elternteile, die Dauer seit der Trennung, die Anzahl der Beziehungen, aus denen Kinder hervorgegangen sind, sowie auf das Alter des Befragten keine nennenswerten Unterschiede zwischen Vätern mit und ohne Angaben bestehen. Auch beim Einkommen ist die Verteilung relativ ähnlich. Lediglich der Anteil derer, die keine Einkünfte angeben, ist unter den Ausfällen größer. Dies steht im Einklang mit der Tatsache, dass der Anteil Erwerbsloser unter den Vätern höher ist, die keine Angaben zum Kontakt machen. Das Bildungsniveau beider Gruppen ist sehr ähnlich ausgeprägt. Väter, die nicht in die Berechnungen integriert werden können, sind häufiger mit der Kindsmutter verheiratet gewesen. Da die frühere Familienform der Eltern Einfluss auf das sozio-emotionale Sorgehandeln hat, können die Ausfälle gegebenenfalls zu einer Unterschätzung der Care-Dimension im deutschen Fall führen. Die in der Analyse unberücksichtigten Fälle weisen ferner stärkere Ausfälle mit Blick auf die Trennungsregelung auf. Werden nur die gültigen Prozent betrachtet, weisen beide Gruppen ähnliche Verteilungen der Unterhaltsfestlegung auf. Die Sorgerechtsfestlegung gelingt den Vätern ohne Angaben jedoch häufiger einvernehmlich und seltener unter Beteiligung eines Gerichts. Zusammen mit den nachgewiesenen Zusammenhängen liegt hier die Vermutung einer Unterschätzung väterlichen Sorgehandelns in Form von Care nahe. Aufgrund der hohen Ausfälle gilt dies jedoch nur unter großer Vorsicht. Ähnliche Annahmen können für die Form des Sorgerechts getroffen werden. Väter, die die Kontakthäufigkeit nicht angeben, weisen häufiger gemeinsames Sorgerecht auf als Väter, die in die Berechnungen integriert werden können. Aufgrund der relativ kleinen Fallzahl und der sonst sehr ähnlichen Merkmale im Vergleich zu Vätern, deren Informationen vorliegen, ist jedoch zu vermuten, dass die vermuteten Effekte nicht allzu groß sind. 
Die Tatsache, dass Väter, die in den anderen Teilvariablen des Care-Indizes keine Angaben aufweisen, zugunsten einer höheren Fallzahl dennoch in den Berechnungen berücksichtigt werden, kann zu einer Unterschätzung des tatsächlichen Sorgehandelns und Verzerrungen in den Ergebnissen führen. Die Annahme, dass der Vater mit fehlenden Informationen diese Form des väterlichen Sorgehandelns nicht ausübt, scheint zwar theoretisch plausibel, endgültig abgesichert werden kann sie nicht. Insgesamt zeigen sich die jeweiligen Care-Indizes als gute - relativ robuste - Indikatoren der sozio-emotionalen Dimension väterlichen Sorgehandelns im vorliegenden Datenmaterial.

Neben der z.T. unterschiedlichen Erfassung der sozio-emotionalen Dimension väterlichen Sorgehandelns können die länderspezifischen Ergebnisse ferner der variierenden Erhebung finanzieller Aspekte geschuldet sein. Innerhalb der britischen Untersuchung wird zwischen verschiedenen Formen von Zahlungsunregelmäßigkeiten differenziert. So können die Angaben der aktuell Zahlenden denen, die nie gezahlt oder die die Leistungen eingestellt haben, gegenübergestellt werden. In Deutschland und Norwegen liegen dagegen Informationen zu Zahlungsschwierigkeiten im Wesentlichen nur dichotom vor. Dabei werden deutsche Väter gefragt, ob es in der Vergangenheit schon einmal vorkam, dass sie ihren Zahlungsverpflichtungen nicht nachkommen konnten. Diese retrospektive Erfassung kann durch Erinnerungseffekte Verzerrungen aufweisen. Einmalig ausbleibende Unterhaltsleistungen oder länger zurückliegende Zahlungsprobleme können aufgrund von Erinnerungslücken so einer Erfassung entgehen. Insgesamt können Ereignisse zeitlich falsch eingeordnet, erinnert oder nachträglich rationalisiert werden. Die Zahlungsprobleme werden damit unterschätzt. Ferner kann auf diese Weise nicht unterschieden werden, ob Väter in der Vergangenheit ihren Zahlungsverpflichtungen nicht nachgekommen sind oder ob sie es derzeit nicht tun. Im norwegischen Fall wird nach dem Auftreten von Unterhaltsschulden gefragt. Diese stellen eine besondere Form von ausbleibenden finanziellen Transfers dar, denn sie setzen die Beteiligung der Unterhaltsbehörde voraus. Nur in Fällen, in denen die Mutter Unterhaltsvorschuss beantragt, entstehen Unterhaltsschulden. Fraglich ist, ob mit der ersten Unregelmäßigkeit der Zahlung behördlicher Beistand angefordert wird. Damit würden hier speziell die Problemfälle erfasst, bei denen längerfristig Unterhaltsprobleme auftreten. Es werden so die stärkeren Zahlungsschwierigkeiten abgebildet, die höchst wahrscheinlich seltener auftreten. Eltern, die Zahlungsschwierigkeiten privat lösen, werden auf diese Weise nicht in die Analyse integriert. Das kann zu einer Unterschätzung der Zahlungsprobleme in Norwegen führen. Dagegen ist jedoch einschränkend einzuwenden, dass in 90\% der Fälle norwegische Elternpaare den Transfer des Unterhalts (freiwillig) über die zuständige Behörde regeln. Die unterschiedliche Operationalisierung von Unterhaltsproblemen in den Datensätzen könnte einen Teil der nationalen Unterschiede in der Häufigkeitsverteilung erklären. So zeigen die britischen Väter deutlich 
mehr Variation in der dichotomisierten Erfassung von Zahlungsschwierigkeiten (vorhanden vs. nicht vorhanden) und einen höheren Anteil an Problemen. ${ }^{231}$

Zur Erfassung der abhängigen Variablen Cash als Zahlungsprobleme ist generell anzumerken, dass insgesamt die empirische Erhebung von Informationen, die im Zusammenhang mit der finanziellen Situation des Befragten stehen, problematisch ist. Diese Schwierigkeiten erhöhen sich, wenn es um das Versäumnis von Unterhaltszahlungen geht. Die nur schwach ausgeprägte Varianz der Antworten sowie die hohe Ausfälle könnten mit dem Phänomen sozialer Erwünschtheit zusammenhängen. Antwortverweigerungen können damit begründet werden, dass Väter sich schämen, wenn sie Unterhaltszahlungen nicht geleistet haben, obwohl sie verpflichtet wären. Sie haben damit nicht nur gegen eine gesetzliche Regelung, die sie zur Zahlung verpflichtet, verstoßen, sondern ebenso gegen mehr oder weniger stark institutionalisierte Normen. Dies kann die zum einen die male breadwinner-Norm sein, nach der der Mann innerhalb seiner Familie vornehmlich für die finanzielle und materielle Versorgung seiner Familie verantwortlich ist. Es kann sich zum anderen aber auch um eine allgemeiner formulierte Norm der elterlichen Verantwortung handeln, nach der Eltern - Mütter wie Väter - für den materiellen Unterhalt ihrer Familie zu sorgen haben. Bleiben Unterhaltszahlungen aus - aus welchen Gründen auch immer werden diese Normen nicht erfüllt. Darüber hinaus können Antworten verweigert bzw. verfälscht werden, weil die Väter institutionelle Konsequenzen fürchten. Sie glauben, ihre Angaben werden an die zuständigen Behörden weitergegeben, so dass ihnen Nachzahlungen und erhöhte Forderungen drohen. Auch dies kann zu ausbleibenden Antworten oder Beschönigungen der Unterhaltssituation führen. Für alle drei Datensätze ist anzunehmen, dass das finanzielle Sorgehandeln in Form von Zahlungsschwierigkeiten eher unter- als überschätzt wird. So geben die Nachtrennungseltern je nach Perspektive unterschiedliche Niveaus des Sorgehandelns der Väter an. Dabei neigen Väter dazu, mehr Unterstützungsleistungen anzugeben als Mütter nach ihren Angaben erhalten. Des Weiteren

\footnotetext{
${ }^{231}$ Darüber hinaus weist der britische Datensatz weitere Besonderheiten auf, die zu den Ergebnissen geführt haben können. So wurde bereits auf die Schwierigkeiten der Zuordnung der Angaben zu den jeweiligen Elternschaften hingewiesen (Kap. 7.2 Die britische Untersuchung nach Bradshaw und Mitarbeitern). Es kann nicht ausgeschlossen werden, dass Angaben zu unterschiedlichen Beziehungen in der vorliegenden Analyse zusammengebracht werden. Des Weiteren kann die komplizierte Fragebogenführung zu verschiedenen Beziehungen mit Blick auf die Kontaktmuster und zur letzten Beziehung mit Blick auf finanzielle Unterstützung zu Verwirrungen und gegebenenfalls „falschen“ Angaben unter den Befragten geführt haben. Darüber hinaus ist der britische Fragebogen am stärksten ausdifferenziert. Dies liefert einerseits zahlreiche, sehr aufschlussreiche Informationen, die auch für die anderen beiden Untersuchungen hilfreich sein könnten, doch gleichzeitig besteht die Gefahr starker - und eventuell auch systematischer - Ausfälle. Die extreme Länge des Fragebogens (insgesamt 94 Seiten) könnte Abbrüche der Interviews provoziert haben. Aus den Daten wird leider nicht ersichtlich, warum viele Angaben zur zweiten und dritten Elternschaft fehlen.
} 
ist davon auszugehen, dass die Väter, die gar keinen Unterhalt zahlen eher die Beantwortung eines Fragebogens verweigern als Väter, die ihren Zahlungsverpflichtungen nachkommen. Dabei sind - ähnlich wie bei der Care-Variablen - auch bei der Erfassung der finanziellen Aspekte väterlichen Sorgehandelns im britischen Fall - ein wenig überraschend - nur sechs fehlende Angaben zu verzeichnen. In Deutschland geben dagegen 47 und in Norwegen 24 Väter keine Antwort. ${ }^{232}$ Die systematische Betrachtung der deutschen Ausfälle bei der Angabe von Zahlungsproblemen ergibt kaum Unterschiede zwischen den Gruppen der antwortenden und nicht antwortenden Väter in Merkmalen wie dem väterlichen Einkommen, der Erwerbstätigkeit, der Anzahl an Unterhaltspflichten, der Partnersituation des Vaters, dem früheren Familienstand der Eltern, der Dauer seit der Trennung, der Anzahl der gescheiterten Beziehungen, aus denen Kinder hervorgegangen sind sowie dem Alter des Vaters. Auch die Entfernung der Elternhäuser ist ähnlich wie bei Vätern, die keine Angaben machen. Hier sind lediglich stärkere Informationsdefizite in der Prädiktorvariable auszumachen als bei Vätern, die Angaben zu möglichen Zahlungsschwierigkeiten machen. Väter, für die keine Informationen zum Unterhaltsverhalten vorliegen, zeigen ein etwas höheres Bildungsniveau, leben eher mit Kindern in einer Haushaltsgemeinschaft und haben tendenziell seltener mit der Kindesmutter zusammengelebt. Väter ohne Angaben wissen häufiger nicht, ob die Kindsmutter in einer neuen Partnerschaft lebt und geben etwas seltener eine einvernehmliche Festlegung des Sorgerechts an. Sowohl bei der Sorgerechtsfestlegung wie auch bei der Form des Sorgerechts weisen diese Väter mehr Ausfälle auf als Väter, die geantwortet haben. Ferner liegen so gut wie keine Informationen zur Unterhaltsfestlegung in der Gruppe der Ausfälle vor.

Der Vergleich der norwegischen Männer ohne Angaben mit den Vätern, die Auskunft zu ihrer Zahlungspraxis erteilt haben, zeigt kaum Unterschiede in den Niveaus des Einkommens, der Erwerbstätigkeit, der Arbeitszeit, der Anzahl der getrennt lebenden Kinder, der Festlegung des Unterhalts, der festgelegten Form des Umgangs, dem freundschaftlichen Verhältnis zwischen den Eltern, der Anzahl getrennt gelebter Elternschaften sowie dem Alter des Vaters. Auch die Entfernung zwischen den Wohnorten ist sehr ähnlich ausgestaltet; keiner der Väter ohne Angaben lebt jedoch weiter als sechs Stunden von seinen Kindern entfernt. Des Weiteren sind Väter, zu denen keine Informationen vorliegen, tendenziell geringer qualifiziert, leben eher mit anderen Personen in einer Haushaltsgemeinschaft, sind seltener mit der Kindsmutter verheiratet gewesen, haben insgesamt länger in der elterlichen Partnerschaft gelebt, besitzen seltener eine schriftliche Vereinbarung zum Umgang mit dem Kind und sind eher länger getrennt lebend. Väter, die nichts zu ihren

${ }^{232}$ Dies entspricht in beiden Fällen gut 4\% der Stichprobe. 
Unterhaltsproblemen ausgesagt haben, wissen ferner seltener über die finanzielle Situation der Kindsmutter Bescheid. Aufgrund der geringen Anzahl an norwegischen Ausfällen dürfen die wenigen Unterschiede jedoch insgesamt nicht überbewertet werden.

Neben den unterschiedlichen Operationalisierungen der Kriterien können die unterschiedlichen Ergebnisse insbesondere in der multiplen Analyse z.T. auf die verschiedenen Prädiktoren, die in den nationalen Modellen integriert werden, zurückzuführen sein. So fehlen im Datensatz von Forsa Informationen zur Aushandlung und Ausgestaltung des Umgangs. Es liegen lediglich Angaben zum Sorgerecht, der rechtlichen Grundlage väterlicher Fürsorge, vor. Des Weiteren gibt es keine Informationen zur väterlichen Arbeitszeit, der Dauer der elterlichen Beziehung vor ihrem Scheitern und der ökonomischen Situation, die in den anderen Datensätzen Berücksichtigung finden und z.T. auch Effekte aufweisen. Die Briten haben - wie bereits bei den abhängigen Variablen - den umfassendsten Satz an Erklärungsgrößen erhoben. Dennoch liegen keine Informationen zur Regelung des Sorgeund Umgangsrechts vor. Im norwegischen Datenmaterial fehlen die Angaben zur Haushaltszusammensetzung und Partnersituation des Vaters sowie zur Sorgerechtsfestlegung und -vereinbarung. ${ }^{233}$ Zwar ist davon auszugehen, dass die Aufnahme der fehlenden Variablen in das Modell - die Mehrheit der berücksichtigten Variablen liegt in allen drei Datensätzen vor - nicht sämtliche nationale Unterschiede nivelliert. Dennoch kann die Integration identischer Prädiktoren zu veränderten Ergebnissen führen. So erwies sich z.B. die freundschaftliche Beziehung zwischen den Eltern zum Zeitpunkt der Befragung sowohl in Großbritannien wie auch in Norwegen als ein wichtiger Erklärungsfaktor väterlichen Sorgehandelns. Dieser Einfluss müsste für deutsche Väter überprüft werden. Das Gleiche gilt für die Regelung des Sorge- und Umgangsrecht in Großbritannien.

\subsection{Die Zusammensetzung der Stichproben}

Nationale Unterschiede in den Ergebnissen können sich ebenso durch die jeweilige Zusammensetzung der Stichproben ergeben. Die Autoren der Forsa-Untersuchung verweisen auf die Unterschiede zwischen den Stichproben der befragten Unterhaltspflichtigen und berechtigten. So weisen die unterhaltspflichtigen Elternteile weniger unterhaltsberechtigte Kinder auf. Eine höhere Anzahl an Partnerschaften, aus denen Kinder hervorgegangen sind, tritt in dieser Stichprobe noch seltener auf als bei den unterhaltsberechtigten Eltern. Des Weiteren ist der Anteil der Geschiedenen auf Kosten des Anteils der Befragten, die

233 Diese Angaben könnten im norwegischen Fall jedoch von untergeordneter Bedeutung sein, da das gemeinsame Sorgerecht eine lange Tradition besitzt. 
nie mit dem unterhaltsberechtigten Elternteil zusammengelebt haben, etwas höher (Forsa 2002: 35). Über Unterschiede und Gemeinsamkeiten der antwortenden Väter und der Väter, die eine Beteiligung an der Studie verweigert haben, werden keine Angaben gemacht.

Der Vergleich der antwortenden norwegischen Väter mit denen, die eine Auskunft verweigerten, weist laut den Autoren auf repräsentative Ähnlichkeiten in Alter und Zahl der Kinder, die Unterhaltsansprüche besitzen, hin. Das Einkommensniveau variiert jedoch. Väter mit geringerem Einkommen sind seltener zur Durchführung eines Interviews bereit. Des Weiteren kann angenommen werden, dass die 10\% der Nachtrennungsväter, die nicht durch die für Unterhaltszahlungen zuständige nationale Versicherungsbehörde erfasst werden, eher höheren Einkommensklassen zuzuordnen sind (Skevik/Hyggen 2002: 125). Insgesamt unterschätzt die Stichprobe damit die beiden Extreme der Einkommensverteilung: Väter mit niedrigem und Väter mit besonders hohem Einkommen (Skevik 2006a: 122). Darüber hinaus wird das Sample durch eine leichte Überrepräsentanz von Arbeitern mit geringem Ausbildungsniveau charakterisiert, während typische Elternpaare der Mittelschicht mit geringem Konfliktpotential leicht unterschätzt werden (Skevik/Hyggen 2002: 125; Skevik 2006a: 122).

Auch Bradshaw und Mitarbeiter haben in ihrer Untersuchung die Merkmale der antwortenden und sich der Befragung verweigernden Väter verglichen. Es werden Unterschiede hinsichtlich des Familienstandes und der sozialen Schicht festgestellt. Innerhalb der britischen Stichprobe fehlen allein stehende Männer aus der ungelernten Arbeiterklasse und Arbeitslose. Darüber hinaus sind die Väter, die entweder nichts von ihrer Vaterschaft wissen oder diese innerhalb einer Befragung nicht zugeben wollen, empirisch nicht zu erfassen - dies gilt ebenso für den deutschen und norwegischen Fall. Daher verweisen die britischen Autoren darauf, die Repräsentativität ihrer Daten nur unter Vorsicht anzunehmen. Sie vermuten, dass weniger als die Hälfte der tatsächlichen getrennt lebenden Väter dies auf Anfrage auch zugibt (Bradshaw et al. 1999: 11ff., 22). Auskunftsverweigerungen sind bei Untersuchungen, die sich mit familiensoziologischen Fragestellungen beschäftigen, häufiger, weil diese einen der wichtigsten persönlichen Lebensbereiche betreffen. Sie sind eng verbunden mit der Identität, dem Selbstbild und Selbstwertgefühl des Befragten (Hill 2005: 168). Umso überraschender ist es, dass im britischen und auch norwegischen Datensatz so wenig Antworten zur abhängigen Variablen im Interviewverlauf ausgefallen sind. Es ist zu vermuten, dass Nachtrennungsväter eher eine Befragung gänzlich verweigern, als im Interview einzelne Fragen zur Unterhalts- und Sorgepraxis auszulassen.

Der Vergleich der Zusammensetzung der nationalen Stichproben, die in die vorliegende Analyse integriert wurden, zeigt, dass norwegische Väter deutlich seltener in unmittelbarer Nachbarschaft zu ihren Ex-Partnerinnen leben. Dafür weisen sie häufiger eine 
Reisedistanz von 30 bis 120 Minuten auf. In den anderen Kategorien, die die räumliche Entfernung ausdrücken, ähneln sich die Stichproben. Dies kann den geographischen Begebenheiten Norwegens geschuldet sein. Ein Umzug in eine andere Stadt ist meist aufgrund der dünnen Besiedelung des Landes mit längeren Wegstrecken zum alten Wohnort verbunden. Die Ergebnisse haben jedoch gezeigt, dass dies sich nicht auf das väterliche Sorgehandeln in Norwegen auswirkt. Nationale Unterschiede ergeben sich ferner mit Blick auf das Einkommen. Aufgrund der sehr unterschiedlichen Erfassung sind die Kategorisierungen nur schwer zu vergleichen. ${ }^{234}$ Auffällig ist jedoch der relativ hohe Anteil der britischen Väter ohne Einkommen im Vergleich zur deutschen Stichprobe. Dafür ist die höchste Einkommensklasse im britischen Fall fast doppelt so stark besetzt wie im deutschen. Systematische Aussagen über die nationalen Unterschiede sind jedoch aufgrund der Erfassung von Netto- und Bruttobeträgen für sehr unterschiedliche Zeiträume nicht möglich. Ferner sind im britischen Datensatz deutlich mehr Erwerbslose als in der norwegischen und deutschen Studie zu finden. Dies könnte eine Erklärung für den höheren Anteil von Unterhaltsproblemen unter britischen Vätern sein. Gleichzeitig geben britische Väter häufiger als norwegische Arbeitszeiten über 40 Stunden pro Woche an. Ähnlich wie beim Einkommen gestaltet sich der Vergleich der jeweiligen Bildungsniveaus aufgrund der nationalen Spezifika in den Bildungsabschlüssen schwierig. Dennoch zeigt die britische Stichprobe einen im Vergleich zu den deutschen Vätern sehr hohen Anteil an Vätern ohne Abschluss. Innerhalb der deutschen Stichprobe sind die besser Qualifizierten im Vergleich zu den anderen beiden Stichproben etwas stärker vertreten. Die deutschen Väter leben ferner seltener nach der Trennung mit Kindern zusammen als britische. Britische Väter sehen sich öfter als deutsche und norwegische mit Unterhaltsverpflichtungen für mehrere Kinder konfrontiert. Die höheren Zahlungsforderungen könnten eine weitere Erklärung für das häufigere Auftreten von Zahlungsschwierigkeiten im britischen Fall darstellen. Die Partnersituationen beider Elternteile gestalten sich über die Nationen ähnlich. Knapp die Hälfte aller Väter befinden sich in einer neuen Partnerschaft. Die Angaben der Väter zur Beziehungssituation der Ex-Partnerin weisen einen leicht höheren Anteil neuer Partnerschaften unter den Kindsmüttern aus. In allen Datensätzen ist diese Variable durch hohe Ausfälle gekennzeichnet. Dies ist insofern nicht verwunderlich, als dass die meisten davon durch Unwissenheit zustande kommen. Getrennt lebende Partner sind über ihre jeweiligen neuen Beziehungen nicht zwingend informiert. Der Anteil der Eltern, die vor der Trennung nicht zusammengelebt haben, ist im norwegischen Fall etwas höher als in den anderen Datensätzen. Der Prozentsatz an unehelich zusammenlebenden

${ }^{234}$ Siehe dazu ausführlich Kap. 9.1.1 Der Einfluss der Prädiktoren der physischen Umwelt. 
Eltern ist in Norwegen deutlich stärker ausgeprägt. Britische und deutsche Väter weisen dagegen starke Ähnlichkeiten in der Stichprobenzusammensetzung nach früherem Familienstand auf. Innerhalb des norwegischen Samples finden sich im Vergleich zum britischen Fall etwas seltener Paare, die länger als fünf Jahre in einer Partnerschaft gelebt haben. Des Weiteren geben norwegische Väter häufiger als britische an, dass sie ein freundschaftliches Verhältnis zur Kindsmutter pflegen. Der Anteil erst kurz zurückliegender Trennungen ist im deutschen Fall deutlich kleiner als in den beiden anderen Stichproben. Dafür sind Trennungen, die bis zu zwei Jahre her sind, häufiger vertreten. In allen drei Datensätzen lebt die Mehrheit der Väter von Kindern getrennt, die aus einer Beziehung entstanden sind. Britische Väter geben jedoch deutlich häufiger an, mehr als eine Elternschaft aus vergangenen Beziehungen zu haben. Auffällig ist ferner die Altersstruktur der norwegischen Stichprobe. Keine der befragten Väter sind jünger als 35 Jahre alt. Diese Altersklasse ist auch in den anderen beiden Datensätzen nur schwach besetzt, aber dies gilt im norwegischen Fall auch für die nächst jüngere Alterskategorie, die in Deutschland und insbesondere in Großbritannien mehr Fälle aufweist. Insgesamt sind über $80 \%$ der norwegischen Befragten älter als 55 Jahre. $^{235}$ Der Einfluss des Alters wird in der vorliegenden Untersuchung nicht analysiert, dennoch kann angenommen werden, dass z.B. älteren Vätern andere, v.a. finanzielle Ressourcen zur Verfügung stehen als jungen Berufsanfängern. Des Weiteren werden sie andere Prioritäten in dieser Phase ihres Lebens setzen. Die Auswirkungen auf das väterliche Sorgehandeln sind nicht zuletzt auch abhängig vom Alter des Kindes bzw. dem Alter des Vaters zum Zeitpunkt der Geburt. ${ }^{236}$

Die einzelnen Erklärungsfaktoren zeigen nationale Variationen in ihren Häufigkeitsverteilungen, die jedoch aufgrund der Probleme, die mit der Vergleichsperspektive auf die drei Datensätze verbunden sind, nicht überschätzt werden dürfen. Dennoch liefern die Unterschiede zwischen den Stichproben Hinweise für die unterschiedlichen Häufigkeiten in den abhängigen Variablen; die Differenzen in den Erklärungsmodellen für väterliches Sorgehandeln in Form von Care und Cash erklären sie nur bedingt. Es muss offen bleiben, ob es sich bei den Unterschieden in den Stichproben um Unstimmigkeiten in der Stichprobenziehung - und damit methodische Fehlerquellen - handelt, oder ob norwegische Väter in Nachtrennungsfamilien generell andere Merkmale aufweisen als deutsche oder britische. Dann wäre zu fragen, warum dies der Fall ist. Insgesamt können zur Repräsen-

${ }^{235}$ Zur genauen Darstellung der Zusammensetzung der jeweiligen Stichproben siehe im Anhang Tabellen B.1 bis B.3.

236 Zum Erkenntnisgewinn einer Längsschnittperspektive siehe Kap. 12 Ausblick: Weiterführende Forschungsansätze. 
tativität der einzelnen Stichproben keine gesicherten Aussagen getroffen werden. Dies ist u.a. der mangelnden Erfassung von Nachtrennungsvätern in der amtlichen Statistik geschuldet (u.a. Bradshaw et al. 1999: 11ff.). ${ }^{237}$ Es kann in den nationalen Kontexten jeweils nur geschätzt werden, wie viele Nachtrennungsväter es tatsächlich gibt und welche Merkmale diese aufweisen. Darüber hinaus sind schiefe Verteilungen in vielen bekannten Studien der Familiensoziologie ein Problem. Sie verringern eventuell die Varianz der Variablen sowie die Erklärungskraft der statistischen Modelle insbesondere auf multipler Ebene (Hill 2005: 173). Mit Blick auf die Care-Variable zeigen alle drei Datensätze akzeptable Verteilungen. Im britischen Fall könnte die geringe Variation in der CashVariablen zu Verzerrungen führen.

Aufgrund mangelnder Alternativen wird das vorliegende Datenmaterial aus Deutschland, Großbritannien und Norwegen für eine Sekundäranalyse genutzt. Trotz der aufgeführten Defizite in den Daten und den Herausforderungen, die mit der Vergleichsperspektive auf unabhängig entwickelte nationale Untersuchungen verbunden sind, kann eine erste Antwort auf die forschungsleitende Frage, was väterliches Sorgehandeln in Nachtrennungsfamilien bestimmt, gegeben werden. Darüber hinaus liefern die Ergebnisse ebenso wie die kritischen Überlegungen zahlreiche Ansatzpunkte für weiterführende Forschungsfragen, die im folgenden Kapitel abschließend dargestellt werden.

${ }^{237}$ Dies gilt auch für den deutschen Fall. Väter, die getrennt von ihren Kindern leben, werden als solche nicht in der amtlichen Statistik geführt. Sie gelten als Single-Haushalte, so lange sie nicht mit einer neuen Familie zusammenleben. Angaben zur getrennt lebenden Familien werden nicht erfasst. Lediglich Gerichtsakten geben Aufschluss über Merkmale von Nachtrennungsvätern in einer amtlichen Form. Hier werden jedoch nur jene Fälle aufgeführt, die ein hohes Konfliktpotential aufweisen und gerichtliche Hilfe in Anspruch genommen haben. Auch hier kann nicht von einer repräsentativen Erfassung ausgegangen werden. 


\section{Ausblick: Weiterführende Forschungsansätze}

In den Ausführungen zur Einordnung der Ergebnisse ist bereits auf die Lücken zwischen den und innerhalb der Untersuchungen hingewiesen worden. Nachstehende Tabelle 12.1 zeigt diese noch einmal zusammenfassend auf einen Blick. Dabei werden ferner Dimensionen aufgelistet, die in keinem der drei Datensätze Berücksichtigung finden. Neuere Forschungen können zunächst von den jeweils erfassten Aspekten der einzelnen Datensätze profitieren und des Weiteren aus dem theoretischen Modell neue Dimensionen und Determinanten väterlichen Sorgehandelns ableiten.

Alle drei Untersuchungen haben weitreichende Aspekte des väterlichen Sorgehandelns sowohl in Form von Care als auch von Cash erhoben. Doch keine der Studien ist all umfassend, d.h. die einzelnen Untersuchungen weisen auf Variablen hin, die sich in den anderen beiden nicht finden. Darüber hinaus können weitere Aspekte ausgemacht werden, die in künftigen Untersuchungen berücksichtigt werden sollten. Dazu zählt mit Blick auf die Care-Erfassung zunächst die stärkere Berücksichtigung der Qualität des väterlichen Kontakts. Die Erfassung der Kontaktpraxis in den Studien konzentriert sich v.a. auf die Kontakthäufigkeit, die die quantitative Dimension väterlichen Sorgehandelns abbildet. Dabei wird vernachlässigt, dass nicht nur die Häufigkeit der Besuche quantitativ eine Rolle spielt, sondern auch ihre zeitliche Länge erfasst werden müsste. Dies wird lediglich im britischen Datensatz berücksichtigt. Die Kontakthäufigkeit stellt ferner nur einen Aspekt des väterlichen Sorgehandelns dar. Darüber hinaus müssen weitere qualitative Merkmale der Vater-Kind-Interaktion in die Analyse integriert werden, wie dies mit der Konstruktion der Care-Indizes in der vorliegenden Analyse gemacht wurde. Der Vorwurf der Beschränkung auf quantitative Merkmale gilt auch für die Art der Erfassung der einzelnen Aktivitäten, die der Vater mit seinem Kind unternimmt. Deutsche Väter werden gefragt, ob sie jemals eine der aufgeführten Unternehmungen mit ihrem Kind gemacht haben, während britische und norwegische Väter die Aktivitäten des letzten Besuchs angeben. In allen drei Datensätzen besteht die Möglichkeit einer Mehrfachnennung. Daraus wird jedoch weder die Häufigkeit der einzelnen Unternehmungen - über mehrere Besuche hinweg - deutlich, noch welchen zeitlichen Anteil die einzelnen Aktivitäten an der gemeinsam verbrachten Zeit eingenommen haben. Dies macht eine wesentlichen Aspekt für die Bewertung der Intensität väterlichen Kümmerns aus.

Zur stärkeren Berücksichtigung der Qualität des väterlichen Sorgehandelns kann darüber hinaus z.B. die Aufnahme von Alltagshandlungen zählen. Ein Merkmal väterlichen Sorgehandelns nach der elterlichen Trennung ist der Verlust von Alltäglichkeit. Dieser sollte differenzierter erfasst werden. Zwar werden Väter in allen drei Studien nach der Ausge- 
staltung ihrer Besuche gefragt, und es werden auch alltägliche Aktivitäten wie „gemeinsame Mahlzeiten“, „Einkaufen“ oder „Unterstützung bei Schulaufgaben“ genannt, unklar bleibt jedoch z.B., welchen zeitlichen Anteil diese im Vergleich zu Freizeitaktivitäten ausmachen. Ferner wird im deutschen und britischen Datensatz nach der väterlichen Partizipation an wichtigen Entscheidungen, die das Kind betreffen, erfasst. In der britischen Studie finden sich darüber hinaus Angaben zur Beteiligung an Chauffeurdiensten, Baby Sitting oder auch Schulveranstaltungen. Die Frage nach der Ausgestaltung des letzten Besuches lässt Väter vielleicht eher an besondere (Freizeit-) Aktivitäten denken und weniger an alltägliche Routinen. Letztere sollten als wichtiger Bestandteil väterlichen Sorgehandelns systematischer erfasst werden. Neben den alltäglichen Aktivitäten kann ferner differenzierter nach Übernachtungsbesuchen gefragt werden. Die norwegische Untersuchung erfasst die Anzahl der Übernachtungen des Kindes beim Vater im letzten Monat. Dabei ist jedoch zwischen Übernachtungen am Wochenende und unter der Woche zu differenzieren. Zur Alltäglichkeit väterlichen Handelns zählt ferner die väterliche Beteiligung an Erziehungsaufgaben. Auch diese kann stärker direkt erfasst werden. Hier ist ebenso von Interesse, inwieweit der Vater ein vertrauensvoller Ansprechpartner für sein Kind ist. Dies sind Fragen, die mit Blick auf die Care-Komponenten väterlichen Sorgehandelns in künftigen Untersuchungen stärker berücksichtigt werden könnten.

Die Cash-Dimension wird in der vorliegenden Untersuchung ausschließlich als auftretende Zahlungsprobleme operationalisiert. Dabei bleibt insbesondere im deutschen und norwegischen Fall die konkrete Ausgestaltung dieser Unterhaltsschwierigkeiten offen. Unklar ist sowohl, ob es sich bei den Problemen um Unregelmäßigkeiten in den Zahlungen, zu geringe Beträge oder gänzlich ausbleibende Unterhaltsleistungen handelt, als auch wie häufig diese Schwierigkeiten auftreten. Kritisch ist an dieser Stelle jedoch einmal mehr auf die Schwierigkeiten der Erfassung finanzieller Aspekte in der Umfrageforschung zu verweisen. Fraglich ist, ob eine differenziertere Erhebung nicht stärkere Ausfälle bewirken würde. 
Tab. 12.1: Überblick der theoretisch denkbaren und tatsächlich erfassten Variablen nach Datensätzen.

\begin{tabular}{|c|c|c|c|c|}
\hline \multirow{2}{*}{\multicolumn{2}{|c|}{\begin{tabular}{l|l} 
& \\
ABHÄNGIGE VARIABLEN
\end{tabular}}} & \multicolumn{3}{|c|}{ Datensätze } \\
\hline & & \multirow[t]{2}{*}{ FS } & \multirow[t]{2}{*}{ BS } & \multirow[t]{2}{*}{ NV } \\
\hline \multirow{7}{*}{$\begin{array}{l}\text { Care } \\
\text { Kontakt } \\
\text { (Quantität) }\end{array}$} & & & & \\
\hline & Häufigkeit des Kontakts & + & + & $(+)$ \\
\hline & Dauer des Kontakts & - & + & - \\
\hline & Letzter Kontakt & - & + & + \\
\hline & Veränderung des Kontaktes & - & + & - \\
\hline & Zufriedenheit mit Kontakt & - & + & + \\
\hline & Gründe für Kontaktmangel (kurzfristig) & - & + & $(+)$ \\
\hline \multirow{5}{*}{$\begin{array}{l}\text { Beziehung } \\
\text { (Qualität) }\end{array}$} & Andere Kontaktformen (Telefon, Post,...) & - & + & + \\
\hline & Aktivitäten & + & + & + \\
\hline & Urlaub & + & + & - \\
\hline & Partizipation an wichtigen Entscheidungen & + & + & - \\
\hline & Alltagsbeteiligung & - & + & - \\
\hline Cash & & FS & BS & NV \\
\hline \multirow[t]{6}{*}{ Unterhaltspraxis } & Zahlungen? Ja/Nein & - & + & - \\
\hline & Höhe der Zahlungen & + & + & + \\
\hline & Zahlungsprobleme ja/nein & + & + & + \\
\hline & Art der Zahlungsprobl. (Regelmäßigkeit, Häufigkeit, Höhe) & - & $(+)$ & - \\
\hline & Unterhaltsvorschuss & + & n.r. & + \\
\hline & Finanzieller Anteil des anderen Elternteils & - & $(+)$ & - \\
\hline \multirow{4}{*}{$\begin{array}{l}\text { "freiwillige"/ } \\
\text { andere } \\
\text { Leistungen }\end{array}$} & Häufigkeit informeller Zahlungen & - & - & - \\
\hline & Höhe informeller Zahlungen & - & + & - \\
\hline & $\begin{array}{l}\text { Andere Formen materieller Unterstützung (z.B. Geschenke, } \\
\text { Kleidung) }\end{array}$ & - & + & - \\
\hline & Reisekosten & - & - & + \\
\hline \multirow{2}{*}{\multicolumn{2}{|c|}{$\begin{array}{l}\text { UNABHÄNGIGE VARIABLEN } \\
\text { Vater }\end{array}$}} & FS & BS & NV \\
\hline & & & & \\
\hline \multirow[t]{3}{*}{ Charakteristika } & Alter & + & + & + \\
\hline & $\begin{array}{l}\text { Familienorientierte Einstellungen (z.B. Vaterschaftskonzept, } \\
\text { Familien- vs. Berufsorientierung, Bedeutung der Familie) }\end{array}$ & - & - & - \\
\hline & Wohnort (Stadt/Land, Nord/Süd/Ost/West) & + & $(+)$ & + \\
\hline \multirow[t]{8}{*}{ Ressourcen } & Einkommen & + & + & + \\
\hline & Arbeitsmarktbeteiligung: Arbeitszeit & - & + & + \\
\hline & Arbeitsmarktbeteiligung: Karriereorientierung & - & - & - \\
\hline & Sozialleistungen & - & + & + \\
\hline & Bildungsniveau & + & + & + \\
\hline & Zeitverwendung (Arbeit, Freizeit, Familie) & - & - & - \\
\hline & Neue Partnerschaft (Kohabitation und/oder Ehe) & + & + & - \\
\hline & Haushaltszusammensetzung & $(+)$ & + & $(-)$ \\
\hline \multicolumn{2}{|l|}{ KINDSMUTTER } & FS & BS & NV \\
\hline \multirow[t]{5}{*}{ Ressourcen } & Erwerbstätigkeit & + & + & + \\
\hline & Ökonomische Situation & - & + & + \\
\hline & Inanspruchnahme Sozialhilfe & - & + & - \\
\hline & Neue Partnerschaft & + & + & + \\
\hline & Unterhalt vom Kindsvater & + & + & - \\
\hline
\end{tabular}

+ = erfasst;

(+) = z.T. erfasst;

$(-)=$ indirekt/rudimentär erfasst,

- $\quad$ = nicht erfasst;

n.r. $=$ nicht relevant

$\mathrm{FS}=$ Forsa-Studie;

BS = Studie von Bradshaw und Mitarbeiter;

NV = NOVA-Studie

Quelle: eigene Darstellung. 
Tab. 12.1 (Forts.): Überblick der theoretisch denkbaren und tatsächlich erfassten Variablen nach Datensätzen.

\begin{tabular}{|c|c|c|c|c|}
\hline \multicolumn{2}{|c|}{ BEZIEHUNG DER ELTERN VOR DER TRENNUNG } & \multirow{2}{*}{$\begin{array}{c}\text { FS } \\
+ \\
\end{array}$} & \multirow{2}{*}{$\begin{array}{c}\text { BS } \\
+ \\
\end{array}$} & \multirow{2}{*}{$\begin{array}{c}\text { NV } \\
+ \\
\end{array}$} \\
\hline & Lebensform (Kohabitation und/oder Ehe) & & & \\
\hline & Haushaltszusammensetzung & - & - & - \\
\hline & Arbeitsteilung & - & - & - \\
\hline & Dauer der Beziehung & - & + & + \\
\hline \multicolumn{2}{|c|}{ BEZIEHUNG DER ELTERN WÄHREND DER TRENNUNG } & FS & BS & NV \\
\hline & Konfliktniveau & $(+)$ & $(-)$ & $(+)$ \\
\hline \multirow{4}{*}{$\begin{array}{l}\text { Aushandlung } \\
\text { Cash }\end{array}$} & Zahlungsvereinbarung (Ja/Nein) & + & + & + \\
\hline & Aushandlung des Unterhalts (mit/ohne Hilfe) & + & + & + \\
\hline & Gründe für Nicht-Regelung & + & - & - \\
\hline & Zahlungen trotz Nicht-Regelung & + & $(+)$ & - \\
\hline \multirow[t]{6}{*}{ Regelung Cash } & Niveau der Regelung (Höhe, Häufigkeit) ( $\neq$ Praxis) & + & + & + \\
\hline & Art der Regelung (fester Betrag/Anteil) & + & - & - \\
\hline & Zufriedenheit mit Unterhaltsregelung & - & + & + \\
\hline & Gründe für Unzufriedenheit & - & + & + \\
\hline & Zeitpunkt der Regelung & + & - & - \\
\hline & Erfahrung mit Behörden & $(-)$ & + & $(-)$ \\
\hline \multirow{2}{*}{$\begin{array}{l}\text { Aushandlung } \\
\text { Care }\end{array}$} & Aushandlung des Sorgerechts & + & - & - \\
\hline & Aushandlung des Umgangsrechts & - & - & + \\
\hline \multirow{3}{*}{ Regelung Care } & Sorgerechtsarrangement & + & - & - \\
\hline & Veränderungen des Sorgerechts über die Zeit & - & - & - \\
\hline & Form des vereinbarten Umgangs & - & - & + \\
\hline \multicolumn{2}{|c|}{ BEZIEHUNG DER ELTERN NACH DER TRENNUNG } & FS & BS & NV \\
\hline & Dauer seit der Trennung & + & + & + \\
\hline & Freundschaftliches Verhältnis & - & + & + \\
\hline & Entfernung zwischen elterlichen Wohnorten & + & + & + \\
\hline \multicolumn{2}{|l|}{ SONSTIGES } & FS & BS & NV \\
\hline \multirow[t]{3}{*}{ Kind/er } & Geschlecht & + & + & + \\
\hline & Alter & + & + & + \\
\hline & Anzahl & + & + & + \\
\hline \multirow[t]{3}{*}{ Soziales Umfeld } & Herkunftsfamilie (Großeltern, Geschwister) & - & + & - \\
\hline & Weiteres Verwandtschaftssystem & - & - & - \\
\hline & Soziales Netz (Freunde, Nachbarn, Kollegen) & - & $(-)$ & - \\
\hline \multicolumn{5}{|c|}{$\begin{array}{ll}+ & =\text { erfasst; } \\
(+) & =\text { z.T. erfasst; } \\
(-) & =\text { indirekt/rudimentär erfasst } \\
- & =\text { nicht erfasst; } \\
\text { n.r. } & =\text { nicht relevant } \\
\text { FS } & =\text { Forsa-Studie; } \\
\text { BS } & =\text { Studie von Bradshaw und Mitarbeiter; } \\
\text { NV } & =\text { NOVA-Studie }\end{array}$} \\
\hline \multicolumn{5}{|c|}{ Quelle: eigene Darstellung. } \\
\hline
\end{tabular}

Über die Zahlungsschwierigkeiten hinaus liefert der britische Datensatz einen hilfreichen Ansatz zur Ausdifferenzierung der Zahlungspraxis. Neben der Höhe der Unterhaltsleistungen und den Zahlungsproblemen, d.h. dem Abweichen von Zahlungsvereinbarungen, finden sich hier Angaben zu „informalen“ - wie es die Autoren selbst nennen finanziellen Unterstützungsleistungen. Dazu zählen materielle Gaben die nicht formal vereinbart wurden, wie z.B. in Form von Geschenken, Einkaufstouren, Ansparungen für das Kind oder eines zusätzlichen Taschengeldes während der Besuche. Dieses finanzielle Sorgehandeln wird in den anderen beiden Datensätzen nicht berücksichtigt. Es stellt sich die 
Frage, inwiefern sich stärker freiwillig motivierte finanzielle Aufwendungen vom formalen Unterhalt unterscheiden. Werden sie eventuell als Kompensation ausbleibender Unterhaltszahlungen genutzt oder additiv geleistet?

Für die Analyse väterlichen Sorgehandelns in Form von Care und Cash insgesamt wäre es interessant den Ist- und Soll-Zustand der Sorgepraxis zu vergleichen. Mit Blick auf die finanzielle Unterstützung werden deutsche Väter gefragt, wie viel Unterhalt vereinbart wurde. Wie viel sie tatsächlich zahlen, wird darüber hinaus nicht erfasst. Ähnliche Überlegungen gelten auch für die Kontakthäufigkeit. In allen drei Datensätzen geben die Väter an, wie häufig sie ihre Kinder sehen bzw. wann sie das letzte Mal Kontakt gehabt haben. Es bestehen jedoch keinerlei Informationen darüber, ob dies dem vereinbarten Umgang entspricht. Interessant wäre die Differenz zwischen Anspruch - in Form von vereinbarter Regelung zwischen beiden Elternteilen - und Realität - tatsächlicher Kontaktpraxis - zu untersuchen. Warum geben Väter freiwillig mehr? Warum verbringen Väter weniger Zeit mit ihren Kindern als ihnen formal zugesprochen wird? Warum engagieren sich Väter über den vereinbarten Kontakt hinaus, und wo liegen die Grenzen dieses Mehr an Engagement?

Mit Blick auf die zu erklärenden Größen wäre ferner der Entwicklungsprozess des individuellen Handelns der Väter im Zeitverlauf von Interesse. Die vorliegende Untersuchung kann nur rein statische Ergebnisse vorweisen. Die Väter werden zu ihrem Sorgehandeln, wie es unmittelbar dem Zeitpunkt der Erhebung vorausgegangen ist, befragt. Väterliches Sorgehandeln verändert sich jedoch, wie dargestellt, in Abhängigkeit von der Lebensphase und dem Entwicklungsstand sowohl des Vaters als auch des Kindes. Eine Untersuchung des väterlichen Sorgehandelns über die gesamte Lebensspanne in Form einer Längsschnittuntersuchung würde Erkenntnisse über Unterschiede in der väterlichen Sorge zwischen verschiedenen Kindern einer Partnerschaft (erstes vs. zweites Kind), zwischen Kindern aus verschiedenen Beziehungen zu unterschiedlichen Zeitpunkten im Lebenslauf (erste vs. zweite Elternschaft) oder zwischen Kindern, die einen unterschiedlichen Status aufweisen (leibliche vs. adoptierte vs. soziale Kinder) liefern.

Neben zusätzlichen Aspekten des zu erklärenden Phänomens väterlichen Sorgehandelns in Nachtrennungsfamilien können ferner mit Hilfe des theoretischen Grundmodells weitere Erklärungsfaktoren benannt werden, die in keiner der drei Untersuchungen zu finden sind. Dazu zählt zunächst eine (stärkere) Erfassung der inneren Bedingungen. Die Prädiktoren, die hier dem Überzeugungssystem zugeordnet werden (die ökonomische Situation der Mutter, Dauer seit der Trennung und Zahl der Elternschaften), weisen nur sehr begrenzt Zusammenhänge mit dem väterlichen Sorgehandeln in Form von Care und Cash auf. Einstellungen des Vaters werden in der Thesentestung - aufgrund der Datenlage - nicht direkt berücksichtigt. Ein Teil des inneren Überzeugungssystems, der hier von besonderer Be- 
deutung sein könnte, ist z.B. das individuelle Vaterschaftskonzept des Befragten. Es wird auf die Bedeutung makro-sozialer Vaterleitbilder innerhalb nationaler Familienpolitiken ebenso verwiesen, ${ }^{238}$ wie auf die Entwicklung verschiedener Typologien von Vaterschaftskonzepten in der Literatur. ${ }^{239}$ Es stellt sich die Frage, welche individuellen Vaterschaftskonzepte für Nachtrennungsväter handlungsrelevant sind. Wichtig für die Erklärung des Sorgehandelns könnte sein, wie die Männer sich selbst als Vater - nach der elterlichen Trennung - verstehen, wie sie ihre Funktion innerhalb der Familie und für ihre Kinder definieren. Sehen sie ihre Aufgabe primär in der finanziellen Unterstützung in Form eines Ernährers oder teilen sie das Verständnis der sog. „neuen“ Väter, die darüber hinaus auch Betreuung und Erziehung als ihre Aufgabe betrachten? Damit könnten eventuell einige der nationalen Unterschiede im väterlichen Sorgehandeln erklärt werden. Vielleicht sehen sich norwegische Väter eher als caring fathers als z.B. deutsche oder britische Väter, die sich primär als Versorger konzeptionalisieren? Mit dem individuell vertretenen Ernährermodell eng verbunden ist die generelle Familienorientierung des Mannes, d.h. welche Prioritisierung von Lebensbereichen er vornimmt. In welchem Verhältnis stehen jeweils die individuelle Karriere-, Freizeit- und Familienorientierung? Berufliche Karriereplanung hängt eng zusammen mit familialen Lebenszielen.

Die jeweiligen Einstellungen wirken sich somit auf die Ausgestaltung väterlichen Sorgehandelns in der alltäglichen Praxis aus (Herlth 2002: 592), dennoch ist beides nicht gleichzusetzen. Empirische Untersuchungen in den unterschiedlichsten sozialwissenschaftlichen Themenbereichen verweisen immer wieder auf die Diskrepanz zwischen Einstellungen und dem tatsächlichen Handeln. Neben den Einstellungen wäre es daher interessant, wie der Nachtrennungsvater über seine familialen Verpflichtungen hinaus seine Zeit ausgestaltet. Auf die Bedeutung der Ressource Zeit wird im theoretischen Modell ausführlich hingewiesen. ${ }^{240}$ Im britischen und norwegischen Datensatz wird in diesem Zusammenhang die individuelle Arbeitszeit des Vaters berücksichtigt. Darüber hinaus wäre jedoch die Zeitverwendung für andere Aktivitäten, wie Hausarbeit oder Freizeitgestaltung von Interesse.

Die vorangehenden Ausführungen zur Erweiterung der Datensätze um individuelle Einstellungen und Zeitverwendungen verweisen auf eine weitere wichtige Erklärungsgröße

\footnotetext{
238 Siehe ausführlich Kap. 6.2.1 Institutioneller Kontext deutscher, britischer und norwegischer (Nachtrennungs-)Väter.

${ }^{239}$ Im Detail dazu Kap. 3.1 (Nachtrennungs-)Väter in der wissenschaftlichen Literatur.

${ }^{240}$ Vergleiche Kap. 4.2.2.1 Die physische Umwelt: Ressourcen und Restriktionen.
} 
väterlichen Sorgehandelns nach der elterlichen Trennung. Das jeweilige Vaterschaftskonzept sowie die individuelle Familien- und Karriereorientierung entstehen nicht erst mit dem Scheitern der elterlichen Partnerschaft. Sie bestehen bereits vor der Trennung. Zwar können sich dadurch Routinen z.B. aufgrund eines fehlenden familialen Alltags verändern und auch Prioritäten können sich in dieser Lebensphase wandeln, dennoch ist das väterliche Sorgehandeln sowie damit verbundene familiale Einstellungen vor der Trennung von Bedeutung für die Sorgepraxis danach. Wenn der Vater v.a. die Funktion des „Versorgers“ inne hat, dann ist er bereits vor der Trennung zum größten Teil physisch vom Alltag des Kindes ausgeschlossen und besitzt eher die Rolle eines Feierabend- und Wochenend-Vaters. Bei einer egalitären Arbeitsteilung nimmt der Vater stärker am Kindsalltag teil (Amendt 2004: 164; Palkovitz 1997: 214). Ein Vater, der vor dem Verlassen des gemeinsamen Haushalts eine enge Bindung zu seinem Kind aufgebaut hat, wird diese wahrscheinlicher auch nach der Trennung intensiv pflegen als ein Vater, der zuvor die Betreuung und Erziehung seiner Kinder primär der Partnerin überlassen hat. Damit wäre es für die Analyse väterlichen Sorgehandelns in Nachtrennungsfamilien wichtig zu erfassen, wie die Arbeitsteilung beider Partner vor der Trennung ausgesehen hat. ${ }^{241}$ Die geschlechtsspezifische Arbeitsteilung kann ferner nationale Unterschiede in die Analyse integrieren. So wird, wie dargestellt, die Gleichstellung von Männern und Frauen in Norwegen bereits länger (sozial)politisch forciert, als dies z.B. in Deutschland oder Großbritannien der Fall ist. Dies könnte sich - wenn auch mit Einschränkungen, wie empirische Untersuchungen zeigen (Skrede 2001: 8; Kitterød/Kjeldstad 2003: 49) - im Handeln norwegischer Väter niederschlagen. Insgesamt - so zeigen die vorliegenden empirischen Ergebnisse - sollte generell die Beziehung zwischen den Eltern vor, während und nach der Trennung systematisch berücksichtigt werden.

Die Aspekte der Arbeitsteilung vor der Trennung, individuelle Einstellungen zur Vaterschaft sowie der Zeitverwendung der Nachtrennungsväter verweisen auf einen Ansatzpunkt für weiterführende Untersuchungen väterlichen Sorgehandelns generell. So könnte es sinnvoll sein, das Handeln von Vätern in unterschiedlichen Familienformen zu vergleichen. Der Forschungsstand weist darauf hin, dass Väter und die „Neuartigkeit“ ihres Handelns zunehmend wissenschaftliches Interesse weckt. Wenig Kenntnisse bestehen dabei über die reine Zeitverwendung hinaus, wie das individuelle Vaterschaftskonzept in die Praxis umgesetzt wird. Interessant wäre in diesem Zusammenhang eine systematische Untersuchung, wie sich väterliches Sorgehandeln in Form von Care und Cash in verschiedenen Familienarrangements erklären und unterscheiden lässt. Dabei wäre z.B. an

${ }^{241}$ Eine Längsschnittstudie würde diese Perspektive ermöglichen. 
einen Vergleich verschiedener Familienformen zu denken. Als Vergleichseinheiten kämen dabei z.B. die sog. Zwei-Eltern- bzw. Kernfamilien mit beiden biologischen Elternteilen in einer Haushaltsgemeinschaft, die sog. „soziale“ Vaterschaft, d.h. ein Mann, der mit einer neuen Partnerin und ihren Kindern zusammenlebt und Nachtrennungsfamilien als getrennt gelebte Vaterschaft, wie sie in der vorliegenden Arbeit untersucht werden, in Frage. Insbesondere in Familien mit beiden leiblichen Eltern in einem Haushalt oder Familien mit sozialer Vaterschaft ist die Differenzierung und Ausgestaltung von Care und Cash - ohne die starke gesetzliche Trennung von Unterhaltspflichten und Sorgerechten wie bei getrennt lebenden Vätern - interessant. Daraus kann abgeleitet werden, wie stark Männer im Alltag immer noch als Ernährer handeln und wie sie ihre sozio-emotionale Fürsorge darüber hinaus ausgestalten.

Ziel der Analyse war primär die Bestimmung der Determinanten väterlichen Sorgehandelns. Dabei wurde anhand eines individuellen Handlungsmodells Hypothesen formuliert, die die Einflussfaktoren benennen. Makro-sozialen Einflüssen, so die theoretisch fundierte Hypothese, kam dabei eine vorstrukturierende Bedeutung zu. Die Ergebnisse legen die Vermutung nahe, dass die nationalen Kontexte, eine größere Rolle spielen als zunächst angenommen. Welche Dimensionen dieser Kontexte wie auf das väterliche Sorgehandeln wirken, kann mit Hilfe der vorliegenden Daten nicht näher geprüft werden. Unter den erhobenen Variablen finden sich wenige, die nationalstaatlichen Besonderheiten z.B. des elterlichen Trennungsprozesses abbilden. Diese sind v.a. im britischen Datensatz kaum zu finden. Generell weisen die Variablen, die die Festlegung von Unterhalt und Sorge- bzw. Umgangsrechten erfassen, in allen drei Studien Defizite auf. Künftige Untersuchungen sollten ihre forschungsleitende Perspektive verstärkt auf die Erklärung nationaler Unterschiede lenken. Die Berücksichtigung gleicher Aspekte sowohl auf Seiten des väterlichen Sorgehandelns als auch auf Seiten der Erklärungsgrößen in verschiedenen nationalen Kontexten erleichtert die ländervergleichende Perspektive.

Die vergleichende Umfrageforschung befindet sich im Spagat zwischen vielen Fällen auf der Mikro- und wenigen Fällen auf der Makroebene (Pickel 2003: 152). Das dargestellte statistische Vorgehen umfasst eine Beziehungsanalyse auf Individualebene. Es werden Zusammenhänge zwischen individuellen Merkmalen, Eigenschaften und Handlungsweisen überprüft; einzelne Aspekte des Akteurs in Relation zueinander gesetzt. Diese Form der Analyse ermöglicht jedoch kaum strukturelle Determinanten der institutionellen Rahmenbedingungen systematisch und sinnvoll in die Berechnungen zu integrieren. Inwieweit eine Care betonende Vaterpolitik sich direkt auf das individuelle Handeln aus- 
wirkt, kann empirisch nur schwer überprüft werden. ${ }^{242}$ Dies basiert nicht zuletzt auf der fehlenden Erfassung der Aspekte innerhalb der Umfrageforschung. Doch Informationen der Aggregatebene können auf der individuellen Analyseebene nutzbar gemacht werden, indem kulturelle und strukturelle Umweltbedingungen als Kollektivumfeld des Einzelnen in ihrer Bedeutung für individuelle Einstellungen und Handlungen einbezogen werden. Hier wäre z.B. an gepoolte Individualregressionen unter Berücksichtigung der Länder als Dummyvariable oder den Ländern zugeordnete Strukturvariablen zu denken. Die sog. Länderdummies geben darüber Aufschluss, inwieweit sich die Häufigkeitsverteilung der zu erklärenden Variable aufgrund von nationalen Charakteristika signifikant voneinander unterscheiden (ebd.: 169f.). Bei einer ausreichend großen Zahl an untersuchten Nationen könnten auf diese Weise Ländereffekte quantifiziert werden. ${ }^{243}$ Subjektive Vergleichsdaten gewinnen in den komparativ arbeitenden Sozialwissenschaften zunehmend an Bedeutung. Umfragedaten ermöglichen dabei, Aussagen über kulturelle Aspekte und Unterschiede zwischen Gesellschaften zu treffen und gleichzeitig „den Bürger“ in den Vergleich nationaler Kontexte einzubeziehen (ebd.: 152). Eine größere Anzahl untersuchter Länder könnte bisher vernachlässigte Nationen, z.B. aus Ost- und Südeuropa integrieren. Es wäre denkbar, dass hier andere Vaterschaftskonzepte auf nationaler aber auch auf individueller Ebene zu finden sind.

Die vorgeschlagene Erweiterung der Untersuchungseinheiten auf Länderebene und die gepoolte Regressionsanalyse zur Aufdeckung länderspezifischer Einflüsse lässt jedoch weiter offen, welche Kontextbedingungen genau die Unterschiede in den Ergebnissen verursachen (Pickel 2003: 170). Ein theoretisch fundierter, systematischer Ländervergleich bleibt unerlässlich. Makro-soziale Strukturen müssen auch theoretisch stärker systematisiert berücksichtigt werden, indem z.B. kulturelle und historische Besonderheiten in die Untersuchung integriert werden, die bisher vernachlässigt wurden (Kaufmann/Schulze 2002: 4). Die vorangestellten Ausführungen bezogen sich primär auf die politische und rechtliche Perspektive nationalstaatlicher Unterschiede. Zur Berücksichtigung makro-sozialer Einflüsse in Form von individuellen Angaben müsste der Analyse zunächst ein systematischer Ländervergleich mit umfassender Darstellung der makro-sozialen Rahmung getrennt lebender Väter vorausgehen. Es ist in der Wissenschaft anerkannt, dass die Bedeutung politischer Kontexte stark variiert und sehr komplex ist (Ellingsæter 2003: 422). So zeigen sich bereits in den vorliegenden Daten aufgrund von Reformen und damit ver-

\footnotetext{
${ }^{242}$ Siehe dazu ausführlich Kap. 6.1.2 Der Einfluss des makro-sozialen Kontexts.

${ }^{243}$ Ein Pooling der Daten in einem großen Datensatz ist aufgrund der unterschiedlichen Erfassungsmodalitäten und Datenlücken nicht möglich. Damit können Ländereffekte nicht quantifiziert werden.
} 
bundenen Übergangsregelungen unterschiedliche rechtliche Rahmenbedingungen für verschiedene Kohorten getrennt lebender Väter in einer Stichprobe. Darüber hinaus können scheinbar gleiche politische Maßnahmen je nach nationalem Kontext unterschiedliche Bedeutungen annehmen und zu verschiedenen Konsequenzen führen (Ellingsæter 2003: 423).

Eine Ergänzung des Modells muss damit sowohl auf theoretischer wie auf empirischer Ebene erfolgen. Als Beispiele können hier die bereits erwähnten individuellen Vaterschaftskonzepte oder im britischen Fall die bisher ausgebliebene und generell unterspezifizierte Erfassung der institutionellen Trennungsregelungen von Sorge- und Umgangsrecht genannt werden. Insgesamt muss auch im deutschen und norwegischen Datensatz systematischer zwischen Sorge- und Umgangsrechten, sowohl in ihrem Aushandlungsprozess wie auch in der festgelegten Form, differenziert werden. Die Erfassung der Nutzung von Institutionen im Trennungsprozess kann in allen drei Datensätzen erweitert werden. Meist wird sie lediglich dichotom (beansprucht vs. nicht beansprucht) erfasst. Offen bleibt die Ausgestaltung der Inanspruchnahme, die Beurteilung ihrer Nützlichkeit oder auch die Gründe für die (Nicht-) Beanspruchung. Neben nationalstaatlichen Unterschieden können ferner Variationen väterlichen Sorgehandelns in unterschiedlichen Subgruppen bestehen (Duncan/ Strell 2004: 42). Hier wäre an Differenzen zwischen unterschiedlichen Schichten oder regionalen Kontexten (Stadt vs. Land) zu denken.

Die vorangestellten Ausführungen - insbesondere einer Längsschnitt- bzw. Länder vergleichenden Untersuchung - erfordern jedoch hohe zeitliche und finanzielle Ressourcen, wenn alle genannten Desiderate innerhalb einer Studie Berücksichtigung finden sollen. Dennoch können auch Teilaspekte für künftige Forschungsvorhaben fruchtbar gemacht werden. Insgesamt sind noch einige Fragen zur Ausgestaltung des väterlichen Sorgehandelns sowohl innerhalb von Nachtrennungsfamilien als auch in verschiedenen makrosozialen Kontexten offen. 


\section{Anhang}

\section{A. Konstruktion der Care-Indizes}

\section{A.1. Der Care-Index der Forsa-Studie}

Tab. A.1.1: Intensität der einzelnen Aktivitäten im deutschen Care-Index

Tab. A.1.2: Rangfolge der Aktivitäten und Gewichtung im deutschen CareIndex

\section{A.2. Der Care-Index der Bradshaw-Studie}

Tab. A.2.1: Intensität der einzelnen Aktivitäten im britischen Care-Index

Tab. A.2.2: Rangfolge der Aktivitäten und Gewichtung im britischen CareIndex

Tab. A.2.3: Gewichtung der restlichen Komponenten des britischen CareIndex

\section{A.3. Der Care-Index der NOVA-Studie}

Tab. A.3.1: Intensität der einzelnen Aktivitäten im norwegischen CareIndex.

Tab. $\quad$ A.3.2: Rangfolge der Aktivitäten und Gewichtung im norwegischen Care-Index

\section{B. Häufigkeitsverteilungen der unabhängigen Variablen nach Datensatz}

Tab. B.1: Zusammensetzung der Stichprobe für die Sekundäranalyse der Forsa-Studie.

Tab. B.2: Zusammensetzung der Stichprobe für die Sekundäranalyse der Bradshaw-Studie.

Tab. 


\section{A Konstruktion der Care-Indizes}

\section{A.1. Der Care-Index der Forsa-Studie}

Tab. A.1.1: Intensität der einzelnen Aktivitäten im deutschen Care-Index.

\begin{tabular}{|c|c|c|c|c|c|c|c|c|c|c|}
\hline & $\begin{array}{l}\frac{5}{0} \\
\frac{0}{\pi} \\
\frac{0}{5} \\
\frac{\pi}{5} \\
\frac{0}{0} \\
\frac{0}{0} \\
\frac{5}{2} \\
\frac{\pi}{0}\end{array}$ & 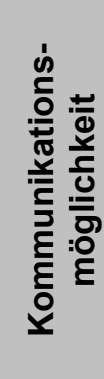 & 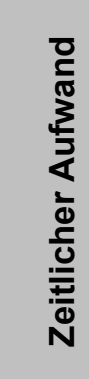 & 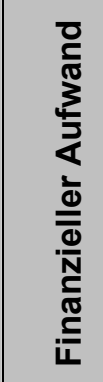 & 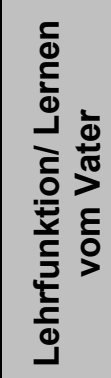 & 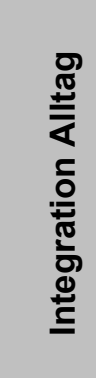 & 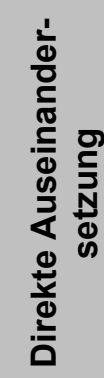 & 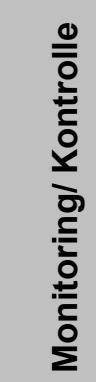 & $\begin{array}{l}\stackrel{q}{\xi} \\
\stackrel{\xi}{\xi} \\
\stackrel{⿹}{\xi}\end{array}$ & 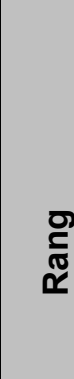 \\
\hline AktivitätlGewicht & $1 x$ & $2 x$ & $2 x$ & $1 x$ & $3 x$ & $2 x$ & $3 x$ & $3 x$ & & \\
\hline Ausflüge/ & $2-3$ & $1-2$ & $2-3$ & $2-3$ & $1-2$ & 1 & $1-2$ & 2 & 30 & 7 \\
\hline Spaziergänge & 1 & 3 & 1 & 0 & $1-2$ & 2 & 3 & 1 & 29,5 & 8 \\
\hline Spielen & $0-1$ & $1-2$ & 1 & 0 & 2 & 3 & 3 & 3 & 35,5 & 4 \\
\hline Fernsehen & 0 & 0 & $1-2$ & 0 & $0-1$ & 3 & 0 & 1 & 13,5 & 12 \\
\hline $\begin{array}{l}\text { Lernen für Schule/ } \\
\text { Hausaufgaben machen }\end{array}$ & 0 & 2 & $1-2$ & 0 & 3 & 3 & 3 & 3 & 40 & 2 \\
\hline Unterhalten & 0 & 3 & 1 & 0 & 3 & 3 & 3 & $1-2$ & 36,5 & 3 \\
\hline Gemeinsames Hobby & $2-3$ & $1-2$ & $2-3$ & $1-2$ & $1-2$ & 2 & $1-2$ & 2 & 31 & 6 \\
\hline PC spielen/arbeiten & 0 & $0-1$ & $1-2$ & 0 & 2 & 3 & 1 & $1-2$ & 23,5 & 10 \\
\hline Sport & 1 & $1-2$ & $1-2$ & $0-1$ & 1 & 2 & 2 & 2 & 26,5 & 9 \\
\hline Kino & 1 & 0 & 2 & 2 & 0 & $1-2$ & 0 & $0-1$ & 11,5 & 14 \\
\hline $\begin{array}{l}\text { Freunde/Verwandte } \\
\text { besuchen }\end{array}$ & 2 & 1 & $1-2$ & 1 & 0 & 2 & 1 & 1 & 18 & 11 \\
\hline Gemeinsame Mahlzeit & 1 & 3 & $1-2$ & 1 & $1-2$ & 3 & 2 & 2 & 33,5 & 5 \\
\hline Musik hören & 0 & $1-2$ & $1-2$ & 0 & 0 & 3 & 1 & 0 & 12 & 13 \\
\hline $\begin{array}{l}\text { Gemeinsamer Urlaub / } \\
\text { Ferien }\end{array}$ & 3 & 3 & 3 & 3 & 2 & 0 & 3 & 3 & 42 & 1 \\
\hline
\end{tabular}

Kodierung: $0=$ keine Bedeutung; $1=$ gering, $2=$ moderat, $3=$ hoch

Quelle: eigene Darstellung. 
Tab. A.1.2: Rangfolge der Aktivitäten und Gewichtung im deutschen Care-Index.

\begin{tabular}{|c|l|c|}
\hline Nr. & \multicolumn{1}{|c|}{ Aktivität } & Gewicht in Care \\
\hline 1. & Gemeinsamer Urlaub/ Ferien & 6 \\
\hline 2. & Lernen für Schule/ Hausaufgaben machen & 6 \\
\hline 3. & Unterhalten & 5 \\
\hline 4. & Spielen & 5 \\
\hline 5. & Gemeinsame Mahlzeiten & 5 \\
\hline 6. & Gemeinsames Hobby & 4 \\
\hline 7. & Ausflüge & 4 \\
\hline 8. & Spaziergänge & 4 \\
\hline 9. & Sport & 4 \\
\hline 10. & PC spielen/ arbeiten & 1 \\
\hline 11. & Freunde/ Verwandte besuchen & 1 \\
\hline 12. & Fernsehen & 1 \\
\hline 13. & Musik hören & 1 \\
\hline 14. & Kino & 1 \\
\hline
\end{tabular}

Quelle: eigene Darstellung. 


\section{A.2. Der Care-Index der Bradshaw-Studie}

Tab. A.2.1: Intensität der einzelnen Aktivitäten im britischen Care-Index

\begin{tabular}{|c|c|c|c|c|c|c|c|c|c|c|}
\hline & 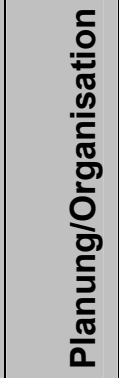 & 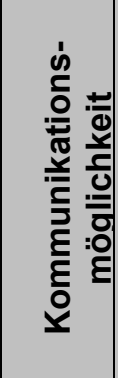 & 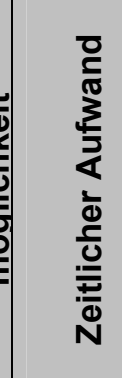 & 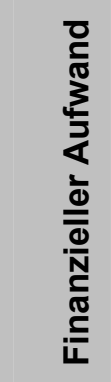 & 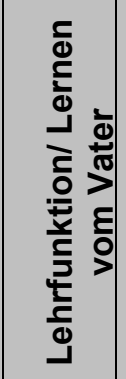 & 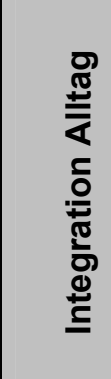 & 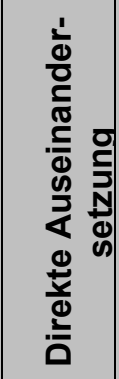 & 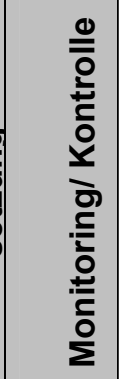 & 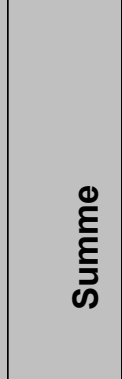 & 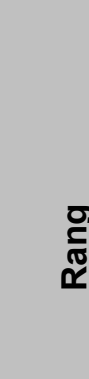 \\
\hline Aktivität|Gewicht & $1 x$ & $2 x$ & $2 x$ & $1 x$ & $3 x$ & $2 x$ & $3 x$ & $3 x$ & & \\
\hline Ausflüge & $2-3$ & $1-2$ & $2-3$ & $2-3$ & $1-2$ & 1 & $1-2$ & 2 & 30 & 4 \\
\hline Spielen & $0-1$ & $1-2$ & 1 & 0 & 2 & 3 & 3 & 3 & 35,5 & 2 \\
\hline Fernsehen & 0 & 0 & $1-2$ & 0 & $0-1$ & 3 & 0 & 1 & 13,5 & 8 \\
\hline $\begin{array}{l}\text { Besondere Freude } \\
\text { gemacht (treats) }\end{array}$ & $1-2$ & 1 & 1 & 1 & 1 & $0-1$ & 1 & 1 & 18,5 & 6 \\
\hline Einkaufen & 1 & 2 & 2 & 2 & 1 & 3 & $2-3$ & 2 & 33,5 & 3 \\
\hline Freunde/Verw.besuche & 2 & 1 & $1-2$ & 1 & 0 & 2 & 1 & 1 & 18 & 7 \\
\hline Spielfreunde zu Besuch & 2 & 1 & $1-2$ & 1 & 0 & 2 & 1 & 1 & 18 & 7 \\
\hline Sport & 1 & $1-2$ & 1-2 & $0-1$ & 1 & 2 & 2 & 2 & 26,5 & 5 \\
\hline Kino & 1 & 0 & 2 & 2 & 0 & 1-2 & 0 & $0-1$ & 11,5 & 9 \\
\hline $\begin{array}{l}\text { Gemeinsamer Urlaub / } \\
\text { Ferien }\end{array}$ & 3 & 3 & 3 & 3 & 2 & 0 & 3 & 3 & 42 & 1 \\
\hline
\end{tabular}

Kodierung: $0=$ keine Bedeutung; $1=$ gering, $2=$ moderat, $3=$ hoch

Quelle: eigene Darstellung. 
Tab.A.2.2: Rangfolge der Aktivitäten und Gewichtung im britischen Care-Index.

\begin{tabular}{|c|l|c|}
\hline Nr. & \multicolumn{1}{|c|}{ Aktivität } & Gewicht in Care \\
\hline 1. & Ferien & 6 \\
\hline 2. & Spiele & 5 \\
\hline 3. & Einkaufen & 4 \\
\hline 4. & Ausflüge & 4 \\
\hline 5. & Sport & 4 \\
\hline 6. & Besondere Freude gemacht & 2 \\
\hline 7. & Freunde/ Verwandte besucht & 1 \\
\hline 8. & Fernsehen & 1 \\
\hline 9. & Kino & 1 \\
\hline
\end{tabular}

Quelle: eigene Darstellung.

Tab. A.2.3: Gewichtung der restlichen Komponenten des britischen Care-Index.

\begin{tabular}{|c|l|c|}
\hline Nr. & \multicolumn{1}{|c|}{ Partizipation } & Gewicht in Care \\
\hline 1. & Hausaufgabenhilfe & 6 \\
\hline 2. & Teilnahme an Schulaktivitäten & 6 \\
\hline 3. & Schulbringdienst & 2 \\
\hline 4. & Chauffeur für Kinder & 2 \\
\hline 5. & Baby Sitting & 2 \\
\hline
\end{tabular}

Quelle: eigene Darstellung. 


\section{A.3. Der Care-Index der NOVA-Studie}

Tab. A.3.1: Intensität der einzelnen Aktivitäten im norwegischen Care-Index.

\begin{tabular}{|c|c|c|c|c|c|c|c|c|c|c|}
\hline & 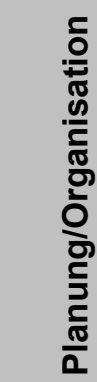 & 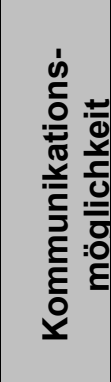 & 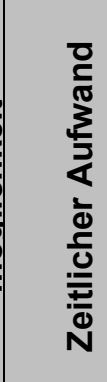 & 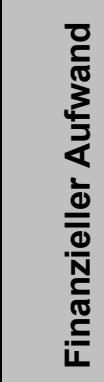 & 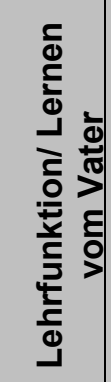 & 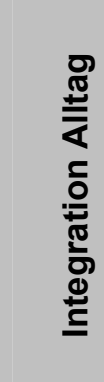 & 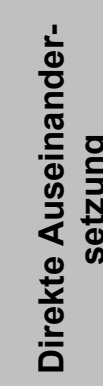 & 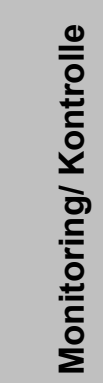 & ڤ્ & 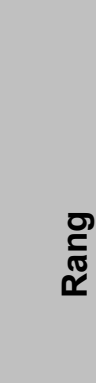 \\
\hline Aktivität|Gewicht & $1 x$ & $2 x$ & $2 x$ & $1 x$ & $3 x$ & $2 x$ & $3 x$ & $3 x$ & & \\
\hline Ausflüge & $2-3$ & $1-2$ & $2-3$ & $2-3$ & $1-2$ & 1 & $1-2$ & 2 & 30 & 5 \\
\hline Spielen & $0-1$ & $1-2$ & 1 & 0 & 2 & 3 & 3 & 3 & 35,5 & 2 \\
\hline Fernsehen & 0 & 0 & $1-2$ & 0 & $0-1$ & 3 & 0 & 1 & 13,5 & 8 \\
\hline Hausaufgaben machen & 0 & 2 & $1-2$ & 0 & 3 & 3 & 3 & 3 & 40 & 1 \\
\hline Einkaufen & 1 & 2 & 2 & 2 & 1 & 3 & $2-3$ & 2 & 33,5 & 4 \\
\hline PC spielen/Internet & 0 & $0-1$ & $1-2$ & 0 & 2 & 3 & 1 & $1-2$ & 23,5 & 7 \\
\hline Sport & 1 & $1-2$ & $1-2$ & $0-1$ & 1 & 2 & 2 & 2 & 26,5 & 6 \\
\hline Kino & 1 & 0 & 2 & 2 & 0 & $1-2$ & 0 & $0-1$ & 11,5 & 9 \\
\hline vorlesen & 0 & $1-2$ & $1-2$ & 0 & 3 & 3 & 3 & $1-2$ & 34,5 & 3 \\
\hline $\begin{array}{l}\text { Gemeinsame Mahlzeit/ } \\
\text { sonstige Hausarbeit }\end{array}$ & 1 & 3 & $1-2$ & 1 & $1-2$ & 3 & 2 & 2 & 33,5 & 4 \\
\hline
\end{tabular}

Kodierung: $0=$ keine Bedeutung; $1=$ gering, $2=$ moderat, $3=$ hoch

Quelle: eigene Darstellung. 
Tab. A.3.2: Rangfolge der Aktivitäten und Gewichtung im norwegischen Care-Index.

\begin{tabular}{|c|l|c|}
\hline Nr. & \multicolumn{1}{|c|}{ Aktivität } & Gewicht in Care \\
\hline 1. & Hausaufgaben machen & 6 \\
\hline 2. & Spielen & 5 \\
\hline 3. & vorlesen & 5 \\
\hline 4. & Gemeinsame Mahlzeiten/Hausarbeit & 4 \\
\hline 5. & Eusflüge & 4 \\
\hline 6. & Sport & 4 \\
\hline 7. & PC spielen/ Internet & 1 \\
\hline 8. & Fernsehen & 1 \\
\hline 9. & Kino & 1 \\
\hline
\end{tabular}

Quelle: eigene Darstelung. 
B. Häufigkeitsverteilungen der unabhängigen Variablen nach Datensatz.

Tab. B.1: Zusammensetzung der Stichprobe für die Sekundäranalyse der ForsaStudie.

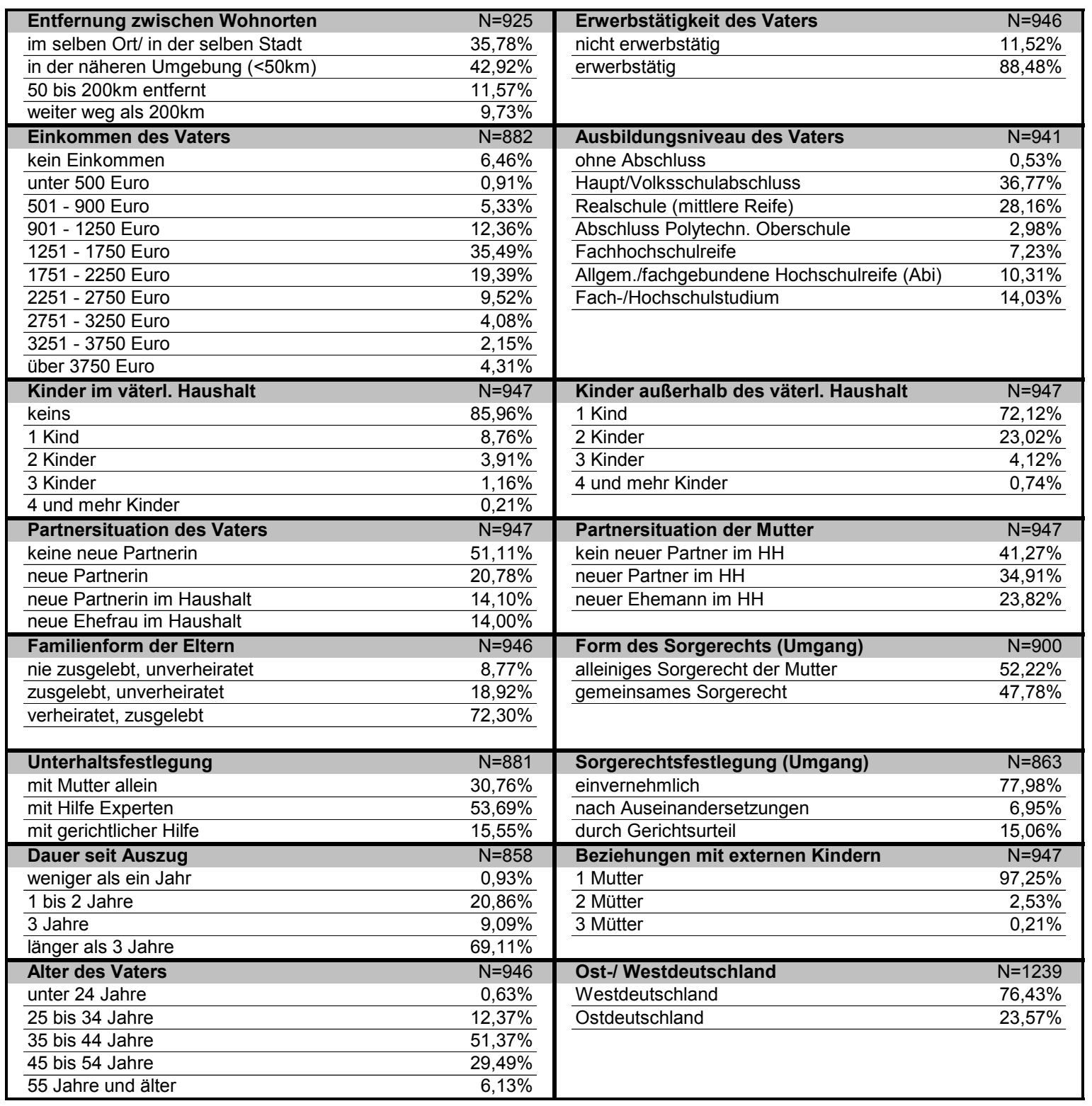

Quelle: eigene Berechnung. 
Tab. B.2: Zusammensetzung der Stichprobe für die Sekundäranalyse der BradshawStudie.

\begin{tabular}{|c|c|c|c|}
\hline Entfernung zwischen Wohnorten & $\mathrm{N}=550$ & Erwerbstätigkeit des Vaters & $\mathrm{N}=575$ \\
\hline unter $5 \mathrm{~km}$ & $38,36 \%$ & nicht erwerbstätig & $29,39 \%$ \\
\hline 5 bis $50 \mathrm{~km}$ & $39,27 \%$ & erwerbstätig & $70,61 \%$ \\
\hline 51 bis $200 \mathrm{~km}$ & $11,82 \%$ & & \\
\hline über $200 \mathrm{~km}$ & $8,18 \%$ & & \\
\hline im Ausland & $2,36 \%$ & & \\
\hline Einkommen des Vaters & $\mathrm{N}=417$ & Ausbildungsniveau des Vaters & $\mathrm{N}=533$ \\
\hline unter 500Euro & $24,94 \%$ & keine & $32,08 \%$ \\
\hline 500-900Euro & $2,64 \%$ & CSE/GCE/GCSE & $34,90 \%$ \\
\hline $900-1250$ Euro & $1,92 \%$ & ONC/Diplom & $5,07 \%$ \\
\hline 1250-1750Euro & $8,39 \%$ & BTECH/ A level & $6,94 \%$ \\
\hline 1750-2250Euro & $19,42 \%$ & HNC/ Diplom & $3,00 \%$ \\
\hline $2250-2750$ Euro & $14,39 \%$ & degree & $9,57 \%$ \\
\hline 2740-3250Euro & $11,99 \%$ & Postgraduate & $8,44 \%$ \\
\hline 3250-3750Euro & $6,24 \%$ & & \\
\hline über 3750 Euro & $2,88 \%$ & & \\
\hline Ökonomische Situation des Vaters & $\mathrm{N}=573$ & Arbeitszeit des Vaters & $\mathrm{N}=575$ \\
\hline sehr gut & $9,95 \%$ & nicht erwerbstätig & $29,39 \%$ \\
\hline gut & $18,85 \%$ & unter 30 Std & $3,30 \%$ \\
\hline ok & $42,58 \%$ & 30 bis $40 \mathrm{Std}$ & $27,30 \%$ \\
\hline schlecht & $13,79 \%$ & über 40 Std & $40,00 \%$ \\
\hline sehr schlecht & $14,83 \%$ & & \\
\hline Kinder im väterl. Haushalt & $\mathrm{N}=577$ & Kinder außerhalb des väterl. Haushalt & $\mathrm{N}=545$ \\
\hline kein Kind & $67,42 \%$ & 1 Kind & $54,13 \%$ \\
\hline ein Kind & $16,29 \%$ & 2 Kinder & $36,51 \%$ \\
\hline zwei Kinder & $10,57 \%$ & 3 Kinder & $7,34 \%$ \\
\hline drei Kinder & $3,64 \%$ & 4 und mehr Kinder & $2,02 \%$ \\
\hline vier und mehr Kinder & $2,08 \%$ & & \\
\hline Partnersituation des Vaters & $\mathrm{N}=386$ & Partnersituation der Mutter & $\mathrm{N}=507$ \\
\hline keine neue Partnerin im $\mathrm{HH}$ & $55,67 \%$ & keine neue Partnerschaft & $42,01 \%$ \\
\hline neue Partnerin im $\mathrm{HH}$ & $20,59 \%$ & neue feste Partnerschaft & $57,99 \%$ \\
\hline neue Ehefrau im $\mathrm{HH}$ & $23,73 \%$ & & \\
\hline Familienform der Eltern & $\mathrm{N}=575$ & Dauer der Beziehung & $N=560$ \\
\hline nicht zus.gelebt & $7,13 \%$ & nie zusammengelebt & $7,32 \%$ \\
\hline unverheiratet, zus.gelebt & $18,09 \%$ & unter 5 Jahren & $23,21 \%$ \\
\hline \multirow[t]{3}{*}{ verheiratet, zus.gelebt } & $74,78 \%$ & 5 bis 10 Jahre & $38,04 \%$ \\
\hline & & 11 bis 16 Jahre & $23,04 \%$ \\
\hline & & über 16 Jahre & $8,39 \%$ \\
\hline Unterhaltsfestlegung & $\mathrm{N}=416$ & Freundschaft zwischen Eltern & $\mathrm{N}=570$ \\
\hline zwischen Eltern & $42,07 \%$ & nicht freundschaftlich & $43,16 \%$ \\
\hline mit Hilfe Dritter & $57,93 \%$ & freundschaftlich & $56,84 \%$ \\
\hline Ökonomische Situation der Mutter & $\mathrm{N}=457$ & Dauer seit Auszug & $\mathrm{N}=480$ \\
\hline Komfortabler Standard & $35,67 \%$ & weniger als $1 \mathrm{Jahr}$ & $10,42 \%$ \\
\hline mittlerer Standard (doing alright) & $30,63 \%$ & 1 bis 2 Jahre & $10,21 \%$ \\
\hline zurecht kommend & $21,44 \%$ & 2 bis 3 Jahre & $9,17 \%$ \\
\hline Situation schwierig & $8,32 \%$ & länger als 3 Jahre & $70,21 \%$ \\
\hline Situation sehr schwierig & $3,94 \%$ & & \\
\hline Alter des Vaters & $\mathrm{N}=568$ & Beziehungen mit externen Kindern & $\mathrm{N}=461$ \\
\hline unter 24 Jahre & $2,99 \%$ & aus 1 Beziehung & $70,28 \%$ \\
\hline 25 bis 34 Jahre & $37,32 \%$ & aus 2 Beziehungen & $26,46 \%$ \\
\hline 35 bis 44 Jahre & $37,85 \%$ & aus 3 Beziehungen und mehr & $3,25 \%$ \\
\hline 45 bis 54 Jahre & $19,37 \%$ & & \\
\hline 55Jahre und älter & $2,46 \%$ & & \\
\hline
\end{tabular}

Quelle: eigene Berechnung. 
Tab. B.3: Zusammensetzung der Stichprobe für die Sekundäranalyse der NOVAStudie.

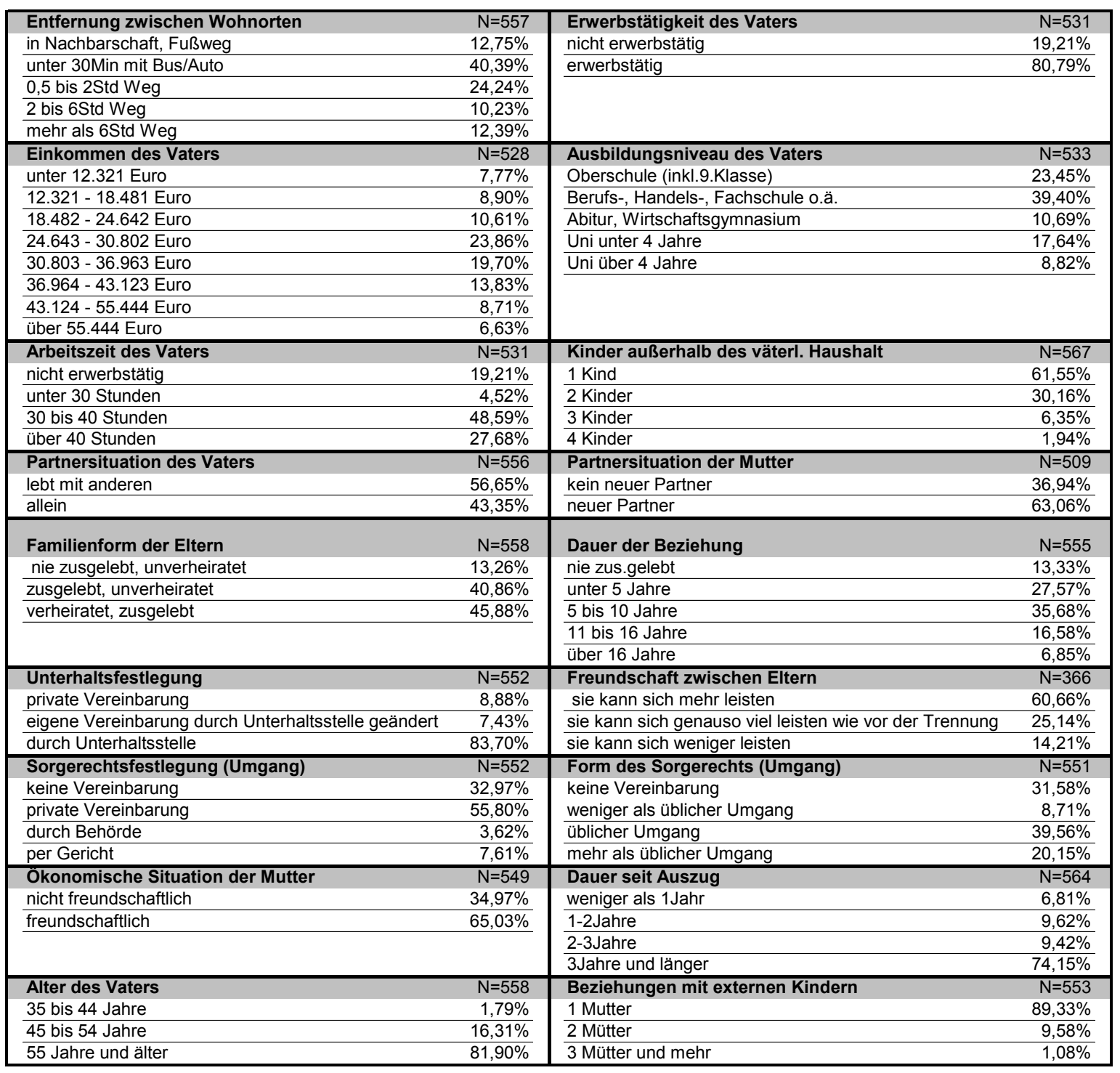

Quelle: eigene Berechnung. 


\section{Literaturverzeichnis}

Amato, Paul R. (1998): More Than Money? Men's Contribution to Their Children's Lives, in: Booth, Alan; Crouter, Ann C. (Hrsg.): Men in Families. When Do They Get Involved? What Difference Does It Make?, Nahwah, New Jersey, London: Lawrence Erlbaum Associates Publishers, S. 241-278.

Amendt, Gerhard (2004): Scheidungsväter, Band 6, Schriftenreihe des Instituts für Geschlechter- und Generationenforschung, Bremen: Universität Bremen.

Amendt, Gerhard (2003): Forschungsprojekt: Vätererfahrungen nach der Trennung vom Ehe- oder Lebenspartner, 1. Bericht, Institut für Geschlechter- und Generationenforschung, Universität Bremen, im Internet unter: http://www.g-i-sa.de/res.php?id=404 (letzter Zugang 09.2.2008).

Andreß, Hans-Jürgen; Bergloh, Barbara; Güllner, Miriam; Wilking, Katja (2003): Wenn aus Liebe rote Zahlen werden. Über die wirtschaftlichen Folgen von Trennung und Scheidung, Wiesbaden: Westdeutscher Verlag.

Andreß, Hans-Jürgen; Hagenaars, Jacques A.; Kühnel, Steffen (1997): Analyse von Tabellen und kategorialen Daten. Log-lineare Modelle, latente Klassenanalyse, logistische Regression und GSK-Ansatz, Berling (u.a.): Springer.

Babka von Gostomski, Christian (1999): Die Rolle von Kindern bei Ehescheidungen, in: Klein, Thomas; Kopp, Johannes (Hrsg.): Scheidungsursachen aus soziologischer Sicht, Familie und Gesellschaft, Band 2, Würzburg: Ergon Verlag, S. 203-232.

Backhaus, Klaus; Erichson, Bernd; Plinke, Wulff; Weiber, Rolf (2006): Multivariate Analysemethoden. Eine anwendungsorientierte Einführung, 11. Auflage, Berlin, Heidelberg, New York: Springer.

Barlow, Anne; Duncan, Simon; James, Grace (2002): New Labour, the Rationality Mistake and Family Policy in Britain, in: Carling, Alan; Duncan, Simon; Edwards, Rosalind (Hrsg.): Analysing Families. Morality and Rationality in Policy and Practice, London, New York: Routledge, S. 110-128.

Baur, Nina (2004): Kreuztabellen und Kontingenzanalyse, in: Baur, Nina; Fromm, Sabine (Hrsg.): Datenanalyse mit SPSS für Fortgeschrittene. Ein Arbeitsbuch, Wiesbaden: Verlag für Sozialwissenschaften, S. 157-190.

Becker, Gary S (1981): A Treatise on Family, Cambridge/ Ma and London: Harvard University Press. 
Becker, Gary S. (1992): Die ökonomische Sicht menschlichen Verhaltens, in: Pies, Ingo (Hrsg.): (1996): Familie, Gesellschaft und Politik - die ökonomische Perspektive, Tübingen: J.C.B. Mohr (Paul Siebeck), S. 21-49.

Becker, Gary S. (1988): Familienökonomik und Makro-Verhalten, in: Pies, Ingo (Hrsg.) (1996): Familie, Gesellschaft und Politik - die ökonomische Perspektive, Tübingen: JCB Mohr (Paul Siebeck), S. 117-136.

Becker, Gary S. (1985): Eine ökonomische Analyse der Familie, in: Pies, Ingo (Hrsg.): (1996): Familie, Gesellschaft und Politik - die ökonomische Perspektive, Tübingen: JCB Mohr (Paul Siebeck), S. 101-116.

Beckh, Katharina; Walper, Sabine (2002): Stiefkinder und ihre Beziehung zu den Eltern: Ein Fokus auf die Rolle von leiblichen und Stiefvater, in: Bien, Walter; Hartl, Angela; Teubner, Markus (Hrsg.): Stieffamilien in Deutschland. Eltern und Kinder zwischen Normalität und Konflikt, Deutsches Jugendinstitut Familien-Survey, Band 10, , Opladen: Leske und Budrich, S. 201-228.

Bennett, Fren (2006): Paying for Children: Current Issues and Implications for Policy Debates, in: Lewis, Jane (Hrsg.): Children, Changing Families and Welfare States, Cheltenham, Northampton: Edward Elgar, S. 110-134.

Bergman, Helena; Hobson, Barbara (2002): Compulsory Fatherhood: The Coding of Fatherhood in the Swedish Welfare State, in: Hobson, Barbara (Hrsg.): Making Men into Fathers: Men, Masculinities and the Social Politics of Fatherhood, Cambridge: Cambridge University Press, S. 92-124.

Bien, Walter; Hartl, Angela; Teubner, Markus (Hrsg.) (2002): Stieffamilien in Deutschland. Eltern und Kinder zwischen Normalität und Konflikt, Deutsches Jugendinstitut Familien-Survey, Band 10, Opladen: Leske und Budrich.

Björnberg, Ulla (2006): Paying for the Costs of Children in 8 North European Countries: Ambivalent Trends, in: Lewis, Jane (Hrsg.): Children, Changing Families and Welfare States, Cheltenham, Northampton: Edward Elgar, S. 90-109.

Booth, Alan; Crouter, Ann C. (Hrsg.) (1998): Men in Families. When Do They Get Involved? What Difference Does It Make?, Nahwah/ New Jersey, London: Lawrence Erlbaum Associates, Publishers.

Bradshaw, Jonathan; Stimson, Carol (1996): Non Resident Fathers, [computer file], Colchester, Essex: UK Data Archive [distributor], SN: 3978.

Bradshaw, Jonathan; Stimson, Carol; Skinner, Christiane; Williams, Julie (1999): Absent Fathers?, London, New York: Routledge. 
Bradshaw, Jonathan; Hatland, Aksel (Hrsg.) (2006): Social Policy, Employment, and Family Change in Comparative Perspektive, Cheltenham, Northhampton.

Brandth, Berit; Kvande, Elin (2003): Father presence in childcare, in: Jensen, An-Magritt; McKee, Lorna (Hrsg.): Children and the Changing Family. Between Transformation and Negotiation, Future of Childhood series, London, New York: Routledge, S. 6176.

Braver, Sanford L.; Wolchik, Sharlene A.; Sandler, Irwin N.; Sheets, Virgil ,L. (1993): A Social Exchange Model of Nonresidential Parent Involvement, in: Depner, Charlene E., Bray, James H. (Hrsg.): Non-residential parenting: New Vistas in Family Living, Newsbury Park, London, New Delhi: Sage Publications, S. 87-108.

Bray, James H.; Berger, Sandra H. (1993): Non-residential Parent-Child Relationships Following Divorce and Remarriage. A Longitudinal Perspective, in: Depner, Charlene E., Bray, James H. (Hrsg.): Non-residential parenting: New Vistas in Family Living, Newsbury Park, London, New Delhi: Sage Publications, S. 156-181.

Brosius, Felix (1998): professionelle Statistik unter Windows, Bonn: International Thomson Publishing.

Brüderl, Josef (2006): Was kann familiensoziologische Theorie?, in: Zeitschrift für Familienforschung, Jg. 18, Heft 2/2006, S. 206-211.

Brüderl, Josef (2004): Die Überprüfung von Rational-Choice-Modellen mit Umfragedaten, in: Diekmann, Andreas ; Voss, Thomas (Hrsg.): Rational Choice Theorie in den Sozialwissenschaften, München: Oldenbourg, S. 163-180.

Bühl, Achim; Zöfel, Peter (2005): Einführung in die moderne Datenanalyse unter Windows, 9. Auflage, München: Pearson Studium.

Bühl, Walter L (1994): Institution, in: Fuchs-Heinritz, Werner; Lautmann, Rüdiger; Rammstedt, Otthein; Wienold, Hanns (Hrsg.): Lexikon zur Soziologie, 3. Auflage, Opladen: Westdeutscher Verlag, S. 302-303.

Bühl, Walter L (1988): Institution, in: Fuchs, Werner; Klima, Rolf; Lautmann, Rüdiger; Rammstedt, Otthein; Wienold, Hanns (Hrsg.): Lexikon zur Soziologie, 2.Auflage, Opladen: Westdeutscher Verlag, S. 345-346.

BuMinJFG (Bundesministerium für Jugend, Familie und Gesundheit) (1977): Zur Situation Alleinstehender. Repräsentativerhebung des emnid-Instituts, Bielefeld: EMNID.

Burkart, Günter (1994): Die Entscheidung zur Elternschaft. Eine empirische Kritik von Individualisierungs- und Rational-Choice-Theorien, Stuttgart: Ferdinand Enke Verlag. 
Cabrera, Natasha J.; Tamis-LeMonda, Catherine S.; Bradley, Robert H.; Hofferth, Sandra; Lamb, Michael E. (2000): Fatherhood in the Twenty-First Century, in: Child Development, 2000, 71 (1), S. 127-136.

Cheal, David (2002): Sociology of Family Life, New York: Palgrave.

Clarke, Lynda; Henwood, Melanie (1997): Great Britain: The Lone Parent as the New Norm?, in: Kaufmann, Franz-Xaver; Kuijsten, Anton; Schulze, Hans-Joachim; Strohmeier, Klaus Peter (Hrsg.): Family Live and Family Policies in Europe. Volume 1. Structures and Trends in the 1980, Oxford: Clarendon Press, S. 155-94.

Clarke, Lynda; Roberts, Ceridwen (2002): Policy and Rhetoric. The Growing Interest in Fathers and Grandparents in Britain, in: Carling, Alan; Duncan, Simon; Edwards, Rosalind (Hrsg.): Analysing Families. Morality and Rationality in Policy and Practice, London, New York: Routledge, S. 165-182.

Clasen, Jochen (2005): Reforming European Welfare States. Germany and the United Kingdom compared, Oxford: University Press.

Cohen, Theodore F. (1993): What Do Fathers Provide? Reconsidering the Economic and Nurturant Dimensions of Men as Parents, in: Hood, Jane (Hrsg.): Research on Men and Masculinities, Newsbury Park, London, New Delhi: Sage Publications, S. 1-22.

Coleman, James (1990): Foundations of Social Theory, Cambridge Mass.: Belknap/Harvard University Press.

Corden, Anne (2001): Comparing Child Maintenance Systems: Conceptual and Methodological Issues, in: International Journal Social Research Methodology, 4 (4), S. 287-300.

Corden, Anne (1999): Making Child Maintenance Regimes Work, Family and Parenthood: Policy \& Practice, Joseph Rowntree Foundation, London: Family Policy Studies Centre.

Crompton, Rosemary (1999): Familienpolitik. Eine praxisorientierte Gesamtdarstellung der Grundlagen, Handlungsfelder und Probleme, Weinheim, München: Juventa Verlag.

Crow, Graham (2002): Families, Moralities, Rationalities and Social Change, in: Carling, Alan; Duncan, Simon; Edwards, Rosalind (Hrsg.): Analysing Families. Morality and Rationality in Policy and Practice, London, New York: Routledge, S. 285-296.

Daly, Mary (2004): Changing Conceptions of Family and Gender Relations in European Welfare States and the Third Way, in: Lewis, Jane; Surrender, Rebecca (Hrsg.): Welfare State Change. Towards a Third Way?, Oxford: Oxford University Press, S. 135-154. 
Depner, Charlene E.; Bray, James H. (1993b): Nonresidential Parenting. Multidimensional Aproaches in Research, Policy, and Practice, in: Depner, Charlene E., Bray, James H. (Hrsg.): Non-residential parenting: New Vistas in Family Living, Newsbury Park, London, New Delhi: Sage Publications, S. 182-201.

Depner, Charlene E.; Bray, James H. (Hrsg.) (1993a): Nonresidential parenting: new vistas in family living, Newsbury Park, London, New Delhi: Sage Publications.

Dethloff, Nina; Martiny, Dieter (2004): National Report Germany concerning the CEFL Questionaire on Parental Responsibility, im Internet unter: http://www2.law.uu.nl/priv/cefl/Reports/pdf2/Germany.pdf (letzter Zugang 09.2.2008).

Diekmann, Andreas (2000): Empirische Sozialforschung. Grundlagen, Methoden, Anwendungen, Hamburg: Rowohlt.

Dienel, Christiane (2002): Parental Leave. A Short Guide for Employers and Employees, URN No. 06/566, im Internet unter: www.dti.gov.uk/employment/employmentlegislation/employment-guidance/page18480.html (letzter Zugang 05.10.2007).

Döge, Peter (2006): Männer - Paschas und Nestflüchter? Zeitverwendung von Männern in der Bundesrepublik, Opladen: Barbara Budrich.

Dollahite, D. C.; Hawkins, A. J.; Brotherson, S. E. (1997): Fatherwork: A Conceptual Ethic of Fathering as Generative Work, in: Hawkins, Alan J.; Dollahite, David C. (Hrsg.): Generative Fathering: Beyond Deficit Perspectives, Thousand Oaks, London, New Delhi: Sage Publications, S. 17-35.

Donzelot, Jacques (1979): The Policing of Families: Welfare versus the State, London: Hutchinson.

Dorbritz, Jürgen (2004): Kinderwünsche in Europa. Keine Kinder mehr gewünscht?, in: BiB-Mitteilungen 25 (3), S.10-17, im Internet unter: http://www.bibdemographie.de/publikat/bib-mit3_2004.pdf (letzter Zugang 09.2.2008).

Drinck, Barbara (2005): Vatertheorien. Geschichte und Perspektive, Opladen: Barbara Budrich.

Duncan, Simon; Strell, Monika (2004): Combining Lone Motherhood and Paid Work: The Rationality Mistake and Norwegian Social Policy, in: Journal of European Social Policy, Vol 14 (1), S. 41-54.

Ellingsæter, Anne Lise (1999): Dual Breadwinners between State and Market, in: Crompton, Rosemary (Hrsg.): Restructuring Gender Relations and Employment. The Decline of the Male Breadwinner, Oxford: Oxford University Press, S. 40-59. 
Ellingsæter, Anne Lise (2003): The Complexity of Family Policy Reform. The Case of Norway, in: European Societies, 5, S. 419-443.

Ellingsæter, Anne Lise; Leira, Arnlaug (2006): Epilogue: Scandinavian policies of parenthood - a success story?, in: Ellingsæter, Anne Lise; Leira, Arnlaug (Hrsg.): Politicising Parenthood in Scandinavia. Gender Relations in Welfare States, Bristol: Policy Press, S. 265-277.

Erler, Wolfgang (2006): Germany, in: Moss, Peter; O’Brien, Margaret (Hrsg.): International Review of Leave Policies and Related Research in 2006, Department of Trade and Industry, Employment Relations Research Series No. 57, S. 123-131, im Internet unter: www.dti.gov.uk/files/file31948.pdf (letzter Zugang 05.10.2007).

Esser, Hartmut (2006): Affektuelles Handeln: Emotionen und das Modell der FrameSelektion, in: Schützeichel, Rainer (Hrsg.): Emotionen und Sozialtheorie. Disziplinäre Ansätze, Frankfurt am Main, New York: Campus Verlag, S. 143-174.

Esser, Hartmut (2004): Sinn, Kultur und „Rational Choice“, in: Jaeger, Friedrich; Straubhaar, Jürgen (Hrsg.): Handbuch der Kulturwissenschaften, Band 2, Paradigmen und Disziplinen, Stuttgart [u.a.]: Metzler, S. 249-265.

Esser, Hartmut (2002): Ehekrisen: Das (Re-)Framing der Ehe und der Anstieg der Scheidungsraten, in: Zeitschrift für Soziologie, Jg. 31, Heft 6, Dezember 2002, S. 472-496.

Esser, Hartmut (2001): Soziologie. Spezielle Grundlagen, Bd. 6: Sinn und Kultur, Frankfurt Main, New York: Campus.

Esser, Hartmut (2000c): Soziologie. Spezielle Grundlagen, Bd. 5: Institutionen, Frankfurt Main, New York: Campus.

Esser, Hartmut (2000b): Soziologie. Spezielle Grundlagen, Bd. 4: Opportunitäten und Restriktionen, Frankfurt Main, New York: Campus.

Esser, Hartmut (2000a): Soziologie. Spezielle Grundlagen, Bd. 3, Soziales Handeln, Frankfurt Main, New York: Campus.

Esser, Hartmut (1999): Soziologie. Spezielle Grundlagen, Bd. 1: Situationslogik und Handeln, Frankfurt Main, New York: Campus. 
Eydal, Guðný Björk (2006): Policies and Caring Fathers in the Nordic Countries, Paper presented at the WELLCHI Network Conference 2: "Well-being of Children and Labour Markets in Europe. Different Kinds of Risks Resulting from Various Structures and Changes in the Labour Markets”, 31.03.-01.04.2006, Centre for Globalisation and Governance, University of Hamburg, im Internet unter: www.ciimu.org/webs/wellchi/conference_2/streamb/bjork.pdf (letzter Zugang 11.10.2007).

Eydal, Guðný Björk (2005): Childcare Policies of the Nordic Welfare States: Different Paths to Enable Parents to Earn and Care?, in: Pfau-Effinger, Birgit; Geissler, Birgit (Hrsg.): Care and Social Integration in European Societies, Bristol: Policy Press, S. 153-172.

Federkeil, Gero (1997): The Federal Republic of Germany: Polarization of Family Structure, in: Kaufmann, Franz-Xaver; Kuijsten, Anton; Schulze, Hans-Joachim; Strohmeier, Klaus Peter (Hrsg.): Family Live and Family Policies in Europe, Volume 1, Structures and Trends in the 1980, Oxford: Clarendon Press, S. 77-113.

Feldhaus, Michael; Huinink, Johannes (2005): Längsschnittliche Beziehungs- und Familienforschung. Darstellung eines Forschungsprogramms, in: Busch, Friedrich W.; Nave-Herz, Rosemarie (Hrsg.): Familie und Gesellschaft. Beiträge zur Familienforschung, Oldenburg: Bibliotheks- und Informationssystem der Carl von Ossietzky Universität Oldenburg (BIS), S. 187-205.

Ferrarini, Tommy (2006): Families, States and Labour Marktets. Institutions, Causes and Consequences of Family Policy in Post-War Welfare States, Cheltenham, Northampton: Edward Elgar.

Finch, Naomi (2003): Family Policy in the UK, third report for the project 'Welfare Policies and Employment in the Context of Family Change', im Internet unter: www.york.ac.uk/inst/spru/research/nordic/ukpolicy.pdf (letzter Zugang 26.10.2007).

Folbre, Nancy (1994): Who Pays for the Kids? Gender and the Structure of Constraints, London, New York: Routledge.

Forsa (Gesellschaft für Sozialforschung und statistische Analysen) (2002): Unterhaltszahlungen für minderjährige Kinder in Deutschland, Bundesministerium für Familie, Senioren, Frauen und Jugend, in: Schriftenreihe Band 228, S. .

Forste, Renate (2002): Where Are All the Men? A Conceptual Analysis of the Role of Men in Family Formation, in: Journal of Family Issues, 23 (5), July 2002, S. 579-600.

Fthenakis, Wassilos (1999): Engagierte Vaterschaft: die sanfte Revolution in der Familie, LBS-Initiative Junge Familie, Opladen: Leske und Budrich. 
Fthenakis, Wassilos E. (2002): Mehr als Geld? Zur (Neu-)Konzeptionalisierung väterlichen Engagements, in: Fthenakis, Wassilos E.; Textor, Martin R. (Hrsg.): Mutterschaft, Vaterschaft, Weinheim, Basel: Beltz Verlag, S. 90-119.

Fthenakis, Wassilos E.; Kalicki, Bernhard; Peitz, Gabriele (2002): Paare werden Eltern. Ergebnisse Der LBS-Familien-Studie, Opladen: Leske und Budrich.

Funder, Kathleen; Harrison, Margaret; Weston, Ruth (Hrsg.) (1993): Settling Down. Pathways of Parents after Divorce, Melbourne: Australian Institute of Family Studies.

Fux, Beat (2002): Which Models of the Family are Encouraged or Discouraged by Different Family Policies, in: Kaufmann, Franz-Xaver; Kuijsten, Anton; Schulze, HansJoachim; Strohmeier, Klaus Peter (Hrsg.): Family Live and Family Policies in Europe, Volume 2, Problems and Issues in Comparative Perspective, Oxford: Clarendon Press, S. 363-418.

Gerlach, Irene (2004): Familienpolitik, Wiesbaden: VS Verlag für Sozialwissenschaften.

Glover, Judith (2002): The 'Balance Model'. Theorising Women's Employment Behaviour, in: Carling, Alan; Duncan, Simon; Edwards, Rosalind (Hrsg.): Analysing Families. Morality and Rationality in Policy and Practice, London, New York: Routledge, S. 251-267.

Goldscheider, Frances K.; Kaufman, Galye (1996): Fertility and Commitment: Bringing Men Back In, in: Population and Development Review, Vol. 22, Supplement: Fertility in the United States: New Patterns, S. 87-99.

Greshoff, Rainer; Schimank, Uwe (2003): Die integrative Sozialtheorie von Hartmut Esser, im Internet unter: http://www.fernuni-hagen.de/SOZ/ (letzter Zugang 09.2.2008).

Greve, Bent (2000): Family policy in the Nordic countries, in: Pfenning, Astrid; Bahle, Thomas (Hrsg.): Families and Family Policies in Europe. Comparative Perspectives, Frankfurt/Main, Berlin, Bern, Brüssel, New York, Oxford, Wien: Peter Lang, Europäischer Verlag der Wissenschaften, S. 90-103.

Hantrais, Linda (1999): Comparing Family Policies in Europe, in: Clasen, Jochen (Hrsg.): Comparative Social Policy: Concepts, Theories and Methods, Oxford, Malden: Blackwell Publishers, S. 95-113.

Hantrais, Linda; Lohkamp-Himmighofen, Marlene (1999): Changing Family Forms, Law and Policy, Fifth Series: Interaction between Socio-Demographic Change, Social and Economic Policies in the European Union, Leicestershire: Cross-National Research Group. 
Harrison, Margaret (1993): Patterns of Maintenance Payment over Time, in: Funder, Kathleen; Harrison, Margaret; Weston, Ruth (Hrsg.): Settling Down. Pathways of Parents after Divorce, Melbourne: Australian Institute of Family Studies, S. 116134.

Hartl, Angela (2002b): Die Beziehung des Stiefkindes zu seinem außerhalb lebenden Elternteil, in: Bien, Walter; Hartl, Angela; Teubner, Markus (Hrsg.): Stieffamilien in Deutschland. Eltern und Kinder zwischen Normalität und Konflikt, DJI: FamilienSurvey, Band 10, Opladen: Leske und Budrich, S. 177-197.

Hartl, Angela (2002a): Zur Lebenssituation von Stiefkindern, in: Bien, Walter; Hartl, Angela; Teubner, Markus (Hrsg.): Stieffamilien in Deutschland. Eltern und Kinder zwischen Normalität und Konflikt, DJI: Familien-Survey, Band 10, Opladen: Leske und Budrich, S. 147-175.

Hatland, Aksel; Mayhew, Emese (2006): Parental Obligations, in: Bradshaw, Jonathan; Hatland, Aksel (Hrsg.): Social Policy, Employment and Family Change in Comparative Perspective, Northampton: Edward Elgar, S. 79-95.

Herlth, Alois (2002): Ressourcen der Vaterrolle. Familiale Bedingungen der Vater-KindBeziehung, in: Walter, Heinz (Hrsg.): Männer als Väter: Sozialwissenschaftliche Theorie und Empirie, Gießen: Psychosozial-Verlag, S. 585-608.

Hildenbrand, Bruno (2002): Der abwesende Vater als strukturelle Herausforderung in der familialen Sozialisation, in: Walter, Heinz (Hrsg.): Männer als Väter: Sozialwissenschaftliche Theorie und Empirie, Gießen: Psychosozial-Verlag, S. 743672.

Hill, Paul B. (2005): Methodenprobleme in der Ehe- und Familiensoziologie, in: Busch, Friedrich W.; Nave-Herz, Rosemarie (Hrsg.): Familie und Gesellschaft. Beiträge zur Familienforschung, Oldenburg: Bibliotheks- und Informationssystem der Carl von Ossietzky Universität Oldenburg (BIS), S. 165-186.

Hill, Paul B.; Kopp, Johannes (2004): Familiensoziologie. Grundlagen und theoretische Perspektiven, 3. überarbeitete Auflage, Wiesbaden: Verlag für Sozialwissenschaften.

Hill, Paul B.; Kopp, Johannes (1999): Ehescheidung: Historische Entwicklungen und theoretischen Erklärungen, in: Klein, Thomas; Kopp, Johannes (Hrsg.): Scheidungsursachen aus soziologischer Sicht, Familie und Gesellschaft, Band 2, Würzburg: Ergon Verlag, S. 23-42.

Himmelweit, Susan (2002): Economic Theory, Norms and the Care Gap or: Why Do Economists Become Parents?, in: Carling, Alan; Duncan, Simon; Edwards, Rosalind (Hrsg.): Analysing Families. Morality and Rationality in Policy and Practice, London, New York: Routledge, S. 231-250. 
Hobson, Barbara (Hrsg.) (2002): Making Men into Fathers - Men, Masculinities and the Social Politics of Fatherhood, Cambridge: Cambridge University Press.

Hobson, Barbara; Duvander, Ann-Zofie; Halldén, Karin (2006): Men and Women’s Agency and Capabilities to Create a Worklife Balance in Diverse and Changing Institutional Contexts, in: Lewis, Jane (Hrsg.): Children, Changing Families and Welfare States, Cheltenham, Northampton: Edward Elgar, S. 267-295.

Hobson, Barbara; Morgan, David (2002): Introduction, in: Hobson, Barbara (Hrsg.): Making Men into Fathers: Men, Masculinities and the Social Politics of Fatherhood, Cambridge: Cambridge University Press, S. 1-21.

Jaursch, Stefanie (2003): Erinnertes und aktuelles Erziehungsverhalten von Müttern und Vätern: Intergenerationale Zusammenhänge und kontextuelle Faktoren, InauguralDissertation in der Philosophischen, München: Fakultät I der Friedrich-AlexanderUniversität Erlangen-Nürnberg.

Jensen, An-Magritt (2006): Fatherhood - Between Ideology and Demography, in: Varanka, Jouni; Forslund, Maria (Hrsg.): Possibilities and Challenges? Men’s Reconciliation of Work and Family Life - Conference Report, Norden: Nordic Council of Ministers, S. 37-45.

Jensen, An-Magritt (1999): Partners and Parents in Europe: A Gender Divide, in: Leira, Arnlaug (Hrsg.): Family Change: Practices, Policies, and Values. Comparative Social Research, Vol. 18, Stanford: Family Change Jai Press, S. 1-29.

Kalicki, Bernhard; Peitz, Gabriele; Fthenakis, Wassilos F (2002): Subjektive Elternschaftskonzepte und faktische Rollenausübung: Theoretische Überlegungen und empirische Befunde, in: Fthenakis, Wassilos F.; Textor, Martin R. (Hrsg.): Mutterschaft, Vaterschaft, Weinheim, Basel: Beltz Verlag, S. 170-183.

Kaufmann, Franz-Xaver (2002): Politics and Policies towards the Family in Europe: A Framework and an Inquiry into their Differences and Convergences, in: Kaufmann, Franz-Xaver; Kuijsten, Anton; Schulze, Hans-Joachim; Strohmeier, Klaus Peter (Hrsg.): Family Live and Family Policies in Europe. Volume 2. Problems and Issues in Comparative Perspective, Oxford: Clarendon Press, S. 419-490.

Kaufmann, Franz-Xaver; Schulze, Hans-Joachim (2002): Comparing Family Life in the Frame of National Policies: An Introduction, in: Kaufmann, Franz-Xaver; Kuijsten, Anton; Schulze, Hans-Joachim; Strohmeier, Klaus Peter (Hrsg.): Family Live and Family Policies in Europe. Volume 2. Problems and Issues in Comparative Perspective, Oxford: Clarendon Press, S. 1-18. 
Kilkey, Majella (2004): The 'Citizen-Worker' Model and Assumptions about Men, Work and Care, Paper contributed to the stream "Family Policy in European Countries: Assumptions and Outcomes”, ESPAnet conference, European Social Policy: Meeting the Needs of a New Europe, 9.-11.09.2004, University of Oxford, Oxford, UK, im Internet unter: www.apsoc.ox.ac.uk/Espanet/espanetconference/ papers/ppr.10A.MK.pdf (letzter Zugang 05.10.2007).

Kilkey, Mariella (2000): Mothers Between Paid Work and Care. The policy regime in twenty countries, Aldershot, Burlington USA, Singapore, Sydney: Ashgate.

Kitterød, Ragni Hege; Kjeldstad, Randi (2003): A new father's role? Employment patterns among Norwegian fathers in 1991 - 2001, in: Economic Survey, 1/2003, im Internet unter: www.ssb.no/english/subjects/08/05/10/es/200301/kitterod.pdf (letzter Zugang 26.10.2007), S. $39-51$.

Kjeldstad, Randi (2000): Employment Strategy Policies and Lone Parenthood: The Case of Norway, in: Social Politics: International Studies in Gender, State \& Society, Volume 7, Number 3, S. 343-371.

Knijn, Trudie (1995): Hat die Vaterschaft noch eine Zukunft? Eine theoretische Betrachtung zu veränderter Vaterschaft, in: Ambruster, Christof; Müller, Ursula; Stein-Hilbers, Marlene (Hrsg.): Neue Horizonte? Sozialwissenschaftliche Forschung über Geschlechter und Geschlechterverhältnisse, Geschlecht und Gesellschaft, Band 1, Opladen: Leske und Budrich, S. 171-192.

Knijn, Trudie; Ostner, Ilona; Schmitt, Christoph (2007): Männer und (ihre) Kinder. Einstellungen zur Elternschaft im Ländervergleich, in: Lange, Andreas; Lettke, Frank (Hrsg.): Generationen und Familien. Analysen - Konzepte - gesellschaftliche Spannungsfelder, Frankfurt/Main: Suhrkamp, S. 189-222.

Knijn, Trudie; Selten, Peter; (2002): Transformations of fatherhood: The Netherlands, in: Hobson, Barbara (Hrsg.): Making Men into Fathers: Men, Masculinities and the Social Politics of Fatherhood, Cambridge: Cambridge University Press, S. 168-187.

Kolbe, Wiebke (2002): Elternschaft im Wohlfahrtsstaat. Schweden und die Bundesrepublik im Vergleich 1945-2000, Frankfurt, New York: Campus Verlag.

Kolbe, Wiebke (2001): Vaterschaftskonstruktionen im Wohlfahrtsstaat: Schweden und die Bundesrepublik in historischer Perspektive, in: Döge, Peter; Meuser, Michael (Hrsg.): Männlichkeit und soziale Ordnung. Neuere Beiträge zur Geschlechterforschung, Opladen: Leske und Budrich, S. 183-199.

Kudera, Werner (2002): Neue Väter, neue Mütter - neue Arrangements der Lebensführung, in: Walter, Heinz (Hrsg.): Männer als Väter: Sozialwissenschaftliche Theorie und Empirie, Gießen: Psychosozial-Verlag, S. 145-185. 
Kühnel, Steffen; Bamberg, Sebastian (1998): Überzeugungssysteme in einem zweistufigen Modell rationaler Handlungen. Das Beispiel umweltgerechteren Verkehrsverhaltens, in: Zeitschrift für Soziologie, Jg. 27, Heft 4, 4.8.1998, S. 256-270.

Kühnel, Steffen-M; Krebs, Dagmar (2001): Statistik für die Sozialwissenschaften. Grundlagen, Methoden, Anwendungen, Hamburg: Rowohlt.

Kuijsten, Anton; Schulze, Hans-Joachim (1997): The Netherlands: The Latent Family, in: Kaufmann, Franz-Xaver; Kuijsten, Anton; Schulze, Hans-Joachim; Strohmeier, Klaus Peter (Hrsg.): Family Live and Family Policies in Europe. Volume 1. Structures and Trends in the 1980s, Oxford: Clarendon Press, S. 253-301.

Kuijsten, Anton; Strohmeier, Klaus Peter (1997): Ten Countries in Europe: An Overview, in: Kaufmann, Franz-Xaver; Kuijsten, Anton; Schulze, Hans-Joachim; Strohmeier, Klaus Peter (Hrsg.): Family Live and Family Policies in Europe. Volume 1. Structures and Trends in the 1980s, Oxford: Clarendon Press, S. 394-423.

Kunz, Volker (2004): Rational Choice, Frankfurt, New York: Campus Verlag.

Lamb, Michael (1987): The Father's Role: Cross-Cultural Perspectives, Hillsdale/New Jersey: Erlbaum.

Lammi-Taskula, Johanna (2006): Nordic Men on Parental Leave: Can the Welfare State Change Gender Relations?, in: Ellingsæter, Anne Lise; Leira, Arnlaug (Hrsg.): Politicising Parenthood in Scandinavia. Gender Relations in Welfare States , Bristol: Policy Press, S. 79-99.

Lange, Andreas (2003): Konturen des „postfordistischen Papas“, in: ZSE, 23, Jg. 2003, Heft 3, S. 320-322.

Leip, Evelyn (2004): Geburtenentwicklung und gesellschaftliche Struktur. Zur Reichweite von Rational Choice-Ansätzen am Beispiel des generativen Verhaltens, Inauguraldissertation zur Erlangung des Akademischen Grades eines Dr. phil., vorgelegt dem Fachbereich 12 Sozialwissenschaften der Universität Mainz, im Internet unter: http://deposit.ddb.de/cgi-bin/dokserv?idn=971995109\&dok_var =d1\&dok_ext=pdf\&filename=971995109.pdf (letzter Zugang 09.2.2008).

Leira, Arnlaug (2006): Parenthood Change and Policy Reform in Scandinavia, 1970s 2000s, in: Ellingsæter, Anne Lise; Leira, Arnlaug (Hrsg.): Politicising Parenthood in Scandinavia. Gender Relations in Welfare States , Bristol: Policy Press, S. 27-51.

Leira, Arnlaug (1999): Introduction, in: Leira, Arnlaug (Hrsg.): Family Change: Practices, Policies, and Values. Comparative Social Research, Vol. 18, Stanford: Jai Press, S. ix-xxii. 
Leira, Arnlaug (1996): Parents, Children and the State: Family Obligations in Norway, ISF Report 96: 23, Oslo: Institutt for Samfunnsforskning (ISF).

Leitner, Sigrid (1999): Frauen und Männer Im Wohlfahrtsstaat: Zur Strukturellen Umsetzung von Geschlechterkonstruktionen in sozialen Sicherungssystemen, Frankfurt am Main: Peter Lang, Europäischer Verlag der Wissenschaften.

Leitner, Sigrid; Ostner, Ilona; Schratzenstaller, Margit (Hrsg.) (2003): Wohlfahrtsstaat und Geschlechterverhältnis im Umbruch, Jahrbuch 7 des Zentrum für Europa- und Nordamerikastudien, Wiesbaden: VS Verlag für Sozialwissenschaften.

Leitner, Sigrid; Ostner, Ilona; Schratzenstaller, Margit (2003): Einleitung: Was kommt nach dem Ernährermodell? Sozialpolitik zwischen Re-Kommodifizierung und ReFamilisierung, in: Leitner, Sigrid; Ostner, Ilona; Schratzenstaller, Margit (Hrsg.): Wohlfahrtsstaat und Geschlechterverhältnis im Umbruch. Was kommt nach dem Ernährermodell, Jahrbuch für Europa- und Nordamerika-Studien, 7, Wiesbaden: VS Verlag für Sozialwissenschaften, S. 9-27.

Lewis, Jane (2003): Auf dem Weg zur „Zwei-Erwerbstätigen"-Familie, in: Leitner, Sigrid; Ostner, Ilona; Schratzenstaller, Margit (Hrsg.): Wohlfahrtsstaat und Geschlechterverhältnis im Umbruch. Was kommt nach dem Ernährermodell, Jahrbuch für Europa- und Nordamerika-Studien, 7, Wiesbaden: VS Verlag für Sozialwissenschaften, S. 62-84.

Lewis, Jane (2002): The Problem of Fathers: Policy and Behavior in Britain, in: Hobson, Barbara (Hrsg.): Making Men into Fathers: Men, Masculinities and the Social Politics of Fatherhood, Cambridge: Cambridge University Press, S. 125-149.

Lewis, Jane (2001): Orientations to Work and the Issue of Care, in: Millar, Jane; Rowlingson, Karen (Hrsg.): Lone parents, employment and social policy. Crossnational comparisons, Bristol: The Policy Press, S. 153-168.

Lewis, Jane (1997): Lone Mothers: The British Case, in: Lewis, Jane (Hrsg.): Lone Mothers in European Welfare Regimes. Shifting Policy Logics, London/ Philadelphia: Jessica Kingsley Publishers, S. 50-75.

Lindenberg, Siegwart (1981): Erklärung als Modellbau: Zur soziologischen Nutzung von Nutzentheorien, in: Schulte, Werner (Hrsg.): Soziologie in der Gesellschaft, Bremen: Universität Bremen, S. 20-35.

Lødrup, Peter; Sverdrup, Tone (2004): National Report Norway concerning the CEFL Questionaire on Parental Responsibility, im Internet unter: http://www2.law.uu.nl/priv/cefl/Reports/pdf2/Norway.pdf (letzter Zugang 09.2.2008). 
Lowe, Nigel (2004): National Report England \& Wales concerning the CEFL Questionaire on Parental Responsibility, im Internet unter: http://www2.law.uu.nl/priv/ cefl/Reports/pdf2/England.pdf (letzter Zugang 09.2.2008).

Marsiglio, William (1991): Paternal Engagement Activities with Minor Children, in: Journal of Marriage and the Family 53 (November 1991), S. 973 - 986.

Marsiglio, William; Amato, Paul; Day, Randal D.; Lamb, Michael E. (2000): Scholarship on Fatherhood in the 1990s and Beyond, in: Journal fo Marriage and the Familiy 62, No. 1, (November 2000), S. 1173-1191.

Marten, Carina (2007a): Aktivierungspotential Alleinerziehende? Das Reziprozitätsverhältnis zwischen allein erziehenden Müttern und dem Wohlfahrtsstaat, in: Marten, Carina; Scheuregger, Daniel (Hrsg.): Reziprozität und Wohlfahrtsstaat. Anlysepotential und sozialpolitische Relevanz, LeverkusenOpladen: Barbara Budrich, S. 195-226.

Marten, Carina (2007b): Towards a European Father Model? The Role of Fathers Within Family Policies in Germany, Great Britain and Norway, Artikel präsentiert auf dem ESPAnet Young Researchers Workshop “The European Social Model and Beyond”, Goettingen, December 6th - 8th, 2007, unveröffentlicht.

Martin, Claude (1995): Father, Mother and the Welfare State. Family and Social Transfers after Marital Breakdown, in: Journal of European Social Policy (1995), 5 (1), S. $43-63$.

Matzner, Michael (2004): Vaterschaft aus der Sicht von Vätern, Wiesbaden: Verlag für Sozialwissenschaften.

Matzner, Michael (1998): Vaterschaft heute: Klischees und soziale Wirklichkeit, Frankfurt/Main, New York: Campus Verlag.

Mau, Steffen (2002): Wohlfahrtsregime als Reziprozitätsarrangements. Versuch einer Typologisierung, in: Berliner Journal Soziologie, Heft 3, 2002, S. 345-364.

May, Christina (2007): Reziprozität zwischen Generationen im Wohlfahrtsstaat, in: Marten, Carina; Scheuregger, Daniel (Hrsg.): Reziprozität und Wohlfahrtsstaat. Anlysepotential und sozialpolitische Relevanz, Leverkusen-Opladen: Barbara Budrich, S. 147-171.

McClelland, David (1961): The Achieving Society, Princeton NJ: Van Nostrand.

Meyer, Traute (2005): Political Actors and the Modernisation of Care Policies in Britain and Germany, in: Pfau-Effinger, Birgit; Geissler, Birgit (Hrsg.): Care and Social Integration in European Societies, Bristol: Policy Press, S. 281-305. 
Miebach, Bernhard (2006): Soziologische Handlungstheorien. Eine Einführung, 2. Auflage, Wiesbaden: VS Verlag für Sozialwissenschaften.

Millar, Jane (1999): Obligations and Autonomy in Social Welfare, in: Crompton, Rosemary (Hrsg.): Restructuring Gender Relations and Employment. The Decline of the Male Breadwinner, Oxford: Oxford University Press, S. 26-39.

Moch, Matthias (2002): Beziehungen zwischen geschiedenen Vätern und ihren erwachsenen Töchtern, in: Walter, Heinz (Hrsg.): Männer als Väter: Sozialwissenschaftliche Theorie und Empirie, Gießen: Psychosozial-Verlag, S. 643683.

Morgan, David (2002): Sociological Perspectives on the Family, in: Carling, Alan; Duncan, Simon; Edwards, Rosalind (Hrsg.): Analysing Families. Morality and Rationality in Policy and Practice, London, New York: Routledge, S. 147-164.

Moss, Peter; O’Brien, Margaret (2006): United Kingdom, in: Moss, Peter; O’Brien, Margaret (Hrgs.): International Review of Leave Policies and Related Research in 2006, Department of Trade and Industry, Employment Relations Research Series No. 57 , im Internet unter: www.dti.gov.uk/files/file31948.pdf (letzter Zugang 05.10.2007, 217-228).

Napp-Peters, Anneke (1995): Familien nach der Scheidung, München: Verlag Antje Kunstmann.

Nauck, Bernhard (1989): Individualistische Erklärungsansätze in der Familienforschung: Die Rational-Choice Basis von Familienökonomie, Ressourcen- und Austauschtheorie, in: Nave-Herz, Rosemarie; Markefka, Manfred (Hrsg.): Handbuch der Familien- und Jugendforschung, Band 1: Familienforschung, Neuwied, Frankfurt am Main: Luchterhand, S. 45-61.

Nave-Herz, Rosemarie (1989): Gegenstandsbereich und historische Entwicklung der Familienforschung, in: Nave-Herz, Rosemarie; Markefka, Manfred (Hrsg.): Handbuch der Familien- und Jugendforschung, Band 1: Familienforschung, Neuwied und Frankfurt/M: Luchterhand Verlag, S. 1-17.

NIA (National Insurance Administration) (2007): The Rights of Parents of Small Children in Norway, im Internet unter: www.regjeringen.no/upload/BLD/ Veiledning\%20og\%20brosjyrer/2007/270636_the_rights_of_parents_of_small_child ren_2007_english.pdf (letzter Zugang 26.10.2007).

Niedermayer, Oskar; Widmaier, Ulrich (2003): Quantitativ vergleichende Methoden, in: Berg-Schlosser, Dirk/Müller-Rommel, Ferdinand (Hrsg.): Vergleichende Politikwissenenschaft: ein einführendes Studienbuch, Opladen: Leske \& Budrich, S. 77-101. 
O’Brien, Margaret (2006): Fathers, Family Life and Work: Can Fathers Have It All?, Paper presented at the WELLCHI Network Conference 2 "Well-being of Children and Labour Markets in Europe. Different Kinds of Risks Resulting from Various Structures and Changes in the Labour Markets”, 31.03.-01.04.2006, Centre for Globalisation and Governance, University of Hamburg, im Internet unter: www.ciimu.org/webs/wellchi/conference_2/streamb/brien.pdf (letzter Zugang 05.10.2007).

Oláh, Livia; Bernhardt, Eva M.; Goldscheider, Frances K. (2002): Co residential Paternal Roles in Industrialized Countries: Sweden, Hungary and the United States, in: Hobson, Barbara (Hrsg.): Making Men into Fathers: Men, Masculinities and the Social Politics of Fatherhood, Cambridge: Cambridge University Press, S. 25-67.

Opielka, Michael (2004): Sozialpolitik. Grundlagen und vergleichende Perspektiven, Hamburg: Rowohlt.

Opp, Karl-Dieter (1999): Contending Conceptions of the Theory of Rational Action, in: Journal of Theoretical Politics, 11 (2), S. 171-202.

Opp, Karl-Dieter (1986): Das Modell des Homo Sociologicus. Eine Explikation und eine Konfrontation mit dem utilitaristischen Verhaltensmodell, in: Analyse \& Kritik, Jg. 8, Heft 2, S. 1-27.

Ostner, Ilona (2007): Pflichten von Eltern und Kindern im Wohlfahrtsstaat - Aktuelle Trends und vergleichende Perspektiven, in: Marten, Carina; Scheuregger, Daniel (Hrsg.): Reziprozität und Wohlfahrtsstaat. Anlysepotential und sozialpolitische Relevanz, Leverkusen-Opladen: Barbara Budrich, S. 225-242.

Ostner, Ilona (2004): What are children for? Reciprocity and solidarity between the generation, in: Knijn Trudie Knijn; Komter, Afke (Hrsg.): Solidarity between the generations, Cheltenham: Edward Elgar, S. 167-184.

Ostner, Ilona (2002): A new role for fathers? The German case, in: Hobson, Barbara (Hrsg.): Making Men into Fathers: Men, Masculinities and the Social Politics of Fatherhood, Cambridge: Cambridge University Press, S. 150-167.

Ostner, Ilona (1997): Lone Mothers - the Case of Germany, in: Lewis, Jane (Hrsg.): Lone Mothers in the European Union, London: Jessica Kingsley, S. 21-49.

Ostner, Ilona; Kupka, Peter; Raabe, Cerstin (1995): Wege in die Ehe - Bilanzierungen bei Spätheiratenden, in: Nauck, Bernhard; Onnen-Isemann (Hrsg.): Familie im Brennpunkt von Wissenschaft und Forschung, Neuwied: Luchterhand, S. 419-436. 
Ostner, Ilona; Reif, Michael; Turba, Hannu; Schmitt, Christoph (2003): Family Policies in Germany, third report for the project 'Welfare Policies and Employment in the Context of Family Change', Drafted for the meeting October 8th - 9th in Utrecht, the Netherlands, im Internet unter: www.york.ac.uk/inst/spru/research/nordic/ gerpoli.PDF (letzter Zugang 26.10.2007).

Ostner, Ilona; Schmitt, Christoph (2008): Family Policies in the Context of Famliy Change. The Nordic Countries in Comparative Perspective, Wiesbaden: VS Verlag für Sozialwissenschaften.

Palkovitz, Rob (1997): Reconstructing Involvement. Expanding Conceptualizations of Men's Caring in Contemporary Families, in: Hawkins, Alan J.; Dollahite, David C. (Hrsg.): Generative Fathering: Beyond Deficit Perspectives, Thousand Oaks, London, New Delhi: Sage Publications, S. 200-216.

Pickel, Gert (2003): Die Verwendung von Individualdaten zum Nationenvergleich: Anmerkungen und Beispiele aus der vergleichenden Forschung, in: Pickel, Susanne; Pickel, Gert; Lauth, Hans-Joachim; Jahn, Detlef (Hrsg.): Vergleichende Politikwissenschaftliche Methoden. Neue Entwicklungen und Diskussionen, Wiesbaden: Westdeutscher Verlag, S. 96-115.

Ribbens McCarthy, Jane; Edwards, Rosalind (2002): The Individual in Public and Private. The Significance of Mothers and Children, in: Carling, Alan; Duncan, Simon; Edwards, Rosalind (Hrsg.): Analysing Families. Morality and Rationality in Policy and Practice, London, New York: Routledge, S. 199-217.

Rollett, Brigitta; Werneck, Harald (2002): Die Vaterrolle in der Kultur der Gegenwart und die väterliche Rollenentwicklung in der Familie, in: Walter, Heinz (Hrsg.): Männer als Väter: Sozialwissenschaftliche Theorie und Empirie, Gießen: PsychosozialVerlag, S. 323-343.

Rudolf, Matthias; Müller, Johannes (2004): Multivariate Verfahren. Eine praxisorientierte Einführung mit Anwendungsbeispielen in SPSS, Göttingen u.a.: Hogrefe Verlag.

Scheiwe, Kirsten (1999): Kinderkosten und Sorgearbeit. Eine rechtsvergleichende Studie, juristische Abhandlungen, Band XXXVI, Frankfurt am Main: Vittorio Klostermann.

Schimank, Uwe (2004): Der akteurzentrierte Institutionalismus, in: Gabriel, Manfred (Hrsg.): Paradigmen der akteurszentrierten Soziologie, Wiesbaden: VS Verlag für Sozialwissenschaften, S. 287-301.

Schmid, Michael (2004): Rationales Handeln und soziale Prozesse. Beiträge zur soziologischen Theoriebildung, Wiesbaden: VS Verlag für Sozialwissenschaften. 
Schmitt, Christian (2005): Kinderlosigkeit von Männern - Geschlechtsspezifische Determinanten ausbleibender Elternschaft, in: Tölke, Angelika; Hank, Karsten (Hrsg.): Männer - Das “vernachlässigte” Geschlecht in der Familienforschung, Wiesbaden: Verlag für Sozialwissenschaften, S. 18-43.

Schnabel, Annette (2006): Sind Emotionen rational? Emotionen als Herausforderung für Rational-Choice-Ansätze, in: Schützeichel, Reiner (Hrsg.): Schützeichel, Reiner: Emotionen und Sozialtheorie. Disziplinäre Ansätze, Frankfurt am Main, New York: Campus Verlag, S. 175-194.

Simon, Herbert A (1955): A Behavioral Model of Rational Choice, in: The Quarterly Journal of Economics 69, S. 99-118.

Simon, Herbert A. (1993): Homo rationalis. Die Vernunft im menschlichen Leben, Frankfurt/M.; New York: Campus.

Skevik, Anne (2006b): Fairness in Child Support Assessments: The Views of Non-resident Fathers in Norway, in: International Journal of law, Policy and the Family, 20 (2), S. 181-200.

Skevik, Anne (2006a): Absent fathers' or 'reorganized families'? Variations in father-child contact after parental break-up in Norway, in: The Sociological Review, 54 (1), S. 114-132.

Skevik, Anne (2003b): Family Policies in Norway, third report for the project "Welfare Policy and Employment in the Context of Family Change", drafted for the meeting 5th - 6th June in Reykjavik, Iceland, im Internet unter: www.york.ac.uk/inst/ spru/research/nordic/norwpoli.PDF (letzter Zugang 26.10.2007).

Skevik, Anne (2003a): The New Family's Vulnerable Vanguard: Child Maintenance Reform in Norway, in: Social Policy and Society 3 (1), Cambridge: Cambridge University Press, S. 11-19.

Skevik, Anne (1998): The state-parent-child relationship after familiy break-ups: Child maintenance in Norway and Britain, in: Flora, Peter (Hrsg.): The state of social welfare 1997: international studies on social insurance, retirement, employment, family policy and healthcare, International Studies on Social Security 4, Aldershot et al. : Ashgate, S. 217-238.

Skevik, Anne; Hyggen, Christer (2002): Samværsfedrenes situasjon. Rapport fra en spørreundersøkelse, Rapport 15/02, Oslo: NOVA (Norsk institutt for forskning om oppvekst, velferd og aldring. 
Skinner, Christine (1999): The Financial Obligations of Non-resident Fathers and the Implications for Social Policy, doctoral thesis at University of York, York: Department of Social Policy and Social Work.

Skocpol, Theda; Somers, Margaret (1980): The Uses of Comparative History in Macrosocial Inquiry, in: Comparative Studies in Society and History, 22, S. 174197.

Skrede, Kari (2001): The other side of the coin? Recent changes in family policy, men's family roles, family formation and family dissolution in Norway, Paper for presentation at the IUSSP XXIV General Population Conference, Salvador Brazil, 18th - 24th August, Session 33: Social policy and demographic behaviour, im Internet unter: www.iussp.org/Brazil2001/s30/S33_03_skrede.pdf (letzter Zugang 26.10.2007).

Smart, Carol; May, Vanessa (2004): Why Can't They Agree? The Underlying Complexity of Contact and Residence Disputes, in: Journal of Social Welfare and Family Law, 26 (4), S. 347-360.

Smart, Carol; Neale, Bren (1999): Family Fragments?, Cambridge: Polity Press.

Snary, John (1993): How Fathers Care for the Next Generation. A Four-Decade Study, Cambridge, London: Harvard University Press.

Streeck, Wolfgang; Thelen, Kathleen (2005): Introduction. Institutional Change in Advanced Political Economies, in: Streeck, Wolfgang; Thelen, Kathleen (Hrsg.): Beyond Continuity. Institutional Change in Advanced Political Economies, New York: University Press, S. 1-39.

Strohmeier, Klaus Peter (2002): Family Policy - How Does It Work?, in: Kaufmann, FranzXaver; Kuijsten, Anton; Schulze, Hans-Joachim; Strohmeier, Klaus Peter (Hrsg.): Family Live and Family Policies in Europe. Volume 2. Problems and Issues in Comparative Perspective, Oxford: Clarendon Press, S. 321-362.

Teubner, Markus (2002): Stieffamilientypen und haushaltsübergreifende Stiefkonstellationen, in: Bien, Walter; Hartl, Angela; Teubner, Markus (Hrsg.): Stieffamilien in Deutschland. Eltern und Kinder zwischen Normalität und Konflikt, DJI: Familien-Survey, Band 10, Opladen: Leske und Budrich, S. 51-82.

Tölke, Angelika (2005): Die Bedeutung von Herkunftsfamilie, Berufsbiografie und Partnerschaften für den Übergang zur Ehe und Vaterschaft, in Tölke, Angelika; Hank, Karsten (Hrsg.): Männer - Das "vernachlässigte” Geschlecht in der Familienforschung, in: Zeitschrift für Familienforschung, Sonderheft 4, S. 98-126. 
Tölke, Angelika; Hank, Karsten (2005b): Das ,vernachlässigte’ Geschlecht in der Familienforschung: Untersuchungen zu Partnerschaft und Elternschaft bei Männern, in: Tölke, Angelika; Hank, Karsten (Hrsg.): Männer - Das "vernachlässigte” Geschlecht in der Familienforschung, in: Zeitschrift für Familienforschung, Sonderheft 4, S. 7-17.

Tölke, Angelika; Hank, Karsten (2005a): Männer - Das ,vernachlässigte’ Geschlecht in der Familienforschung, Zeitschrift für Familienforschung, Sonderheft 4, Wiesbaden: VS Verlag für Sozialwissenschaften.

Vaskovics, Laszlo; Rupp, Nina; Hofmann, Barbara (1997): Lebensverläufe in der Moderne: nichteheliche Lebensgemeinschaften - eine Längsschnittstudie, Opladen: Leske und Budrich.

Vogel, Joachim (1999): Der europäische ,Welfare Mix’. Institutionelle Konfigurationen und Verteilungsergebnisse in der Europäischen Union und Schweden. Eine Längsschnittund vergleichende Perspektive, in: Flora, Peter; Noll, Heinz-Herbert Noll (Hrsg.): Sozialberichterstattung und Sozialstaatsbeobachtung. Individuelle Wohlfahrt und wohlfahrtsstaatliche Institutionen im Spiegel empirischer Analysen, Frankfurt am Main, New York: Campus Verlag, S. 73-110.

Walter, Heinz (2002b): Deutschsprachige Väterforschung - Sondierungen in einem weiten Terrain, in: Walter, Heinz (Hrsg.): Männer als Väter: Sozialwissenschaftliche Theorie und Empirie, Gießen: Psychosozial-Verlag, S. 13-78.

Walter, Heinz (Hrsg.) (2002a): Männer als Väter: sozialwissenschaftliche Theorie und Empirie, Gießen: Psychosozial-Verlag.

Weber, Max (1972) [1922]: Wirtschaft und Gesellschaft. Grundriss der verstehenden Soziologie, 5. Aufl., Tübingen: Mohr.

Weldon, Michelle (2006): The Parental Body in Law: An Examination of how the Working Parent is Conceptualized in UK Labour Law, eSharp Issue 8, im Internet unter: http://www.gla.ac.uk/media/media_41210_en.pdf (letzter Zugang 05.10.2007).

Wittenberg, Reinhard; Cramer, Hans (2003): Datenanalyse mit SPSS für Windows, 3. Auflage, Stuttgart: Lucius \& Lucius.

Yeandle, Sue (1999): Women, Men, and Non-Standard Employment: Breadwinning and Caregiving in Germany, Italy, and the UK, in: Crompton, Rosemary (Hrsg.): Restructuring Gender Relations and Employment. The Decline of the Male Breadwinner, Oxford: Oxford University Press, S. 80-104. 


\section{Lebenslauf}

Persönliche Daten

\section{Schulbildung}

Grundschule Ramsloh

Orientierungsstufe Schulzentrum Saterland

Albertus-Magnus-Gymnasium Friesoythe

Allgemeine Hochschulreife

\section{Carina Marten (geb. Siefken)}

geboren am 15.10.1977

in Oldenburg (Niedersachsen) verheiratet, keine Kinder Staatsangehörigkeit: deutsch

$1984-1988$

$1988-1990$

1990 - 1997

12.06.1997

ab WiSe 1998/99

05.10 .2000

09/2001-02/2002 la-Neuve, Belgien

Diplomarbeit: „Vatersein in Raten. Väterliches Sorgehandeln bei getrennt lebenden, unterhaltsberechtigten Kindern“

Abschluss als Diplom Sozialwissenschaftlerin mit den Prüfungsfächern Soziologie, Sozialpsychologie,

Betriebswirtschaftslehre und Strafrecht

Stipendium der Deutschen Forschungsgesellschaft im Graduiertenkolleg „Die Zukunft des Europäischen

Sozialmodells“, Georg-August-Universität Göttingen

\section{Wissenschaftliche Tätigkeit}

Studentische Hilfskraft am Soziologischen Seminar, GeorgAugust-Universität Göttingen

Studentische Hilfskraft am Methodenzentrum Sozialwissenschaften, Georg-August-Universität Göttingen

Wissenschaftliche Mitarbeiterin am Methodenzentrum Sozialwissenschaften, Georg-August-Universität Göttingen

Wissenschaftliche Hilfskraft am Institut für Soziologie, Georg-August-Universität Göttingen

Wissenschaftliche Mitarbeiterin bei Prof. Dr. Ilona Ostner, Institut für Soziologie, Abteilung II „Politische Soziologie und Sozialpolitik“, Georg-August-Universität Göttingen
SoSe 2000

WiSe 2000/01- SoSe 2001;

SoSe 2002 - WiSe 2003/04

01.06. - 30.09.2004

WiSe 2007/2008

seit März 2008 